\title{
USDA
}

\section{California's Forest Resources: Forest Inventory and Analysis, 2001-2010}
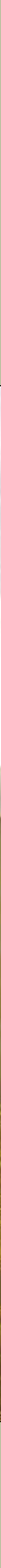
In accordance with Federal civil rights law and U.S. Department of Agriculture (USDA) civil rights regulations and policies, the USDA, its Agencies, offices, and employees, and institutions participating in or administering USDA programs are prohibited from discriminating based on race, color, national origin, religion, sex, gender identity (including gender expression), sexual orientation, disability, age, marital status, family/parental status, income derived from a public assistance program, political beliefs, or reprisal or retaliation for prior civil rights activity, in any program or activity conducted or funded by USDA (not all bases apply to all programs). Remedies and complaint filing deadlines vary by program or incident.

Persons with disabilities who require alternative means of communication for program information (e.g., Braille, large print, audiotape, American Sign Language, etc.) should contact the responsible Agency or USDA's TARGET Center at (202) 720-2600 (voice and TTY) or contact USDA through the Federal Relay Service at (800) 877-8339. Additionally, program information may be made available in languages other than English.

To file a program discrimination complaint, complete the USDA Program Discrimination Complaint Form, AD-3027, found online at http://www.ascr.usda.gov/complaint_filing_cust.html and at any USDA office or write a letter addressed to USDA and provide in the letter all of the information requested in the form. To request a copy of the complaint form, call (866) 632-9992. Submit your completed form or letter to USDA by: (1) mail: U.S. Department of Agriculture, Office of the Assistant Secretary for Civil Rights, 1400 Independence Avenue, SW, Washington, D.C. 20250-9410; (2) fax: (202) 690-7442; or (3) email: program.intake@usda.gov.

USDA is an equal opportunity provider, employer, and lender.

\section{Technical Editors}

Glenn A. Christensen is a forester, Karen L. Waddell is a forester, Sharon M. Stanton is an ecologist, and Olaf Kuegler is a mathematical statistician, U. S. Department of Agriculture, Forest Service, Pacific Northwest Research Station, Forestry Sciences Laboratory, 620 SW Main Street, Suite 400, Portland, OR 97205.

\section{Contributing Authors}

Joel Thompson is a forestry technician, John Chase is a geographic information system specialist, Jeremy Fried is a research forester, and Sarah Jovan is a research ecologist, Forestry Sciences Laboratory, 620 SW Main Street, Suite 400, Portland, OR 97205. Andrew Gray is a research ecologist, Forestry Sciences Laboratory, 3200 SW Jefferson Way, Corvallis, OR 97331.

Cover: Siskiyou Mountains at sunrise, with Preston Peak on the horizon (elevation 7,313 feet, the highest point in this range). Photo by Gerad Dean. 


\section{California's Forest Resources: Forest Inventory and Analysis, 2001-2010}

Glenn A. Christensen,

Karen L. Waddell,

Sharon M. Stanton,

Olaf Kuegler,

Technical Editors

U.S. Department of Agriculture

Forest Service

Pacific Northwest Research Station

Portland, Oregon

General Technical Report PNW-GTR-913

February 2016 



\section{Abstract}

Christensen, Glenn A.; Waddell, Karen L.; Stanton, Sharon M.; Kuegler, Olaf, tech.

eds. 2015. California's forest resources: Forest Inventory and Analysis, 2001-2010.

Gen. Tech. Rep. PNW-GTR-913. Portland, OR: U.S. Department of Agriculture, Forest

Service, Pacific Northwest Research Station. 293 p.

This report highlights key findings from the most recent (2001-2010) data collected by the Forest Inventory and Analysis program across all forest land in California, updating previously published findings from data collected from 2001 through 2005 (Christensen et al. 2008). We summarize and interpret basic resource information such as forest area, ownership, volume, biomass, and carbon stocks; forest structure and function topics such as biodiversity, forest age, and dead wood; disturbance information such as tree damage, fire, and invasive plants; and tree growth, mortality, and removals for timber products. Consistent sampling across different ownerships and forest management regimes now allow comparisons. The appendices provide details on inventory methods and design and include summary tables of data, with statistical error, for the suite of forest characteristics inventoried.

Keywords: Biomass, carbon, dead wood, fire, forest land, insects, invasive plants, inventory, timber volume, timberland, California. 


\section{Contents}

1 What Is the Purpose of This Report?

2 What Is Forest Inventory and Analysis?

2 Why Are California's Forests Important?

5 Where Are California's Forests?

8 What Types of Forest Grow in California?

11 How Much Volume, Biomass, and Carbon Are Stored in California's Forests?

16 How Old Are the Trees in California?

16 Who Owns California's Forests?

23 National Woodland Owner Survey

24 How Much of California's Forest Land Is Available for Harvest?

27 Annual Tree Growth, Removals, and Mortality in California ${ }^{2}$

28 Privately Owned Timberland: Change Between 1991-1994 and 2007-2010

29 State And Local Government Timberland: Change Between 1991-1994 and 2007-2010

29 National Forest System (NFS) Forest Land: Change Between 2001-2006 and 2006-2010

33 How Many Trees Show Signs of Damaging Agents?

35 What Types of Understory Vegetation Are Most Common in California's Forests?

37 What Nonnative Invasive Plants Are Found in California's Forests?

41 How Has Fire Influenced California's Forests?

43 How Productive Are California's Forests?

43 California's Timberlands

46 Nontimber Forest Product Species

46 What Is the Extent of Dead Wood in California's Forests?

50 Conclusion

51 Common and Scientific Plant Names

56 Acknowledgments

56 Metric and English Equivalents

57 Literature Cited

59 Glossary

67 Appendix 1-Inventory Design and Methods

73 Appendix 2-Summary Data Tables 


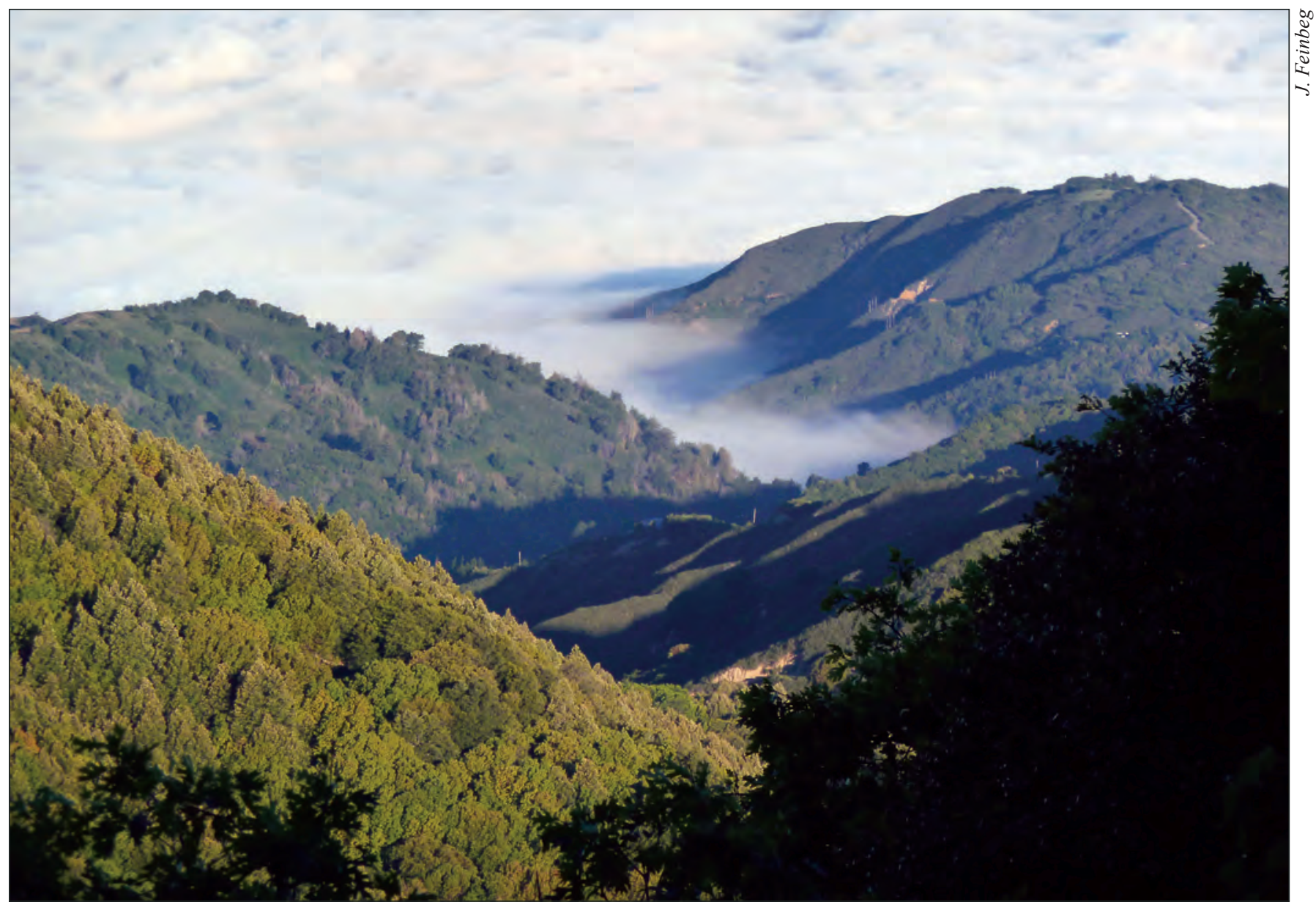

Monterey County, California.

\section{What Is the Purpose of This Report?}

This report presents a summary of California's forest resources across all ownerships and forest types. It highlights key forest characteristics, summarized from inventory field plots sampled across California over a period of 10 years (2001 through 2010). Basic resource information includes forest area, ownership, land use, biomass, biodiversity, dead wood, and forest health issues.

Pacific Northwest Forest Inventory and Analysis (PNW-FIA) implemented an annual sampling strategy in California in 2001, measuring a systematic sample each year that represents 10 percent of all plots in the state.
This report updates the previous California's Forest Resources, 2001-2005: Five-Year Forest Inventory and Analysis Report (Christensen et al. 2008), summarizing and averaging the most recent inventory information that includes 100 percent of all FIA plots in the state. Therefore, these data are not independent from those published in the earlier report, but are a part of the same 10-year inventory estimate and should be treated as one full cycle of data. Statistical estimates of change in overall tree growth, removals, and mortality are provided in this report. 


\section{What Is Forest Inventory and Analysis?}

The FIA program was created within the U.S. Department of Agriculture, Forest Service in 1928 to conduct unbiased assessments of all the Nation's forested lands for use in economic and forest management planning. The program was charged with collecting forest data on a series of permanent field plots, compiling and making data available, and providing research and interpretations from those data. Four FIA units are responsible for inventories of all forested lands in the continental United States, Alaska, Hawaii, Puerto Rico, Guam, American Samoa, Palau, the U.S. Virgin Islands, and Pacific Island groups including the Marshall Islands, Federated States of Micronesia, and Northern Mariana Islands.

Starting in 2000, as required by the Agricultural Research, Extension, and Education Reform Act of 1998 (the Farm Bill), FIA implemented a new standardized national inventory method in which a portion of all plots in each state were measured each year. Appendix 1 explains the differences between the previous and current inventory methods. The effect of the change is that, for the first time in 70 years, all FIA units are using a common plot design, a common set of measurement protocols, and a standard database design for compilation and distribution of data. Under this unified approach, FIA now provides unbiased estimates of a wide variety of forest conditions over all forested lands in the United States in a consistent and timely manner.

This report covers all forested lands in California, with estimates representing averaged values for all inventory plots visited by field crews between 2001 and 2010 (figs. 1 and 2). The base set of field plots is spaced at about 3-mile intervals on a hexagonal grid, spanning both publicly and privately owned forests, and including lands reserved from industrial wood production (e.g., national parks, wilderness areas, and natural areas). The annual inventory involves a cycle of measurements for 10 systematic subsamples, each representing about 10 percent of the approximately 6,000 forest land plots in California (figs. 3 and 4). Additional information about annual inventories is available in appendix 1 of this report and at http://fia.fs.fed.us/.

\section{Why Are California's Forests Important?}

Californians derive a variety of economic, ecological, and social benefits from the state's forests. Historically, the state's economy was based largely upon natural resources, and today forests and timber still play a key role in California's economy and culture. Since statehood, California has ranked as high as second for softwood lumber production in the Nation, along with Oregon and Washington (Morgan et al. 2012). The industry has gone through significant changes such as private forests shifting from old-growth to second-growth forest management, and a decline in harvests from federal timberlands by as much as 75 percent during the 1990s. Currently, the industry is experiencing a rise in wood harvested as biomass for energy production. Biomass power plants generate electricity by burning wood waste from sawmills and logging operations. California's forests also provide many nontimber forest products (NTFPs) and financial benefits to the state from ecosystem services such as the regulation of water quality and quantity, carbon sequestration, and soil creation and retention.

Ecological benefits of the structure and function of forested ecosystems include providing habitat for plants and animals such as fish by filtering runoff to streams, providing shade that helps control water temperatures, and contributing to the food web. Forested ecosystems also increase water absorption and retention of soils, and complete nutrient cycling.

There are also less tangible social benefits that can be more difficult to quantify. Forests are often the location for recreational, cultural, and spiritual activities. California is home to some of the Nation's most treasured national forests and internationally known national parks including Yosemite, Redwood, and Sequoia and Kings Canyon (fig. 5). In 2013, the state's 26 national parks were visited by over 35 million people and contributed over $\$ 1.5$ billion in economic benefit from tourism in 2012 (NPS 2014). West coast culture values the natural world, and people living there tend to have strong cultural and spiritual values concerning forested communities. Many of those living in the Western States consider outdoor recreation an important part of their lifestyle and enjoy significantly greater access to publically managed forests than in other regions of the United States. 


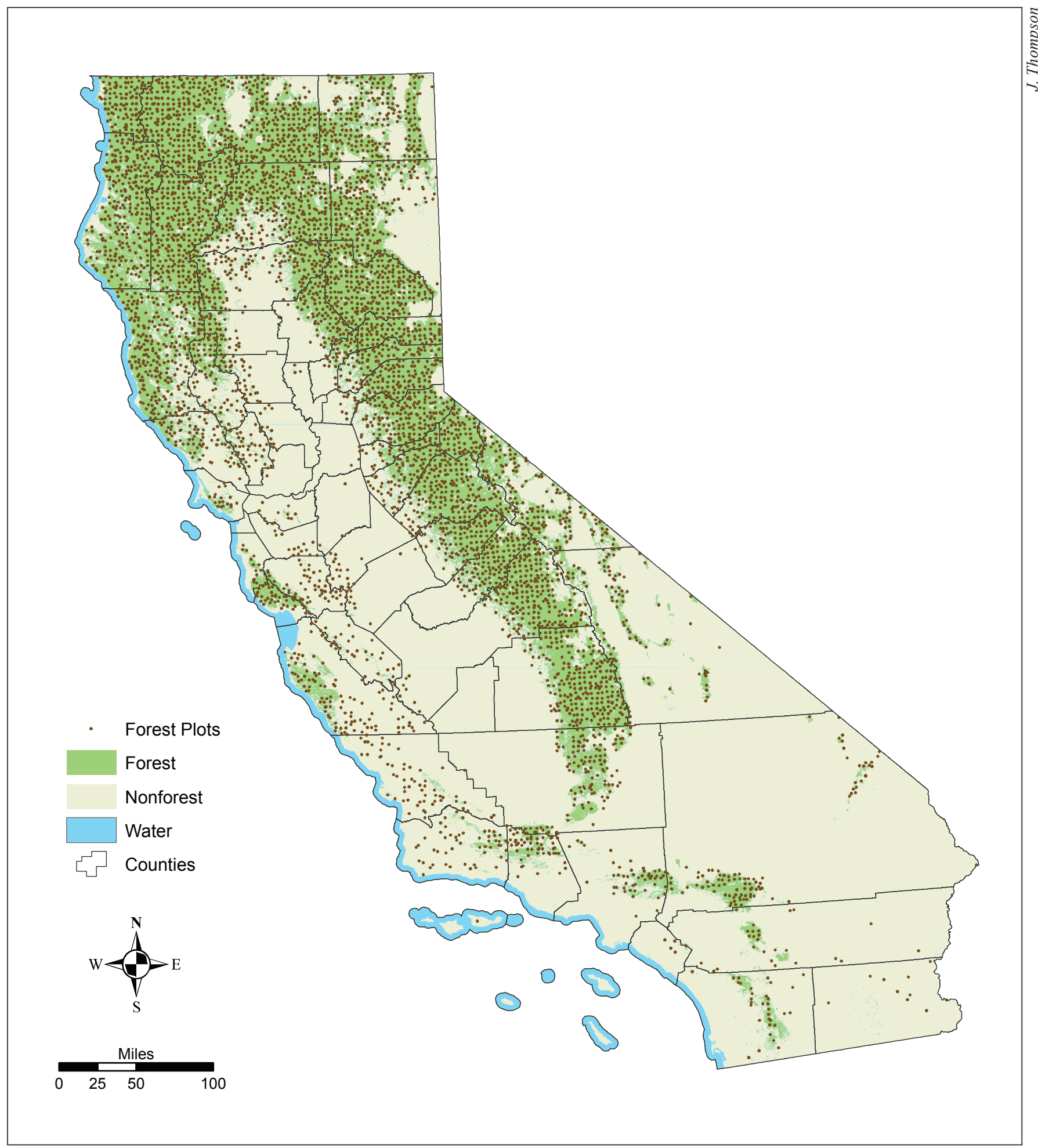

Figure 1-Forested plots measured between 2001 and 2010 provide the data used in this report. Locations are approximate (forest/ nonforest geographic information system layer: Blackard et al. 2008). 


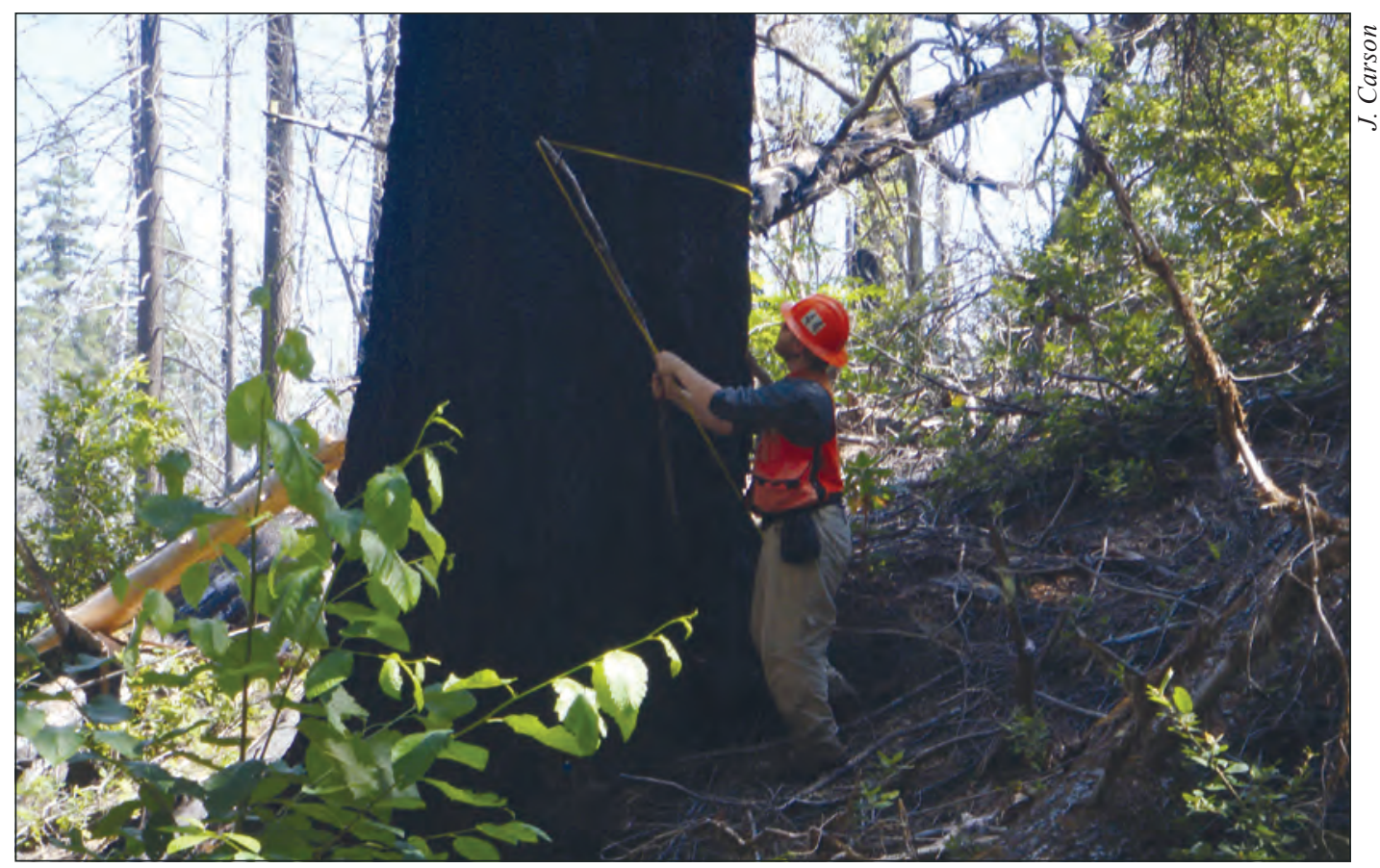

Figure 2-Forest Inventory and Analysis field crews take a wide variety of measurements on each plot visited.

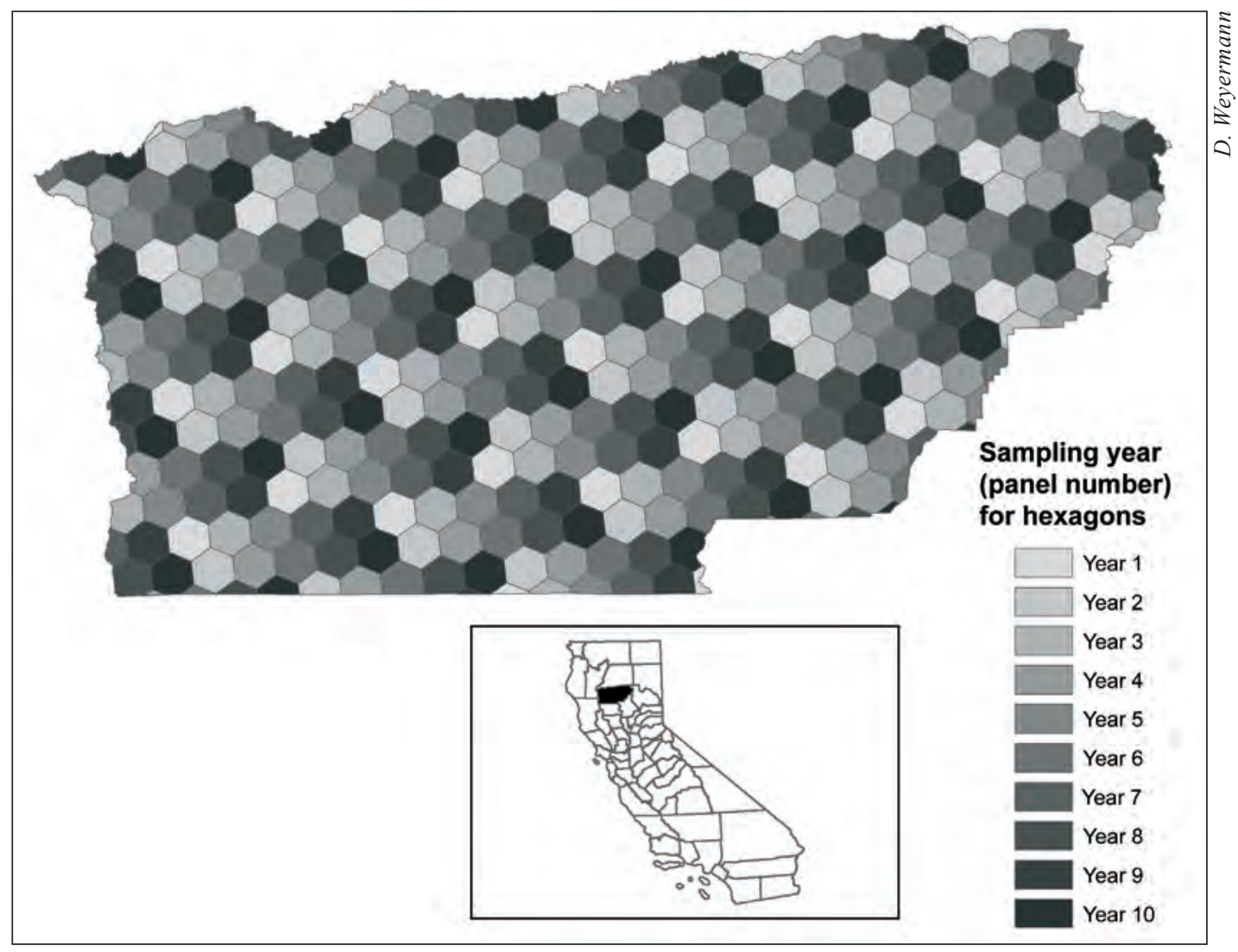

Figure 3-Example of the hexagonal grid that serves as the foundation of field plot assignment, with the cells shaded by which one of the 10 annual measurement panels it belongs to, magnified for Tehama County, California. All plots in one panel are sampled in 1 year. 


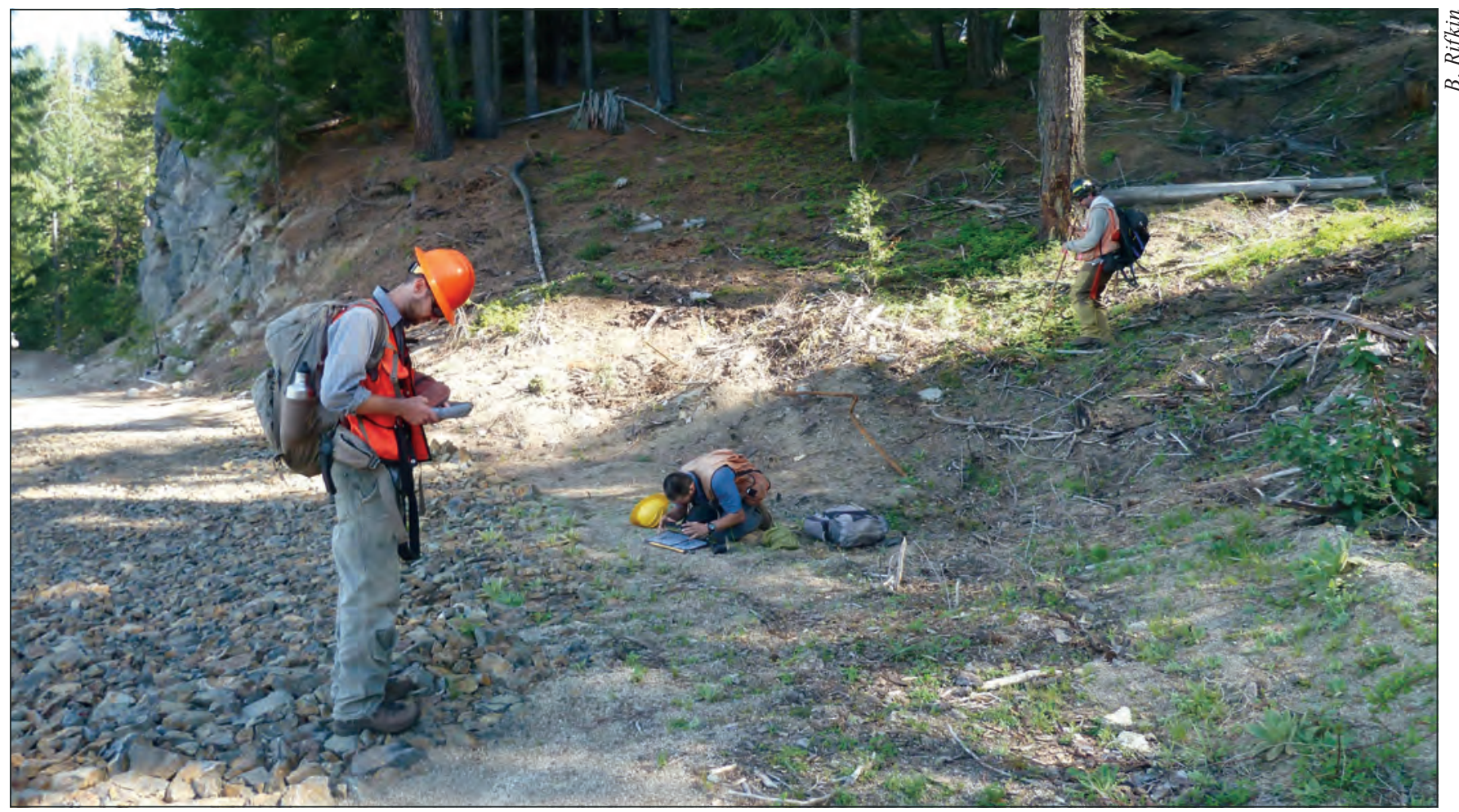

Figure 4-Forest Inventory and Analysis field crews using equipment to accurately determine location of a plot for measurement.

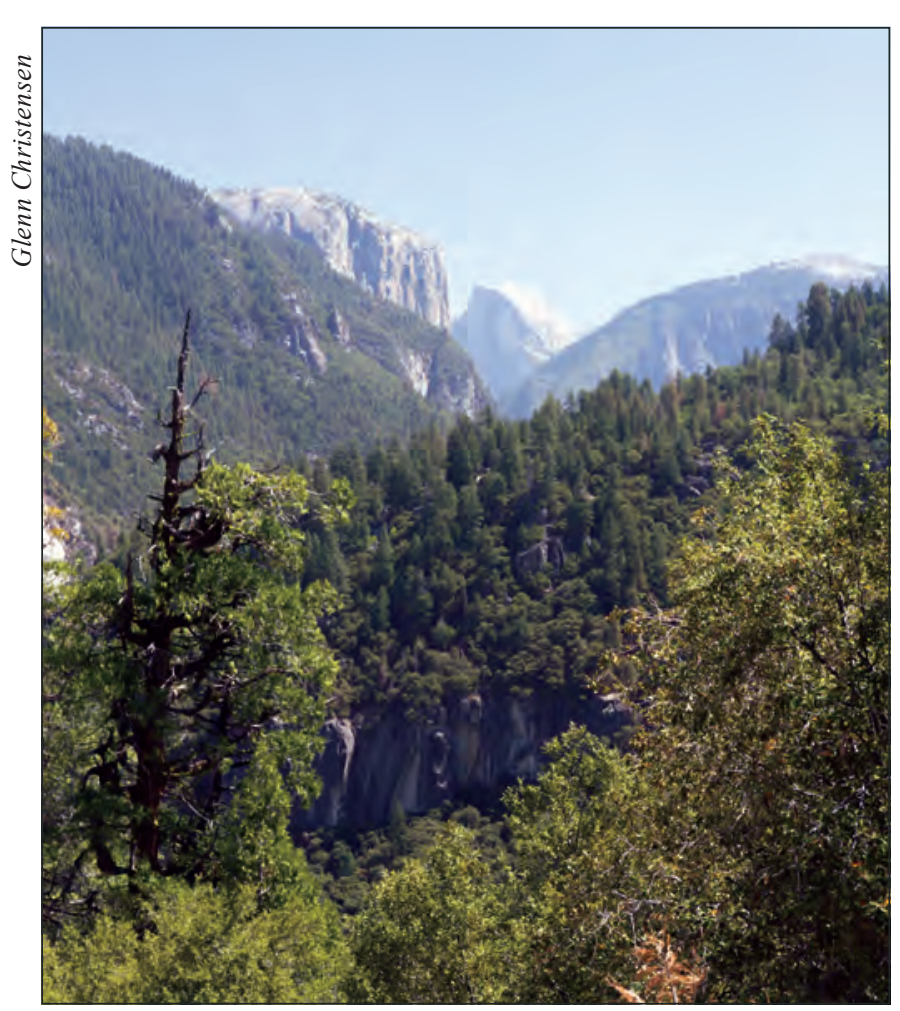

Figure 5-Yosemite National Park is just one of 26 national parks enjoyed by millions of visitors annually throughout the state.

\section{Where Are California's Forests?}

Almost a third of California is forested. The total land area is about 100 million acres, with 33 million forested acres.

Forest land is defined as land that is at least 10 percent stocked by forest trees of any size, or land formerly having such tree cover and not currently developed for a nonforest use (see "Glossary" for a detailed definition). Most of the forests are found in the mountainous areas such as the Klamath, Sierra Nevada, and Coast ranges, and in the cool, mesic fog belt along the state's north and central coasts (fig. 6). The Klamath Mountains ecosection has the highest percentage of forested land in the state ( 90 percent), followed by the Southern Cascades and Sierra Nevada ecosections, each being about 75 percent forested (figs. 7 and 8).

By county, Trinity has the most forest. Just over 90 percent of this county is forested. Alpine, Plumas, and Sierra Counties also rank highly, with over 80 percent of their land area covered by forest. 


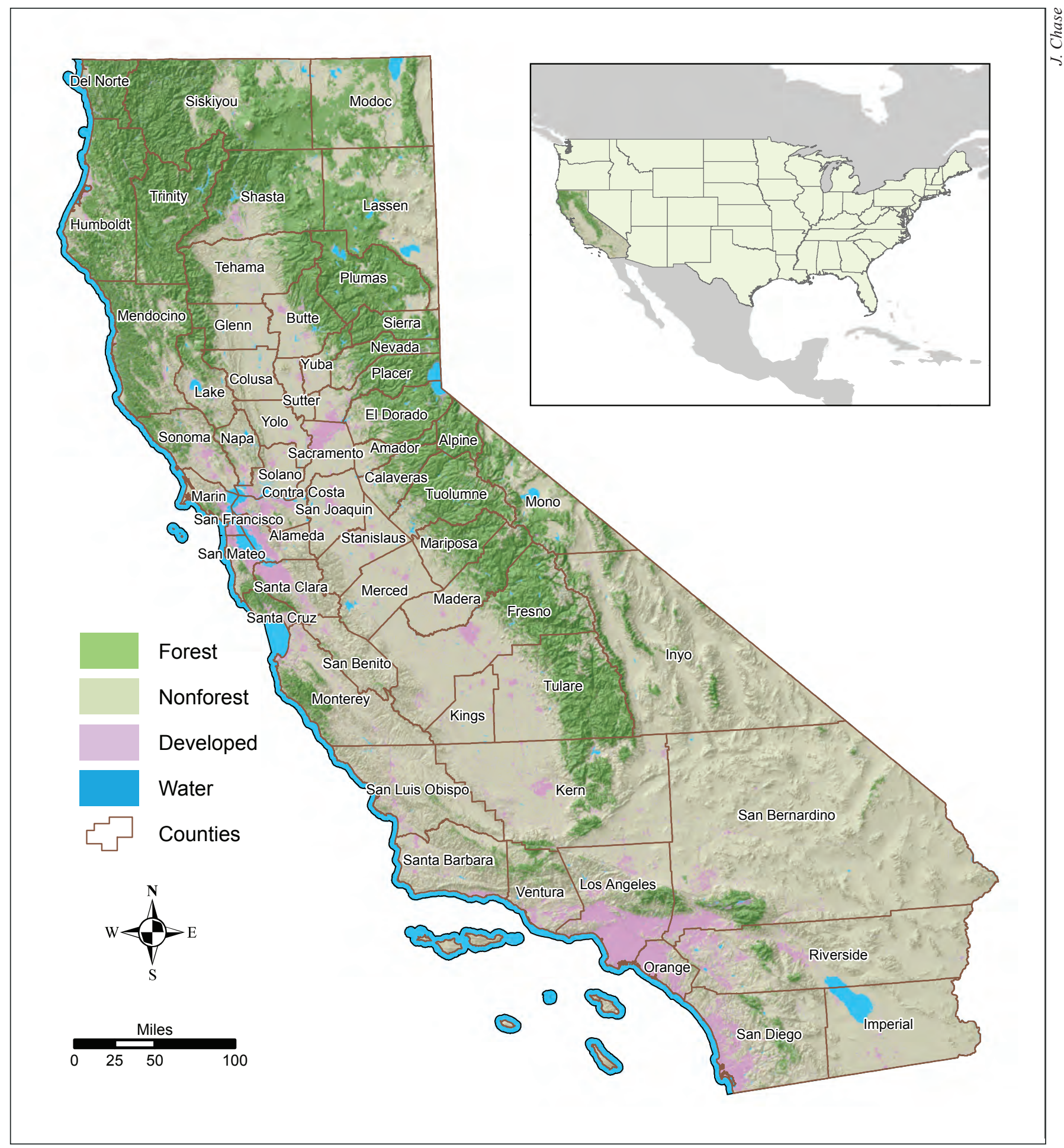

Figure 6-California land cover and counties (forest/nonforest geographic information system layer: Blackard et al. 2008; landcover layer: U.S. Geological Survey (USGS) Multi-Resolution Land Characteristics Consortium National Land Cover Dataset, Homer et al. 2007; relief layer: USGS National Elevation Dataset, Gesch et al. 2002). 


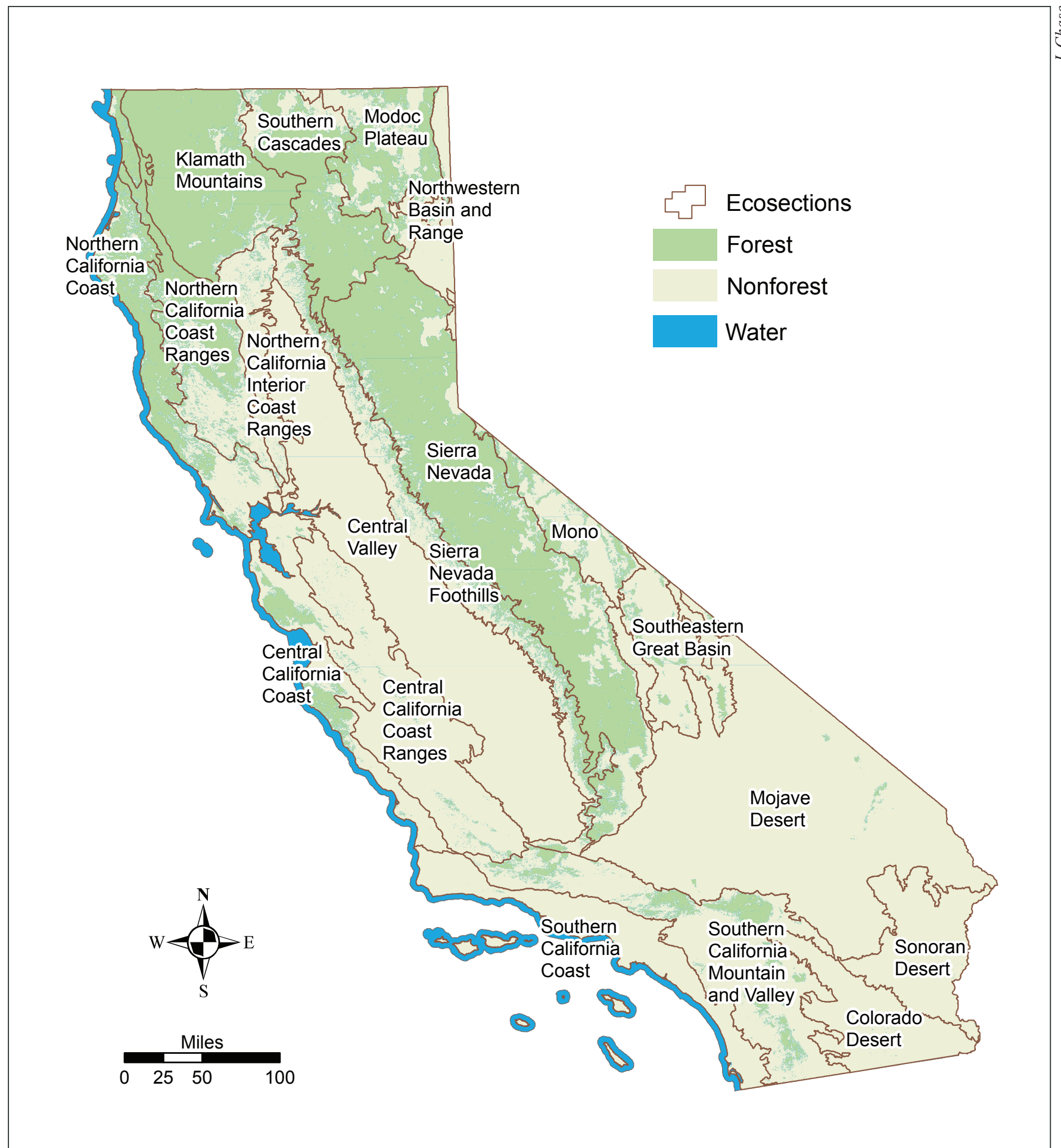

Figure 7-California ecosections (ecosection geographic information system [GIS] layer: McNab et al. 2005; forest/nonforest GIS layer: Blackard et al. 2008). 


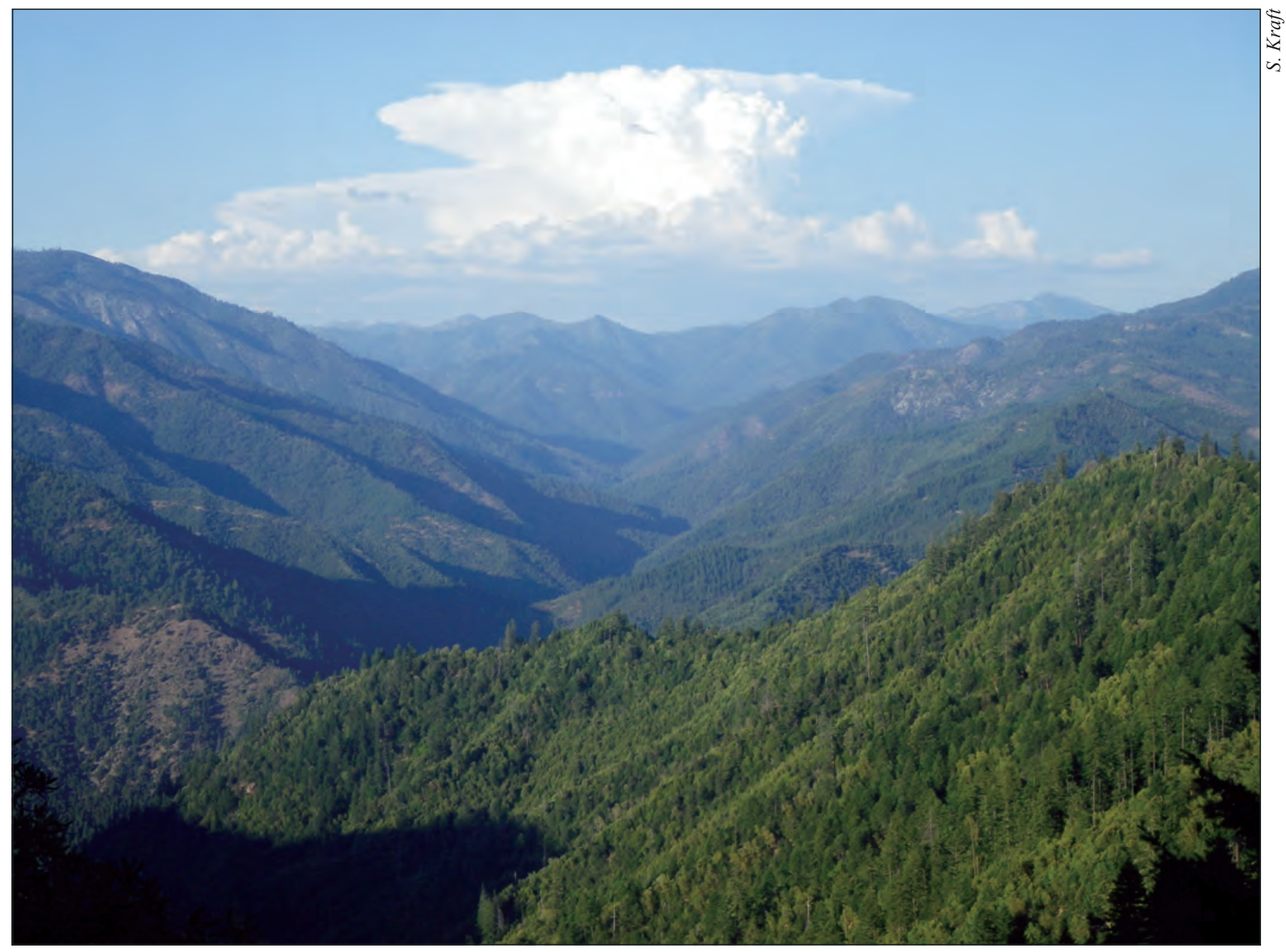

Figure 8-Counties in the northern part of the state have the majority of forested area such as Siskiyou County as shown here.

\section{What Types of Forest Grow in California?}

The environment and vegetation along the Pacific coast are among the most diverse in North America, and California's distinct combinations of climate, elevation, and soil types create a unique mix of ecoregions and forest types. Forest Inventory and Analysis field crews identified 86 tree species in California. About 58 percent of California's forests are dominated by coniferous forest types - predominantly the California mixed-conifer group covering 7.8 million acres (24 percent of all forested land area), ponderosa pine covering 2.2 million acres ( 7 percent), and other western softwoods groups covering about 2 million acres (6 percent) (fig. 9). A forest type group is a combination of forest types that share closely associated species and similar productivity characteristics. The mixed-conifer group may be composed of several western conifers including Douglas-fir, ponderosa pine, sugar pine, Jeffrey pine, incense cedar, white fir, red fir, and other true fir species (see "Common and Scientific Plant Names").

Hardwood forest types cover an additional 13 million acres (40 percent of forested land area). The major hardwood forest types are western oak (9.5 million acres), tanoak/laurel ( 2 million acres), and other hardwoods (608,000 acres) (figs. 10 and 11). The remaining 2 percent of forested land is classified as nonstocked (less than 10 percent stocked by trees, see "Glossary" for detailed definition). 


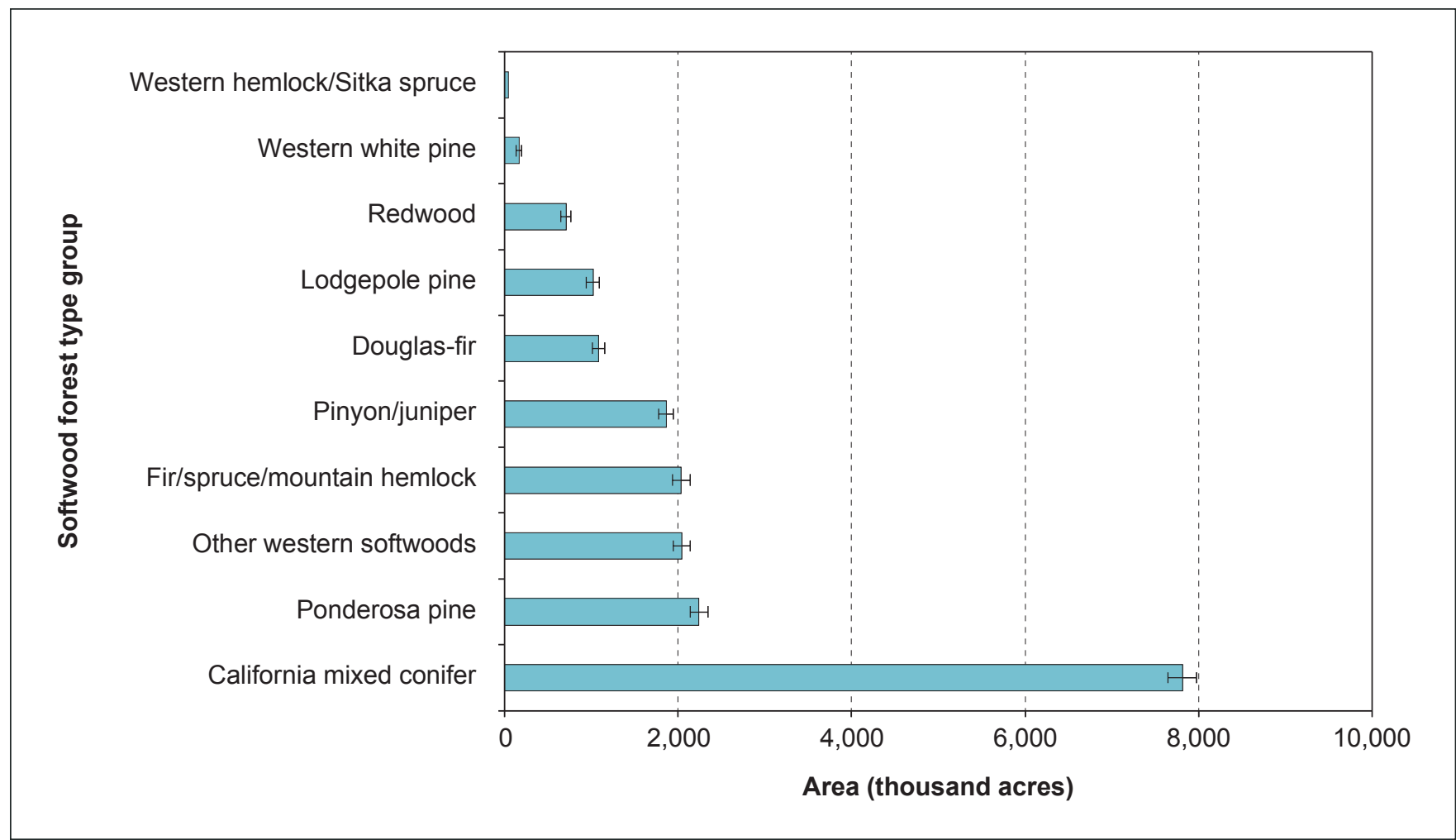

Figure 9-Area of softwood forest type groups on forest land in California, 2001-2010.

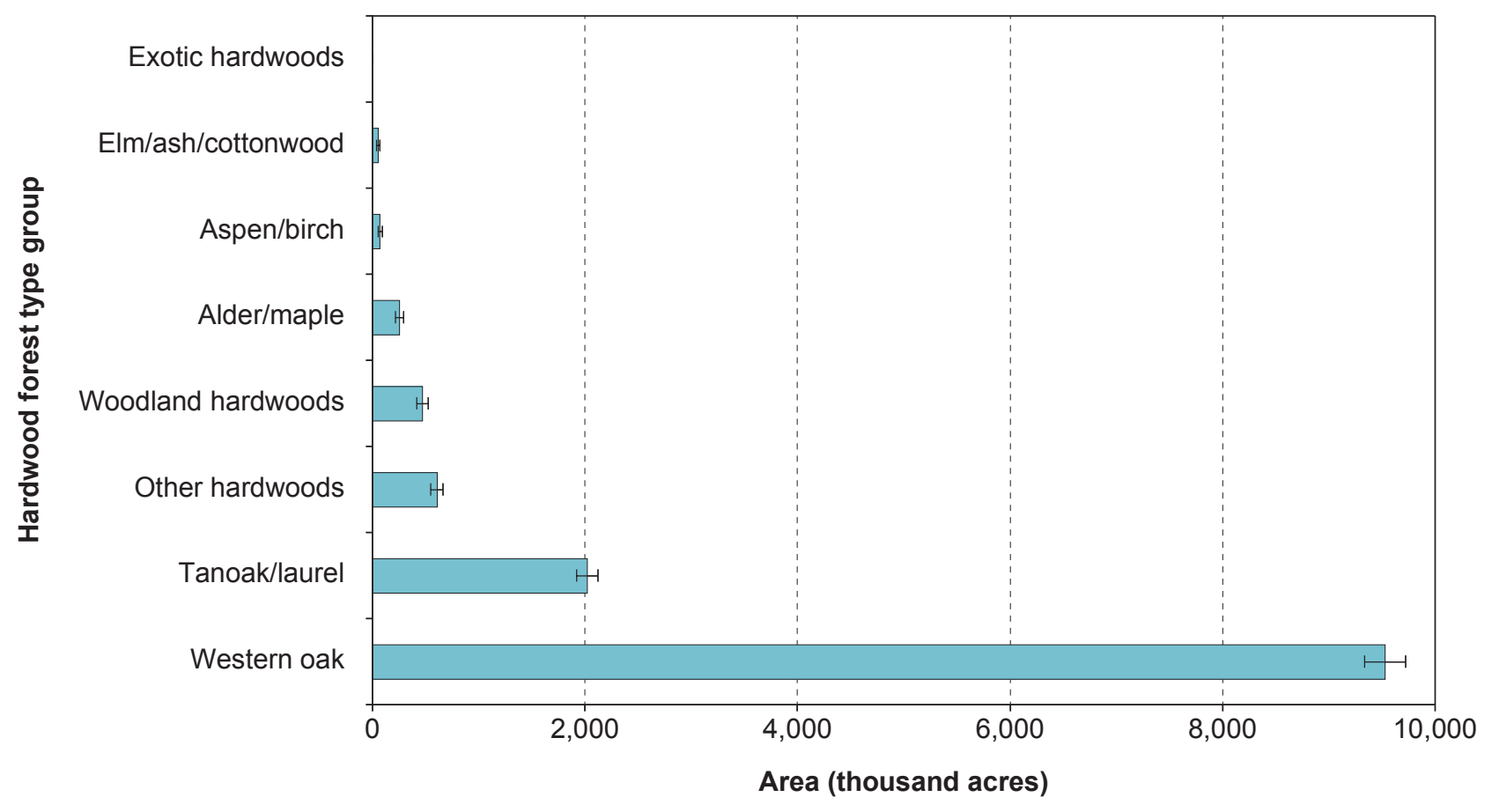

Figure 10-Area of hardwood forest type groups on forest land in California, 2001-2010. 


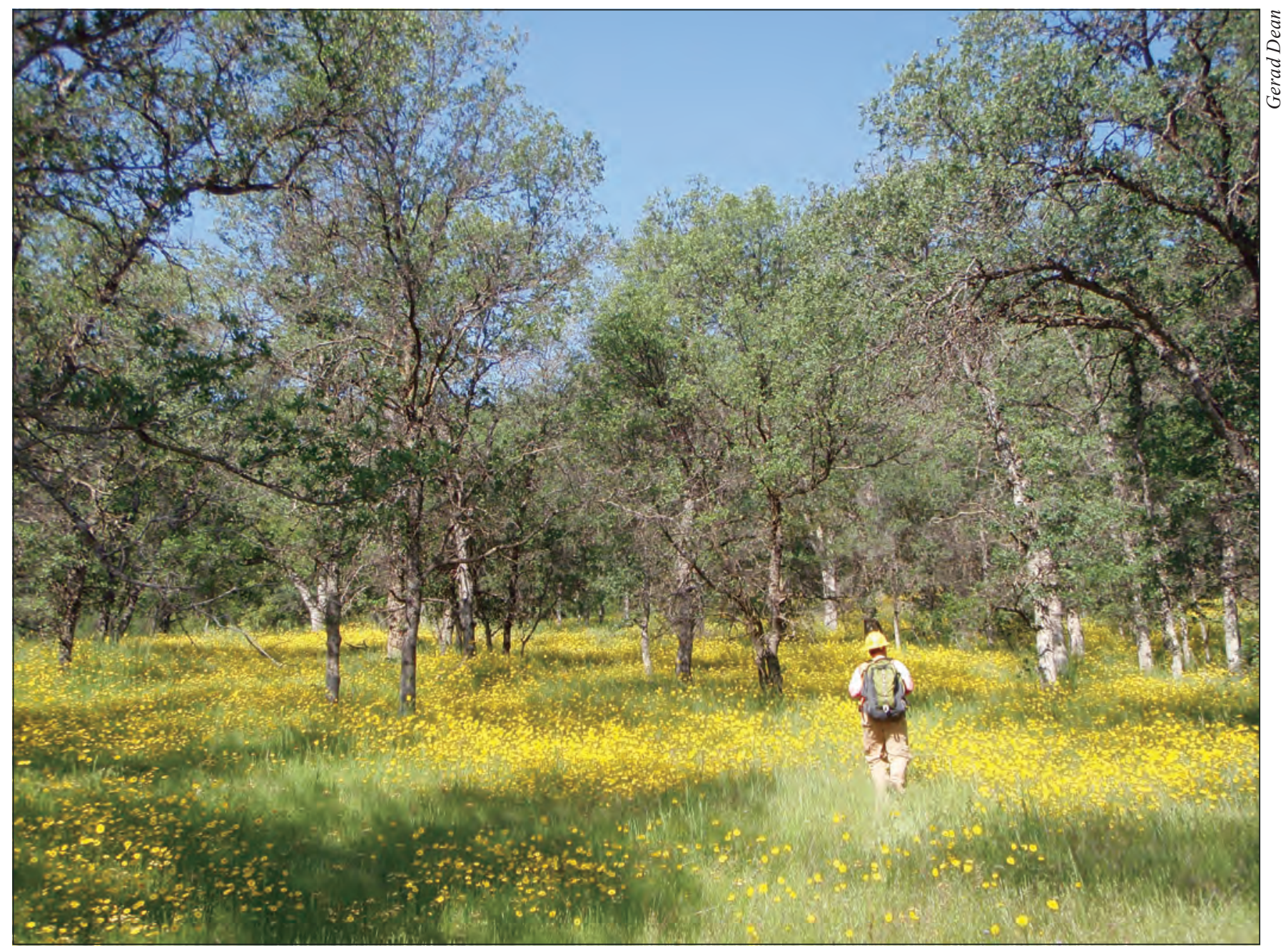

Figure 11-An example of oak woodland forest type found in Fresno County. 


\section{How Much Volume, Biomass, and Carbon Are Stored in California's Forests?}

The volume of wood in California's forests is an indicator of forest productivity, vigor, and structure. Biomass, carbon storage, and capacity for production of wood products can all be estimated from current volume. California has about 100 billion net cubic feet (465 billion Scribner board feet) of wood volume on forest land.

The greatest proportion of wood volume is found in three softwood tree species groups-Douglas-fir, true firs, and pines - which collectively make up 65 percent of all live-tree volume on California forest land; the remaining 35 percent is in softwood species such as redwood and incense cedar, or hardwood species such as oaks and tanoaks. The majority (55 percent) of live-tree volume is on Forest Service land, followed by individual private owners (18 percent) and corporate (14 percent) owners (figs. 12 and 13). State and federal forest land tends to have more volume per acre, on average, than privately owned forest land. By diameter class, state and federal forests tend to accumulate a greater proportion of total volume in larger diameter classes while private ownerships tend to concentrate relatively more volume in smaller diameter classes (fig. 14).
It is important to note that estimates of volume in standing dead trees are derived from gross cubic foot volume which, by definition and because it is calculated from total, not actual, height, includes rotten and form defect and missing bole volume. Missing volume in dead trees can be considerable. For example, for a previously harvested or broken topped tree for which a stump or snag remnant taller than 6 feet remains, the volume calculation uses estimated total height of an unharvested, unbroken tree. The resulting bias in dead tree volume estimates precludes meaningful comparison of standing dead and live tree volume, as live tree calculations use net volume which omits cull and missing portions of tree boles.

Forest biomass and carbon accumulate in live trees, snags, and down wood in a complex pattern across $\mathrm{Cal}-$ ifornia (fig. 15). Biomass estimates from comprehensive forest inventories are essential for quantifying the amount and distribution of carbon stocks, evaluating sources of sustainable fuel, and tracking net primary productivity. Total estimated biomass in live trees and dead wood across California is 2.5 billion tons. Forest biomass represents the aboveground woody biomass of trees in tons per acre. This biomass is the accumulated oven-dry weight of the stem, bark, stump, top, and live woody branches. The biomass of

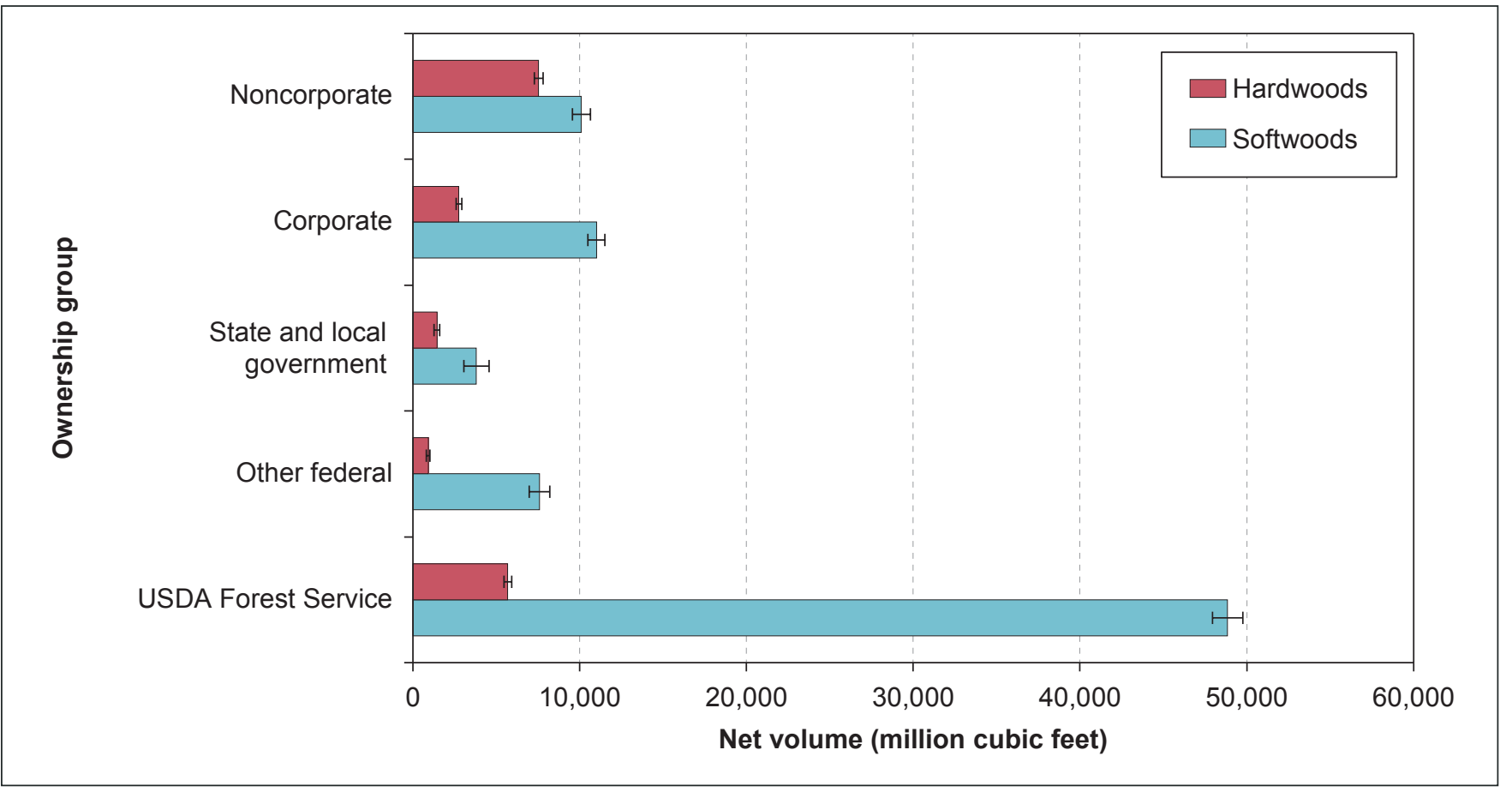

Figure 12-Net cubic volume of all live trees by ownership group on forest land in California, 2001-2010. 


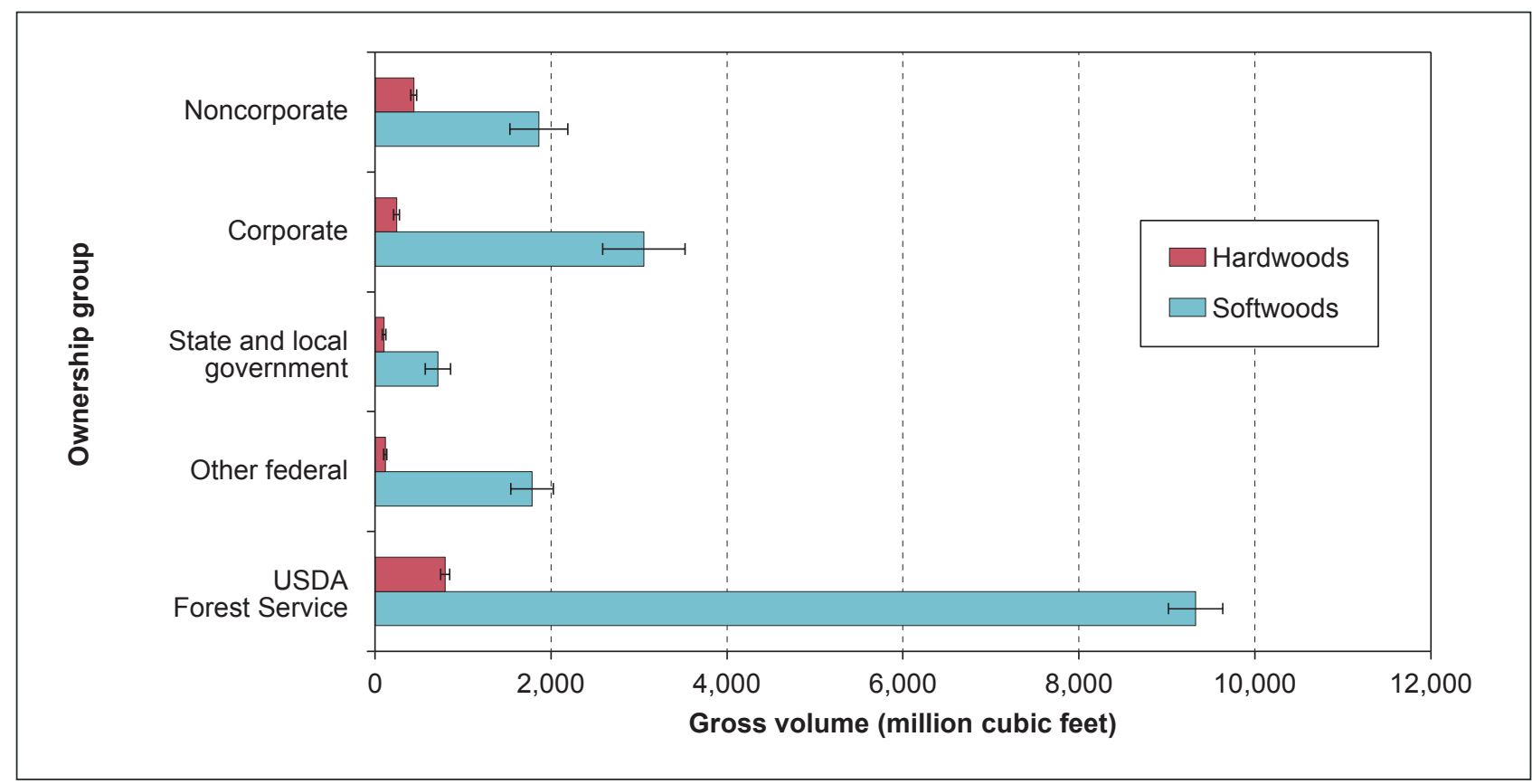

Figure 13-Gross cubic volume of all dead trees by ownership group on forest land in California, 2001-2010.

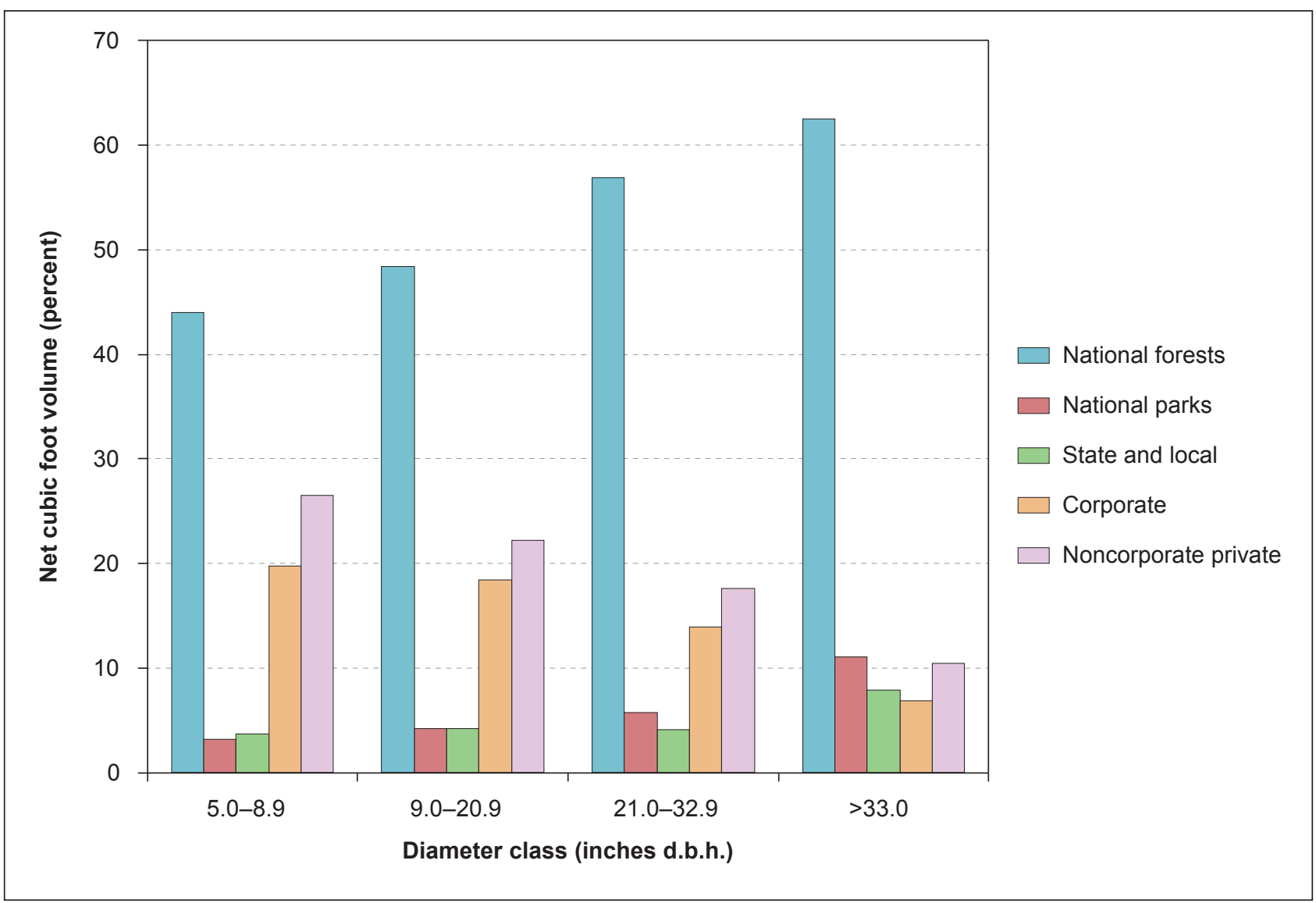

Figure 14-Percentage of volume by diameter class and ownership group on forest land in California, 2001-2010. 
foliage, cones, fruits, and roots was not calculated and is not included in this estimate of aboveground biomass.

There is nearly 10 times as much biomass in live trees compared to dead trees. Over 2 billion tons of biomass and about 1 billion megagrams of carbon have accumulated in live trees $\geq 1$ inch diameter at breast height (d.b.h.), primarily on unreserved forest land (fig. 16). The majority of this biomass (53 percent) is on land managed by the Forest Service. Reserved forest land, such as wilderness areas and national parks, contains about 22 percent of California's live-tree biomass. Statewide, softwood forest types have more than double the amount of biomass and carbon as hardwood types.

Oaks are the most abundant tree species in California; however, Douglas-fir contributes the most to total live-tree biomass and carbon storage. The more than 461 million tons of Douglas-fir biomass represent about 209 million megagrams of carbon sequestered in live trees. Live biomass is heavily concentrated in large softwood trees $(>21$ inches d.b.h.). In contrast, most of the biomass in hardwood species is contained in smaller trees (5 to 17 inches d.b.h.).

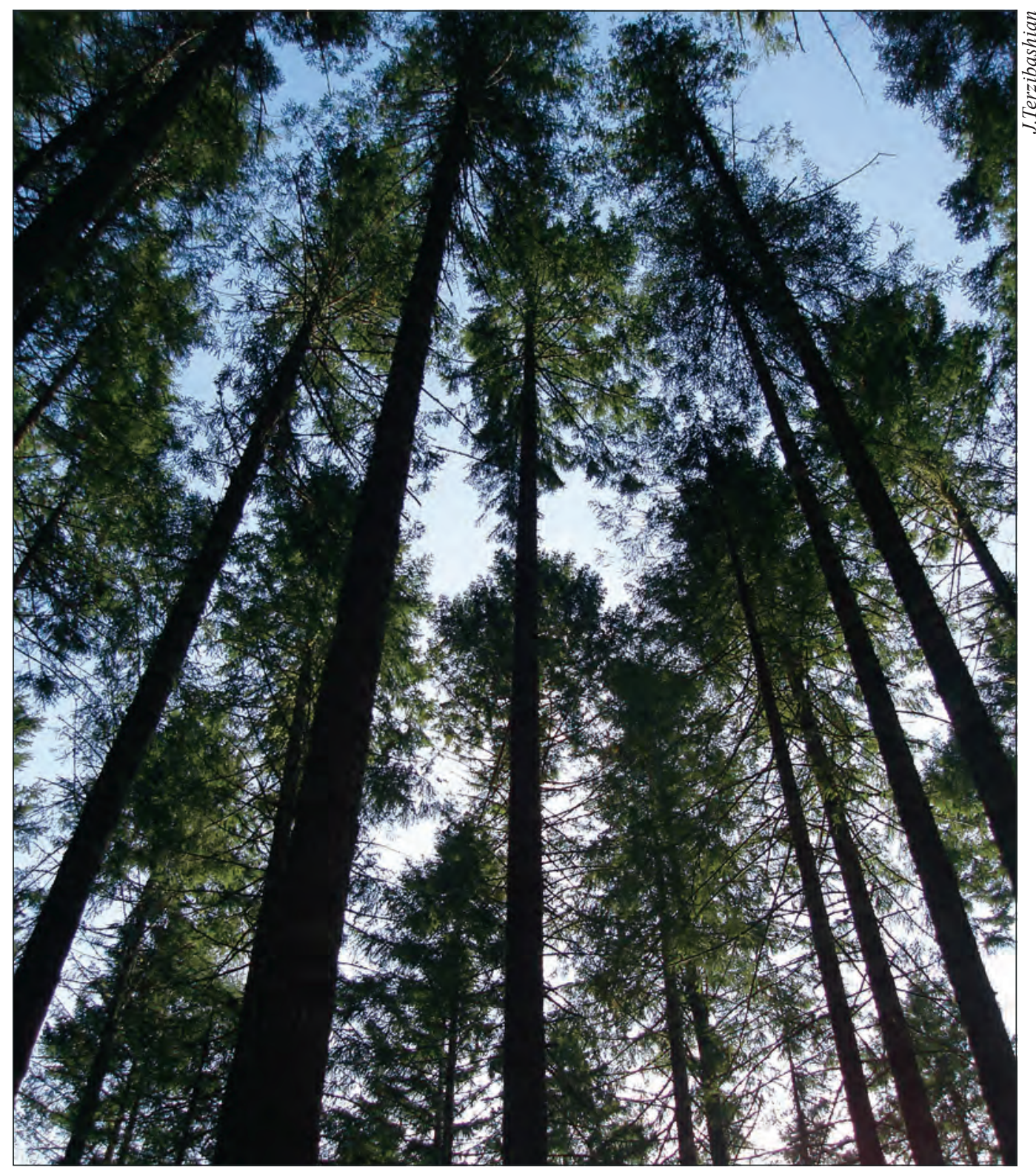

Figure 15-Biomass and carbon accumulate in standing live trees as well as overall contributions from dead trees still standing or fallen to the forest floor. 


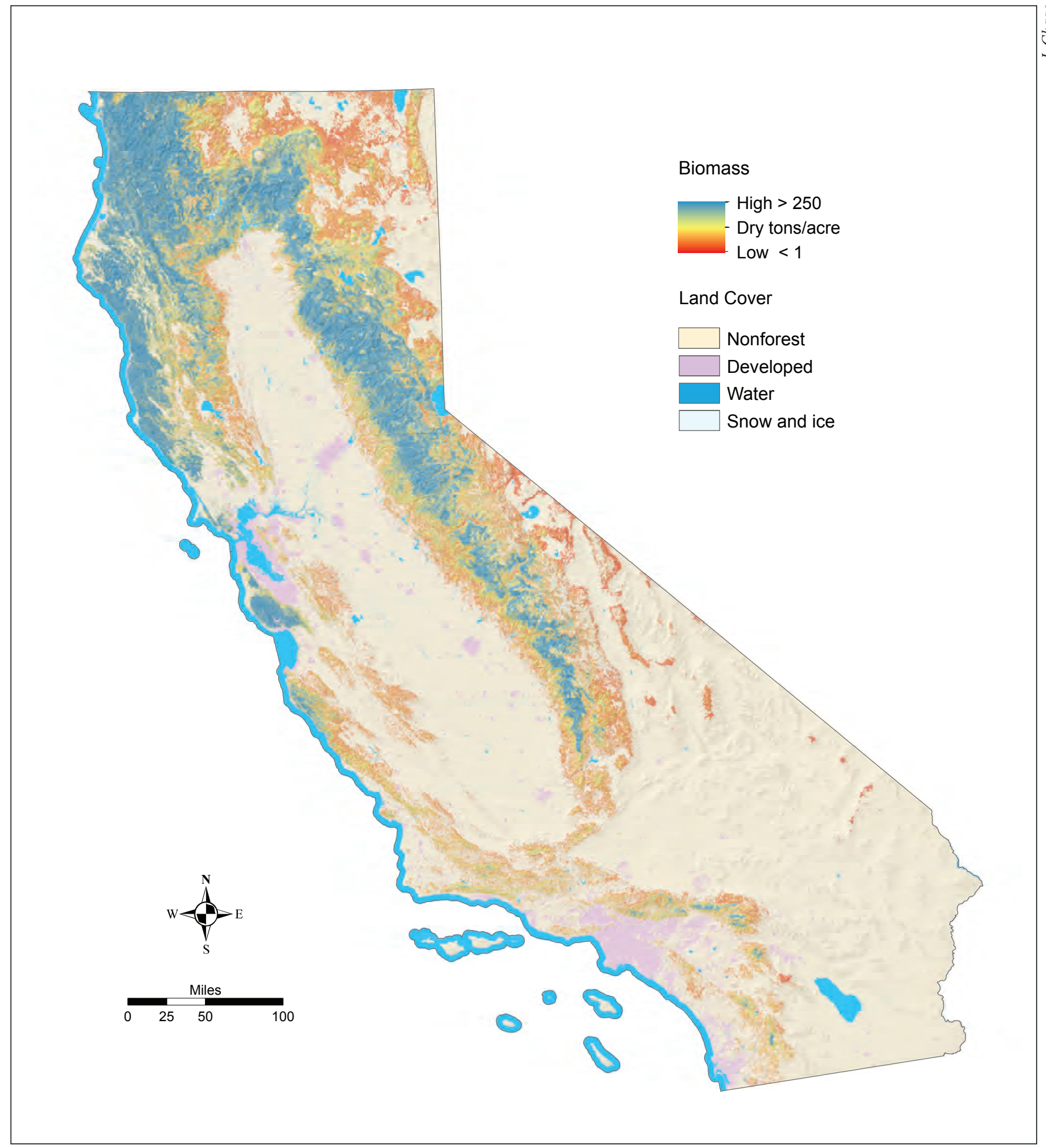

Figure 16-Estimated live-tree biomass (dry/tons per acre) in California, 2001-2010 (forest/nonforest geographic information system layer: Blackard et al. 2008; landcover layer: U.S. Geological Survey (USGS) Multi-Resolution Land Characteristiccs Consortium National Land Cover Dataset, Homer et al. 2007; relief layer: USGS National Elevation Dataset, Gesch et al. 2002). Forest biomass represents the aboveground woody biomass of trees in tons per acre. This biomass is the accumulated oven-dry weight of the stem, bark, stump, top, and live woody branches. The biomass of foliage, cones, fruits, and roots was not calculated and is not included in this estimate of aboveground biomass. 


\section{Research Application-Accurate Forest Carbon Standards Prove Elusive ${ }^{\mathbf{1}}$}

Recent interest in obtaining accurate estimates of forest carbon stocks and stock change has been intense, driven in large part by forest carbon offset markets and those who trade in them. Measuring forest carbon flux as assessed by change in carbon stocks, relative to a baseline or "business as usual" scenario, is an oft-sought metric but is not yet standardized. For tracking flux at broad (state and regional) scales and for generating baseline reference values, comprehensive forest inventories like Forest Inventory and Analysis that are based on remeasured, permanent sample plots have the potential to provide the most accurate estimates. However, converting these measurements into carbon stock and flux introduces several distinct types of uncertainty: measurement, sampling, model, and model-selection error. Rigorous quality control can minimize measurement error, and large samples are the antidote to sampling error. Model error, the uncertainty not explained by the variables included in a model, can be accounted for when model creators provide error information, but model-selection error, which arises from the availability of multiple potentially suitable models, can introduce substantial bias (inaccuracy) and is not easily resolved.

Model-selection error arises from the need to estimate aboveground carbon stores for each tree by selecting from among what, for a common commercially important species like Douglas-fir, can be thousands of alternative computation pathways. Under the most optimistic scenario of a sensitivity analysis of live-tree carbon stores in northwest Oregon, model-selection error, defined as half the prediction range (56 to 119 teragrams of carbon [Tg C]) expressed as a percentage of the prediction envelope midpoint, was 37 percent (Melson et al. 2011). When models are applied to tree measurements (e.g., height and diameter) that lie outside the range of data from which models were developed, which is all but unavoidable when seeking estimates for large trees or less common species, model-selection error can be far greater. Given that sampling error for the northwest Oregon analysis implied a 95 percent confidence interval of 6 percent, and that measurement error is consistently far smaller, model selection accounts for most of the uncertainty in live-tree carbon stores.

Despite these limitations, forest carbon estimates can be extremely helpful to understanding forest carbon dynamics and the net climate implications of forest management, disturbance, and utilization, for example. However, the accuracy and applicability of the carbon models available today are difficult to establish and, as such, these models are limited as tools for monetizing the impacts of carbon-offset projects. Potential exists for a model with substantial bias or significant errors to be selected from the mélange available, on a less than objective basis, to support a particular offset transaction.

\section{Citation}

Melson, S.L.; Harmon, M.E.; Fried, J.S.; Domingo,

J.B. 2011. Estimates of live-tree carbon stores in the Pacific Northwest are sensitive to model selection. Carbon Balance and Management 6(2). http://www. treesearch.fs.fed.us/pubs/39998.

${ }^{1}$ Author: Jeremy Fried. 


\section{How Old Are the Trees in California?}

The FIA field crews estimate stand age from the average age of predominant overstory trees, derived from field counts of annual rings in increment cores. There are notable exceptions of older stands in the state (figs. 17 and 18), but overall the stand ages tend to be younger, with nearly half of the state's forests being less than 80 years old (47 percent). Almost 75 percent of the stands are less than 120 years old (fig. 19). Older stands are more commonly softwood forest types (fig. 20).

Fifteen percent (4.3 million acres) of forest stands across California are older than 160 years, and about 9 percent (2.5 million acres) are older than 200 years. California mixed-conifer stands older than 160 years account for 7 percent of total forest acreage and 3 percent of the acreage in stands older than 200 years. The remaining combined forest types with stand ages in excess of 160 years make up 10 percent of total forest area. The vast majority of older forests are California mixed-conifer on publicly owned land in national forests and national parks.

Western white pine forest types have a large proportion of their acreage in older stands: 62 percent of California's white pine is older than 160 years, about 104,000 acres. Lodgepole pine forests also tend to be older, with 40 percent of these stands older than 160 years, about 404,000 acres.

California mixed-conifer is the most common softwood forest type in terms of acreage, however only 21 percent of these forests are older than 160 years, about 1.6 million acres. Western oaks are the most common hardwood forest type by acreage, and only about 5 percent are older than 160 years, about 323,000 acres (fig. 21).

\section{Who Owns California's Forests?}

Ownership has a strong influence on how forests are managed and used. Federal owners must consider multiple management objectives including water resources, wildlife, recreation, conservation, biological diversity, and wood products. Corporate and other private owners often focus on more specific outcomes, such as aesthetics, wood production, or real estate investment, within state and federal regulations and incentives.

Federal agencies such as the Forest Service, Bureau of Land Management (BLM), and the National Park Service
(NPS) manage more than half the forested land in California, about 57 percent (fig. 22). Federal lands tend to be at higher elevations and contain older forests, and therefore contain bigger trees on less productive sites. About 3 percent of federal forest land is considered highly productive, while 9 percent of private lands fall into that category. Federal lands comprise the vast majority of the 5.9 million acres of reserved forest lands (those withdrawn by law from production of wood products).

Other publicly owned forest lands include state and county forests and those administered by other federal agencies, such as the U.S. Fish and Wildlife Service, the Department of Defense, and the Department of Energy. California's state-owned forest lands, managed by multiple agencies, encompass 730,000 acres. Forest lands managed by state and local governments (about 3 percent of California's forests) include a considerable area of highly productive redwood forests, as well as a great proportion of low-productivity forests that are managed primarily for habitat and watershed protection objectives. About 10 percent of the area is on high-productivity sites with about 57 percent on low-productivity sites.

Accounting for about 39 percent of the forested land, private ownership (corporate and noncorporate) is distributed throughout the state (figs. 23 and 24). Private owners include families, individuals, conservation and natural resource organizations, unincorporated partnerships, associations, clubs, corporations, and Native American tribes. Private lands tend to contain a higher proportion of productive land, and its forests tend to be in younger age classes. The vast majority of private owners (about 99 percent) own parcels of 500 acres or fewer, making up 40 percent of the area of privately owned forest land.

Corporations manage 14 percent of California's forests. Recently, some large, publicly owned timber companies reorganized into real estate investment trusts (REIT) and timberland investment management organizations (TIMO). The REITs directly own forest land, whereas TIMOs manage lands owned by investors. The REITs and TIMOs own forest land as investment vehicles that compete with and complement alternative investments; these entities may or may not own wood-processing facilities. 


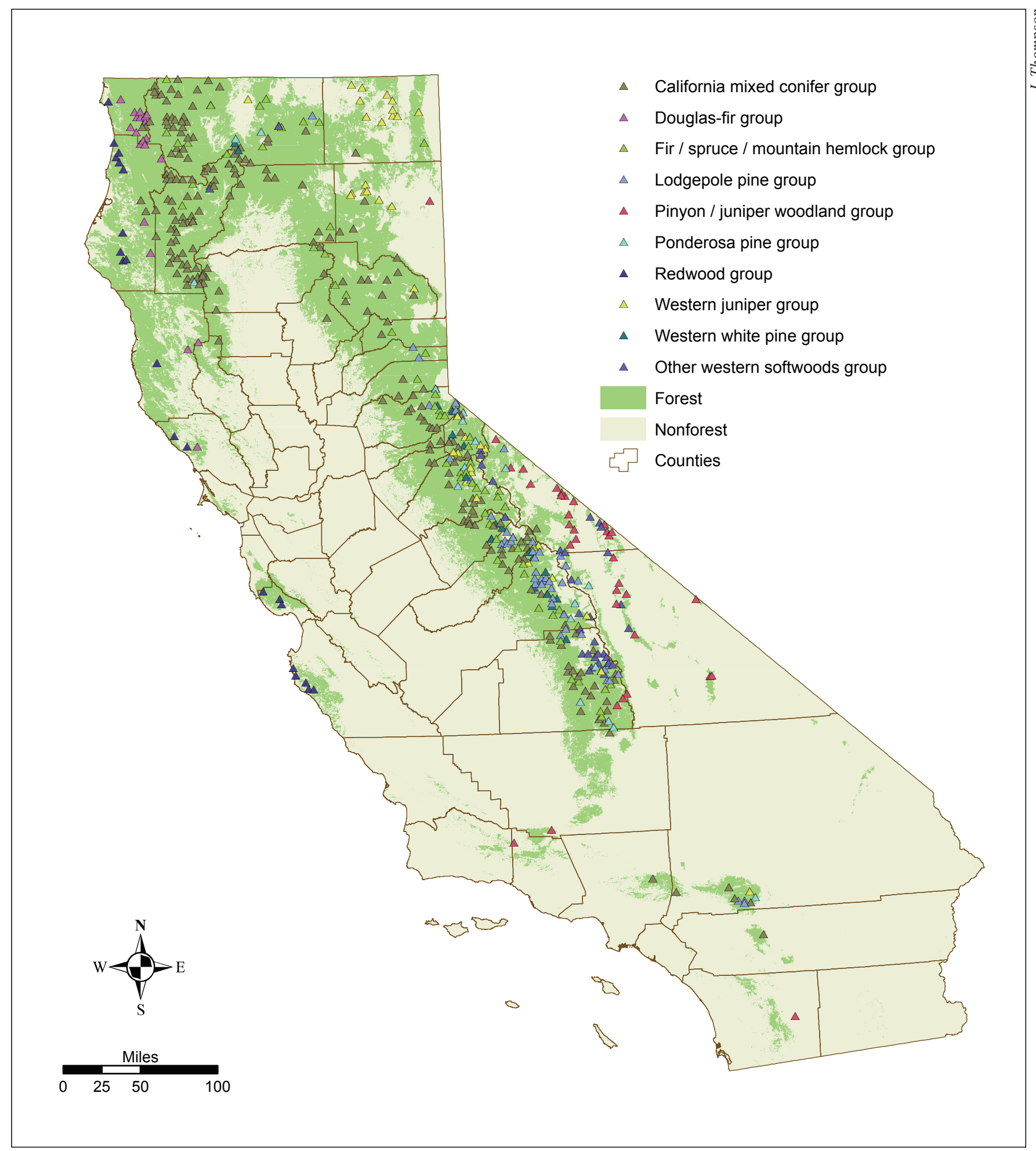

Figure 17-Distribution of softwood stands >200 years old in California, 2001-2010 (forest/nonforest geographic information system layer: Blackard et al. 2008). 


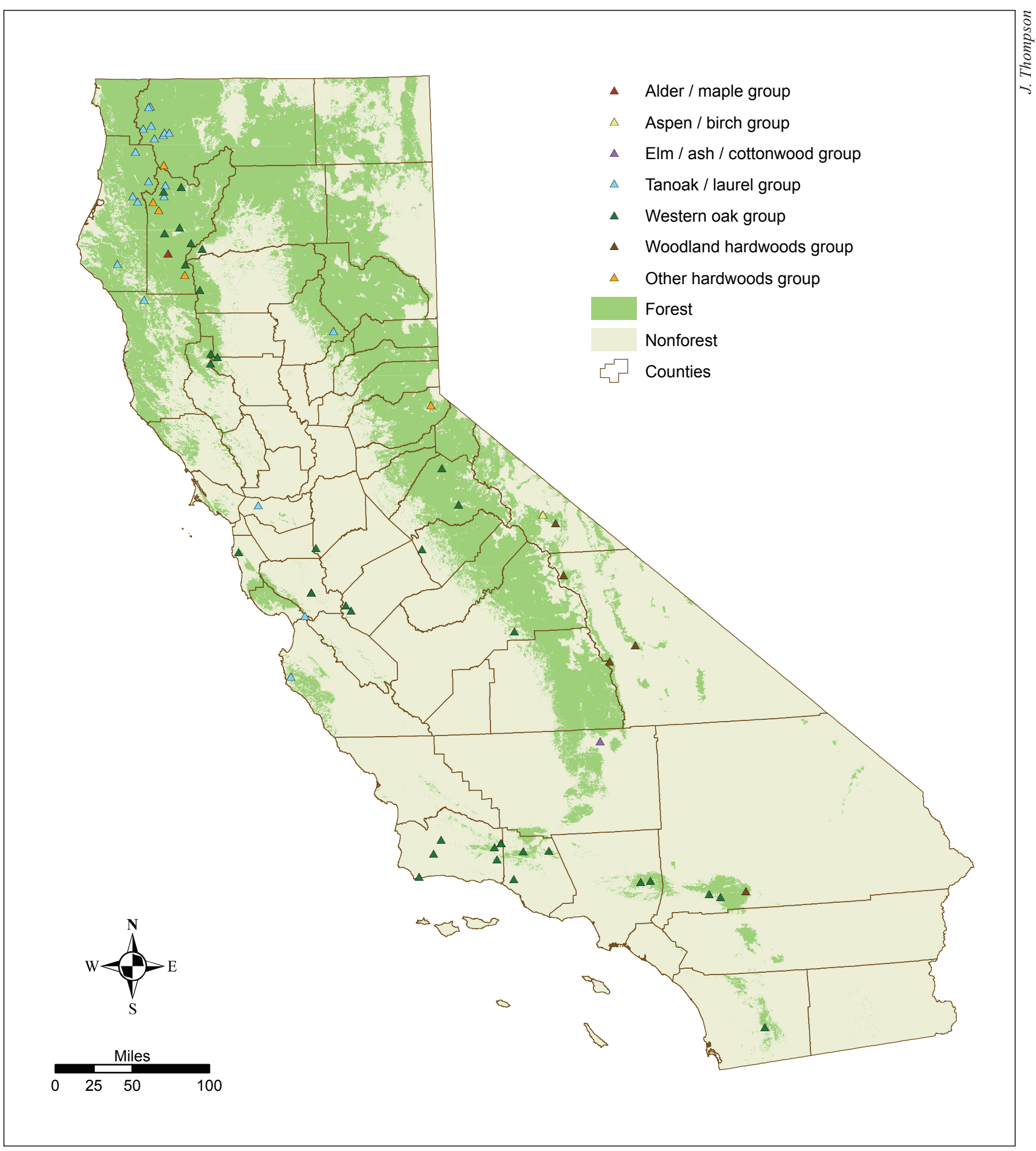

Figure 18-Distribution of hardwood stands $>200$ years old in California, 2001-2010 (forest/nonforest geographic information system layer: Blackard et al. 2008). 


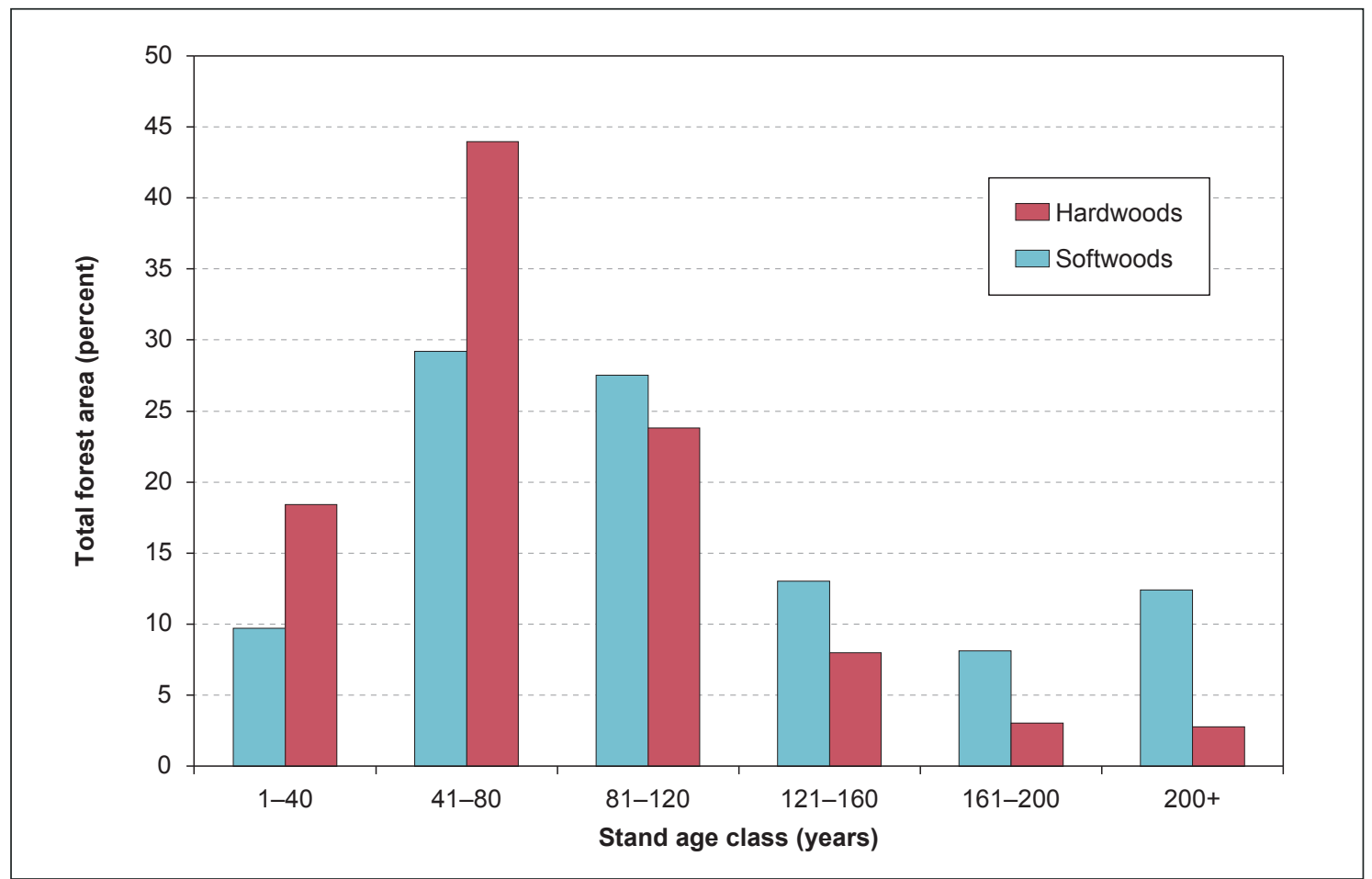

Figure 19-Percentage of forest area by stand age class for softwood and hardwood forest types in California, 2001-2010.

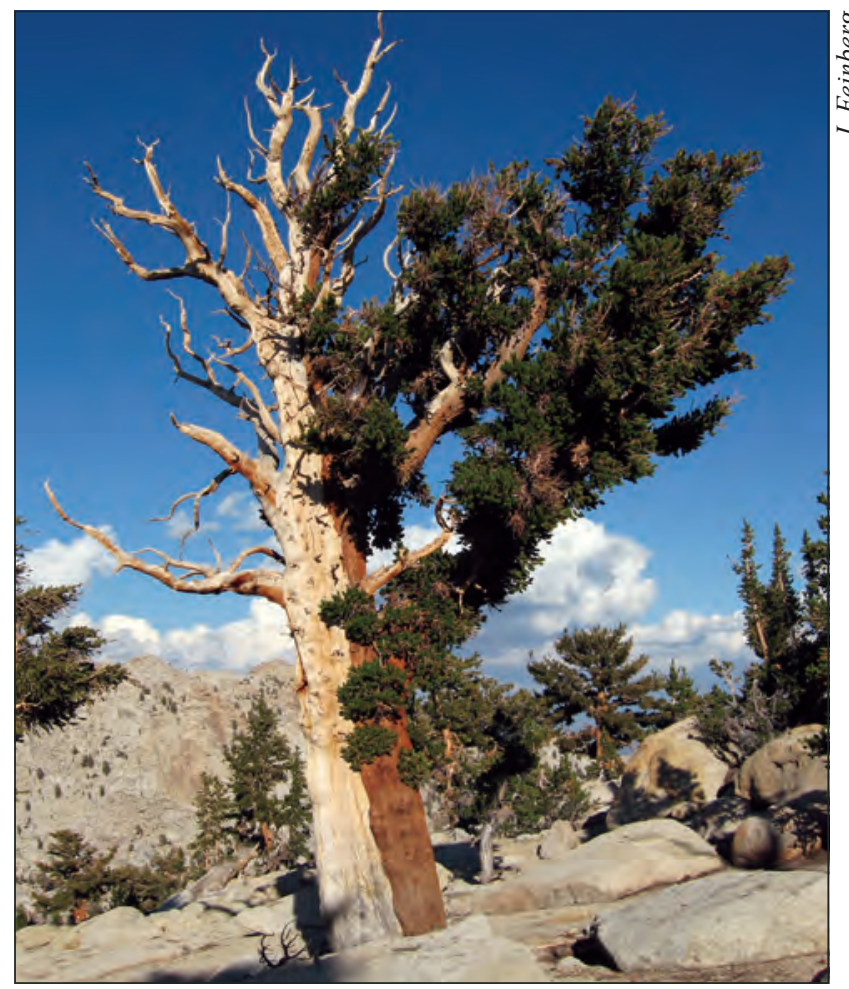

Figure 20-Endemic to California and closely related to bristlecone pine, the foxtail pine can exceed 1,000 years of age. 


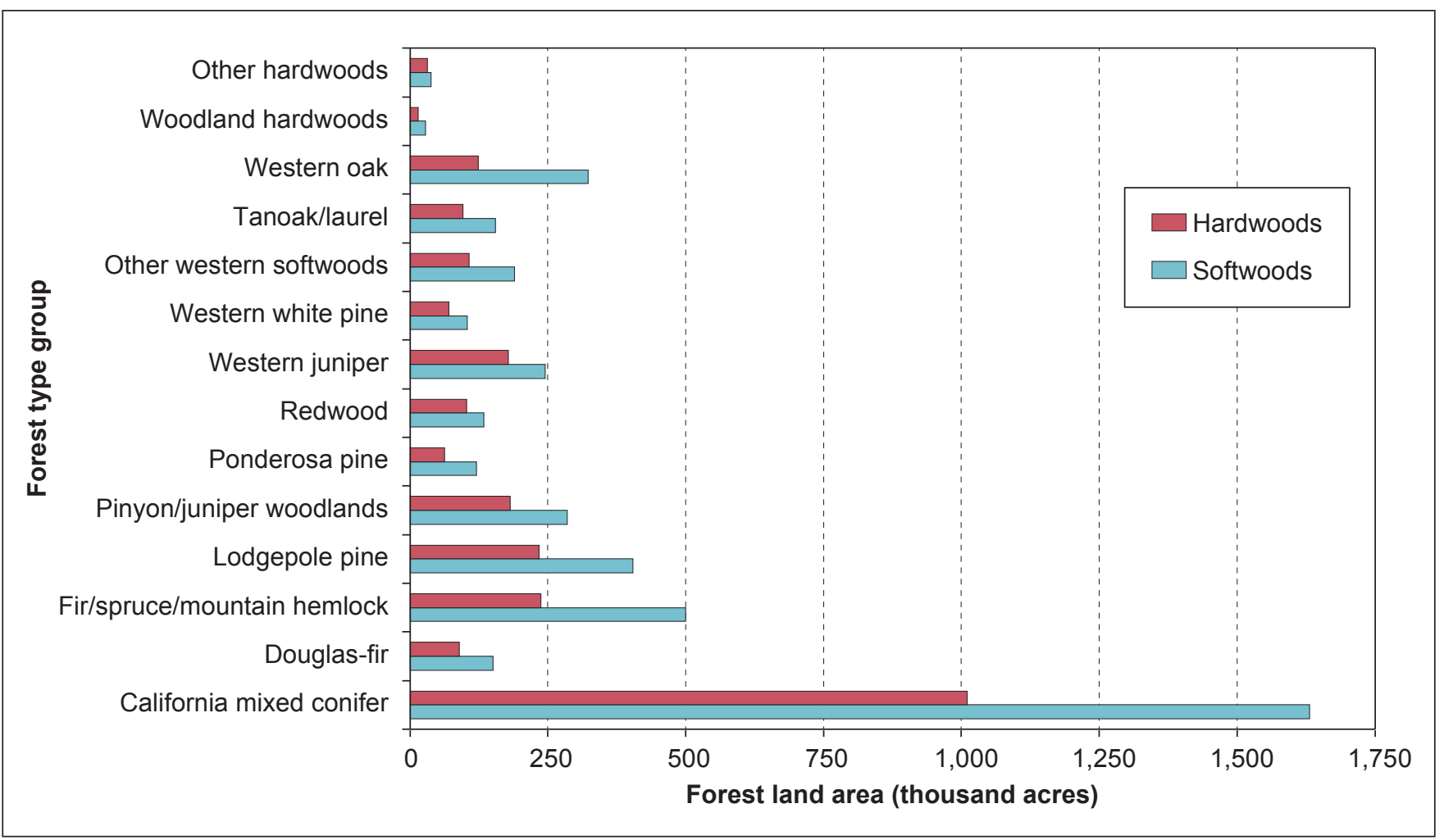

Figure 21-Total forest area by forest type group for stands 160+ and 200+ years old in California, 2001-2010.

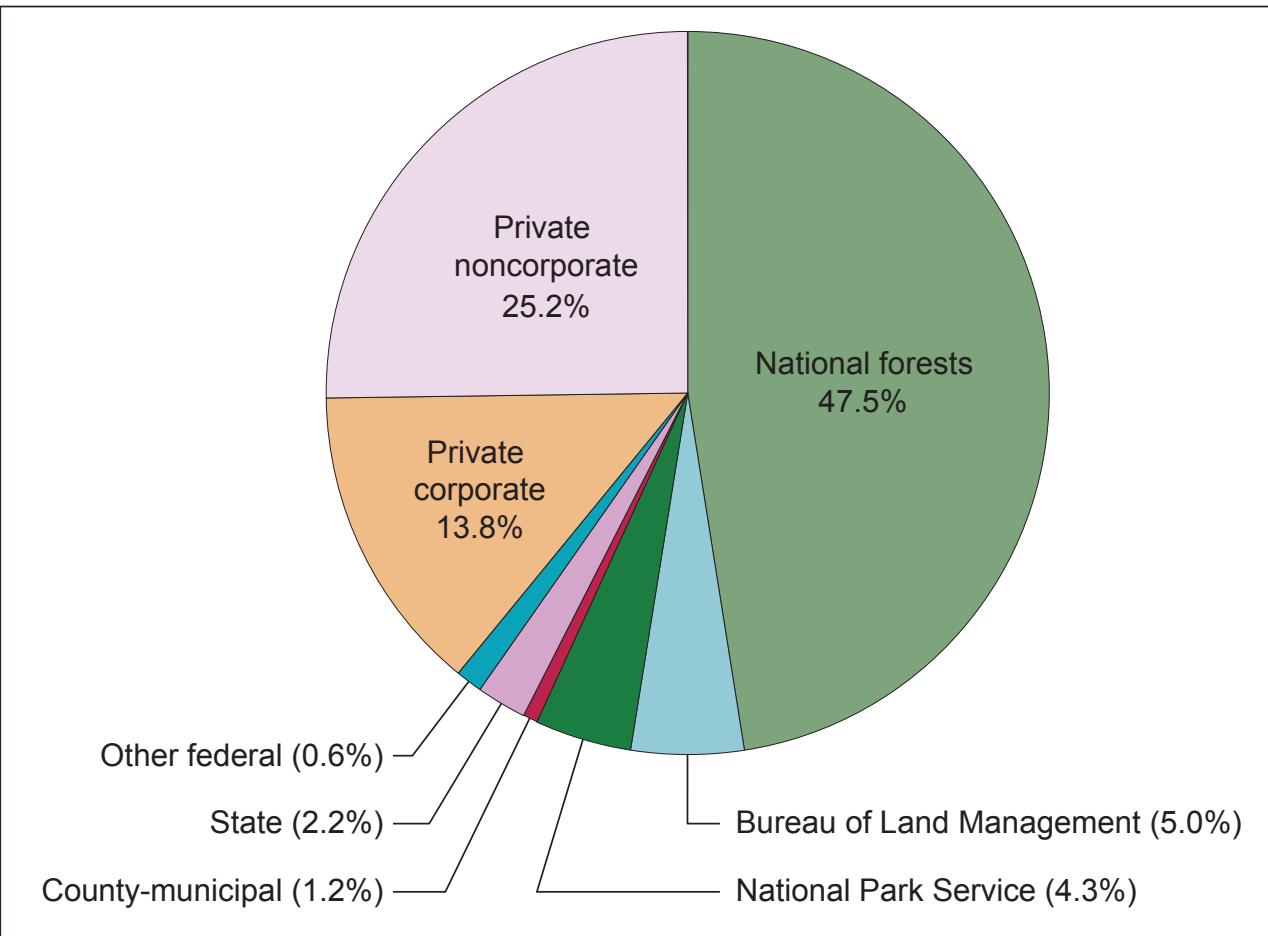

Figure 22-Percentage of forest land by owner group in California, 2001-2010. 


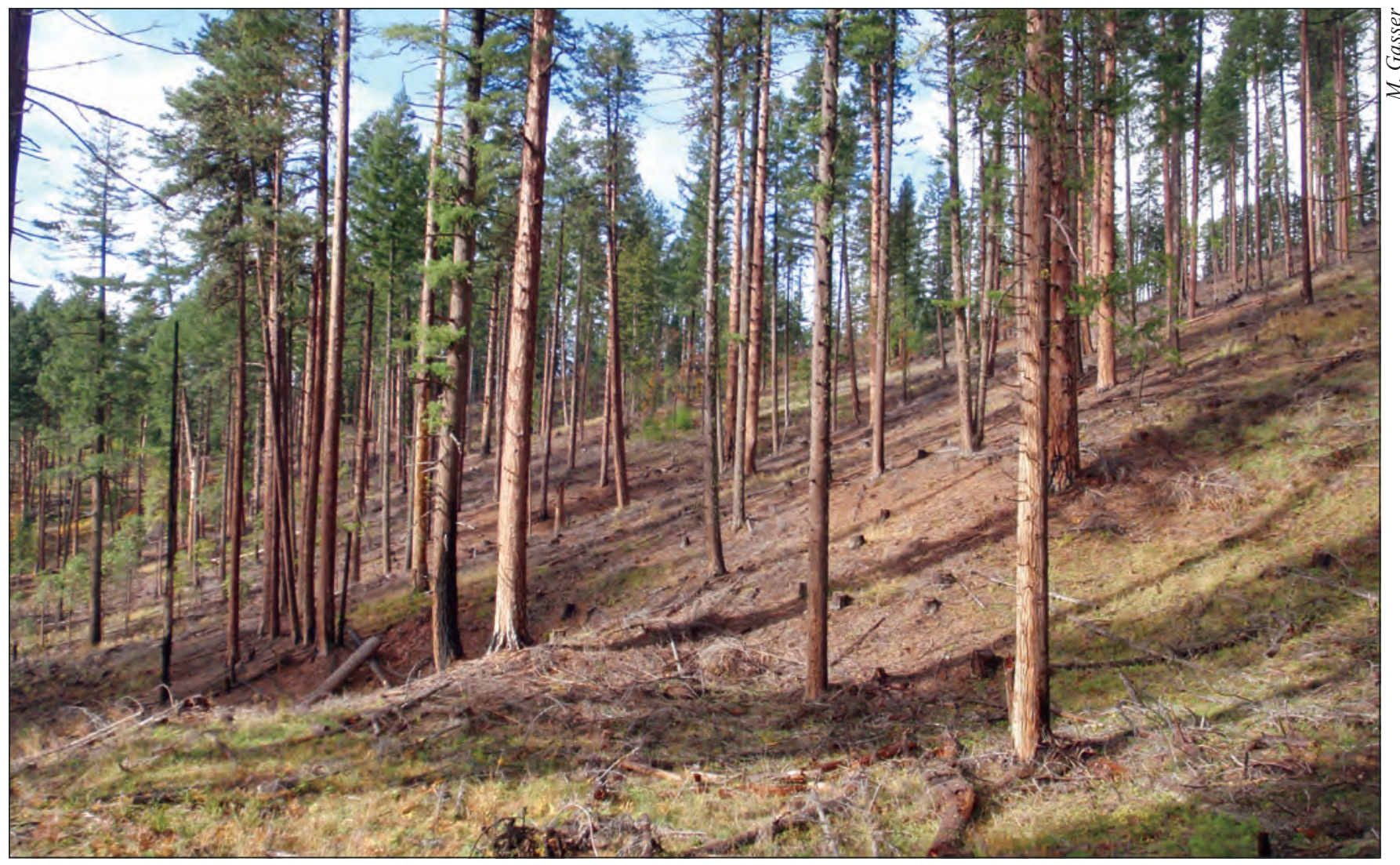

Figure 23-About 39 percent of California's forest land is owned by private individuals or corporations. 


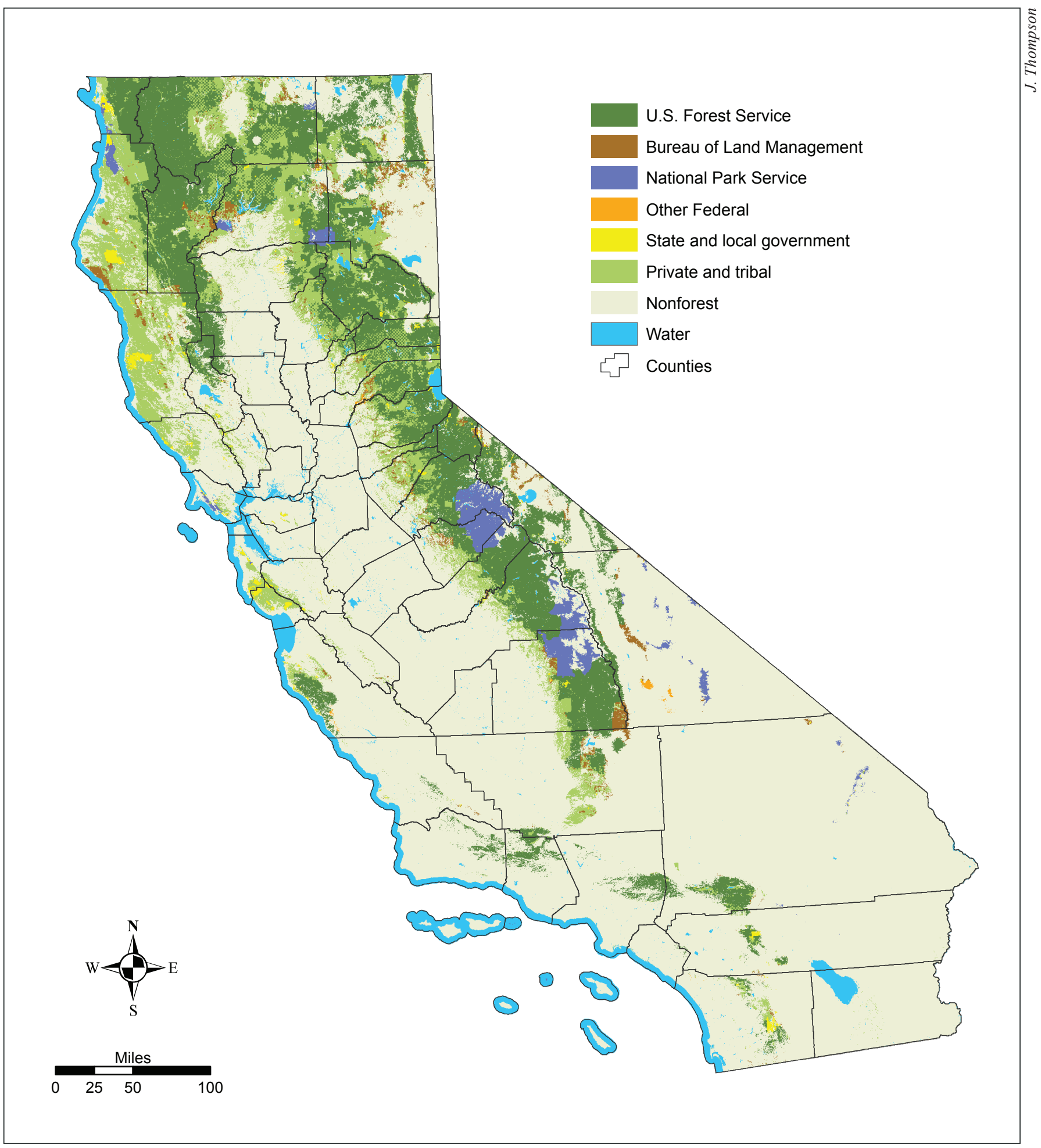

Figure 24-California forest ownership categories (forest/nonforest geographic information system layer: Blackard et al. 2008; ownership layer courtesy CAL FIRE). 


\section{National Woodland Owner Survey}

The National Woodland Owner Survey, a mail-in questionnaire-based survey conducted by FIA, provides some insight into private family forest owners and their concerns, current forest management, and future intentions (Butler et al. 2005). Between 2011 and 2013, there were 166 responses to these surveys in California. About 98 percent of family owners surveyed (those with ownerships at least 10 acres in size) own parcels of 500 acres or less, making up 61 percent of the area of family-owned forest land (fig. 25). About 5 percent of the owners surveyed had written management plans. Wildfire ranked as the leading concern of respondents along with trespassing/poaching, passing the land intact to heirs, and property taxes. Of the 25 percent that had harvested trees within the last 5 years, the leading reason was to reduce fire hazard ( 38 percent). Reducing fire hazard was also the leading reason they will likely harvest trees in the next 5 years ( 31 percent). Family woodland owners are also concerned about invasive plants. Removing invasive plants was the second leading reason landowners would likely harvest trees in the next 5 years. Keeping forests as forests ranked high among small woodland owners. When asked if they would rather keep their woodland as forest or sell if offered a reasonable price, 70 percent of respondents indicated they would choose to keep their land as forest.

The ownership survey revealed the following demographics for California family forest landowners:

- The majority of primary owners are male (80 percent).

- Diversity among primary owners is low (89 percent are Caucasian).

- Primary ownership is aging, with 55 percent of the primary owners being older than 65 years.

- Only 24 percent of the primary owners are younger than 55 years.

- Most owners are retired (58 percent).

- Most owners are well educated: 63 percent of the primary owners have earned a bachelor's or graduate degree.

- Only 19 percent have owned their land for more than 25 years.

- 38 percent use their land as part of their primary residence.

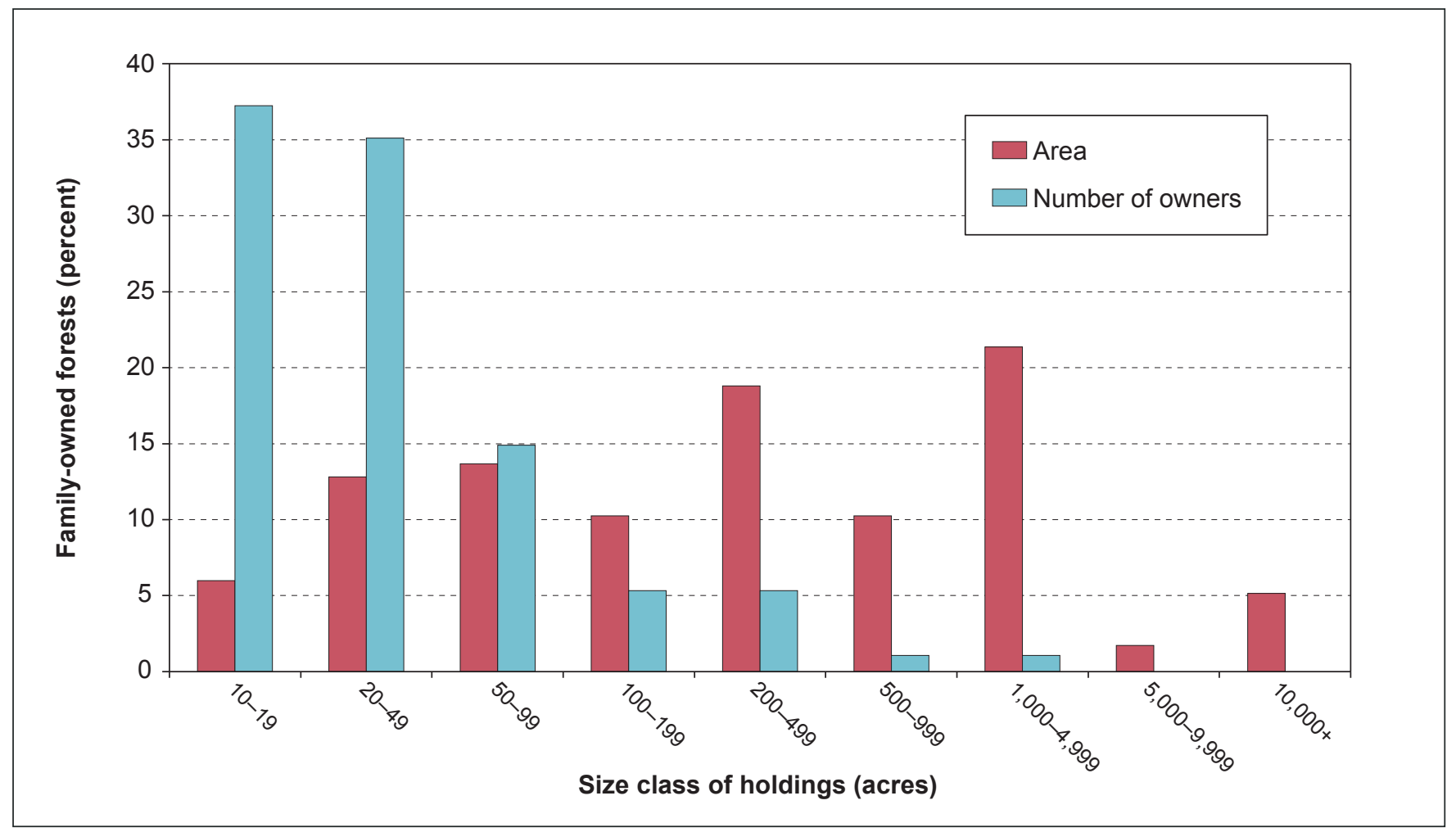

Figure 25-Percentage of area and percentage of number of family-owned forest holdings by size class in California, $2011-2013$. 


\section{How Much of California's Forest Land Is Available for Harvest?}

About 17 million of the 33 million forested acres in California can be considered timberland. Forest is considered timberland if it is growing on ground capable of significant annual tree growth and considered available for timber management. This is land potentially available for production of wood products and does not include acreages locally withdrawn from timber harvest or other active management. About 18 percent, nearly 6 million acres, of California's forests are unavailable for timber production (fig. 26).

The majority of timberland in California is publicly owned, about 56 percent. The Forest Service alone administers over 9 million acres. State and local governments manage the smallest fraction of public timberland in the state at less than 1 percent. Private ownership, both corporate and individual owners, covers about 7.5 million acres of timberland (fig. 27).

By tree species group, most of the wood volume found on timberland is in conifer species, about 92 percent. The primary conifer species found on California's timberlands is Douglas-fir (fig. 28). By volume, this species has about 32 percent of all board-foot volume, over 103 billion Scribner board feet (about 17.6 billion cubic feet). Tanoak and oak species groups make up the majority of the wood volume found in hardwood species. There is about 24 billion Scribner board feet (about 5 billion cubic feet) found in all hardwood tree species (fig. 29).

The average harvestable volume per acre on timberland for the state is about 19,000 Scribner board feet $(3,419$ cubic feet per acre). Harvestable volume is wood volume found in sawtimber trees (live commercial species at least 9.0 inches d.b.h. for softwoods and 11.0 inches d.b.h. for hardwoods) on timberland. Despite managing the least amount of acreage in timberland, state and local governments have some of the greatest volumes per acre with the average being about 29,000 Scribner board feet per acre (5,122 cubic feet per acre) (fig. 30). Private ownerships (corporate and individual owners) tend to manage their timberland with less volume per acre on average, about 16,000 Scribner board feet per acre $(2,914$ cubic feet per acre).

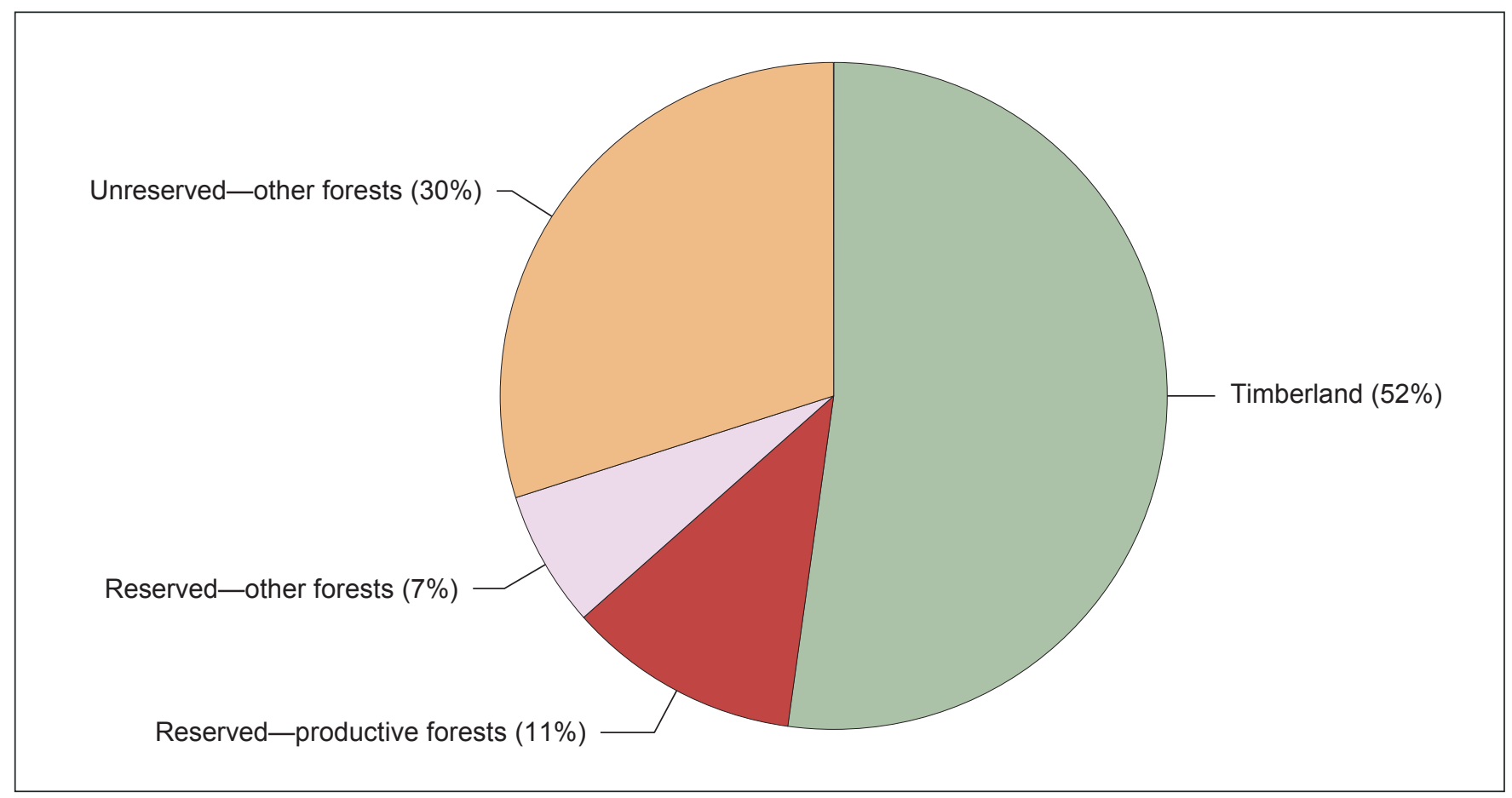

Figure 26-Proportion of forest land area by current land status in California, 2001-2010. 


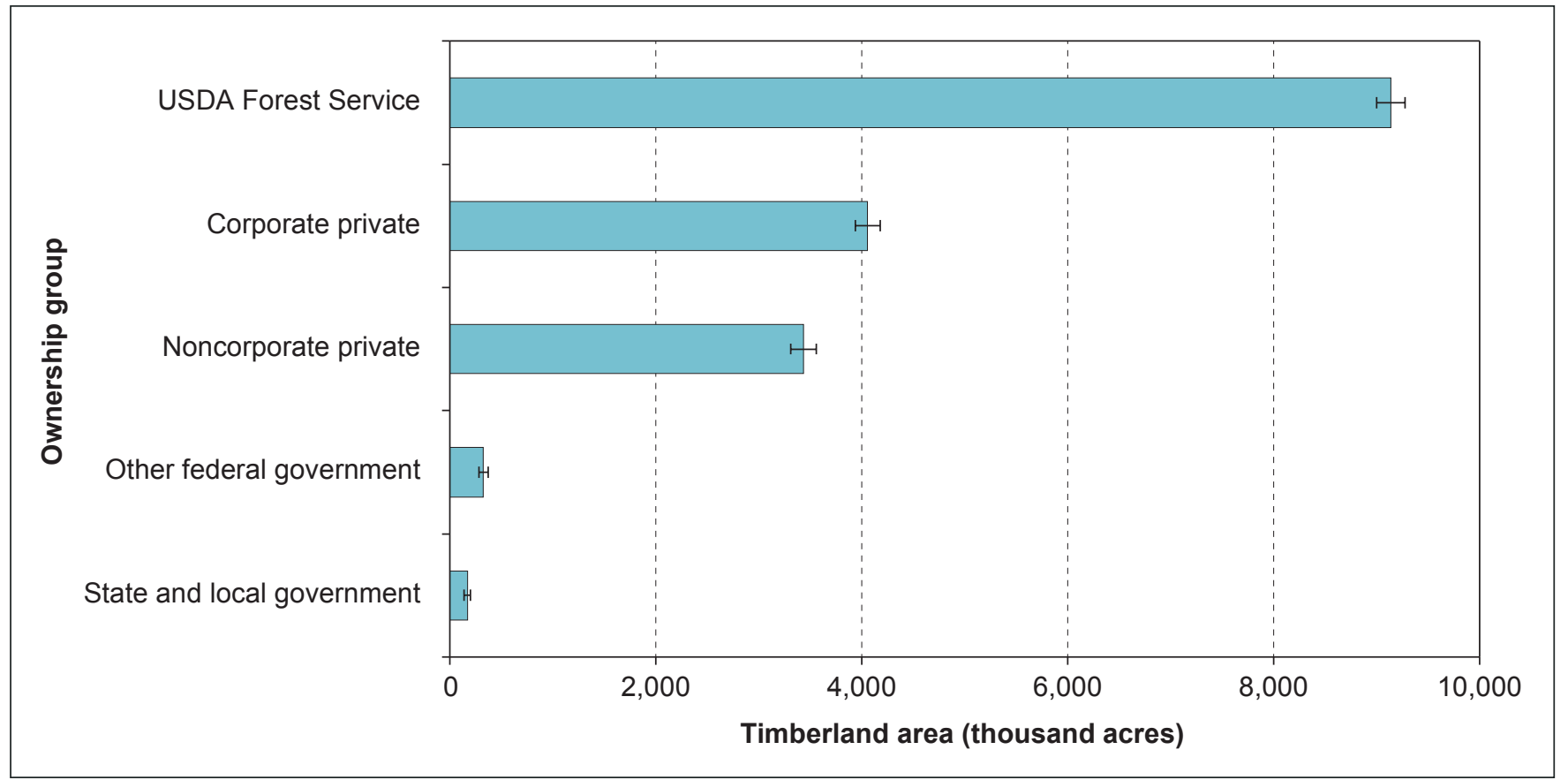

Figure 27-Timberland area by ownership in, California 2001-2010.

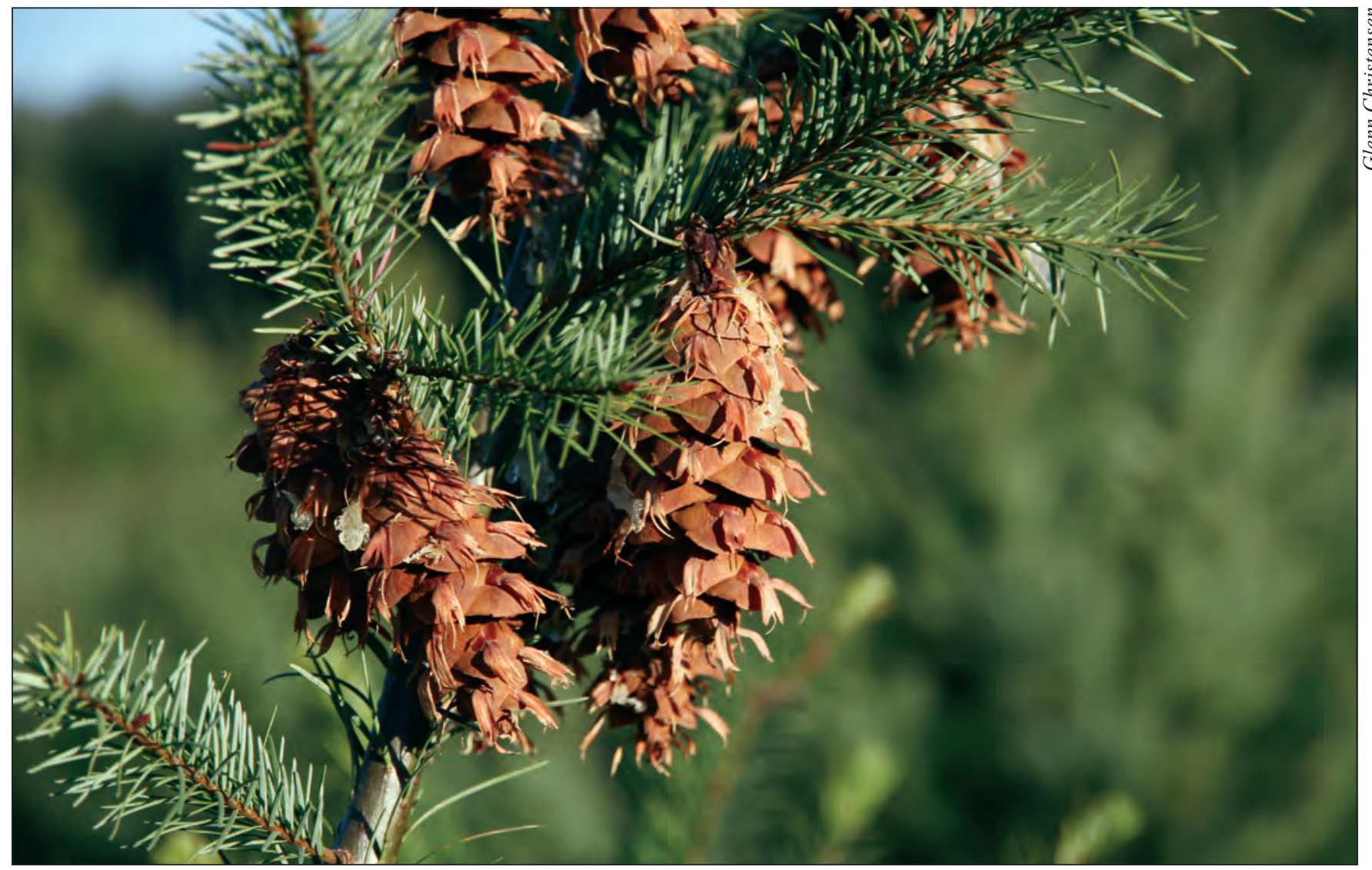

Figure 28-Douglas-fir is the primary conifer species found on California's timberlands. 


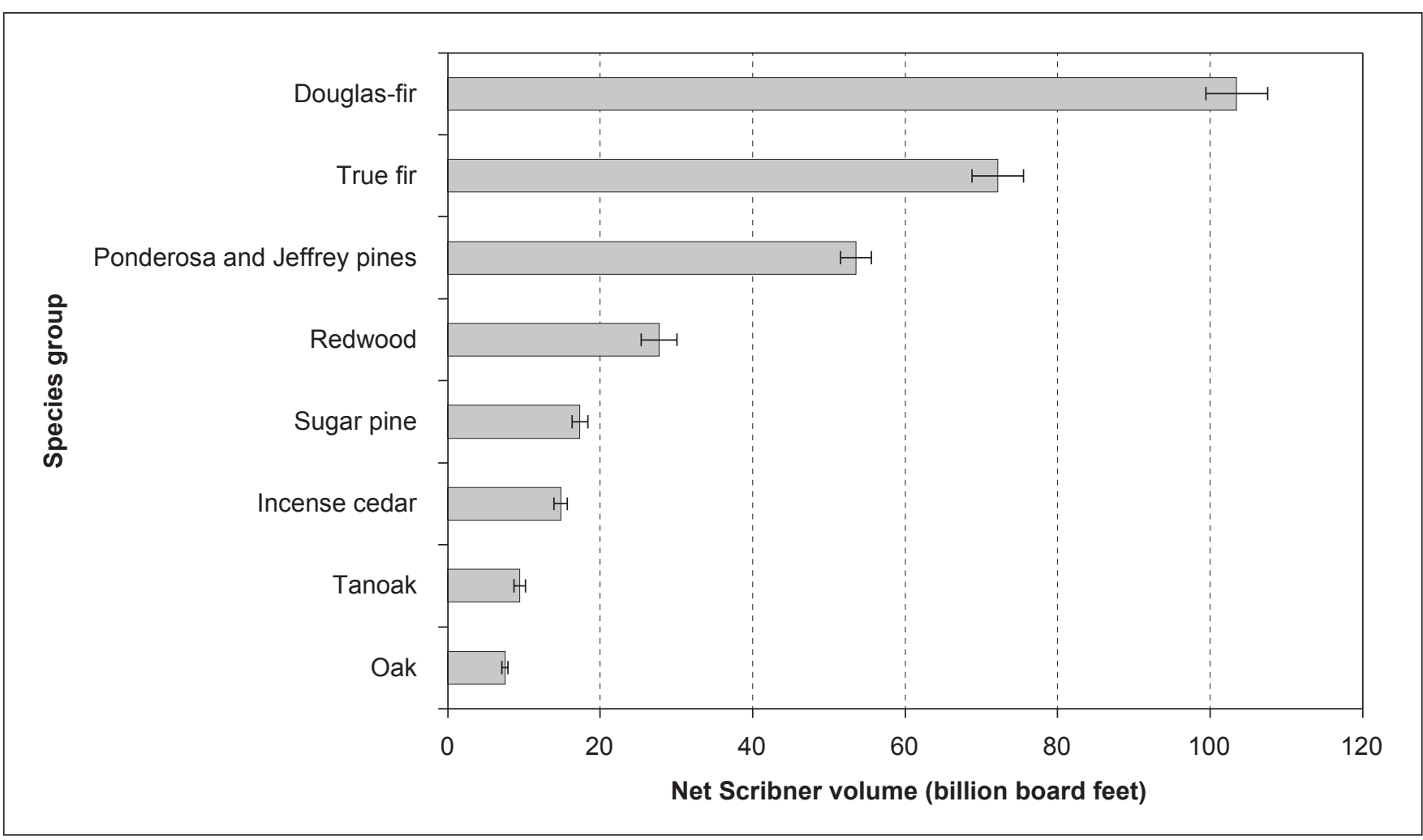

Figure 29-Scribner board-foot volume found on timberland by species group (those contributing at least 2 percent of total volume) in California, 2001-2010.

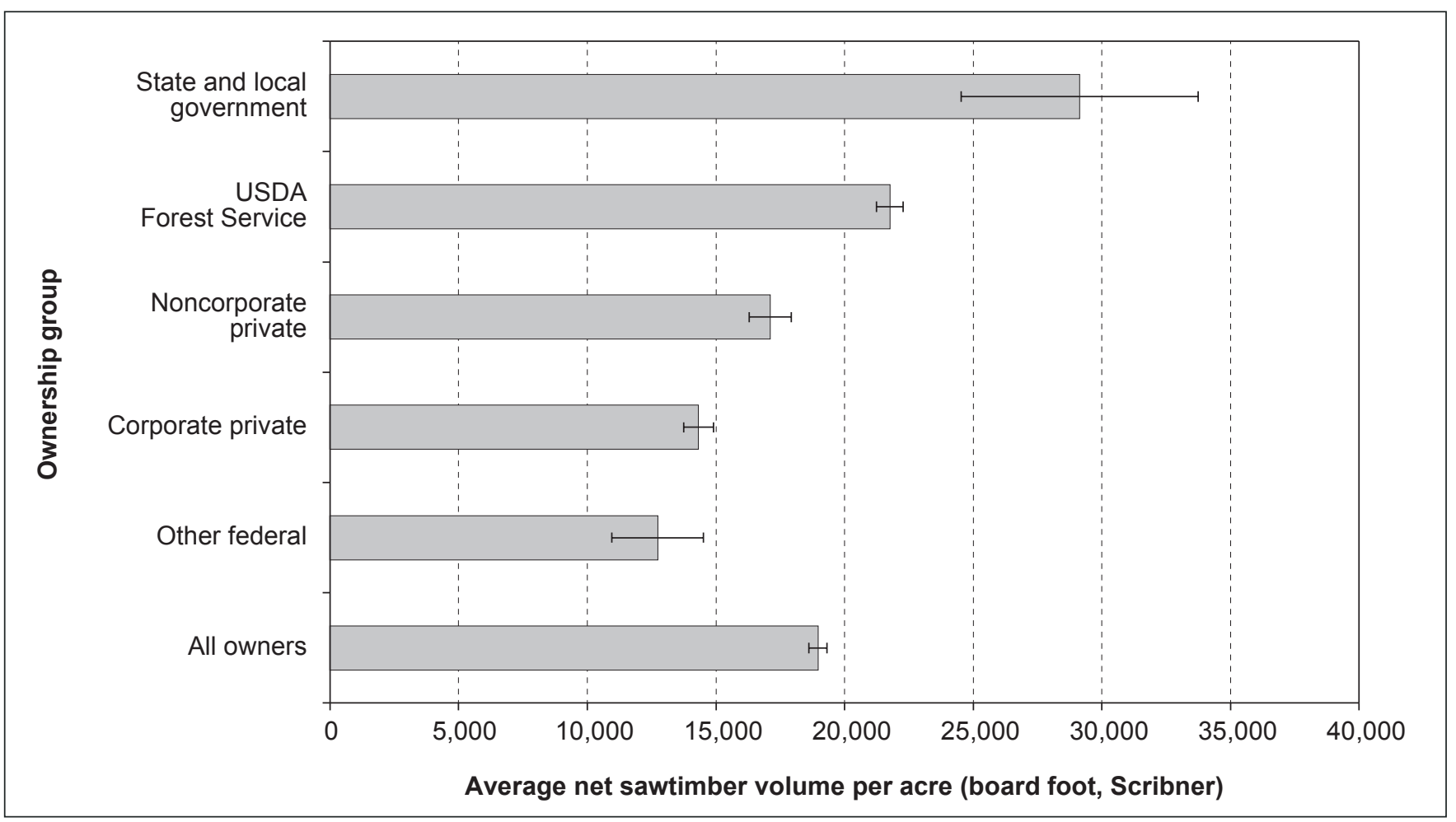

Figure 30-Average sawtimber tree volume per acre by ownership group (net board feet, Scribner) in California, 2001-2010. 


\section{Annual Tree Growth, Removals, and Mortality in California ${ }^{2}$}

Changes in wood volume over time are an indicator of sustainability and can be explained by examining tree growth, removals (harvesting), and mortality. These components are key factors in understanding how management, ecology, and climate change interact across the landscape. It is important to take into account that private forests are distinctly different from Forest Service forests in management practice as well as vegetation type, productivity, and topographic location. For example, the Forest Service manages vast areas of wilderness and other reserved land that cannot be compared directly with privately owned forests managed for production of wood products.

When the sum of all tree removals and mortality exceeds growth, total tree volume declines. In small areas, removals undertaken to reduce fire hazard or deter pest infestations may sometimes exceed growth in the short run.
This trend, however, may be reversed in the long run by protecting against catastrophic mortality (fig. 31). Conversely, widespread mortality caused by bark beetles, for example, may offset growth gains and thus prevent or delay attainment of a stand's desired future condition and reduce the production of ecological benefits such as sequestration of carbon or economic benefits such as forest products.

Previously, FIA conducted a forest inventory between 1991 and 1994 on private, state, and local government timberland in California. Owing to numerous differences between the current inventory and those prior measurements, comparing current volume estimates with those published from previous inventories will not produce valid change estimates. Differences include, for example, sampling different areas (forest land vs. timberland, with or without reserved land) and using different definitions (e.g., growing stock). To estimate volume change despite these differences, we estimated net change based on revisited

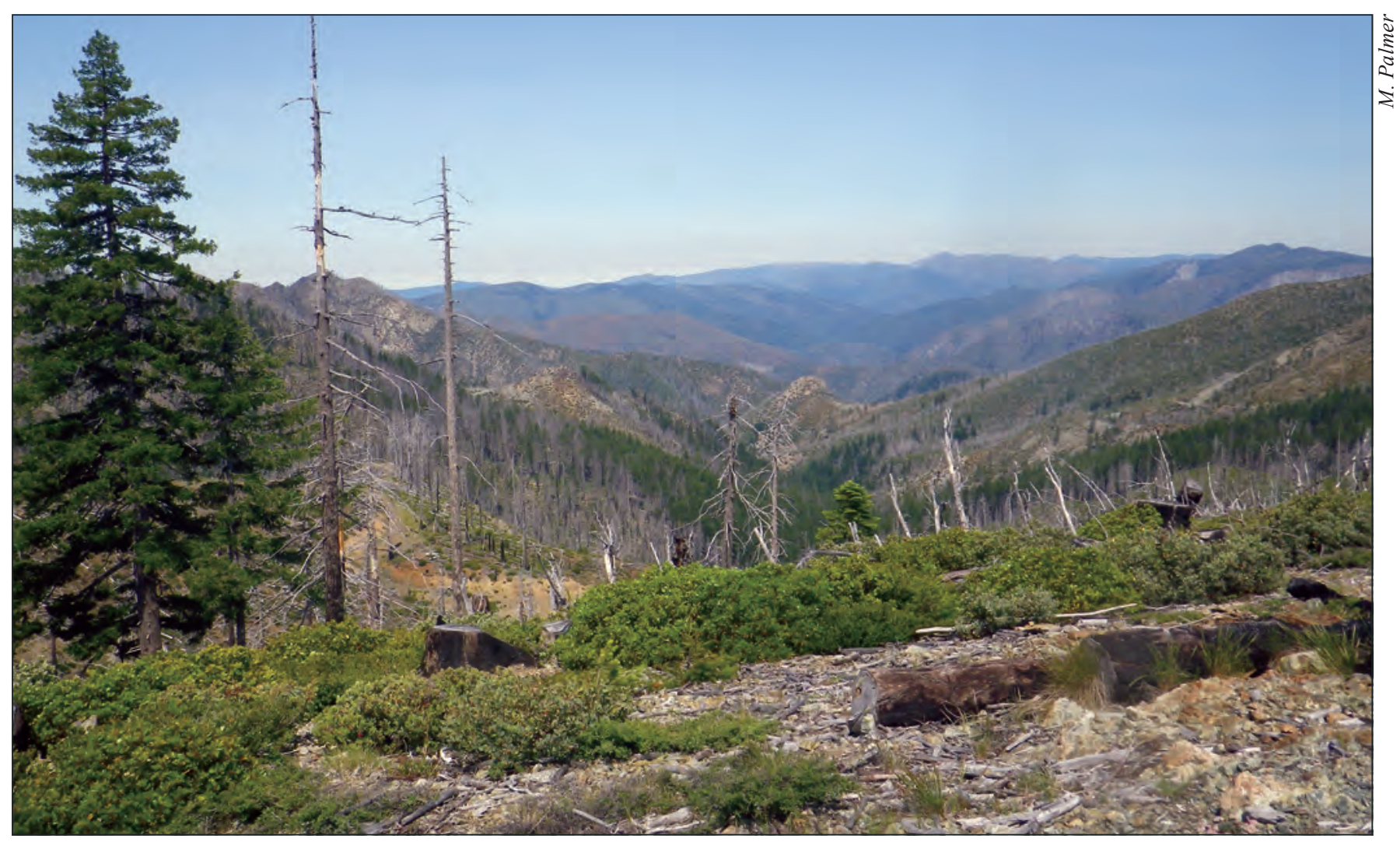

Figure 31-Growth of trees is offset by harvesting and mortality. Tree mortality shown here was caused by wildfire.

\footnotetext{
2 Author: Olaf Kuegler
} 
plots, relying on the algorithms and definitions of today's annual inventory. Between 2007 and 2010, FIA remeasured a subset of the 1991-1994 inventory (280 plots). The remeasurement period ranged from 13 to 19 years with an average of 16.3 years. We estimated tree growth, removals, and mortality using a subset of remeasured trees that were alive in 1991-1994 and 5 inches or more d.b.h. from the remeasured plots.

To calculate volume and biomass changes on forest land managed by the Forest Service, we relied on remeasured plots under the current FIA field design, which included all forest land (1,627 plots). These plots were first measured between 2001 and 2006 and remeasured about 5 years later between 2006 and 2010. Because timberland is a subset of forest land, we were able to estimate changes for timberland as well as for Forest Service lands. Additional information on timber harvest removals supporting California's primary forest products industry can be found in Morgan et al. (2012).

\section{Privately Owned Timberland: Change Between 1991-1994 and 2007-2010}

Annual volume growth on noncorporate privately owned timberland significantly exceeds removals and mortality in California, currently estimated at $45.5 \mathrm{ft}^{3} \cdot \mathrm{ac}^{-1} \cdot \mathrm{yr}^{-1}$ (fig. 32). On corporate privately owned timberland, volume growth also exceeded removals and mortality, currently estimated at $8.6 \mathrm{ft}^{3} \cdot \mathrm{ac}^{-1} \cdot \mathrm{yr}^{-1}$; however, the difference is not statistically significant from zero. Overall, we found annual volume growth on private timberland (noncorporate and corporate) significantly exceeded removals and mortality by $24.4 \mathrm{ft}^{3} \cdot \mathrm{ac}^{-1} \cdot \mathrm{yr}^{-1}$. While net annual volume growth (annual growth minus annual mortality) is similar on corporate and noncorporate timberland (currently estimated at $74.9 \mathrm{ft}^{3} \cdot \mathrm{ac}^{-1} \cdot \mathrm{yr}^{-1}$ versus $\left.69.7 \mathrm{ft}^{3} \cdot \mathrm{ac}^{-1} \cdot \mathrm{yr}^{-1}\right)$, removals on corporate timberland are about two and a half times as high as on noncorporate timberland $\left(66.3 \mathrm{ft}^{3}\right.$ versus $\left.24.2 \mathrm{ft}^{3} \cdot \mathrm{ac}^{-1} \cdot \mathrm{yr}^{-1}\right)$.

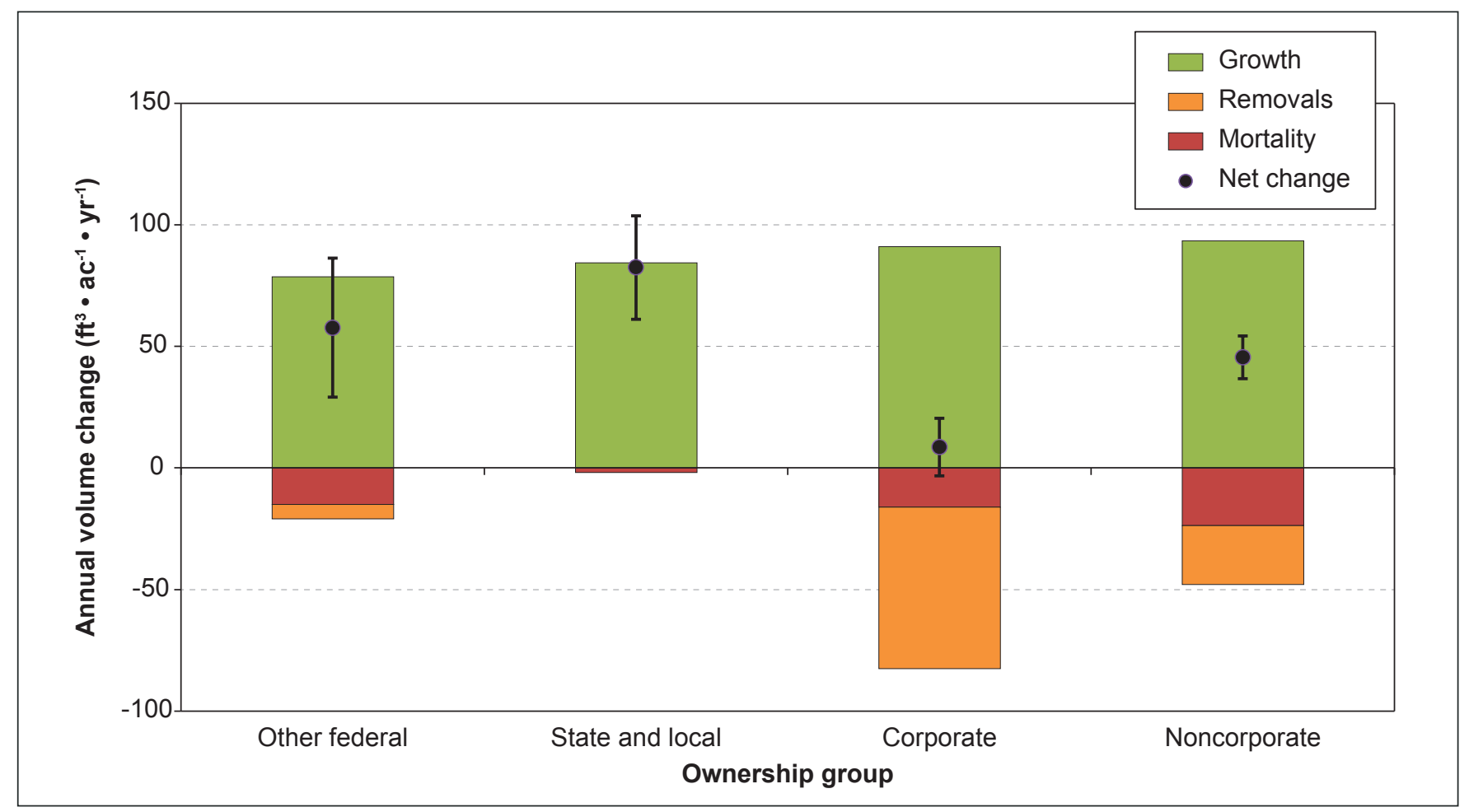

Figure 32-Average annual change in volume (cubic feet) growth, removals and mortality per acre per year on non-national forest timberland between 1991-1994 and 2007-2010 by ownership in California (error bars represent sampling error). 
State And Local Government Timberland: Change Between 1991-1994 and 2007-2010

On timberland managed by state agencies and local municipalities, annual volume growth significantly exceeded mortality and harvest by $82.5 \mathrm{ft}^{3} \cdot \mathrm{ac}^{-1} \cdot \mathrm{yr}^{-1}$. Annual mortality was very low on timberland within these ownerships, currently estimated at $1.9 \mathrm{ft}^{3} \cdot \mathrm{ac}^{-1} \cdot \mathrm{yr}^{-1}$, and no harvested trees were found on these remeasured plots (tables 1 through 4).

\section{National Forest System (NFS) Forest Land: Change Between 2001-2006 and 2006-2010}

Across all NFS forest land, annual volume growth significantly exceeded mortality and removals by about 11.3 $\mathrm{ft}^{3} \cdot \mathrm{ac}^{-1} \cdot \mathrm{yr}^{-1}$ (fig. 33). Average annual mortality rates on NFS land, estimated at $46.0 \mathrm{ft}^{3} \cdot \mathrm{ac}^{-1} \cdot \mathrm{yr}^{-1}$, were relatively high compared to private ownerships (fig. 34). However, the timeframe for assessment of change between NFS and private ownerships is vastly different and therefore cannot be compared directly. Removal of tree volume is very low on NFS forest land, currently estimated at $2.3 \mathrm{ft}^{3} \cdot \mathrm{ac}^{-1} \cdot \mathrm{yr}^{-1}$.

Although not statistically significant, average annual cubic feet volume loss through tree mortality exceeded growth on reserved forest land (64.2 versus $46.8 \mathrm{ft}^{3} \cdot \mathrm{ac}^{-1}$. $\left.\mathrm{yr}^{-1}\right)$. However, biomass mortality was significantly less than growth. Over the long term, growth should equal mortality if no trees are being harvested. However, over shorter periods, volume loss through disturbances such as fire or insect infestations can cause overall tree mortality to be higher than growth. For example, California experienced large wildfires during the measurement period, especially in 2008. For that year, we estimate about 11.3 percent (SE 3.2 percent) of the tree volume on reserved land being affected (but not necessarily killed) by fire compared to an average of 0.9 percent (SE 0.2 percent) of tree volume per year during the period 1995 to 2007 (tables 5 through 8).

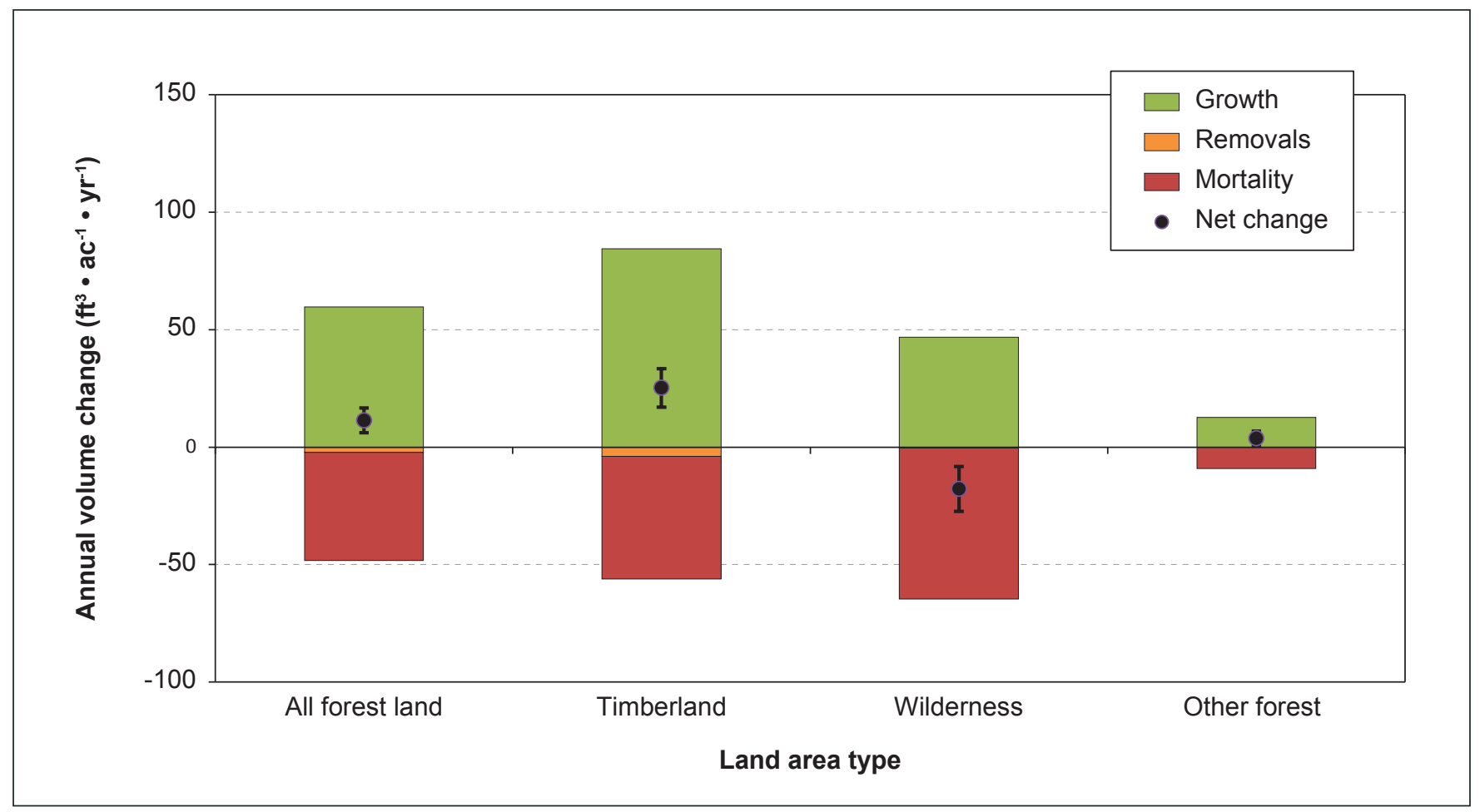

Figure 33-Average annual change in volume (cubic feet) growth, removals, and mortality per year on national forest land between 2001-2006 and 2006-2010 by land status in California (error bars represent sampling error). 
Table 1-Average annual volume growth, removals, and mortality per acre per year on non-national forest timberland between 1991-1994 and 2007-2010 in California

\begin{tabular}{|c|c|c|c|c|c|c|c|c|c|c|}
\hline & \multirow{2}{*}{\multicolumn{2}{|c|}{ Other federal }} & \multirow{2}{*}{\multicolumn{2}{|c|}{ State and local }} & \multicolumn{6}{|c|}{ Private } \\
\hline & & & & & \multicolumn{2}{|c|}{ Corporate } & \multicolumn{2}{|c|}{ Noncorporate } & \multicolumn{2}{|c|}{ Total private } \\
\hline & Mean & SE & Mean & SE & Mean & SE & Mean & SE & Mean & SE \\
\hline & \multicolumn{10}{|c|}{ Cubic feet per acre per year } \\
\hline Growth & 78.52 & 25.10 & 84.33 & 21.77 & 91.05 & 6.28 & 93.36 & 7.85 & 92.04 & 4.87 \\
\hline Mortality & 14.96 & 5.95 & 1.85 & 0.49 & 16.18 & 3.06 & 23.64 & 3.50 & 19.37 & 2.30 \\
\hline Removals & 5.90 & 6.17 & - & - & 66.28 & 8.73 & 24.21 & 4.05 & 48.32 & 5.30 \\
\hline Change & $57.67^{a}$ & 28.62 & $82.48^{a}$ & 21.28 & 8.59 & 11.81 & $45.51^{a}$ & 8.73 & $24.35^{a}$ & 7.65 \\
\hline
\end{tabular}

- $=$ not available.

${ }^{a}$ Estimate is significant, different from zero at the 95 percent significance level.

Table 2-Average annual volume (cubic feet) growth, removals, and mortality per year on non-national forest timberland between 1991-1994 and 2007-2010 in California

\begin{tabular}{|c|c|c|c|c|c|c|c|c|c|c|}
\hline & \multirow{2}{*}{\multicolumn{2}{|c|}{ Other federal }} & \multirow{2}{*}{\multicolumn{2}{|c|}{ State and local }} & \multicolumn{6}{|c|}{ Private } \\
\hline & & & & & \multicolumn{2}{|c|}{ Corporate } & \multicolumn{2}{|c|}{ Noncorporate } & \multicolumn{2}{|c|}{ Total private } \\
\hline & Total & SE & Total & SE & Total & SE & Total & SE & Total & SE \\
\hline & \multicolumn{10}{|c|}{ Thousand cubic feet per year } \\
\hline Growth & 13,313 & 6,652 & 6,023 & 3,995 & 392,152 & 29,935 & 299,613 & 28,465 & 691,765 & 38,368 \\
\hline Mortality & 2,536 & 1,429 & 132 & 88 & 69,693 & 13,417 & 75,870 & 12,105 & 145,564 & 17,602 \\
\hline Removals & 1,000 & 1,075 & - & - & 285,460 & 38,060 & 77,697 & 13,757 & 363,157 & 39,858 \\
\hline Change & 9,777 & 6,179 & 5,891 & 3,907 & 36,998 & 50,921 & $146,046^{a}$ & 28,148 & $183,044^{a}$ & 57,697 \\
\hline
\end{tabular}

$-=$ not available.

${ }^{a}$ Estimate is significant, different from zero at the 95 percent significance level.

Table 3-Average annual biomass (tons) growth, removals, and mortality per acre per year on non-national forest timberland between 1991-1994 and 2007-2010 in California

\begin{tabular}{|c|c|c|c|c|c|c|c|c|c|c|}
\hline & \multirow{2}{*}{\multicolumn{2}{|c|}{ Other federal }} & \multirow{2}{*}{\multicolumn{2}{|c|}{ State and local }} & \multicolumn{6}{|c|}{ Private } \\
\hline & & & & & \multicolumn{2}{|c|}{ Corporate } & \multicolumn{2}{|c|}{ Noncorporate } & \multicolumn{2}{|c|}{ Total private } \\
\hline & Mean & SE & Mean & $\mathbf{S E}$ & Mean & SE & Mean & $\mathbf{S E}$ & Mean & SE \\
\hline & \multicolumn{10}{|c|}{ Short tons per acre per year } \\
\hline Growth & 1.48 & 0.46 & 1.68 & 0.44 & 1.86 & 0.13 & 1.88 & 0.16 & 1.87 & 0.10 \\
\hline Mortality & 0.33 & 0.14 & 0.06 & 0.02 & 0.36 & 0.06 & 0.50 & 0.07 & 0.42 & 0.05 \\
\hline Removals & 0.13 & 0.13 & - & - & 1.32 & 0.17 & 0.47 & 0.08 & 0.96 & 0.11 \\
\hline Change & $1.02^{a}$ & 0.52 & $1.62^{a}$ & 0.42 & 0.17 & 0.24 & $0.90^{a}$ & 0.17 & $0.49^{a}$ & 0.15 \\
\hline
\end{tabular}

$-=$ not available.

${ }^{a}$ Estimate is significant, different from zero at the 95 percent significance level. 
Table 4-Average annual biomass (tons) growth, removals, and mortality per year on non-national forest timberland between 1991-1994 and 2007-2010 in California

\begin{tabular}{|c|c|c|c|c|c|c|c|c|c|c|}
\hline & \multirow{2}{*}{\multicolumn{2}{|c|}{ Other federal }} & \multirow{2}{*}{\multicolumn{2}{|c|}{ State and local }} & \multicolumn{6}{|c|}{ Private } \\
\hline & & & & & \multicolumn{2}{|c|}{ Corporate } & \multicolumn{2}{|c|}{ Noncorporate } & \multicolumn{2}{|c|}{ Total private } \\
\hline & Total & SE & Total & SE & Total & SE & Total & SE & Total & SE \\
\hline & \multicolumn{10}{|c|}{ Thousand short tons per year } \\
\hline Growth & 250 & 125 & 120 & 80 & 7,990 & 608 & 6,033 & 572 & 14,023 & 776 \\
\hline Mortality & 57 & 32 & 4 & 3 & 1,552 & 281 & 1,609 & 241 & 3,161 & 360 \\
\hline Removals & 21 & 23 & - & - & 5,689 & 757 & 1,520 & 270 & 7,209 & 792 \\
\hline Change & 172 & 113 & 116 & 77 & 749 & 1,030 & $2,903^{a}$ & 552 & $3,653^{a}$ & 1,159 \\
\hline
\end{tabular}

- $=$ not available.

${ }^{a}$ Estimate is significant, different from zero at the 95 percent significance level.

Table 5-Average annual volume (cubic feet) growth, removals, and mortality per acre per year on National Forest System land between 2001-2006 and 2006-2010

\begin{tabular}{|c|c|c|c|c|c|c|c|c|}
\hline & \multicolumn{2}{|c|}{ Forest land } & \multicolumn{2}{|c|}{ Timberland } & \multicolumn{2}{|c|}{$\begin{array}{c}\text { Reserved forest } \\
\text { land }\end{array}$} & \multicolumn{2}{|c|}{$\begin{array}{l}\text { Low-productivity } \\
\text { forest land }\end{array}$} \\
\hline & Mean & SE & Mean & SE & Mean & SE & Mean & SE \\
\hline & \multicolumn{8}{|c|}{ Cubic feet per acre per year } \\
\hline Growth & 59.61 & 2.72 & 81.43 & 4.40 & 46.82 & 3.98 & 12.69 & 1.58 \\
\hline Mortality & 45.98 & 4.46 & 52.29 & 6.79 & 64.24 & 9.20 & 9.07 & 2.74 \\
\hline Removals & 2.31 & 0.85 & 3.91 & 1.48 & 0.41 & 0.47 & - & - \\
\hline Change & $11.32^{\mathrm{a}}$ & 5.26 & $25.23^{a}$ & 8.22 & -17.82 & 9.47 & 3.63 & 3.21 \\
\hline
\end{tabular}

$-=$ not available.

${ }^{a}$ Estimate is significant, different from zero at the 95 percent significance level.

Table 6-Average annual volume (cubic feet) growth, removals, and mortality per year on National Forest System land between 2001-2006 and 2006-2010

\begin{tabular}{|c|c|c|c|c|c|c|c|c|}
\hline & \multicolumn{2}{|c|}{ Forest land } & \multicolumn{2}{|c|}{ Timberland } & \multicolumn{2}{|c|}{ Reserved forest land } & \multicolumn{2}{|c|}{$\begin{array}{l}\text { Low-productivity } \\
\text { forest land }\end{array}$} \\
\hline & Total & SE & Total & SE & Total & SE & Total & SE \\
\hline & \multicolumn{8}{|c|}{ Thousand cubic feet per year } \\
\hline Growth & 928,584 & 42,363 & 719,786 & 41,695 & 169,363 & 16,808 & 53,985 & 7,090 \\
\hline Mortality & 716,207 & 69,518 & 462,173 & 60,670 & 232,369 & 35,298 & 38,568 & 11,762 \\
\hline Removals & 36,026 & 13,168 & 34,560 & 13,113 & 1,466 & 1,686 & - & - \\
\hline Change & $176,352^{a}$ & 81,908 & $223,053^{a}$ & 72,834 & $-64,472$ & 34,389 & 15,417 & 13,667 \\
\hline
\end{tabular}

$-=$ not available.

${ }^{a}$ Estimate is significant, different from zero at the 95 percent significance level. 
Table 7-Average annual biomass (tons) growth, removals, and mortality per acre per year on National Forest System land between 2001-2006 and 2006-2010

\begin{tabular}{|c|c|c|c|c|c|c|c|c|}
\hline & \multicolumn{2}{|c|}{ Forest land } & \multicolumn{2}{|c|}{ Timberland } & \multicolumn{2}{|c|}{$\begin{array}{c}\text { Reserved forest } \\
\text { land }\end{array}$} & \multicolumn{2}{|c|}{$\begin{array}{l}\text { Low-productivity } \\
\text { forest land }\end{array}$} \\
\hline & Mean & SE & Mean & SE & Mean & SE & Mean & SE \\
\hline & \multicolumn{8}{|c|}{ Short tons per acre per year } \\
\hline Growth & 1.13 & 0.05 & 1.52 & 0.08 & 0.90 & 0.07 & 0.29 & 0.03 \\
\hline Mortality & 0.97 & 0.09 & 1.09 & 0.14 & 1.35 & 0.20 & 0.23 & 0.08 \\
\hline Removals & 0.04 & 0.02 & 0.08 & 0.03 & 0.01 & 0.01 & - & - \\
\hline Change & 0.12 & 0.10 & $0.36^{a}$ & 0.16 & $-0.45^{a}$ & 0.20 & 0.06 & 0.09 \\
\hline
\end{tabular}

$-=$ not available.

${ }^{a}$ Estimate is significant, different from zero at the 95 percent significance level.

Table 8-Average annual biomass (tons) growth, removals, and mortality per year on National Forest System land between 2001-2006 and 2006-2010.

\begin{tabular}{|c|c|c|c|c|c|c|c|c|}
\hline & \multicolumn{2}{|c|}{ Forest land } & \multicolumn{2}{|c|}{ Timberland } & \multicolumn{2}{|c|}{ Reserved forest land } & \multicolumn{2}{|c|}{$\begin{array}{l}\text { Low-productivity } \\
\text { forest land }\end{array}$} \\
\hline & Total & SE & Total & SE & Total & SE & Total & SE \\
\hline & \multicolumn{8}{|c|}{ Thousand short tons per year } \\
\hline Growth & 17,576 & 788 & 13,456 & 779 & 3,258 & 306 & 1,215 & 155 \\
\hline Mortality & 15,051 & 1,433 & 9,594 & 1,229 & 4,870 & 750 & 977 & 329 \\
\hline Removals & 698 & 247 & 669 & 246 & 29 & 34 & - & - \\
\hline Change & 1,827 & 1,631 & $3,193^{a}$ & 1,419 & $-1,642^{a}$ & 725 & 238 & 362 \\
\hline
\end{tabular}

$-=$ not available.

${ }^{a}$ Estimate is significant, different from zero at the 95 percent significance level. 


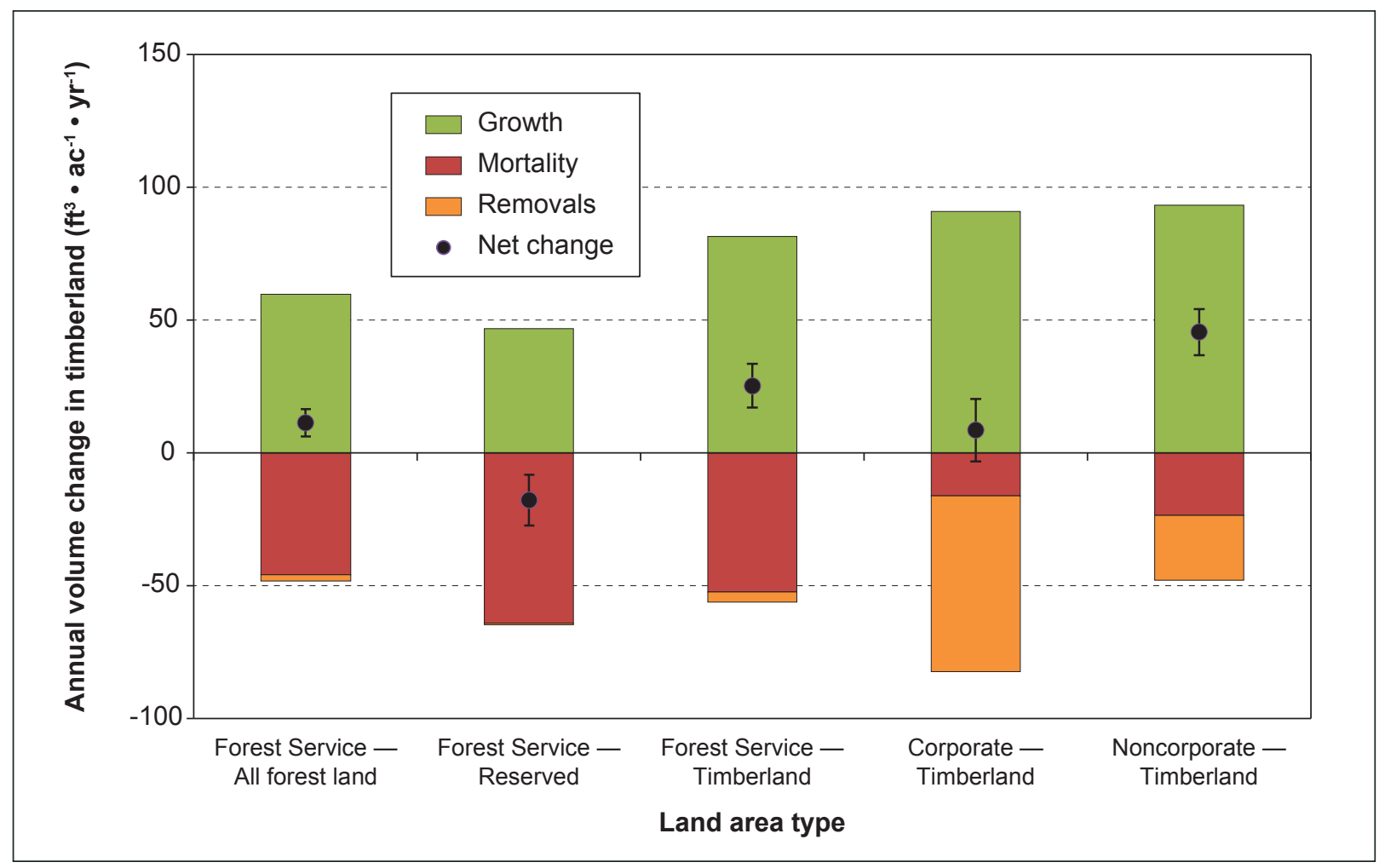

Figure 34-Combined average annual change in volume (cubic feet) growth, removals, and mortality per acre per year on national forest land between 2001-2006 and 2006-2010 by land status compared to privately owned timberland between 1991-1994 and 2007-2010 in California (error bars represent sampling error). Although volume changes are on an annual peracre basis, it is important to note that Forest Service estimates of change cover a different timeframe than private timberland.

\section{How Many Trees Show Signs of Damaging Agents?}

Detecting damage to trees is a critical means to assess overall forest health in terms of resiliency, diversity, function, and resource sustainability. Mortality and disturbance resulting from damage inflicted by native insects, disease, or abiotic stressors such as fire and weather events are agents of natural succession, promoting growth, regeneration, and diversity in California's forests. Natural disturbance resulting from these damages can affect ecosystem structure, composition, and function. Forest Inventory and Analysis crews collect data on damaging agents for each measured live tree.

Overall, about 19 percent of all live trees $\geq 1$ inch d.b.h. were affected by one or more damaging agents. Recorded agents included damage from animals, bark beetles, cankers, insect defoliators, dwarf mistletoe, leafy mistletoe, foliage diseases, stem decays, root disease, and weather-related injury. Physical damage or injury was most commonly recorded, affecting about 68 percent of damaged trees. Stem decays were recorded on about 19 percent of the affected tree volume (fig. 35). Dwarf mistletoe and root diseases each affected about 11 percent of the tree volume (fig. 36). All other recorded damage agents individually contributed less than 3 percent of the affected tree volume. Thirteen percent of the most commonly found conifer species in California (Douglas-fir) had some form of damage. White fir, the next most common conifer, had about a 28 percent incident rate. For hardwood species, tanoak, the most common hardwood species, had about an 11 percent damage rate. Canyon live oak, the next most common hardwood species, had a damage rate of about 15 percent. 


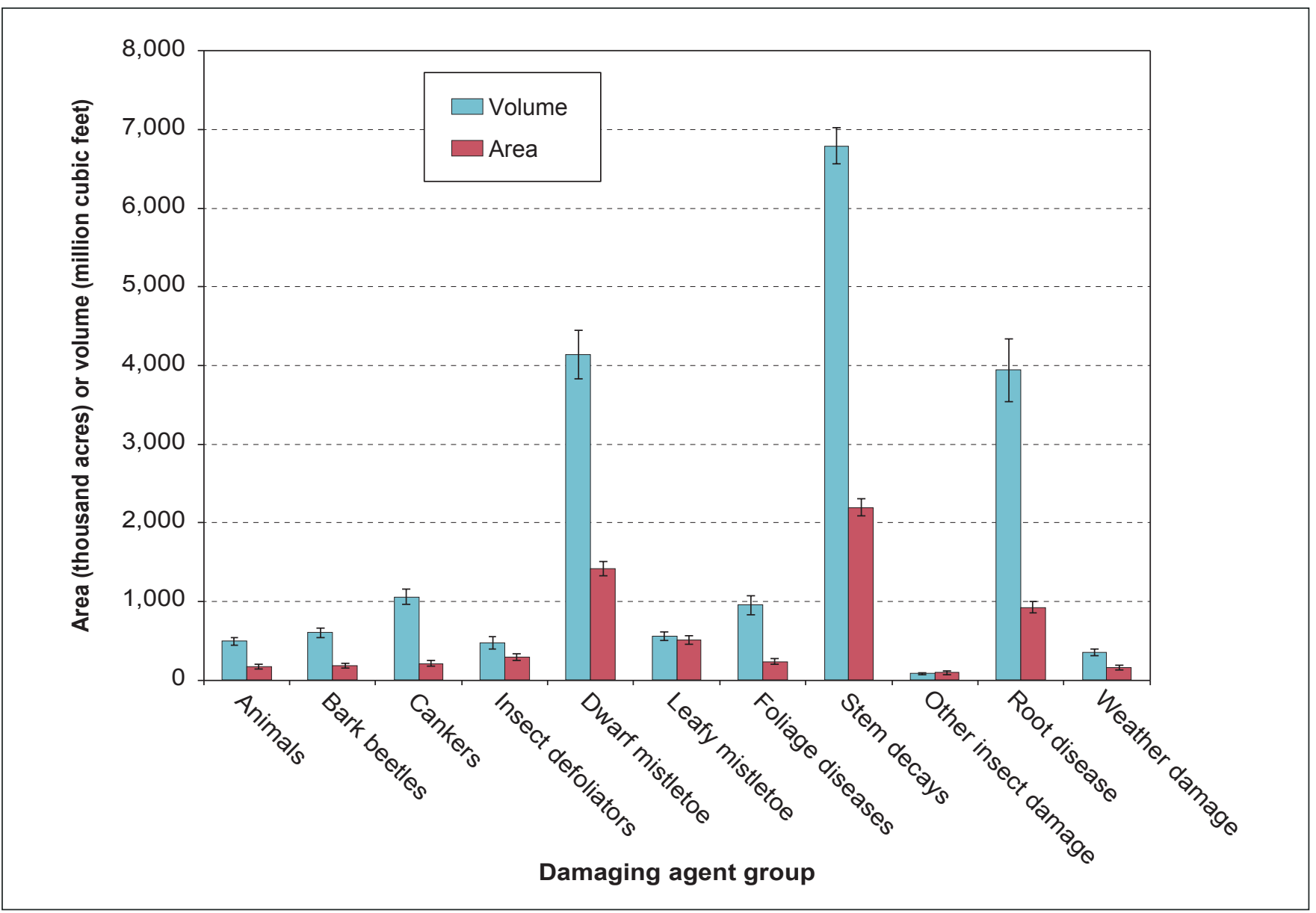

Figure 35-Area and volume of live trees affected by one or more agents on forest land in California, 2001-2010; volume is gross volume of live trees $>5$ inches diameter at breast height; acres are those with $>25$ percent of the basal area with damage.

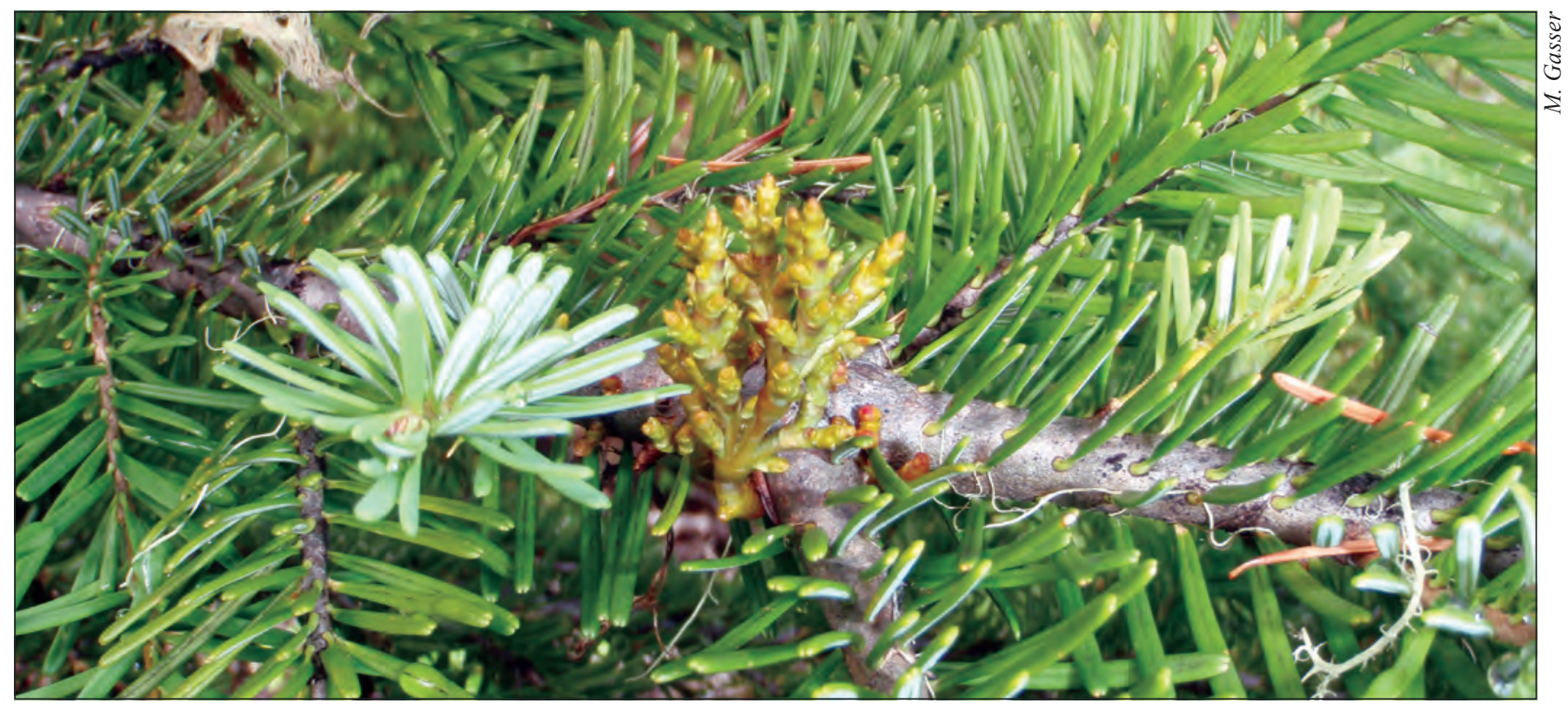

Figure 36-Grand fir branch showing mistletoe infection. 


\section{What Types of Understory Vegetation Are Most Common in California's Forests?}

Understory vegetation is an important structural component in all forest ecosystems. The life-form type and density of cover from bare soil up into the tree crowns influences wildlife habitat, wildfire behavior, and competition between native and nonnative plants. Forest Inventory and Analysis crews sampled understory vegetation on each subplot on forest land (fig 37). Total cover was estimated for tree seedlings and saplings $<5$ inches d.b.h., shrubs, forbs, and graminoids. Total cover of all four of these life forms and of bare mineral soil was estimated.

Cover of understory vegetation in California was greater in hardwood forest types than softwood types. Within each cover type, hardwood forests tended to have greater vegetation cover in both shrub and graminoid cover types and the least area of bare soil (fig. 38). The most shrub cover within hardwood forests is found within the moist forest types, the elm/ash/cottonwood group, and the alder/ maple group (fig. 39). Each of these forest types tended to

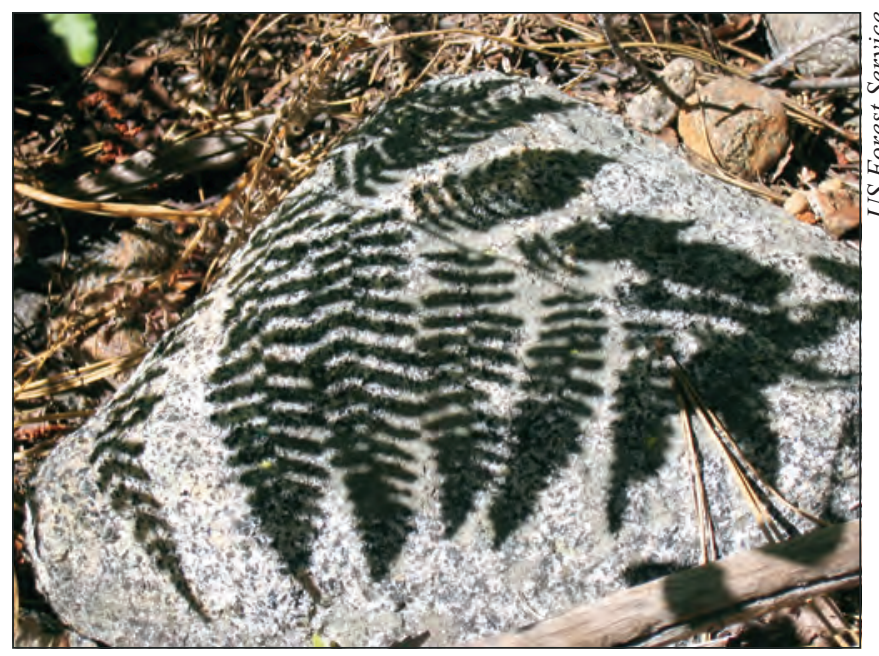

Figure 37-Bracken fern, as outlined in this shadow, is a common understory plant found throughout the state.

have an average shrub cover of more than 30 percent. The greatest cover of seedlings and saplings within hardwood forests was in aspen/birch forest types and averaged about 13 percent cover. For softwoods, Douglas-fir forests tended to have the greatest cover of seedlings and saplings but at

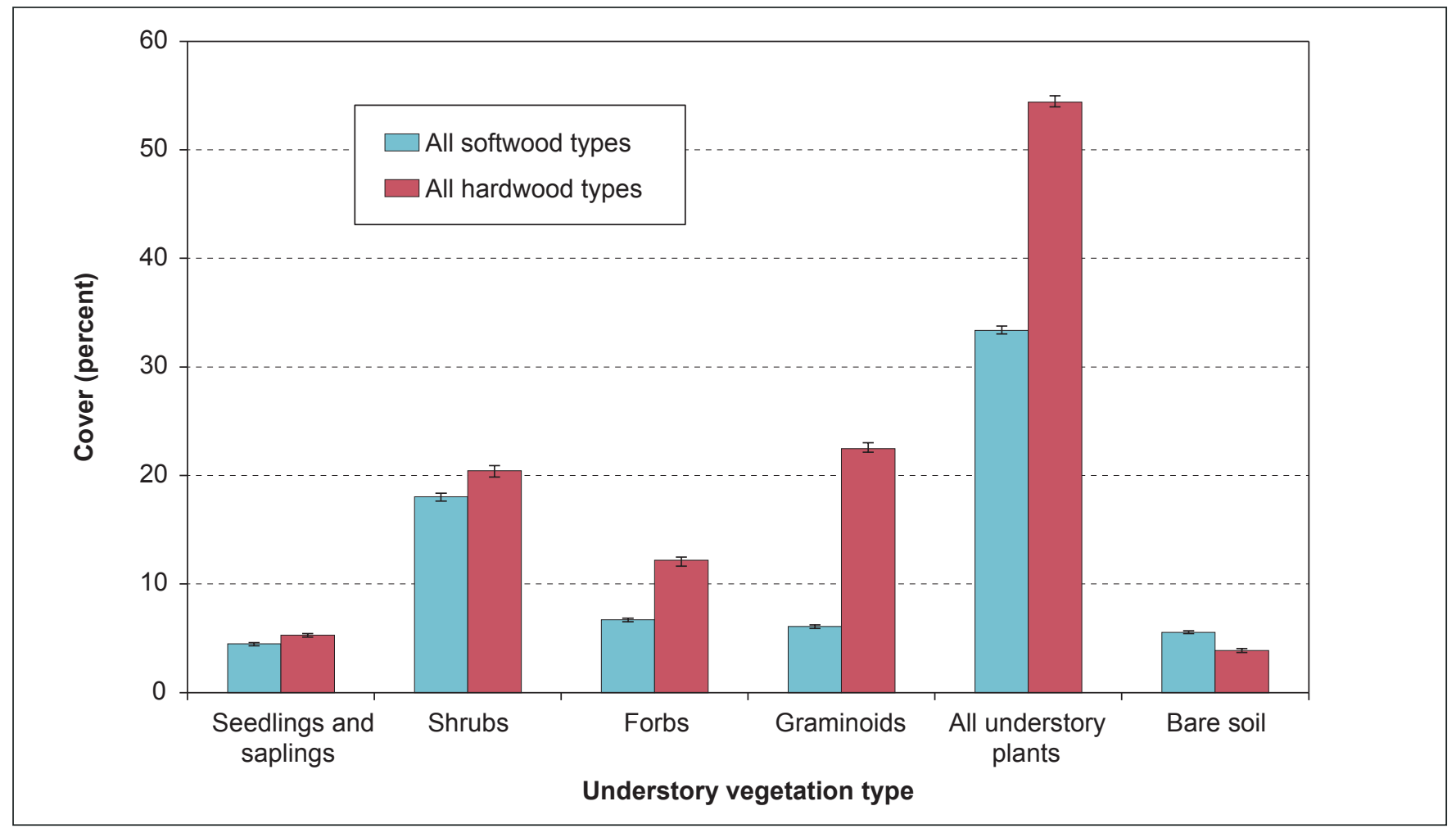

Figure 38-Cover of vegetation life forms and bare soil by softwood and hardwood forests on forest land in California, 2001-2010. 
only about 9 percent cover. For all understory life forms within the softwood forests, redwood and hemlock/Sitka spruce forests had the densest cover; each averaged more than 50 percent cover of understory vegetation. Average percentage of cover was the greatest for all understory life forms in younger forests ranging from 0 to 39 years old (fig. 40). Average cover for all understory plants tended to decline with increasing forest age.

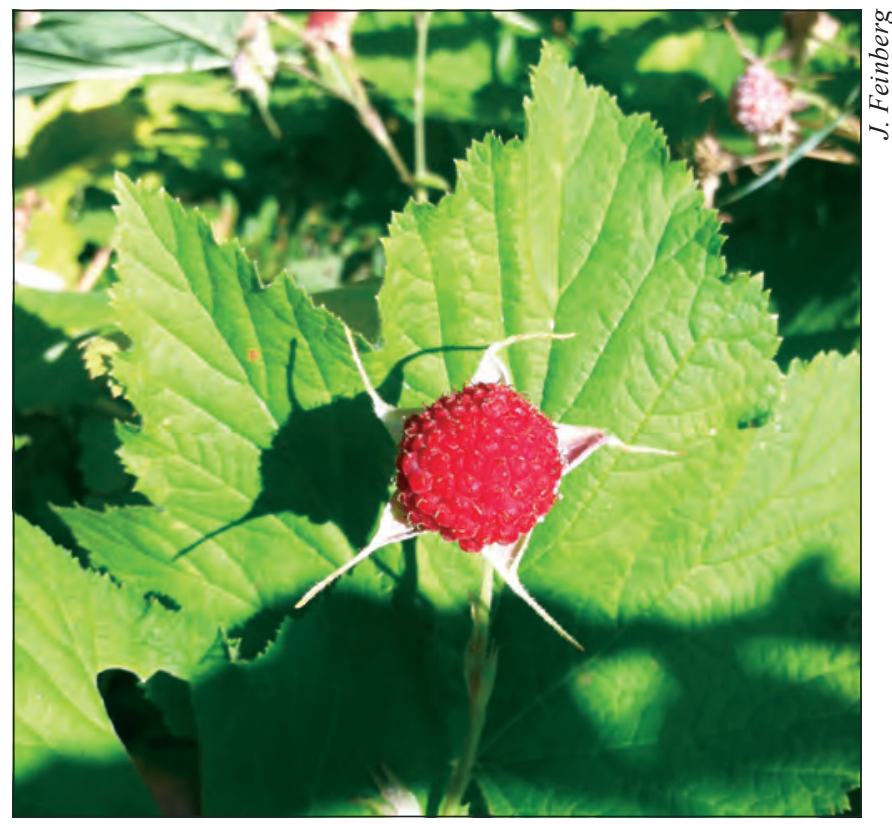

Figure 39-Native to California and found in the understory typically where it is cool and moist, thimbleberry is related to the cultivated red raspberry.

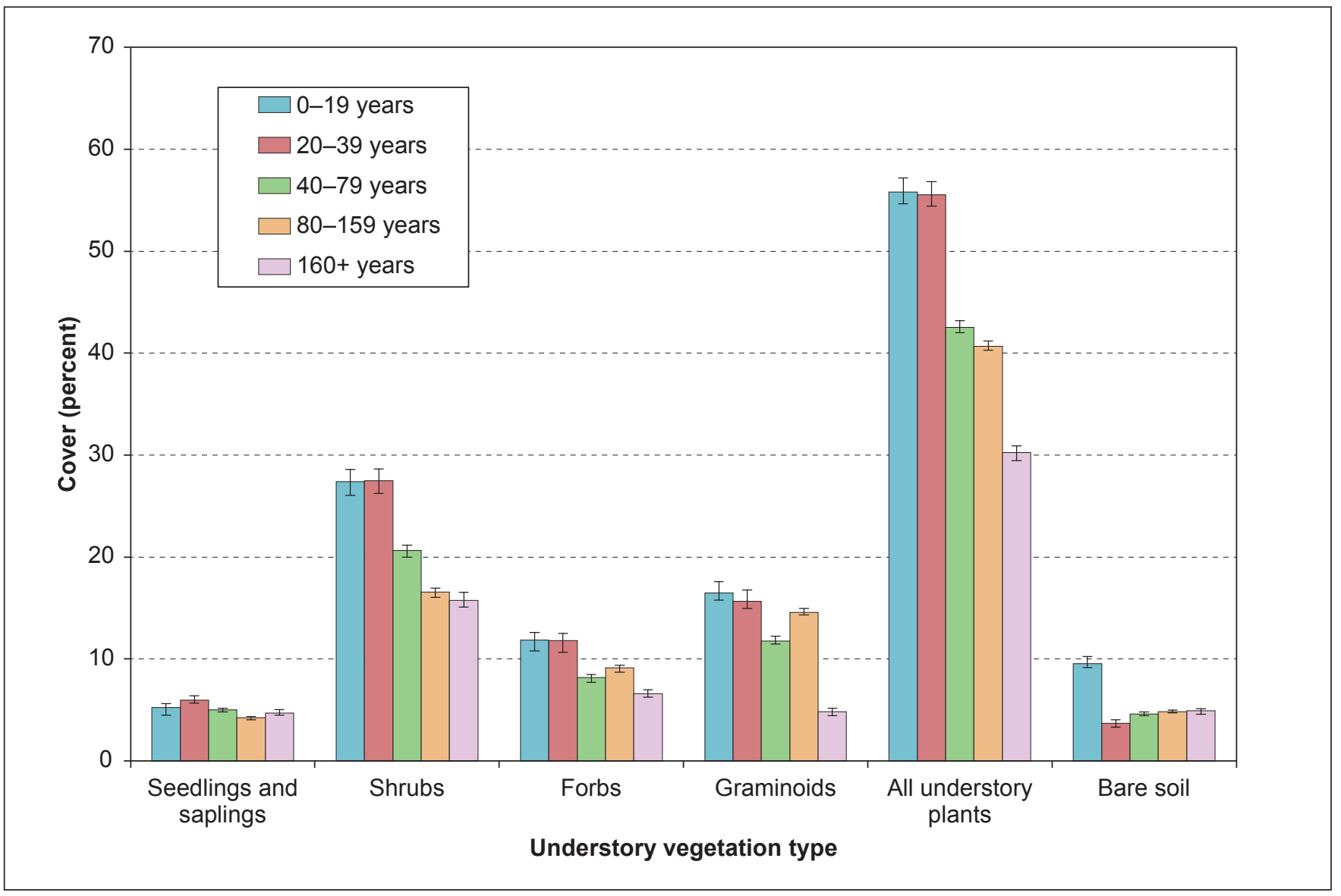

Figure 40-Cover of vegetation life forms and bare soil by forest age class on forest land in California, 2001-2010. 


\section{What Nonnative Invasive Plants Are Found in California's Forests?}

Invasive nonnative plant species pose a threat to native forests throughout California. The aggressive spread of introduced species to forested areas formerly occupied by native plants changes the composition, structure, and function in both natural and managed ecosystems. Because they directly compete for habitat with native species, including those desired for their ecological and potential economic benefits to the state, considerable efforts to contain their undesirable effects must be expended. While most local effects from nonnative species are easier to quantify, without a statewide sampling effort such as the FIA plots, it would be very difficult to understand the extent of these species across all forested landscapes and ownerships. Forest Inventory and Analysis crews estimate the cover of the three plant species with the highest cover in each of three life forms; shrub, forb, and graminoid - as well as of any other species with $\geq 3$ percent cover. Because the definition of "invasive" is subjective, all readily identifiable species that were listed as nonnative to the United States (USDA NRCS 2000) were selected for analysis.

Twenty-five different nonnative species were recorded on 5,575 forested plots. Cheatgrass was found on 11 percent of all sampled plots and was the most commonly recorded nonnative species (see "Scientific and Common Plant Names"). Cheatgrass also affects the largest area, about 185,000 acres (figs. 41 and 42). Of the forb species, spreading hedgeparsley is the most commonly found and is estimated to cover about 88,000 acres of forest land. Himalayan blackberry is the most abundant nonnative shrub species found in California's forests. We estimated this species to cover about 42,000 acres of forest land.

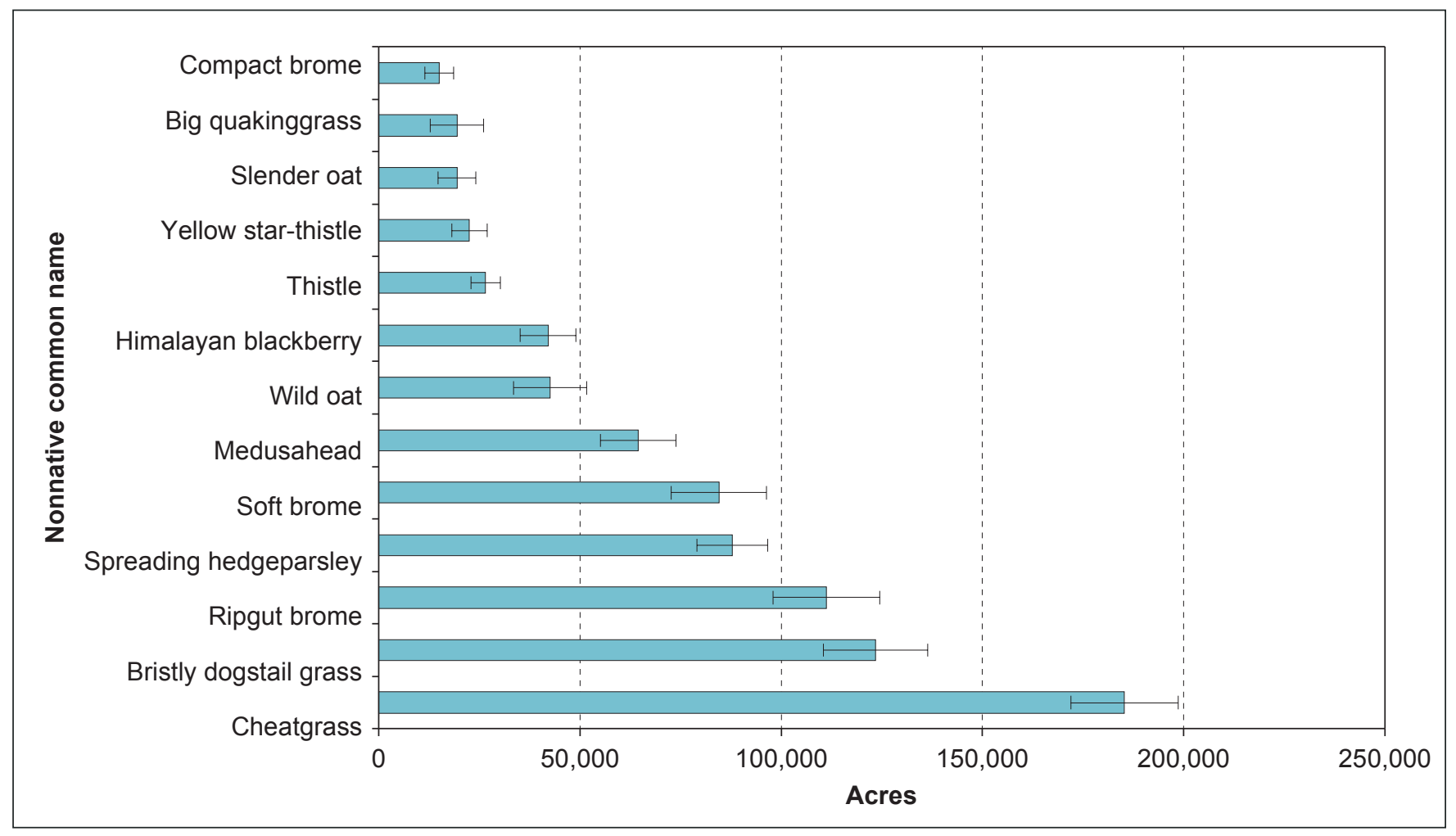

Figure 41-Most frequently found nonnative invasive species on forest land in California, 2001-2010. Only those nonnative species estimated to cover at least 10,000 acres are shown. 


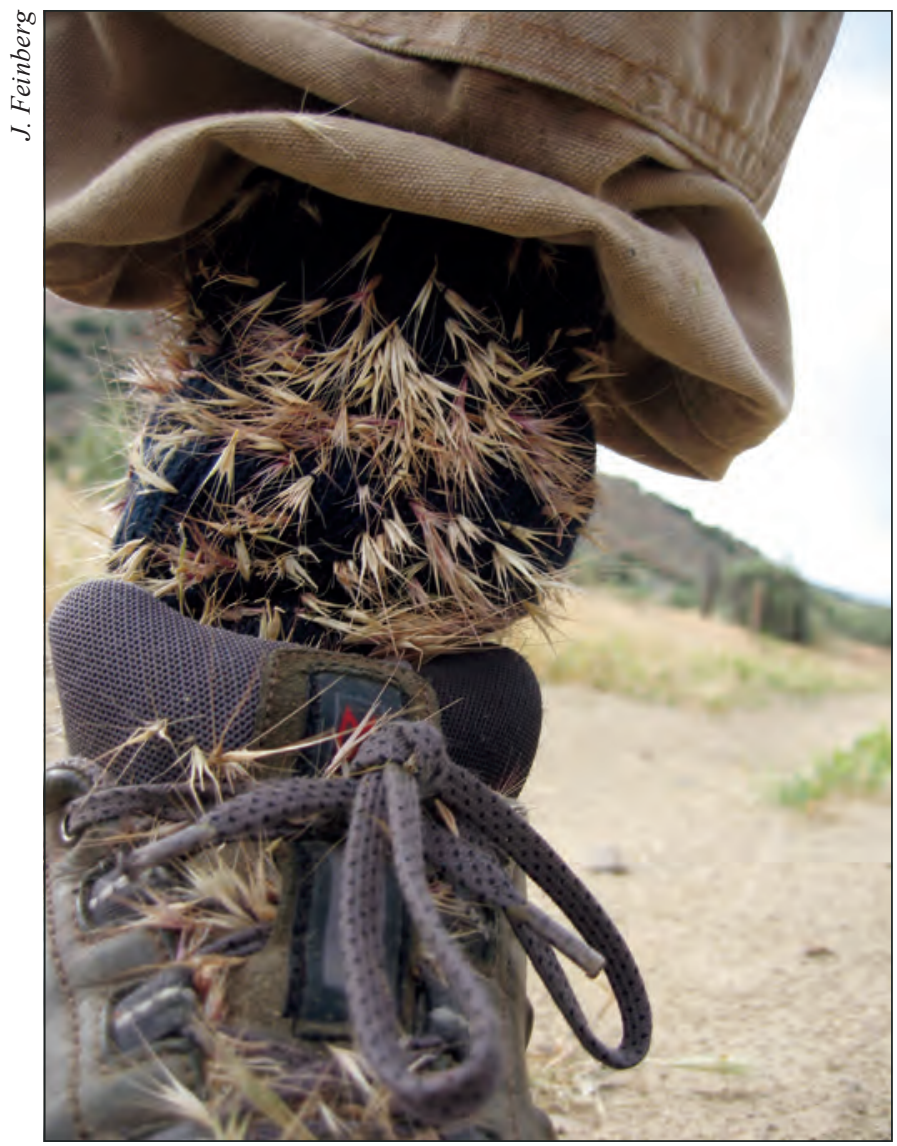

Figure 42-Cheatgrass is the most common nonnative invasive species found on forest land in the state. It uses "hitchhiking" as one mode of seed transport.

\section{Research Application-Nonnative Invasive plants of the Pacific Coast ${ }^{3}$}

Nonnative plants affect the composition and function of natural and managed forests and have large economic costs through degraded land use and eradication expense. Nevertheless, we don't have much comprehensive information on the abundance, distribution, and impact of nonnative invasive plants. The first step to assessing the problem and then dealing with it is to be able to identify the most problematic species from among the thousands of plant species found in California's forests. In collaboration with the University of Washington, the Institute of Applied Ecology, and botanical experts across the west coast, FIA developed a prioritized list of nonnative invasive plants affecting forest lands in the Pacific coastal states of California, Oregon, and Washington, and provided enough detail in nontechnical language, along with photos of different stages of plant development, to allow reliable identification in the field (Gray et al. 2011). The final list of 56 species was designed to capture species believed to be most prevalent or problematic for use by strategic forest inventories and novice botanists.

\section{Citation}

Gray, A.N.; Barndt, K.; Reichard, S.H. 2011.

Nonnative invasive plants of Pacific coast forests: a field guide for identification. Gen. Tech. Rep. PNW-GTR-817. Portland, OR: U.S. Department of Agriculture, Forest Service, Pacific Northwest Research Station. 91 p.

\footnotetext{
${ }^{3}$ Author: Andrew Gray.
} 


\section{Research Application-}

\section{Lichen-Based Indicators of Nitrogen Pollution in California's Forests ${ }^{4}$}

Nitrogen $(\mathrm{N})$ is the most common chemical element in living tissues after oxygen, carbon, and hydrogen. Most terrestrial and marine systems are naturally N-limited, making $\mathrm{N}$ a fundamental driver of ecosystem productivity. For this reason, excess $\mathrm{N}$ from anthropogenic sources (e.g., motor vehicle exhaust, crop fertilizers, emissions from livestock) can profoundly reshape forest structure and function (Pardo et al. 2011). Species decline or increase depending on their capacity to utilize the new $\mathrm{N}$ and remain competitive. In parts of Mediterranean California, $\mathrm{N}$ alters the fire cycle by increasing fuel buildup of fast-growing understory plants and leaf senescence of negatively impacted species (Fenn et al. 2010). Lichens have become the canaries in the coal mine for mapping $\mathrm{N}$ because even small $\mathrm{N}$ increases cause the replacement of native lichen floras by "weedy" eutrophic lichen species (Jovan et al. 2012; fig. 43). Our recent research demonstrates the accuracy of lichen-based $\mathrm{N}$ estimates by comparing lichen metrics with $\mathrm{N}$ pollutant measurements.

Lichens were first recognized as "health meters for the air" in 1866 by Finnish botanist William Nylander. Today large-scale monitoring programs commonly use lichens as a budget-friendly supplement to instrumented air quality monitors, which may cost $\$ 10,000$ or more to operate annually. The FIA program monitors tree-dwelling lichens at a subset of grid sites, amounting to over 5,500 observations made nationwide since 1998. Continually improving accuracy has increased the utilization and benefit of lichen-based estimates of pollution to policymakers and managers tasked with deciding on allowable pollutant emissions.

The San Bernardino Mountains (SBM) in particular have become a natural laboratory for studying lichen indicators for $\mathrm{N}$ and refining techniques. Excess $\mathrm{N}$ is a key air quality and ecological issue here; the SBM form the leeward boundary of the Los Angeles Basin and include forests

\footnotetext{
${ }^{4}$ Author: Sarah Jovan.
}

receiving the highest $\mathrm{N}$ levels recorded for North America, up to $70 \mathrm{~kg} \mathrm{~N}$ per hectare per year at one site (as compared to natural background levels of 1 to $2 \mathrm{~kg} \mathrm{~N}$ per hectare per year). Lichen and instrument-based monitoring began in the 1970s (Sigal and Nash 1983, Temple et al. 2005) along an east-west transect of $\mathrm{N}$ deposition (Fenn et al. 2010).

Scientists from the U.S. Forest Service monitor an unprecedented array of air pollutants along the transect, allowing us to challenge some widely held assumptions about how lichens respond to N. We found that eutrophs increase on tree trunks as a function of total $\mathrm{N}$ deposition (i.e., the totality of $\mathrm{N}$ pollutants washed from the atmosphere in wet and dry deposition; fig. 44). The high correlation between eutrophs and total $\mathrm{N}\left(\mathrm{r}^{2}=0.94\right)$ demonstrates how eutroph abundance may be used for high-accuracy $\mathrm{N}$ mapping. Additionally, lichen metrics are a better representation of $\mathrm{N}$ deposition than ammonia measurements, especially in the SBM where the $\mathrm{N}$ regime is dominated by oxidized N pollutants. Cost-effective tools like this greatly

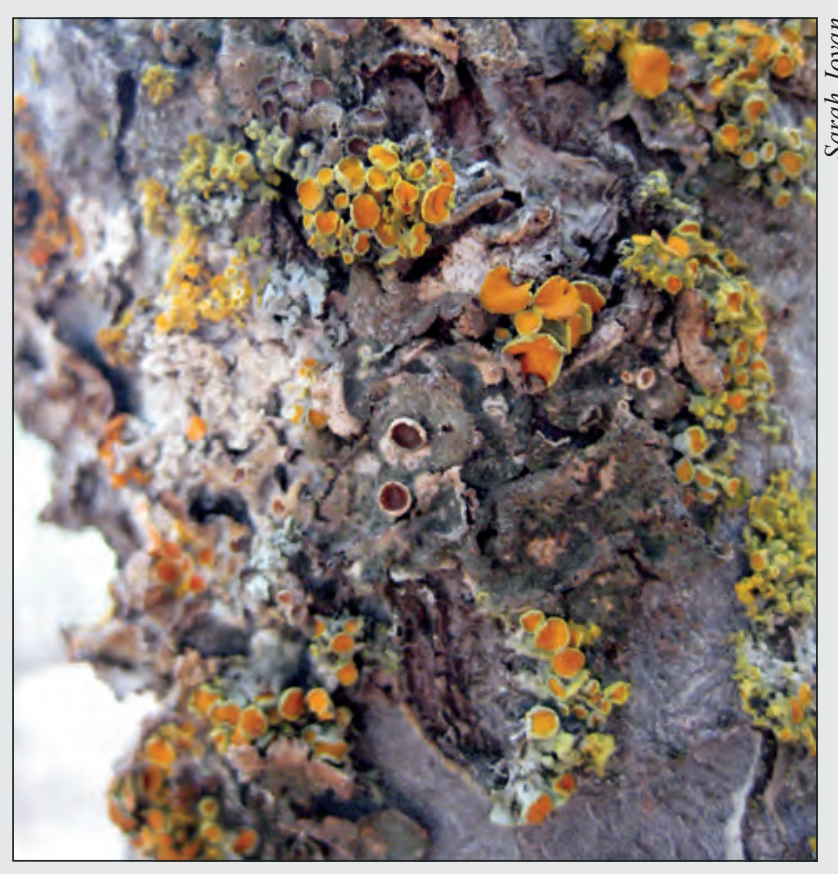

Figure 43 -At one nitrogen $(\mathrm{N})$-affected site, the sunburst lichen (orange) thrives while the less N-tolerant camouflage lichen (green and bleached white) appears stressed. 

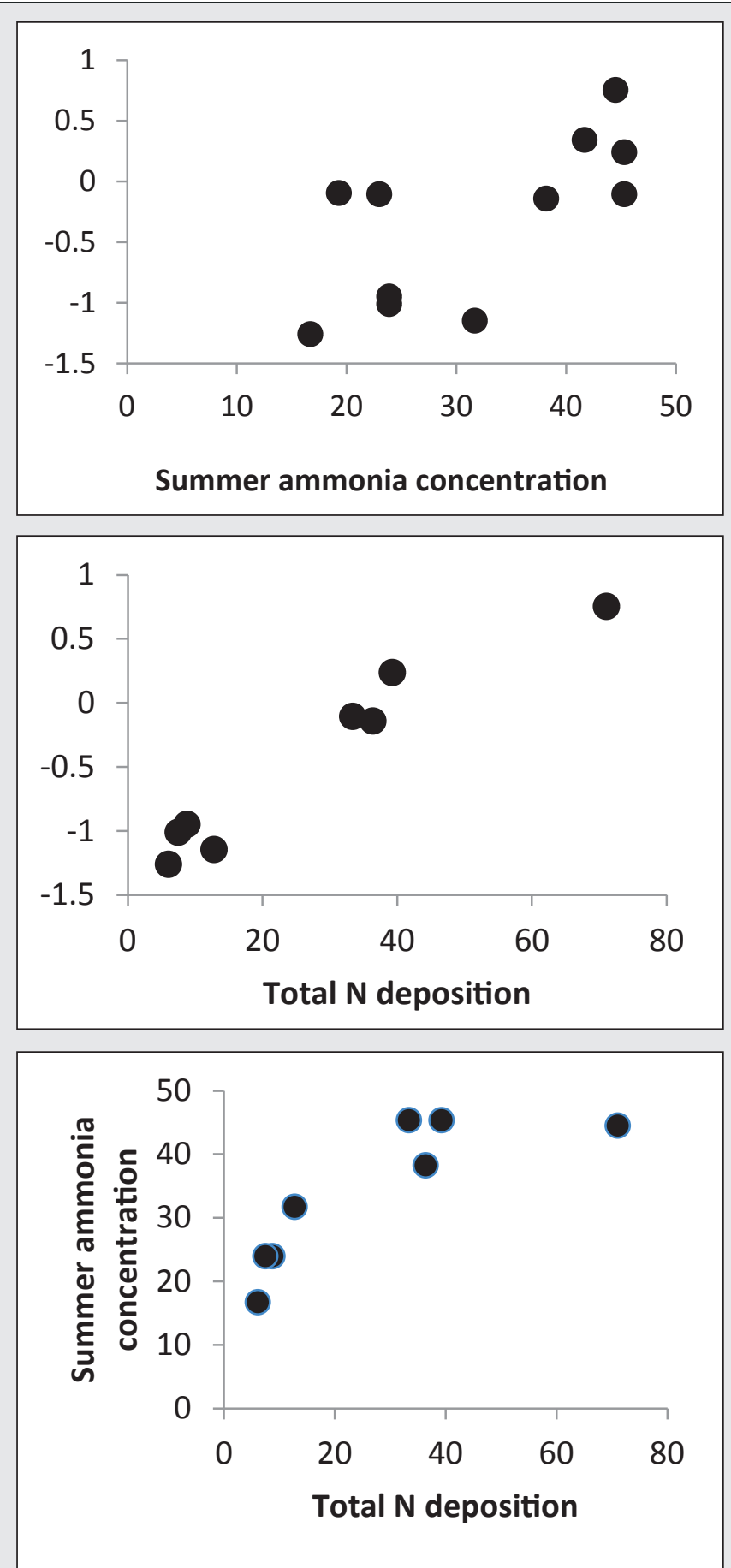

Figure 44-Scatterplots showing a strong correlation between an index of eutroph lichens and total nitrogen $(\mathrm{N})$ as measured in throughfall under a Pinus ponderosa canopy (A). The eutroph index was moderately correlated with summer ammonia concentrations (B), one component of total N. Oxidized forms of N (i.e., $\mathrm{NO}_{\mathrm{x}}$ ) are prevalent at high $\mathrm{N}$ sites, lessening the relationship between total $\mathrm{N}$ and ammonia. increase the scope and intensity of information available to managers on the distribution of $\mathrm{N}$ inputs across the landscape, leading to more informed decisions. This is critical for California where $\mathrm{N}$-compromised ecosystems are commonly reported (Fenn et al. 2010) and widely spaced instrumented networks can't capture the variability in N. For more information, see Jovan et al. 2012.

\section{Citations}

Fenn, M.E.; Allen, E.B.; Weiss, S.B.; Jovan, S.;

Geiser, L.H.; Tonnesen,G.S.; Johnson,R.F.;

Rao, L.E.; Gimeno, B.S.; Yuan, F.; Meixner, T.;

Bytnerowicz, A. 2010. Nitrogen critical loads and management alternatives for $\mathrm{N}$-impacted ecosystems in California. Journal of Environmental Management. 91(12): 2404-2423.

Jovan, S.; Riddell, J.; Padgett, P.E.; Nash, T.H., III; 2012. Eutrophic lichens respond to multiple forms of $\mathrm{N}$ : implications for critical levels and critical loads research. Ecological Applications. 22(7): 1910-1922.

Pardo, L.H.; Fenn, M.E.; Goodale, C.L.; Geiser, L.H.; Driscoll, C.T.; Allen, E.; Baron, J.S.; Bobbink, R.; Bowman, W.D.; Clark, C.M.; Emmett, B.; Gilliam, F.S.; Greaver, T.L.; Hall, S.J.; Lilleskov, E.A.; Liu, L.; Lynch, J.A.; Nadelhoffer, K.J.; Perakis, S.S.; Robin-Abbott, M.J.; Stoddard, J.L.; Weathers, K.C.; Dennis, R.L. 2011. Effects of nitrogen deposition and empirical nitrogen critical loads for ecoregions of the United States. Ecological Applications. 21(8): 3049-3082.

Sigal, L.L.; Nash, T.H., III 1983. Lichen communities on conifers in southern California mountains: an ecological survey relative to oxidant air pollution. Ecology. 64: 1343-1354.

Temple, P.J.; Bytnerowicz, A.; Fenn, M.E.; Poth, M.A. 2005. Air pollution impacts in the mixed conifer forests of southern California. In: Kus, B.E.; Beyers, J.L., tech. coords. Planning for biodiversity: bringing research and management together. Gen. Tech. Rep. PSW-GTR-195. Albany, CA: U.S. Department of Agriculture, Forest Service, Pacific Southwest Research Station: 145-164. 
How Has Fire Influenced California's Forests?

Wildland fire plays a significant role in shaping California's forests (fig. 45). As the population continues to grow, we increase our reliance on healthy forests and the ecosystem services they provide, such as clean water, wildlife habitat, carbon sequestration, wood products, and recreation. Understanding how to manage wildland fire hazards to threatened resources and forest land becomes ever more critical. To measure the overall impact of wildfire on forest land across the state, FIA crews estimate evidence of surface and crown fire that occurred since the previous plot visit, usually 5 to 10 years earlier. Compiling these disturbance estimates for all visited plots allows us to determine the average forest area and percentage of forest land burned each year in California.

Between 1995 and 2008, the estimated average annual forested area that burned was 0.75 percent, or about 249,000 acres (SE: 16,159 acres) across the state. By region, there is high variability from year to year. In 2008, the northern interior region had a record fire year where over 800,000 acres of forest land burned, pushing the statewide total to over 1 million acres that year. Following a prolonged drought, these mostly lightning-caused fires started early in the season in rugged inaccessible terrain, allowing them to burn an unprecedented area of forest land. This estimate of total area burned for 2008 parallels the 1.37 million acres reported by the California Department of Forestry and Fire Protection (2010), which also includes nonforested areas such as range and grasslands not currently measured by FIA. No clear annual statistical trends are found between regions, except for the jump in acres burned in 2008, owing to high regional variability (fig. 46).

Because fire is a relatively rare event at a single point on the landscape, the number of plots where evidence of fire is observed is small, and, in some years, crews have recorded no fire-damaged plots in an entire region such as the north coast. Also, since 2001 when FIA started measuring 10 percent of all plots annually, the sample size basis for any given year changes. For example, a 2000 fire incident could be recorded on any plot because all plots were installed between 2001 and 2010. However, a fire incident from 2009 can only be observed on plots measured in 2010. Thus, estimates for 2009 fire incidents have a higher sampling error because the sampling intensity is only about $1 / 10$ as compared to the 2000 fire incident estimate. Because of the changing sample size, the estimates of error also change. The most recent estimates, which are based on the smallest sample sizes, also tend to have the largest estimated error.

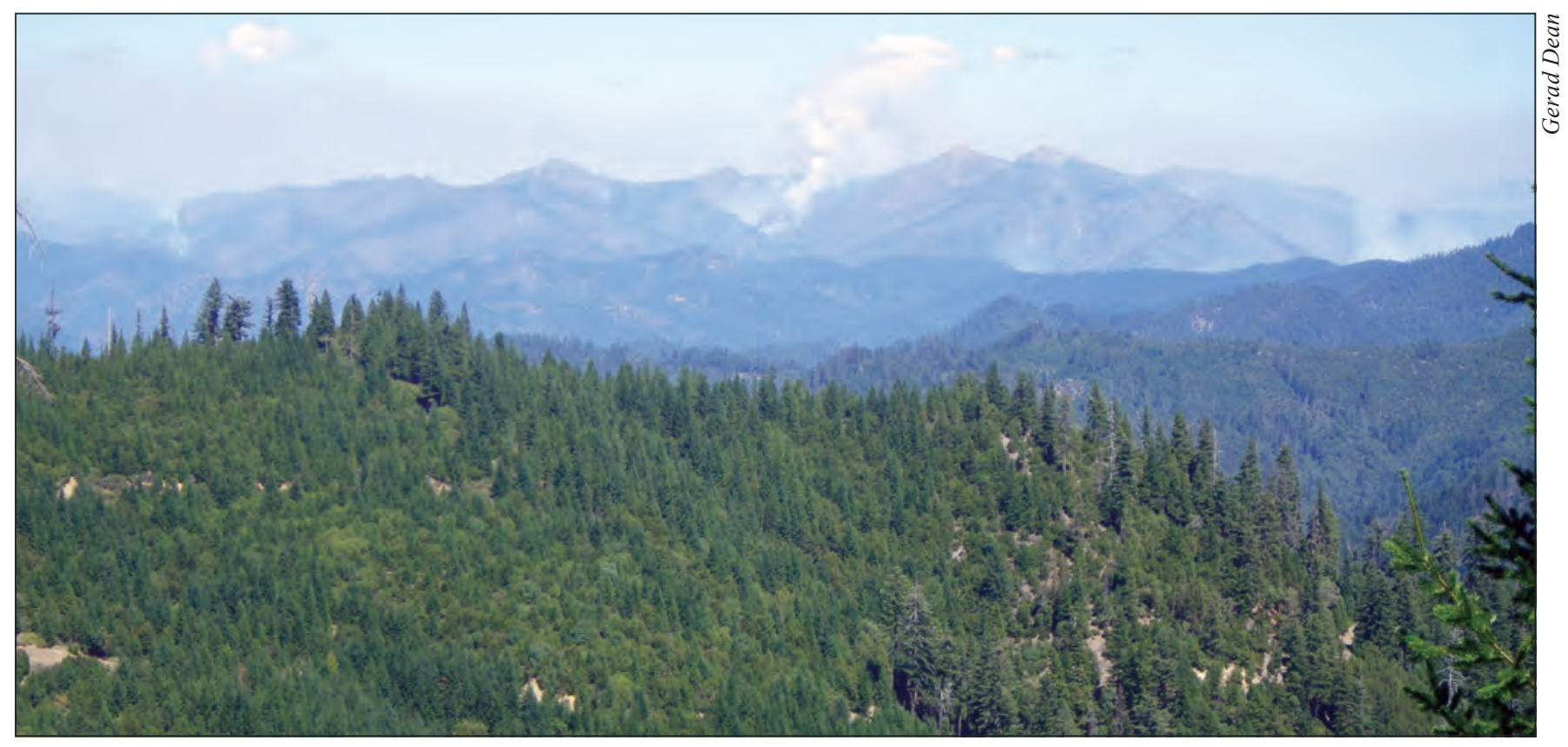

Figure 45-Late-summer smoke from multiple wildfires that can be seen burning in the forests of Siskiyou County. 


\section{Research Application-Modeling Fuel Treatment Opportunities and Outcomes ${ }^{5}$}

Owing to factors such as fire exclusion and reduced management activity on public lands due to declining resources, most dry mixed-conifer forests across the West rate high in fire hazard and low in resilience. While there is great interest in restoring the health and resilience of these forests, there is considerable variation in how these forests respond to mechanical fuel treatment and in the associated costs.

To gauge the likely success and economic viability of landscape-scale fuels management designed to restore health and resiliency to historically harvested and burned forests, we used BioSum (FIA's tool for Bioregional Inventory Originated Simulation Under Management) * to model effectiveness and costs of a handful of "generic" mechanical treatment prescriptions applied to 5,000 FIA inventory plots that provide a spatially balanced and statistically representative sample of dry mixed-conifer forests in California,

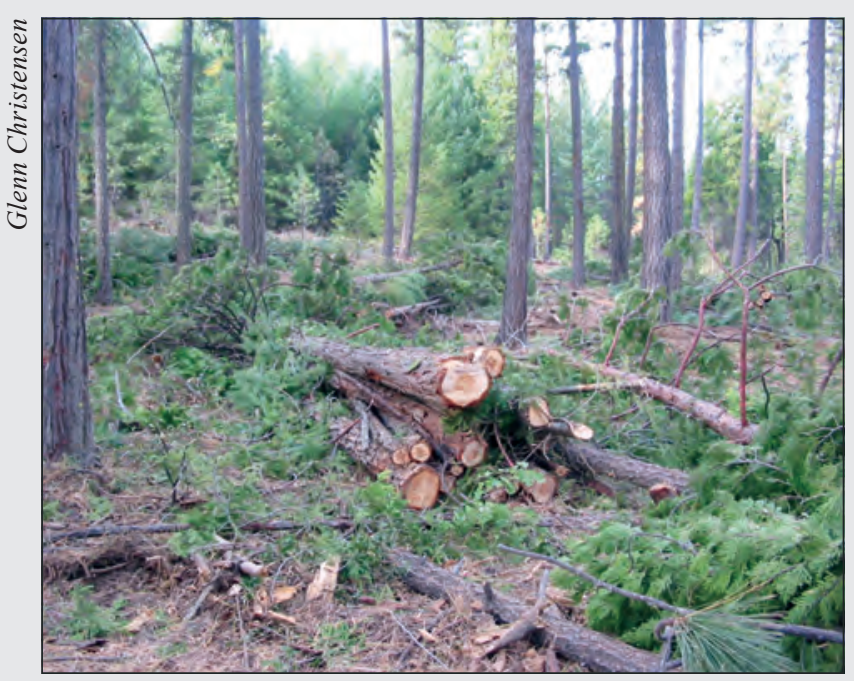

Figure 47-Data collected at Forest Inventory and Analysis plots are used in models that simulate mechanical fuel treatments, similar to this mechanized thinning, as the basis of research to evaluate the likely success and economic viability of landscape-scale fuels management.
Oregon, Washington, Idaho, Montana, and Utah (Jain et al. 2012) (fig. 47). We found that for up to a third of the forest area, implementing the locally most effective generic treatment led to more resilient forests over time as gauged by crown fire potential and surface flame length, lower forest carbon emissions, and greater retention of the live trees that constitute an important component of wildlife habitat.

In Douglas-fir, true fir, pine, and larch forests, modeling revealed that mechanical fuel treatments can recover, on average, 1,000 to $2,000 \mathrm{ft}^{3} \cdot \mathrm{ac}^{-1}$ of merchantable wood and earn net revenue of over $\$ 1,000$ per acre, even after covering $\$ 1,000$ to $\$ 2,000$ per acre in onsite treatment cost. Sales of energy wood-the tops and limbs of all harvested trees as well as the entirety of nonmerchantable trees - account for about a fifth of the total product value, so while somewhat helpful in achieving feasibility, bioenergy has limited potential for making a significant difference in financing fuel treatment at the broad scale. Work is ongoing to test other kinds of mechanical treatments and hybrid treatments that also involve fire and animals to explore cost effectiveness of fuels management in these forests.

\section{Citation}

Jain, T.; Battaglia, M.; Han-Sup, H.; Graham, R.; Keyes, C.; Fried, J.; Sandquist, J. 2012. A comprehensive guide to fuels management practices for dry mixed-conifer forests in the northwestern United States. Gen. Tech. Rep. RMRS-GTR-292. Fort Collins, CO: U.S. Department of Agriculture, Forest Service, Rocky Mountain Research Station. 331 p. http://www.treesearch.fs.fed.us/pubs/42150.

\footnotetext{
${ }^{5}$ Author: Jeremy Fried

*http://biosum.info
} 


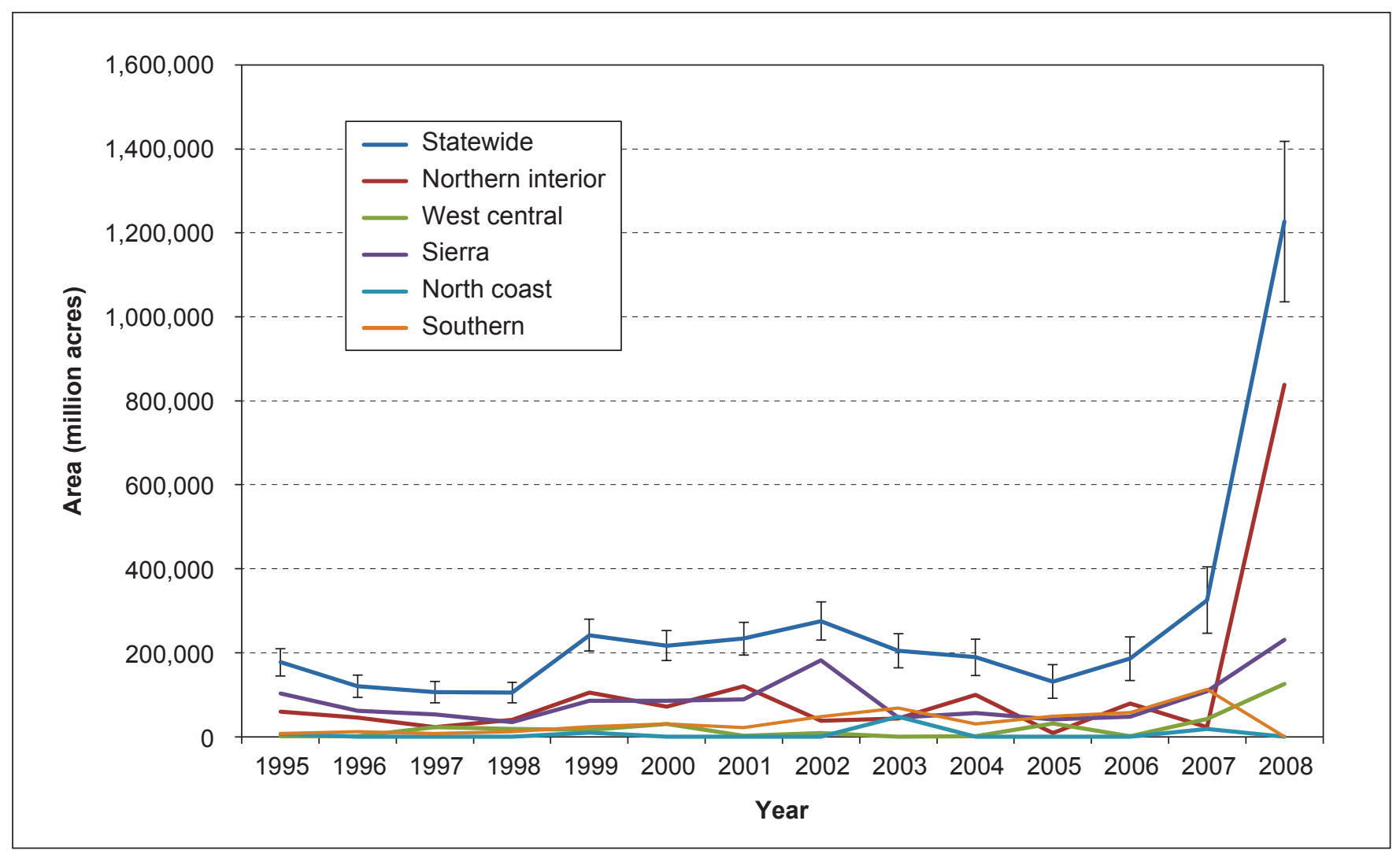

Figure 46-Area of forest burned by ecosection group (see fig. 7) on forest land in California, 1995-2008. Note: 2009 is not included in the chart because it is too small of a sample size and includes only a single year of plot measurements from 2010 (10 percent of all plots in the 10 -year cycle), when no crew reported a plot affected by fire the previous year.

\section{How Productive Are California's Forests?}

\section{California's Timberlands}

Productivity is measured by site class, an indicator of a forest's potential annual growth that can be used to describe the overall potential of timberland to produce wood. Most of California's timberland grows on moderately productive sites, classified here as 85 to 164 cubic feet per acre per year of volume. About 54 percent (9.3 million acres) of the state's timberland area falls into this category. The most productive sites, those with a potential growth rate of more than 164 cubic feet per acre per year, occupy nearly 10 percent $(1.7$ million acres) of the timberland area.

California mixed conifer, the prevalent forest type in the state, has about 64 percent of its area in the moderate site class (fig. 48). Of the softwood forest types, the redwood group has the greatest proportion of its timberland area in the most productive classes, about 64 percent (fig. 49). For the least productive sites ( 20 to 84 cubic feet per acre per year), western juniper has the greatest proportion of its acreage in these classes, over 90 percent. Most of the timberland area for hardwood forest types is in the moderately productive site classes, about 55 percent.

Timberland managed by the Forest Service is mostly on moderately productive site classes: about 51 percent is found on site classes between 85 to 164 cubic feet per acre per year (fig. 50). However, a large portion, about 44 percent, of Forest Service-managed timberland is found on the lowest site classes. Private ownerships also are primarily found on moderately productive sites, about 48 percent. Private ownerships tend to have a greater proportion of timberland in the highest classes, nearly 13 percent of all private timberland. Together these timberlands contribute to the state's total timber harvest, which was 1.7 billion board feet in 2006 (Morgan et al. 2012). Total sales value was about $\$ 1.5$ billion in 2006, with lumber accounting for 64 percent of the total (Morgan et al. 2012). 


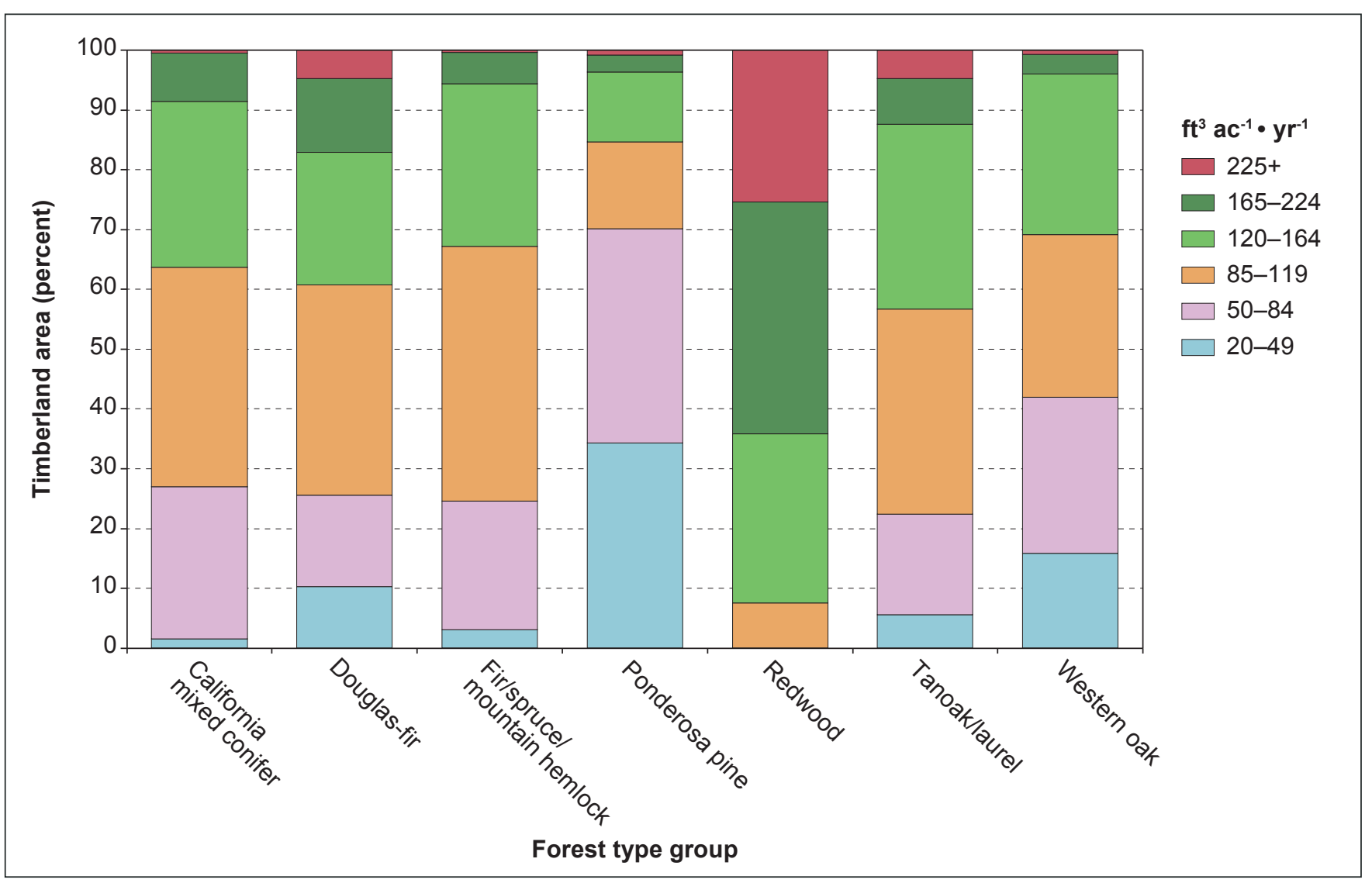

Figure 48-Percentage of timberland area by major forest type groups and site class in California, 2001-2010.

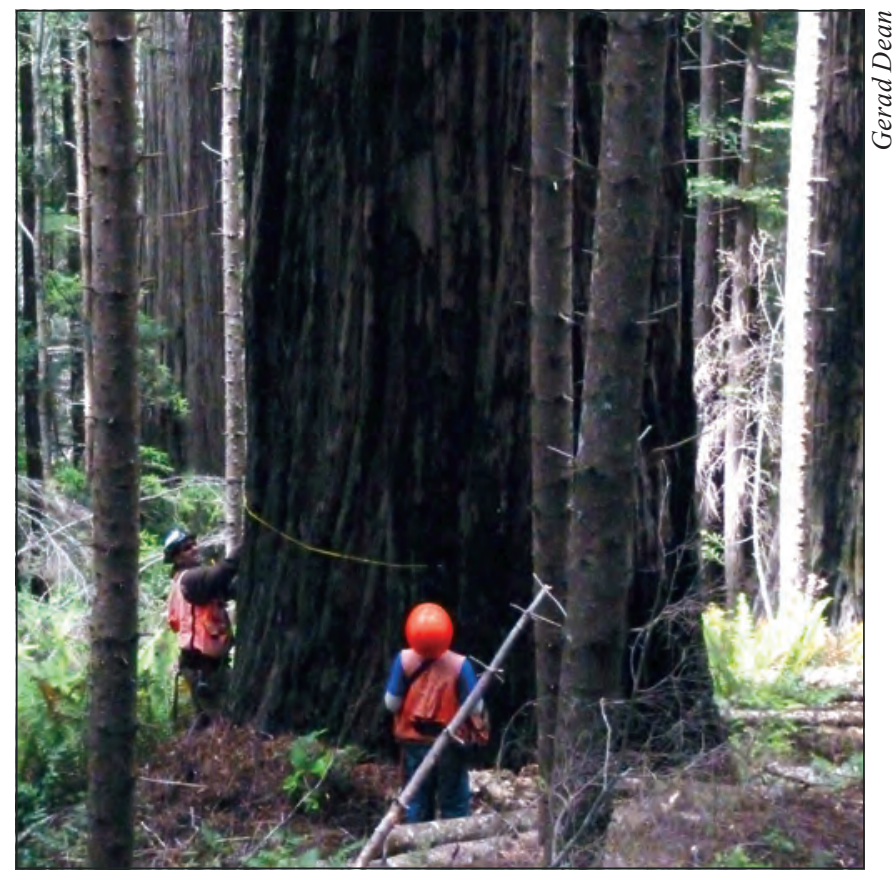

Figure 49-The redwood forest type, as seen here in Humboldt County, has the greatest proportion of timberland area in the most productive site class. 


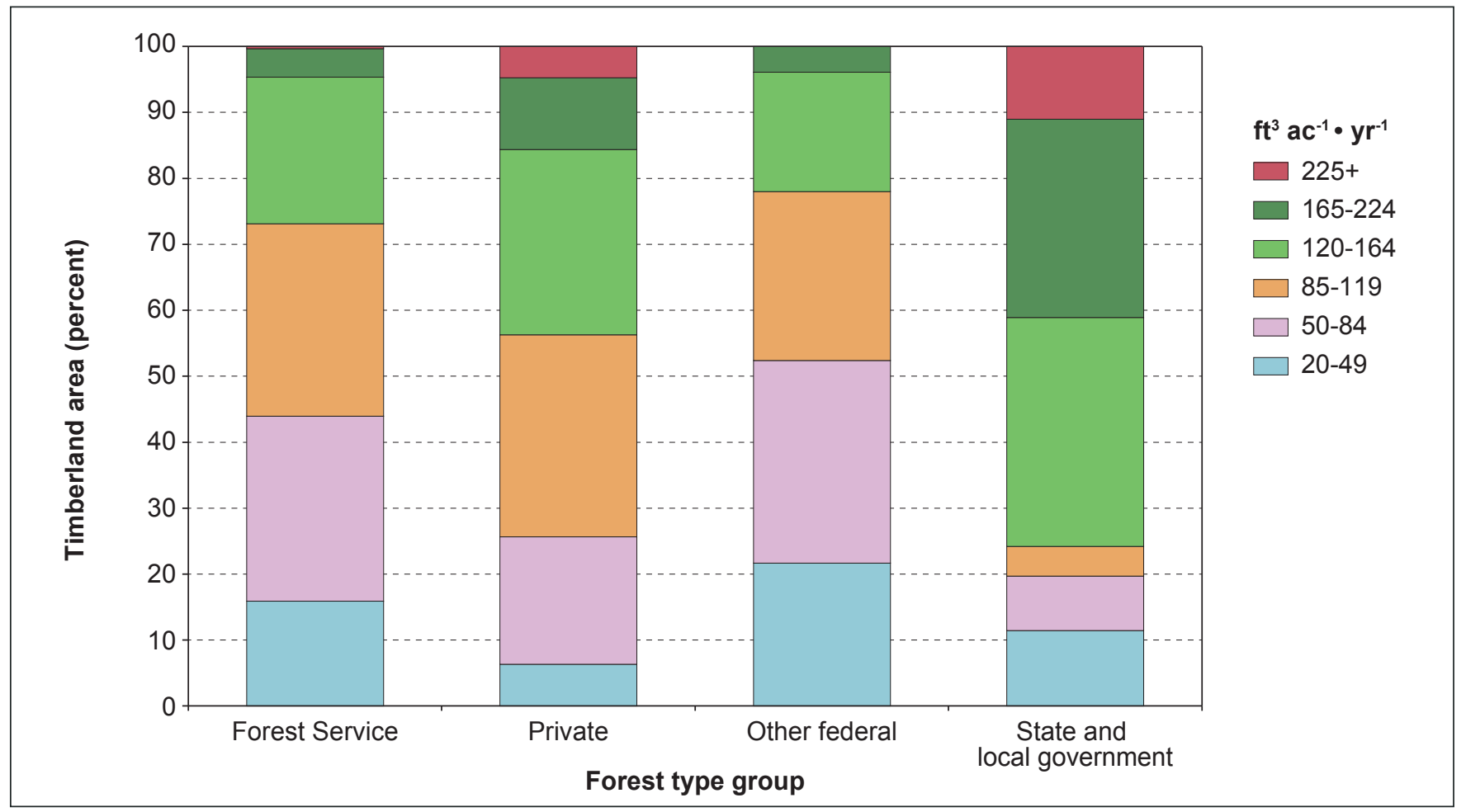

Figure 50-Percentage of timberland area by ownership group and site class in California, 2001-2010.

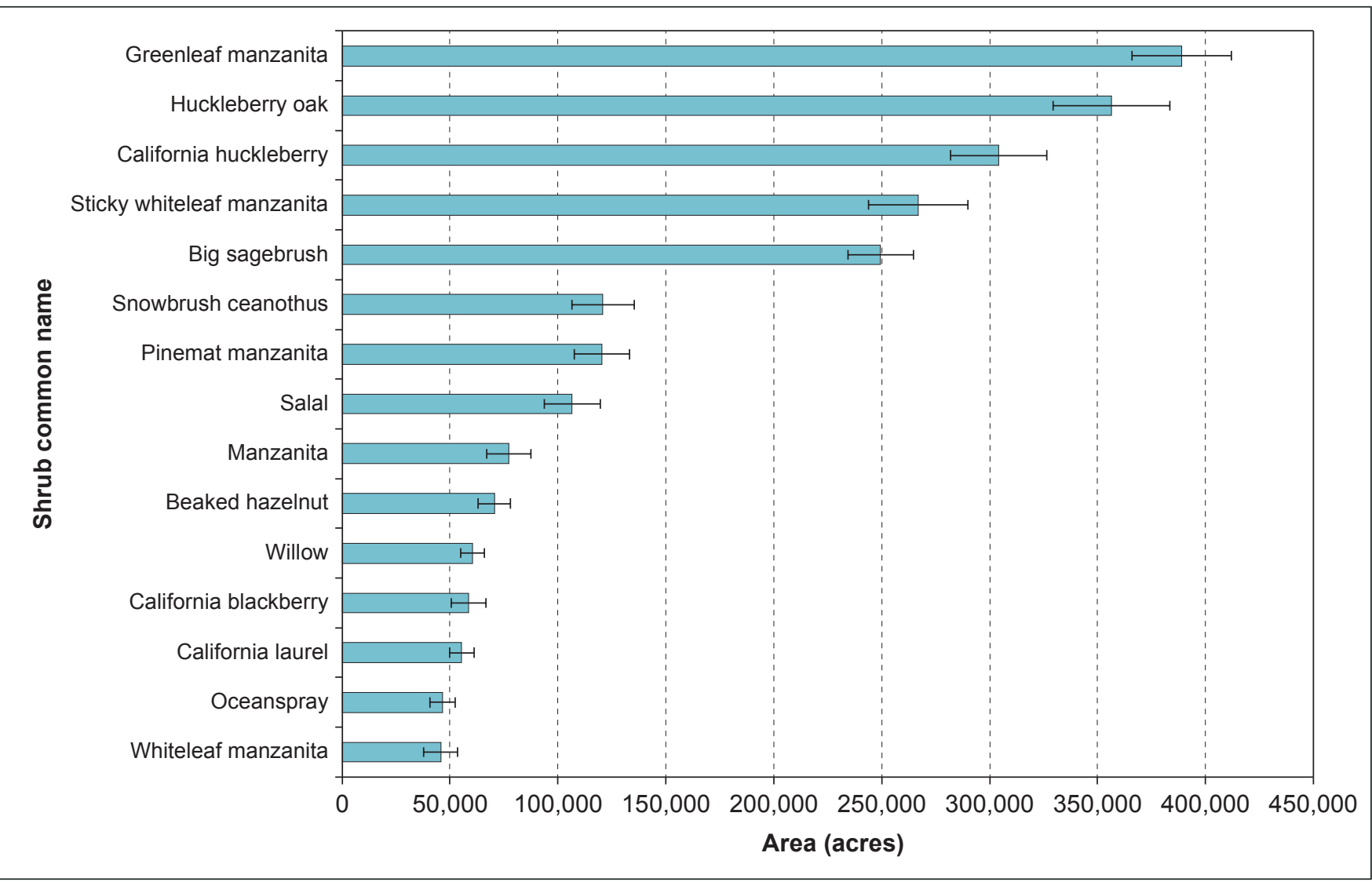

Figure 51-Total shrub cover (acres) of forest land by selected species used in nontimber forest products in California, 2001-2010. 


\section{Nontimber Forest Product Species}

Nontimber forest products (NTFP) are those forest products not traditionally included as timber-based products, but still economically and ecologically important to the state. The NTFPs are derived from many species ranging from tree seedlings and saplings to shrubs and herbs.

Swordfern is the herbaceous species with the greatest cover (235,000 acres [SE: 19,000 acres]). The next most common herb species is brackenfern, which covers about 138,000 acres (SE: 10,000). Together, these two species occupy about 70 percent of the forested area covered by herbs in California. The shrubs with the greatest cover were greenleaf manzanita, covering about 389,000 acres (SE: 23,000), and huckleberry oak, which covers about 356,000 acres (SE: 27,000; fig. 51). Tanoak seedlings and saplings were the most abundant of the NTFP species, covering about 315,000 acres (SE: 17,000).

\section{What Is the Extent of Dead Wood in California's Forests?}

Dead wood, standing as snags or fallen on the forest floor, is an important component of forest structure and wildlife habitat. Forest Inventory and Analysis plot measurements are well suited to estimate dead wood throughout all forest types and ownerships across the state. Just as the FIA data are used to help describe plant biodiversity in California's forests, including characteristics of special habitat types such as old forests and riparian corridors, and status of forest components such as tree crowns, soils, and understory vegetation, the collected information can also describe the extent of dead wood in California's forests.

Dead trees fill many ecologically important roles in forests. Dead wood in a forest provides habitat for wildlife and fungi, improves soil fertility through nutrient cycling and moisture retention, adds to fuel loads, and is a key structural element in mature forests. Large-scale disturbances from fire and insect outbreaks can have a profound effect on the total dead wood present, which is an important parameter for forest managers. Dead wood resources are complex and must be assessed from a variety of perspectives - too much can be viewed as a fire hazard, and too little can be viewed as a loss of habitat. Forest Inventory and
Analysis provides estimates of the amount of both standing dead trees (snags) and down wood (logs) (fig. 52).

There are approximately 498 million tons of dead wood biomass on forest land across California, about 233 million tons of standing snags and another 265 million tons of down wood. By comparison, there are about 2.2 billion tons of live tree biomass found on these forests, nearly 10 times more biomass. The majority of dead wood is found on forest land managed by the Forest Service: about 57 percent of all dead wood biomass in the state is found on this ownership (fig. 53). Private ownerships, both corporate and individual noncorporate, account for about 30 percent of the dead wood biomass. Together, other federal and state/local governments make up the remaining 13 percent of deadwood. On a per-acre basis, Forest Service lands average about 18 snags per acre and about 16 tons of down wood biomass per acre (fig. 54). This is in contrast to private ownerships, which average about 10 snags per acre but average similar amounts of down wood biomass, about 14 tons per acre. By stand age, maximum total biomass per acre in standing dead trees occurs in stands 161 to 200 years old for softwood forest types at about 30 tons per acre (fig. 55).

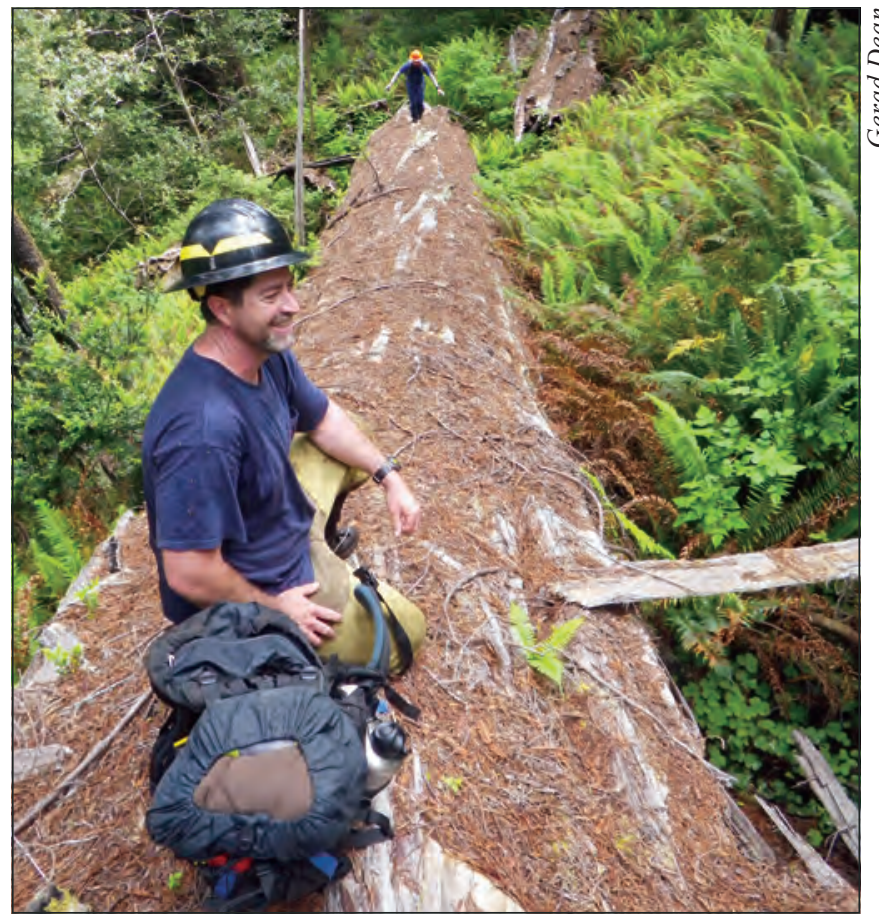

Figure 52 - Large down logs, such as this massive log from a fallen redwood tree in Humboldt County, contribute to key habitat and structural elements in mature forests of the region. 


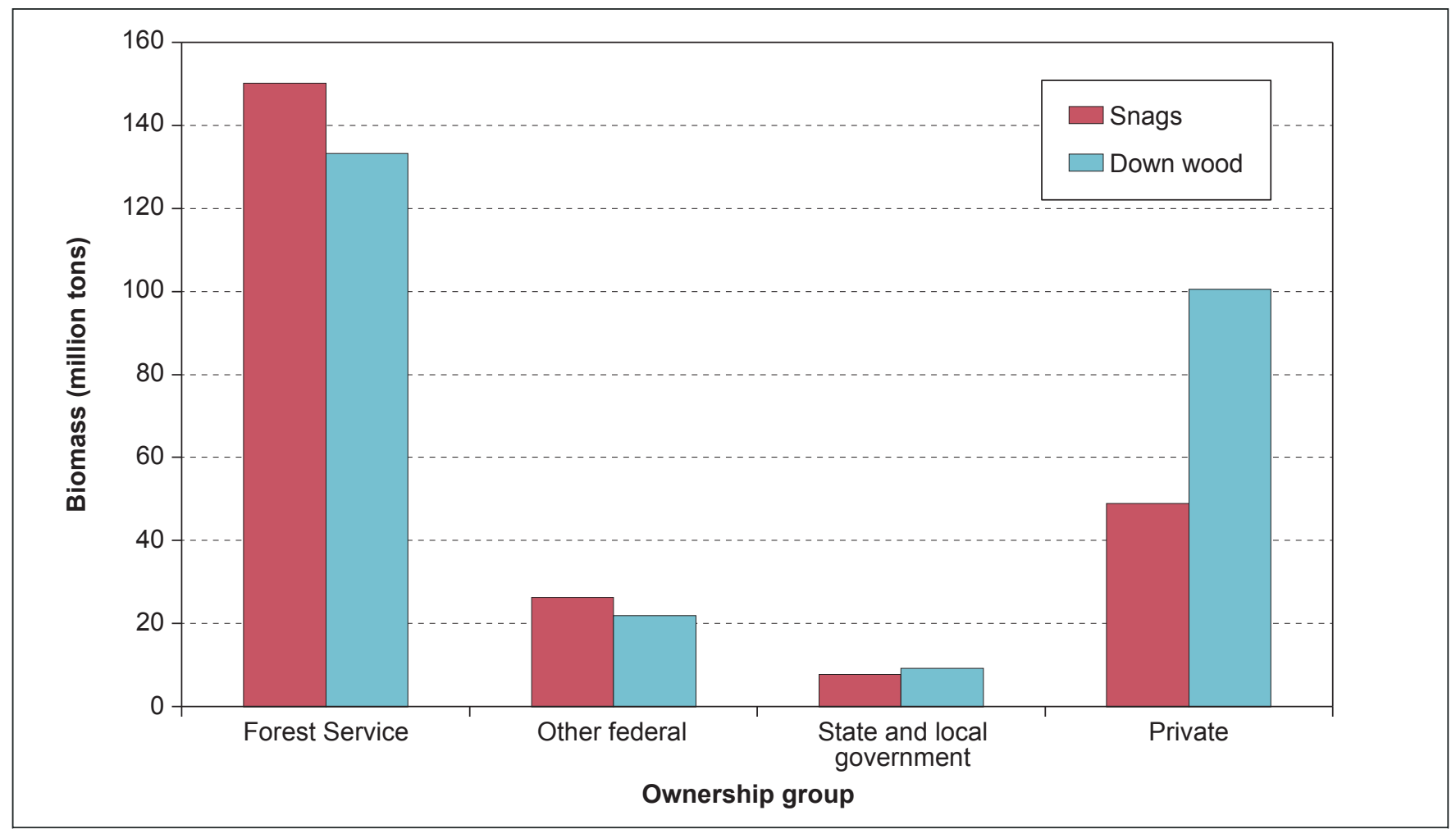

Figure 53-Total biomass (million tons) in standing dead trees (snags) and down wood by ownership group in California, 2001-2010.

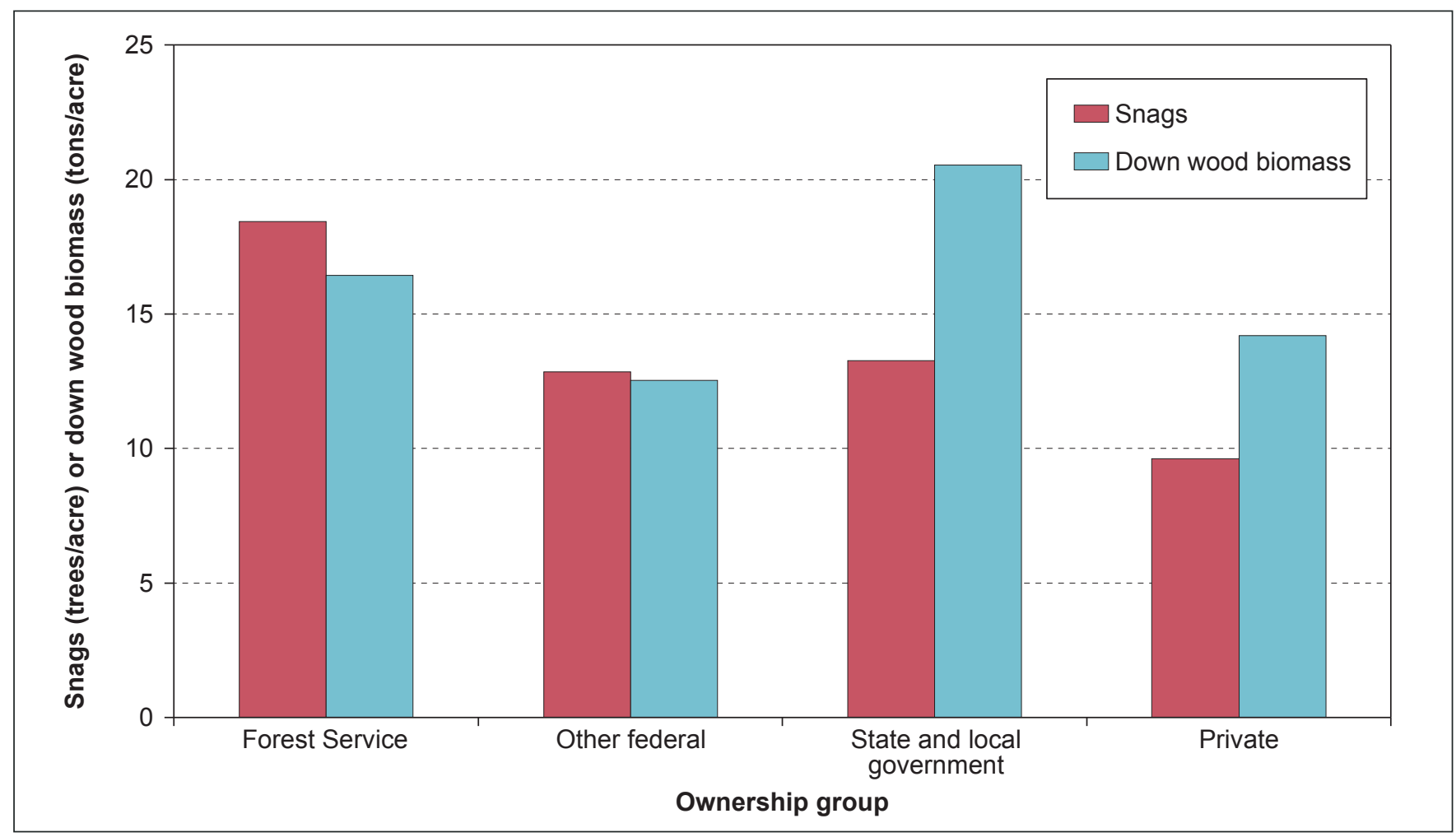

Figure 54- Average number of standing dead trees (snags) per acre and down wood biomass (tons) per acre by ownership group in California, 2001-2010. 


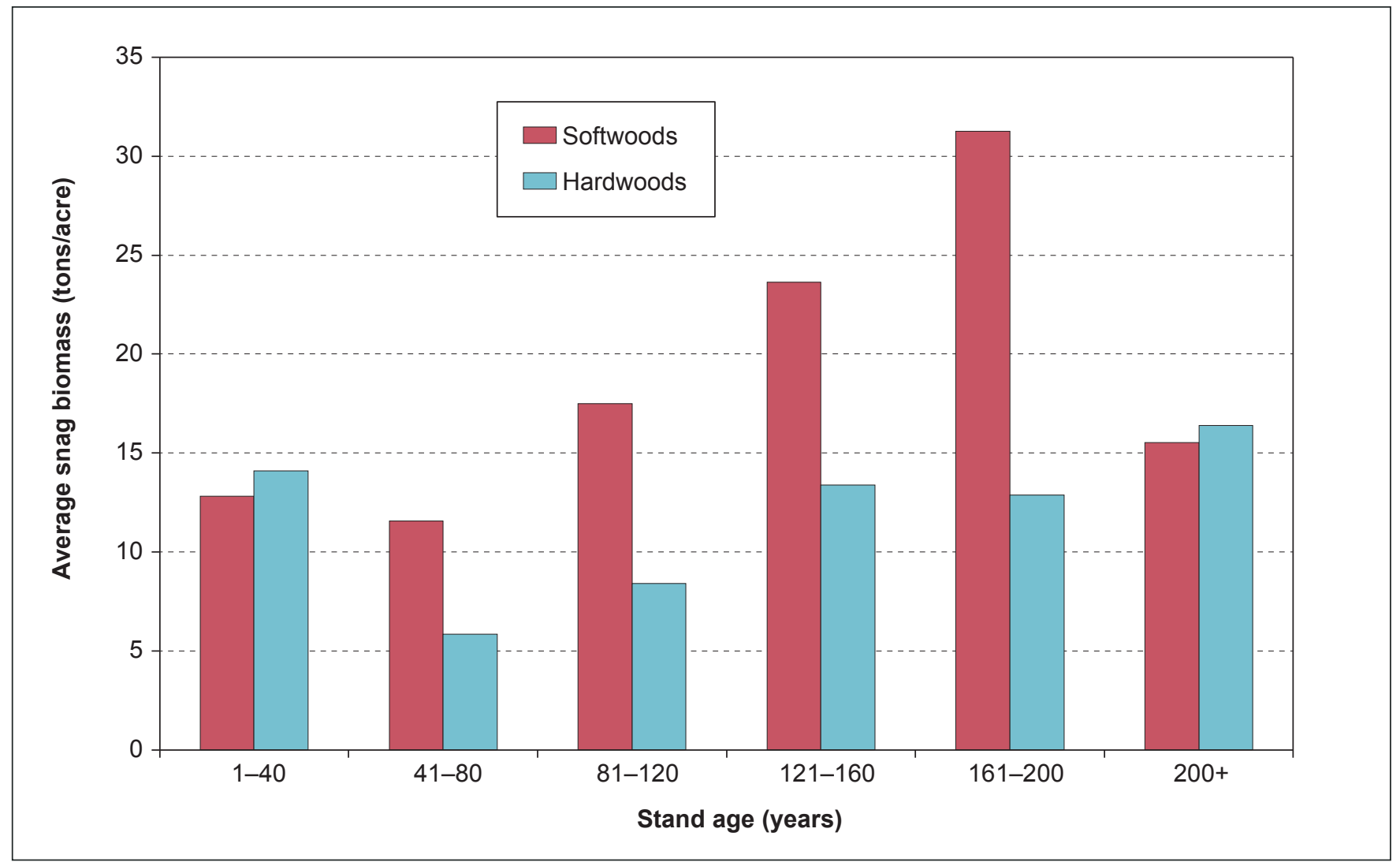

Figure 55-Average dead tree (snag) biomass (tons) per acre by stand age for softwood and hardwood forest types in California, 2001-2010. 


\section{Research Application-Monitoring Fisher Habitat ${ }^{6}$}

Every species of forest wildlife has unique features it seeks out to make its home. The systematic sample of all forest lands by Forest Inventory and Analysis (FIA) provides powerful opportunities to identify what kinds of forests those species are selecting. The fisher (Martes pennanti) is a forest carnivore in the weasel family that is rare in California (fig. 56). In a collaboration between the Pacific Southwest Research Station, Humboldt State University, and FIA, the forests that fishers used for "resting" (refuge for prolonged periods) in northern California were identified by following the locations of several individuals that had been captured and fitted with radio collars. Plots were installed at those fisher resting sites that exactly matched the FIA inventory protocols. We then compared the characteristics of plots that fishers used to the characteristics of plots in the surrounding landscape.

We found that fishers were selecting forests with high canopy closure, older trees, high basal area, large down wood, and abundant hardwoods. These fisher habitat preferences in the north coast were similar to those found in a study for the Sierra Nevada, suggesting general features that managers could conserve or promote on the landscape to maintain future populations of fishers, in combination with other key attributes affecting fishers like abundance of food, predators, and pathogens. The habitat models, tied to the national FIA plot design, will allow us to monitor future changes in fisher habitat as FIA inventories are remeasured.

\section{Citation}

Zielinski, W.J.; Dunk, J.R.; Gray, A.N. 2012.

Estimating habitat value using forest inventory data: the fisher (Martes pennanti) in northwestern California. Forest Ecology and Management. 275: 35-42. http://www.treesearch.fs.fed.us/pubs/41478.

\footnotetext{
${ }^{6}$ Author: Andrew Gray.
}
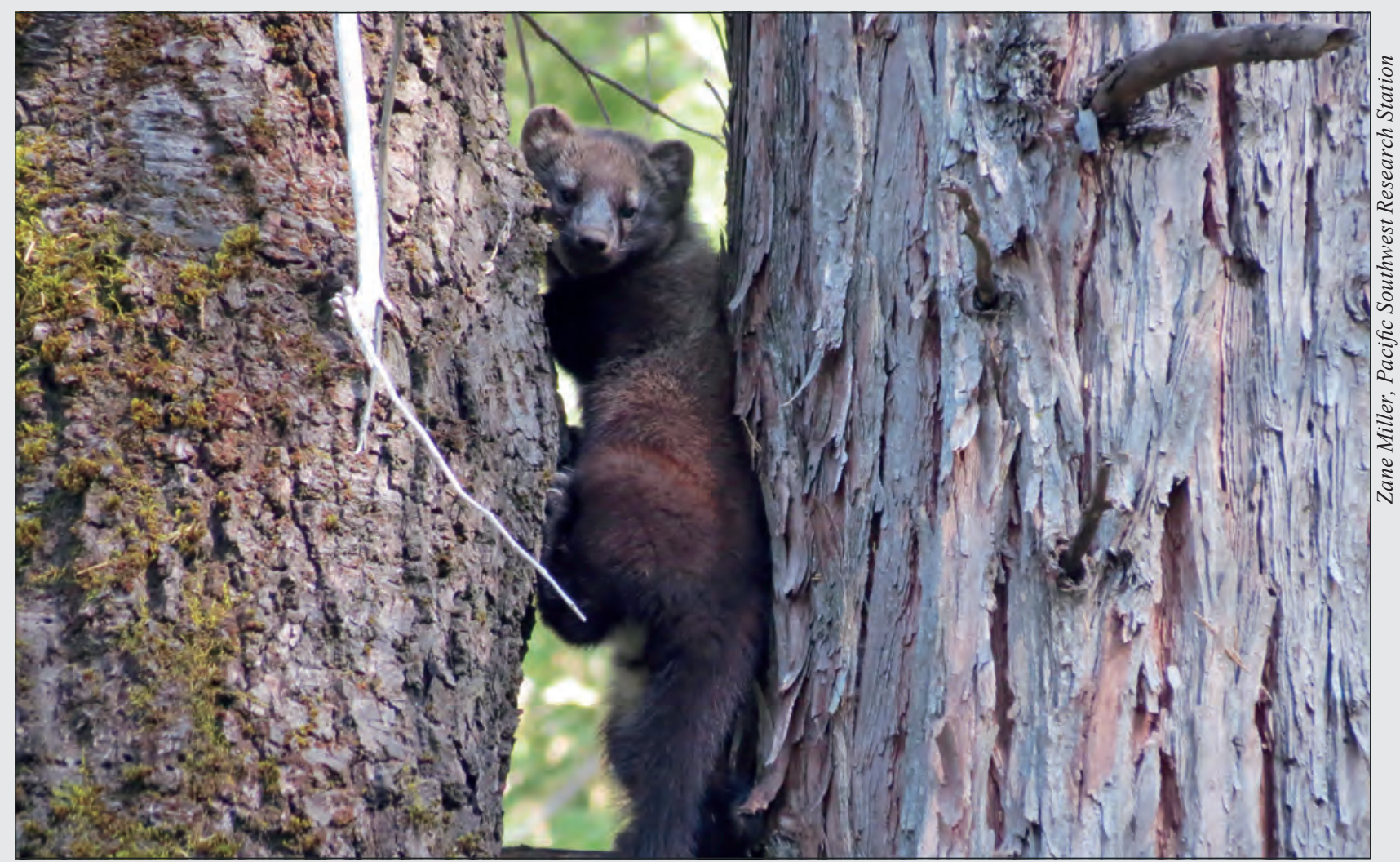

Figure 56-A fisher (Martes pennanti) in trees. 


\section{Conclusion}

This report presents an updated overview of California's

forest resources, highlighting information that is new as well as confirming previously known information. We expect some readers will be eager to see more indepth research and analysis on selected topics to fully understand current status, change, and relationships in California's forests. We want to stress that the data in this report are not independent from those published in a previous report that covered the period 2001-2005 (Christensen et al. 2008). The values presented here cannot be compared to the previous report or that data set to assess change in forest resources over the last decade; these data are a continuation of the full 10-year cycle. The first 10 -year cycle is now complete for all forested plots measured from 2001 to 2010. All future measurements are remeasurements assessing the change that occurred during the 10 years between visits. Remeasured plots tell us everything from impacts of disturbances such as wildfire and changes in land use, to specifically how much each tree has grown. Armed with these data, we can begin quantifying trends in California's forests at multiple scales. From assessing change in the growth and survival of understory seedlings to large, landscape-level changes facing forests from future challenges like a growing population and a changing climate.

The annual FIA inventory will continue into the future provided funding and support for it are maintained. As directed by the 1998 Farm Bill (Section 253[c] of the Agriculture Research, Extension, and Education Reform Act of 1998), findings from the inventory will be published no more frequently than every 5 years. 


\section{Common and Scientific Plant Names}

\begin{tabular}{|c|c|c|}
\hline Life form & Common name & Scientific name \\
\hline \multirow[t]{39}{*}{ Trees: } & Alder & Alnus spp. \\
\hline & Ash & Fraxinus spp. \\
\hline & Aspen, quaking aspen & Populus tremuloides Michx. \\
\hline & Bigcone Douglas-fir & Pseudotsuga macrocarpa (Vasey) Mayr \\
\hline & Bigleaf maple & Acer macrophyllum Pursh \\
\hline & Birch & Betula spp. \\
\hline & Bishop pine & Pinus muricata D. Don \\
\hline & Bitter cherry & Prunus emarginata (Dougl. ex Hook.) D. Dietr. \\
\hline & Black cottonwood & $\begin{array}{l}\text { Populus balsamifera L. ssp. trichocarpa (Torr. \& A. Gray } \\
\text { ex Hook.) Brayshaw }\end{array}$ \\
\hline & Blue oak & Quercus douglasii Hook. \& Arn. \\
\hline & Boxelder & Acer negundo L. \\
\hline & Brewer spruce & Picea breweriana S. Wats. \\
\hline & Bristlecone pine & Pinus aristata Engelm. \\
\hline & California black oak & Quercus kelloggii Newberry \\
\hline & California buckeye & Aesculus californica (Spach) Nutt. \\
\hline & California juniper & Juniperus californica Carr. \\
\hline & California nutmeg, California torreya & Torreya californica Torr. \\
\hline & California red fir & Abies magnifica A. Murr. \\
\hline & California sycamore & Platanus racemosa Nutt. \\
\hline & California white oak & Quercus lobata Née \\
\hline & California-laurel & Umbellularia californica (Hook. \& Arn.) Nutt. \\
\hline & Canyon live oak & Quercus chrysolepis Liebm. \\
\hline & Cedar & Thuja spp. \\
\hline & Cherry and plum species & Prunus spp. \\
\hline & Chinkapin oak & Quercus muehlenbergii Engelm. \\
\hline & Coast live oak, California live oak & Quercus agrifolia Née \\
\hline & Chokecherry & Prunus virginiana $\mathrm{L}$. \\
\hline & Cottonwood & Populus spp. \\
\hline & Coulter pine & Pinus coulteri D. Don \\
\hline & Curl-leaf mountain mahogany & Cercocarpus ledifolius Nutt. \\
\hline & Cypress & Cupressus spp. \\
\hline & Desert ironwood, tesota, Arizona ironwood & Olneya tesota A. Gray \\
\hline & Douglas-fir & Pseudotsuga menziesii (Mirbel) Franco \\
\hline & Elm & Ulmus spp. \\
\hline & Engelmann oak & Quercus engelmannii Greene \\
\hline & Engelmann spruce & Picea engelmannii Parry ex Engelm. \\
\hline & Eucalyptus & Eucalyptus spp. \\
\hline & Foxtail pine & Pinus balfouriana Grev. \& Balf. \\
\hline & Fremont cottonwood & Populus fremontii S. Wats. \\
\hline
\end{tabular}




\begin{tabular}{|c|c|c|}
\hline Life form & Common name & Scientific name \\
\hline & Giant chinguapin, golden chinguapin & Chrysolepis chrysophylla (Dougl. ex Hook.) Hjelmqvist \\
\hline & Giant sequoia & Sequoiadendron giganteum (Lindl.) Buchh. \\
\hline & Grand fir & Abies grandis (Dougl. ex D. Don) Lindl. \\
\hline & Gray pine, ghost pine & Pinus sabiniana Dougl. ex Dougl. \\
\hline & Great Basin bristlecone pine & Pinus longaeva D.K. Bailey \\
\hline & Hawthorn & Crataegus spp. \\
\hline & Hemlock & Tsuga spp. \\
\hline & Honey mesquite & Prosopis glandulosa Torr. \\
\hline & Incense cedar & Calocedrus decurrens (Torr.) Florin \\
\hline & Interior live oak & Quercus wislizeni A. DC. \\
\hline & Jeffrey pine & Pinus jeffreyi Grev. \& Balf. \\
\hline & Knobcone pine & Pinus attenuata Lemmon \\
\hline & Limber pine & Pinus flexilis James \\
\hline & Lodgepole pine & Pinus contorta Dougl. ex Loud. \\
\hline & Maple & Acer spp. \\
\hline & Mesquite & Prosopis spp. \\
\hline & Modoc cypress, Baker cypress & Cupressus bakeri Jeps. \\
\hline & Monterey cypress & Cupressus macrocarpa Hartw. ex Gord. \\
\hline & Monterey pine & Pinus radiata $\mathrm{D}$. Don \\
\hline & Mountain hemlock & Tsuga mertensiana (Bong.) Carr. \\
\hline & Noble fir & Abies procera Rehd. \\
\hline & Northern California black walnut & Juglans hindsii Jeps. ex R. E. Sm. \\
\hline & Oak & Quercus spp. \\
\hline & Oregon ash & Fraxinus latifolia Benth. \\
\hline & Oregon crabapple & Malus fusca (Raf.) Schneid. \\
\hline & Oregon white oak & Quercus garryana Dougl. ex Hook. \\
\hline & Pacific dogwood & Cornus nuttallii Audubon ex Torr. \& Gray \\
\hline & Pacific madrone & Arbutus menziesii Pursh \\
\hline & Pacific silver fir & Abies amabilis (Dougl. ex Loud.) Dougl. ex Forbes \\
\hline & Pacific yew & Taxus brevifolia Nutt. \\
\hline & Pine, pinyon & Pinus spp. \\
\hline & Ponderosa pine & Pinus ponderosa P.\& C. Lawson \\
\hline & Port Orford cedar & Chamaecyparis lawsoniana (A. Murr.) Parl. \\
\hline & Red alder & Alnus rubra Bong. \\
\hline & Redcedar, juniper & Juniperus spp. \\
\hline & Redwood, coast redwood & Sequoia sempervirens (Lamb. ex D. Don) Endl. \\
\hline & Rocky mountain maple & Acer glabrum Torr. \\
\hline & Russian olive & Elaeagnus angustifolia $\mathrm{L}$. \\
\hline & Sargent's cypress & Cupressus sargentii Jepson \\
\hline & Screwbean mesquite & Prosopis pubescens Benth. \\
\hline & Shasta red fir & Abies magnifica A. Murr. var. shastensis Lemmon \\
\hline
\end{tabular}




\begin{tabular}{|c|c|c|}
\hline Life form & Common name & Scientific name \\
\hline & Singleleaf pinyon & Pinus monophylla Torr. \& Frém. \\
\hline & Sitka spruce & Picea sitchensis (Bong.) Carr. \\
\hline & Southern California black walnut & Juglans californica S. Watson \\
\hline & Spruce & Picea spp. \\
\hline & Subalpine fir & Abies lasiocarpa (Hook.) Nutt. \\
\hline & Sugar pine & Pinus lambertiana Dougl. \\
\hline & Sweetgum & Liquidambar styraciflua $\mathrm{L}$. \\
\hline & Tanoak & Lithocarpus densiflorus (Hook. \& Arn.) Rehd. \\
\hline & Tasmanian bluegum & Eucalyptus globulus Labill. \\
\hline & True fir species & Abies spp. \\
\hline & Twoneedle pinyon, Colorado pinyon & Pinus edulis Engelm. \\
\hline & Utah juniper & Juniperus osteosperma (Torr.) Little \\
\hline & Walnut & Juglans spp. \\
\hline & Washoe pine & Pinus washoensis Mason \& Stockwell \\
\hline & Western hemlock & Tsuga heterophylla (Raf.) Sarg. \\
\hline & Western honey mesquite & Prosopis glandulosa Torr. \\
\hline & Western juniper & Juniperus occidentalis Hook. \\
\hline & Western larch & Larix occidentalis Nutt. \\
\hline & Western redcedar & Thuja plicata Donn ex D. Don \\
\hline & Western white pine & Pinus monticola Dougl. ex D. Don \\
\hline & White alder & Alnus rhombifolia Nutt. \\
\hline & White fir & Abies concolor (Gord. \& Glend.) Lindl. ex Hildebr. \\
\hline & Whitebark pine & Pinus albicaulis Engelm. \\
\hline \multirow[t]{17}{*}{ Shrubs: } & Blue elderberry & Sambucus nigra L. ssp. cerulea (Raf.) R. Bolli \\
\hline & California huckleberry & Vaccinium ovatum Pursh \\
\hline & California yerba santa & Eriodictyon californicum (Hook. \& Arn.) Torr. \\
\hline & Chamise & Adenostoma fasciculatum Hook. \& Arn. \\
\hline & Creeping barberry & Mahonia repens (Lindl.) G. Don \\
\hline & Currant spp. & Ribes spp. \\
\hline & Cutleaf blackberry & Rubus laciniatus Willd. \\
\hline & Dwarf mistletoe & Arceuthobium spp. \\
\hline & Dwarf Oregon grape & Mahonia nervosa (Pursh) Nutt. \\
\hline & English holly & Ilex aquifolium $\mathrm{L}$. \\
\hline & English ivy & Hedera helix L. \\
\hline & European black elderberry & Sambucus nigra L. \\
\hline & Greanleaf manzanita & Arctostaphylos patula Greene \\
\hline & Hairy manzanita & Arctostaphylos columbiana Piper \\
\hline & Himalayan blackberry & Rubus discolor Weihe \& Nees \\
\hline & Kinnikinnick & Arctostaphylos uva-ursi (L.) Spreng. \\
\hline & Manzanita & Arctostaphylos spp. \\
\hline
\end{tabular}




\begin{tabular}{|c|c|c|}
\hline Life form & Common name & Scientific name \\
\hline & Oregon boxleaf & Paxistima myrsinites (Pursh) Raf. \\
\hline & Oregon grape & Mahonia aquifolium (Pursh) Nutt. \\
\hline & Pacific ninebark & Physocarpus capitatus (Pursh) Kuntze \\
\hline & Pinemat manzanita & Arctostaphylos nevadensis Gray \\
\hline & Pipsissewa & Chimaphila umbellata (L.) W. Bart. \\
\hline & Pursh's buckthorn & Frangula purshiana (DC.) Cooper \\
\hline & Red elderberry & Sambucus racemosa $\mathrm{L}$. \\
\hline & Rose & Rosa spp. \\
\hline & Salal & Gaultheria shallon Pursh \\
\hline & Scotch broom & Cytisus scoparius (L.) Link \\
\hline & Scouler's willow & Salix scouleriana Barratt ex Hook. \\
\hline & Skunkbush & Rhus trilobata Nutt. \\
\hline & Snowberry & Symphoricarpos spp. \\
\hline & Snowbrush ceanothus & Ceanothus velutinus Dougl. ex Hook. \\
\hline & Sticky whiteleaf manzanita & Arctostaphylos viscida Parry \\
\hline & Thinleaf huckleberry & Vaccinium membranaceum Dougl. ex Torr. \\
\hline & Vine maple & Acer circinatum Pursh \\
\hline & Willow & Salix spp. \\
\hline \multirow[t]{20}{*}{ Forbs: } & Brackenfern & Pteridium aquilinum (L.) Kuhn \\
\hline & British Columbia wildginger & Asarum caudatum Lindl. \\
\hline & Bull thistle & Cirsium vulgare (Savi) Ten. \\
\hline & Canada thistle & Cirsium arvense (L.) Scop. \\
\hline & Common beargrass & Xerophyllum tenax (Pursh) Nutt. \\
\hline & Common yarrow & Achillea millefolium $\mathrm{L}$. \\
\hline & Hairy cat's ear & Hypochaeris radicata $\mathrm{L}$. \\
\hline & Heartleaf arnica & Arnica cordifolia Hook. \\
\hline & Horsetail & Equisetum spp. \\
\hline & Mugwort & Artemisia douglasiana Bess. \\
\hline & Pacific trillium & Trillium ovatum Pursh \\
\hline & Purple foxglove & Digitalis purpurea $\mathrm{L}$. \\
\hline & Spreading hedgeparsley & Torilis arvensis (Huds.) Link \\
\hline & St. John's wort & Hypericum perforatum $\mathrm{L}$. \\
\hline & Stinging nettle & Urtica dioica $\mathrm{L}$. \\
\hline & Swordfern & Polystichum munitum (Kaulfuss) K. Presl \\
\hline & Thistle & Cirsium spp. \\
\hline & Western pearly everlasting & Anaphalis margaritacea (L.) Benth. \\
\hline & Western wormwood & Artemisia ludoviciana Nutt. \\
\hline & Yellow star-thistle & Centaurea solstitialis $\mathrm{L}$. \\
\hline
\end{tabular}




\begin{tabular}{lll}
\hline Life form & Common name & Scientific name \\
\hline Graminoids: & Bristly dogstail grass & Cynosurus echinatus L. \\
& Cheatgrass & Bromus tectorum L. \\
Common velvetgrass & Holcus lanatus L. \\
Compact brome & Bromus madritensis L. \\
False brome & Brachypodium sylvaticum (Huds.) Beauv. \\
Medusahead & Taeniatherum caput-medusae (L.) Nevski \\
Orchardgrass & Dactylis glomerata L. \\
Ripgut brome & Bromus diandrus Roth \\
Silver hairgrass & Aira caryophyllea L. \\
Slender oat & Avena barbata Pott ex Link \\
Soft brome & Bromus hordeaceus L. \\
Wild oat & Avena fatua L. \\
& \\
Beard lichen & Usnea hirta (L.) F.H. Wigg. \\
Beard lichens & Usnea spp. \\
Brown-eyed sunshine lichen & Vulpicida canadensis (Rasanen) J. E. Mattsson \& M.J. Lai \\
Crottle & Parmelia saxatilis (L.) Ach. \\
Lace lichen & Ramalina menziesii Taylor \\
Lungwort lichen & Lobaria pulmonaria (L.) Hoffm. \\
Old man's beard & Bryoria fremontii (Tuck.) Brodo \& D. Hawksw. \\
Orange wall lichen & Xanthoria polycarpa (Hoffm.) Rieber \\
Rosette lichen & Physcia adscendens (Fr.) H. Olivier \\
Witch's hair lichen & Alectoria sarmentosa (Ach.) Ach. \\
Wolf lichen & Letharia vulpina (L.) Hue \\
& \\
\hline
\end{tabular}




\section{Acknowledgments}

The authors wish to thank all FIA crew members operating in California during the 2001-2010 field seasons for their hard work, significant input to improved protocol, and persistent attention to data quality throughout 10 years of plot measurements. We would also like to thank members of the Information Management team for compiling the California raw plot measurements into meaningful data for analysis. Like the field measurements, it's also this team's commitment to data quality that made this report possible. Additionally, thanks are due to the reviewers of this manuscript for their invaluable comments.

\section{Metric and English Equivalents}

\begin{tabular}{lcl} 
When you know: & Multiply by: & To find: \\
\hline Inches & 2.54 & Centimeters \\
Feet & 0.3048 & Meters \\
Miles & 1.609 & Kilometers \\
Acres & 0.405 & Hectares \\
Board feet & 0.0024 & Cubic meters \\
Cubic feet & 0.0283 & Cubic meters \\
Cubic feet per acre & 0.06997 & Cubic meters per hectare \\
Square feet & 0.0929 & Square meters \\
Square feet per acre & 0.229 & Square meters per hectare \\
Ounce & 28349.5 & Milligrams \\
Pounds & 0.453 & Kilograms \\
Pounds per cubic foot & 16.018 & Kilograms per cubic meter \\
Tons per acre & 2.24 & Megagrams per hectare \\
Degrees Fahrenheit & $\left({ }^{\circ} \mathrm{F}-32\right) / 1.8$ & Degrees Celsius \\
British thermal units $(B t u)$ & 0.000293 & Kilowatt hours \\
Pounds per cubic foot & 0.016 & Grams per cubic centimeter
\end{tabular}




\section{Literature Cited}

Air and Waste Management Association. 1998.

Recognition of air pollution injury to vegetation: a pictorial atlas. $2^{\text {nd }}$ ed. Sec. 2.0-Ozone. Pittsburgh, PA.

Azuma, D.L.; Dunham, P.A.; Hiserote, B.A.; Veneklase, C.F. 2004. Timber resource statistics for eastern Oregon, 1999. Resour. Bull. PNW-RB-238. Portland, OR: U.S. Department of Agriculture, Forest Service, Pacific Northwest Research Station. 42 p.

Bechtold, W.A.; Patterson, P.L. 2005. The enhanced Forest Inventory and Analysis Program-national sampling design and estimation procedures. Gen. Tech. Rep. GTRSRS-80. Asheville, NC: U.S. Department of Agriculture, Forest Service, Southern Research Station. 85 p.

Blackard, J.; Finco, M.; Helmer, E.; Holden, G.; Hoppus, M.; Jacobs, D.; Lister, A.; Moisen, G.; Nelson, M.; Riemann, R.; Ruefenacht, B.; Salajanu, D.; Weyermann, D.; Winterberger, K.; Brandeis, T.; Czaplewski, R.; McRoberts, R.; Patterson, P.; Tymcio, R. 2008. Mapping U.S. forest biomass using nationwide forest inventory data and moderate resolution information. Remote Sensing of Environment. 112(4): 1658-1677.

Butler, B.J.; Leatherberry, E.C.; Williams, M.S. 2005. Design, implementation, and analysis methods for the national woodland owner survery. Gen. Tech. Rep. NE-GTR-336. Newtown Square, PA: U.S. Department of Agriculture, Forest Service, Northeastern Research Station. $43 \mathrm{p}$.

California Department of Forestry and Fire Protection, Fire and Resource Assessment Program [FRAP)]. 2010. California's forests and rangelands: 2010 strategy report. 2010. http://frap.fire.ca.gov/data/assessment2010/ pdfs/Strategyreport7-157FINAL.pdf. (5 January 2015).

Campbell, S.J.; Wanek, R.; Coulston, J.W. 2007.

Ozone injury in west coast forests: results of 6 years of monitoring. Gen. Tech. Rep. PNW-GTR-722. Portland, OR: U.S. Department of Agriculture, Forest Service, Pacific Northwest Research Station. 53 p.
Christensen, G.A.; Campbell, S.J.; Fried, J.S., tech. eds. 2008. California's forest resources, 2001-2005: fiveyear Forest Inventory and Analysis report. Gen. Tech. Rep. PNW-GTR-763. Portland, OR: U.S. Department of Agriculture, Forest Service, Pacific Northwest Research Station. 183 p.

Cleland, D.T.; Avers, P.E.; McNab, W.H.; Jensen, M.E.; Bailey, R.G.; King, T.; Russell, W.E. 1997. National hierarchical framework of ecological units. In: Boyce, M.S.; Haney, A., eds. Ecosystem management: applications for sustainable forest and wildlife resources. New Haven, CT: Yale University Press: 181-200.

Franklin, J.F.; Hall, F.; Laudenslayer, W. 1986. Interim definitions for old-growth Douglas-fir and mixed conifer forests in the Pacific Northwest and California. Res. Note. PNW-RN-447. Portland, OR: U.S. Department of Agriculture, Forest Service, Pacific Northwest Research Station. $15 \mathrm{p}$.

Gesch, D.; Oimoen, M.; Greenlee, S.; Nelson, C.; Steuck, M.; Tyler, D. 2002. The National Elevation Dataset. Photogrammetric Engineering and Remote Sensing. 68: 5-11.

Goheen, E.M.; Willhite, E.A. 2006. Field guide to common diseases and insect pests of Oregon and Washington conifers. R6-NR-FID-PR-01-06. Portland, OR: U.S. Department of Agriculture, Forest Service, Pacific Northwest Region. 327 p.

Harrington, C.A., comp. 2003. The 1930s survey of forest resources in Washington and Oregon. Gen. Tech. Rep. PNW-GTR-584. Portland, OR: U.S. Department of Agriculture, Forest Service, Pacific Northwest Research Station. 123 p.

Helms, J.A., ed. 1998. The dictionary of forestry. Bethesda, MD: Society of American Foresters. 210 p. 
Homer, C.; Dewitz, J.; Fry, J.; Coan, M.; Hossain, N.; Larson, C.; Herold, N.; McKerrow, A.; VanDriel, J.N.; Wickham, J. 2007. Completion of the 2001 National Land Cover Database for the conterminous United States. Photogrammetric Engineering and Remote Sensing. 73: 337-341.

McNab, W.H.; Cleland, D.T.; Freeouf, J.A.; Keys, J.E. Jr.; Nowacki, G.J.; Carpenter, C.A., comps. 2005. Description of ecological subregions: sections of the conterminous United States. [CD-ROM]. Washington, DC: U.S. Department of Agriculture, Forest Service. 80 p.

Moore, D.S.; McCabe, G.P. 1989. Introduction to the practice of statistics. New York: W.H. Freeman and Co. $790 \mathrm{p}$.

Morgan, T.; Brandt, J.; Songster, K.; Keegan, C.; Christensen, G. 2012. California's forest products industry and timber harvest, 2006. PNW-GTR-866. Portland, OR: U.S. Department of Agriculture, Forest Service, Pacific Northwest Research Station. 48 p.

National Park Service Website. http://www.nps.gov. (5 January 2015).
Smith, W.B.; Miles, P.D.; Vissage, J.S.; Pugh, S.A. 2004. Forest resources of the United States, 2002. Gen. Tech. Rep. NC-GTR-241. St. Paul, MN: U.S. Department of Agriculture, Forest Service, North Central Research Station. 137 p.

\section{U.S. Department of Agriculture, Forest Service [USDA} FS]. 2006. Forest Inventory and Analysis glossary. On file with: Pacific Northwest Forest Inventory and Analysis Program, 620 SW Main Street, Suite 400, Portland, OR 97205.

\section{U.S. Department of Agriculture, Forest Service [USDA} FS]. 2007. Forest Inventory and Analysis Program: field instructions for the annual inventory of Washington, Oregon, and California. Portland, OR: Pacific Northwest Research Station.

\section{U.S. Department of Agriculture, Natural Resources} Conservation Service [USDA NRCS]. 2000. The PLANTS database. Baton Rouge, LA: National Plant Data Center. http://plants.usda.gov. (5 January 2015). 


\section{Glossary}

abiotic - Pertaining to nonliving factors such as temperature, moisture, and wind.

aerial photography - Imagery acquired from an aerial platform (typically aircraft or helicopter) by means of a specialized large-format camera with well-defined optical characteristics. The geometry of the aircraft orientation at the time of image acquisition is also recorded. The resultant photograph will be of known scale, positional accuracy, and precision. Aerial photography for natural resource use is usually either natural color or color-infrared, and is filmbased or acquired using digital electronic sensors.

air quality index - Value or set of values derived from a multivariate model that examines the composition of lichen communities at each plot to provide a relative estimate of air quality.

artificial regeneration-An artificially regenerated stand is established by planting or artificial seeding.

aspect-Compass direction that a slope faces.

basal area-The cross-sectional area of a tree's trunk.

biodiversity - Variety and variability among living organisms and the ecological complexes in which they occur. Diversity can be defined as the number of different items and their relative frequencies. http://www.epa.gov/ OCEPAterms/bterms.html. (5 January 2015).

bioenergy - Renewable energy made available from materials derived from biological sources. http://en.wikipedia. org/wiki/Bioenergy. (5 January 2015).

biomass - The aboveground weight of wood and bark in live trees 1.0 inch diameter at breast height (d.b.h.) and larger from the ground to the tip of the tree, excluding all foliage. The weight of wood and bark in lateral limbs, secondary limbs, and twigs under 0.5 inch in diameter at the point of occurrence on sapling-size trees is included in the measure, but on poletimber- and sawtimber-sized trees this material is excluded. Biomass is typically expressed as green or oven-dry weight in tons (USDA FS 2006).

biosite index, ozone - A value calculated from the amount and severity of ozone injury at a site (biosite) that reflects local air quality and plant response and therefore potential risk of ozone impact in the area represented by that biosite (Campbell et al. 2007).

board foot - A volume measure of lumber 1 foot wide, 1 foot long, and 1 inch thick ( 12 in $\times 12$ in $\times 1$ in $=144$ cubic inches).

bole-Trunk or main stem of a tree.

carbon mass - The estimated weight of carbon stored within wood tissues. On average, carbon mass values are about half of biomass values for trees, and are summarized as thousand tons or mean tons per acre.

carbon sequestration-Incorporation of carbon dioxide into permanent plant tissues.

coarse woody material-Down dead tree and shrub boles, large limbs, and other woody pieces that are severed from their original source of growth. Coarse woody material also includes dead trees that are supported by roots, severed from roots, or uprooted, and leaning $>45$ degrees from vertical (USDA FS 2006).

corporate forest land-An ownership class of private forest lands owned by a company, corporation, legal partnership, investment firm, bank, timberland investment management organization (TIMO), or real estate investment trust (REIT).

crown - The part of a tree or woody plant bearing live branches or foliage.

crown dieback - Recent mortality of branches with fine twigs, which begins at the terminal portion of a branch and proceeds toward the trunk. Dieback is only considered when it occurs in the upper and outer portions of the tree (USDA FS 2006). 
crown fire-Fire that spreads across the tops of trees or shrubs more or less independently of a surface fire. Crown fires are sometimes classed as running (independent or active) or dependent (passive) to distinguish the degree of independence from the surface fire (Helms 1998).

current gross annual growth - The total growth of a given stand of trees, within a defined area, over the period of 1 year.

cyanolichen - Lichen species containing cyanobacteria, which fixes atmospheric nitrogen into a form that plants can use.

damage - Damage to trees caused by biotic agents such as insects, diseases, and animals or abiotic agents such as weather, fire, or mechanical equipment.

defoliation-Premature removal of foliage.

diameter at breast height (d.b.h.) - The diameter of a tree stem, located at 4.5 feet above the ground (breast height) on the uphill side of a tree. The point of diameter measurement may vary on abnormally formed trees (USDA FS 2006).

diameter at root collar (d.r.c.) - The diameter of a tree (usually a woodland species), measured outside of the bark at the ground line or stem root collar (USDA FS 2006).

dieback - Progressive dying from the extremity of any part of the plant. Dieback may or may not result in death of the entire plant (Helms 1998).

disturbance-Any relatively discrete event in time that disrupts ecosystem, community, or population structure and changes resources, substrate availability, or the physical environment (Helms 1998).

down woody material (DWM) —-Dead material on the ground in various stages of decay, including coarse and fine woody material. Previously named down woody debris (DWD). The DWM indicator for Forest Inventory and Analysis includes measurements of depth of duff layer, litter layer, and overall fuelbed; fuel loading on the microplot; and residue piles (USDA FS 2006). ecological region - A top-level scale in a hierarchical classification of ecological units subdivided on the basis of global, continental, and regional climatic regimes and broad physiography. Ecological regions (ecoregions) are further subdivided into domains, divisions, and provinces. The next level down in the hierarchy, subregion, is divided into ecological sections (ecosections) and subsections (Cleland et al. 1997).

ecosection-A level in a hierarchical classification of ecological units for a geographic area delineated on the basis of similar climate, geomorphic processes, stratigraphy, geologic origin, topography, and drainage systems (Cleland et al. 1997).

ecosystem - A spatially explicit, relatively homogeneous unit of the Earth that includes all interacting organisms and components of the abiotic environment within its boundaries. An ecosystem can be of any size: a log, a pond, a field, a forest, or the Earth's biosphere (Helms 1998).

elevation - Height above a fixed reference point, often the mean sea level.

erosion-The wearing away of the land surface by running water, wind, ice, or other geological agents.

federal forest land - An ownership class of public lands owned by the U.S. government (USDA FS 2006).

fine woody material (FWM) — Down dead branches, twigs, and small tree or shrub boles $<3$ inches in diameter not attached to a living or standing dead source (USDA FS 2006).

fire regime-The characteristic frequency, extent, intensity, severity, and seasonality of fires within an ecosystem (Helms 1998).

fixed-radius plot - A circular sampled area with a specified radius in which all trees of a given size, shrubs, and other items are tallied (USDA FS 2006). 
forb-A broad-leaved herbaceous plant, as distinguished from grasses, shrubs, and trees (USDA FS 2006).

forest industry land - An ownership class of private lands owned by a company or an individual(s) operating a primary wood-processing plant (USDA FS 2006).

forest land — Land that is at least 10 percent stocked by forest trees of any size, or land formerly having such tree cover, and not currently developed for a nonforest use. The minimum area for classification as forest land is 1 acre. Roadside, streamside, and shelterbelt strips of timber must be at least 120 feet wide to qualify as forest land (USDA FS 2006).

forest type - A classification of forest land based on and named for the tree species that forms the plurality of livetree stocking (USDA FS 2006).

forest type group - A combination of forest types that share closely associated species or site requirements (USDA FS 2006).

fuel treatment - Any manipulation or removal of wildland fuels to reduce the likelihood of ignition or to lessen potential fire damage and resistance to control; e.g., lopping, chipping, crushing, piling, and burning. Synonym: fuel modification, hazard reduction (Helms 1998).

fuelwood - Wood salvaged from mill waste, cull logs, branches, etc., and used to fuel fires in a boiler or furnace.

fungus - Member of a group of saprophytic and parasitic organisms that lack chlorophyll, have cell walls made of chitin, and reproduce by spores; includes molds, rusts, mildews, smuts, and mushrooms. Fungi absorb nutrients from the organic matter in which they live. Not classified as plants; instead fungi are placed in the Kingdom: Fungi (Goheen and Willhite 2006).

geospatial - The combination of spatial software and analytical methods with terrestrial or geographic data sets. Often used in conjunction with geographic information systems and geomatics. graminoid - Grasses (family Gramineae or Poaceae) and grasslike plants such as sedges (family Cyperaceae) and rushes (family Juncaceae).

grassland - Land on which the vegetation is dominated by grasses, grasslike plants, or forbs.

greenhouse gas - A gas, such as carbon dioxide or methane, that contributes to potential climate change. http:// www.epa.gov/OCEPAterms/gterms.html. (5 January 2015).

gross volume - Represents the total volume of wood within the bole of a tree and includes rotten, missing and form cull (volume loss due to rotten, missing, and form cull defect has not been deducted). Including estimated missing bole sections may substantially increase gross volume of some trees with significant breakage or previous harvest (USDA Forest Service 2006).

growing-stock-All live trees 5 inches d.b.h or larger that are considered merchantable in terms of saw-log length and grade; excludes rough and rotten cull trees (USDA FS 2006).

hardwood-Tree species belonging to the botanical subdivision Angiospermae, class Dicotyledonous, usually broadleaved and deciduous (USDA FS 2006).

increment borer - An auger-like instrument with a hollow bit and an extractor, used to extract thin radial cylinders of wood (increment cores) from trees having annual growth rings, to determine increment or age (Helms 1998).

interpolation - A method of reallocating attribute data from one spatial representation to another. Kriging is a more complex example that allocates data from sample points to a surface.

invasive plant - A plant that is not native to the ecosystem under consideration and that causes or is likely to cause economic or environmental harm or harm to human, animal, or plant health. http://www.invasivespeciesinfo.gov/docs/council/isacdef.pdf. (5 January 2015). 
ladder fuel-Combustible material that provides vertical continuity between vegetation strata and allows fire to climb into the crowns of trees or shrubs with relative ease. Ladder fuels help initiate and ensure the continuation of a crown fire (Helms 1998).

lichen-An organism consisting of a fungus and an alga or cyanobacterium living in symbiotic association. Lichens look like masses of small, leafy, tufted or crust-like plants (USDA FS 2006).

live trees-All living trees, including all size classes, all tree classes, and both commercial and noncommercial species listed in the FIA field manual (USDA FS 2006).

mean annual increment (MAI) at culmination-A measure of the productivity of forest land expressed as the average increase in cubic feet of wood volume per acre per year. For a given species and site index, the mean is based on the age at which the MAI culminates for fully stocked natural stands. The MAI is based on the site index of the plot (Azuma et al. 2004).

mensuration-Determination of dimensions, form, weight, growth, volume, and age of trees, individually, or collectively, and of the dimensions of their products (Helms 1998). mesic - Describes sites or habitats characterized by intermediate moisture conditions; i.e., neither decidedly wet nor dry.

microclimate - The climate of a small area, such as that under a plant or other cover, differing in extremes of temperature and moisture from the larger climate outside (Helms 1998).

MMBF - A million board feet of wood in logs or lumber.

model-(1) An abstract representation of objects and events from the real world for the purpose of simulating a process, predicting an outcome, or characterizing a phenomenon. (2) Geographic information system (GIS) data representative of reality (e.g., spatial data models), including the arc-node, georelational model, rasters or grids, polygon, and triangu- lar irregular networks (Helms 1998).

mortality - The death of trees from natural causes, or subsequent to incidents such as storms, wildfire, or insect and disease epidemics (Helms 1998).

municipal land — Land owned by municipalities or land leased by them for more than 50 years (USDA FS 2006). national forest lands - Federal lands that have been designated by Executive order or statute as national forest or purchase units and other lands under the administration of the U.S. Department of Agriculture, Forest Service, including experimental areas and Bankhead-Jones Title III lands (Azuma et al. 2004).

Native American lands - Tribal lands and allotted lands held in trust by the federal government. Native American lands are grouped with farmer-owned and miscellaneous private lands as other private lands (Azuma et al. 2004).

native species - Plant species that were native to an American region prior to Euro-American settlement. For vascular plants, they are the species that are not present on the USDA Natural Resources Conservation Service (NRCS) (2000) list of nonnative species (see nonnative species) (USDA NRCS 2000).

net primary production (NPP) - NPP represents the amount of chemical energy that is available to consumers in an ecosystem. It is the remaining energy from gross primary productivity discounting the loss of energy required by primary producers for respiration.

net volume - Gross volume less deductions for sound and rotten defects. Growing-stock net volume is gross volume (in cubic feet) less deductions for rot and missing bole sections on poletimber and sawtimber growing-stock trees. Sawtimber net volume is gross volume (in board feet) less deductions for rot, sweep, crook, missing bole sections, and other defects that affect the use of sawtimber trees for lumber (Azuma et al. 2004). 
noncensus water - Lakes, reservoirs, ponds, and similar bodies of water 1.0 to 4.5 acres; and rivers, streams, canals, etc., 30.0 to 200 feet wide.

noncorporate forest land-Private forest land owned by nongovernmental conservation or natural resource organizations; unincorporated partnerships, associations, or clubs; individuals or families; or Native Americans.

nonforest inclusion-An area that is not forested, is less than 1.0 acre, and does not qualify as its own condition class (USDA FS 2006).

nonforest land - Land that has never supported forests or formerly was forested and currently is developed for nonforest uses. Included are lands used for agricultural crops, Christmas tree farms, cottonwood plantations, improved pasture, residential areas, city parks, constructed roads, operating railroads and their right-of-way clearings, and powerline and pipeline clearings. The area must be at least 1.0 acre and 120.0 feet wide.

nonnative species-Plant species that were introduced to America subsequent to Euro-American settlement. Nonnative vascular plants are present on the USDA Natural Resources Conservation Service list of nonnative species (USDA NRCS 2000).

nonstocked areas-Timberland that is less than 10 percent stocked with live trees. Recent clearcuts scheduled for planting are classified as nonstocked area (Azuma et al. 2004).

nontimber forest products (NTFP) — Species harvested from forests for reasons other than production of timber commodities. Vascular plants, lichens, and fungi are the primary organisms included in NTFPs.

old-growth forest-Old-growth forest is differentiated from younger forest by its structure and composition, and often by its function. Old-growth stands are typified by the presence of large older trees; variety in tree species, sizes, and spacing; multiple canopy layers; high amounts of standing and down dead wood; and broken, deformed, or rotting tops, trunks, and roots (Franklin et al. 1986).

other forest-Forest land that is unproductive (land not capable of producing more than 20 cubic feet of wood per acre per year). In tables in which reserved and unreserved forest land are not broken out, "other forest" includes all reserved forest land as well as unproductive forest land.

other private forest lands - Lands in private ownership and not reported separately. These may include coal companies, land trusts, and other corporate private landowners (USDA FS 2006).

overrun-Difference between the log scale of a shipment of timber and the actual volume of lumber obtained from it. http://forestry.about.com/library/glossary/blforglo.htm. (5 January 2015).

overstory - That portion of the trees, in a forest of more than one story, forming the uppermost canopy layer (Helms 1998).

owner class - A variable that classifies land into categories of ownership. Current ownership classes are listed in the FIA field manual (USDA FS 2006).

owner group - A variable that combines owner classes into the following groups: Forest Service, other federal agency, state and local government, and private. Differing categories of owner group on a plot require different conditions (USDA FS 2006).

ownership - A legal entity having an ownership interest in land, regardless of the number of people involved. An ownership may be an individual; a combination of persons; a legal entity such as corporation, partnership, club, or trust; or a public agency. An ownership has control of a parcel or group of parcels of land (USDA FS 2006).

ozone $\left(\mathrm{O}_{3}\right)$, tropospheric - A regional, gaseous air pollutant produced primarily through sunlight-driven chemical reactions 
of nitrogen oxide $\left(\mathrm{NO}_{2}\right)$ and hydrocarbons in the troposphere (the lowest layer of the atmosphere). Ozone plays a significant role in greenhouse warming and urban smog and causes foliar injury to deciduous trees, conifers, shrubs, and herbaceous species (Air and Waste Management Association 1998). pathogen-Parasitic organism directly capable of causing disease.

photointerpretation (aerial photography)—A process where points or areas of interest on an aerial photograph are studied to determine information about land cover. The FIA program uses photointerpretation to determine whether field plots are forested or not, and the possible forest type and size class, and to analyze land cover and land use changes.

prescribed burn - Deliberate burning of wildland fuels in either their natural or their modified state and under specified environmental conditions, usually to make the site less susceptible to severe wildfire. Synonym: controlled burn, prescribed fire (adapted from Helms 1998).

productive forest land-Forest land that is producing or capable of producing in excess of 20 cubic feet per acre per year of wood at culmination of mean annual increment (MAI) without regard to reserved status (USDA FS 2006).

public land-An ownership group that includes all federal, state, county, and municipal lands (USDA FS 2006).

quadrat - The basic 3.28-square foot sampling unit for the Phase 3 Vegetation Indicator (USDA FS 2006).

rangeland-Expansive, mostly unimproved lands on which a significant proportion of the natural vegetation is native grasses, grass-like plants, forbs, and shrubs. Rangelands include natural grasslands, savannas, shrublands, many deserts, tundra, alpine communities, coastal marshes, and wet meadows. http://en.wikipedia.org/wiki/ Rangeland. (5 January 2015).

regeneration (artificial and natural) - The established progeny from a parent plant, seedlings or saplings existing in a stand, or the act of renewing tree cover by establishing young trees naturally or artificially. May be artificial (direct seeding or planting) or natural (natural seeding, coppice, or root suckers) (adapted from Helms 1998).

remote sensing - Capture of information about the Earth from a distant vantage point. The term is often associated with satellite imagery but also applies to aerial photography, airborne digital sensors, ground-based detectors, and other devices.

reserved forest land - Land permanently reserved from wood products utilization through statute or administrative designation. Examples include national forest wilderness areas and national parks and monuments (USDA FS 2006).

richness - The number of different species in a given area, often referred to at the plot scale as alpha diversity and at the region scale as gamma diversity (USDA NRCS 2000).

riparian-Related to, living in, or associated with a wetland, such as the bank of a river or stream or the edge of a lake or tidewater. The riparian biotic community significantly influences and is influenced by the neighboring body of water (Helms 1998).

sampling error-Difference between a population value and a sample estimate that is attributable to the sample, as distinct from errors due to bias in estimation, errors in observation, etc. Sampling error is measured as the standard error of the sample estimate (Helms 1998).

sapling-A live tree 1.0 to 4.9 inches in diameter (USDA FS 2006).

saw log-A log meeting minimum standards of diameter, length, and defect for manufacture into lumber or plywood. The definition includes logs with a minimum diameter outside bark for softwoods of 7 inches ( 9 inches for hardwoods) (Azuma et al. 2004). 
sawtimber trees - Live softwood trees of commercial species at least 9.0 inches in d.b.h. and live hardwood trees of commercial species at least 11.0 inches in d.b.h. At least 25 percent of the board-foot volume in a sawtimber tree must be free from defect. Softwood trees must contain at least one 12-foot saw log with a top diameter of not less than 7 inches outside bark; hardwood trees must contain at least one 8-foot saw log with a top diameter of not less than 9 inches outside bark (Azuma et al. 2004).

Scribner rule-The common board-foot log rule used to determine sawtimber volume. Scribner volume is estimated in terms of 32-foot logs for softwoods and 16-foot logs for hardwoods.

seedlings - Live trees $<1.0$ inch d.b.h. and at least 6 inches in height (softwoods) or 12 inches in height (hardwoods) (USDA FS 2006).

shrub-Perennial, multistemmed woody plant, usually less than 13 to 16 feet in height, although under certain environmental conditions shrubs may be single-stemmed or taller than 16 feet. Includes succulents (e.g., cacti) (USDA FS 2007).

shrubland-A shrub-dominated vegetation type that does not qualify as forest.

slope-Measure of change in surface value over distance, expressed in degrees or as a percentage (Helms 1998).

snag-Standing dead tree $\geq 5$ inches d.b.h. and $\geq 4.5$ feet in length, with a lean of $<45$ degrees. Dead trees leaning more than 45 degrees are considered to be DWM. Standing dead material shorter than 4.5 feet are considered stumps (USDA FS 2007).

species group - A collection of species used for reporting purposes (USDA FS 2006).

species turnover - A measure of difference in species composition among plots within an area (e.g., ecological section).
Also known as beta diversity. Species turnover is calculated by dividing the total number of species in an area by the mean number of species per plot (USDA NRCS 2000).

specific gravity constants - Ratio of the density (weight per unit volume) of an object (such as wood) to the density of water at $4{ }^{\circ} \mathrm{C}\left(39.2^{\circ} \mathrm{F}\right)$ (Helms 1998).

stand age - Average age of the live dominant and codominant trees in the predominant stand size class (USDA FS 2006).

stand-size class - A classification of stands based on tree size. Large-diameter stands have the majority of trees at least 11.0 inches d.b.h. for hardwoods and 9.0 inches d.b.h. for softwoods; medium-diameter stands have the majority of trees at least 5.0 inches d.b.h. but not as large as large-diameter trees; and small-diameter stands have the majority of trees less than 5.0 inches d.b.h.

state land-An ownership class of public lands owned by states or lands leased by states for more than 50 years (USDA FS 2006).

stocked/nonstocked - In the FIA program, a minimum stocking value of 10 percent live trees is required for accessible forest land (USDA FS 2007).

stocking - (1) At the tree level, the density value assigned to a sampled tree (usually in terms of numbers of trees or basal area per acre), expressed as a percentage of the total tree density required to fully use the growth potential of the land. (2) At the stand level, the sum of the stocking values of all trees sampled (Bechtold and Patterson 2005).

stratification-A statistical tool used to reduce the variance of the attributes of interest by partitioning the population into homogenous strata (Bechtold and Patterson 2005). succession - The gradual supplanting of one community of plants by another (Helms 1998). 
surface fire - A fire that burns only surface fuels, such as litter, loose debris, and small vegetation (Helms 1998).

sustainability - The capacity of forests, ranging from stands to ecoregions, to maintain their health, productivity, diversity, and overall integrity in the long run, in the context of human activity and use (Helms 1998).

terrestrial-Of or relating to the Earth or its inhabitants; of or relating to land as distinct from air or water. http://www. merriam-webster.com/dictionary/terrestrial. (5 January 2015).

timberland-Forest land that is producing or capable of producing $>20$ cubic feet per acre per year of wood at culmination of mean annual increment (MAI). Timberland excludes reserved forest lands (USDA FS 2006).

transect-A narrow sample strip or a measured line laid out through vegetation chosen for study (Helms 1998).

tree-A woody perennial plant, typically large, with a single well-defined stem carrying a more or less definite crown; sometimes defined as attaining a minimum diameter of 3 inches and a minimum height of 15 feet at maturity. For FIA, any plant on the tree list in the current field manual is measured as a tree (USDA FS 2006).

understory - All forest vegetation growing under an overstory (Helms 1998).

unproductive forest land-Forest land that is not capable of producing in excess of 20 cubic feet per acre per year of wood at culmination of MAI without regard to reserved status (USDA FS 2006).

unreserved forest land-Forest land that is not withdrawn from harvest by statute or administrative regulation. Includes forest lands that are not capable of producing in excess of 20 cubic feet per acre per year of industrial wood in natural stands (Smith et al. 2004). vascular plant - A plant possessing a well-developed system of conducting tissue to transport water, mineral salts, and sugars. http://www.biology-online.org/dictionary/ Vascular_plant. (5 January 2015).

wilderness - (1) According to the Wilderness Act of 1964, "a wilderness, in contrast with those areas where man and his works dominate the landscape, is hereby recognized as an area where the earth and its community of life are untrammeled by man, where man himself is a visitor who does not remain." (2) A roadless land legally classified as a component area of the National Wilderness Preservation System and managed to protect its qualities of naturalness, solitude, and opportunity for primitive recreation. Wilderness areas are usually of sufficient size to make maintenance in such a state feasible (Helms 1998).

wildfire-Any uncontained fire, other than prescribed fire, occurring on wildland. Synonym: wildland fire (adapted from Helms 1998).

wildland - Land other than that dedicated for uses such as agriculture, urban, mining, or parks (Helms 1998).

wildland forest - A large continuous tract of forest with few or no developed structures on it. Delineated on aerial imagery for the purpose of detecting land use change. The PNW-FIA program and the Oregon Department of Forestry jointly use a minimum of 640 acres with fewer than five developed structures to designate wildland forest. 


\section{Appendix 1-Inventory Design and Methods} Field Design and Sampling Method

The Pacific Northwest Research Station's Forest Inventory and Analysis (PNW-FIA) unit implemented the new annual inventory across all ownerships in California in 2001. The overall sampling design was a significant change from that of previous periodic inventories; the differences will be discussed more fully below.

In the annual inventory system for the Pacific Northwest (Alaska, Washington, Oregon, and California), the objective is to measure approximately 10 percent of the annual plots across an entire state each year. This annual subsample is referred to as a panel. The plots measured in a single panel are selected to ensure systematic coverage within each county, spanning both publicly and privately owned forests, and including lands reserved from industrial wood production such as national parks, wilderness areas, and natural areas. Estimates of forest attributes can be derived from measurements of a single panel for areas as small as a survey unit or ecosection; however, such estimates are often imprecise because one panel represents only 10 percent of the full inventory sample. More precise statistics are obtained by combining data from multiple panels. Estimates from sampled plots in the 10 panels measured 2001-2010 were combined to produce the statistics in this report. After all panels are initially measured (2010), from 2011 forward each panel is remeasured approximately every 10 years.

The FIA program collects information in three phases. In phase 1, a sample of points is interpreted from remotely sensed imagery, either aerial photos or satellite data, and the landscape is stratified into meaningful groupings, such as forested and nonforested areas, ecologically similar regions, and forest types. In phase 2, field plots are measured for a variety of indicators that describe forest composition, structure, and the physical geography of the landscape. Phase 2 plots are spaced at approximate 3-mile intervals on a hexagonal grid throughout the forest. In phase 3, a 1/16 sample of phase 2 plots is measured to assess forest health/ ecosystem indicators. Phase 3 is currently being modified to be a more flexible system incorporating different levels of effort in response to client needs and budget.

\section{Phase 1}

The goal of phase 1 is to reduce the variance associated with estimates of forest land area and volume by dividing samples into similar groups. Digital imagery collected by remote-sensing satellites is classed into a few similar strata (such as forest or nonforest) by means of standard techniques for image classification, and the total area of each of these strata is used to assign a representative acreage to each sample plot. Source data were derived from digitized 1940s survey maps (Harrington 2003) as well as percentage of tree canopy density derived from Landsat Thematic Mapper (30-m resolution) imagery collected in 2001 (Homer et al. 2007). The resulting classes, or strata (ranging from entirely forested to entirely nonforested, for example), are combined with other forest attributes likely to improve stratification effectiveness, such as owner class. For this report, separate strata are defined for national forest lands outside wilderness that were sampled at a greater density of plots than the FIA standard of 1 plot per 6,000 acres. The resulting strata are collapsed as necessary to ensure that at least 10 plots are in each stratum. Stratified estimation is applied by assigning each plot to one of these collapsed strata and by calculating the area of each collapsed stratum in each estimation unit. The estimates from stratified data are usually more precise than those from unstratified estimates.

\section{Phase 2}

The nationally standardized plot installed at each forested phase 2 location is a cluster of four subplots spaced 120 feet apart (fig. 57). Subplot 1 is in the center, with subplots 2 through 4 uniformly distributed radially around it. Each point serves as the center of a 1/24-acre circular subplot used to sample all trees at least 5.0 inches in diameter at breast height (d.b.h.). A 1/300-acre microplot, with its center located just east of each subplot center, is used to sample trees 1.0 to 4.9 inches d.b.h., as well as seedlings (trees less than 1.0 inch d.b.h.). On all lands in California , a 1/4-acre "macroplot" (58.9-foot radius) around each subplot center is used to tally trees larger than 24 inches d.b.h. On national forests in California, a hectare plot (a 185.1-foot fixed-radius plot centered on subplot 1) is also established to tally 


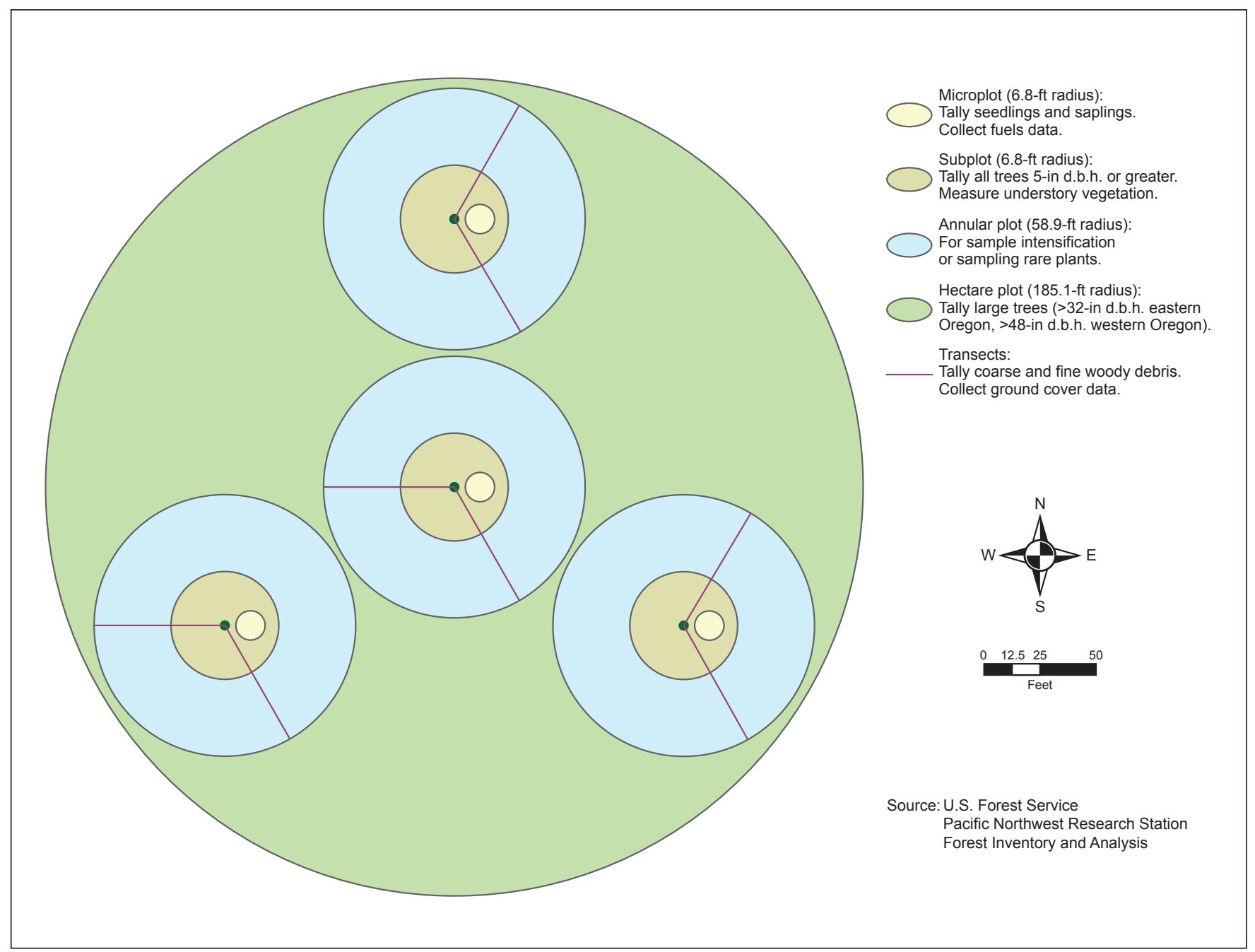

Figure 57-The Forest Inventory and Analysis plot design used in California, 2001-2010. D.b.h. = diameter at breast height.

trees larger than 32 inches d.b.h. in the eastern part of the Northwest Forest Plan area and larger than 48 inches d.b.h. in the western part of the Northwest Forest Plan area.

All phase 2 plots classified by aerial photography as possibly being forested are established in the field without regard to land use or land cover. Field crews delineate areas within the plot that are comparatively less heterogeneous than the plot as a whole with regard to reserved status, owner group, forest type, stand size class, regeneration status, and tree density; these areas are described as condition classes. The process of delineating these condition classes on a fixed-radius plot is called mapping. All measured trees are assigned to the mapped condition class in which they are located.
On phase 2 plots, crews assess physical characteristics such as slope, aspect, and elevation; stand characteristics such as age, size class, forest type, disturbance, site productivity, and regeneration status; and tree characteristics such as tree species, diameter, height, damages, decay, and vertical crown dimensions. They also collect general descriptive information such as soil depth, proximity to water and roads, and the geographic position of the plot in the larger landscape. In California, crews also assess regional variables: height and cover of understory species, the structure of live and dead fuels, and the structure and composition of down wood (see "Core, Core-Optional, and Regional Variables" section below). 
The FIA program sampled 5,575 forested phase 2 plots in California between 2001 and 2010 on the standard national plot grid. Estimates of timber volume and other forest attributes were derived from tree measurements and classifications made at each plot. Volumes for individual tally trees were computed with equations for each of the major species in California. Estimates of growth, removals, and mortality for non-national-forest timberland were determined from the remeasurement of 280 forested sample plots established in previous inventories. Estimates of growth, removals, and mortality for national forest land were determined from the remeasurement of 1,627 forested sample plots first measured by the Pacific Southwest Region of the National Forest System (Region 5) between 2001 and 2006 with the annual FIA design, then remeasured between 2006 and 2010.

\section{Phase 3 Ecosystem Indicators}

More extensive forest health measurements were collected in a 16-week period during the growing season (when most plants are in full leaf and many are flowering) on a subset (1/16) of phase 2 sample locations. At these phase 3 plots, measurements were taken on tree crowns, soils, lichens, down woody material, and (in some years) understory vegetation in addition to the phase 2 variables. One forest health measurement, ozone injury, was conducted on a separate grid with all 65 ozone plots measured annually.

The FIA program sampled 2,513 phase 3 plots in California between 2000 and 2010. The relatively small number of phase 3 samples is intended to serve as a broad-scale detection monitoring system for forest health problems. Budget uncertainties in 2011 resulted in the cessation of phase 3 data collection nationally. Forest Inventory and Analysis units and clients across the Nation have expressed interest in reevaluating our Forest Health program and working together to shape future directions. Therefore, the phase 3 protocols are evolving into a more flexible system that is more fully integrated with the FIA core program.

\section{Core, Core-Optional, and Regional Variables}

The majority of FIA variables collected in California are identical to those collected by FIA elsewhere in the United States - these are national "core" or "core optional" vari- ables (as the name suggests, collection of core optional variables is optional, but, if collected, they must be collected in the same way everywhere). A number of other variables are unique to PNW-FIA. These are "regional" variables and include such items as down woody material and understory vegetation on Phase 2 plots (not to be confused with down woody and understory vegetation on phase 3 plots, which are measured using a slightly different protocol), as well as insect and disease damage, a record of previous disturbance on the plot, and measurements for special studies.

\section{Data Processing}

The data used for this report are stored in the FIA National Information Management System (NIMS). The NIMS provides a means to input, edit, process, manage, and distribute FIA data. It includes a process for data loading, a national set of edit checks to ensure data consistency, an error-correction process, approved equations and algorithms, code to compile and calculate attributes, a table report generator, and routines to populate the presentation database. It applies numerous algorithms and equations to calculate, for example, stocking, forest type, stand size, volume, and biomass. The NIMS also generates estimates and associated statistics based on county areas and stratum weights developed outside of NIMS.

Additional FIA statistical design and estimation techniques are further reviewed in Bechtold and Patterson (2005).

\section{Statistical Estimates}

Throughout this report, we have published sampling errors (SE) for most of our estimates. These SEs account for the fact that we measured only a small sample of the forest (thereby producing a sample-based estimate) and not the entire forest (which is the population parameter of interest). Because of small sample sizes or high variability within the population, some estimates can be very imprecise. The reader is encouraged to take the SE into account when drawing any inference. One way to consider this type of uncertainty is to construct confidence intervals. Customarily, 66- or 95-percent confidence intervals are used. A 95-percent confidence interval means that one can be 95 percent confident that the interval contains the true population parameter of interest. For more details about confidence 
intervals, please consult Moore and McCabe (1989) or other statistical literature.

It is relatively easy to construct approximate 66- or 95-percent confidence intervals by multiplying the SE by 1.0 (for 66-percent confidence intervals) or 1.96 (for 95-percent confidence intervals) and subtracting and adding this to the estimate itself. For example, in table A2 of appendix 2, we estimated the total timberland in California to be 17,128,100 acres with an SE of 185,700. A 95-percent confidence interval for the total timberland area ranges from $16,764,100$ to $17,492,100$ acres.

The reader may want to assess whether or not two estimates are significantly different from each other. The statistically correct way to address this is to estimate the SE of the difference of two estimates, and either construct a confidence interval or use the equivalent z-test. However, this requires the original inventory data. It is often reasonable to assume that two estimates are nearly uncorrelated. For example, plots usually belong to one and only one owner. The correlation between estimates for different owners will be very small. If both estimates can be assumed to be nearly uncorrelated, the SE of the difference can be estimated by

$$
S E_{\text {Difference }}=\sqrt{S E_{\text {Estimate 1 }}^{2}+S E_{\text {Estimate 2 }}^{2}}
$$

Using the SE of the difference, a confidence interval of the difference can be constructed with this method.

If two estimates are based on data that occur on the same plot at the same time, the above equation should not be used. For example, appendix 2 table A36 contains estimates of tree volume by diameter class. If the reader wants to compare the volume of trees in the diameter class 9.0 to 10.9 d.b.h. (5.16 billion cubic feet) with that of trees in the diameter class 21.0 to 22.9 d.b.h. (5.73 billion cubic feet), the covariance between the estimates is not zero and this equation should not be used.

There are two other approaches the reader could possibly consider, but we do not recommend them. The first is to construct a confidence interval for one estimate and evaluate whether the other estimate falls within the interval. The problem is that unless both estimates are highly positively correlated, this approach will lead to a too-small confidence interval. The second approach is to construct confidence intervals for both estimates and determine whether or not they overlap. The problem here is that unless both estimates are highly negatively correlated, this approach will be very conservative. For more complex and indepth analysis, the reader may contact the PNW-FIA unit.

All estimates-means, totals, and their associated SE- are based on the poststratification methods described by Bechtold and Patterson (2005).

\section{Access Denied, Hazardous, or Inaccessible Plots}

Although every effort was made to visit all field plots that were entirely or partially forested, some were not sampled for a variety of reasons. Field crews may have been unable to obtain permission from the landowner to access the plot ("denied access"), and there were some plots that were impossible for crews to safely reach or access ("hazardous/ inaccessible"). Some private landowners deny access to their land, but privately owned plots usually are not as hazardous or inaccessible as plots on public lands. Although permission to visit public lands is almost always granted, some public land lies in higher elevation areas that can be very dangerous or impossible to reach.

This kind of missing data can introduce bias into the estimates if the nonsampled plots tend to be different from the entire population. Plots that are obviously nonforested (based on aerial photos) are rarely visited, and therefore the proportion of denied-access, hazardous, or inaccessible plots is significantly smaller than it is for forested plots.

The poststratification approach outlined in Bechtold and Patterson (2005) removes nonsampled plots from the sample. Estimates are adjusted for plots that are partially nonsampled by increasing the estimates by the nonsampled proportion within each stratum. To reduce the possible bias introduced by nonsampled plots, we delineated five broad strata groups: census water, forested public land, nonforested public land, forested private land, and nonforested private land. Some of these five broad strata groups were further divided into smaller strata to reduce the variance. Percentage of denied-access and hazardous/inaccessible plots for each of the five broad strata groups for California 2001-2010, are shown in the following tabulation: 


\begin{tabular}{lcccc} 
Strata group & Total plots & Denied access & $\begin{array}{c}\text { Hazardous/ } \\
\text { inaccessible }\end{array}$ & $\begin{array}{c}\text { Other } \\
\text { nonsampled }\end{array}$ \\
\hline Census water & 833 & 0.12 & Percent & \\
Private forest & 2,267 & 17.15 & 0 & 0 \\
Private nonforest & 5,977 & 4.88 & 1.71 & 0.62 \\
Public forest & 3,183 & 0.66 & 0.15 & 0.13 \\
Public nonforest & 5,381 & 0.33 & 1.29 & 0.13 \\
$\quad$ Total & 17,641 & 4.08 & 1.28 & 0.06 \\
\hline
\end{tabular}

\section{Timber Products Output Survey}

The timber products information presented in this report was based on a census of California's timber processors and out-of-state processors that use California timber. The census was conducted by the University of Montana's Bureau of Business and Economic Research in cooperation with PNW-FIA (Morgan et al. 2012). Through a written questionnaire or a phone interview, forest products manufacturers provided the following information for each of their facilities: plant production capacity and employment; volume of raw material received, by county and ownership; species of timber received; finished product volumes, types, sales value, and market locations; and utilization and marketing of manufacturing residue. This survey is designed to determine the size and composition of California's timber harvest and forest products industry, the industry's use of forest resources, and the generation and disposition of wood residues.

\section{National Woodland Owner Survey}

This survey of private forest owners (Butler et al. 2005) is conducted annually by the USDA Forest Service FIA Program to increase our understanding of private woodland owners. Questionnaires are mailed to individuals and private groups who own woodlands in which FIA has established forest inventory plots. Nationally, 20 percent of these owners (about 50,000) are contacted each year, and questionnaires with more detail are sent to coincide with national census, inventory, and assessment programs. For California, 432 private noncorporate woodland owners were sent questionnaires, and the 166 that were returned provide the data that were summarized and presented in this report.

\section{Periodic Versus Annual Inventories}

The PNW-FIA program began fieldwork for the fifth inventory of California in 2001. This was the first inventory that used the annual inventory system, in which $1 / 10$ of all forested plots (referred to as one panel) were visited each year. The first statewide panel of field plots was completed in 2001. By 2010, all field plots in the state had been measured, prompting production of another congressionally mandated 5-year analysis of California's forest resources as an update to the previous report (Christensen et al. 2008).

Data from new inventories are often compared with those from earlier inventories to determine trends in forest resources. However, for the comparisons to be valid, the procedures used in the two inventories should ideally be identical. Previous inventories of California's forest resources were completed in 1974, 1983, and 1994. These were periodic inventories in which all timberland plots in the state (outside of national forests and reserved areas such as national parks) were visited within a 2- or 3-year window. The last periodic inventory on national forests was completed in 1999.

As a result of our ongoing efforts to improve the efficiency and reliability of the inventory and to conform to the national annual inventory design adopted by all FIA units, several changes in procedures and definitions have been made since the last California inventory in 1994. These changes included an increase in plot density of about 18 percent, a new plot footprint (changing from a five-subplot configuration, in which about 2.5 acres were sampled, to a four-subplot configuration in which less than 1 acre is sampled) (fig. 58), a new set of nationally consistent measurement protocols, a plot visitation schedule that calls for 


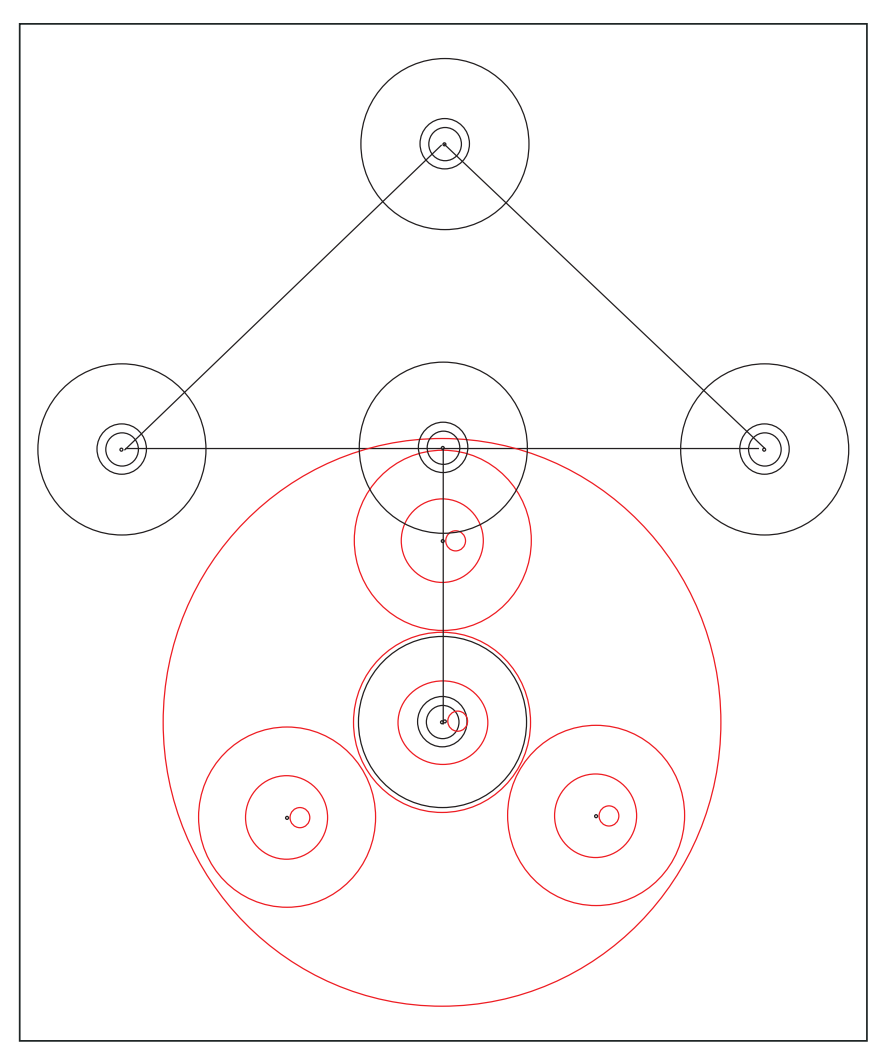

Figure 58-Relationship of periodic (upper, black figure) and annual (lower, red figure) inventory plot designs. Note that only one subplot center overlaps for both designs.

sampling of 10 percent of all forested plots in the state each year, and changes in timberland classification protocols. Although these changes will have little impact on statewide estimates of forest area, timber volume, and tree biomass, they have significantly affected estimates of timberland area (see below) and may affect plot classification variables such as forest type and stand size class, especially for estimates at the county level.

Disparities in timberland area estimates for California between periodic and annual inventories resulted from protocol changes for gathering site index data at each plot. Estimates of timberland area from the annual inventory are noticeably larger than timberland estimates reported from periodic inventories in California. One reason for this is a significant change in the procedures used to classify forest land as either productive timberland or unproductive forest land. In the periodic inventory of the mid 1990s, forest land was often classified using aerial photos or stratified map layers, before plots were assessed in the field. Classifica- tions were based on a number of factors such as species present, density/cover of trees, and geographic location. Timberland was defined as forests capable of producing at least $20 \mathrm{ft}^{3} \cdot \mathrm{ac}^{-1} \cdot \mathrm{yr}^{-1}$ of continuous crops of commercial trees, where "commercial" was defined in terms of size and quality of roundwood suitable for lumber or other manufactured products. All other forests (those not classified as timberland via aerial photos or field assessment) were assigned one of the "unproductive" forest land labels (oak woodland or pinyon-juniper, for example). Site trees were used to determine site index and the associated productivity index (mean annual increment, MAI) on all timberland plots. In some cases, the calculated MAI fell below the 20 $\mathrm{ft}^{3} \cdot \mathrm{ac}^{-1} \cdot \mathrm{yr}^{-1}$ threshold, resulting in several plots being reclassified as unproductive forest.

With the intent of developing a more objective approach to classifying forest land after the advent of annual inventory (which began in California in 2001), FIA began collecting site trees on all forest land, including unproductive land. As before, MAI is the basis for assigning a site class to every forest condition on the plot, which, in turn, is used to determine whether forest land is timberland or unproductive forest. Because there are a limited number of site index equations available for each species, and there can be difficulty in locating a representative site tree on some poor sites, the calculated MAI is sometimes unrepresentative of actual productivity. Thus, FIA now also uses certain tree species as indicators for low-productivity sites similar to what had been done during the periodic inventory. 


\section{Appendix 2-Summary Data Tables}

The following tables contain basic information about the forest resources of California as they relate to the discussions of current forest issues and basic resource information presented in this report. These tables aggregate data to a variety of levels, including county, ecosection, owner group, and forest type, allowing Pacific Northwest Research Station Forest Inventory and Analysis (FIA) inventory results to be applied at various scales and used for various analyses. Many other tables could be generated from the California annual data, but space limits us to just the key ones. Data are also available for download in nonsummarized form at www.fia.fs.fed.us.

The national FIA web site (http://www.fia.fs.fed.us/ tools-data/default.asp) contains a tool for querying the California annual data and generating custom tables or maps. Some of the tables in this appendix contain summaries of regional variables; data for regional variables currently are not included in the national FIA database (FIADB). Additional information on regional variables, field manuals, and local contact information is available at: http:// www.fs.fed.us/pnw/rma/index.php.

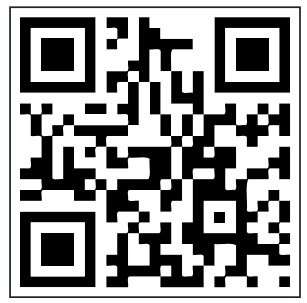

Note that information in the tables presented and in those generated from the FIADB may differ. As new data are added each year to FIADB, any tables generated from it will be based on the current full set of data in FIADB (e.g., 2002-2011, 2003-2012, etc.), whereas tables in this publication contain data from only 2001-2010. The user can take a snapshot of data from FIADB by selecting the desired years and generating tables that are similar, but probably not identical, to those presented here owing to quality assurance updates.

\section{Number of Plots}

Table A2-1-Number of Forest Inventory and Analysis plots measured in California from 2001 through 2010, by sample status, land class, and ownership group

Area

Table A2-2 - Area of sampled land and water, by land status and ownership group, California, 2001-2010

Table A2-3 - Area of forest land, by county and land status, California, 2001-2010

Table A2-4-Area of forest land, by county and ownership group, California, 2001-2010

Table A2-5-Area of forest land, by ownership and land status, California, 2001-2010

Table A2-6-Area of forest land, by forest type group and land status, California, 2001-2010

Table A2-7-Area of forest land, by forest type group and site productivity class, California, 2001-2010

Table A2-8 - Area of forest land, by forest type group, ownership group, and land status, California, 2001-2010

Table A2-9-Area of forest land, by forest type group and stand size class, California, 2001-2010

Table A2-10 - Area of forest land, by forest type group and stand age class, California, 2001-2010

Table A2-11 - Area of forest land by ecological section and land status, California, 2001-2010

Table A2-12 - Area of forest land, by forest type group and stand origin, California, 2001-2010

Table A2-13 - Area of timberland, by forest type group and stand size class, California, 2001-2010 


\section{Number of Live Trees}

Table A2-14-Number of live trees on forest land, by county and land status, California, 2001-2010

Table A2-15-Number of dead trees on forest land, by county and land status, California, 2001-2010

Table A2-16-Number of live trees on forest land, by county and broad species group, California, 2001-2010

Table A2-17-Number of dead trees on forest land, by county and broad species group, California, 2001-2010

Table A2-18 - Number of live trees on forest land, by species group and diameter class, California, 2001-2010

Table A2-19 - Number of dead trees on forest land, by species group and diameter class, California, 2001-2010

Table A2-20 - Number of live trees on forest land, by forest type group and ownership group, California, 2001-2010

Table A2-21 - Number of dead trees on forest land, by forest type group and ownership group, California, 2001-2010

Table A2-22 - Average number of live trees per acre on forest land, by forest type group and ownership group, California, 2001-2010

Table A2-23-Average number of dead trees per acre on forest land, by forest type group and ownership group, California, 2001-2010

Table A2-24-Number of growing-stock trees on timberland, by species group and diameter class, California, 2001-2010

\section{Tree Volume}

Table A2-25-Net volume of live trees on forest land, by ownership and land status, California, 2001-2010

Table A2-26 - Gross volume of dead trees on forest land, by ownership and land status, California, 2001-2010
Table A2-27- Net volume of live trees on forest land, by county and land status, California, 2001-2010

Table A2-28 - Gross volume of dead trees on forest land, by county and land status, California, 2001-2010

Table A2-29 - Net cubic volume of live trees on forest land, by county and broad species group, California, 2001-2010

Table A2-30 - Net volume of live trees on forest land, by forest type group and stand size class, California, 20012010

Table A2-31 - Gross volume of dead trees on forest land, by forest type group and stand size class, California, 20012010

Table A2-32 - Net volume of live trees on forest land, by forest type group and ownership group, California, 20012010

Table A2-33 - Gross volume of dead trees on forest land, by forest type group and ownership group, California, 20012010

Table A2-34 - Net volume of live trees on forest land, by species group and ownership group, California, 2001-2010

Table A2-35-Gross volume of dead trees on forest land, by species group and ownership group, California, 2001-2010

Table A2-36 - Net volume of live trees on forest land, by species group and diameter class, California, 2001-2010

Table A2-37-Gross volume of dead trees on forest land, by species group and diameter class, California, 2001-2010

Table A2-38 - Net volume of live trees on forest land, by forest type group and stand origin, California, 2001-2010

Table A2-39-Gross volume of dead trees on forest land, by forest type group and stand origin, California, 2001-2010

Table A2-40 - Net volume of live trees on forest land, by forest type group and stand age class, California, 2001-2010 
Table A2-41- Gross volume of dead trees on forest land, by forest type group and stand age class, California, 2001-2010 Table A2-42-Average net volume per acre of live trees on forest land, by forest type group and stand age class, California, 2001-2010

Table A2-43-Average gross volume per acre of dead trees on forest land, by forest type group and stand age class, California, 2001-2010

Table A2-44-Average net volume per acre of live trees on forest land, by forest type group and stand size class, California, 2001-2010

Table A2-45-Average gross volume per acre of dead trees on forest land, by forest type group and stand size class, California, 2001-2010

Table A2-46-Average net volume of live trees on forest land, by forest type group and ownership group, California, 2001-2010

Table A2-47-Average gross volume per acre of dead trees on forest land, by forest type group and ownership group, California, 2001-2010

Table A2-48 - Net volume of growing-stock trees on timberland, by species group and diameter class, California, 2001-2010

Table A2-49-Net volume of growing-stock trees on timberland, by species group and ownership group, California, 2001-2010

Table A2-50 - Net volume of sawtimber-sized trees on timberland, by species group and ownership group, California, 2001-2010

Table A2-51-Net board-foot volume (Scribner rule) of sawtimber trees on timberland, by county and ownership group, California, 2001-2010
Table A2-52 - Net board-foot volume (Scribner rule) of sawtimber trees on timberland, by forest type group and ownership group, California, 2001-2010

Table A2-53-Net board-foot volume (Scribner rule) of sawtimber trees on timberland, by forest type group and stand size class, California, 2000-2010

Table A2-54-Net board-foot volume (Scribner rule) of sawtimber trees on timberland, by species group and diameter class, California, 2001-2010

Table A2-55-Net board-foot volume (International 1/4-inch rule) of sawtimber trees on timberland, by species group and diameter class, California, 2001-2010

Table A2-56-Net board-foot volume (International 1/4-inch rule) of sawtimber trees on timberland, by species group and ownership group, California, 2001-2010

Table A2-57-Average net board-foot volume per acre (Scribner rule) of sawtimber trees on timberland, by forest type group and ownership group, California, 2001-2010

Table A2-58-Average net board-foot volume per acre (Scribner rule) of sawtimber trees on timberland, by forest type group and stand size class, California, 2001-2010

\section{Biomass}

Table A2-59-Aboveground biomass of live trees on forest land, by ownership and land status, California, 2001-2010

Table A2-60-Aboveground biomass of dead trees on forest land, by ownership and land status, California, 2001-2010

Table A2-61-Aboveground biomass of live trees on forest land, by county and land status, California, 2001-2010

Table A2-62-Aboveground biomass of dead trees on forest land, by county and land status, California, 2001-2010

Table A2-63-Aboveground biomass of live trees on forest land, by forest type group and ownership group, California, 2001-2010 
Table A2-64-Aboveground biomass of dead trees on forest land, by forest type group and ownership group, California, 2001-2010

Table A2-65-Aboveground biomass of live trees on forest land, by forest type group and stand size class, California, 2001-2010

Table A2-66-Aboveground biomass of dead trees on forest land, by forest type group and stand size class, California, 2001-2010

Table A2-67-Aboveground biomass of live trees on forest land, by forest type group and stand age class, California, 2001-2010

Table A2-68 - Aboveground biomass of dead trees on forest land, by forest type group and stand age class, California, 2001-2010

Table A2-69-Aboveground biomass of live trees on forest land, by species group and diameter class, California, 2001-2010

Table A2-70 - Aboveground biomass of dead trees on forest land, by species group and diameter class, California, 2001-2010

Table A2-71-Aboveground green biomass of live trees on forest land, by ownership and land status, California, 2001-2010

Table A2-72-Aboveground green biomass of live trees on forest land, by species group and diameter class, California, 2001-2010

Table A2-73 - Average aboveground biomass per acre of live trees on forest land, by forest type group and ownership group, California, 2001-2010

Table A2-74-Average aboveground biomass per acre of dead trees on forest land by forest type group and ownership group, California, 2001-2010
Table A2-75-Average aboveground biomass per acre of live trees on forest land, by forest type group and stand size class, California, 2001-2010

Table A2-76-Average aboveground biomass per acre of dead trees on forest land, by forest type group and stand size class, California, 2001-2010

Table A2-77-Average aboveground biomass per acre of live trees on forest land, by forest type group and stand age class, California, 2001-2010

Table A2-78 - Average aboveground biomass per acre of dead trees on forest land, by forest type group and stand age class, California, 2001-2010

\section{Carbon}

Table A2-79—Aboveground carbon mass of live trees on forest land, by ownership and land status, California, 2001-2010

Table A2-80-Aboveground carbon mass of dead trees on forest land, by ownership and land status, California, 2001-2010

Table A2-81-Aboveground carbon mass of live trees on forest land, by forest type group and ownership group, California, 2001-2010

Table A2-82 - Aboveground carbon mass of dead trees on forest land, by forest type group and ownership group, California, 2001-2010

Table A2-83-Aboveground carbon mass of live trees on forest land, by forest type group and stand size class, California, 2001-2010

Table A2-84-Aboveground carbon mass of dead trees on forest land, by forest type group and stand size class, California, 2001-2010

Table A2-85-Aboveground carbon mass of live trees on forest land, by species group and diameter class, California, 2001-2010 
Table A2-86-Aboveground carbon mass of dead trees on forest land, by species group and diameter class, California, 2001-2010

Table A2-87-Average aboveground carbon mass per hectare of live trees on forest land, by forest type group and ownership group, California, 2001-2010

Table A2-88-Average aboveground carbon mass per hectare of dead trees on forest land, by forest type group and ownership group, California, 2001-2010

Table A2-89-Average aboveground carbon mass per hectare of live trees on forest land, by forest type group and stand size class, California, 2001-2010

Table A2-90 - Average aboveground carbon mass per hectare of dead trees on forest land, by forest type group and stand size class, California, 2001-2010

\section{Down Wood}

Table A2-91-Volume of down wood on forest land, by forest type group and down wood diameter class, California, 2001-2010

Table A2-92 - Biomass of down wood on forest land, by forest type group and down wood diameter class, California, 2001-2010

Table A2-93 - Carbon mass of down wood on forest land, by forest type group and down wood diameter class, California, 2001-2010

Table A2-94-Number of pieces of down wood on forest land, by forest type group and down wood diameter class, California, 2001-2010

Table A2-95-Biomass of down wood on forest land, by forest type group, ownership group, and land status,

California, 2001-2010

Table A2-96-Carbon mass of down wood on forest land, by forest type group, ownership group, and land status,

California, 2001-2010
Table A2-97-Biomass of down wood on forest land, by county and land status, California, 2001-2010

Table A2-98 - Carbon mass of down wood on forest land, by county and land status, California, 2001-2010

Table A2-99-Average volume per acre of down wood on forest land by, forest type group and down wood diameter class, California, 2001-2010

Table A2-100 - Average biomass per acre of down wood on forest land, by forest type group and down wood diameter class, California, 2001-2010

Table A2-101-Average carbon mass per hectare of down wood on forest land, by forest type group and down wood diameter class, California, 2001-2010

Table A2-102 - Average number of pieces per acre of down wood on forest land, by forest type group and down wood diameter class, California, 2001-2010

Table A2-103-Average biomass per acre of down wood on forest land, by forest type group, ownership group, and land status, California, 2001-2010

Table A2-104-Average biomass per acre of down wood on forest land, by ownership and land status, California, 2001-2010

Table A2-105-Average biomass per acre of down wood on forest land, by county and land status, California, 20012010

\section{Combined Live Trees and Dead Wood (Standing Dead Trees and Down Wood)}

Table A2-106-Average biomass per acre of all dead wood (dead trees and down wood) on forest land, by county and land status, California, 2001-2010

Table A2-107-Volume of live trees, dead trees, and down wood on forest land, by stand age class, California, 20012010 
Table A2-108 - Biomass of live trees, dead trees, and down wood on forest land, by stand age class, California, 20012010

Table A2-109-Carbon mass of live trees, dead trees and down wood on forest land, by stand age class, California, 2001-2010

Table A2-110 - Average volume per acre of live trees, dead trees, and down wood on forest land, by stand age class, California, 2001-2010

Table A2-111-Average biomass per acre of live trees, dead trees, and down wood on forest land, by stand age class, California, 2001-2010

Table A2-112 - Average carbon mass per hectare of live trees, dead trees, and down wood on forest land, by stand age class, California, 2001-2010

\section{National Forest}

Table A2-113 - Area of forest land, by national forest and land status, California, 2001-2010

Table A2-114 - Net volume of live trees on forest land, by national forest and land status, California, 2001-2010

Table A2-115-Aboveground biomass of live trees on forest land, by national forest and land status, California, 20012010

Table A2-116 - Aboveground carbon mass of live trees on forest land, by national forest and land status, California, 2001-2010

Table A2-117-Biomass of down wood on forest land, by national forest and down wood diameter class, California, 2001-2010

Table A2-118 - Average net volume per acre of live trees on forest land, by national forest and land status, California, 2001-2010
Table A2-119_Average aboveground biomass per acre of live trees on forest land, by national forest and land status, California, 2001-2010

Table A2-120 - Average aboveground carbon mass per hectare of live trees on forest land, by national forest and land status, California, 2001-2010

Table A2-121-Average biomass per acre of down wood on forest land, by national forest and down wood diameter class, California, 2001-2010

\section{Tree Damage}

Table A2-122 - Number of live trees with damage on forest land, by species and type of damage, California, 2001-2010

Table A2-123 - Gross volume of live trees with damage on forest land, by species and type of damage, California, 2001-2010

Table A2-124-Area of forest land with more than 25 percent of basal area damaged, by forest type and type of damage, California, 2001-2010

\section{Timber Products Output and Removals}

Table A2-125-Total roundwood output by product, species group, and source of material, California, 2006

Table A2-126-Volume of timber removals by type of removal, source of material, species group, California, 2006

\section{Nontimber Forest Products}

Table A2-127-Estimated area of forest land covered by the most abundant vascular plant nontimber forest products, by plant group and species, California, 2001-2010

Table A2-128 - Percentage of forested plots with selected lichen nontimber forest products present, by species, California, 2001-2005 


\section{Tree Crowns}

Table A2-129-Estimated mean crown density and other statistics for live trees on forest land, by species group, California, 2001-2010

Table A2-130-Estimated mean foliage transparency and other statistics for live trees on forest land, by species group, California, 2001-2010

Table A2-131-Estimated mean crown dieback and other statistics for live trees on forest land, by species group, California, 2001-2010

\section{Understory Vegetation}

Table A2-132-Estimated area of forest land covered by selected nonnative vascular plant species and number of sample plots, by life form and species, California, 2001-2010

Table A2-133 - Mean cover of understory vegetation on forest land, by forest type group and life form, California 2001-2010

Table A2-134-Mean of understory vegetation by forest type class, age class group, and life form, California 2001-2010

\section{Lichens}

Table A2-135-Summary of lichen community indicator species richness on forest land, by location, California, 1998-2001, 2003

Table A2-136 - Summary of lichen community indicator climate index parameters on forest land, for the moisture gradient of Jovan and McCune's (2004) model, California, 1998-2001, 2003

Table A2-137- Summary of lichen community indicator climate index parameters on forest land, for the temperature gradient of Jovan and McCune's (2004) model. California, 1998-2001, 2003
Table A2-138 - Summary of lichen community indicator air quality index parameters on forest land, by type of parameter, Greater Central Valley, California, 1998-2001, 2003

Table A2-139- Summary of lichen community indicator air quality index parameters on forest land, by type of parameter, Greater Sierra Nevada, California, 1998-2001, 2003

\section{Ozone Injury}

Table A2-140-Ozone injury summary information from ozone biomonitoring plots, by year, California, 2000-2009

\section{Evidence of Fire}

Table A2-141-Forest land area on which evidence of fire was observed, by year and ecosection group, California, 1995-2009 
Table A2-1-Number of Forest Inventory and Analysis plots measured in California from 2001 through 2010, by sample status, land class, and ownership group

\begin{tabular}{|c|c|c|c|c|}
\hline Sampled status and land class & National forest & Other public & Private & Total \\
\hline
\end{tabular}

Sampled plots:

Forest land plots:

Softwood types

\begin{tabular}{rrrr}
2,040 & 468 & 868 & 3,331 \\
708 & 330 & 1,257 & 2,280 \\
115 & 18 & 37 & 170 \\
\hline 2,759 & 799 & 2,090 & 5,575
\end{tabular}

Nonforest land plots

1,074

4,512

6,196

11,742

Unsampled plots:

Denied access

Hazardous

Total

\begin{tabular}{rrrr}
26 & 13 & 714 & 753 \\
207 & 78 & 90 & 375 \\
\hline 234 & 95 & 828 & 1,156
\end{tabular}

All plots

3,555

5,183

8,282

16,837

Note: Totals are not additive because one plot can contain more than one forest type, land class, and sample status. 


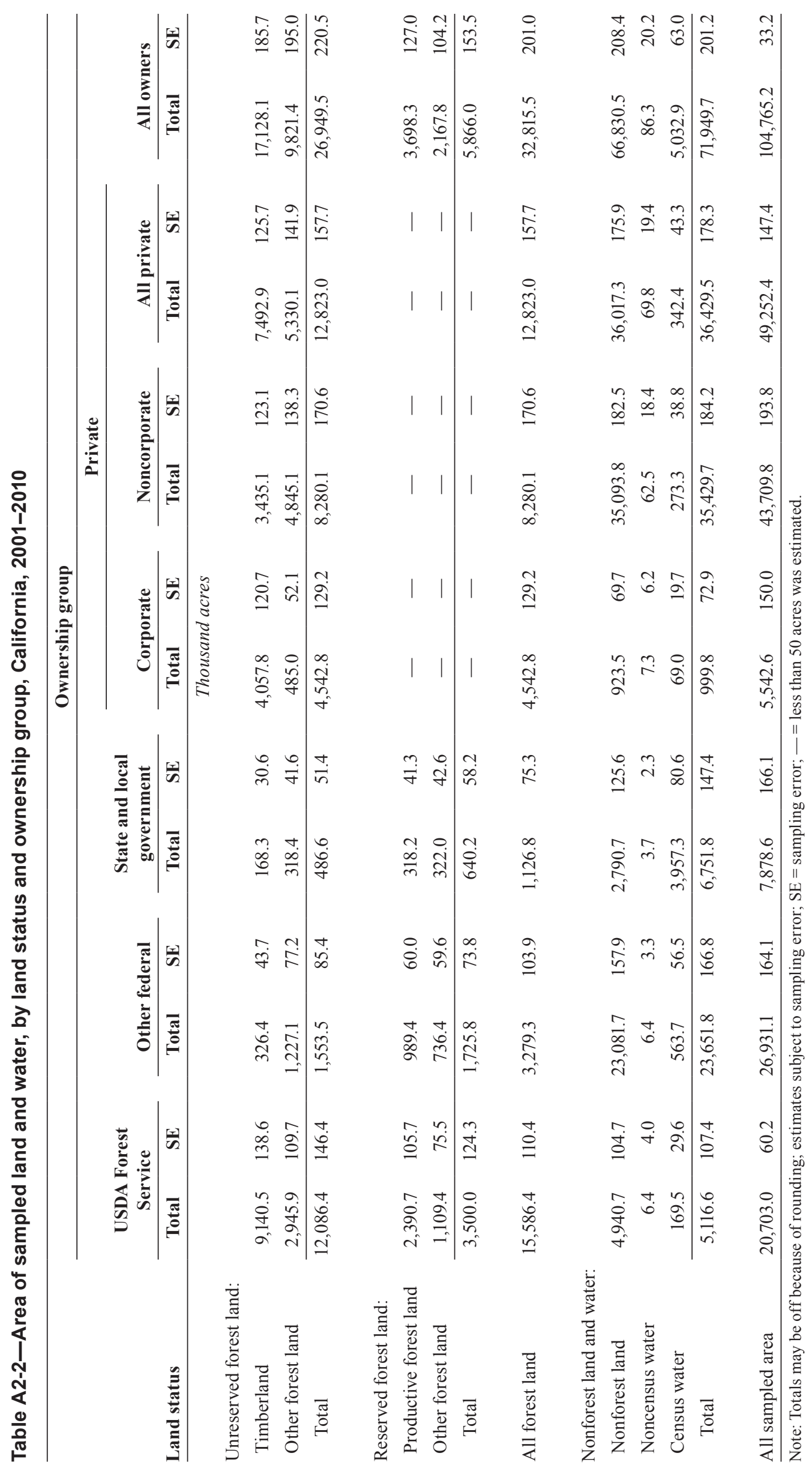




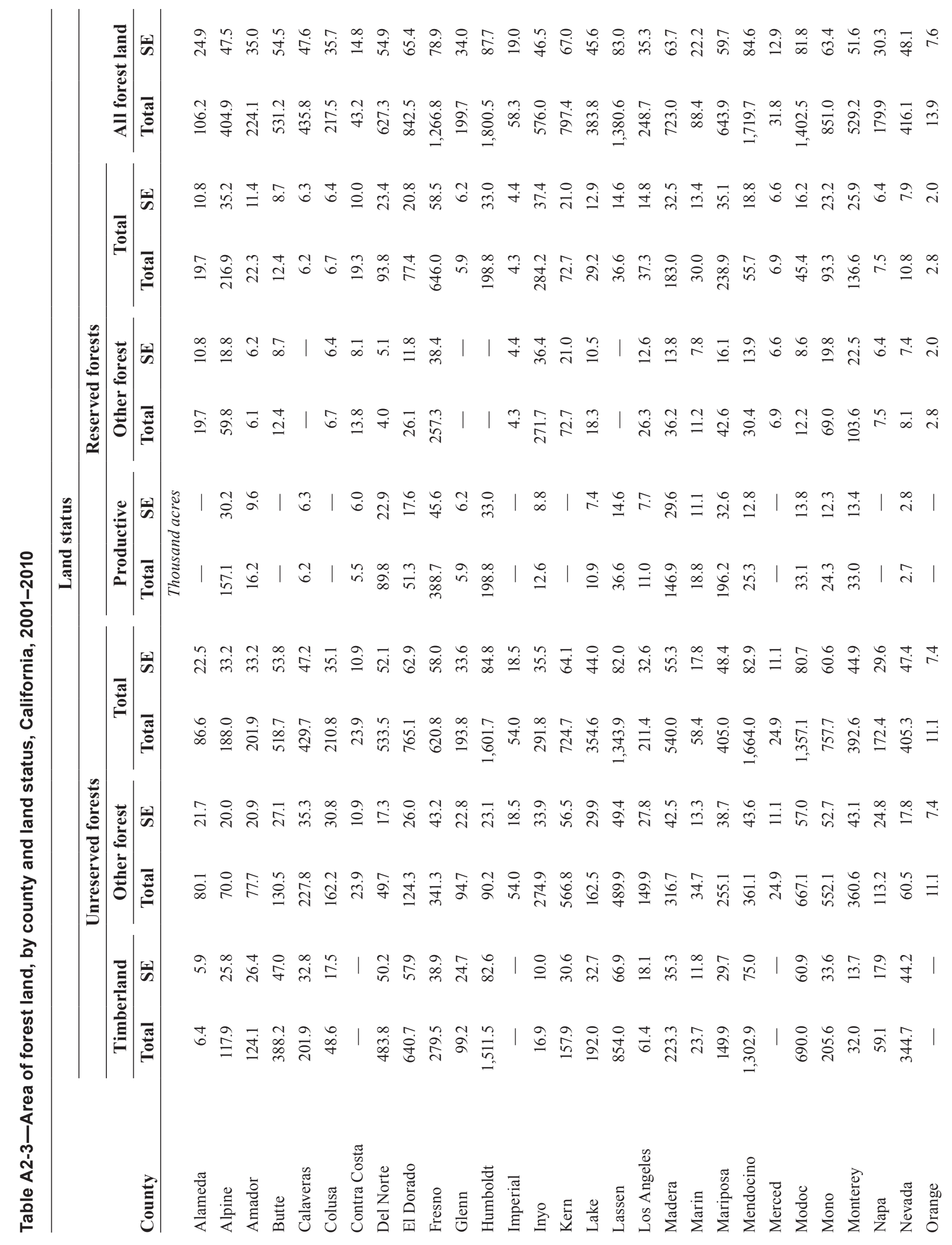




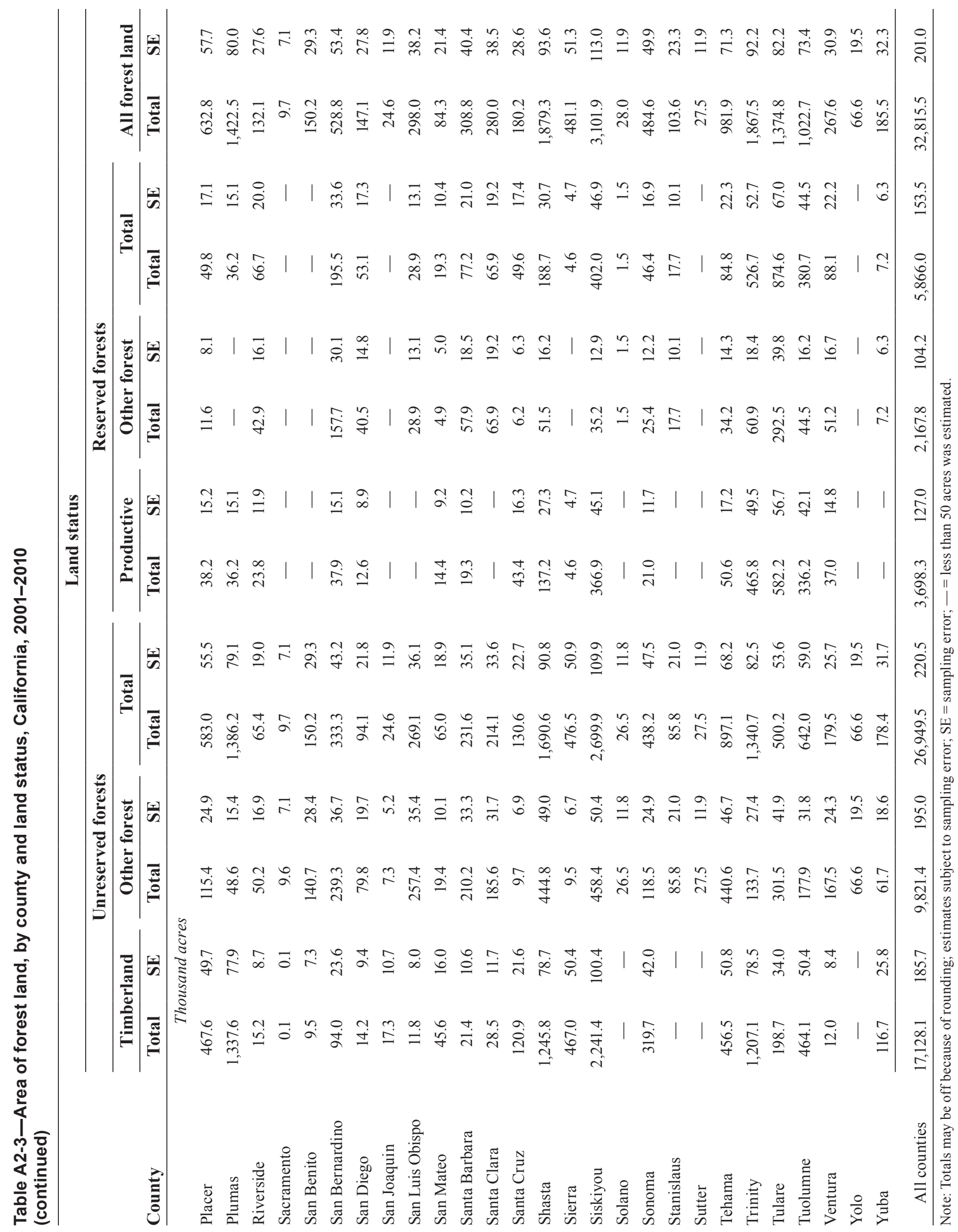


Table A2-4-Area of forest land, by county and ownership group, California, 2001-2010

\begin{tabular}{|c|c|c|c|c|c|c|c|c|c|c|}
\hline \multirow[b]{3}{*}{ County } & \multicolumn{8}{|c|}{ Ownership group } & & \\
\hline & \multicolumn{2}{|c|}{ National forest } & \multicolumn{2}{|c|}{ Other federal } & \multicolumn{2}{|c|}{$\begin{array}{c}\text { State and local } \\
\text { government }\end{array}$} & \multicolumn{2}{|c|}{ Private } & \multicolumn{2}{|c|}{ Total } \\
\hline & Total & SE & Total & SE & Total & SE & Total & SE & Total & SE \\
\hline & \multicolumn{10}{|c|}{ Thousand acres } \\
\hline Alameda & - & - & - & - & 61.5 & 19.1 & 44.7 & 16.2 & 106.2 & 24.9 \\
\hline Alpine & 373.1 & 45.3 & 6.3 & 6.3 & 18.3 & 10.8 & 7.2 & 6.6 & 404.9 & 47.5 \\
\hline Amador & 51.8 & 17.1 & 6.2 & 6.3 & 1.4 & 1.1 & 164.8 & 29.5 & 224.1 & 35.0 \\
\hline Butte & 162.4 & 30.2 & 12.4 & 8.9 & 17.1 & 9.7 & 339.3 & 43.4 & 531.2 & 54.5 \\
\hline Calaveras & 54.3 & 17.8 & 18.9 & 10.8 & 26.4 & 12.9 & 336.3 & 40.9 & 435.8 & 47.6 \\
\hline Colusa & 63.3 & 19.5 & 18.0 & 9.9 & - & - & 136.2 & 28.3 & 217.5 & 35.7 \\
\hline Contra Costa & - & - & 5.5 & 6.0 & 14.3 & 8.1 & 23.4 & 10.9 & 43.2 & 14.8 \\
\hline Del Norte & 421.3 & 41.9 & 9.9 & 8.0 & 36.3 & 15.3 & 159.7 & 31.2 & 627.3 & 54.9 \\
\hline El Dorado & 497.9 & 48.9 & 10.8 & 7.9 & 28.1 & 12.9 & 305.6 & 40.9 & 842.5 & 65.4 \\
\hline Fresno & 831.6 & 61.9 & 183.9 & 32.3 & - & - & 251.3 & 36.9 & $1,266.8$ & 78.9 \\
\hline Glenn & 146.1 & 29.5 & - & - & 4.6 & 4.6 & 49.0 & 16.4 & 199.7 & 34.0 \\
\hline Humboldt & 319.3 & 35.9 & 139.8 & 28.6 & 82.1 & 21.7 & $1,259.2$ & 75.5 & $1,800.5$ & 87.7 \\
\hline Imperial & - & - & 48.0 & 17.4 & 10.3 & 7.6 & - & - & 58.3 & 19.0 \\
\hline Inyo & 323.0 & 32.6 & 245.3 & 32.4 & 7.7 & 7.0 & - & - & 576.0 & 46.5 \\
\hline Kern & 312.1 & 41.4 & 128.4 & 27.8 & 7.7 & 7.0 & 349.2 & 44.2 & 797.4 & 67.0 \\
\hline Lake & 191.3 & 32.2 & 41.7 & 15.3 & 11.4 & 8.1 & 139.4 & 27.4 & 383.8 & 45.6 \\
\hline Lassen & 581.8 & 54.5 & 342.7 & 39.3 & - & - & 456.1 & 49.4 & $1,380.6$ & 83.0 \\
\hline Los Angeles & 196.2 & 31.0 & 6.2 & 6.3 & 18.4 & 9.8 & 27.8 & 12.2 & 248.7 & 35.3 \\
\hline Madera & 361.7 & 44.3 & 64.9 & 19.6 & 6.3 & 6.3 & 290.2 & 40.5 & 723.0 & 63.7 \\
\hline Marin & - & - & 30.0 & 13.4 & 27.5 & 11.7 & 30.9 & 13.4 & 88.4 & 22.2 \\
\hline Mariposa & 150.3 & 29.3 & 256.8 & 36.4 & - & - & 236.8 & 37.2 & 643.9 & 59.7 \\
\hline Mendocino & 165.5 & 30.0 & 35.9 & 14.9 & 96.9 & 24.5 & $1,421.4$ & 74.2 & $1,719.7$ & 84.6 \\
\hline Merced & - & - & 0.2 & 0.2 & 9.0 & 6.8 & 22.6 & 11.0 & 31.8 & 12.9 \\
\hline Modoc & 970.0 & 65.5 & 82.0 & 22.2 & - & - & 350.5 & 43.4 & $1,402.5$ & 81.8 \\
\hline Mono & 685.1 & 55.2 & 118.1 & 26.7 & 29.1 & 13.4 & 18.7 & 10.8 & 851.0 & 63.4 \\
\hline Monterey & 170.5 & 29.0 & 65.6 & 18.4 & 20.3 & 10.0 & 272.9 & 36.8 & 529.2 & 51.6 \\
\hline Napa & - & - & 1.4 & 1.4 & 16.4 & 9.4 & 162.1 & 28.8 & 179.9 & 30.3 \\
\hline Nevada & 170.6 & 31.1 & 9.3 & 7.2 & 13.9 & 8.3 & 222.4 & 34.8 & 416.1 & 48.1 \\
\hline Orange & 1.4 & 1.3 & - & - & 2.8 & 2.0 & 9.8 & 7.3 & 13.9 & 7.6 \\
\hline Placer & 336.9 & 42.7 & 29.7 & 13.2 & 55.4 & 17.9 & 210.7 & 33.3 & 632.8 & 57.7 \\
\hline Plumas & $1,042.8$ & 67.5 & 24.0 & 12.2 & 6.2 & 6.3 & 349.5 & 42.0 & $1,422.5$ & 80.0 \\
\hline Riverside & 40.8 & 15.2 & 43.6 & 16.4 & 25.2 & 12.3 & 22.5 & 10.6 & 132.1 & 27.6 \\
\hline Sacramento & - & - & - & - & - & - & 9.7 & 7.1 & 9.7 & 7.1 \\
\hline San Benito & - & - & 37.6 & 15.5 & - & - & 112.6 & 24.9 & 150.2 & 29.3 \\
\hline San Bernardino & 244.3 & 36.5 & 173.5 & 30.5 & - & - & 111.0 & 25.3 & 528.8 & 53.4 \\
\hline San Diego & 11.6 & 7.0 & 5.0 & 4.0 & 68.3 & 19.7 & 62.2 & 17.8 & 147.1 & 27.8 \\
\hline
\end{tabular}


Table A2-4-Area of forest land, by county and ownership group, California, 2001-2010 (continued)

\begin{tabular}{|c|c|c|c|c|c|c|c|c|c|c|}
\hline \multirow[b]{3}{*}{ County } & \multicolumn{8}{|c|}{ Ownership group } & & \\
\hline & \multicolumn{2}{|c|}{ National forest } & \multicolumn{2}{|c|}{ Other federal } & \multicolumn{2}{|c|}{$\begin{array}{c}\text { State and local } \\
\text { government }\end{array}$} & \multicolumn{2}{|c|}{ Private } & \multicolumn{2}{|c|}{ Total } \\
\hline & Total & SE & Total & SE & Total & SE & Total & SE & Total & SE \\
\hline & \multicolumn{10}{|c|}{ Thousand acres } \\
\hline San Joaquin & - & - & - & - & - & - & 24.6 & 11.9 & 24.6 & 11.9 \\
\hline San Luis Obispo & 40.9 & 14.5 & 29.4 & 12.7 & 22.1 & 11.1 & 205.7 & 31.2 & 298.0 & 38.2 \\
\hline San Mateo & 一 & - & 4.9 & 5.0 & 40.6 & 14.9 & 38.7 & 14.8 & 84.3 & 21.4 \\
\hline Santa Barbara & 172.5 & 30.3 & - & - & 6.6 & 6.7 & 129.8 & 25.9 & 308.8 & 40.4 \\
\hline Santa Clara & - & - & - & - & 110.3 & 24.9 & 169.7 & 30.2 & 280.0 & 38.5 \\
\hline Santa Cruz & - & - & - & - & 57.7 & 18.6 & 122.6 & 22.5 & 180.2 & 28.6 \\
\hline Shasta & 692.7 & 57.5 & 188.0 & 32.0 & 20.0 & 11.4 & 978.5 & 66.9 & $1,879.3$ & 93.6 \\
\hline Sierra & 394.9 & 45.9 & - & - & 4.6 & 4.7 & 81.6 & 21.6 & 481.1 & 51.3 \\
\hline Siskiyou & $2,148.1$ & 89.7 & 56.6 & 18.5 & 17.2 & 10.1 & 879.9 & 65.7 & $3,101.9$ & 113.0 \\
\hline Solano & - & - & - & - & 5.2 & 3.6 & 22.8 & 10.2 & 28.0 & 11.9 \\
\hline Sonoma & - & - & 8.7 & 7.1 & 55.3 & 18.0 & 420.6 & 46.5 & 484.6 & 49.9 \\
\hline Stanislaus & - & - & - & - & 17.7 & 10.1 & 85.8 & 21.0 & 103.6 & 23.3 \\
\hline Sutter & - & - & - & - & - & - & 27.5 & 11.9 & 27.5 & 11.9 \\
\hline Tehama & 328.7 & 42.1 & 23.2 & 11.6 & 20.9 & 11.2 & 609.0 & 55.4 & 981.9 & 71.3 \\
\hline Trinity & $1,374.9$ & 77.1 & 92.3 & 23.4 & - & - & 400.3 & 45.7 & $1,867.5$ & 92.2 \\
\hline Tulare & 762.4 & 61.8 & 356.7 & 39.6 & 6.2 & 6.3 & 249.6 & 38.0 & $1,374.8$ & 82.2 \\
\hline Tuolumne & 493.0 & 50.0 & 312.9 & 40.3 & 6.5 & 6.3 & 210.4 & 34.5 & $1,022.7$ & 73.4 \\
\hline Ventura & 246.9 & 30.1 & 2.1 & 1.8 & - & - & 18.6 & 9.8 & 267.6 & 30.9 \\
\hline Yolo & - & - & - & - & - & - & 66.6 & 19.5 & 66.6 & 19.5 \\
\hline Yuba & 54.7 & 18.1 & 3.1 & 3.1 & 12.9 & 8.4 & 114.8 & 25.4 & 185.5 & 32.3 \\
\hline All counties & $15,586.4$ & 110.4 & $3,279.3$ & 103.9 & $1,126.8$ & 75.3 & $12,823.0$ & 157.7 & $32,815.5$ & 201.0 \\
\hline
\end{tabular}

Note: Totals may be off because of rounding; estimates subject to sampling error; $\mathrm{SE}=$ sampling error; — $—=$ less than 50 acres was estimated. 


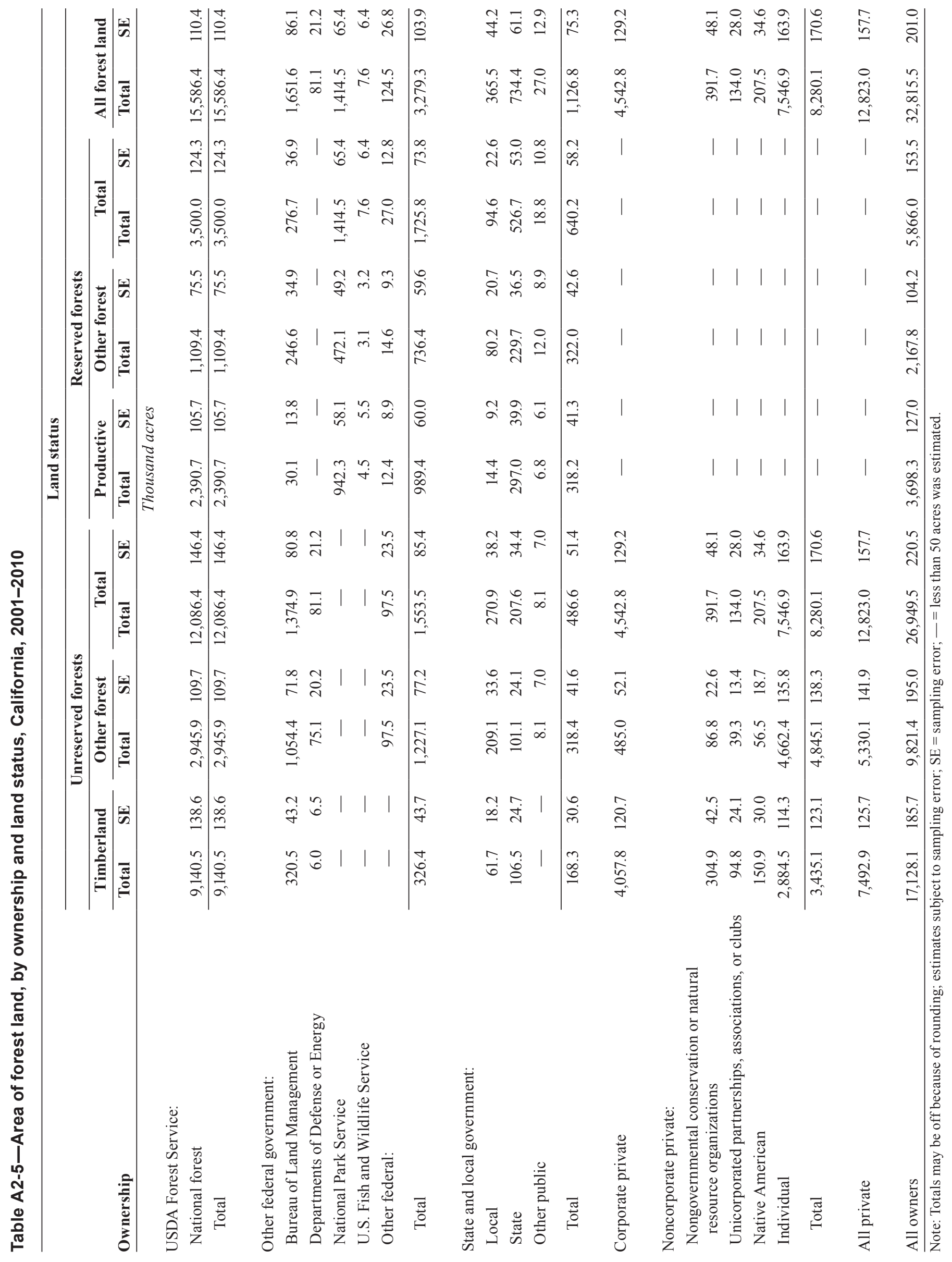




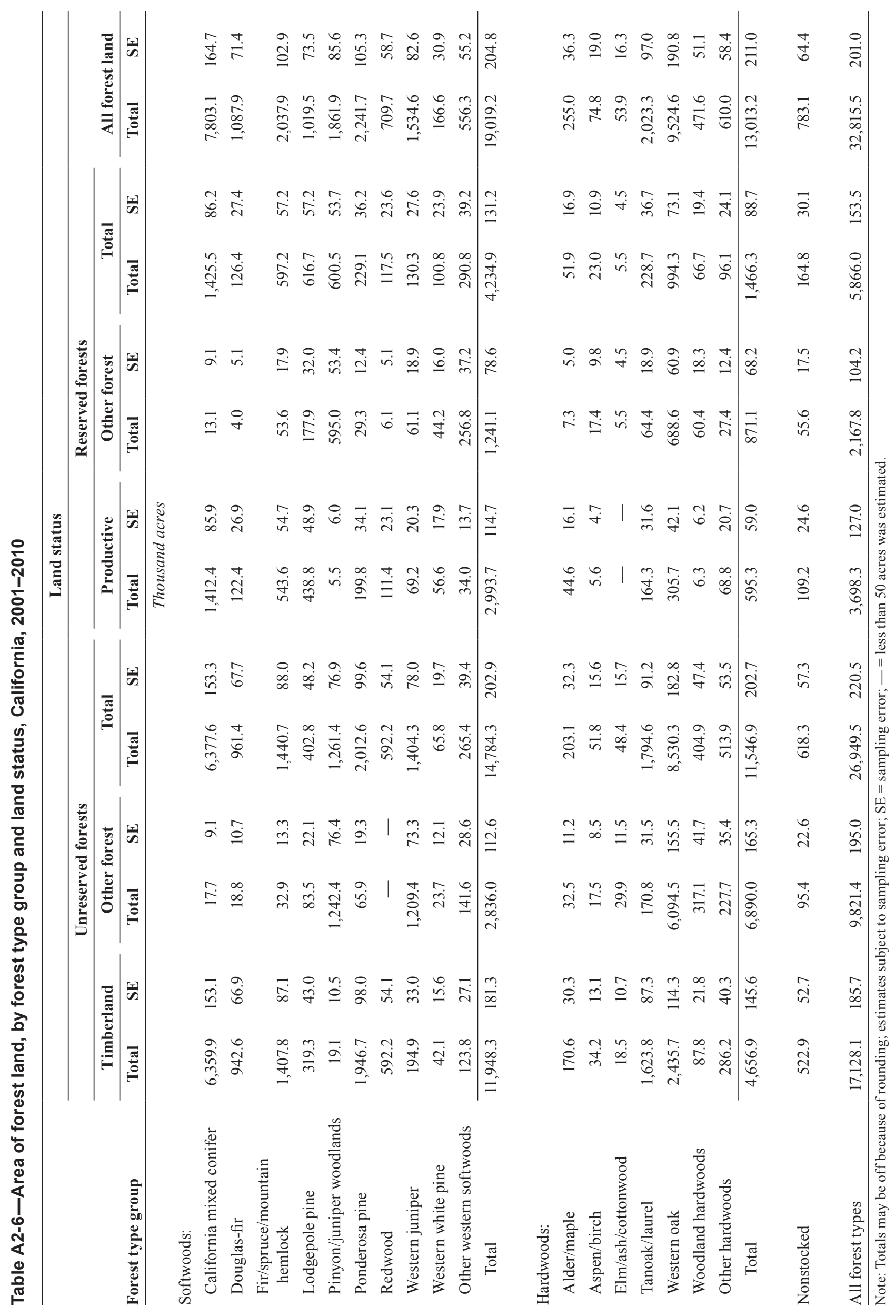




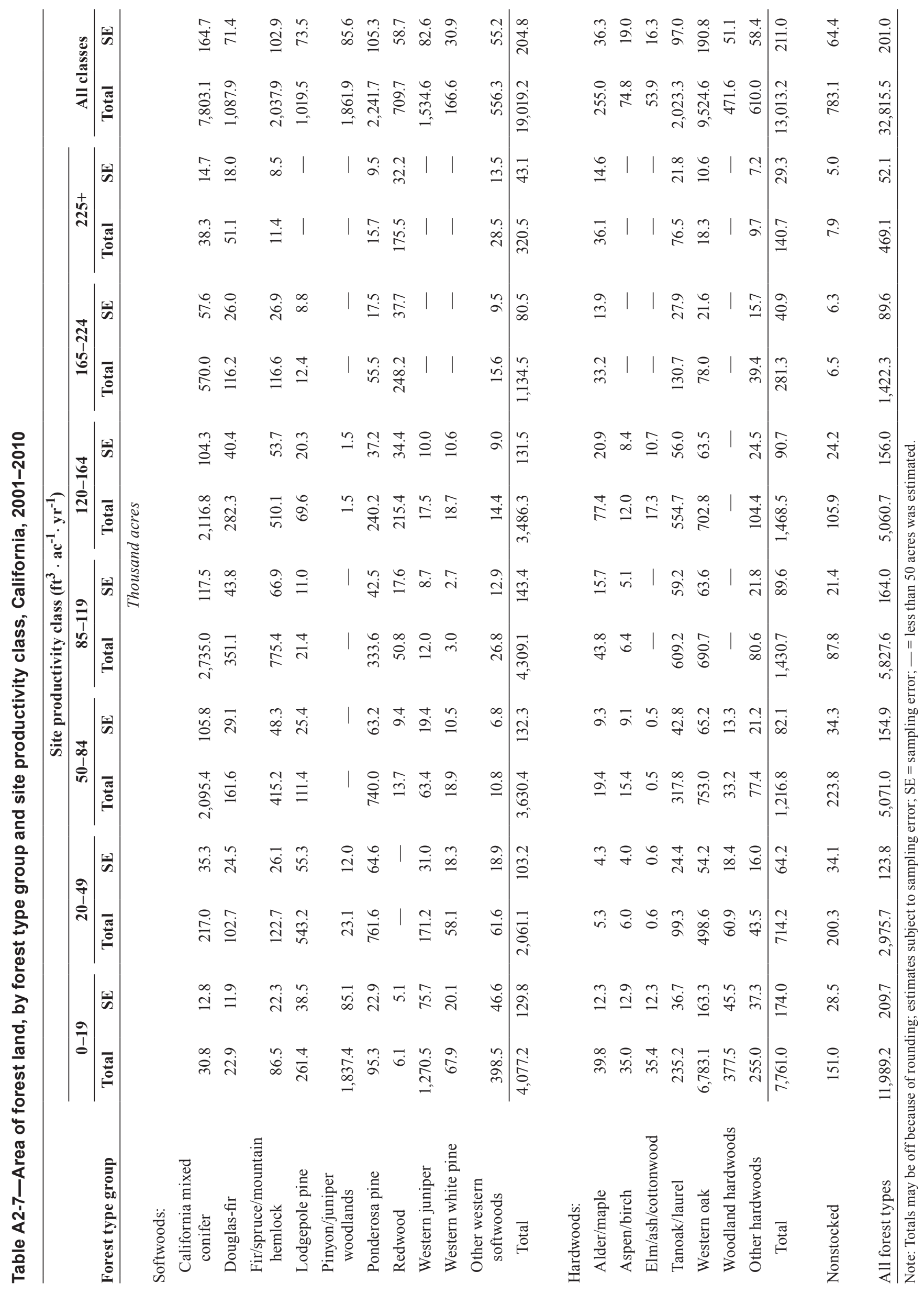




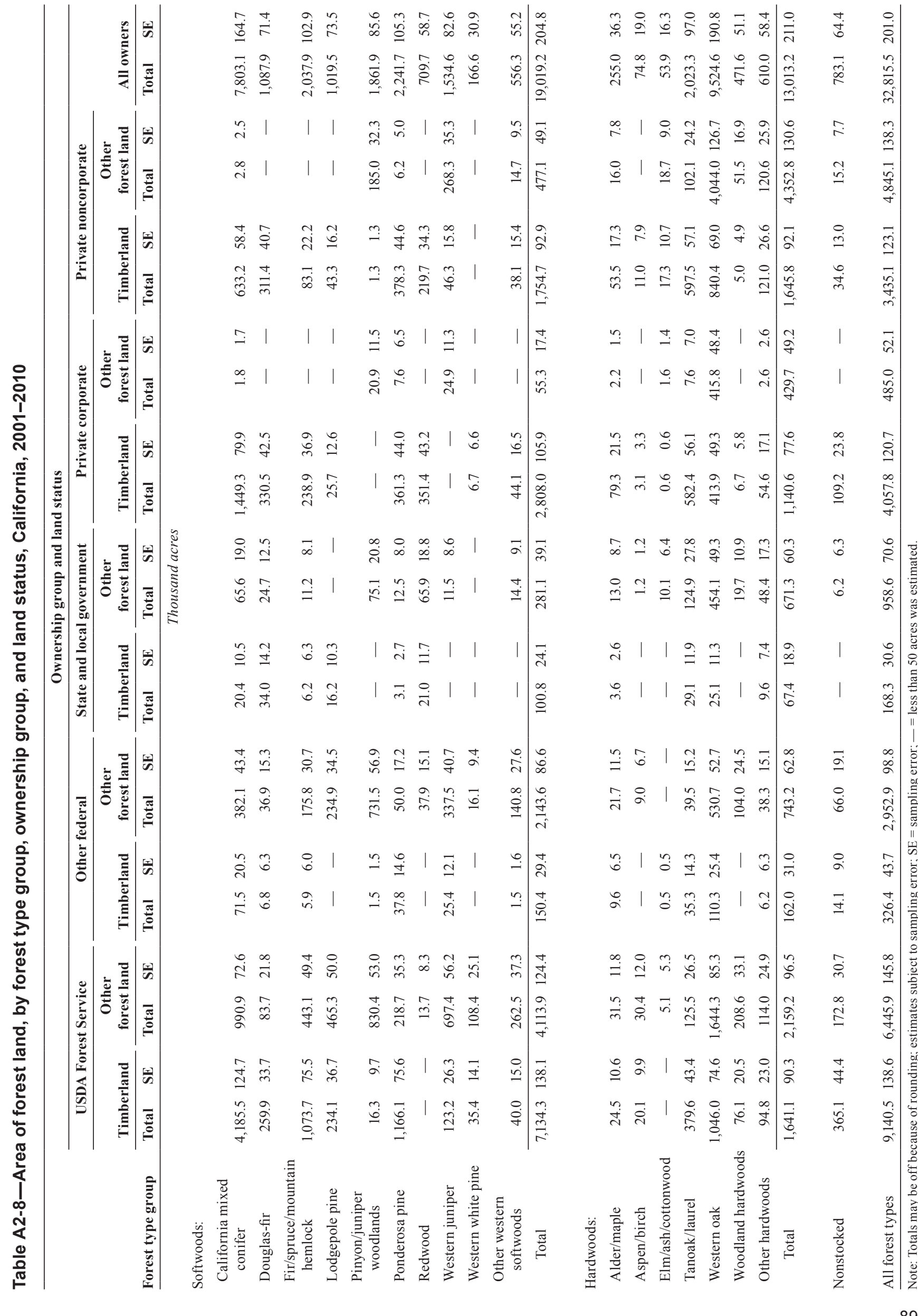


Table A2-9-Area of forest land, by forest type group and stand size class, California, 2001-2010

\begin{tabular}{|c|c|c|c|c|c|c|c|c|}
\hline \multirow[b]{3}{*}{ Forest type group } & \multicolumn{6}{|c|}{ Stand size class } & & \\
\hline & \multicolumn{2}{|c|}{$\begin{array}{c}\text { Large-diameter } \\
\text { stands }\end{array}$} & \multicolumn{2}{|c|}{$\begin{array}{c}\text { Medium- } \\
\text { diameter stands }\end{array}$} & \multicolumn{2}{|c|}{$\begin{array}{c}\text { Small-diameter } \\
\text { stands }\end{array}$} & \multicolumn{2}{|c|}{ All classes } \\
\hline & Total & SE & Total & SE & Total & SE & Total & SE \\
\hline & \multicolumn{8}{|c|}{ Thousand acres } \\
\hline \multicolumn{9}{|l|}{ Softwoods: } \\
\hline California mixed conifer & $7,270.3$ & 161.0 & 178.0 & 31.2 & 354.8 & 44.2 & $7,803.1$ & 164.7 \\
\hline Douglas-fir & 943.3 & 66.5 & 55.4 & 17.4 & 89.2 & 22.5 & $1,087.9$ & 71.4 \\
\hline Fir/spruce/mountain hemlock & $1,900.4$ & 99.5 & 22.7 & 10.4 & 114.7 & 24.8 & $2,037.9$ & 102.9 \\
\hline Lodgepole pine & 933.7 & 70.2 & 42.1 & 15.0 & 43.7 & 15.0 & $1,019.5$ & 73.5 \\
\hline Pinyon/juniper woodlands & $1,527.9$ & 80.7 & 197.6 & 33.9 & 136.4 & 28.5 & $1,861.9$ & 85.6 \\
\hline Ponderosa pine & $1,914.2$ & 98.5 & 176.4 & 30.2 & 151.2 & 28.2 & $2,241.7$ & 105.3 \\
\hline Redwood & 659.9 & 57.1 & 21.6 & 11.3 & 28.2 & 11.1 & 709.7 & 58.7 \\
\hline Western juniper & $1,277.0$ & 78.1 & 120.1 & 25.9 & 137.5 & 29.0 & $1,534.6$ & 82.6 \\
\hline Western white pine & 127.8 & 26.8 & 7.3 & 6.3 & 31.4 & 13.9 & 166.6 & 30.9 \\
\hline Other western softwoods & 373.8 & 45.1 & 72.5 & 20.4 & 110.0 & 25.1 & 556.3 & 55.2 \\
\hline Total & $16,928.3$ & 201.7 & 893.7 & 69.8 & $1,197.2$ & 80.7 & $19,019.2$ & 204.8 \\
\hline
\end{tabular}

Hardwoods:

\begin{tabular}{|c|c|c|c|c|c|c|c|c|}
\hline Alder/maple & 157.4 & 28.9 & 67.9 & 18.4 & 29.7 & 12.4 & 255.0 & 36.3 \\
\hline Aspen/birch & 14.1 & 8.3 & 15.3 & 7.1 & 45.4 & 15.6 & 74.8 & 19.0 \\
\hline Elm/ash/cottonwood & 42.8 & 14.7 & 2.8 & 1.8 & 8.2 & 6.9 & 53.9 & 16.3 \\
\hline Tanoak/laurel & $1,316.8$ & 81.8 & 453.3 & 50.8 & 253.2 & 36.9 & $2,023.3$ & 97.0 \\
\hline Western oak & $4,873.7$ & 150.9 & $3,357.1$ & 128.7 & $1,293.8$ & 84.5 & $9,524.6$ & 190.8 \\
\hline Woodland hardwoods & 384.0 & 46.6 & 60.9 & 18.0 & 26.7 & 10.8 & 471.6 & 51.1 \\
\hline Other hardwoods & 217.1 & 34.6 & 162.2 & 30.6 & 230.7 & 36.1 & 610.0 & 58.4 \\
\hline Total & $7,005.9$ & 175.5 & $4,119.5$ & 141.3 & $1,887.8$ & 100.7 & $13,013.2$ & 211.0 \\
\hline Nonstocked & - & - & - & - & - & - & 783.1 & 64.4 \\
\hline All forest types & $23,934.2$ & 222.7 & $5,013.2$ & 156.0 & $3,085.0$ & 126.3 & $32,815.5$ & 201.0 \\
\hline
\end{tabular}




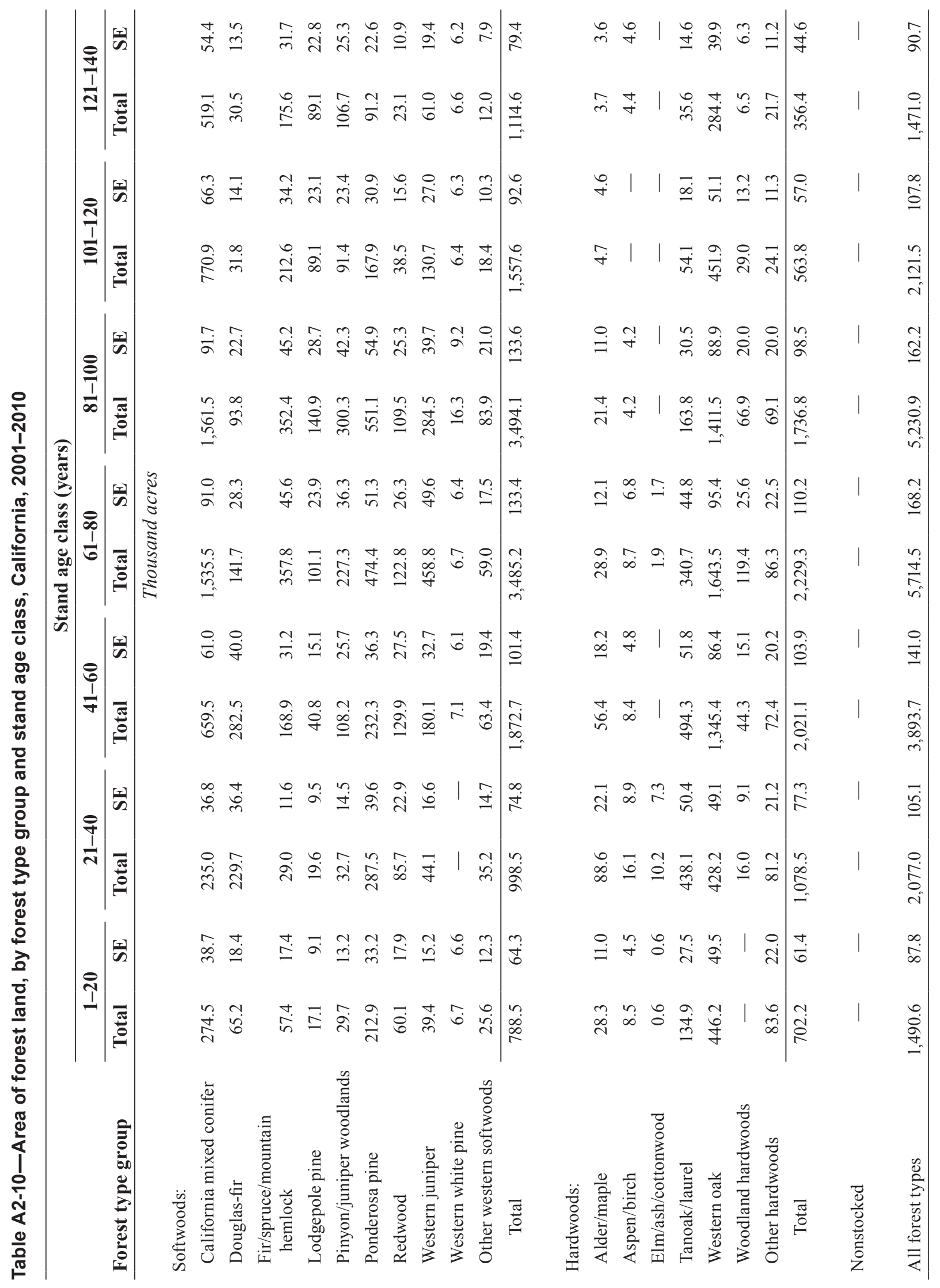




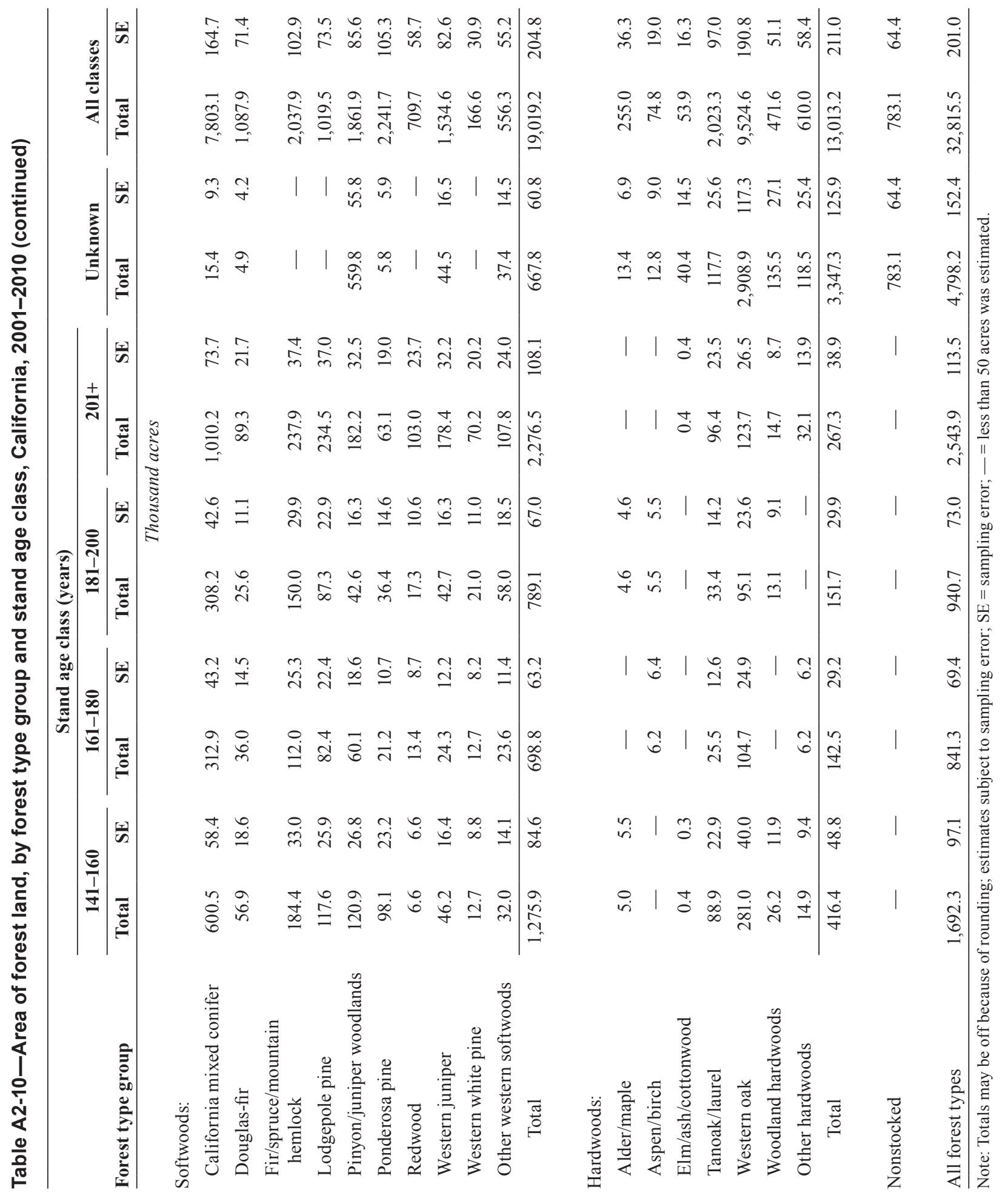




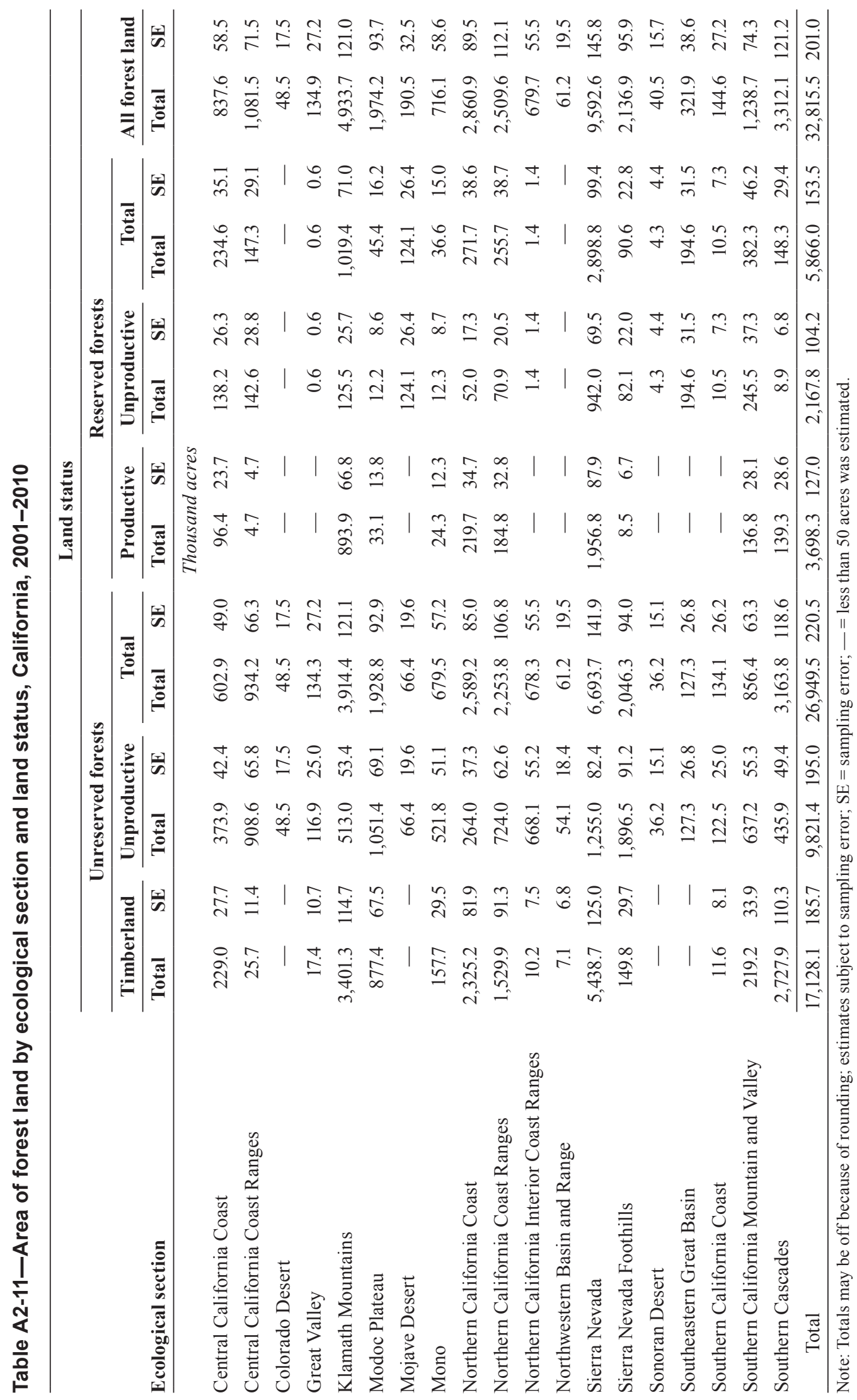


Table A2-12-Area of forest land, by forest type group and stand origin, California, 2001-2010

\begin{tabular}{|c|c|c|c|c|c|c|}
\hline \multirow[b]{3}{*}{ Forest type group } & \multicolumn{4}{|c|}{ Stand origin } & & \\
\hline & \multicolumn{2}{|c|}{ Natural stands } & \multicolumn{2}{|c|}{ Artificial regeneration } & \multicolumn{2}{|c|}{ Total } \\
\hline & Total & SE & Total & SE & Total & SE \\
\hline & \multicolumn{6}{|c|}{ Thousand acres } \\
\hline \multicolumn{7}{|l|}{ Softwoods: } \\
\hline California mixed conifer & $7,413.4$ & 161.7 & 389.7 & 46.8 & $7,803.1$ & 164.7 \\
\hline Douglas-fir & $1,022.3$ & 69.8 & 65.6 & 19.1 & $1,087.9$ & 71.4 \\
\hline Fir/spruce/mountain hemlock & $1,988.6$ & 101.7 & 49.3 & 16.8 & $2,037.9$ & 102.9 \\
\hline Lodgepole pine & $1,012.9$ & 73.1 & 6.6 & 5.3 & $1,019.5$ & 73.5 \\
\hline Pinyon/juniper woodlands & $1,855.3$ & 85.6 & 6.6 & 6.5 & $1,861.9$ & 85.6 \\
\hline Ponderosa pine & $1,761.0$ & 94.6 & 480.7 & 51.1 & $2,241.7$ & 105.3 \\
\hline Redwood & 683.3 & 57.8 & 26.5 & 13.1 & 709.7 & 58.7 \\
\hline Western juniper & $1,510.6$ & 82.2 & 24.0 & 11.8 & $1,534.6$ & 82.6 \\
\hline Western white pine & 159.9 & 30.2 & 6.7 & 6.6 & 166.6 & 30.9 \\
\hline Other western softwoods & 556.3 & 55.2 & - & - & 556.3 & 55.2 \\
\hline Total & $17,963.5$ & 205.7 & $1,055.7$ & 75.3 & $19,019.2$ & 204.8 \\
\hline
\end{tabular}

Hardwoods:

$\begin{array}{lrrrrrr}\text { Alder/maple } & 240.7 & 35.1 & 14.3 & 9.1 & 255.0 & 36.3 \\ \text { Aspen/birch } & 74.8 & 19.0 & - & - & 74.8 & 19.0 \\ \text { Elm/ash/cottonwood } & 53.3 & 16.3 & 0.5 & 0.5 & 53.9 & 16.3 \\ \text { Tanoak/laurel } & 1,858.9 & 93.7 & 164.4 & 30.9 & 2,023.3 & 97.0 \\ \text { Western oak } & 9,407.6 & 189.9 & 117.0 & 25.2 & 9,524.6 & 190.8 \\ \text { Woodland hardwoods } & 470.0 & 51.1 & 1.6 & 1.6 & 471.6 & 51.1 \\ \text { Other hardwoods } & 568.1 & 56.4 & 41.9 & 15.3 & 610.0 & 58.4 \\ \quad & 12,673.5 & 210.0 & 339.7 & 43.3 & 13,013.2 & 211.0 \\ \quad & & & & & & \\ \quad \text { Total } & 678.3 & 60.2 & 104.8 & 23.8 & 783.1 & 64.4 \\ \text { Nonstocked } & & & & & & \\ & 31,315.3 & 212.9 & 1,500.2 & 88.6 & 32,815.5 & 201.0 \\ \text { All forest types } & & & & & & \end{array}$

Note: Totals may be off because of rounding; estimates subject to sampling error; SE = sampling error; — = less than 50 acres was estimated. 
Table A2-13-Area of timberland, by forest type group and stand size class, California, 2001-2010

\begin{tabular}{|c|c|c|c|c|c|c|c|c|}
\hline \multirow[b]{3}{*}{ Forest type group } & \multicolumn{6}{|c|}{ Stand size class } & \multirow{3}{*}{$\begin{array}{c}\begin{array}{c}\text { All } \\
\text { classes }\end{array} \\
\text { Total }\end{array}$} & \multirow[b]{3}{*}{ SE } \\
\hline & \multicolumn{2}{|c|}{$\begin{array}{c}\text { Large-diameter } \\
\text { stands }\end{array}$} & \multicolumn{2}{|c|}{$\begin{array}{c}\text { Medium-diameter } \\
\text { stands }\end{array}$} & \multicolumn{2}{|c|}{$\begin{array}{c}\text { Small-diameter } \\
\text { stands }\end{array}$} & & \\
\hline & Total & SE & Total & SE & Total & SE & & \\
\hline & \multicolumn{8}{|c|}{ Thousand acres } \\
\hline \multicolumn{9}{|l|}{ Softwoods: } \\
\hline California mixed conifer & $5,846.9$ & 148.7 & 178.0 & 31.2 & 335.1 & 43.0 & $6,359.9$ & 153.1 \\
\hline Douglas-fir & 818.6 & 62.5 & 43.0 & 15.0 & 81.0 & 21.6 & 942.6 & 66.9 \\
\hline Fir/spruce/mountain hemlock & $1,328.6$ & 84.5 & 20.0 & 10.2 & 59.1 & 17.5 & $1,407.8$ & 87.1 \\
\hline Lodgepole pine & 270.2 & 39.4 & 27.3 & 12.7 & 21.7 & 10.2 & 319.3 & 43.0 \\
\hline Pinyon/juniper woodlands & 19.1 & 10.5 & - & - & - & - & 19.1 & 10.5 \\
\hline Ponderosa pine & $1,635.1$ & 90.9 & 161.9 & 28.9 & 149.7 & 28.1 & $1,946.7$ & 98.0 \\
\hline Redwood & 542.4 & 52.4 & 21.6 & 11.3 & 28.2 & 11.1 & 592.2 & 54.1 \\
\hline Western juniper & 154.8 & 29.4 & 8.3 & 4.5 & 31.7 & 13.8 & 194.9 & 33.0 \\
\hline Western white pine & 16.7 & 9.3 & 6.3 & 6.2 & 19.1 & 10.9 & 42.1 & 15.6 \\
\hline Other western softwoods & 105.7 & 24.5 & - & - & 18.1 & 9.9 & 123.8 & 27.1 \\
\hline Total & $10,738.2$ & 176.2 & 466.4 & 49.6 & 743.7 & 62.7 & $11,948.3$ & 181.3 \\
\hline \multicolumn{9}{|l|}{ Hardwoods: } \\
\hline Alder/maple & 115.0 & 24.7 & 42.1 & 14.8 & 13.5 & 9.3 & 170.6 & 30.3 \\
\hline Aspen/birch & 9.7 & 6.9 & 9.9 & 6.2 & 14.7 & 9.3 & 34.2 & 13.1 \\
\hline Elm/ash/cottonwood & 17.3 & 10.7 & 0.5 & 0.5 & 0.6 & 0.6 & 18.5 & 10.7 \\
\hline Tanoak/laurel & $1,096.0$ & 74.5 & 347.1 & 44.8 & 180.7 & 31.5 & $1,623.8$ & 87.3 \\
\hline Western oak & $1,507.6$ & 91.8 & 625.3 & 59.4 & 302.8 & 40.9 & $2,435.7$ & 114.3 \\
\hline Woodland hardwoods & 64.1 & 18.9 & 16.9 & 9.4 & 6.9 & 5.9 & 87.8 & 21.8 \\
\hline Other hardwoods & 142.3 & 28.1 & 44.7 & 16.2 & 99.2 & 23.5 & 286.2 & 40.3 \\
\hline Total & $2,951.9$ & 120.9 & $1,086.5$ & 77.5 & 618.4 & 58.0 & $4,656.9$ & 145.6 \\
\hline Nonstocked & - & - & - & - & - & - & 522.9 & 52.7 \\
\hline All forest types & $13,690.1$ & 185.3 & $1,553.0$ & 91.2 & $1,362.1$ & 83.7 & $17,128.1$ & 185.7 \\
\hline
\end{tabular}




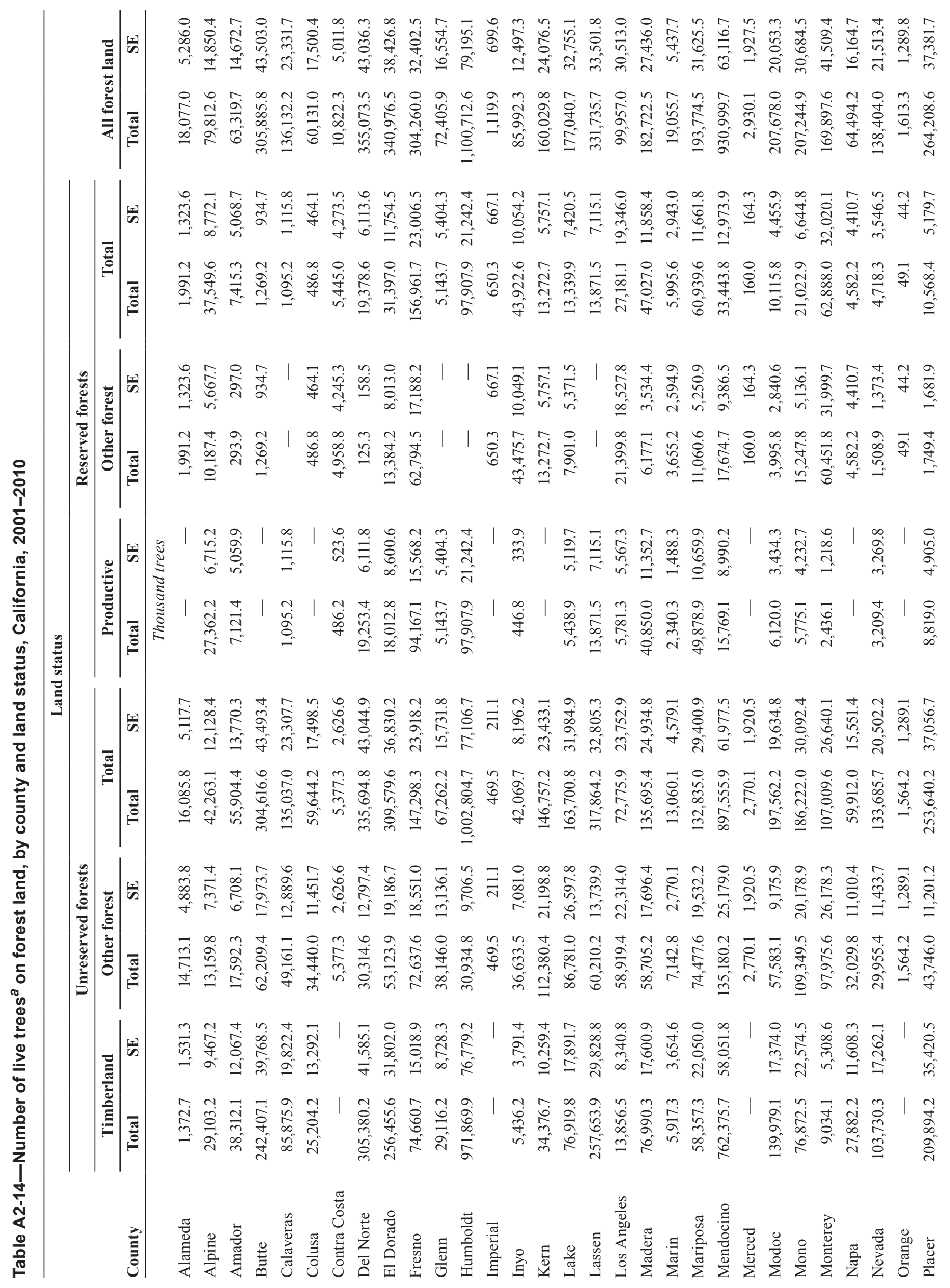




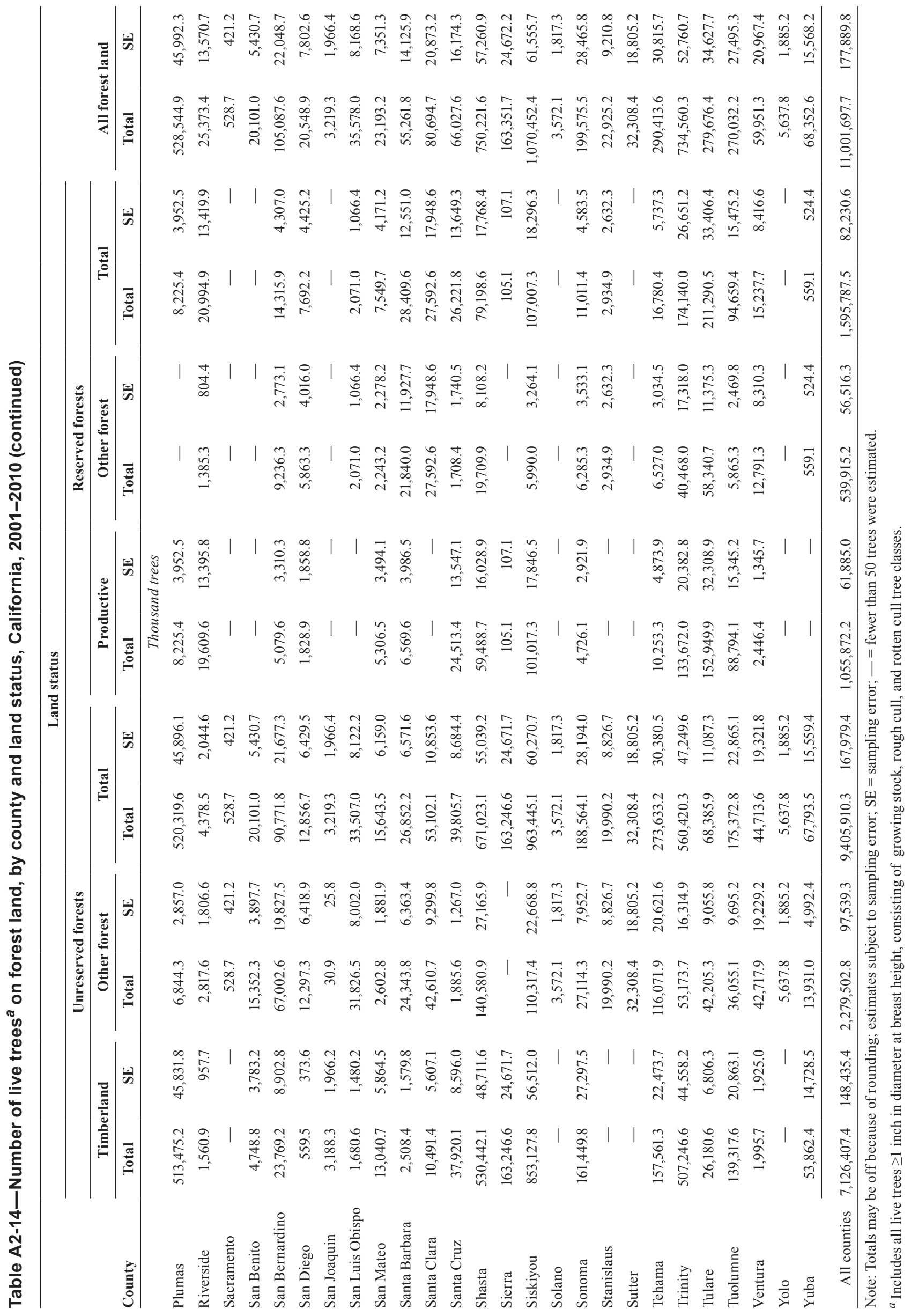




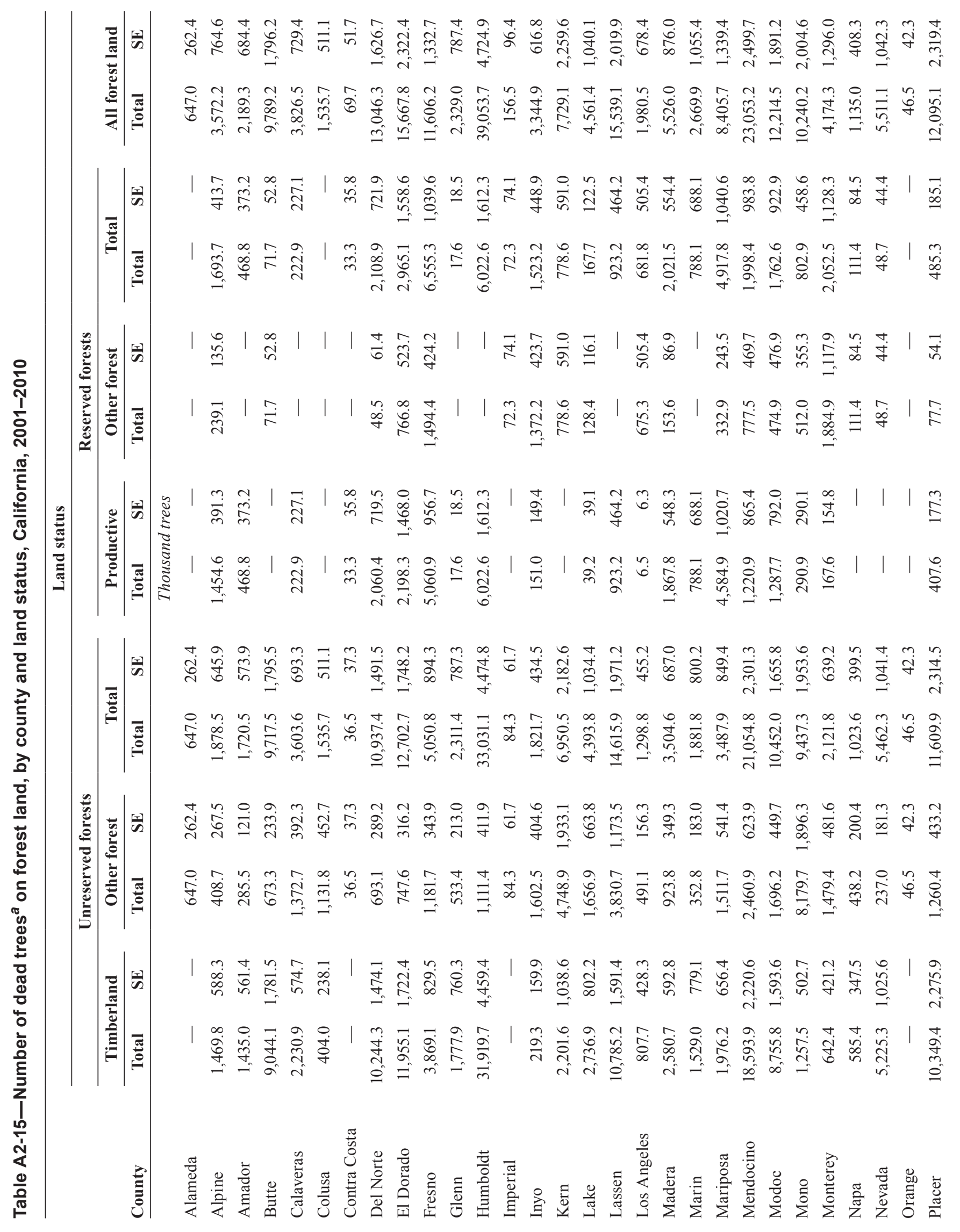




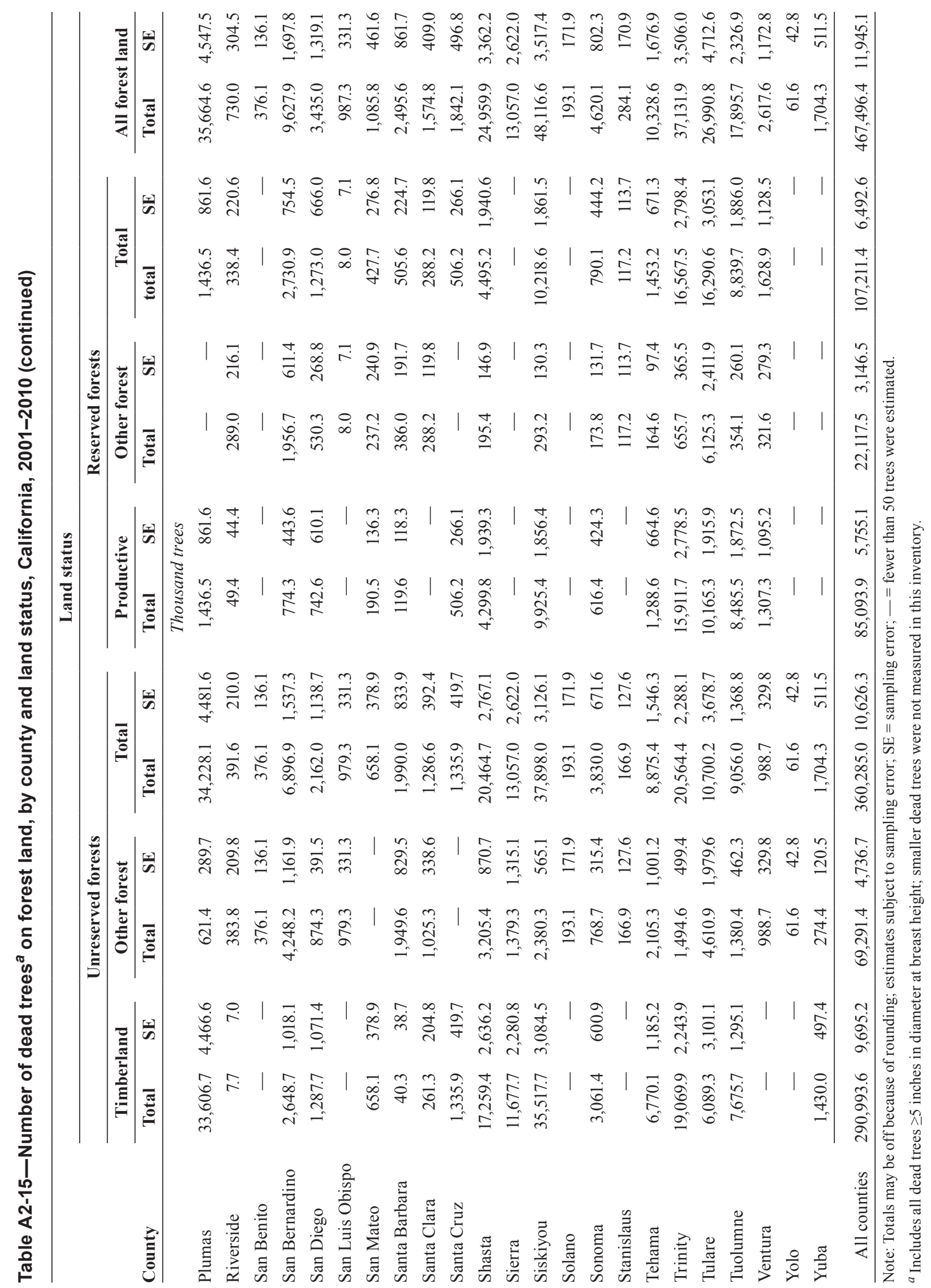


Table A2-16-Number of live trees ${ }^{a}$ on forest land, by county and broad species group, California, 2001-2010

\begin{tabular}{|c|c|c|c|c|c|c|}
\hline \multirow[b]{3}{*}{ County } & \multicolumn{4}{|c|}{ Broad species group } & & \\
\hline & \multicolumn{2}{|c|}{ Softwood species } & \multicolumn{2}{|c|}{ Hardwood species } & \multicolumn{2}{|c|}{ All species groups } \\
\hline & Total & SE & Total & SE & Total & SE \\
\hline & \multicolumn{6}{|c|}{ Thousand trees } \\
\hline Alameda & $1,252.1$ & $1,139.3$ & $16,824.9$ & $5,047.1$ & $18,077.0$ & $5,286.0$ \\
\hline Alpine & $68,235.7$ & $12,937.0$ & $11,576.9$ & $6,909.6$ & $79,812.6$ & $14,850.4$ \\
\hline Amador & $29,999.4$ & $9,606.4$ & $33,320.3$ & $10,441.8$ & $63,319.7$ & $14,672.7$ \\
\hline Butte & $145,729.2$ & $26,797.2$ & $160,156.6$ & $27,657.0$ & $305,885.8$ & $43,503.0$ \\
\hline Calaveras & $63,411.1$ & $15,192.1$ & $72,721.1$ & $17,097.3$ & $136,132.2$ & $23,331.7$ \\
\hline Colusa & $19,686.4$ & $8,281.7$ & $40,444.7$ & $11,690.8$ & $60,131.0$ & $17,500.4$ \\
\hline Contra Costa & 619.2 & 541.8 & $10,203.1$ & $4,869.5$ & $10,822.3$ & $5,011.8$ \\
\hline Del Norte & $143,226.7$ & $21,054.8$ & $211,846.8$ & $32,453.6$ & $355,073.5$ & $43,036.3$ \\
\hline El Dorado & $238,320.3$ & $28,623.7$ & $102,656.2$ & $22,775.1$ & $340,976.5$ & $38,426.8$ \\
\hline Fresno & $187,733.0$ & $21,378.2$ & $116,526.9$ & $23,853.9$ & $304,260.0$ & $32,402.5$ \\
\hline Glenn & $21,576.8$ & $6,434.6$ & $50,829.0$ & $14,307.4$ & $72,405.9$ & $16,554.7$ \\
\hline Humboldt & $429,646.5$ & $40,207.3$ & $671,066.1$ & $58,106.2$ & $1,100,712.6$ & $79,195.1$ \\
\hline Imperial & - & - & $1,119.9$ & 699.6 & $1,119.9$ & 699.6 \\
\hline Inyo & $62,557.9$ & $8,937.2$ & $23,434.4$ & $8,645.1$ & $85,992.3$ & $12,497.3$ \\
\hline Kern & $38,708.3$ & $6,285.0$ & $121,321.5$ & $21,891.4$ & $160,029.8$ & $24,076.5$ \\
\hline Lake & $64,632.3$ & $16,294.9$ & $112,408.5$ & $25,968.4$ & $177,040.7$ & $32,755.1$ \\
\hline Lassen & $291,244.1$ & $30,607.6$ & $40,491.6$ & $12,451.9$ & $331,735.7$ & $33,501.8$ \\
\hline Los Angeles & $4,976.6$ & $1,339.4$ & $94,980.3$ & $30,372.4$ & $99,957.0$ & $30,513.0$ \\
\hline Madera & $105,544.7$ & $18,369.4$ & $77,177.8$ & $18,778.2$ & $182,722.5$ & $27,436.0$ \\
\hline Marin & $3,624.4$ & $1,777.7$ & $15,431.3$ & $4,656.2$ & $19,055.7$ & $5,437.7$ \\
\hline Mariposa & $77,346.5$ & $13,648.8$ & $116,428.0$ & $26,074.2$ & $193,774.5$ & $31,625.5$ \\
\hline Mendocino & $325,315.1$ & $26,841.1$ & $605,684.6$ & $48,300.9$ & $930,999.7$ & $63,116.7$ \\
\hline Merced & - & - & $2,930.1$ & $1,927.5$ & $2,930.1$ & $1,927.5$ \\
\hline Modoc & $182,509.8$ & $18,186.3$ & $25,168.2$ & $6,543.1$ & $207,678.0$ & $20,053.3$ \\
\hline Mono & $133,125.1$ & $20,840.7$ & $74,119.8$ & $21,714.3$ & $207,244.9$ & $30,684.5$ \\
\hline Monterey & $11,963.1$ & $3,976.1$ & $157,934.5$ & $41,054.3$ & $169,897.6$ & $41,509.4$ \\
\hline Napa & $11,244.5$ & $3,068.6$ & $53,249.7$ & $14,320.8$ & $64,494.2$ & $16,164.7$ \\
\hline Nevada & $84,252.5$ & $15,032.4$ & $54,151.6$ & $13,553.0$ & $138,404.0$ & $21,513.4$ \\
\hline Orange & - & - & $1,613.3$ & $1,289.8$ & $1,613.3$ & $1,289.8$ \\
\hline Placer & $176,768.3$ & $31,573.6$ & $87,440.3$ & $15,059.5$ & $264,208.6$ & $37,381.7$ \\
\hline Plumas & $436,925.3$ & $37,861.1$ & $91,619.6$ & $22,139.1$ & $528,544.9$ & $45,992.3$ \\
\hline Riverside & $3,356.3$ & $1,206.5$ & $22,017.1$ & $13,382.0$ & $25,373.4$ & $13,570.7$ \\
\hline Sacramento & - & - & 528.7 & 421.2 & 528.7 & 421.2 \\
\hline San Benito & $2,633.5$ & $1,182.4$ & $17,467.5$ & $5,168.4$ & $20,101.0$ & $5,430.7$ \\
\hline San Bernardino & $33,191.2$ & $6,060.9$ & $71,896.4$ & $20,627.3$ & $105,087.6$ & $22,048.7$ \\
\hline San Diego & $3,155.0$ & $1,754.9$ & $17,393.9$ & $7,475.5$ & $20,548.9$ & $7,802.6$ \\
\hline San Joaquin & 6.2 & 6.3 & $3,213.1$ & $1,966.3$ & $3,219.3$ & $1,966.4$ \\
\hline
\end{tabular}


Table A2-16-Number of live trees ${ }^{a}$ on forest land, by county and broad species group, California, 2001-2010 (continued)

\begin{tabular}{|c|c|c|c|c|c|c|}
\hline \multirow[b]{3}{*}{ County } & \multicolumn{4}{|c|}{ Broad species group } & & \\
\hline & \multicolumn{2}{|c|}{ Softwood species } & \multicolumn{2}{|c|}{ Hardwood species } & \multicolumn{2}{|c|}{ All species groups } \\
\hline & Total & SE & Total & SE & Total & SE \\
\hline & \multicolumn{6}{|c|}{ Thousand trees } \\
\hline San Luis Obispo & $3,268.6$ & $1,188.4$ & $32,309.4$ & $7,989.2$ & $35,578.0$ & $8,168.6$ \\
\hline San Mateo & $6,011.6$ & $2,677.6$ & $17,181.5$ & $5,690.4$ & $23,193.2$ & $7,351.3$ \\
\hline Santa Barbara & $4,935.2$ & $2,043.2$ & $50,326.6$ & $13,788.4$ & $55,261.8$ & $14,125.9$ \\
\hline Santa Clara & $4,496.2$ & $1,993.4$ & $76,198.5$ & $20,582.6$ & $80,694.7$ & $20,873.2$ \\
\hline Santa Cruz & $29,663.5$ & $12,102.5$ & $36,364.0$ & $8,015.3$ & $66,027.6$ & $16,174.3$ \\
\hline Shasta & $407,304.7$ & $35,959.9$ & $342,916.9$ & $40,313.7$ & $750,221.6$ & $57,260.9$ \\
\hline Sierra & $134,720.1$ & $21,779.3$ & $28,631.6$ & $8,117.8$ & $163,351.7$ & $24,672.2$ \\
\hline Siskiyou & $658,274.0$ & $42,471.0$ & $412,178.4$ & $42,350.7$ & $1,070,452.4$ & $61,555.7$ \\
\hline Solano & - & - & $3,572.1$ & $1,817.3$ & $3,572.1$ & $1,817.3$ \\
\hline Sonoma & $69,346.4$ & $14,122.0$ & $130,229.1$ & $19,746.7$ & $199,575.5$ & $28,465.8$ \\
\hline Stanislaus & $4,465.5$ & $2,589.6$ & $18,459.7$ & $8,434.6$ & $22,925.2$ & $9,210.8$ \\
\hline Sutter & - & - & $32,308.4$ & $18,805.2$ & $32,308.4$ & $18,805.2$ \\
\hline Tehama & $132,977.4$ & $18,924.8$ & $157,436.1$ & $21,896.0$ & $290,413.6$ & $30,815.7$ \\
\hline Trinity & $420,118.6$ & $35,583.3$ & $314,441.7$ & $32,216.7$ & $734,560.3$ & $52,760.7$ \\
\hline Tulare & $144,171.7$ & $14,372.2$ & $135,504.7$ & $30,669.7$ & $279,676.4$ & $34,627.7$ \\
\hline Tuolumne & $185,427.6$ & $19,312.0$ & $84,604.6$ & $17,791.0$ & $270,032.2$ & $27,495.3$ \\
\hline Ventura & $15,719.7$ & $3,300.8$ & $44,231.6$ & $20,702.0$ & $59,951.3$ & $20,967.4$ \\
\hline Yolo & 698.5 & 357.4 & $4,939.4$ & $1,662.9$ & $5,637.8$ & $1,885.2$ \\
\hline Yuba & $20,663.6$ & $6,198.2$ & $47,688.9$ & $11,436.0$ & $68,352.6$ & $15,568.2$ \\
\hline All counties & $5,644,780.1$ & $107,341.9$ & $5,356,917.6$ & $144,918.1$ & $11,001,697.7$ & $177,889.8$ \\
\hline
\end{tabular}

Note: Totals may be off because of rounding; estimates subject to sampling error; SE = sampling error; — = fewer than 50 trees were estimated.

${ }^{a}$ Includes all live trees $\geq 1$ inch in diameter at breast height, consisting of growing stock, rough cull, and rotten cull tree classes. 
Table A2-17-Number of dead trees ${ }^{a}$ on forest land, by county and broad species group, California, 2001-2010

\begin{tabular}{|c|c|c|c|c|c|c|}
\hline \multirow[b]{3}{*}{ County } & \multicolumn{4}{|c|}{ Broad species groups } & & \\
\hline & \multicolumn{2}{|c|}{ Softwood species } & \multicolumn{2}{|c|}{ Hardwood species } & \multicolumn{2}{|c|}{ All species groups } \\
\hline & Total & SE & Total & SE & Total & SE \\
\hline & \multicolumn{6}{|c|}{ Thousand trees } \\
\hline Alameda & - & - & 647.0 & 262.4 & 647.0 & 262.4 \\
\hline Alpine & $3,212.1$ & 711.0 & 360.1 & 245.3 & $3,572.2$ & 764.6 \\
\hline Amador & $1,286.3$ & 537.9 & 903.0 & 416.9 & $2,189.3$ & 684.4 \\
\hline Butte & $5,355.8$ & $1,263.6$ & $4,433.4$ & 972.8 & $9,789.2$ & $1,796.2$ \\
\hline Calaveras & $2,328.6$ & 582.1 & $1,497.9$ & 404.4 & $3,826.5$ & 729.4 \\
\hline Colusa & 614.4 & 301.2 & 921.3 & 325.6 & $1,535.7$ & 511.1 \\
\hline Contra Costa & 33.3 & 35.8 & 36.5 & 37.3 & 69.7 & 51.7 \\
\hline Del Norte & $9,104.2$ & $1,339.5$ & $3,942.1$ & 769.2 & $13,046.3$ & $1,626.7$ \\
\hline El Dorado & $12,989.1$ & $2,164.2$ & $2,678.7$ & 620.3 & $15,667.8$ & $2,322.4$ \\
\hline Fresno & $9,946.7$ & $1,253.7$ & $1,659.5$ & 382.5 & $11,606.2$ & $1,332.7$ \\
\hline Glenn & $1,741.7$ & 758.4 & 587.4 & 202.4 & $2,329.0$ & 787.4 \\
\hline Humboldt & $17,188.0$ & $1,936.9$ & $21,865.7$ & $4,117.1$ & $39,053.7$ & $4,724.9$ \\
\hline Imperial & - & - & 156.5 & 96.4 & 156.5 & 96.4 \\
\hline Inyo & $2,476.3$ & 459.1 & 868.7 & 403.4 & $3,344.9$ & 616.8 \\
\hline Kern & $3,809.4$ & $1,234.6$ & $3,919.6$ & $1,685.9$ & $7,729.1$ & $2,259.6$ \\
\hline Lake & $1,554.1$ & 415.9 & $3,007.3$ & 941.4 & $4,561.4$ & $1,040.1$ \\
\hline Lassen & $11,416.1$ & $1,613.8$ & $4,123.1$ & $1,184.7$ & $15,539.1$ & $2,019.9$ \\
\hline Los Angeles & 469.6 & 213.3 & $1,510.9$ & 621.5 & $1,980.5$ & 678.4 \\
\hline Madera & $4,684.6$ & 818.4 & 841.4 & 251.2 & $5,526.0$ & 876.0 \\
\hline Marin & 274.6 & 167.3 & $2,395.3$ & $1,026.9$ & $2,669.9$ & $1,055.4$ \\
\hline Mariposa & $6,419.4$ & $1,157.4$ & $1,986.3$ & 577.5 & $8,405.7$ & $1,339.4$ \\
\hline Mendocino & $9,227.5$ & $1,310.8$ & $13,825.8$ & $1,700.4$ & $23,053.2$ & $2,499.7$ \\
\hline Modoc & $9,128.0$ & $1,534.9$ & $3,086.6$ & 919.4 & $12,214.5$ & $1,891.2$ \\
\hline Mono & $6,337.7$ & $1,465.7$ & $3,902.5$ & $1,120.1$ & $10,240.2$ & $2,004.6$ \\
\hline Monterey & 762.9 & 411.3 & $3,411.5$ & $1,219.8$ & $4,174.3$ & $1,296.0$ \\
\hline Napa & 242.0 & 136.3 & 893.0 & 366.5 & $1,135.0$ & 408.3 \\
\hline Orange & - & - & 46.5 & 42.3 & 46.5 & 42.3 \\
\hline Placer & $9,331.8$ & $1,908.4$ & $2,763.3$ & 722.4 & $12,095.1$ & $2,319.4$ \\
\hline Plumas & $30,649.0$ & $3,933.6$ & $5,015.6$ & $1,598.2$ & $35,664.6$ & $4,547.5$ \\
\hline Riverside & 364.9 & 221.1 & 365.0 & 197.3 & 730.0 & 304.5 \\
\hline San Benito & 82.9 & 63.2 & 293.2 & 118.9 & 376.1 & 136.1 \\
\hline San Bernardino & $7,245.5$ & $1,269.4$ & $2,382.4$ & 690.8 & $9,627.9$ & $1,697.8$ \\
\hline San Diego & $1,110.8$ & 574.6 & $2,324.2$ & $1,135.4$ & $3,435.0$ & $1,319.1$ \\
\hline San Luis Obispo & 107.2 & 62.6 & 880.1 & 319.8 & 987.3 & 331.3 \\
\hline San Mateo & 366.2 & 290.7 & 719.7 & 365.0 & $1,085.8$ & 461.6 \\
\hline Santa Barbara & 498.8 & 217.3 & $1,996.7$ & 830.5 & $2,495.6$ & 861.7 \\
\hline Santa Clara & 218.3 & 112.9 & $1,356.5$ & 369.6 & $1,574.8$ & 409.0 \\
\hline
\end{tabular}


Table A2-17-Number of dead trees ${ }^{a}$ on forest land, by county and broad species group, California, 2001-2010 (continued)

\begin{tabular}{|c|c|c|c|c|c|c|}
\hline \multirow[b]{3}{*}{ County } & \multicolumn{4}{|c|}{ Broad species groups } & & \\
\hline & \multicolumn{2}{|c|}{ Softwood species } & \multicolumn{2}{|c|}{ Hardwood species } & \multicolumn{2}{|c|}{ All species groups } \\
\hline & Total & SE & Total & SE & Total & SE \\
\hline & \multicolumn{6}{|c|}{ Thousand trees } \\
\hline Santa Cruz & 591.5 & 215.6 & $1,250.6$ & 432.3 & $1,842.1$ & 496.8 \\
\hline Shasta & $17,828.6$ & $3,164.6$ & $7,131.3$ & $1,081.6$ & $24,959.9$ & $3,362.2$ \\
\hline Sierra & $10,601.9$ & $2,222.3$ & $2,455.0$ & $1,292.4$ & $13,057.0$ & $2,622.0$ \\
\hline Siskiyou & $36,324.0$ & $2,741.1$ & $11,792.6$ & $1,810.9$ & $48,116.6$ & $3,517.4$ \\
\hline Solano & - & - & 193.1 & 171.9 & 193.1 & 171.9 \\
\hline Sonoma & $1,560.6$ & 425.3 & $3,059.6$ & 610.3 & $4,620.1$ & 802.3 \\
\hline Stanislaus & 129.5 & 121.9 & 154.6 & 119.8 & 284.1 & 170.9 \\
\hline Tehama & $7,104.8$ & $1,205.1$ & $3,223.9$ & 860.5 & $10,328.6$ & $1,676.9$ \\
\hline Trinity & $22,613.4$ & $2,362.2$ & $14,518.5$ & $2,225.9$ & $37,131.9$ & $3,506.0$ \\
\hline Tulare & $19,434.7$ & 3,929.9 & $7,556.1$ & $2,495.9$ & $26,990.8$ & $4,712.6$ \\
\hline Tuolumne & $14,928.3$ & $2,075.4$ & $2,967.4$ & 724.2 & $17,895.7$ & $2,326.9$ \\
\hline Ventura & 808.7 & 268.0 & $1,808.9$ & $1,025.8$ & $2,617.6$ & $1,172.8$ \\
\hline Yolo & 23.9 & 18.1 & 37.7 & 38.8 & 61.6 & 42.8 \\
\hline Yuba & 868.1 & 289.2 & 836.3 & 337.0 & $1,704.3$ & 511.5 \\
\hline All counties & $307,818.1$ & $8,971.4$ & $159,678.3$ & $7,491.7$ & $467,496.4$ & $11,945.1$ \\
\hline
\end{tabular}

Note: Totals may be off because of rounding; estimates subject to sampling error; SE = sampling error; — = fewer than 50 trees were estimated.

${ }^{a}$ Includes all dead trees $\geq 5$ inches in diameter at breast height; smaller dead trees were not measured in this inventory. 


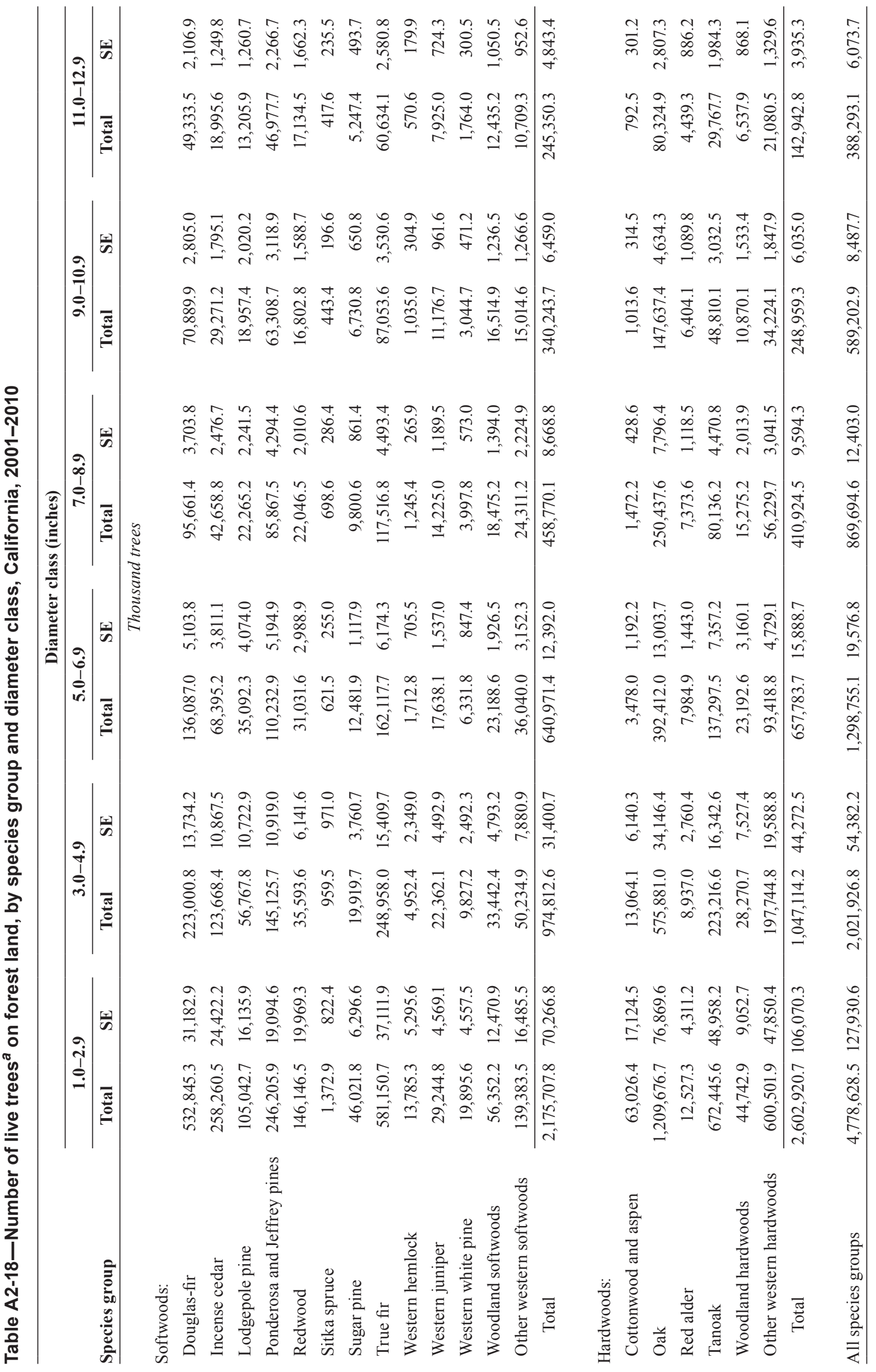




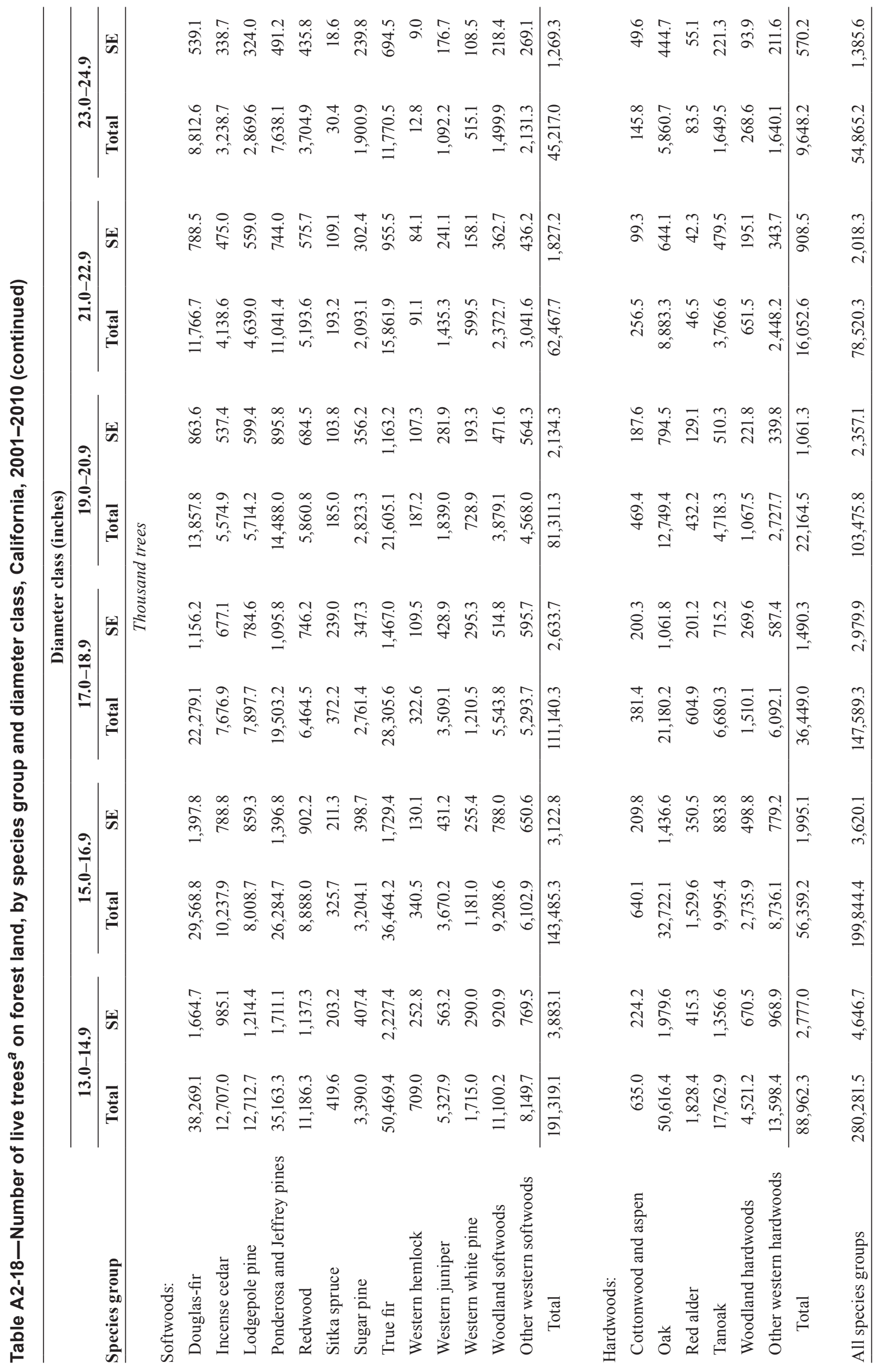




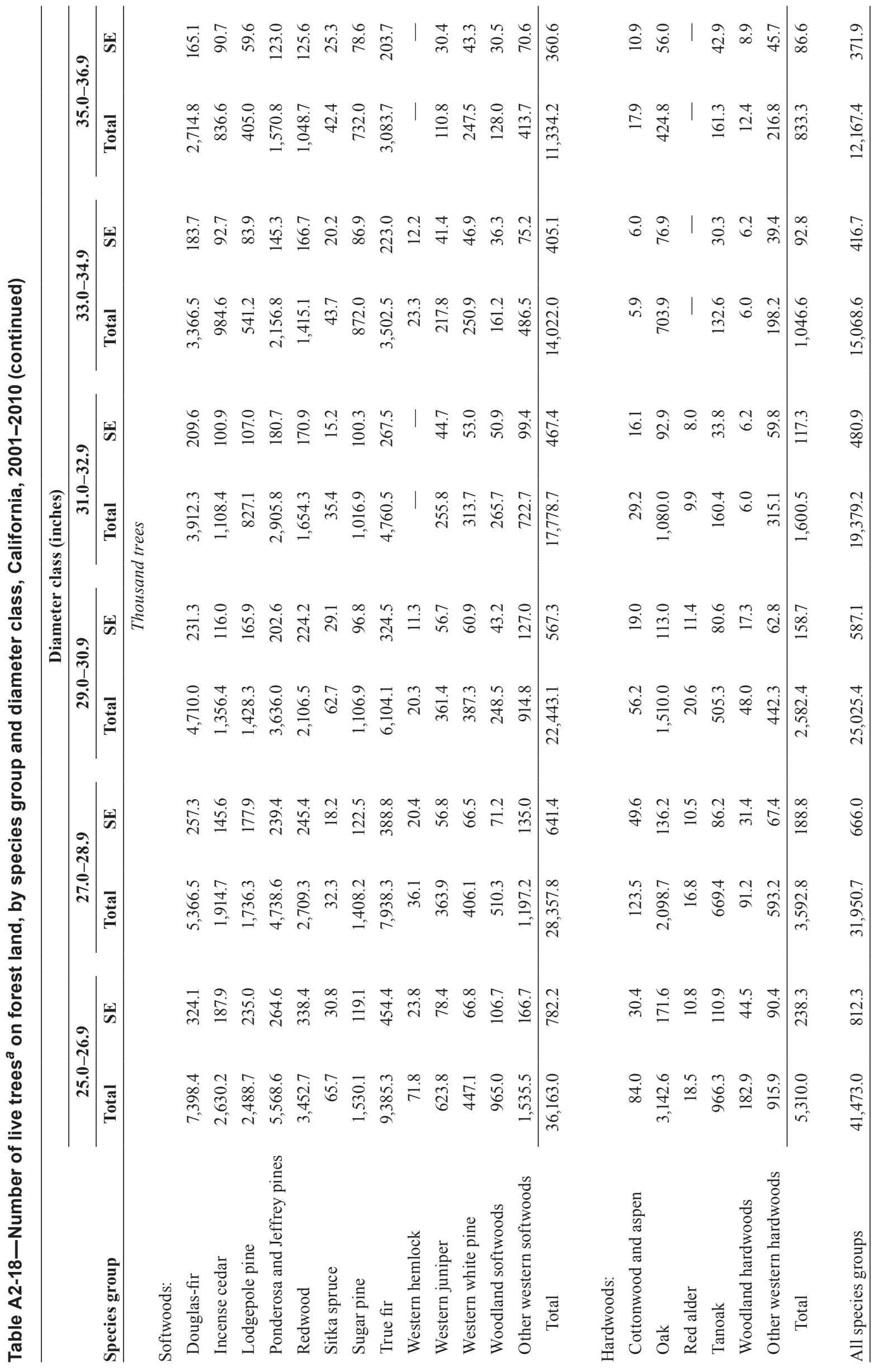




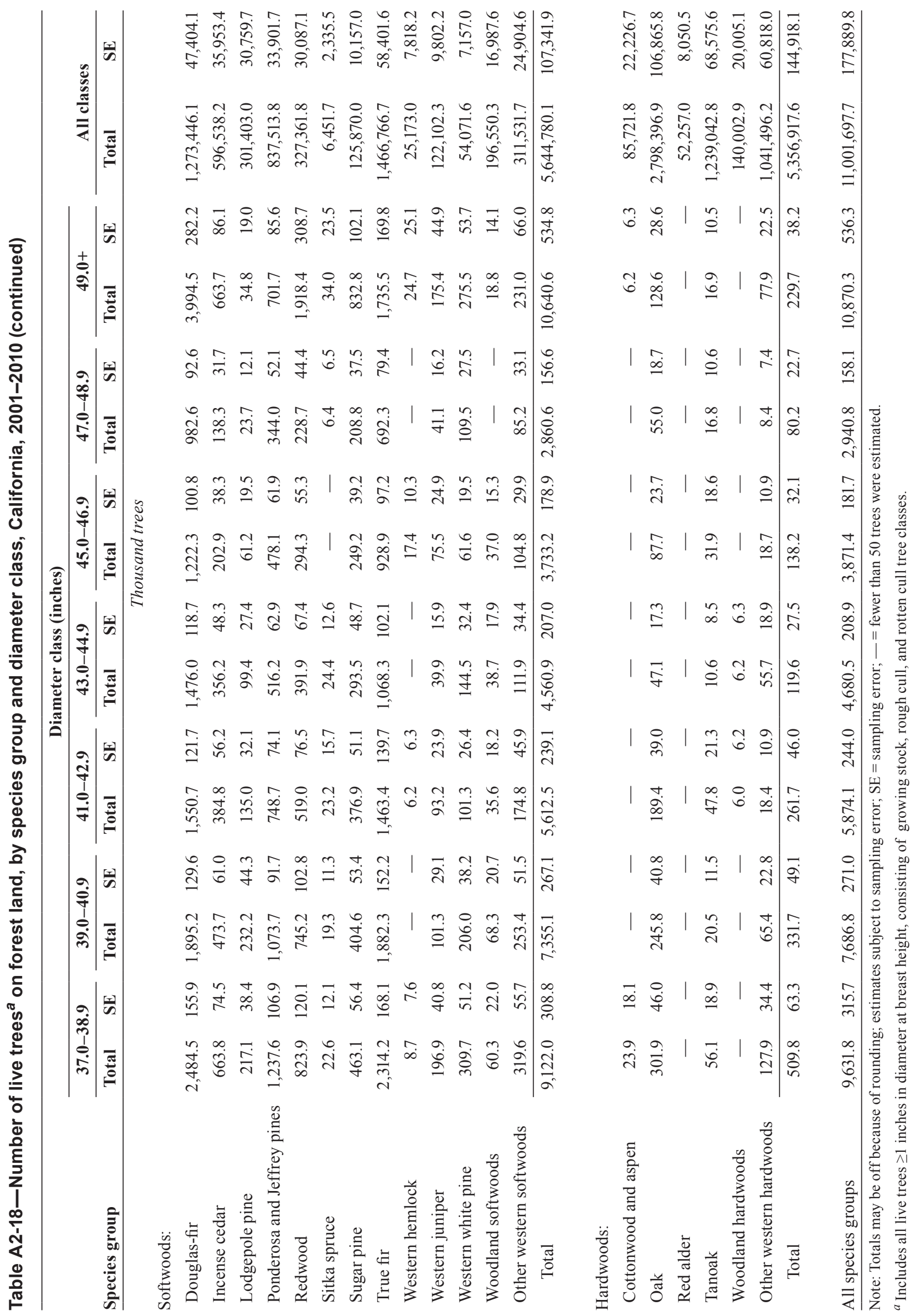




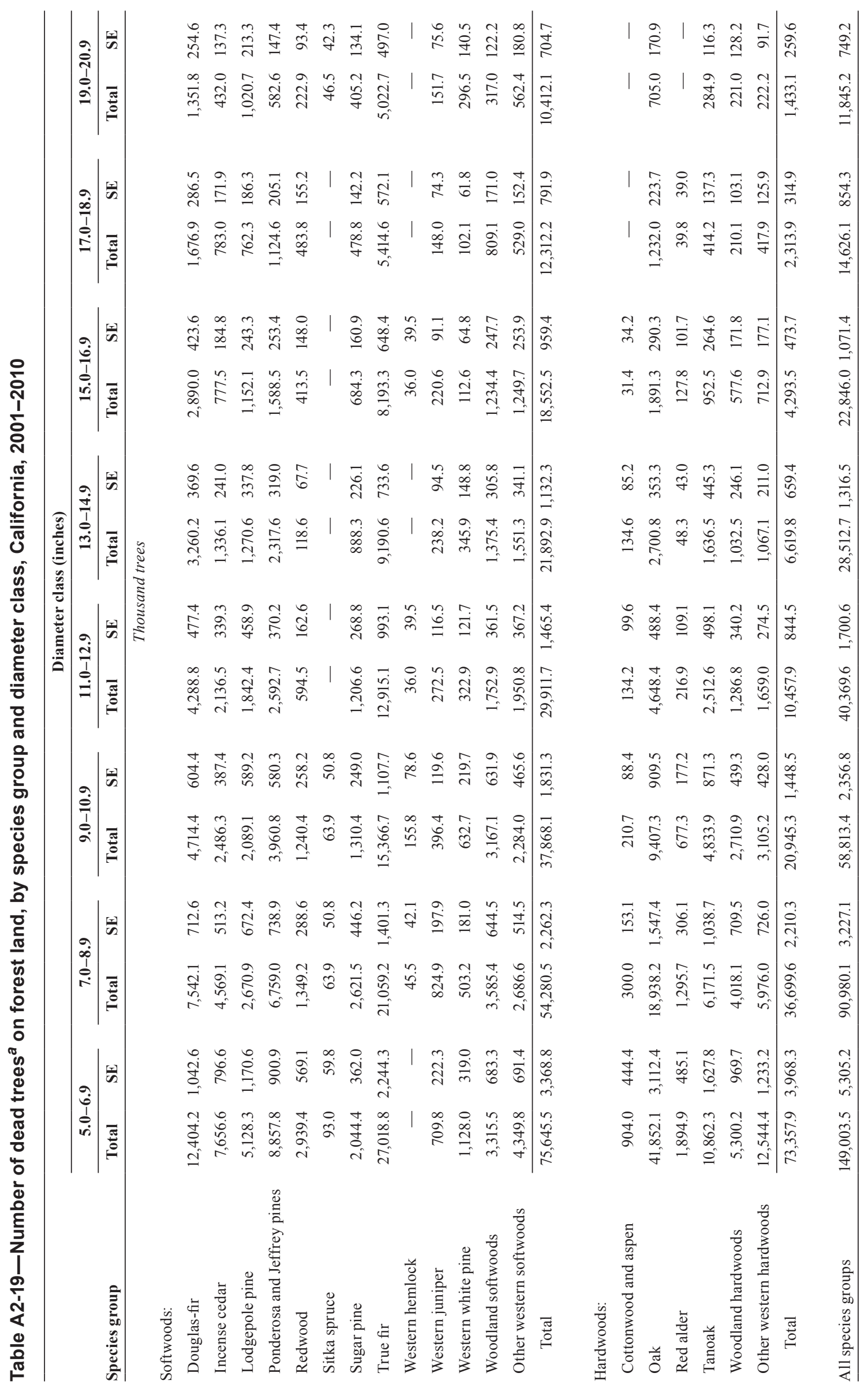




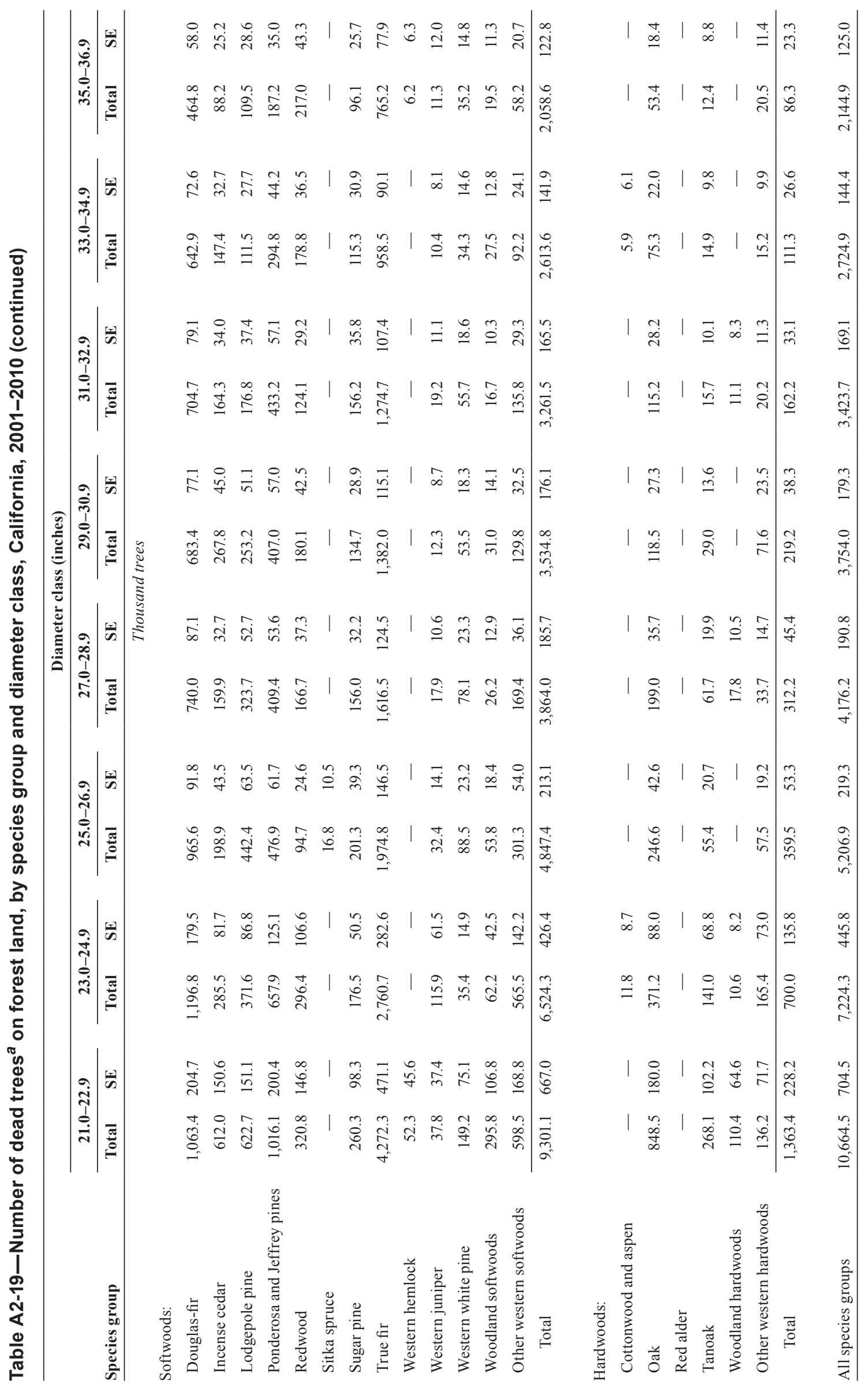




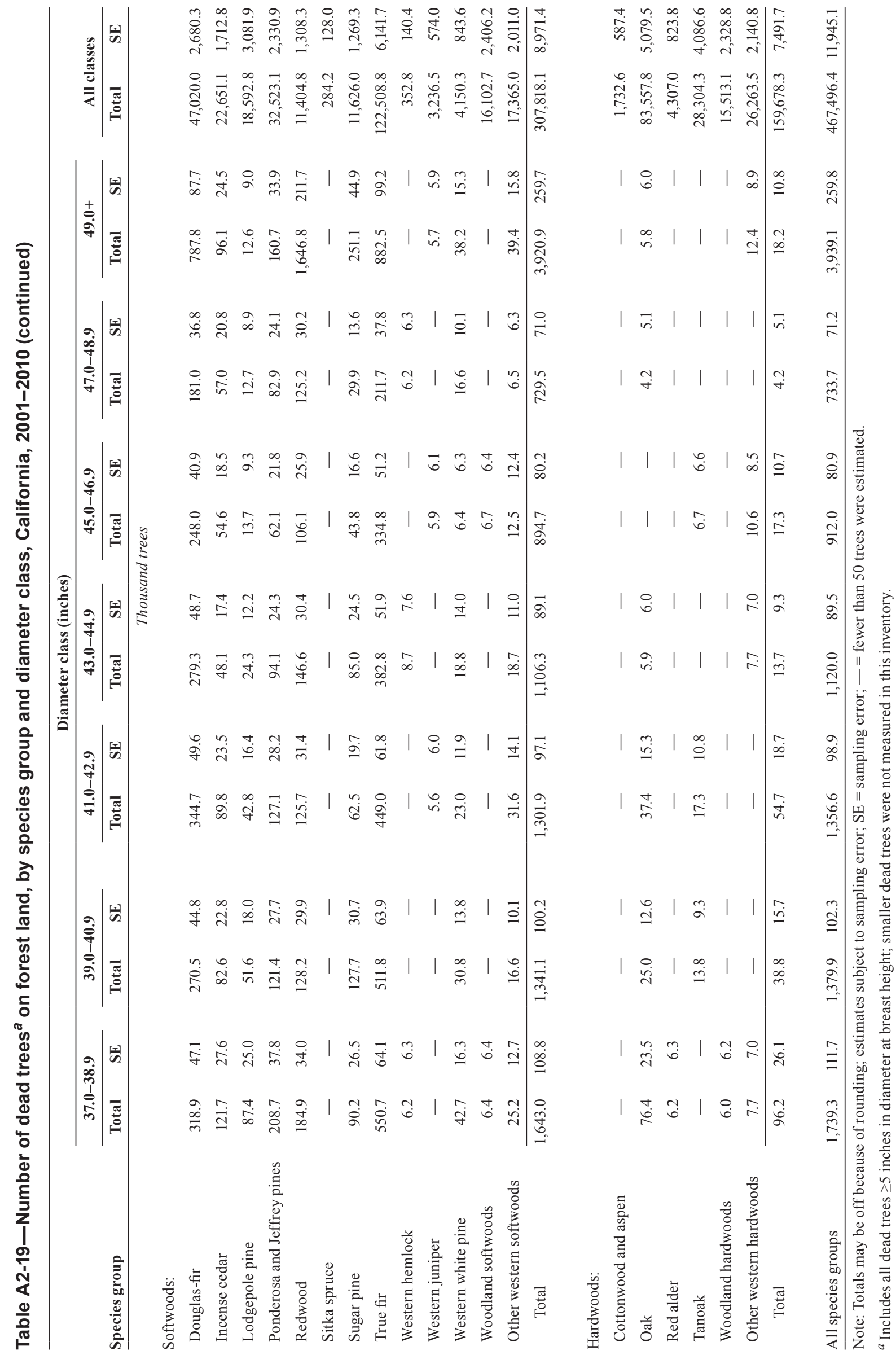




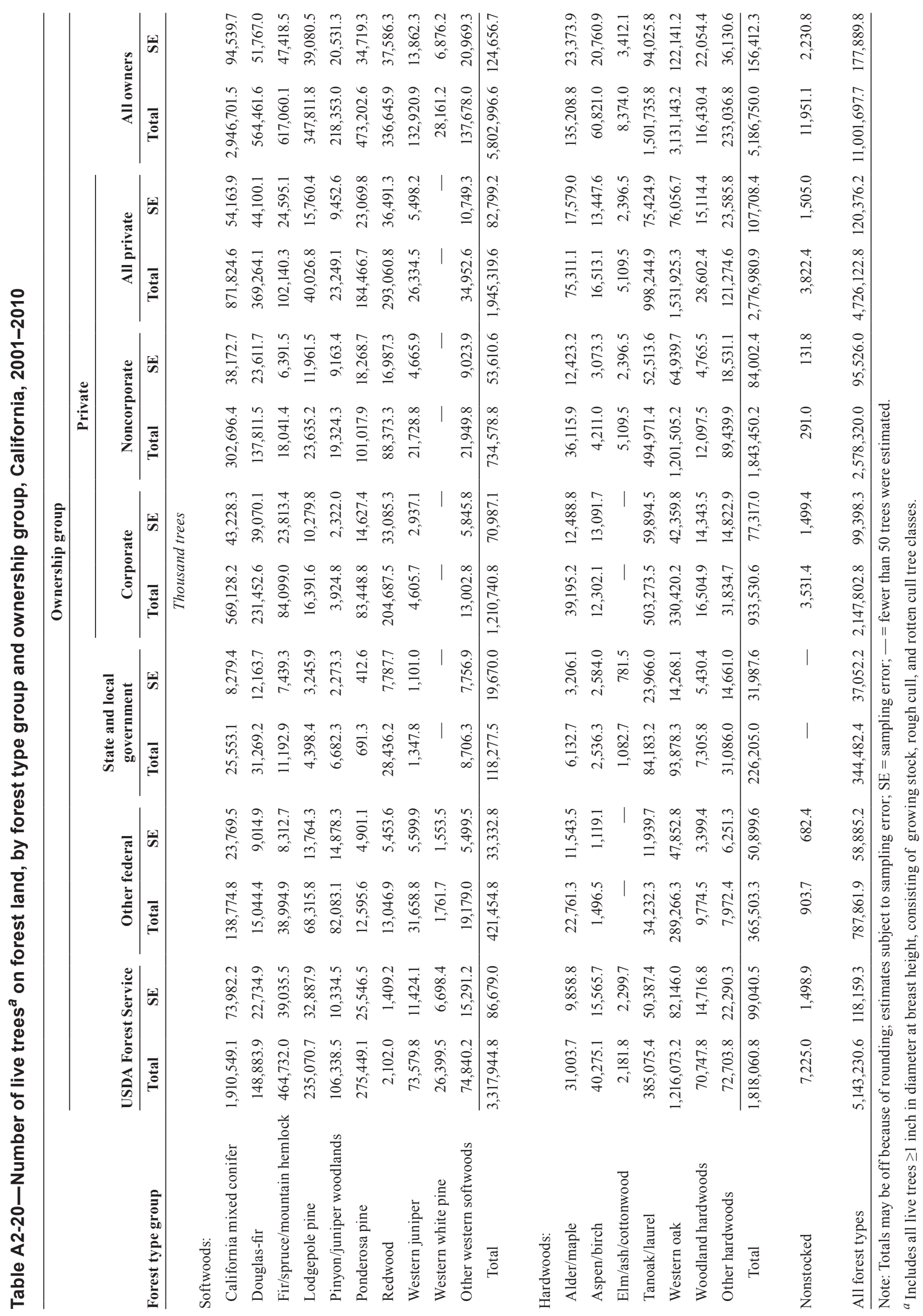




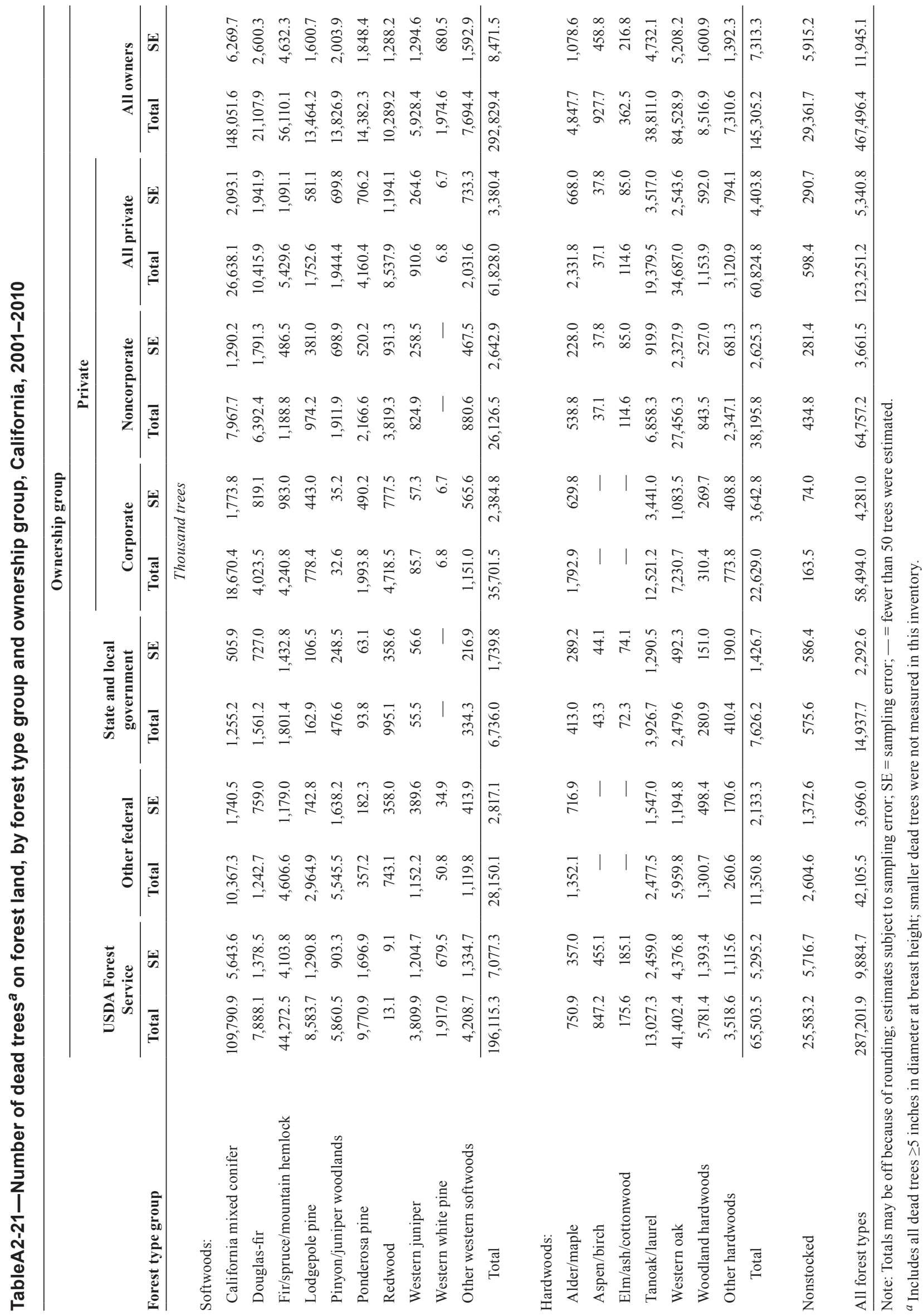




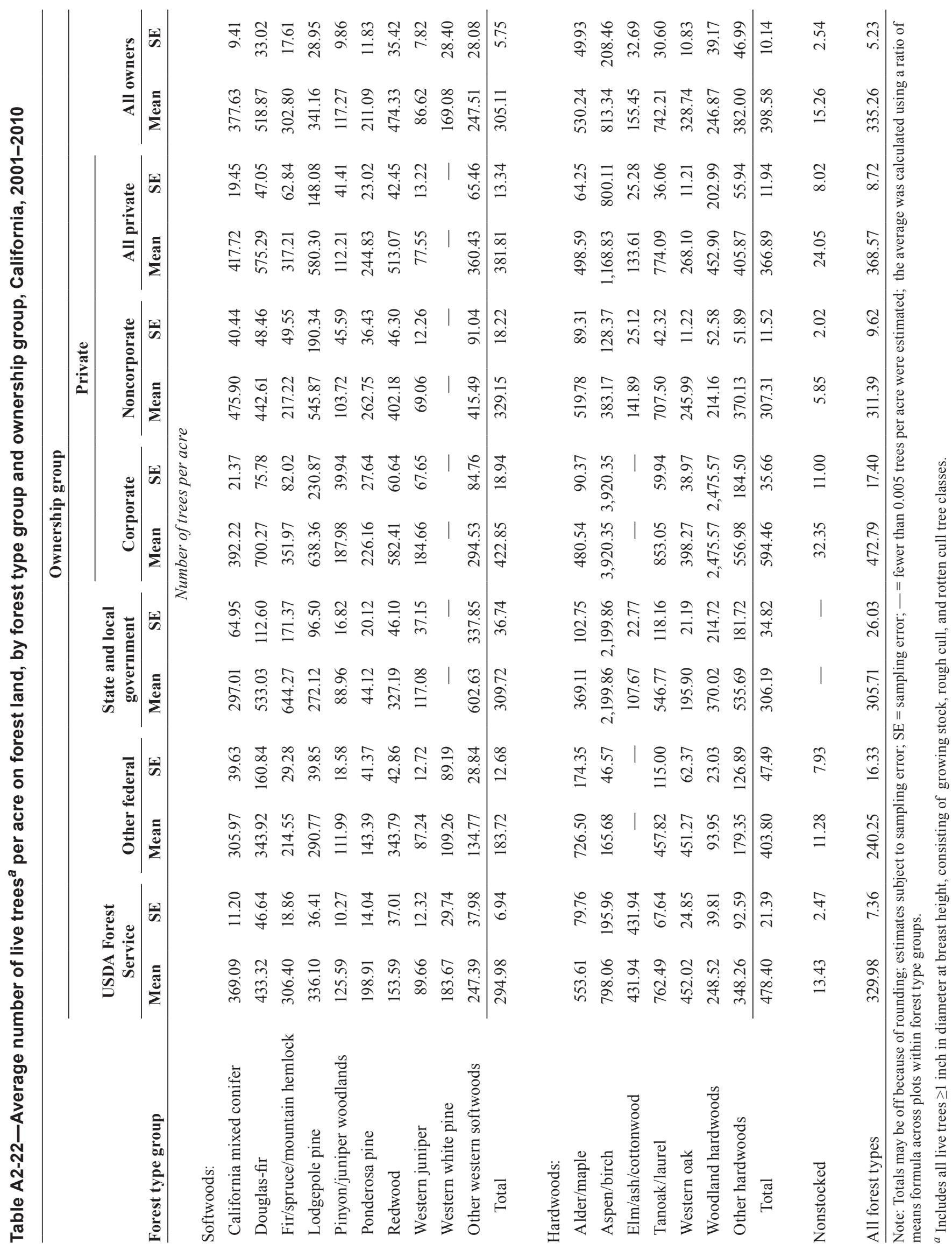




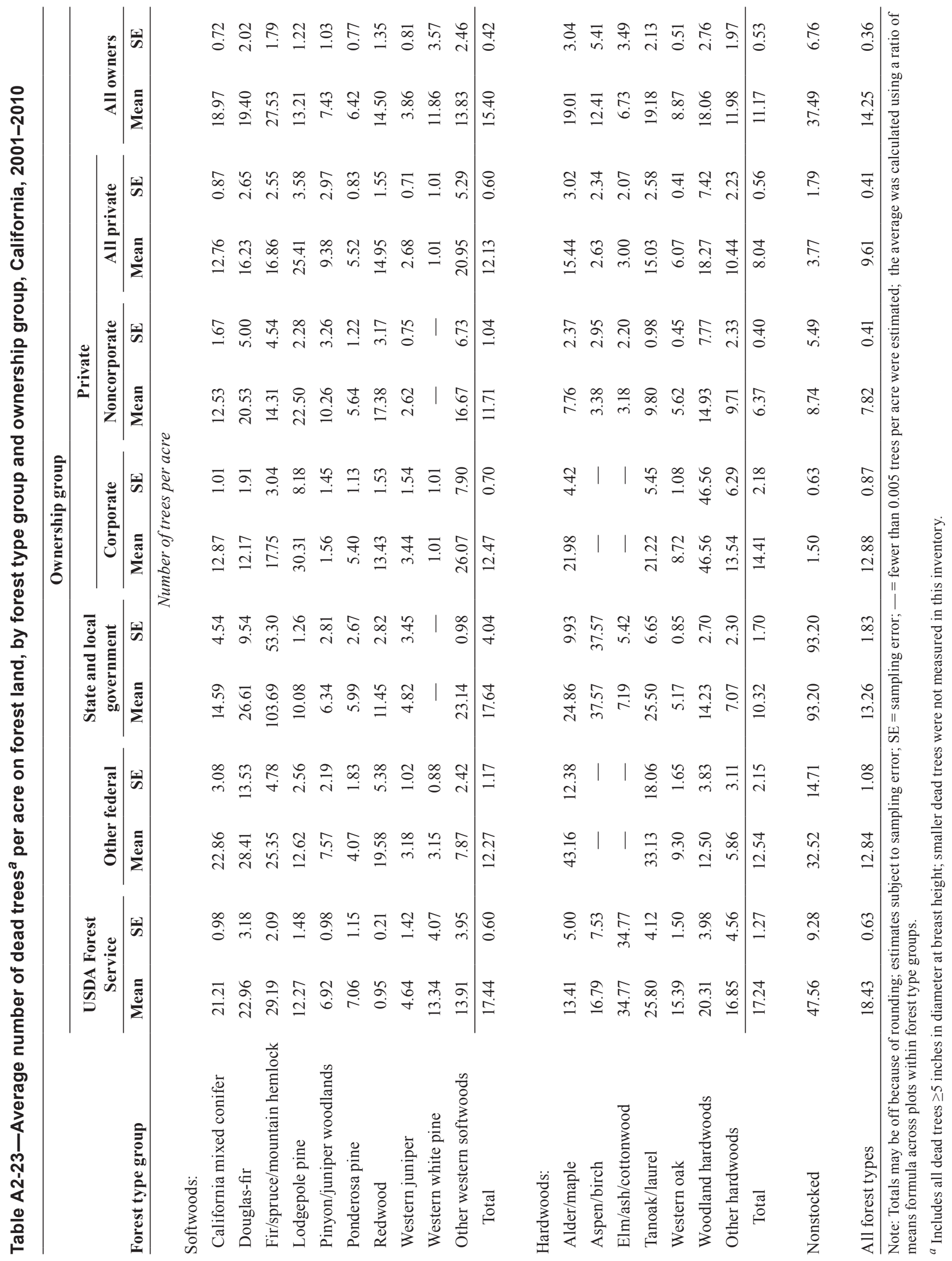




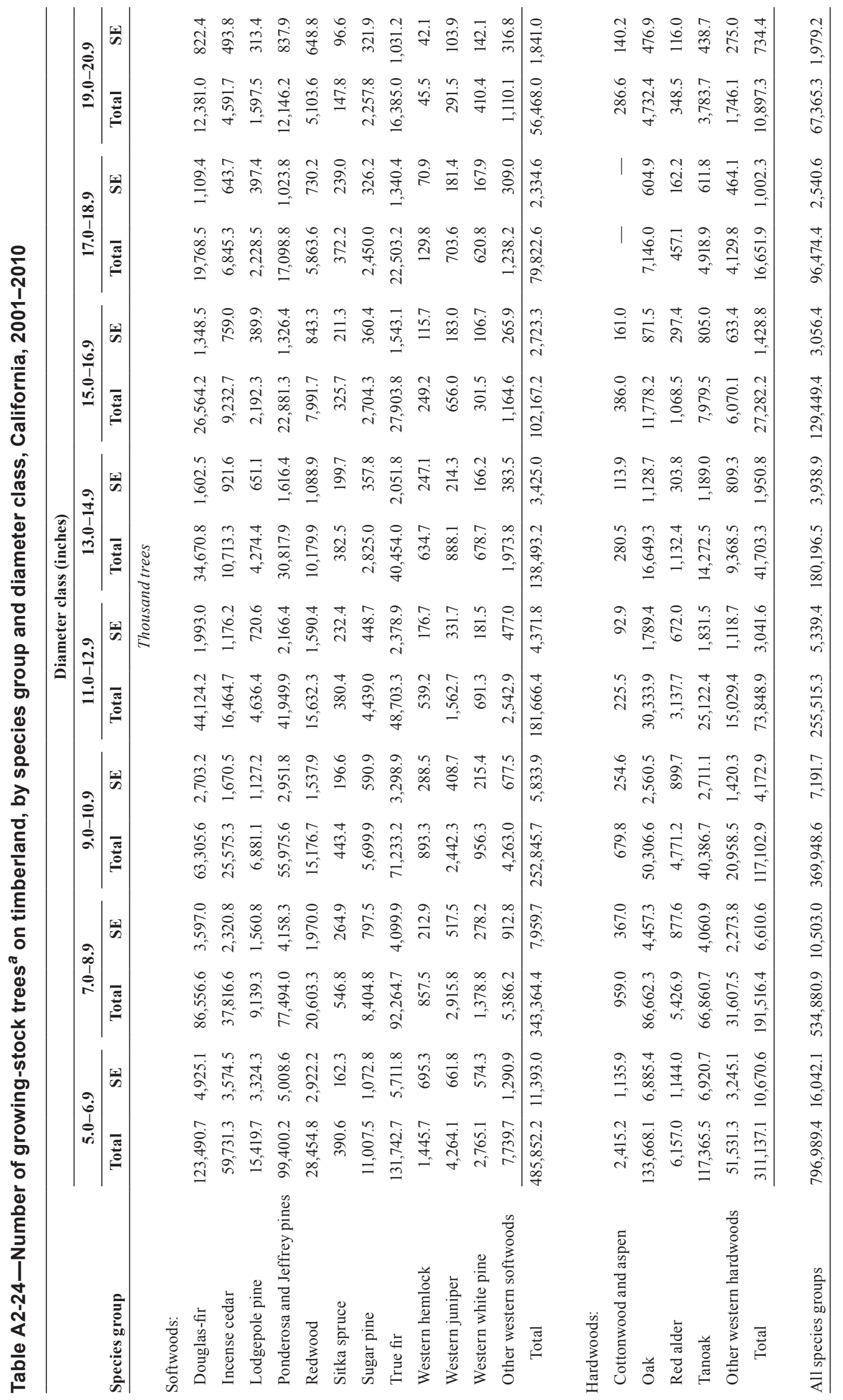




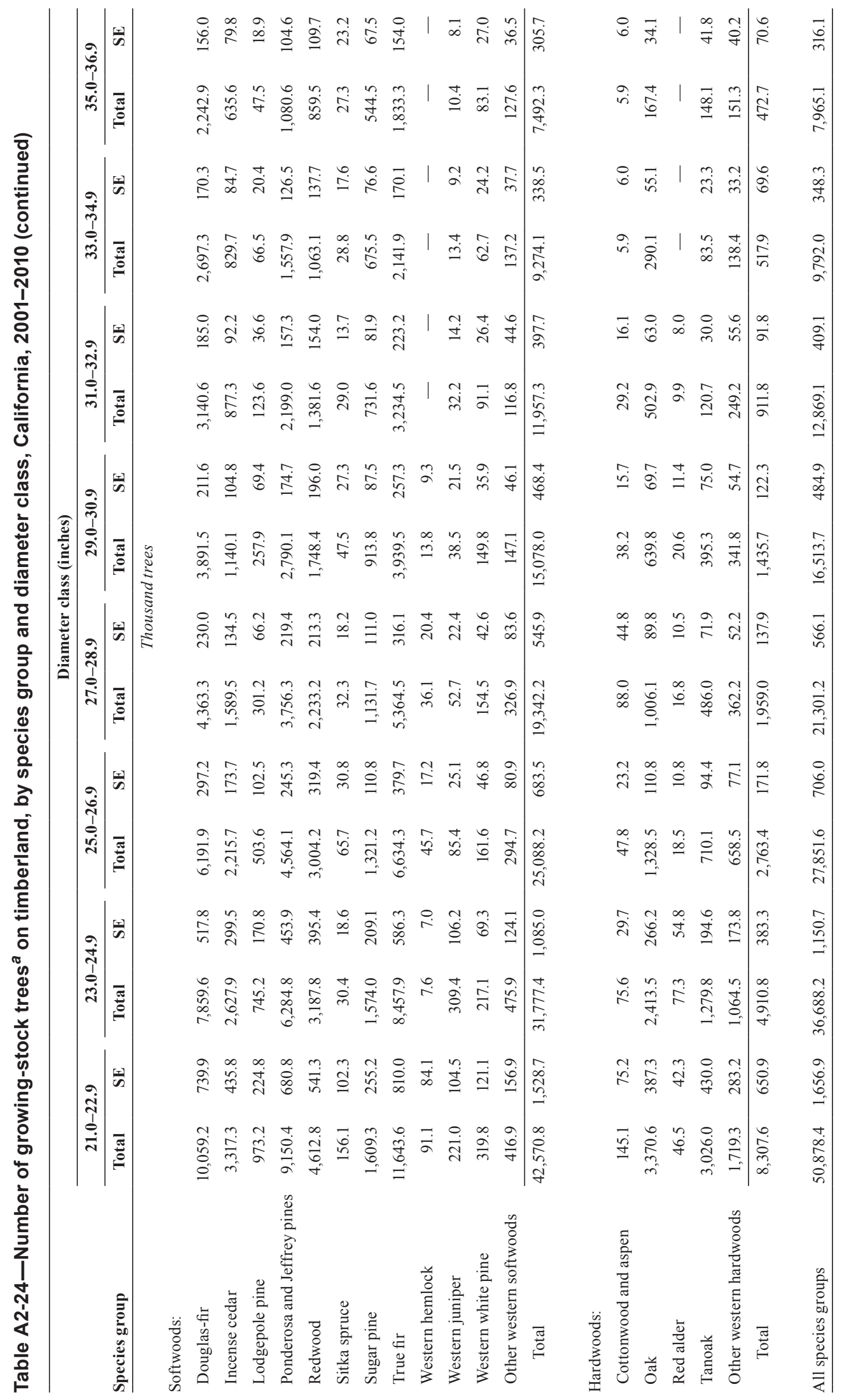




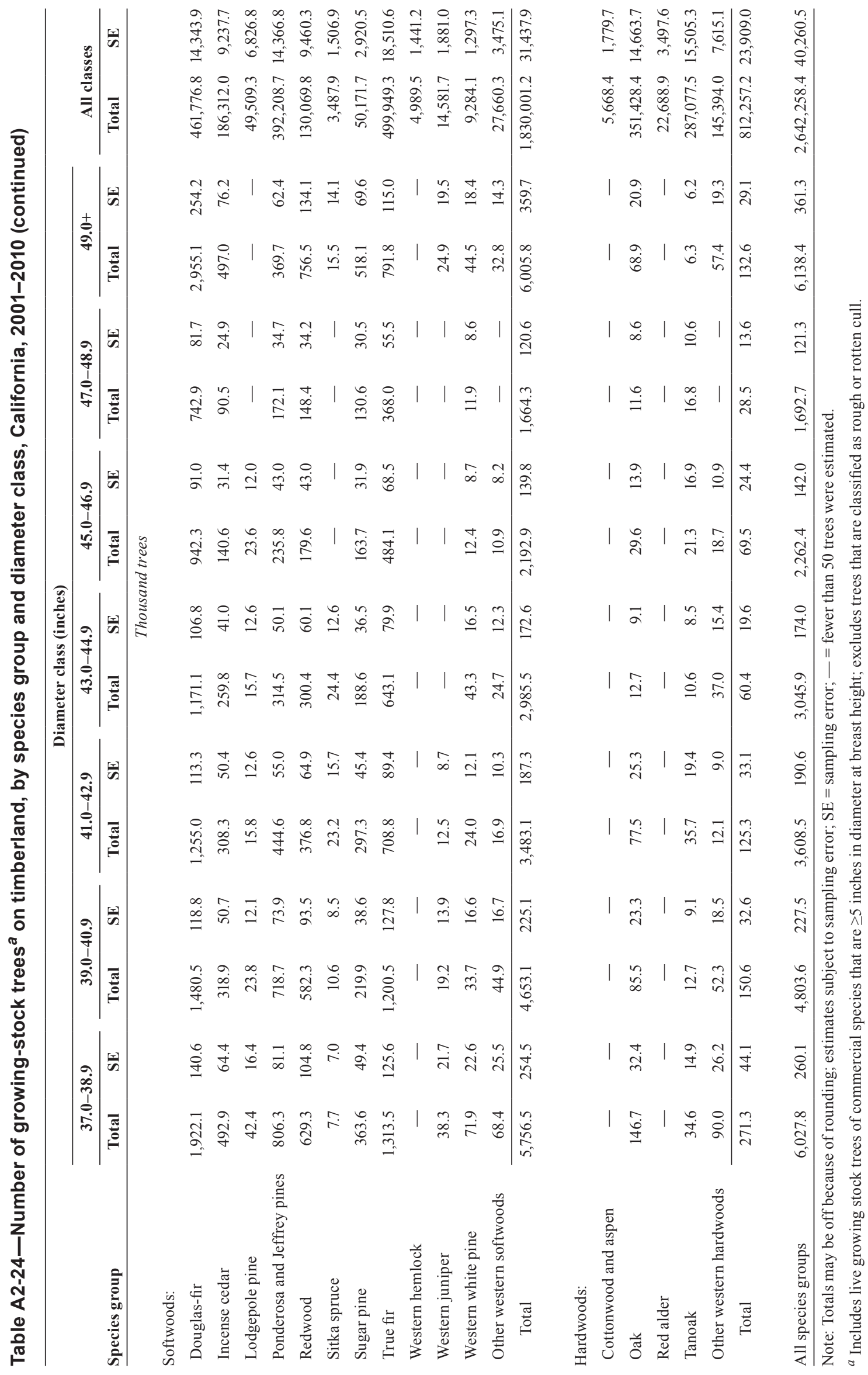




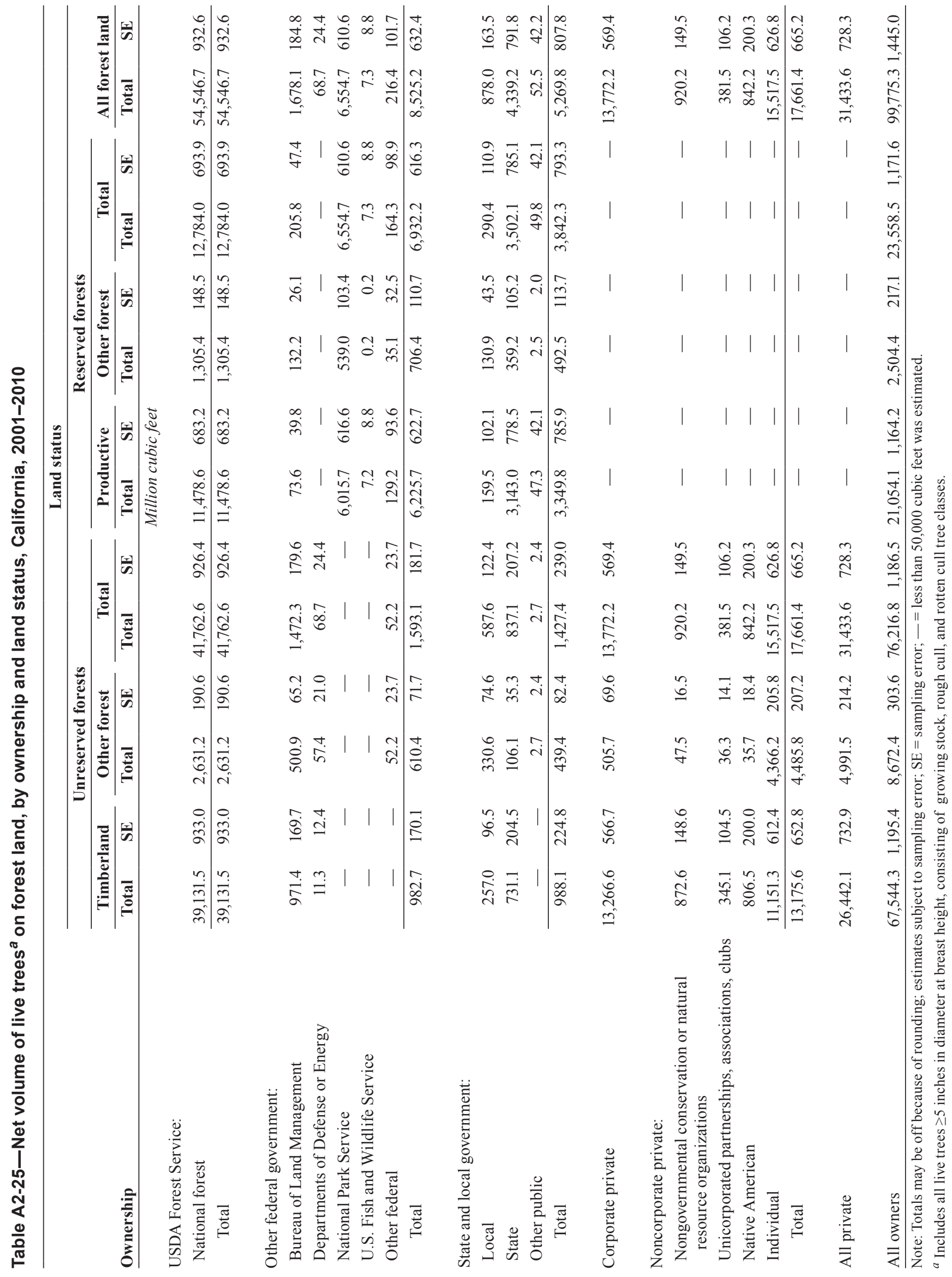




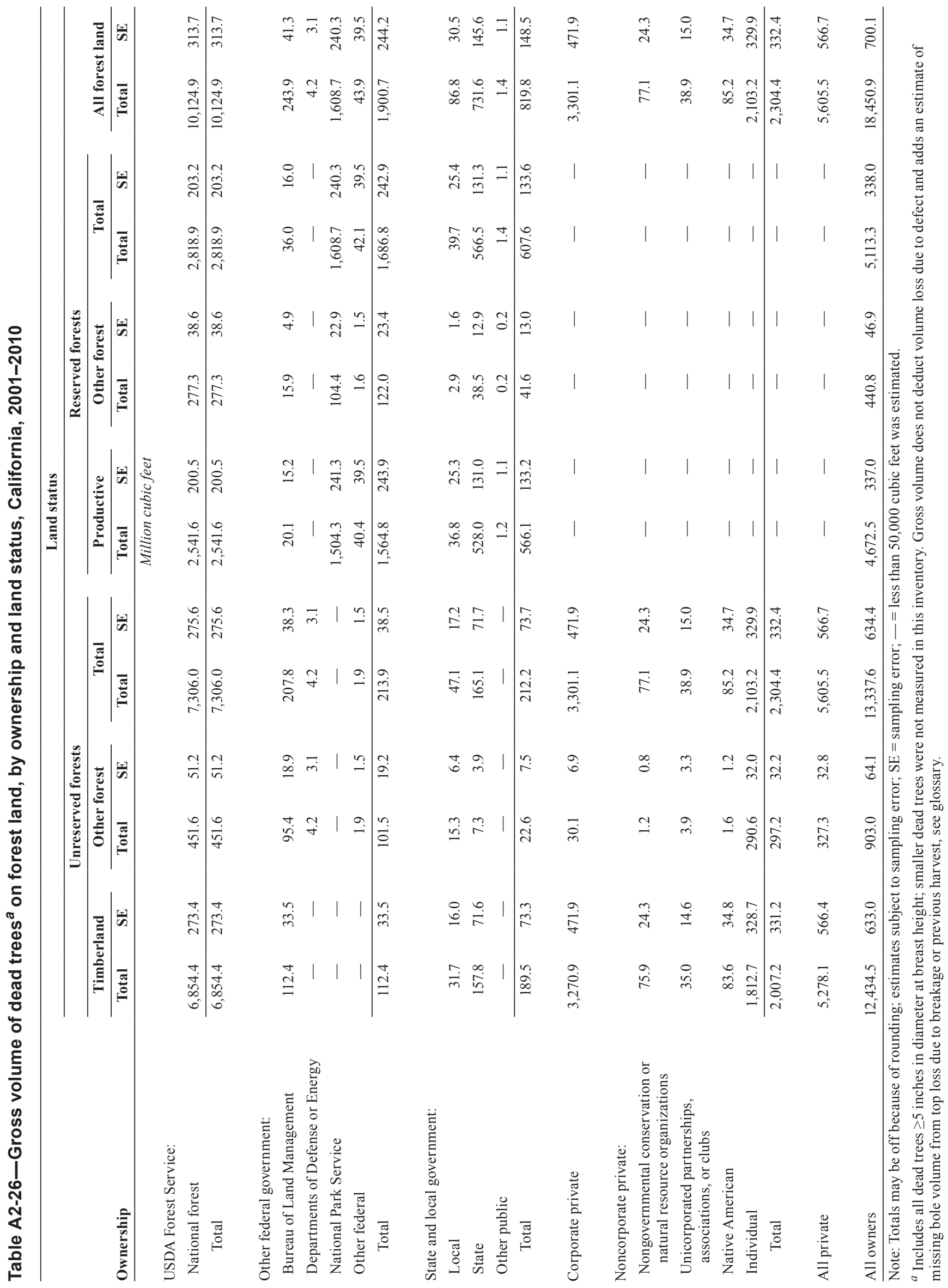




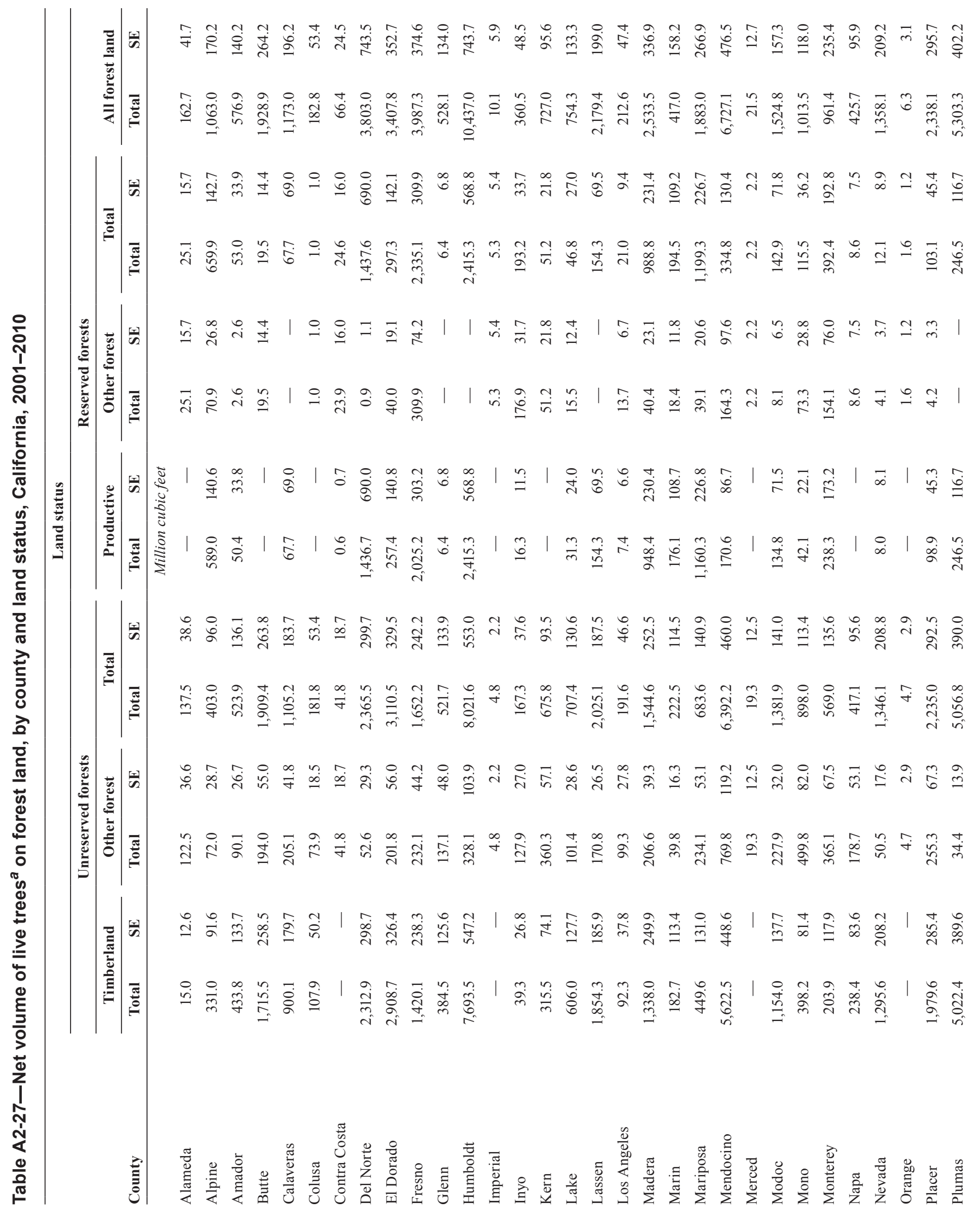




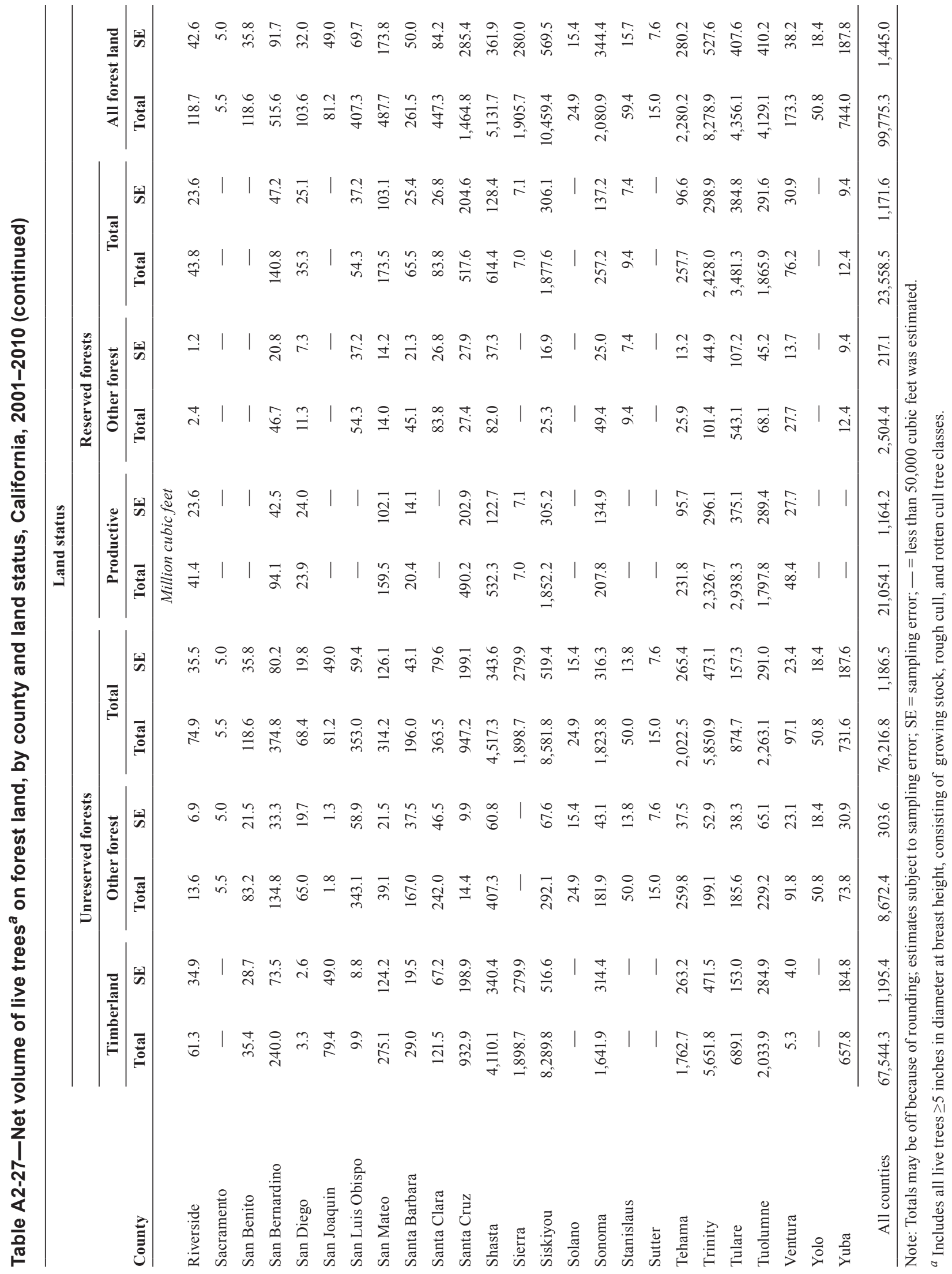




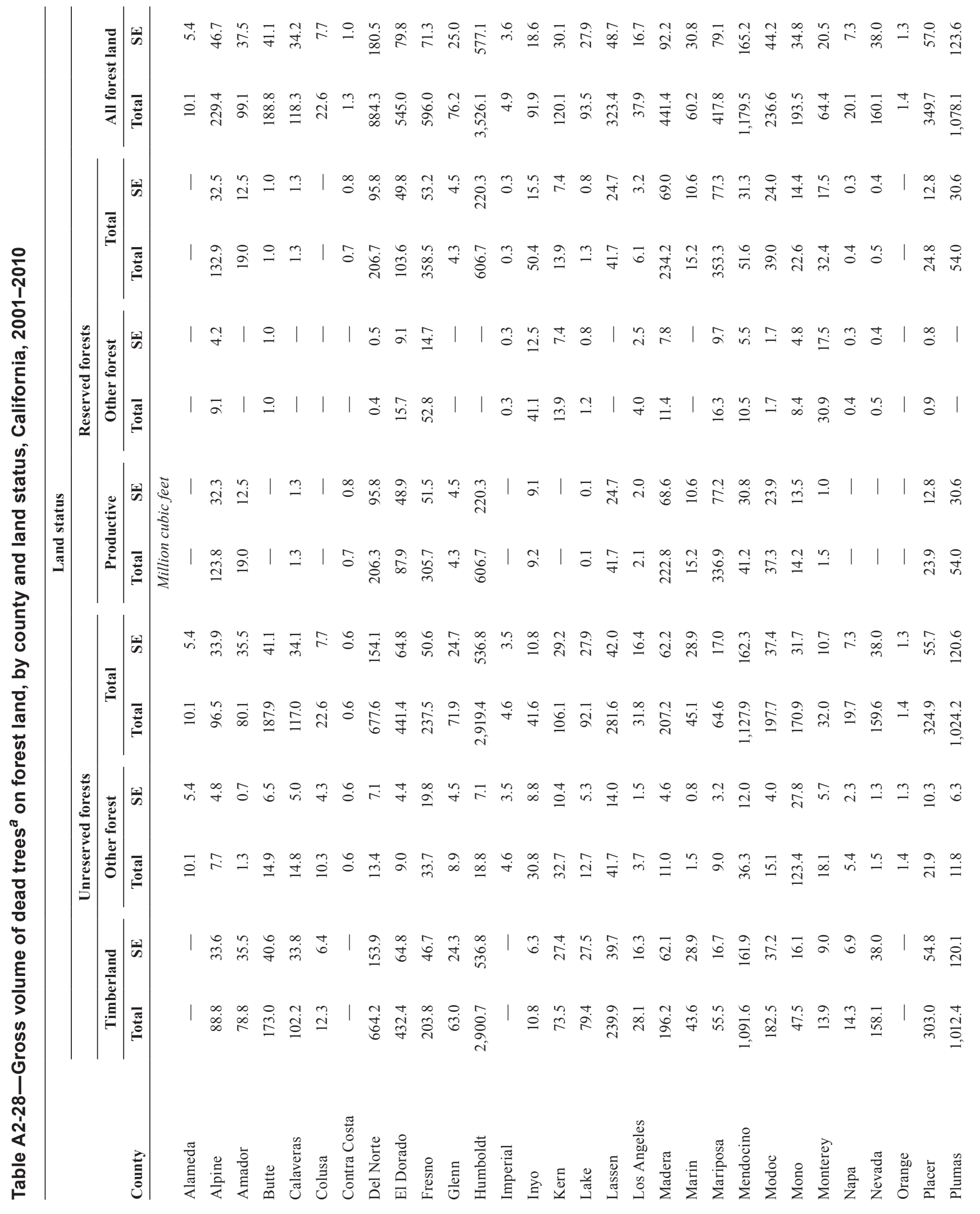




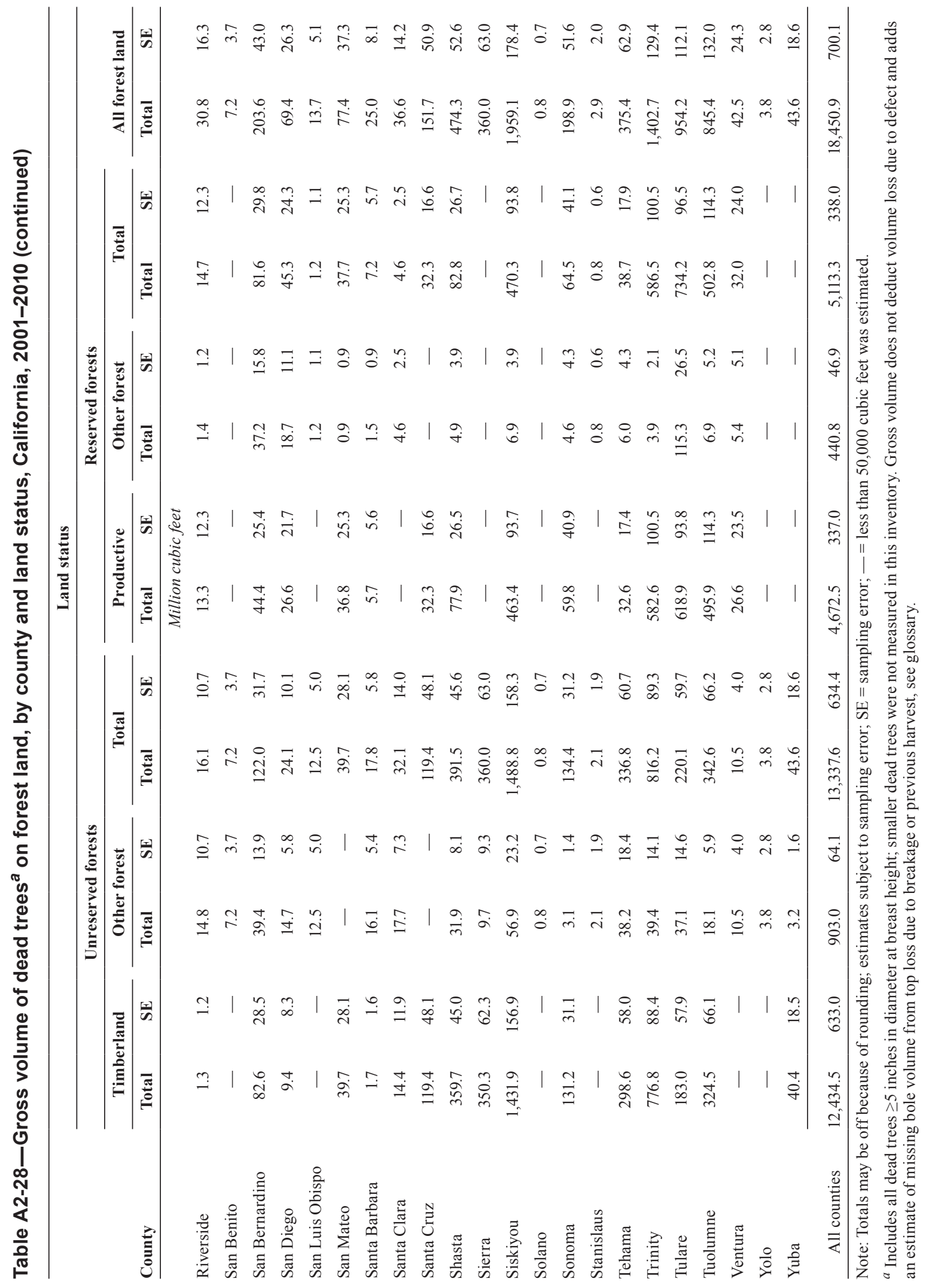


Table A2-29-Net cubic volume of live trees ${ }^{a}$ on forest land, by county and broad species group, California, 2001-2010

\begin{tabular}{|c|c|c|c|c|c|c|}
\hline \multirow[b]{3}{*}{ County } & \multicolumn{4}{|c|}{ Broad species group } & & \\
\hline & \multicolumn{2}{|c|}{ Softwood species } & \multicolumn{2}{|c|}{ Hardwood species } & \multicolumn{2}{|c|}{ All species } \\
\hline & Total & SE & Total & SE & Total & SE \\
\hline & \multicolumn{6}{|c|}{ Million cubic feet } \\
\hline Alameda & 0.1 & 0.1 & 162.6 & 41.7 & 162.7 & 41.7 \\
\hline Alpine & $1,051.4$ & 169.7 & 11.6 & 7.9 & $1,063.0$ & 170.2 \\
\hline Amador & 417.5 & 130.3 & 159.4 & 40.2 & 576.9 & 140.2 \\
\hline Butte & $1,344.8$ & 216.4 & 584.1 & 97.6 & $1,928.9$ & 264.2 \\
\hline Calaveras & 892.7 & 182.0 & 280.3 & 50.1 & $1,173.0$ & 196.2 \\
\hline Colusa & 83.5 & 32.6 & 99.3 & 28.9 & 182.8 & 53.4 \\
\hline Contra Costa & 1.7 & 1.4 & 64.7 & 24.3 & 66.4 & 24.5 \\
\hline Del Norte & $3,172.9$ & 721.2 & 630.1 & 106.1 & $3,803.0$ & 743.5 \\
\hline El Dorado & $3,048.4$ & 337.0 & 359.4 & 57.6 & $3,407.8$ & 352.7 \\
\hline Fresno & $3,725.8$ & 371.7 & 261.5 & 37.0 & $3,987.3$ & 374.6 \\
\hline Glenn & 367.3 & 115.8 & 160.8 & 46.8 & 528.1 & 134.0 \\
\hline Humboldt & $7,700.9$ & 674.7 & $2,736.1$ & 214.6 & $10,437.0$ & 743.7 \\
\hline Imperial & - & - & 10.1 & 5.9 & 10.1 & 5.9 \\
\hline Inyo & 337.1 & 47.0 & 23.4 & 6.7 & 360.5 & 48.5 \\
\hline Kern & 432.4 & 76.5 & 294.6 & 48.2 & 727.0 & 95.6 \\
\hline Lake & 506.2 & 106.8 & 248.0 & 42.4 & 754.3 & 133.3 \\
\hline Lassen & $2,137.1$ & 198.2 & 42.3 & 9.0 & $2,179.4$ & 199.0 \\
\hline Los Angeles & 108.7 & 32.4 & 104.0 & 28.0 & 212.6 & 47.4 \\
\hline Madera & $2,284.6$ & 330.6 & 248.8 & 40.6 & $2,533.5$ & 336.9 \\
\hline Marin & 276.3 & 135.2 & 140.8 & 45.3 & 417.0 & 158.2 \\
\hline Mariposa & $1,542.1$ & 250.8 & 340.9 & 60.5 & $1,883.0$ & 266.9 \\
\hline Mendocino & $4,293.8$ & 385.2 & $2,433.2$ & 187.8 & $6,727.1$ & 476.5 \\
\hline Merced & - & - & 21.5 & 12.7 & 21.5 & 12.7 \\
\hline Modoc & $1,493.9$ & 155.4 & 30.9 & 9.5 & $1,524.8$ & 157.3 \\
\hline Mono & 959.5 & 115.2 & 53.9 & 16.4 & $1,013.5$ & 118.0 \\
\hline Monterey & 514.0 & 205.3 & 447.4 & 66.0 & 961.4 & 235.4 \\
\hline Napa & 200.6 & 61.3 & 225.0 & 54.2 & 425.7 & 95.9 \\
\hline Nevada & $1,149.8$ & 193.2 & 208.3 & 46.1 & $1,358.1$ & 209.2 \\
\hline
\end{tabular}


Table A2-29—Net cubic volume of live trees ${ }^{a}$ on forest land, by county and broad species group, California, 2001-2010 (continued)

\begin{tabular}{|c|c|c|c|c|c|c|}
\hline \multirow[b]{3}{*}{ County } & \multicolumn{4}{|c|}{ Broad species group } & & \\
\hline & \multicolumn{2}{|c|}{ Softwood species } & \multicolumn{2}{|c|}{ Hardwood species } & \multicolumn{2}{|c|}{ All species } \\
\hline & Total & SE & Total & $\mathbf{S E}$ & Total & $\mathbf{S E}$ \\
\hline & \multicolumn{6}{|c|}{ Million cubic feet } \\
\hline Orange & - & - & 6.3 & 3.1 & 6.3 & 3.1 \\
\hline Placer & $1,934.8$ & 275.9 & 403.3 & 73.5 & $2,338.1$ & 295.7 \\
\hline Plumas & $5,107.7$ & 392.3 & 195.7 & 42.9 & $5,303.3$ & 402.2 \\
\hline Riverside & 90.7 & 34.9 & 28.0 & 14.0 & 118.7 & 42.6 \\
\hline Sacramento & - & - & 5.5 & 5.0 & 5.5 & 5.0 \\
\hline San Benito & 21.4 & 8.0 & 97.2 & 29.9 & 118.6 & 35.8 \\
\hline San Bernardino & 404.3 & 80.1 & 111.4 & 26.0 & 515.6 & 91.7 \\
\hline San Diego & 13.6 & 7.1 & 90.1 & 27.2 & 103.6 & 32.0 \\
\hline San Joaquin & 0.1 & 0.1 & 81.1 & 49.0 & 81.2 & 49.0 \\
\hline San Luis Obispo & 17.9 & 6.5 & 389.4 & 67.6 & 407.3 & 69.7 \\
\hline San Mateo & 336.2 & 147.9 & 151.5 & 46.2 & 487.7 & 173.8 \\
\hline Santa Barbara & 50.3 & 19.2 & 211.2 & 43.0 & 261.5 & 50.0 \\
\hline Santa Clara & 82.4 & 46.7 & 364.9 & 57.7 & 447.3 & 84.2 \\
\hline Santa Cruz & $1,053.2$ & 228.5 & 411.6 & 97.2 & $1,464.8$ & 285.4 \\
\hline Shasta & $4,103.8$ & 325.4 & $1,027.9$ & 94.7 & $5,131.7$ & 361.9 \\
\hline Sierra & $1,828.4$ & 272.5 & 77.3 & 25.0 & $1,905.7$ & 280.0 \\
\hline Siskiyou & $9,522.4$ & 537.5 & 937.0 & 90.5 & $10,459.4$ & 569.5 \\
\hline Solano & - & - & 24.9 & 15.4 & 24.9 & 15.4 \\
\hline Sonoma & $1,378.7$ & 292.5 & 702.2 & 84.5 & $2,080.9$ & 344.4 \\
\hline Stanislaus & 10.9 & 5.1 & 48.6 & 13.2 & 59.4 & 15.7 \\
\hline Sutter & - & - & 15.0 & 7.6 & 15.0 & 7.6 \\
\hline Tehama & $1,860.2$ & 264.6 & 419.9 & 53.5 & $2,280.2$ & 280.2 \\
\hline Trinity & $7,068.4$ & 479.2 & $1,210.5$ & 102.3 & $8,278.9$ & 527.6 \\
\hline Tulare & $3,977.5$ & 397.6 & 378.5 & 67.9 & $4,356.1$ & 407.6 \\
\hline Tuolumne & $3,794.3$ & 395.3 & 334.8 & 58.1 & $4,129.1$ & 410.2 \\
\hline Ventura & 114.9 & 31.3 & 58.5 & 18.2 & 173.3 & 38.2 \\
\hline Yolo & 18.9 & 11.6 & 31.9 & 10.3 & 50.8 & 18.4 \\
\hline Yuba & 555.6 & 169.8 & 188.4 & 44.0 & 744.0 & 187.8 \\
\hline All counties & $81,389.8$ & $1,394.2$ & $18,385.5$ & 401.3 & $99,775.3$ & $1,445.0$ \\
\hline
\end{tabular}

Note: Totals may be off because of rounding; estimates subject to sampling error; SE = sampling error; — = less than 50,000 cubic feet was estimated.

${ }^{a}$ Includes all live trees $\geq 5$ inches in diameter at breast height, consisting of growing stock, rough cull, and rotten cull tree classes. 
Table A2-30 - Net volume of live trees ${ }^{a}$ on forest land, by forest type group and stand size class, California, 2001-2010

\begin{tabular}{|c|c|c|c|c|c|c|c|c|}
\hline \multirow[b]{3}{*}{ Forest type group } & \multicolumn{8}{|c|}{ Stand size class } \\
\hline & \multicolumn{2}{|c|}{$\begin{array}{c}\text { Large-diameter } \\
\text { stands }\end{array}$} & \multicolumn{2}{|c|}{$\begin{array}{c}\text { Medium-diameter } \\
\text { stands }\end{array}$} & \multicolumn{2}{|c|}{$\begin{array}{c}\text { Small-diameter } \\
\text { stands }\end{array}$} & \multicolumn{2}{|c|}{ All classes } \\
\hline & Total & SE & Total & SE & Total & $\mathbf{S E}$ & Total & SE \\
\hline & \multicolumn{8}{|c|}{ Million cubic feet } \\
\hline \multicolumn{9}{|l|}{ Softwoods: } \\
\hline California mixed conifer & $36,989.8$ & $1,020.5$ & 203.8 & 45.0 & 134.1 & 25.8 & $37,327.7$ & $1,018.9$ \\
\hline Douglas-fir & $6,117.2$ & 503.9 & 105.3 & 37.9 & 79.5 & 29.9 & $6,302.0$ & 504.7 \\
\hline Fir/spruce/mountain hemlock & $11,305.8$ & 728.5 & 22.9 & 11.7 & 48.0 & 14.4 & $11,376.7$ & 728.9 \\
\hline Lodgepole pine & $3,591.1$ & 324.1 & 76.7 & 35.0 & 6.1 & 3.4 & $3,673.9$ & 326.0 \\
\hline Pinyon/juniper woodlands & 889.0 & 68.7 & 23.1 & 7.4 & 10.1 & 3.5 & 922.3 & 68.3 \\
\hline Ponderosa pine & $5,023.7$ & 325.3 & 87.0 & 18.7 & 24.4 & 8.6 & $5,135.0$ & 325.5 \\
\hline Redwood & $8,030.8$ & 986.2 & 20.8 & 11.7 & 14.2 & 7.4 & $8,065.8$ & 986.1 \\
\hline Western juniper & 728.9 & 71.7 & 24.8 & 7.7 & 9.5 & 4.5 & 763.2 & 72.0 \\
\hline Western white pine & 377.1 & 92.4 & 1.6 & 1.5 & 15.6 & 9.5 & 394.3 & 92.9 \\
\hline Other western softwoods & 999.1 & 176.6 & 26.9 & 11.0 & 9.0 & 3.5 & $1,035.0$ & 177.2 \\
\hline Total & $74,052.6$ & $1,500.4$ & 592.8 & 74.4 & 350.4 & 44.8 & $74,995.8$ & $1,493.7$ \\
\hline
\end{tabular}

Hardwoods:

\begin{tabular}{|c|c|c|c|c|c|c|c|c|}
\hline Alder/maple & 817.2 & 166.3 & 181.6 & 60.5 & 17.0 & 10.6 & $1,015.8$ & 176.8 \\
\hline Aspen/birch & 39.9 & 25.3 & 22.8 & 13.4 & 11.3 & 6.0 & 74.0 & 29.3 \\
\hline Elm/ash/cottonwood & 121.6 & 53.7 & 0.5 & 0.4 & 2.6 & 2.4 & 124.8 & 53.8 \\
\hline Tanoak/laurel & $7,250.6$ & 529.1 & $1,170.5$ & 151.4 & 136.9 & 35.6 & $8,558.0$ & 539.0 \\
\hline Western oak & $9,588.4$ & 401.6 & $3,485.0$ & 191.1 & 339.5 & 38.7 & $13,412.9$ & 428.4 \\
\hline Woodland hardwoods & 233.9 & 36.7 & 23.3 & 9.2 & 2.5 & 1.7 & 259.7 & 37.9 \\
\hline Other hardwoods & $1,028.0$ & 208.3 & 191.5 & 54.7 & 33.0 & 8.5 & $1,252.5$ & 215.2 \\
\hline Total & $19,079.5$ & 685.5 & $5,075.2$ & 252.8 & 542.9 & 54.6 & $24,697.7$ & 694.8 \\
\hline Nonstocked & - & - & - & - & 一 & - & 81.8 & 15.4 \\
\hline All forest types & $93,132.1$ & $1,485.5$ & $5,668.0$ & 261.9 & 893.4 & 70.1 & $99,775.3$ & $1,445.0$ \\
\hline
\end{tabular}

Note: Totals may be off because of rounding; estimates subject to sampling error; SE = sampling error; - - less than 50,000 cubic feet was estimated.

${ }^{a}$ Includes all live trees $\geq 5$ inches in diameter at breast height, consisting of growing stock, rough cull, and rotten cull tree classes. 
Table A2-31-Gross volume of dead trees ${ }^{a}$ on forest land, by forest type group and stand size class, California, 2001-2010

\begin{tabular}{|c|c|c|c|c|c|c|c|c|}
\hline \multirow[b]{3}{*}{ Forest type group } & \multicolumn{8}{|c|}{ Stand size class } \\
\hline & \multicolumn{2}{|c|}{$\begin{array}{c}\text { Large-diameter } \\
\text { stands }\end{array}$} & \multicolumn{2}{|c|}{$\begin{array}{c}\text { Medium-diameter } \\
\text { stands }\end{array}$} & \multicolumn{2}{|c|}{$\begin{array}{c}\text { Small-diameter } \\
\text { stands }\end{array}$} & \multicolumn{2}{|c|}{ All classes } \\
\hline & Total & SE & Total & SE & Total & SE & Total & SE \\
\hline & \multicolumn{8}{|c|}{ Million cubic feet } \\
\hline \multicolumn{9}{|l|}{ Softwoods: } \\
\hline California mixed conifer & $5,770.1$ & 240.2 & 63.1 & 22.7 & 118.7 & 37.8 & $5,952.0$ & 242.9 \\
\hline Douglas-fir & $1,045.5$ & 178.9 & 7.4 & 4.7 & 92.8 & 39.4 & $1,145.7$ & 182.6 \\
\hline Fir/spruce/mountain hemlock & $2,331.4$ & 190.4 & 21.1 & 16.6 & 192.9 & 103.5 & $2,545.4$ & 216.1 \\
\hline Lodgepole pine & 582.2 & 67.0 & 29.2 & 21.5 & 0.6 & 0.7 & 611.9 & 70.4 \\
\hline Pinyon/juniper woodlands & 120.4 & 14.6 & 22.9 & 11.8 & 10.3 & 5.2 & 153.5 & 19.2 \\
\hline Ponderosa pine & 298.8 & 34.3 & 9.3 & 4.4 & 19.7 & 11.0 & 327.8 & 36.2 \\
\hline Redwood & $2,787.4$ & 527.7 & 53.4 & 31.3 & 34.5 & 20.6 & $2,875.3$ & 531.6 \\
\hline Western juniper & 111.0 & 24.3 & 1.2 & 1.0 & 0.3 & 0.2 & 112.6 & 24.4 \\
\hline Western white pine & 99.3 & 27.2 & 0.5 & 0.5 & 5.4 & 3.7 & 105.2 & 27.5 \\
\hline Other western softwoods & 432.4 & 211.2 & 6.4 & 3.6 & 20.5 & 16.1 & 459.3 & 211.8 \\
\hline Total & $13,578.5$ & 655.8 & 214.6 & 49.2 & 495.7 & 120.5 & $14,288.7$ & 666.3 \\
\hline
\end{tabular}

Hardwoods:

\begin{tabular}{|c|c|c|c|c|c|c|c|c|}
\hline Alder/maple & 234.2 & 100.1 & 83.9 & 47.7 & 10.4 & 6.6 & 328.5 & 111.1 \\
\hline Aspen/birch & 5.1 & 4.0 & 4.2 & 2.7 & 0.6 & 0.5 & 9.9 & 4.9 \\
\hline Elm/ash/cottonwood & 2.1 & 1.6 & - & - & 1.4 & 1.3 & 3.5 & 2.1 \\
\hline Tanoak/laurel & 894.4 & 99.6 & 143.9 & 35.5 & 267.4 & 127.0 & $1,305.7$ & 163.6 \\
\hline Western oak & 890.9 & 70.1 & 314.0 & 35.4 & 373.0 & 107.1 & $1,577.9$ & 131.7 \\
\hline Woodland hardwoods & 91.3 & 18.4 & 25.5 & 13.8 & - & - & 116.7 & 23.0 \\
\hline Other hardwoods & 108.1 & 29.7 & 16.4 & 5.9 & 37.7 & 24.3 & 162.3 & 38.9 \\
\hline Total & $2,226.1$ & 159.2 & 587.9 & 70.6 & 690.5 & 167.8 & $3,504.5$ & 238.8 \\
\hline Nonstocked & - & - & - & - & - & - & 657.7 & 132.6 \\
\hline All forest types & $15,804.5$ & 664.1 & 802.5 & 85.7 & $1,186.2$ & 206.2 & $18,450.9$ & 700.1 \\
\hline
\end{tabular}




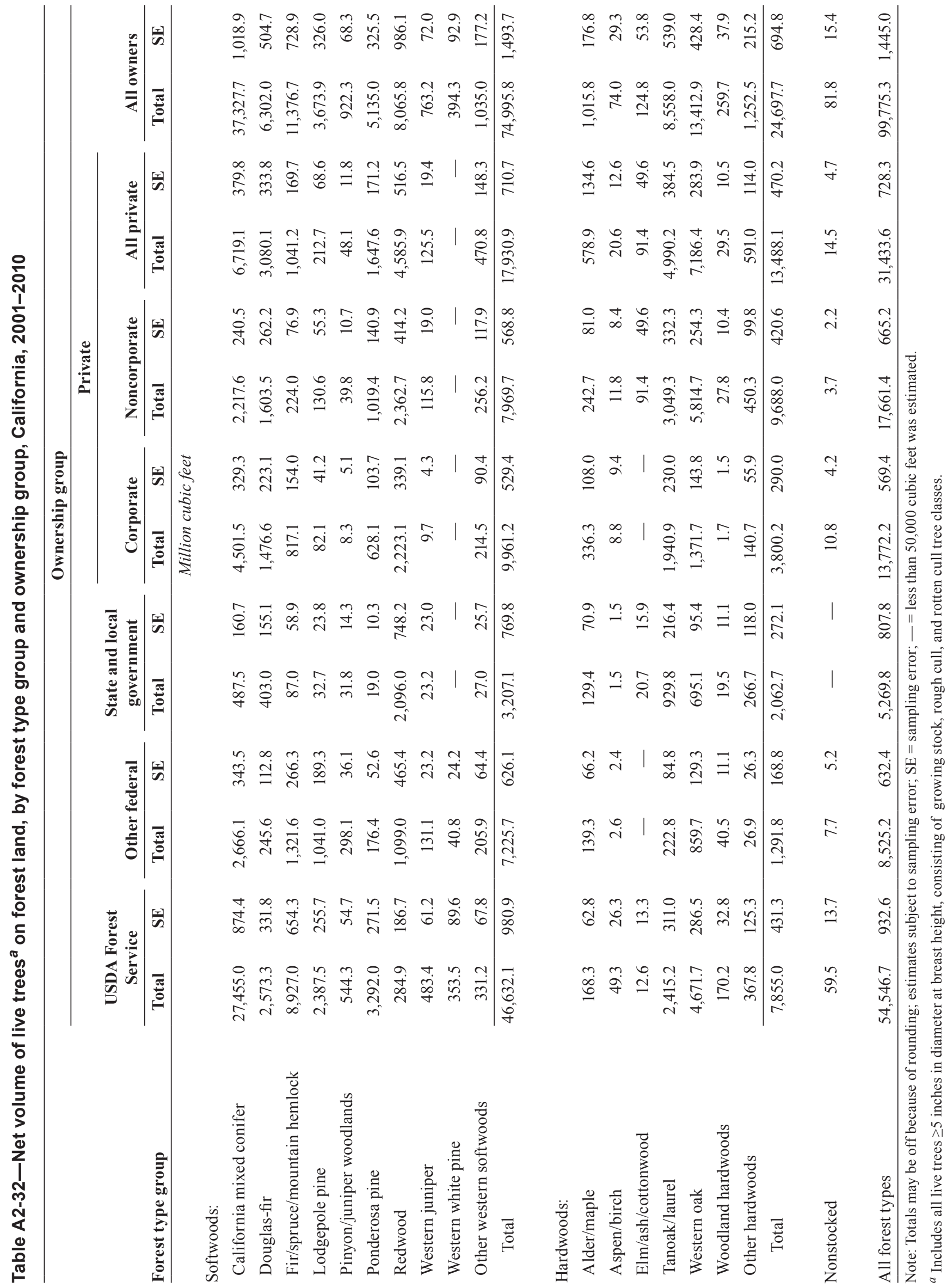




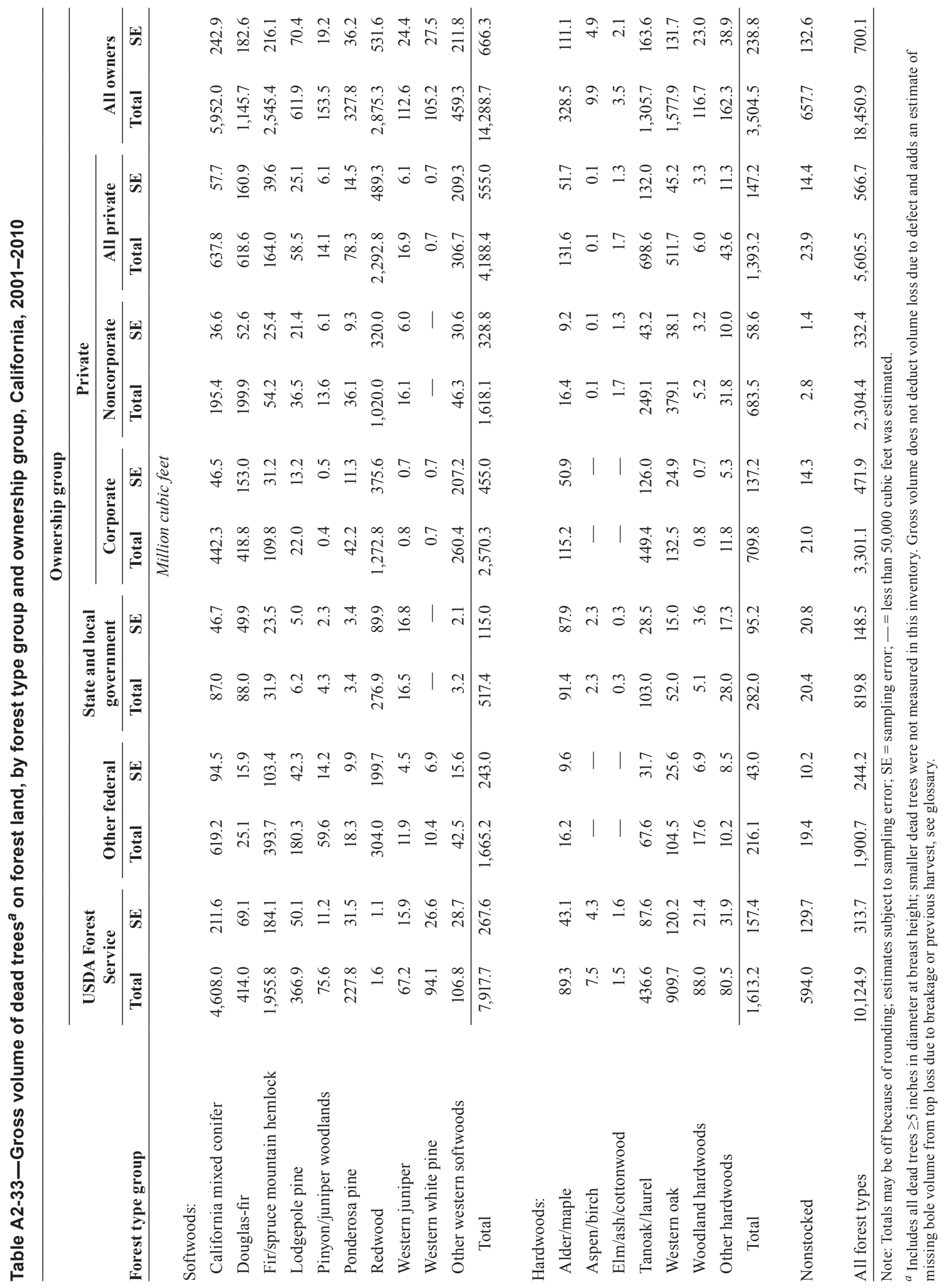




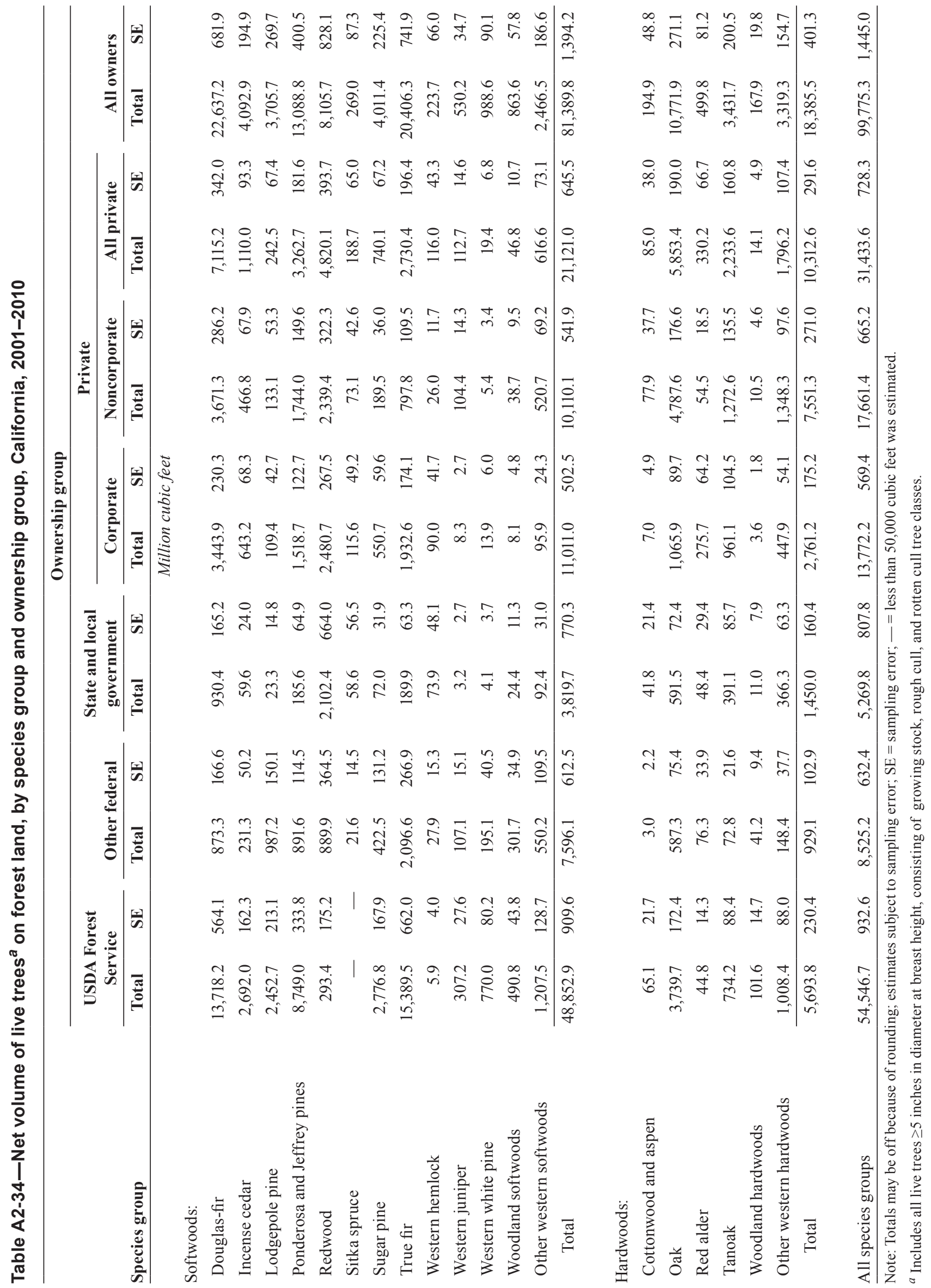




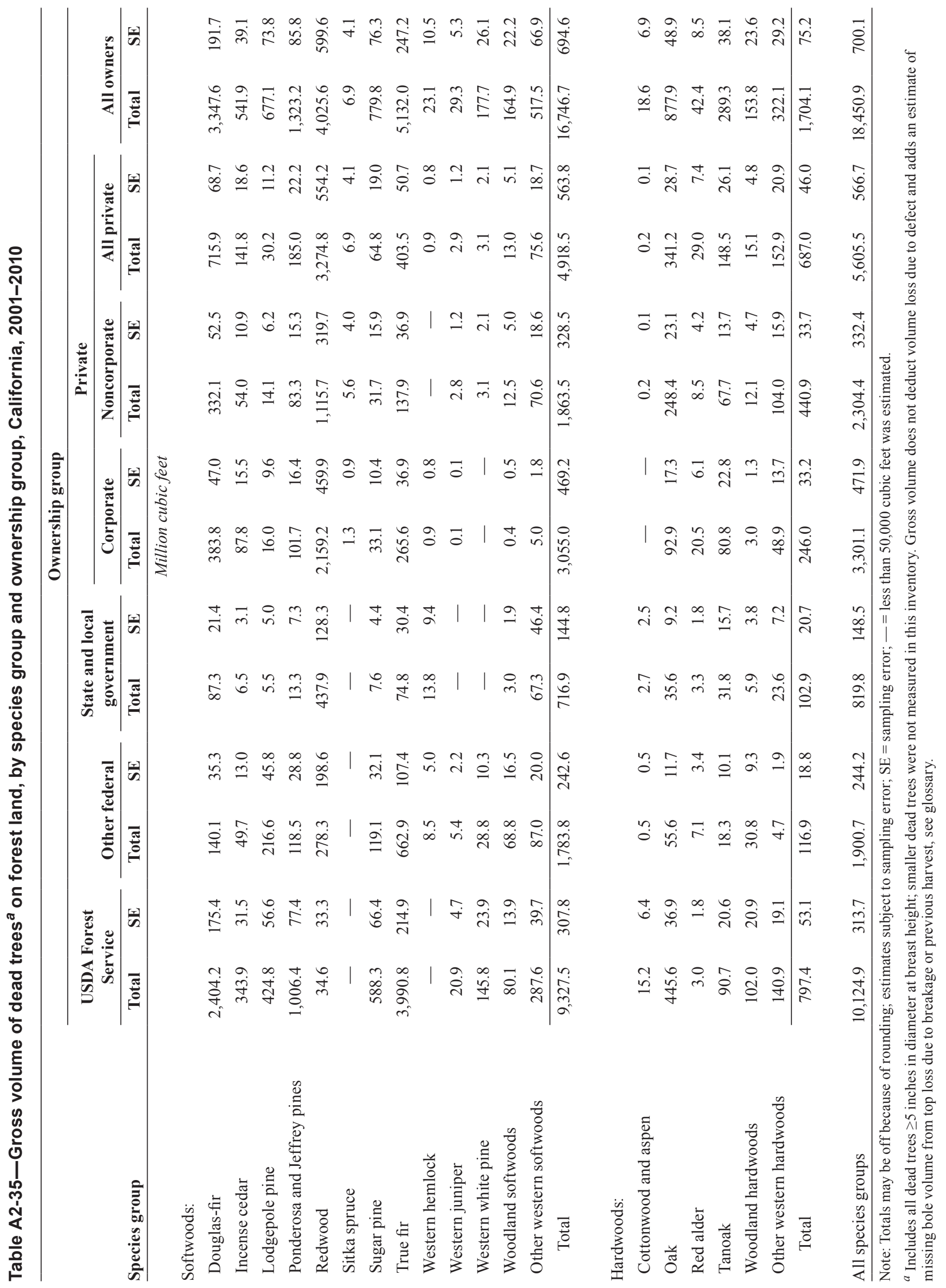




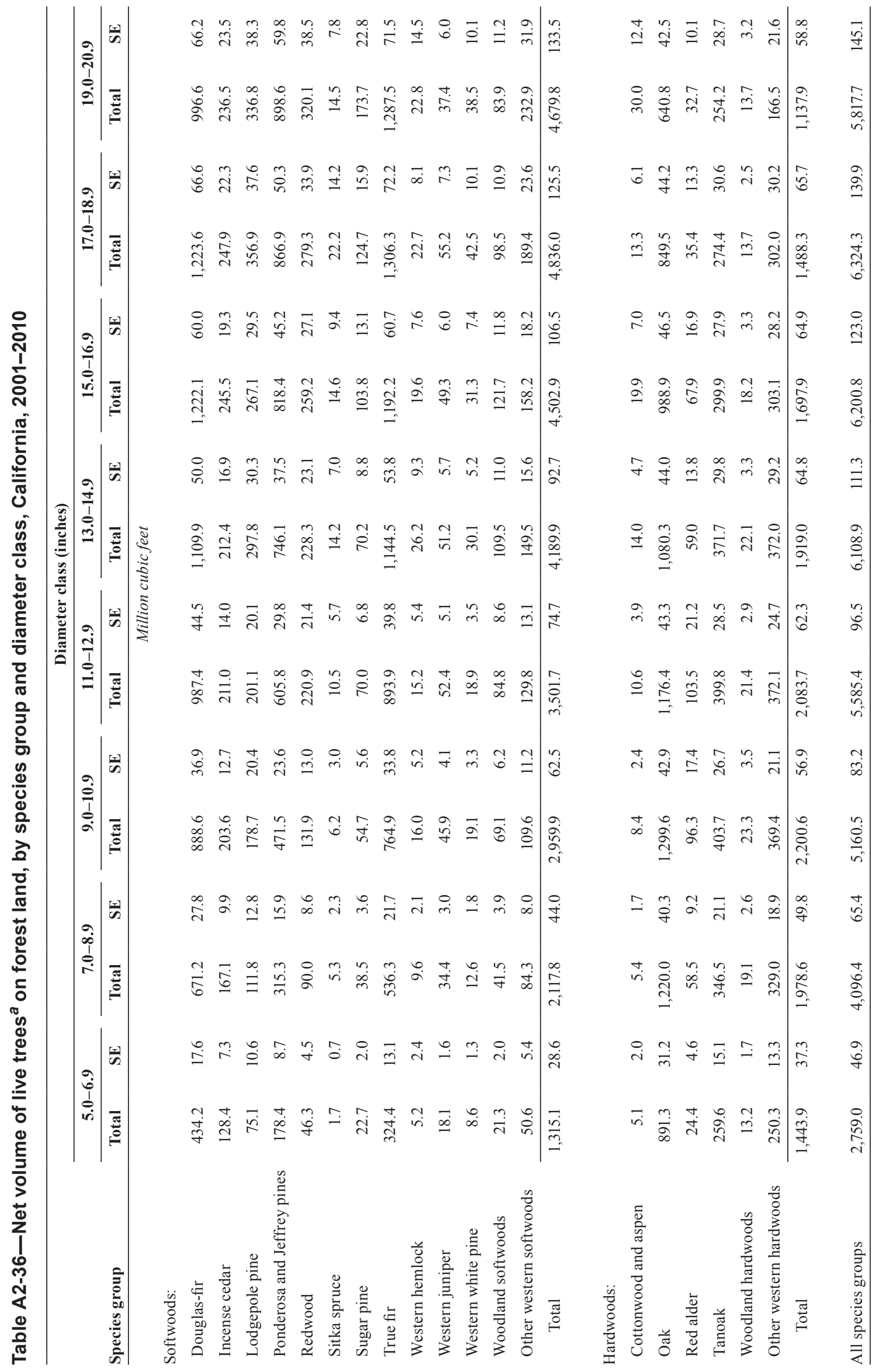




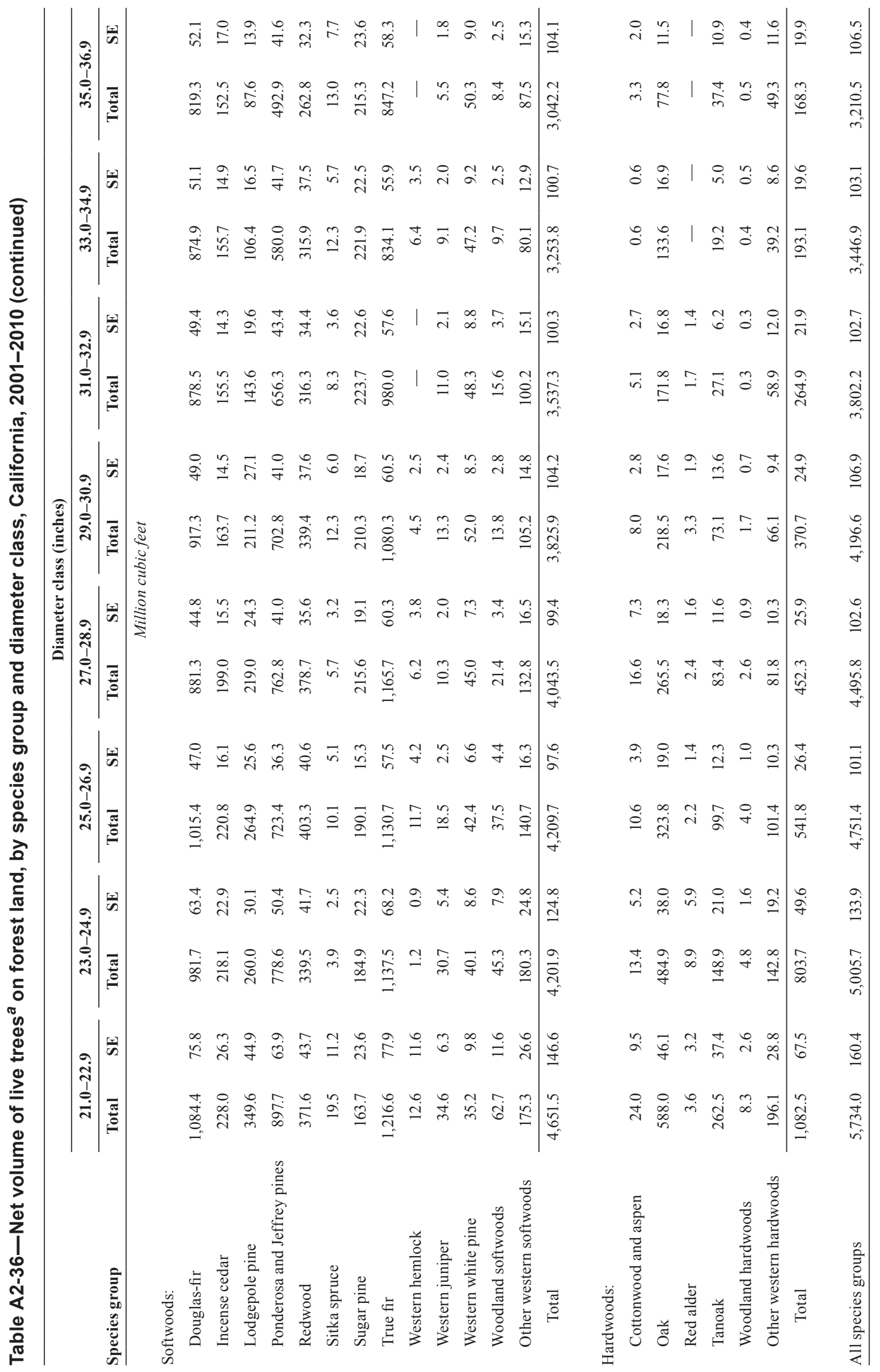




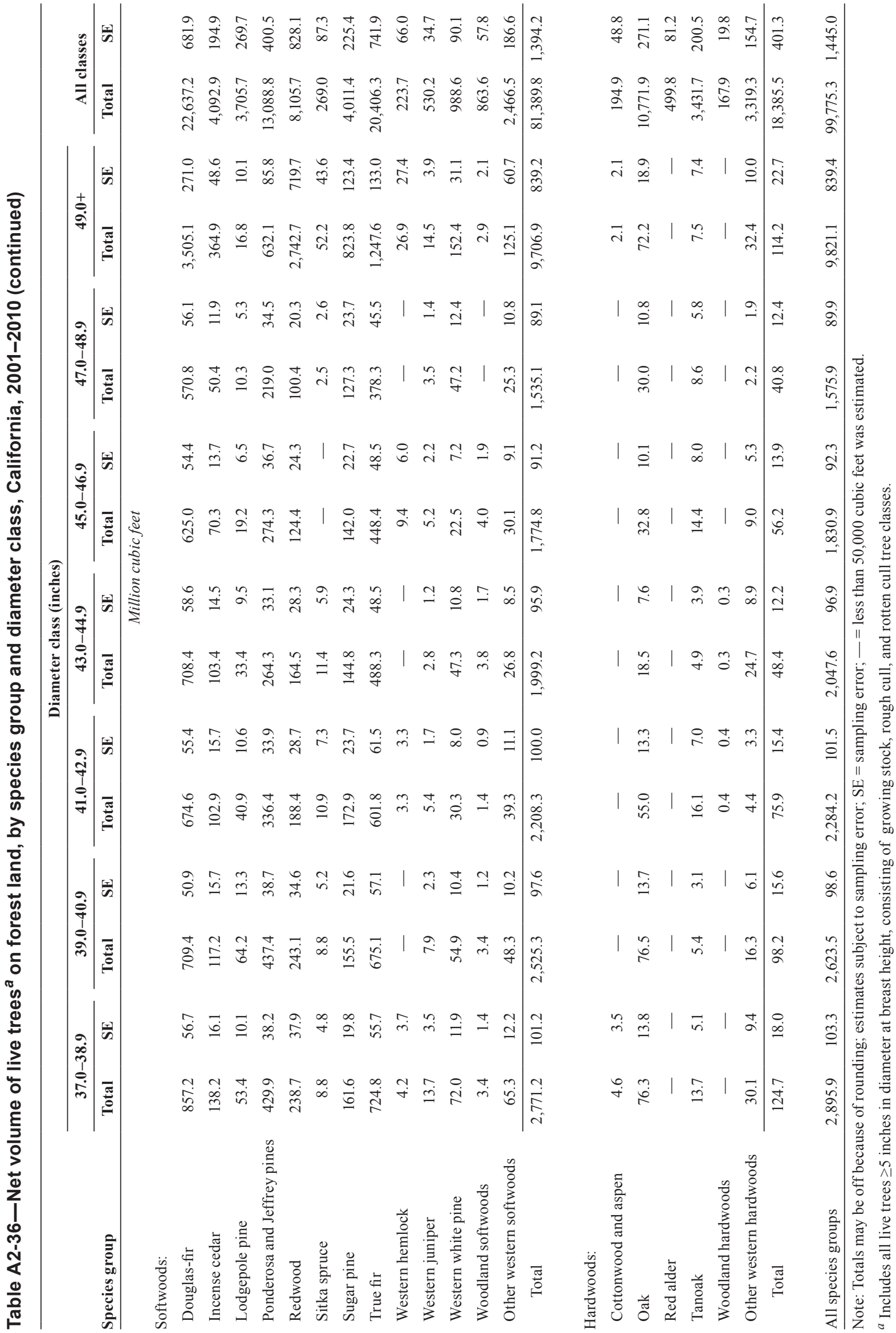




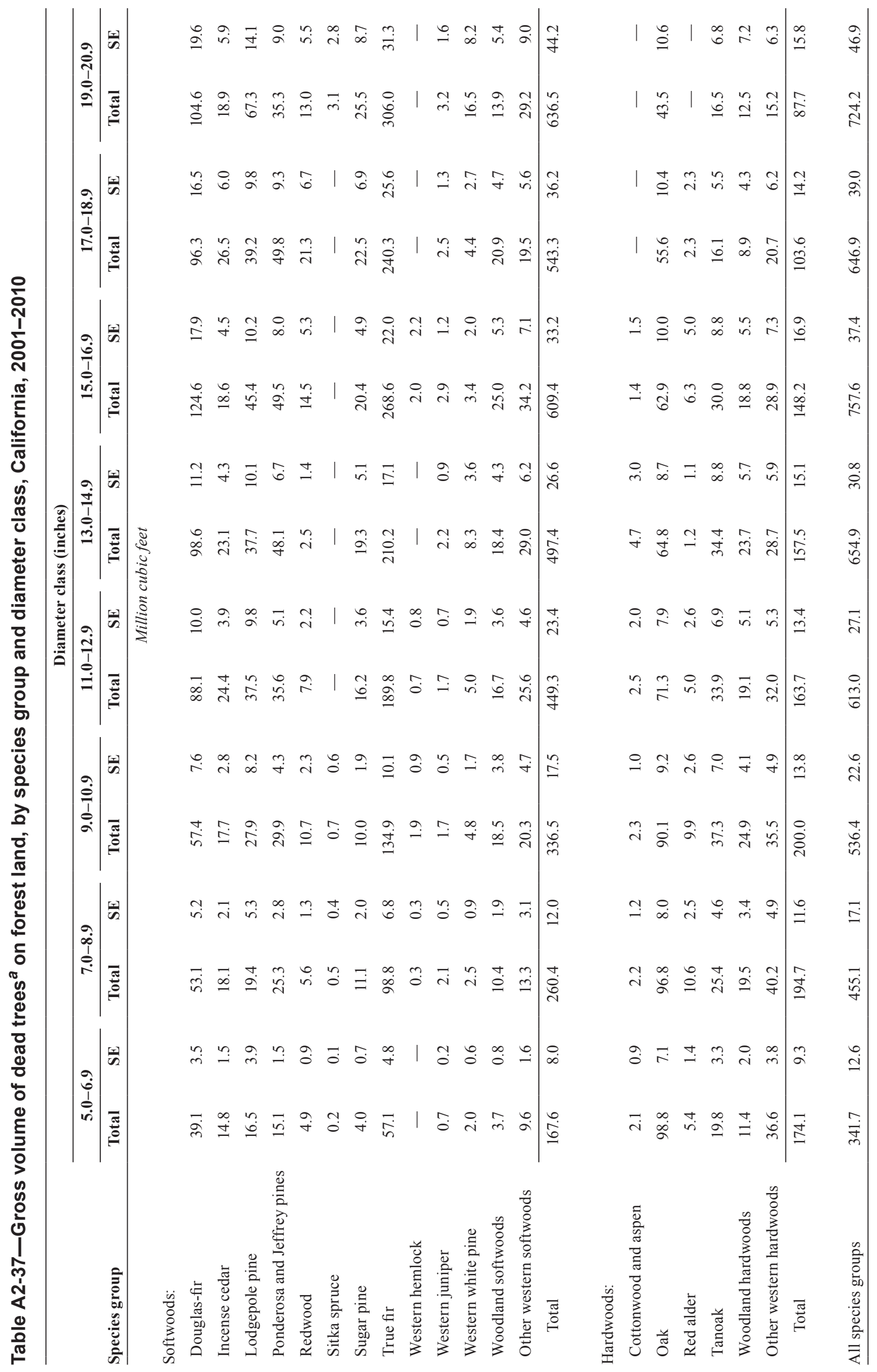




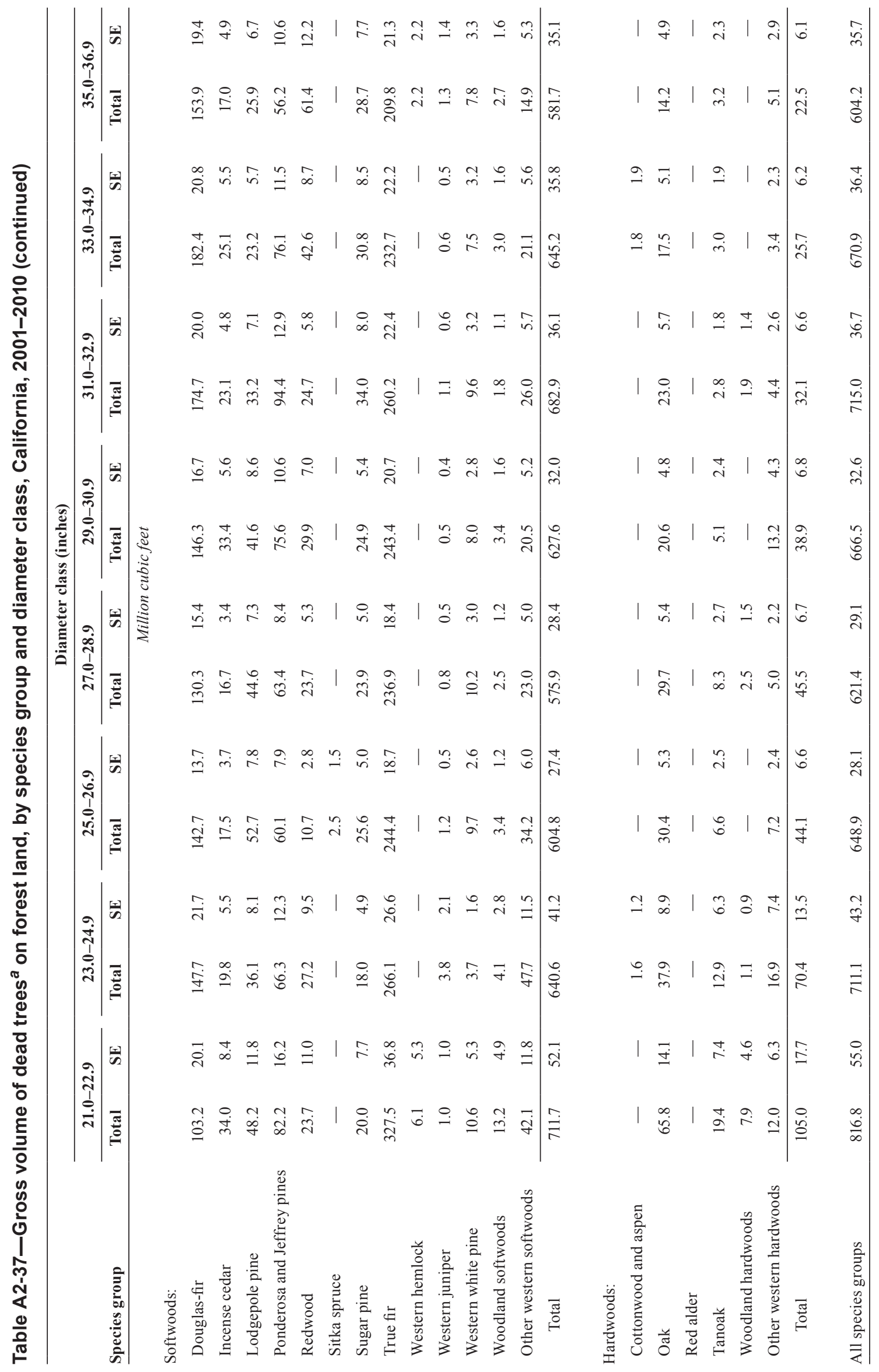




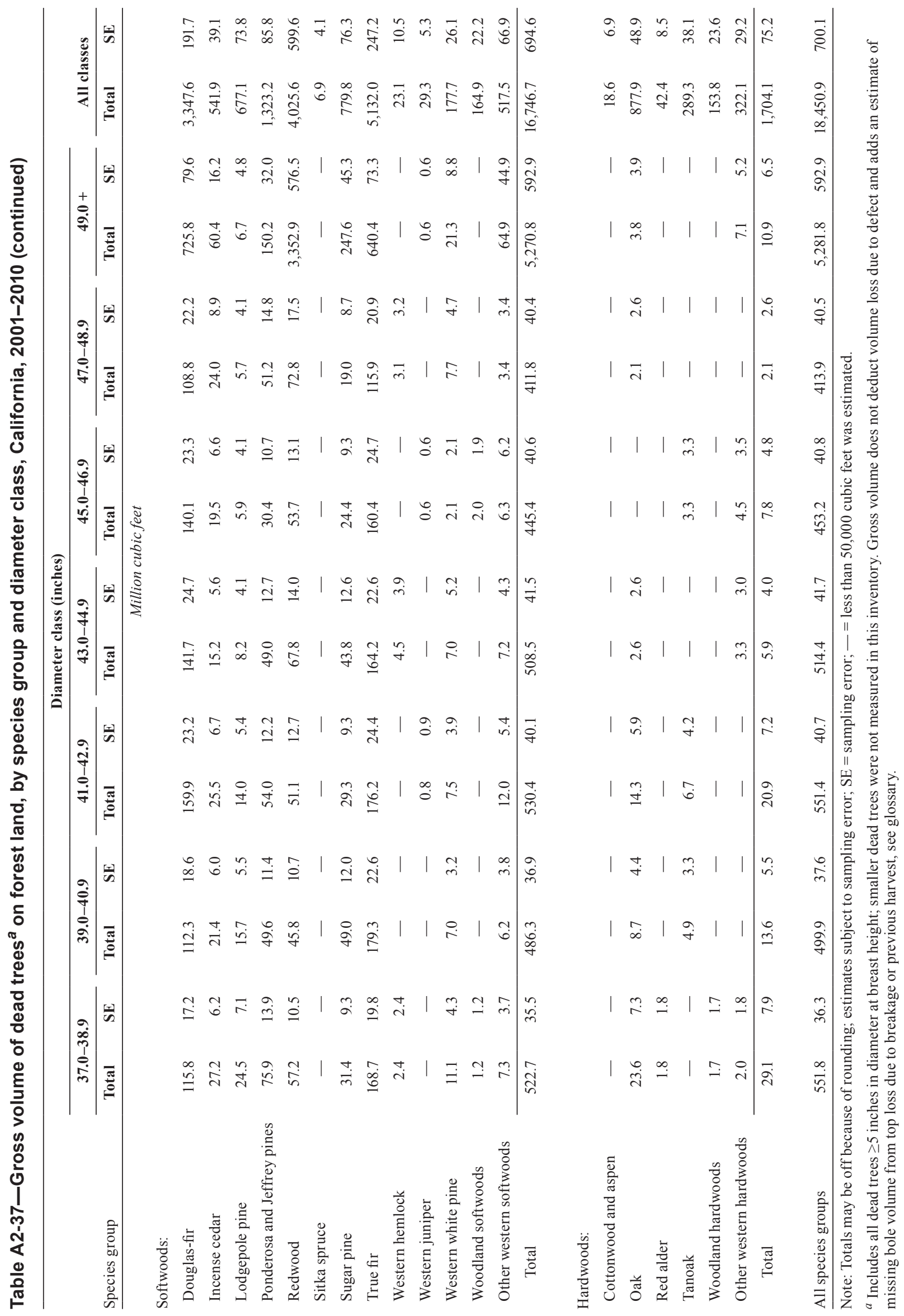


Table A2-38-Net volume of live trees ${ }^{a}$ on forest land, by forest type group and stand origin, California, 2001-2010

\begin{tabular}{|c|c|c|c|c|c|c|}
\hline \multirow[b]{3}{*}{ Forest type group } & \multicolumn{4}{|c|}{ Stand origin } & & \\
\hline & \multicolumn{2}{|c|}{ Natural stands } & \multicolumn{2}{|c|}{ Artificial regeneration } & \multicolumn{2}{|c|}{ Total } \\
\hline & Total & SE & Total & SE & Total & SE \\
\hline & \multicolumn{6}{|c|}{ Million cubic feet } \\
\hline \multicolumn{7}{|l|}{ Softwoods: } \\
\hline California mixed conifer & $36,925.2$ & $1,018.8$ & 402.6 & 79.8 & $37,327.7$ & $1,018.9$ \\
\hline Douglas-fir & $6,139.3$ & 502.7 & 162.6 & 62.7 & $6,302.0$ & 504.7 \\
\hline Fir/spruce/mountain hemlock & $11,286.1$ & 727.6 & 90.5 & 49.8 & $11,376.7$ & 728.9 \\
\hline Lodgepole pine & $3,673.9$ & 326.0 & - & - & $3,673.9$ & 326.0 \\
\hline Pinyon/juniper woodlands & 913.3 & 67.8 & 9.0 & 8.9 & 922.3 & 68.3 \\
\hline Ponderosa pine & $4,603.5$ & 311.1 & 531.5 & 104.2 & $5,135.0$ & 325.5 \\
\hline Redwood & $7,792.9$ & 973.0 & 272.9 & 183.0 & $8,065.8$ & 986.1 \\
\hline Western juniper & 757.6 & 71.9 & 5.5 & 4.6 & 763.2 & 72.0 \\
\hline Western white pine & 394.3 & 92.9 & - & - & 394.3 & 92.9 \\
\hline Other western softwoods & $1,035.0$ & 177.2 & - & - & $1,035.0$ & 177.2 \\
\hline Total & $73,521.1$ & $1,489.5$ & $1,474.7$ & 238.6 & $74,995.8$ & $1,493.7$ \\
\hline
\end{tabular}

Hardwoods:

\begin{tabular}{|c|c|c|c|c|c|c|}
\hline Alder/maple & 939.2 & 167.4 & 76.6 & 57.1 & $1,015.8$ & 176.8 \\
\hline Aspen/birch & 74.0 & 29.3 & - & - & 74.0 & 29.3 \\
\hline Elm/ash/cottonwood & 124.8 & 53.8 & - & - & 124.8 & 53.8 \\
\hline Tanoak/laurel & $8,373.0$ & 538.6 & 185.1 & 53.6 & $8,558.0$ & 539.0 \\
\hline Western oak & $13,330.2$ & 428.2 & 82.6 & 25.6 & $13,412.9$ & 428.4 \\
\hline Woodland hardwoods & 259.7 & 37.9 & - & - & 259.7 & 37.9 \\
\hline Other hardwoods & $1,224.8$ & 214.2 & 27.7 & 20.7 & $1,252.5$ & 215.2 \\
\hline Total & $24,325.6$ & 693.5 & 372.0 & 84.8 & $24,697.7$ & 694.8 \\
\hline Nonstocked & 70.1 & 14.7 & 11.7 & 4.6 & 81.8 & 15.4 \\
\hline All forest types & $97,916.8$ & $1,447.8$ & $1,858.4$ & 253.2 & $99,775.3$ & $1,445.0$ \\
\hline
\end{tabular}


Table A2-39-Gross volume of dead trees ${ }^{a}$ on forest land, by forest type group and stand origin, California, 2001-2010

\begin{tabular}{|c|c|c|c|c|c|c|}
\hline \multirow[b]{3}{*}{ Forest type group } & \multicolumn{4}{|c|}{ Stand origin } & & \\
\hline & \multicolumn{2}{|c|}{ Natural stands } & \multicolumn{2}{|c|}{ Artificial regeneration } & \multicolumn{2}{|c|}{ Total } \\
\hline & Total & SE & Total & SE & Total & SE \\
\hline & \multicolumn{6}{|c|}{ Million cubic feet } \\
\hline \multicolumn{7}{|l|}{ Softwoods: } \\
\hline California mixed conifer & $5,884.8$ & 242.5 & 67.2 & 21.0 & $5,952.0$ & 242.9 \\
\hline Douglas-fir & $1,135.8$ & 182.7 & 9.8 & 3.8 & $1,145.7$ & 182.6 \\
\hline Fir/spruce/mountain hemlock & $2,535.8$ & 216.0 & 9.6 & 6.6 & $2,545.4$ & 216.1 \\
\hline Lodgepole pine & 611.9 & 70.4 & - & - & 611.9 & 70.4 \\
\hline Pinyon/juniper woodlands & 152.9 & 19.2 & 0.7 & 0.7 & 153.5 & 19.2 \\
\hline Ponderosa pine & 299.7 & 35.0 & 28.1 & 9.4 & 327.8 & 36.2 \\
\hline Redwood & $2,838.6$ & 531.2 & 36.7 & 25.5 & $2,875.3$ & 531.6 \\
\hline Western juniper & 112.2 & 24.3 & 0.3 & 0.3 & 112.6 & 24.4 \\
\hline Western white pine & 104.5 & 27.5 & 0.7 & 0.7 & 105.2 & 27.5 \\
\hline Other western softwoods & 459.3 & 211.8 & - & - & 459.3 & 211.8 \\
\hline Total & $14,135.5$ & 666.1 & 153.2 & 35.1 & $14,288.7$ & 666.3 \\
\hline
\end{tabular}

Hardwoods:

\begin{tabular}{|c|c|c|c|c|c|c|}
\hline Alder/maple & 208.2 & 59.2 & 120.3 & 94.1 & 328.5 & 111.1 \\
\hline Aspen/birch & 9.9 & 4.9 & - & - & 9.9 & 4.9 \\
\hline Elm/ash/cottonwood & 3.5 & 2.1 & 一 & 一 & 3.5 & 2.1 \\
\hline Tanoak/laurel & $1,212.7$ & 158.8 & 93.0 & 40.8 & $1,305.7$ & 163.6 \\
\hline Western oak & $1,561.8$ & 131.5 & 16.1 & 7.3 & $1,577.9$ & 131.7 \\
\hline Woodland hardwoods & 116.7 & 23.0 & - & - & 116.7 & 23.0 \\
\hline Other hardwoods & 140.3 & 35.0 & 22.0 & 16.9 & 162.3 & 38.9 \\
\hline Total & $3,253.2$ & 215.7 & 251.3 & 104.2 & $3,504.5$ & 238.8 \\
\hline Nonstocked & 629.2 & 130.0 & 28.5 & 25.7 & 657.7 & 132.6 \\
\hline All forest types & $18,017.8$ & 692.7 & 433.1 & 112.8 & $18,450.9$ & 700.1 \\
\hline
\end{tabular}




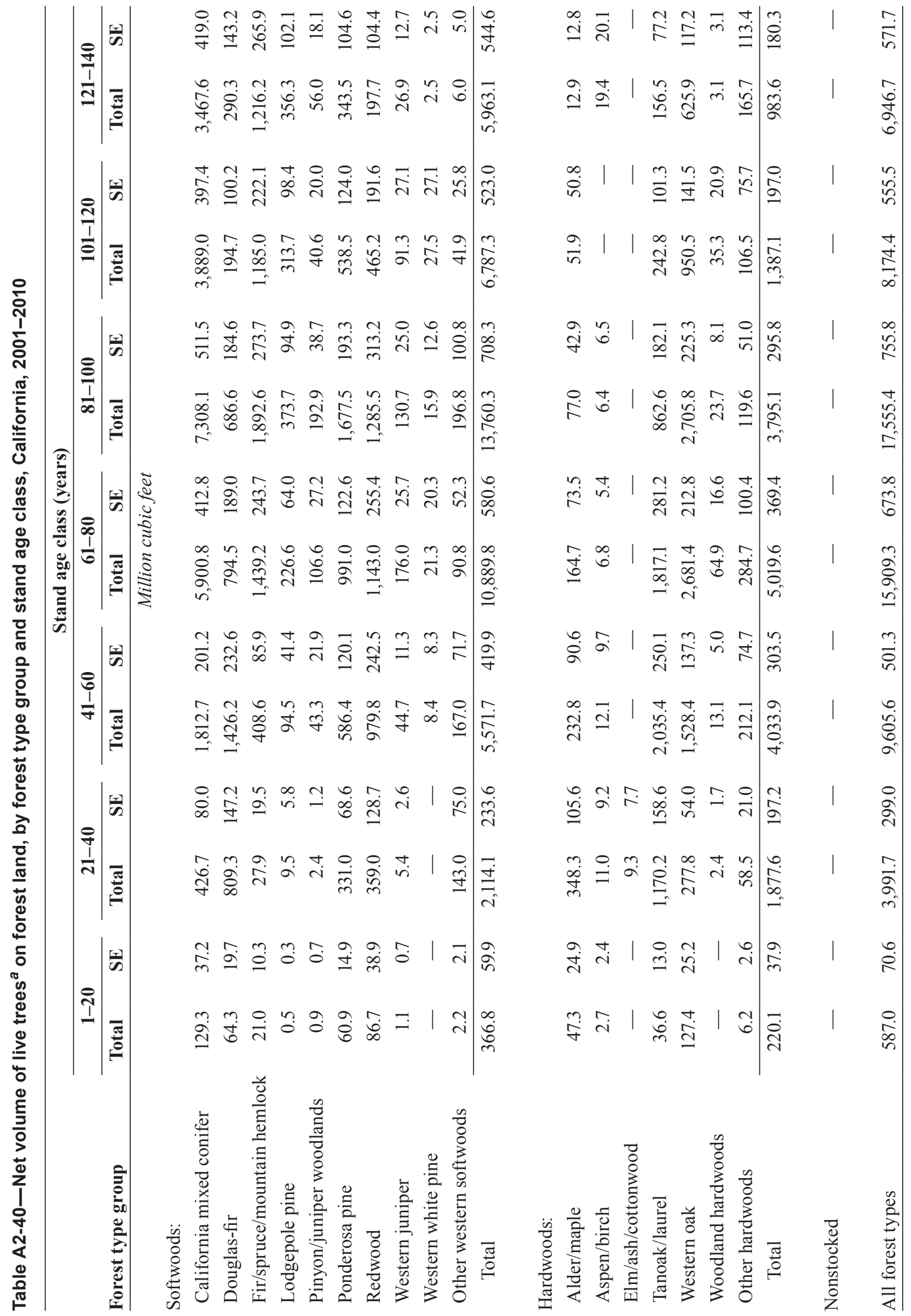




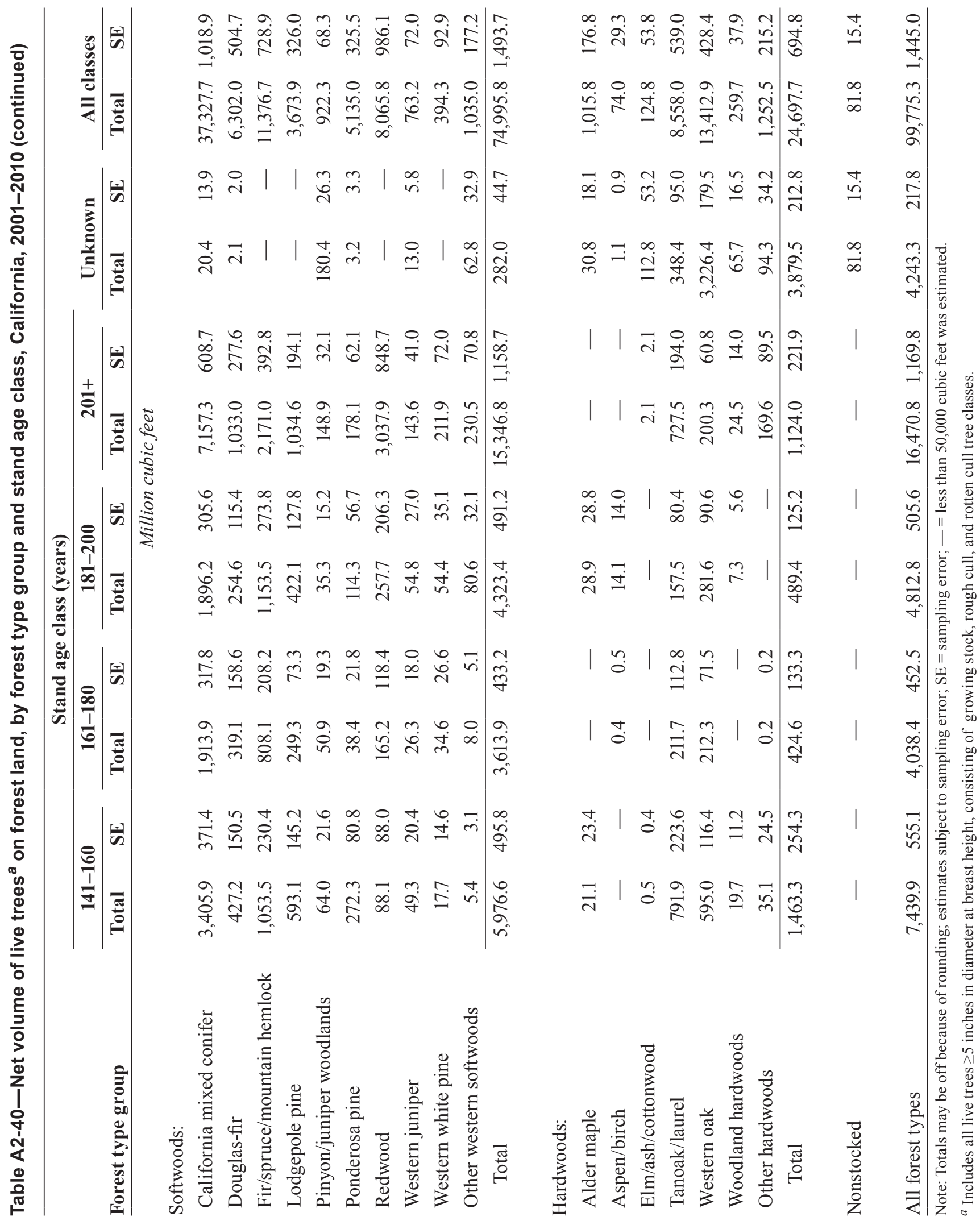




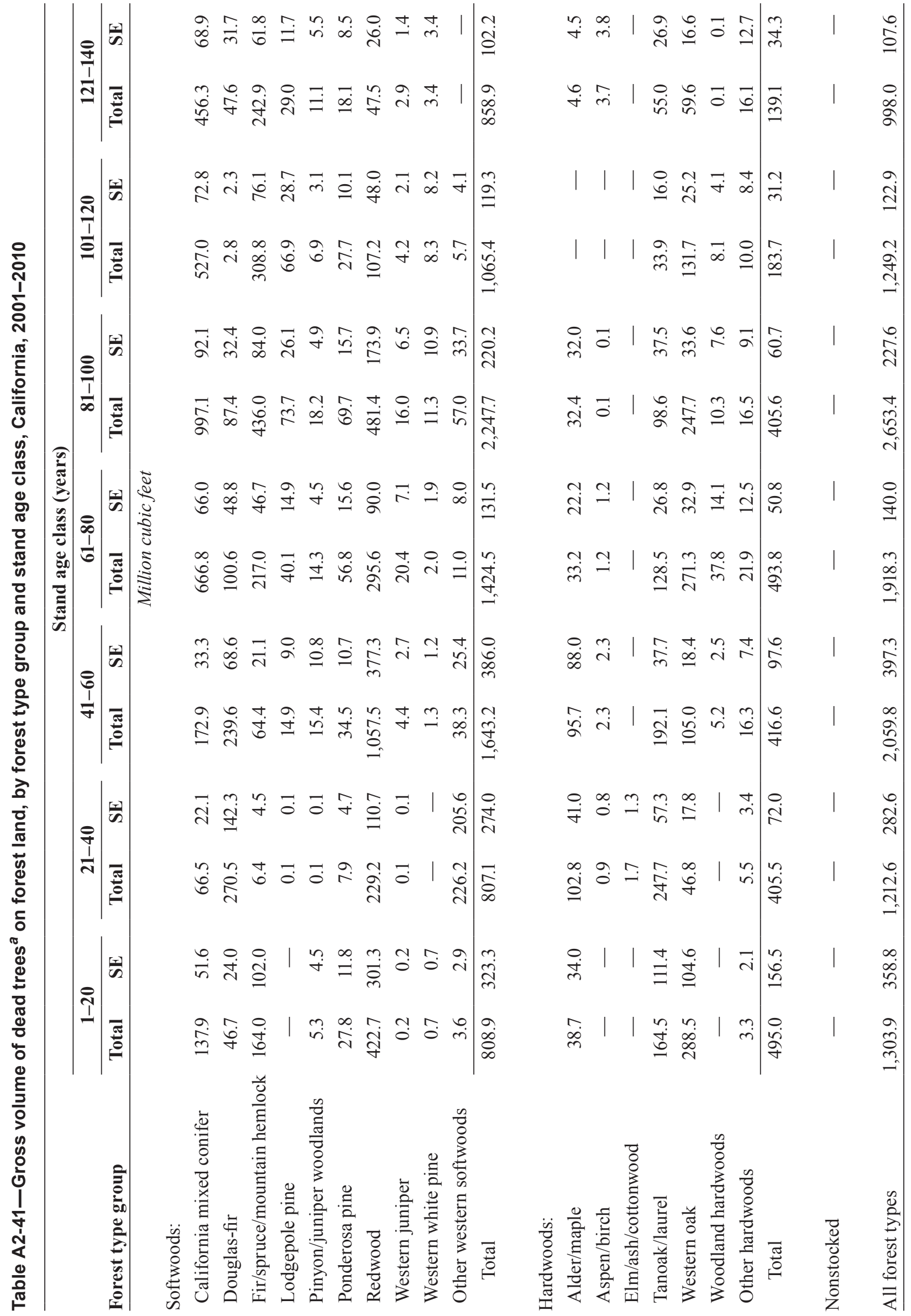




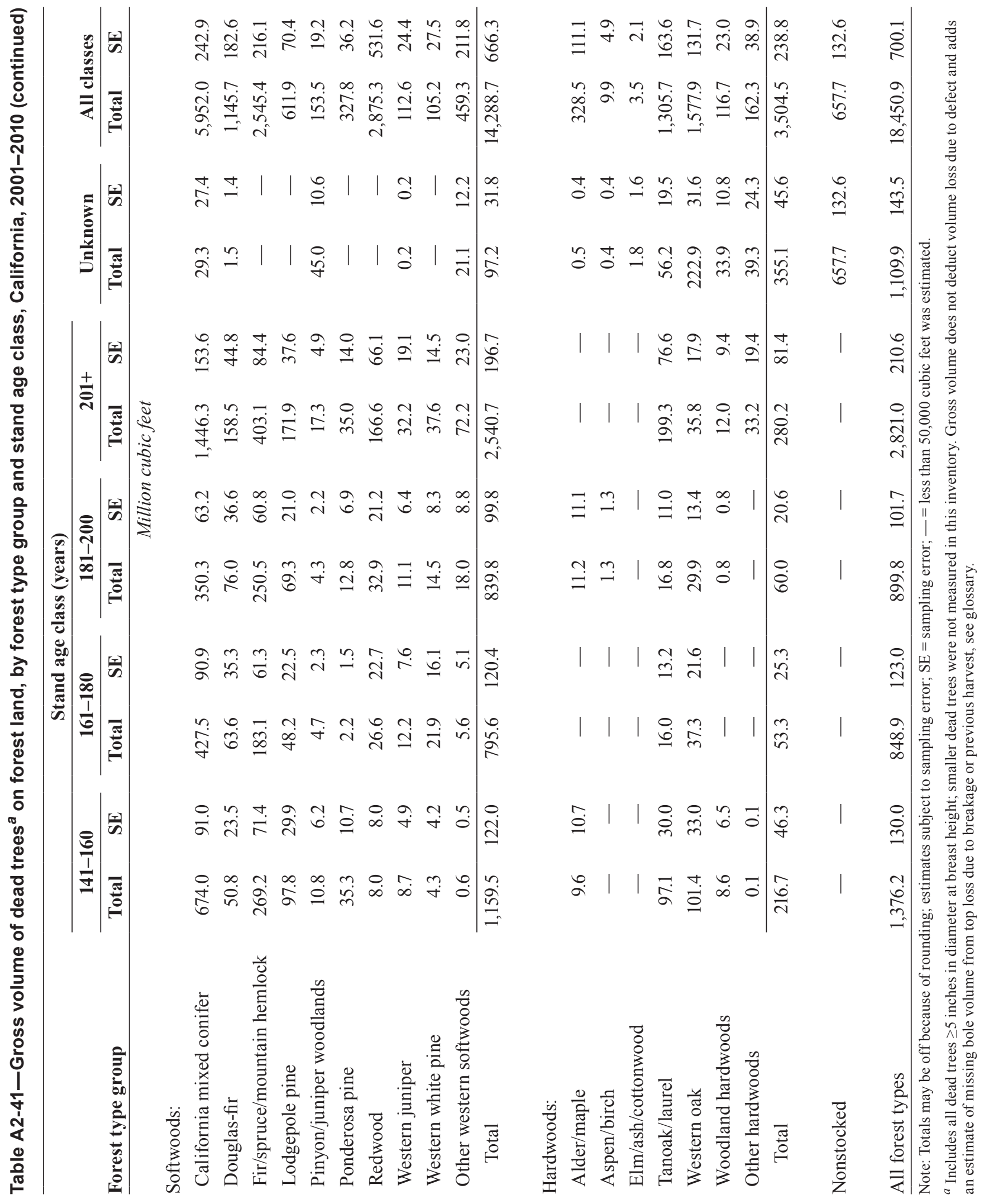




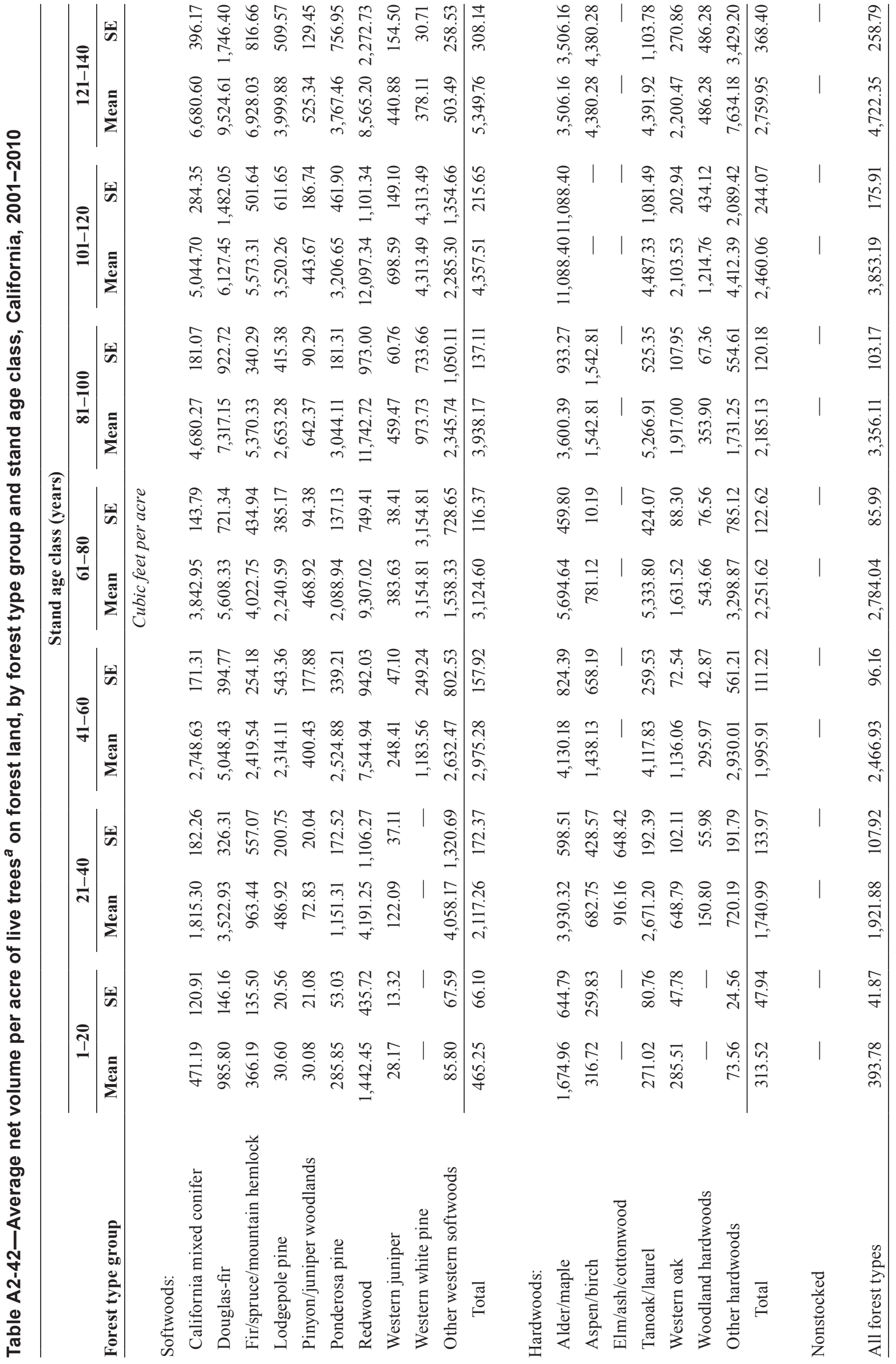




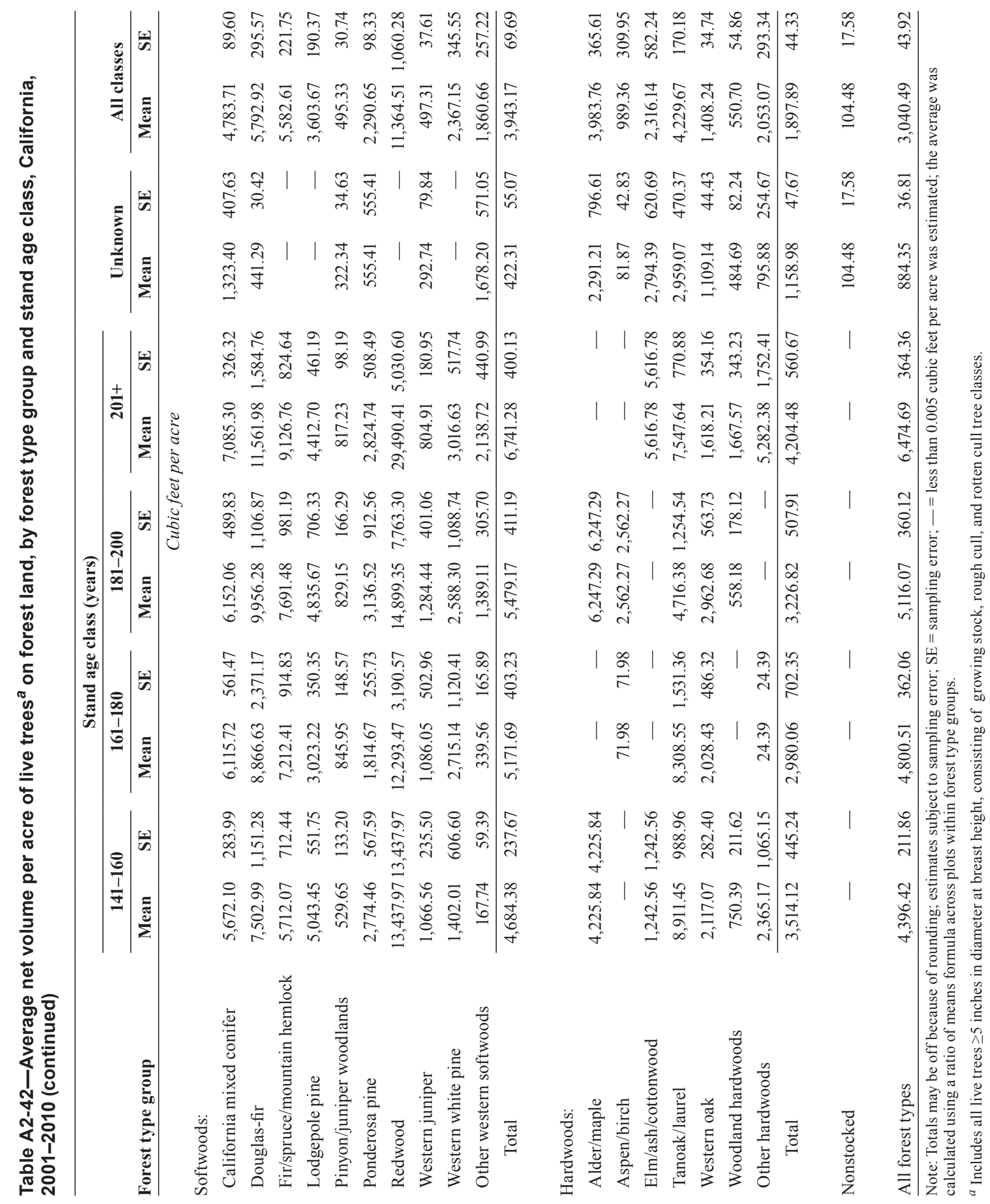




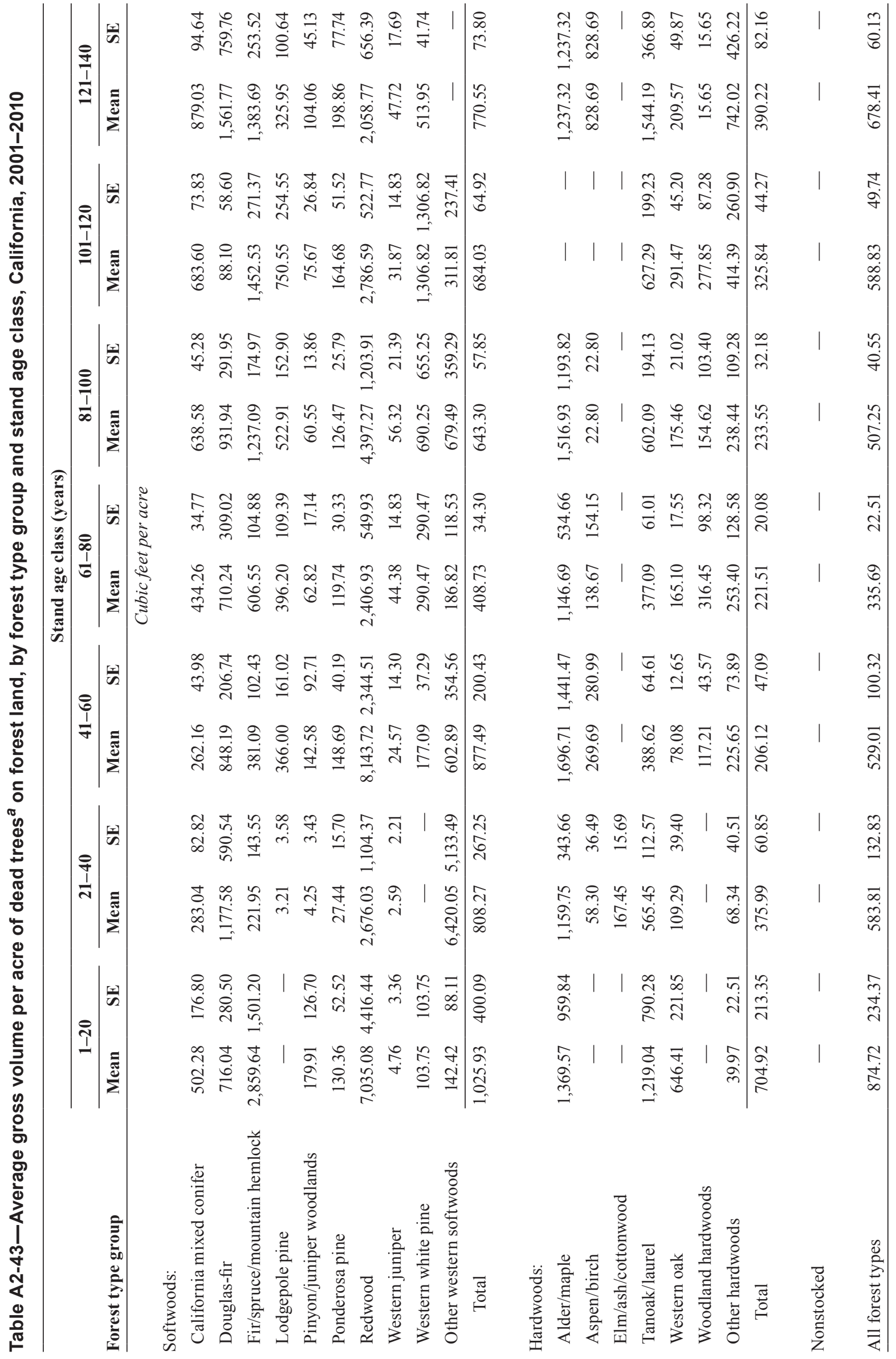




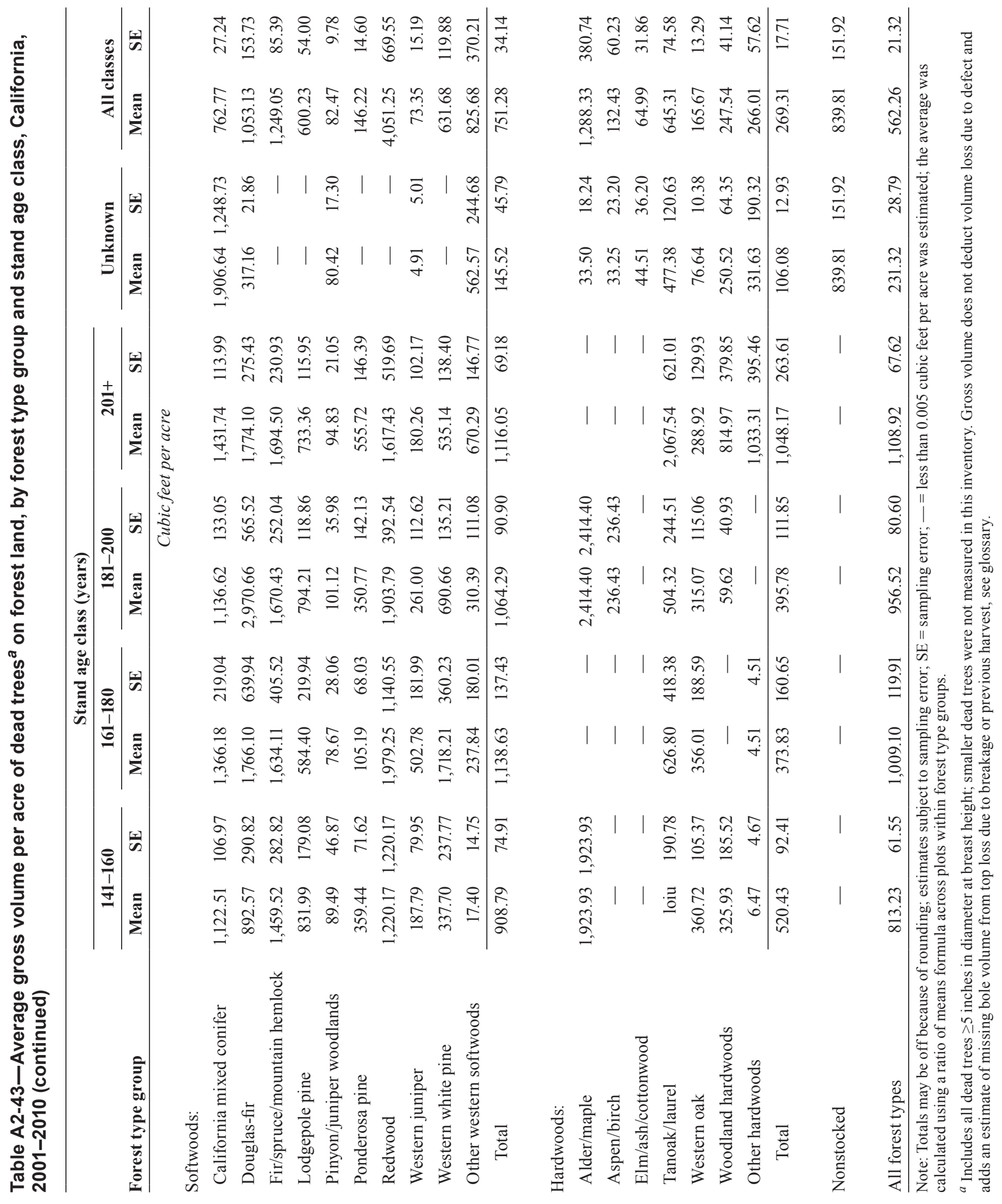


Table A2-44-Average net volume per acre of live trees ${ }^{a}$ on forest land, by forest type group and stand size class, California, 2001-2010

\begin{tabular}{|c|c|c|c|c|c|c|c|c|}
\hline \multirow[b]{3}{*}{ Forest type group } & \multicolumn{6}{|c|}{ Stand size class } & & \\
\hline & \multicolumn{2}{|c|}{$\begin{array}{l}\text { Large-diameter } \\
\text { stands }\end{array}$} & \multicolumn{2}{|c|}{$\begin{array}{c}\text { Medium-diameter } \\
\text { stands }\end{array}$} & \multicolumn{2}{|c|}{$\begin{array}{c}\text { Small-diameter } \\
\text { stands }\end{array}$} & \multicolumn{2}{|c|}{ All classes } \\
\hline & Mean & SE & Mean & SE & Mean & SE & Mean & SE \\
\hline & \multicolumn{8}{|c|}{ Cubic feet per acre } \\
\hline \multicolumn{9}{|l|}{ Softwoods: } \\
\hline California mixed conifer & $5,087.79$ & 91.78 & $1,145.33$ & 150.09 & 377.89 & 56.11 & $4,783.71$ & 89.60 \\
\hline Douglas-fir & $6,485.15$ & 308.97 & $1,899.38$ & 333.53 & 891.43 & 238.75 & $5,792.92$ & 295.57 \\
\hline Fir/spruce/mountain hemlock & $5,949.07$ & 226.47 & $1,009.45$ & 238.03 & 418.03 & 82.23 & $5,582.61$ & 221.75 \\
\hline Lodgepole pine & $3,846.18$ & 198.87 & $1,822.19$ & 430.59 & 139.53 & 56.79 & $3,603.67$ & 190.37 \\
\hline Pinyon/juniper woodlands & 581.86 & 35.36 & 116.90 & 33.12 & 74.40 & 20.45 & 495.33 & 30.74 \\
\hline Ponderosa pine & $2,624.49$ & 105.53 & 493.06 & 75.35 & 161.18 & 47.42 & $2,290.65$ & 98.33 \\
\hline Redwood & $12,169.43$ & $1,118.13$ & 962.56 & 273.79 & 503.88 & 131.85 & $11,364.51$ & $1,060.28$ \\
\hline Western juniper & 570.81 & 43.11 & 206.55 & 56.88 & 68.75 & 28.86 & 497.31 & 37.61 \\
\hline Western white pine & $2,949.81$ & 368.59 & 213.40 & 43.73 & 496.02 & 214.73 & $2,367.15$ & 345.55 \\
\hline Other western softwoods & $2,672.86$ & 342.82 & 370.54 & 103.81 & 81.93 & 28.12 & $1,860.66$ & 257.22 \\
\hline Total & $4,374.49$ & 76.02 & 663.28 & 66.28 & 292.72 & 31.90 & $3,943.17$ & 69.69 \\
\hline
\end{tabular}

Hardwoods:

\begin{tabular}{|c|c|c|c|c|c|c|c|c|}
\hline Alder/maple & $5,192.83$ & 391.98 & $2,674.72$ & 493.12 & 573.14 & 221.43 & $3,983.76$ & 365.61 \\
\hline Aspen/birch & $2,832.62$ & 644.08 & $1,490.17$ & 385.74 & 248.57 & 103.80 & 989.36 & 309.95 \\
\hline Elm/ash/cottonwood & $2,841.91$ & 582.22 & 187.59 & 173.41 & 314.76 & 31.46 & $2,316.14$ & 582.24 \\
\hline Tanoak/laurel & $5,506.03$ & 205.61 & $2,582.35$ & 163.39 & 540.77 & 115.73 & $4,229.67$ & 170.18 \\
\hline Western oak & $1,967.38$ & 55.13 & $1,038.08$ & 40.36 & 262.44 & 24.50 & $1,408.24$ & 34.74 \\
\hline Woodland hardwoods & 609.08 & 61.16 & 382.70 & 131.29 & 94.71 & 43.52 & 550.70 & 54.86 \\
\hline Other hardwoods & $4,733.97$ & 590.95 & $1,180.65$ & 244.55 & 143.13 & 29.40 & $2,053.07$ & 293.34 \\
\hline Total & $2,723.35$ & 71.37 & $1,231.99$ & 43.87 & 287.61 & 24.39 & $1,897.89$ & 44.33 \\
\hline Nonstocked & - & - & 一 & - & - & 一 & 104.48 & 17.58 \\
\hline All forest types & $3,891.17$ & 57.07 & $1,130.61$ & 38.63 & 289.59 & 19.40 & $3,040.49$ & 43.92 \\
\hline
\end{tabular}

Note: Totals may be off because of rounding; estimates subject to sampling error; SE = sampling error; - $=$ less than 0.005 cubic feet per acre was estimated; the average was calculated using a ratio of means formula across plots within forest type groups.

${ }^{a}$ Includes all live trees $\geq 5$ inches in diameter at breast height, consisting of growing stock, rough cull, and rotten cull tree classes. 
Table A2-45-Average gross cubic volume per acre of dead trees ${ }^{a}$ on forest land, by forest type group and stand size class, California, 2001-2010

\begin{tabular}{|c|c|c|c|c|c|c|c|c|}
\hline \multirow[b]{3}{*}{ Forest type group } & \multicolumn{6}{|c|}{ Stand size class } & & \\
\hline & \multicolumn{2}{|c|}{$\begin{array}{l}\text { Large-diameter } \\
\text { stands }\end{array}$} & \multicolumn{2}{|c|}{$\begin{array}{c}\text { Medium-diameter } \\
\text { stands }\end{array}$} & \multicolumn{2}{|c|}{$\begin{array}{c}\text { Small-diameter } \\
\text { stands }\end{array}$} & \multicolumn{2}{|c|}{ All classes } \\
\hline & Mean & SE & Mean & SE & Mean & SE & Mean & $\mathbf{S E}$ \\
\hline & \multicolumn{8}{|c|}{ Cubic feet per acre } \\
\hline \multicolumn{9}{|l|}{ Softwoods: } \\
\hline California mixed conifer & 793.66 & 28.63 & 354.86 & 110.35 & 334.50 & 98.91 & 762.77 & 27.24 \\
\hline Douglas-fir & $1,108.35$ & 173.91 & 134.31 & 69.93 & $1,040.11$ & 360.16 & $1,053.13$ & 153.73 \\
\hline Fir/spruce/mountain hemlock & $1,226.78$ & 77.14 & 930.69 & 580.18 & $1,680.95$ & 813.14 & $1,249.05$ & 85.39 \\
\hline Lodgepole pine & 623.53 & 54.77 & 693.00 & 436.16 & 13.39 & 14.16 & 600.23 & 54.00 \\
\hline Pinyon/juniper woodlands & 78.77 & 8.83 & 115.65 & 56.11 & 75.76 & 34.35 & 82.47 & 9.78 \\
\hline Ponderosa pine & 156.10 & 15.94 & 52.76 & 24.22 & 130.20 & 69.15 & 146.22 & 14.60 \\
\hline Redwood & $4,223.86$ & 713.55 & $2,470.74$ & 497.06 & $1,223.85$ & 646.18 & $4,051.25$ & 669.55 \\
\hline Western juniper & 86.95 & 18.09 & 10.27 & 8.16 & 2.19 & 1.27 & 73.35 & 15.19 \\
\hline Western white pine & 776.63 & 142.48 & 68.84 & 14.11 & 172.51 & 88.49 & 631.68 & 119.88 \\
\hline Other western softwoods & $1,156.86$ & 544.00 & 87.96 & 44.33 & 186.16 & 138.66 & 825.68 & 370.21 \\
\hline Total & 802.12 & 37.59 & 240.09 & 51.56 & 414.02 & 96.87 & 751.28 & 34.14 \\
\hline
\end{tabular}

Hardwoods:

\begin{tabular}{|c|c|c|c|c|c|c|c|c|}
\hline Alder/maple & $1,488.37$ & 559.34 & $1,235.65$ & 564.52 & 349.53 & 139.83 & $1,288.33$ & 380.74 \\
\hline Aspen/birch & 359.47 & 189.25 & 277.46 & 167.49 & 13.15 & 8.79 & 132.43 & 60.23 \\
\hline Elm/ash/cottonwood & 48.93 & 34.51 & 一 & - & 170.64 & 17.05 & 64.99 & 31.86 \\
\hline Tanoak/laurel & 679.20 & 61.95 & 317.39 & 68.88 & $1,056.10$ & 477.43 & 645.31 & 74.58 \\
\hline Western oak & 182.81 & 13.02 & 93.53 & 9.86 & 288.28 & 80.47 & 165.67 & 13.29 \\
\hline Woodland hardwoods & 237.64 & 37.95 & 418.55 & 206.19 & 一 & - & 247.54 & 41.14 \\
\hline Other hardwoods & 497.86 & 106.84 & 101.31 & 31.30 & 163.58 & 101.50 & 266.01 & 57.62 \\
\hline Total & 317.74 & 21.15 & 142.72 & 16.34 & 365.78 & 86.67 & 269.31 & 17.71 \\
\hline Nonstocked & - & - & - & - & - & - & 839.81 & 151.92 \\
\hline All forest types & 660.33 & 27.40 & 160.08 & 16.28 & 384.50 & 64.91 & 562.26 & 21.32 \\
\hline
\end{tabular}

Note: Totals may be off because of rounding; estimates subject to sampling error; $\mathrm{SE}=$ sampling error; — $=$ less than 0.005 cubic feet per acre was estimated; the average was calculated using a ratio of means formula across plots within forest type groups.

${ }^{a}$ Includes all dead trees $\geq 5$ inches in diameter at breast height; smaller dead trees were not measured in this inventory. Gross volume does not deduct volume loss due to defect and adds an estimate of missing bole volume from top loss due to breakage or previous harvest, see glossary. 


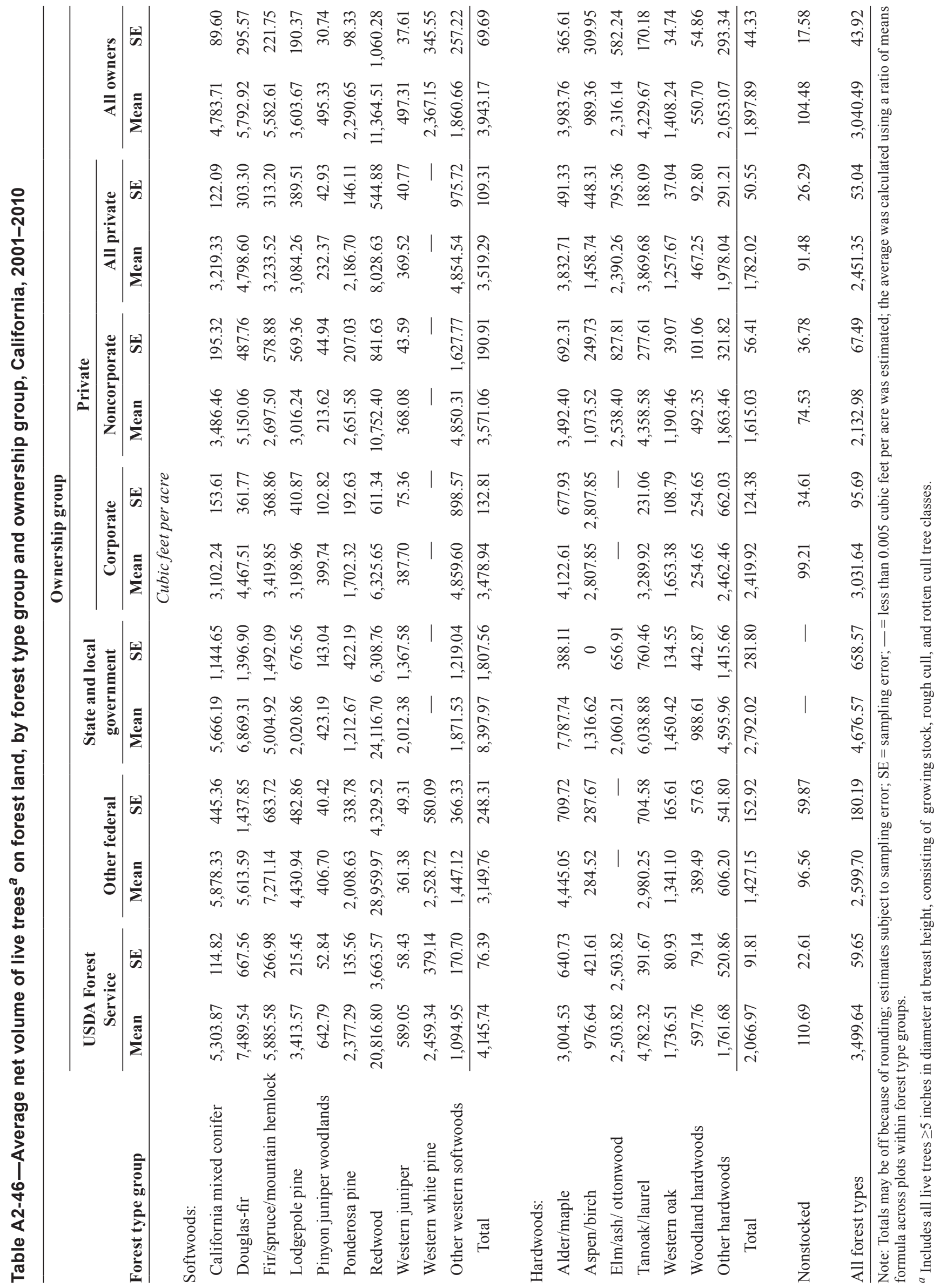




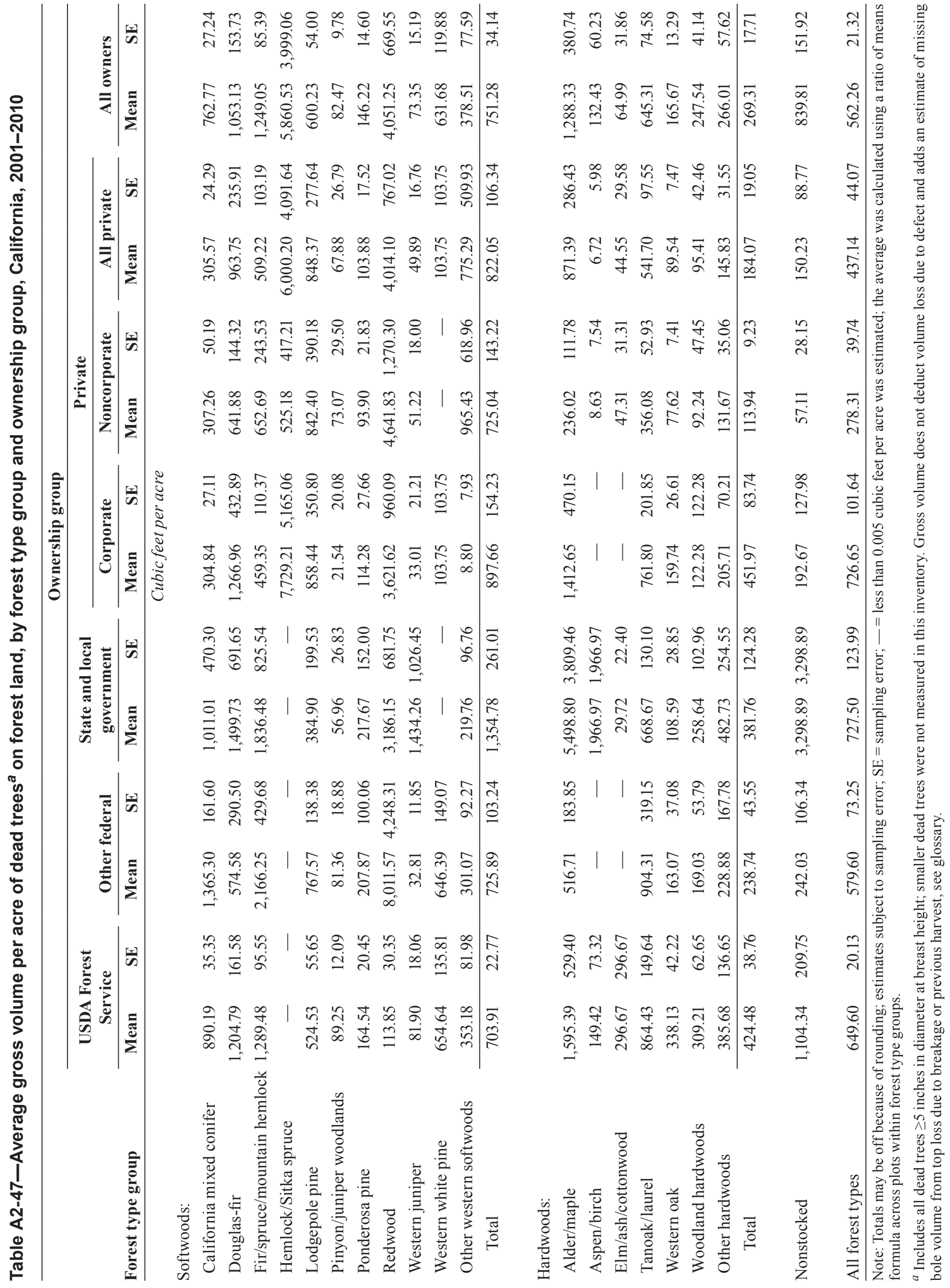




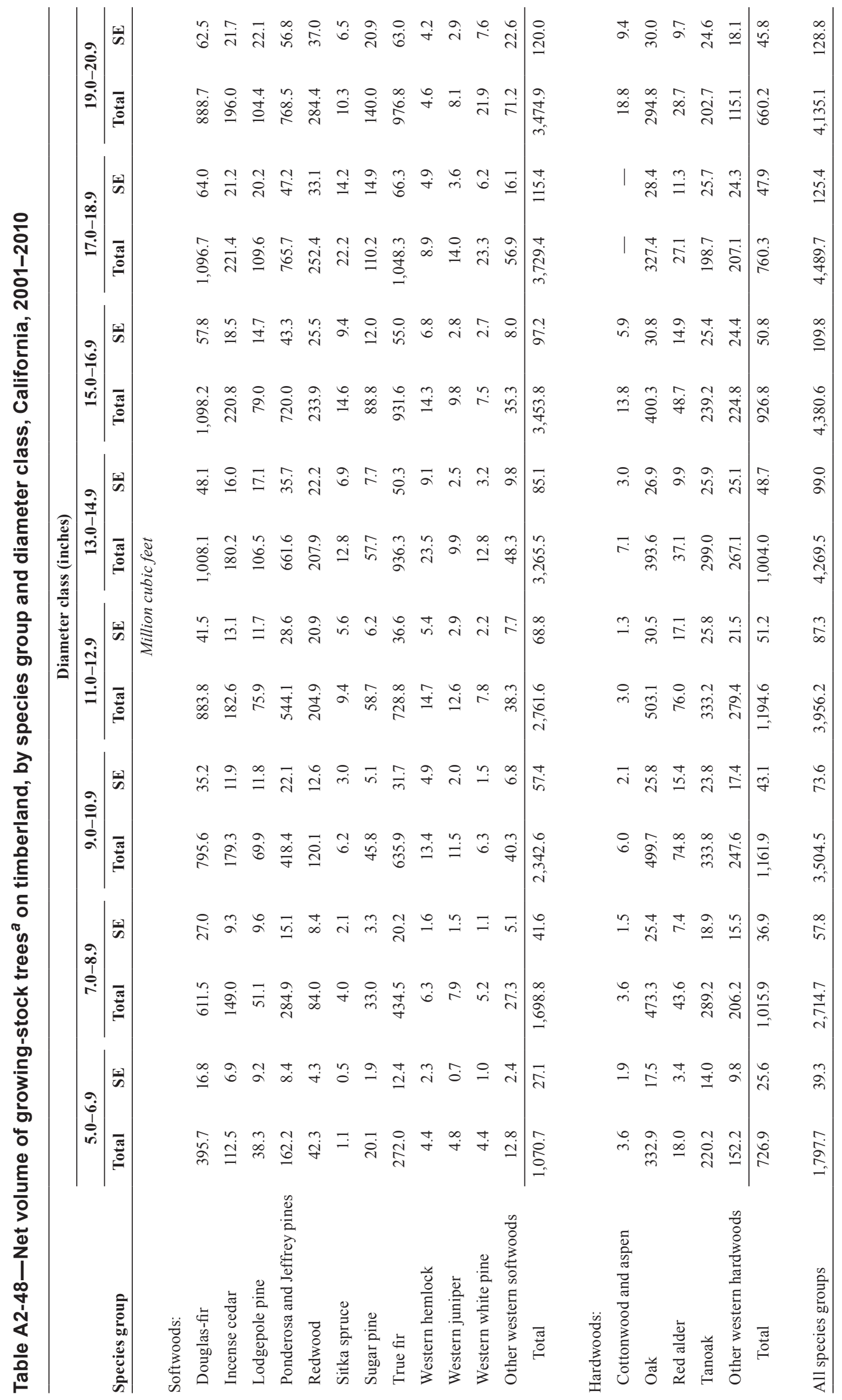




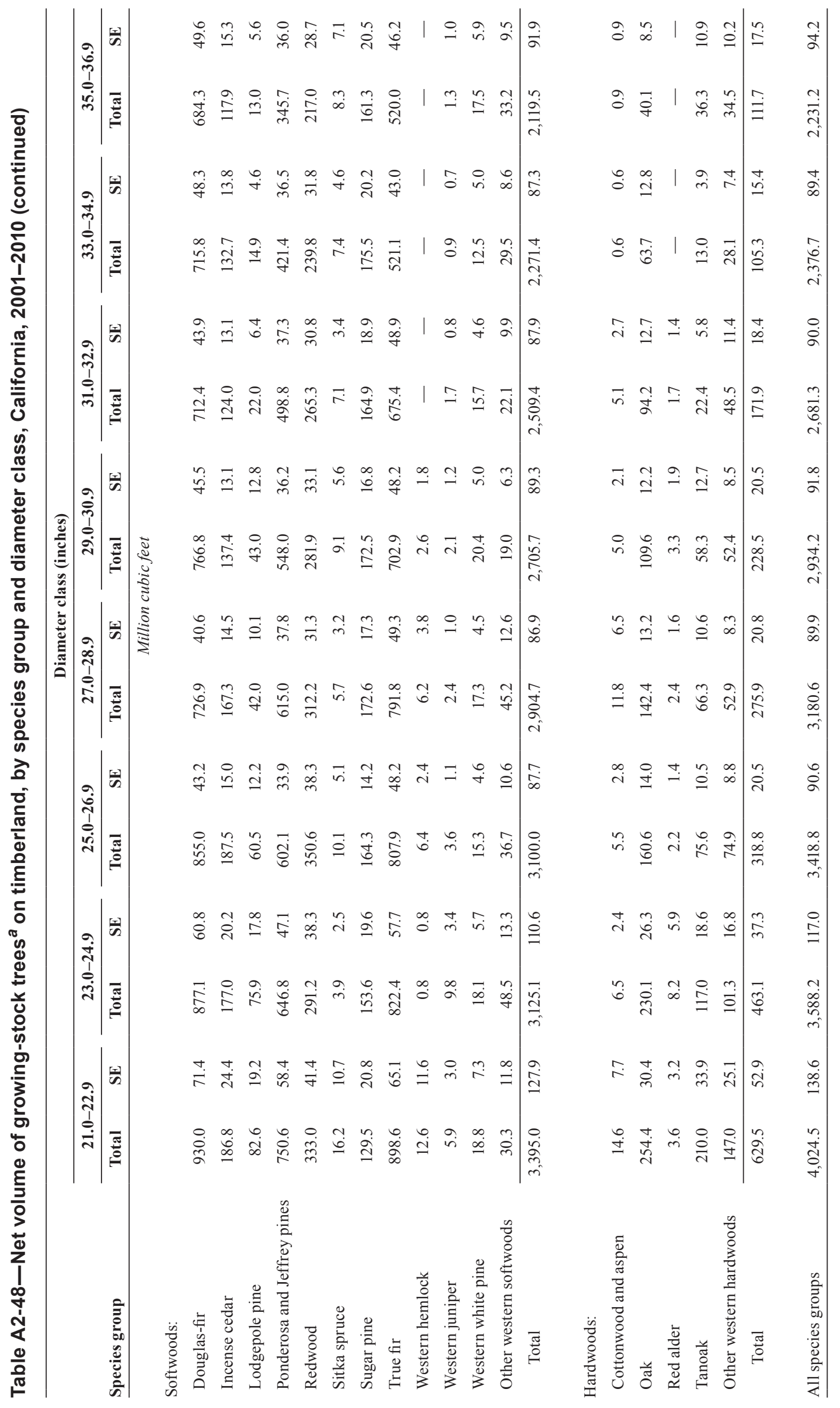




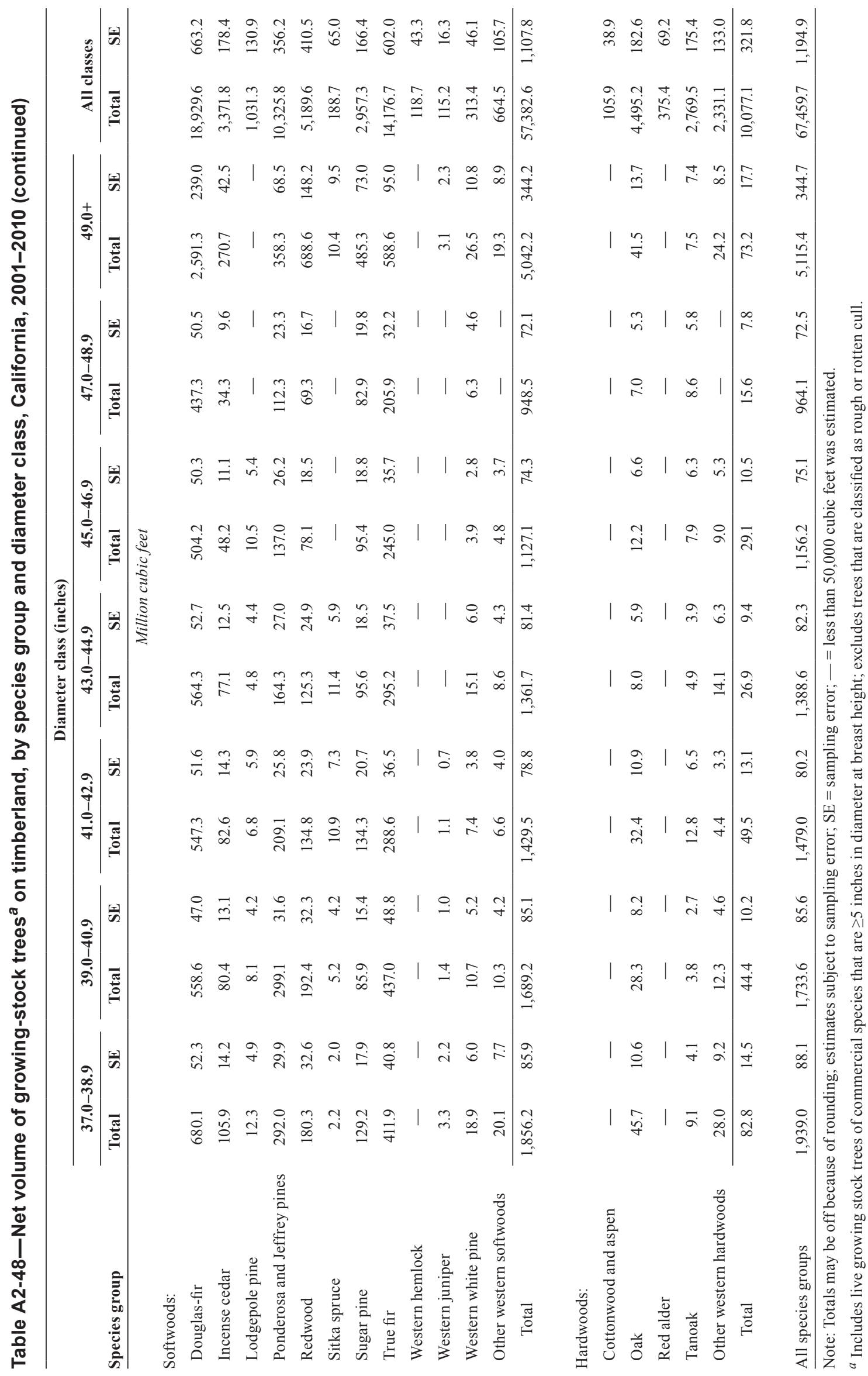




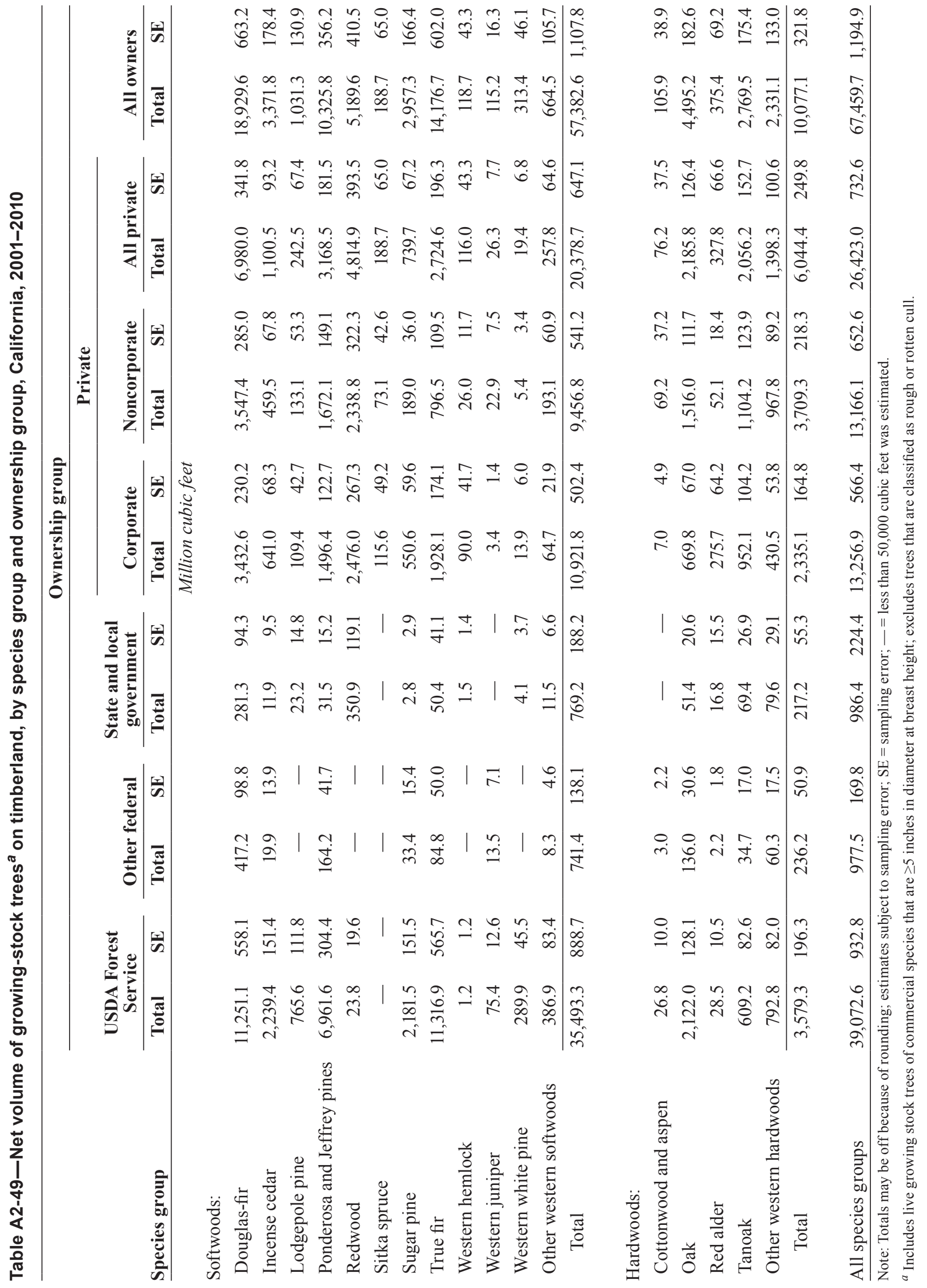




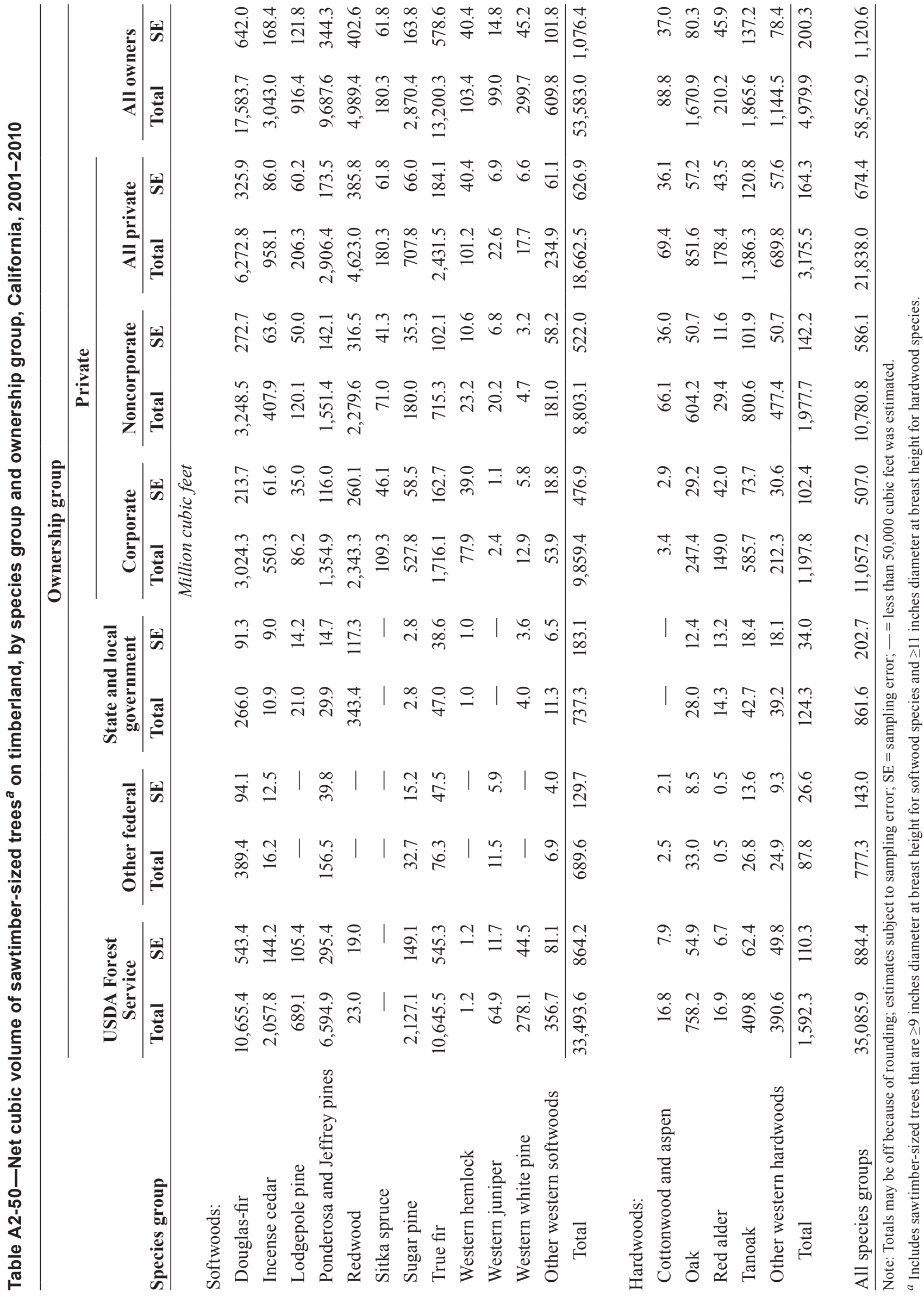


Table A2-51-Net board-foot volume (Scribner rule) of sawtimber trees ${ }^{a}$ on timberland, by county and ownership group, California, 2001-2010

\begin{tabular}{|c|c|c|c|c|c|c|c|c|c|c|}
\hline \multirow[b]{3}{*}{ County } & \multicolumn{8}{|c|}{ Ownership group } & & \\
\hline & \multicolumn{2}{|c|}{ National forest } & \multicolumn{2}{|c|}{ Other federal } & \multicolumn{2}{|c|}{$\begin{array}{l}\text { State and local } \\
\text { government }\end{array}$} & \multicolumn{2}{|c|}{ Private } & \multicolumn{2}{|c|}{ All owners } \\
\hline & Total & SE & Total & SE & Total & SE & Total & SE & Total & SE \\
\hline & \multicolumn{10}{|c|}{ Million board feet, Scribner rule } \\
\hline Alameda & - & - & - & - & 16.4 & 14.4 & 16.6 & 19.0 & 33.0 & 23.9 \\
\hline Alpine & $1,552.0$ & 470.6 & - & - & - & - & 77.5 & 70.5 & $1,629.5$ & 475.8 \\
\hline Amador & 911.0 & 439.9 & 35.0 & 35.6 & - & - & $1,025.6$ & 435.3 & $1,971.5$ & 680.0 \\
\hline Butte & $5,003.3$ & $1,171.4$ & 170.3 & 173.5 & - & 一 & $2,727.0$ & 580.0 & $7,900.5$ & $1,318.6$ \\
\hline Calaveras & $1,465.1$ & 620.8 & - & - & - & - & $2,913.0$ & 708.2 & $4,378.1$ & 943.4 \\
\hline Colusa & 173.9 & 126.4 & - & - & - & - & 212.0 & 152.5 & 385.9 & 198.1 \\
\hline Del Norte & $7,654.5$ & $1,424.9$ & - & - & 384.0 & 249.2 & $3,023.5$ & 649.5 & $11,062.0$ & $1,585.1$ \\
\hline El Dorado & $11,940.3$ & $1,695.6$ & 207.8 & 174.8 & 0.2 & 0.2 & $2,633.4$ & 609.1 & $14,781.7$ & $1,809.5$ \\
\hline Fresno & $7,451.1$ & $1,365.8$ & - & - & - & - & 346.1 & 182.1 & $7,797.2$ & $1,377.9$ \\
\hline Glenn & $1,649.2$ & 644.4 & - & - & - & - & 149.2 & 129.7 & $1,798.5$ & 657.3 \\
\hline Humboldt & $9,322.2$ & $1,823.4$ & 847.1 & 412.2 & 128.9 & 131.3 & $25,631.9$ & $2,313.2$ & $35,930.1$ & $2,960.9$ \\
\hline Inyo & 158.6 & 131.4 & - & - & 29.5 & 26.9 & - & - & 188.1 & 134.1 \\
\hline Kern & $1,137.3$ & 320.4 & 4.0 & 3.6 & - & - & 137.2 & 69.2 & $1,278.6$ & 328.9 \\
\hline Lake & $1,869.0$ & 533.3 & 132.9 & 110.6 & 70.2 & 71.5 & 459.7 & 185.2 & $2,531.8$ & 579.5 \\
\hline Lassen & $5,469.2$ & 799.7 & 197.7 & 90.1 & - & - & $2,408.5$ & 392.7 & $8,075.3$ & 896.4 \\
\hline Los Angeles & 332.4 & 147.0 & - & - & - & - & - & - & 332.4 & 147.0 \\
\hline Madera & $7,073.8$ & $1,461.1$ & - & - & - & - & 369.6 & 245.7 & $7,443.4$ & $1,481.6$ \\
\hline Marin & - & - & - & - & 317.6 & 275.1 & 702.4 & 615.4 & $1,019.9$ & 674.1 \\
\hline Mariposa & $1,282.4$ & 572.0 & 8.3 & 8.7 & - & - & 747.3 & 323.1 & $2,037.9$ & 657.0 \\
\hline Mendocino & $2,010.9$ & 586.2 & 101.5 & 80.2 & $2,043.2$ & 906.1 & $20,613.4$ & $2,045.5$ & $24,769.0$ & $2,322.2$ \\
\hline Modoc & $3,596.4$ & 572.9 & 75.2 & 73.9 & - & - & $1,113.5$ & 225.8 & $4,785.1$ & 623.2 \\
\hline Mono & $1,674.2$ & 371.2 & 16.1 & 16.4 & - & - & 71.7 & 76.3 & $1,762.1$ & 379.3 \\
\hline Monterey & 68.7 & 65.2 & - & - & - & - & 919.7 & 605.9 & 988.4 & 609.4 \\
\hline Napa & - & - & - & - & - & - & $1,065.0$ & 390.0 & $1,065.0$ & 390.0 \\
\hline Nevada & $3,491.8$ & 880.5 & 40.4 & 36.1 & 31.0 & 27.7 & $2,553.5$ & 613.9 & $6,116.7$ & $1,073.4$ \\
\hline Placer & $7,336.5$ & $1,502.8$ & 143.2 & 89.9 & 146.6 & 111.6 & $2,203.3$ & 580.5 & $9,829.6$ & $1,611.8$ \\
\hline Plumas & $19,634.8$ & $1,935.6$ & 41.6 & 29.9 & - & - & $4,993.7$ & 812.1 & $24,670.1$ & $2,092.1$ \\
\hline Riverside & 80.0 & 79.1 & - & - & - & - & 196.6 & 134.9 & 276.6 & 156.4 \\
\hline San Benito & - & - & - & - & - & - & 59.9 & 60.8 & 59.9 & 60.8 \\
\hline San Bernardino & 858.2 & 300.7 & - & - & - & - & 200.7 & 145.5 & $1,058.9$ & 334.0 \\
\hline San Diego & 0.5 & 0.5 & - & - & - & - & 4.4 & 4.0 & 4.9 & 4.0 \\
\hline San Joaquin & - & - & - & - & - & - & 349.6 & 215.6 & 349.6 & 215.6 \\
\hline $\begin{array}{r}\text { San Luis } \\
\text { Obispo }\end{array}$ & - & - & - & - & - & - & 6.6 & 5.8 & 6.6 & 5.8 \\
\hline San Mateo & - & - & - & - & 9.1 & 8.1 & $1,370.7$ & 683.5 & $1,379.7$ & 683.6 \\
\hline
\end{tabular}


Table A2-51-Net board-foot volume (Scribner rule) of sawtimber trees ${ }^{a}$ on timberland, by county and ownership group, California, 2001-2010 (continued)

\begin{tabular}{|c|c|c|c|c|c|c|c|c|c|c|}
\hline \multirow[b]{3}{*}{ County } & \multicolumn{8}{|c|}{ Ownership group } & & \\
\hline & \multicolumn{2}{|c|}{ National forest } & \multicolumn{2}{|c|}{ Other federal } & \multicolumn{2}{|c|}{$\begin{array}{l}\text { State and local } \\
\text { government }\end{array}$} & \multicolumn{2}{|c|}{ Private } & \multicolumn{2}{|c|}{ All owners } \\
\hline & Total & SE & Total & SE & Total & SE & Total & SE & Total & SE \\
\hline & \multicolumn{10}{|c|}{ Million board feet, Scribner rule } \\
\hline Santa Barbara & 69.6 & 58.8 & - & - & - & - & 20.2 & 18.2 & 89.8 & 61.6 \\
\hline Santa Clara & - & - & - & - & 418.9 & 344.4 & 160.7 & 115.2 & 579.6 & 359.6 \\
\hline Santa Cruz & - & - & - & - & 407.1 & 366.1 & $4,446.3$ & $1,032.5$ & $4,853.3$ & $1,111.0$ \\
\hline Shasta & $9,256.9$ & $1,304.3$ & 811.9 & 399.5 & 534.0 & 367.7 & $7,743.4$ & 910.3 & $18,346.2$ & $1,693.5$ \\
\hline Sierra & $8,300.3$ & $1,457.6$ & - & - & - & - & $1,235.0$ & 446.0 & $9,535.2$ & $1,521.6$ \\
\hline Siskiyou & $34,369.9$ & $2,746.6$ & 70.5 & 67.4 & 132.9 & 123.5 & $6,603.8$ & 820.8 & $41,177.0$ & $2,872.9$ \\
\hline Sonoma & - & - & 11.9 & 13.0 & 94.5 & 89.2 & $7,738.9$ & $1,804.9$ & $7,845.3$ & $1,807.1$ \\
\hline Tehama & $5,952.7$ & $1,276.5$ & - & - & - & - & $2,770.3$ & 606.4 & $8,722.9$ & $1,427.2$ \\
\hline Trinity & $23,127.0$ & $2,514.3$ & $1,241.5$ & 463.9 & - & - & $3,924.6$ & 673.1 & $28,293.1$ & $2,640.9$ \\
\hline Tulare & $3,288.9$ & 803.6 & - & - & - & - & 363.6 & 219.7 & $3,652.5$ & 833.1 \\
\hline Tuolumne & $9,068.1$ & $1,513.0$ & - & - & - & - & $1,539.2$ & 458.9 & $10,607.4$ & $1,582.3$ \\
\hline Ventura & 27.4 & 21.8 & - & - & - & - & - & - & 27.4 & 21.8 \\
\hline Yuba & $2,247.8$ & 938.3 & - & - & 137.7 & 135.6 & $1,055.4$ & 466.7 & $3,440.8$ & $1,054.0$ \\
\hline
\end{tabular}

$\begin{array}{llllllllll}\text { All counties } 198,905.6 & 5,448.1 & 4,156.8 & 797.6 & 4,901.7 & 1,185.7 & 116,904.1 & 3,990.5 & 324,868.2 & 6,807.1\end{array}$

Note: Totals may be off because of rounding; estimates subject to sampling error; $\mathrm{SE}=$ sampling error; — = less than 50,000 board feet was estimated.

${ }^{a}$ Includes sawtimber-sized trees that are $\geq 9$ inches diameter at breast height for softwood species and $\geq 11$ inches diameter at breast height for hardwood species. 


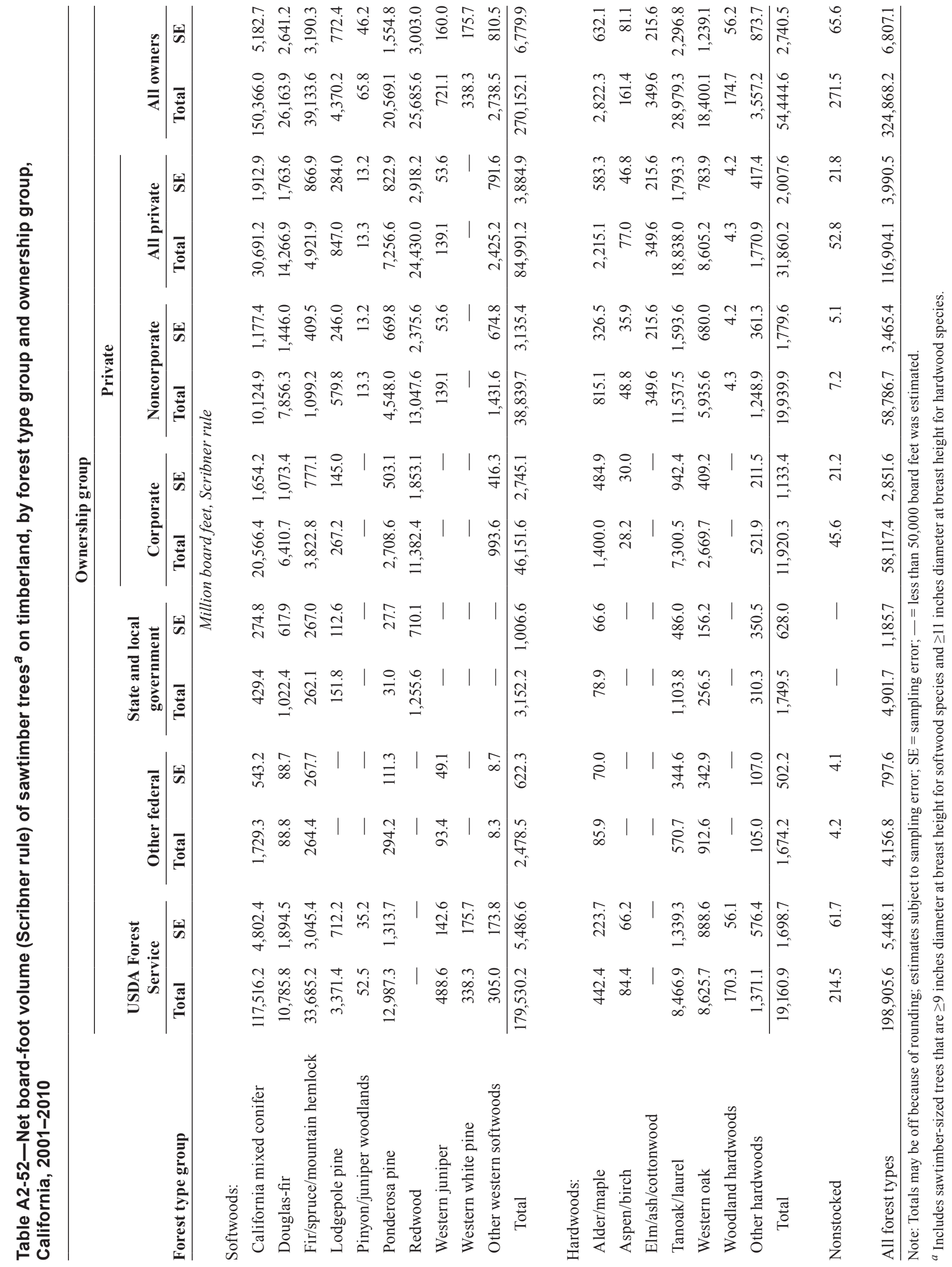


Table A2-53-Net board-foot volume (Scribner rule) of sawtimber trees ${ }^{a}$ on timberland, by forest type group and stand size class, California, 2000-2010

\begin{tabular}{|c|c|c|c|c|c|c|c|c|}
\hline \multirow[b]{3}{*}{ Forest type group } & \multicolumn{8}{|c|}{ Stand size class } \\
\hline & \multicolumn{2}{|c|}{$\begin{array}{l}\text { Large-diameter } \\
\text { stands }\end{array}$} & \multicolumn{2}{|c|}{$\begin{array}{c}\text { Medium-diameter } \\
\text { stands }\end{array}$} & \multicolumn{2}{|c|}{$\begin{array}{c}\text { Small-diameter } \\
\text { stands }\end{array}$} & \multicolumn{2}{|c|}{ All classes } \\
\hline & Total & SE & Total & SE & Total & SE & Total & SE \\
\hline & \multicolumn{8}{|c|}{ Million board feet, Scribner rule } \\
\hline \multicolumn{9}{|l|}{ Softwoods: } \\
\hline California mixed conifer & $149,435.2$ & $5,185.9$ & 420.0 & 99.0 & 510.9 & 119.1 & $150,366.0$ & $5,182.7$ \\
\hline Douglas-fir & $25,557.7$ & $2,640.9$ & 231.0 & 85.0 & 375.2 & 182.2 & $26,163.9$ & $2,641.2$ \\
\hline Fir/spruce/mountain hemlock & $38,961.2$ & $3,189.4$ & 49.0 & 27.5 & 123.4 & 52.9 & $39,133.6$ & $3,190.3$ \\
\hline Lodgepole pine & $4,181.2$ & 766.2 & 184.7 & 97.2 & 4.3 & 4.3 & $4,370.2$ & 772.4 \\
\hline Pinyon/juniper woodlands & 65.8 & 46.2 & - & - & - & - & 65.8 & 46.2 \\
\hline Ponderosa pine & $20,360.4$ & $1,554.9$ & 130.7 & 38.5 & 78.0 & 33.3 & $20,569.1$ & $1,554.8$ \\
\hline Redwood & $25,615.4$ & $3,003.6$ & 34.3 & 21.2 & 35.8 & 22.4 & $25,685.6$ & $3,003.0$ \\
\hline Western juniper & 693.6 & 158.9 & 7.8 & 7.4 & 19.7 & 17.8 & 721.1 & 160.0 \\
\hline Western white pine & 290.3 & 170.8 & 2.1 & 2.1 & 45.8 & 40.9 & 338.3 & 175.7 \\
\hline Other western softwoods & $2,738.5$ & 810.5 & - & - & - & - & $2,738.5$ & 810.5 \\
\hline Total & $267,899.3$ & $6,789.6$ & $1,059.6$ & 170.7 & $1,193.1$ & 231.0 & $270,152.1$ & $6,779.9$ \\
\hline
\end{tabular}

Hardwoods:

\begin{tabular}{|c|c|c|c|c|c|c|c|c|}
\hline Alder/maple & $2,484.6$ & 615.3 & 279.9 & 138.9 & 57.7 & 40.4 & $2,822.3$ & 632.1 \\
\hline Aspen/birch & 94.5 & 70.6 & 48.9 & 35.9 & 18.0 & 17.4 & 161.4 & 81.1 \\
\hline Elm/ash/cottonwood & 349.6 & 215.6 & - & - & - & - & 349.6 & 215.6 \\
\hline Tanoak/laurel & $26,070.9$ & $2,289.2$ & $2,446.8$ & 393.1 & 461.6 & 154.1 & $28,979.3$ & $2,296.8$ \\
\hline Western oak & $15,583.2$ & $1,191.8$ & $2,367.8$ & 354.6 & 449.0 & 125.8 & $18,400.1$ & $1,239.1$ \\
\hline Woodland hardwoods & 159.6 & 55.5 & 9.5 & 7.0 & 5.5 & 6.0 & 174.7 & 56.2 \\
\hline Other hardwoods & $3,317.8$ & 868.6 & 174.3 & 92.7 & 65.1 & 28.7 & $3,557.2$ & 873.7 \\
\hline Total & $48,060.3$ & $2,726.0$ & $5,327.3$ & 553.6 & $1,057.1$ & 205.5 & $54,444.6$ & $2,740.5$ \\
\hline Nonstocked & - & - & - & - & - & - & 271.5 & 65.6 \\
\hline All forest types & $315,959.7$ & $6,868.7$ & $6,386.8$ & 577.6 & $2,250.2$ & 308.3 & $324,868.2$ & $6,807.1$ \\
\hline
\end{tabular}




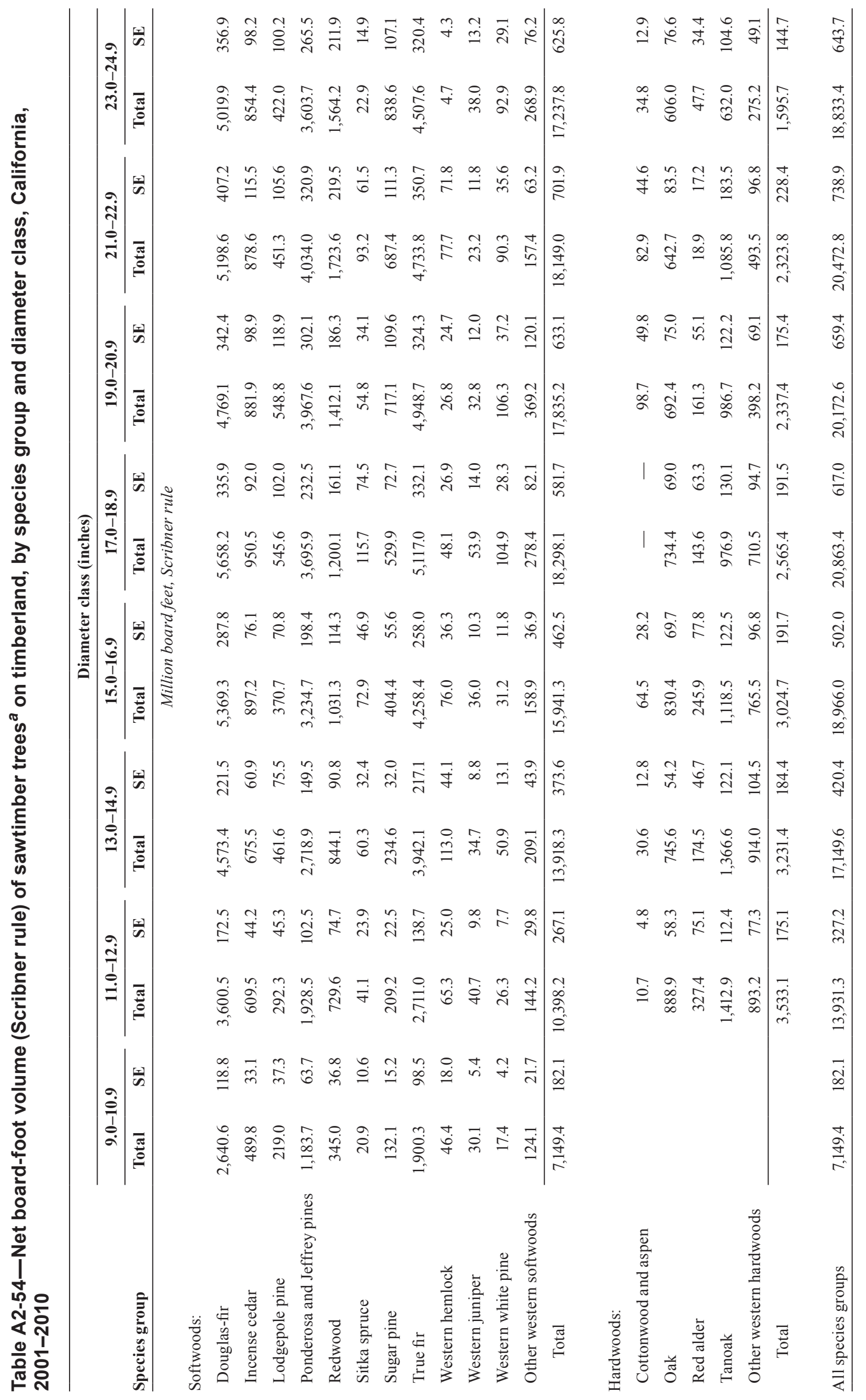




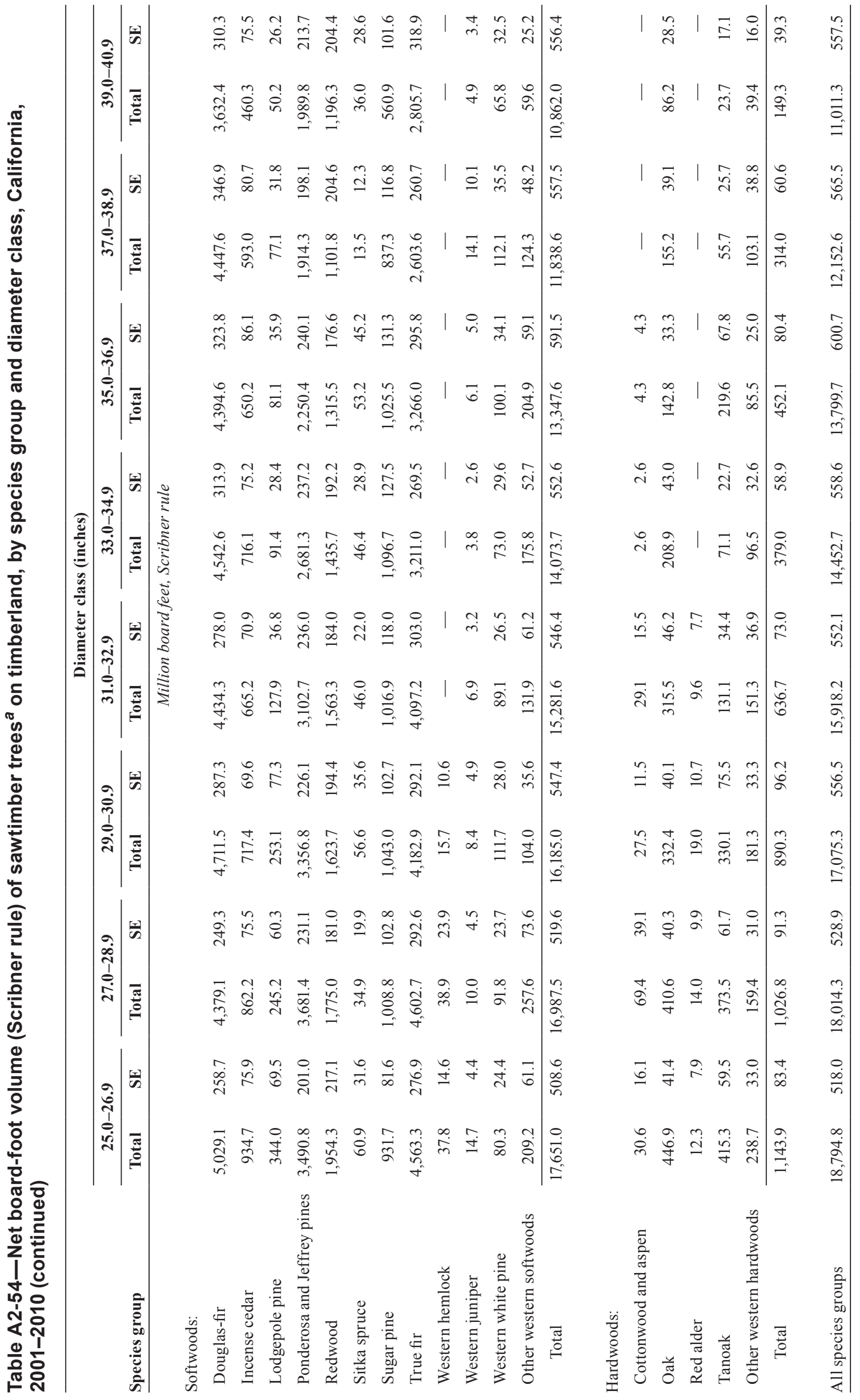




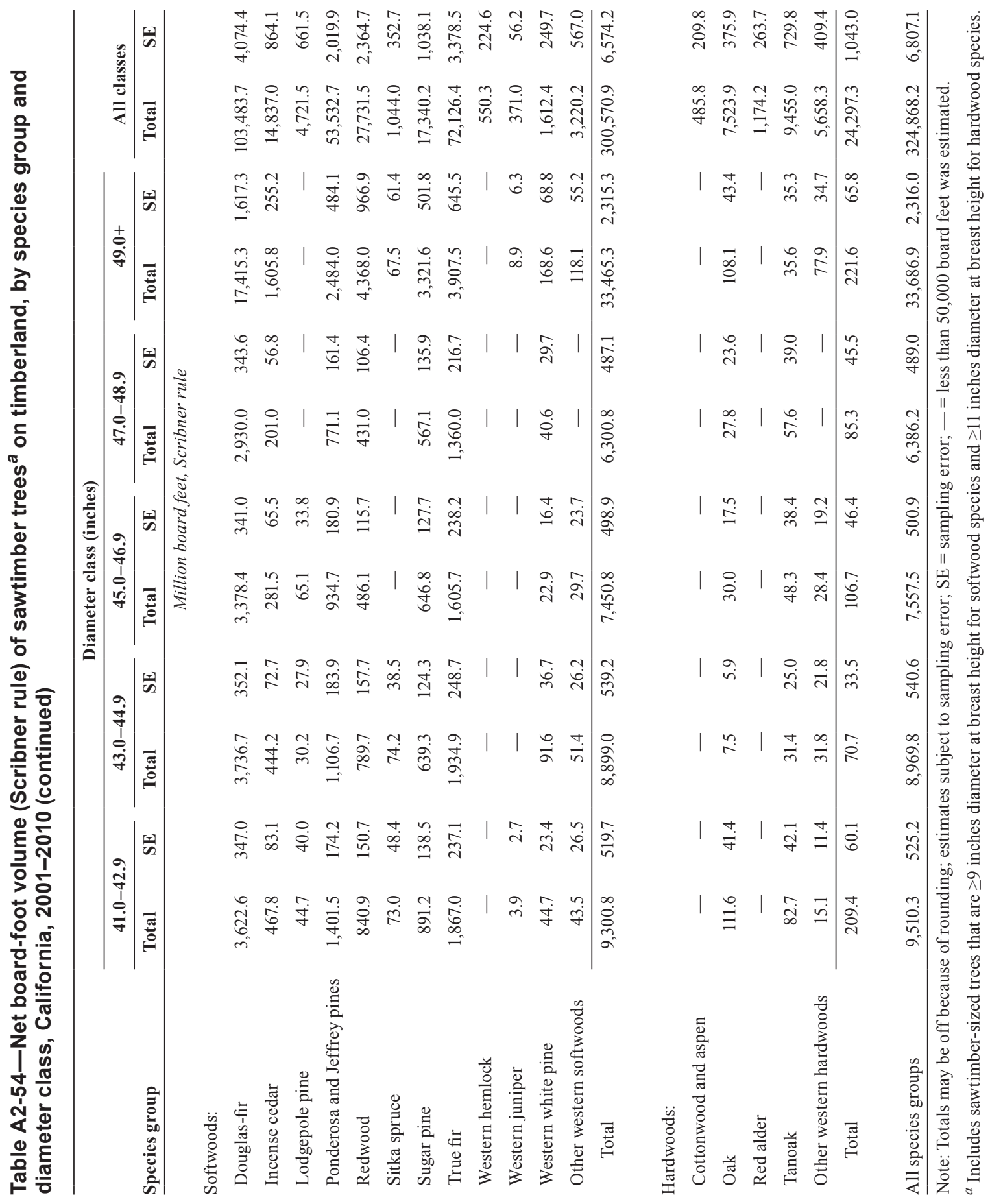




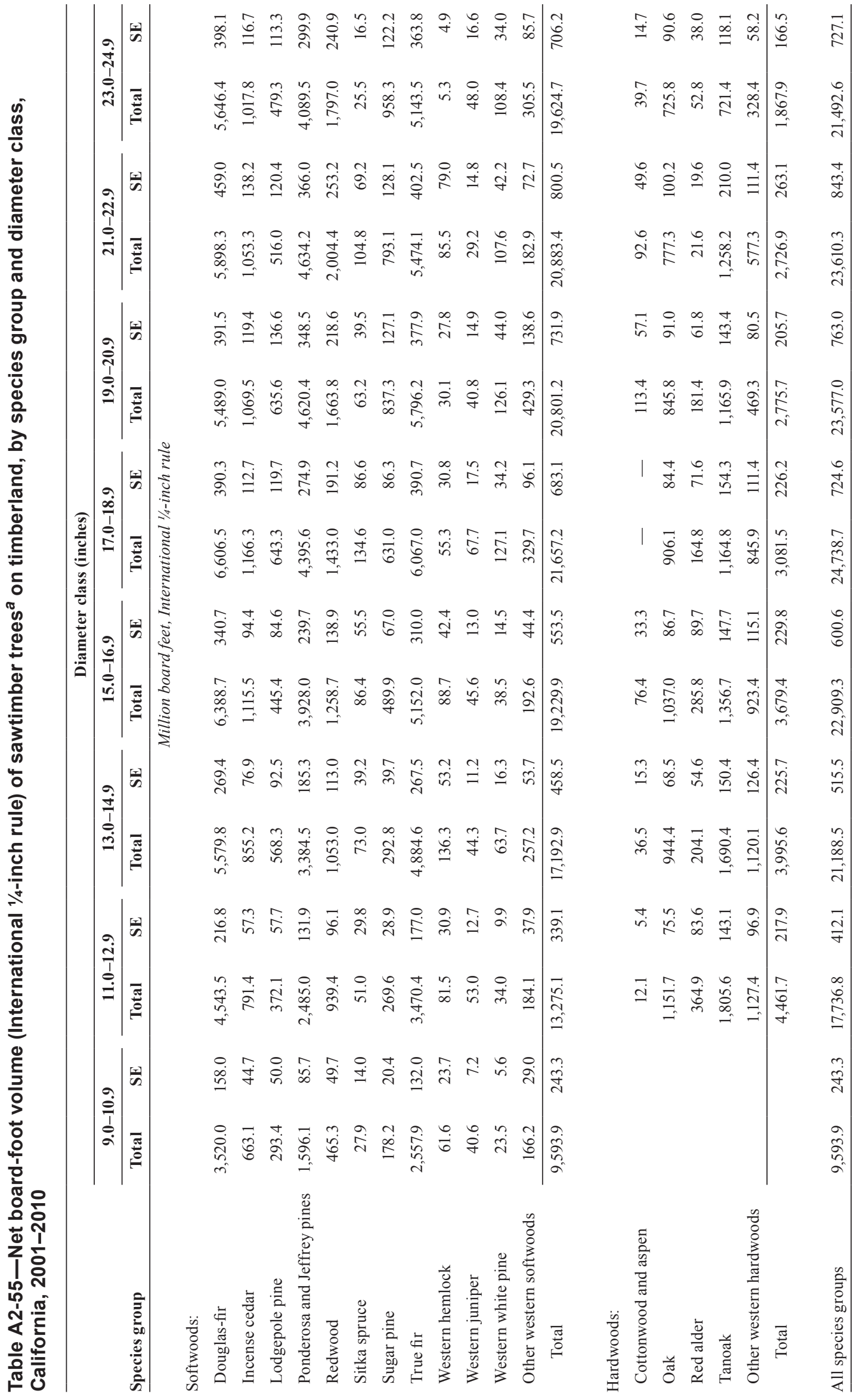




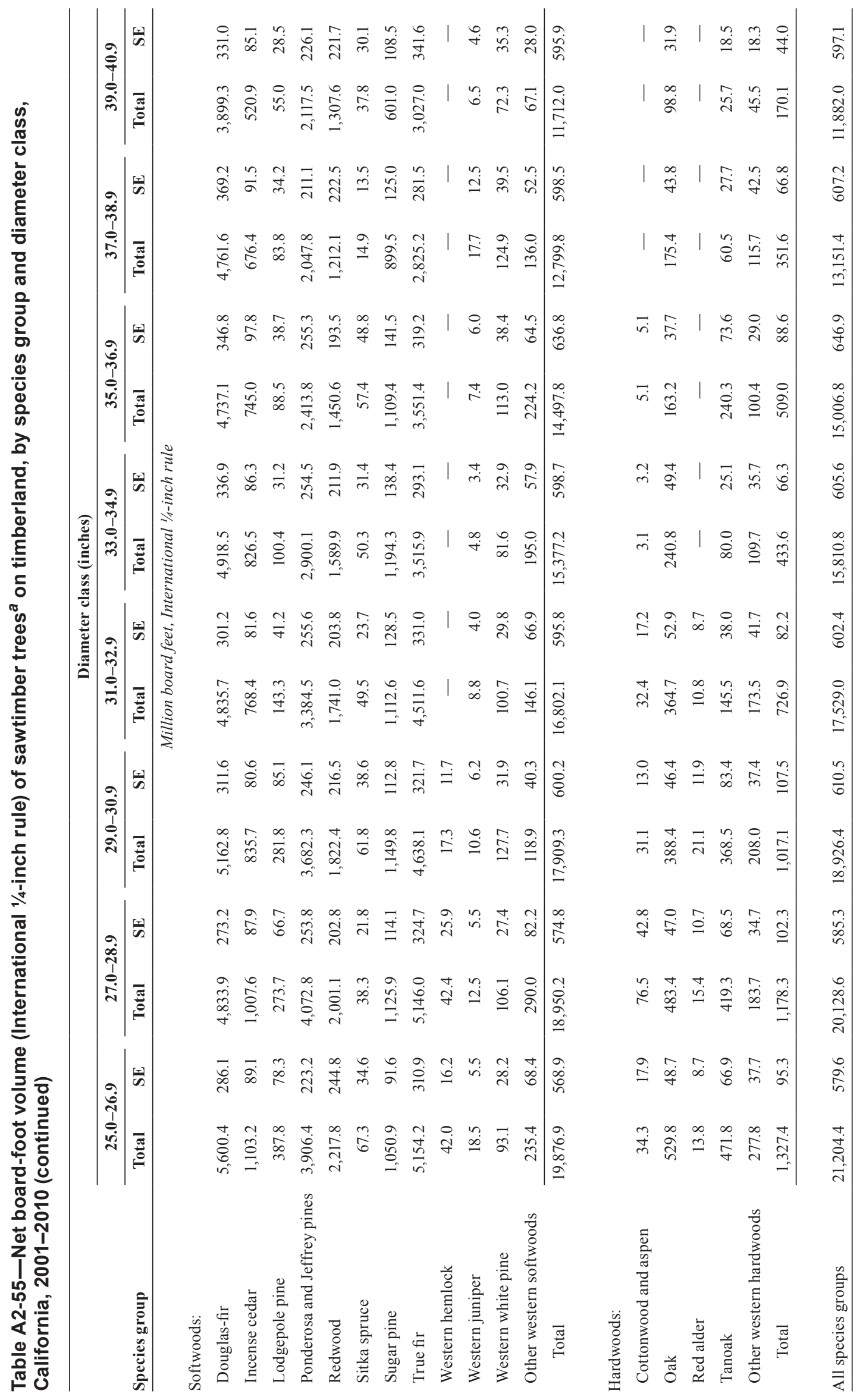




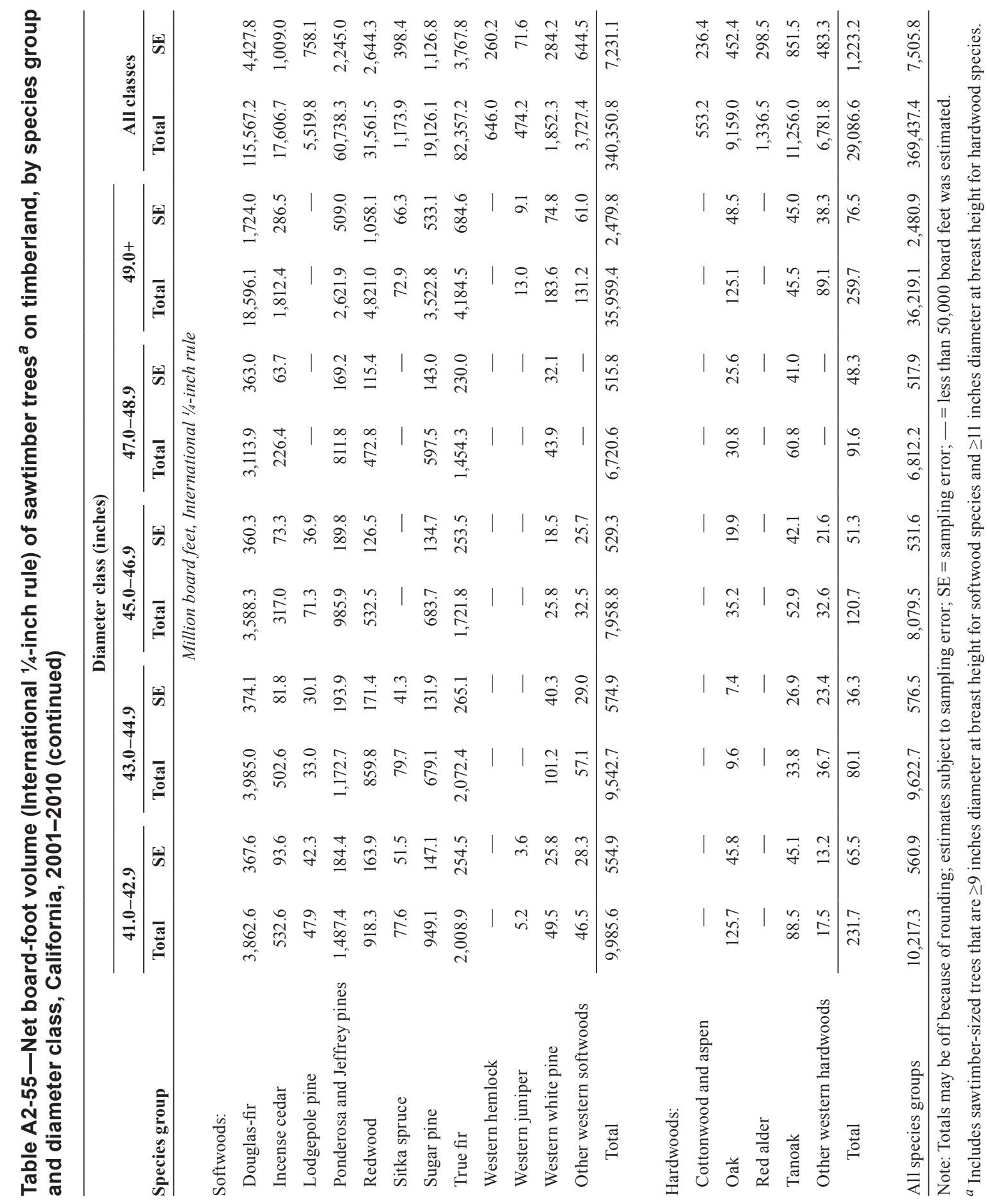




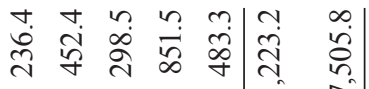

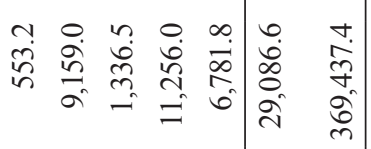

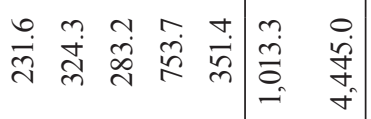

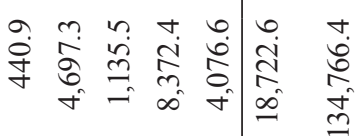

ฮ

m

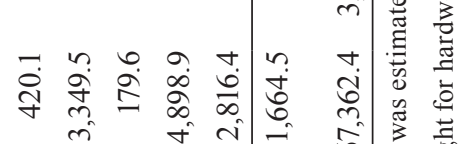

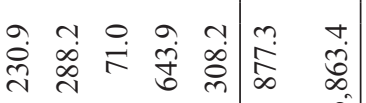

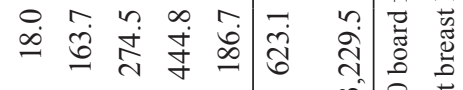

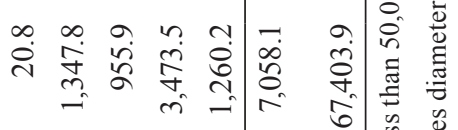

|

ㅇㅇㅇ응

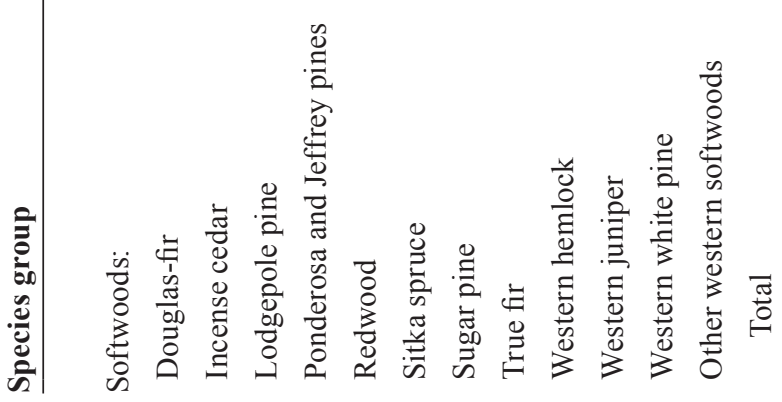

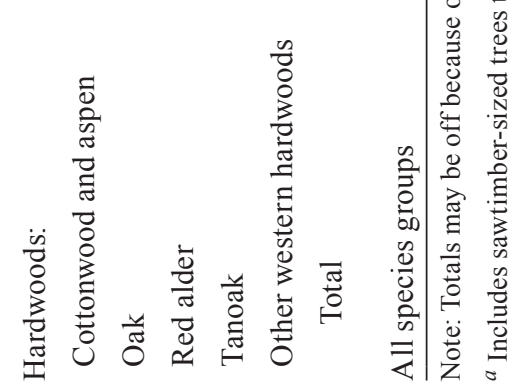




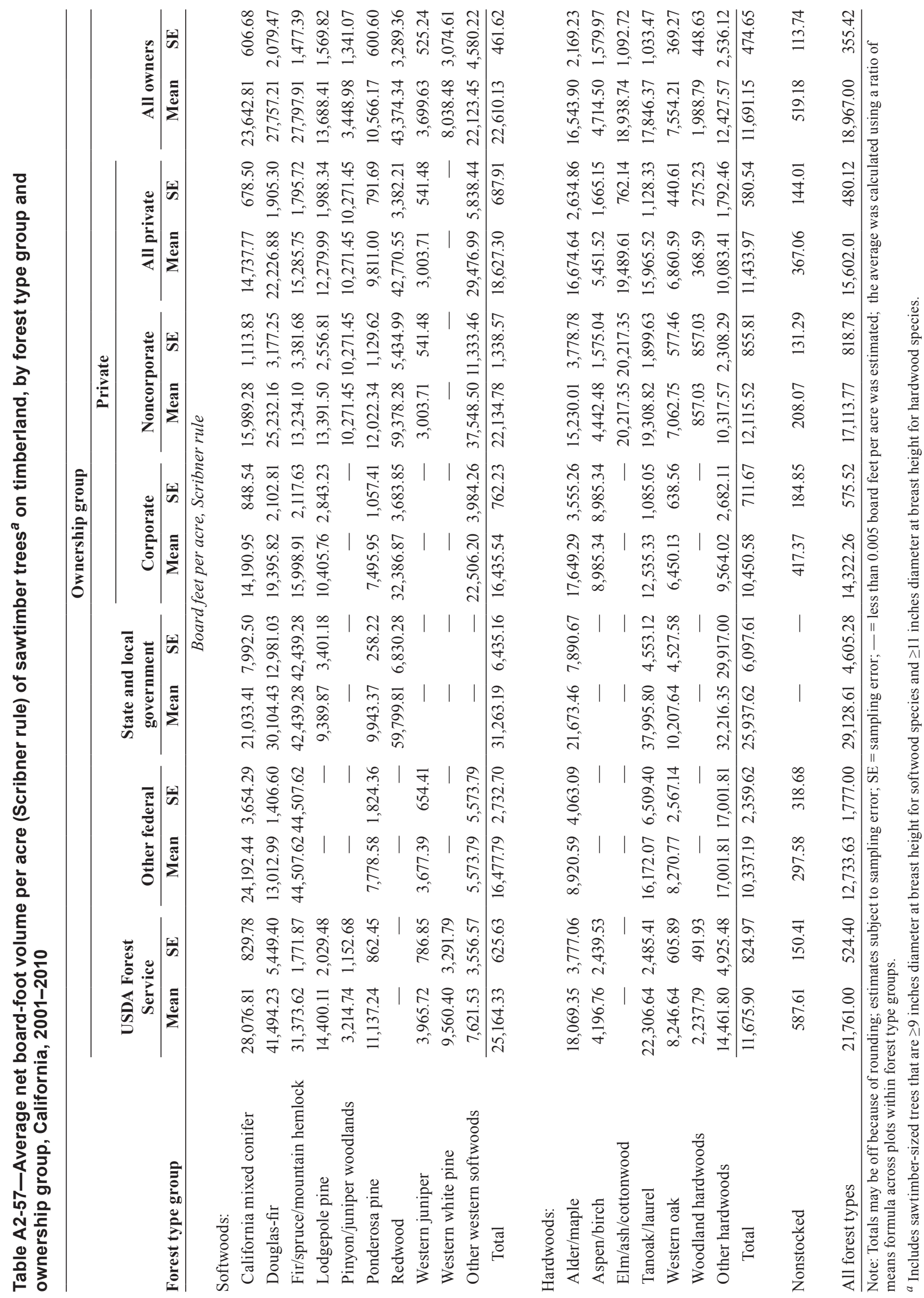


Table A2-58-Average net board-foot volume per acre (Scribner rule) of sawtimber trees ${ }^{a}$ on timberland, by forest type group and stand size class, California, 2001-2010

\begin{tabular}{|c|c|c|c|c|c|c|c|c|}
\hline \multirow[b]{3}{*}{ Forest type group } & \multicolumn{6}{|c|}{ Stand size class } & & \\
\hline & \multicolumn{2}{|c|}{$\begin{array}{c}\text { Large-diameter } \\
\text { stands }\end{array}$} & \multicolumn{2}{|c|}{$\begin{array}{l}\text { Medium-diameter } \\
\text { stands }\end{array}$} & \multicolumn{2}{|c|}{$\begin{array}{c}\text { Small-diameter } \\
\text { stands }\end{array}$} & \multicolumn{2}{|c|}{ All classes } \\
\hline & Mean & SE & Mean & SE & Mean & SE & Mean & SE \\
\hline & \multicolumn{8}{|c|}{ Board feet per acre, Scribner rule } \\
\hline \multicolumn{9}{|l|}{ Softwoods: } \\
\hline California mixed conifer & $25,558.08$ & 634.04 & $2,360.21$ & 381.13 & $1,524.66$ & 297.79 & $23,642.81$ & 606.68 \\
\hline Douglas-fir & $31,221.05$ & $2,265.16$ & $5,375.71$ & $1,008.49$ & $4,630.75$ & $1,842.87$ & $27,757.21$ & $2,079.47$ \\
\hline Fir/spruce/mountain hemlock & $29,324.54$ & $1,517.70$ & $2,446.58$ & 569.98 & $2,087.03$ & 604.41 & $27,797.91$ & $1,477.39$ \\
\hline Lodgepole pine & $15,472.27$ & $1,744.23$ & $6,757.30$ & $1,854.88$ & 199.34 & 172.20 & $13,688.41$ & $1,569.82$ \\
\hline Pinyon/juniper woodlands & $3,448.98$ & $1,341.07$ & - & 一 & - & - & $3,448.98$ & $1,341.07$ \\
\hline Ponderosa pine & $12,452.07$ & 664.11 & 807.16 & 218.63 & 520.82 & 199.61 & $10,566.17$ & 600.60 \\
\hline Redwood & $47,229.38$ & $3,408.61$ & $1,585.99$ & 579.83 & $1,270.83$ & 555.07 & $43,374.34$ & $3,289.36$ \\
\hline Western juniper & $4,479.47$ & 579.50 & 935.13 & 662.94 & 621.88 & 482.70 & $3,699.63$ & 525.24 \\
\hline Western white pine & $17,340.76$ & $1,547.89$ & 338.44 & 338.44 & $2,402.99$ & $1,698.14$ & $8,038.48$ & $3,074.61$ \\
\hline Other western softwoods & $25,903.85$ & $4,990.31$ & - & - & - & - & $22,123.45$ & $4,580.22$ \\
\hline Total & $24,948.30$ & 493.49 & $2,271.73$ & 285.20 & $1,604.39$ & 277.22 & $22,610.13$ & 461.62 \\
\hline
\end{tabular}

Hardwoods:

\begin{tabular}{lrrrrrrrr} 
Alder/maple & $21,612.85$ & $2,389.25$ & $6,646.26$ & $2,195.55$ & $4,271.70$ & 518.83 & $16,543.90$ & $2,169.23$ \\
Aspen/birch & $9,775.23$ & $1,452.35$ & $4,931.88$ & $1,655.57$ & $1,229.54$ & 836.58 & $4,714.50$ & $1,579.97$ \\
Elm/ash/cottonwood & $20,217.35$ & $20,217.35$ & - & - & - & - & $18,938.74$ & $1,092.72$ \\
Tanoak/laurel & $23,788.13$ & $1,304.42$ & $7,048.59$ & 659.14 & $2,554.34$ & 729.75 & $17,846.37$ & $1,033.47$ \\
Western oak & $10,336.33$ & 490.47 & $3,786.68$ & 437.61 & $1,482.87$ & 363.48 & $7,554.21$ & 369.27 \\
Woodland hardwoods & $2,489.31$ & 567.75 & 565.44 & 256.22 & 806.14 & 281.93 & $1,988.79$ & 448.63 \\
Other hardwoods & $23,311.81$ & $4,095.86$ & $3,899.83$ & $1,443.17$ & 655.93 & 250.36 & $12,427.57$ & $2,536.12$ \\
\multicolumn{1}{c}{ Total } & $16,280.94$ & 657.75 & $4,902.96$ & 365.19 & $1,709.24$ & 290.78 & $11,691.15$ & 474.65
\end{tabular}

Nonstocked

414.84

$4,112.69 \quad 279.87$

$1,652.00$

$201.03 \quad 18,967.00$

355.42

Note: Totals may be off because of rounding; estimates subject to sampling error; SE = sampling error; — = less than 0.005 board feet per acre was estimated; the average was calculated using a ratio of means formula across plots within forest type groups.

${ }^{a}$ Includes sawtimber-sized trees that are $\geq 9$ inches diameter at breast height for softwood species and $\geq 11$ inches diameter at breast height for hardwood species. 


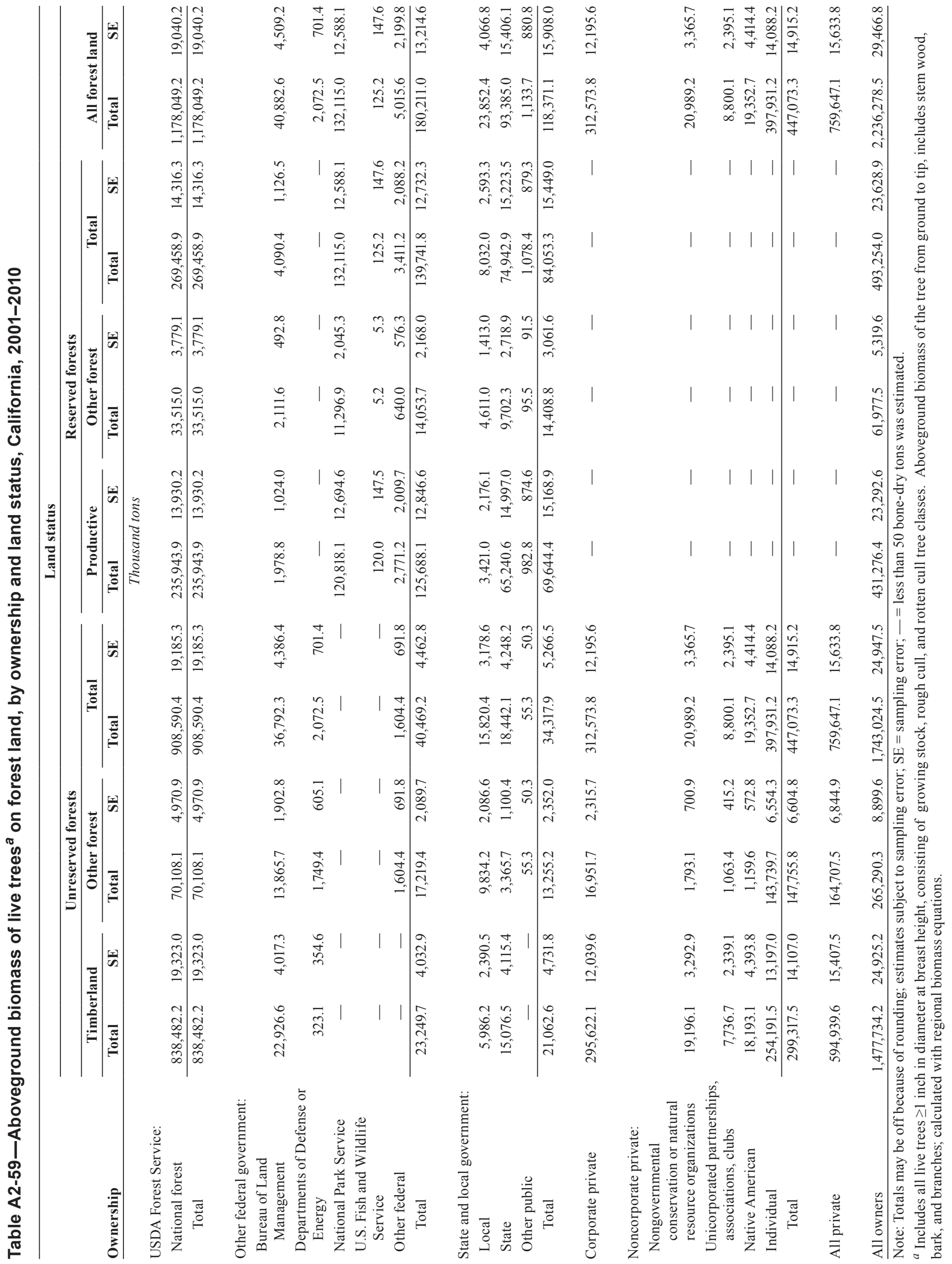




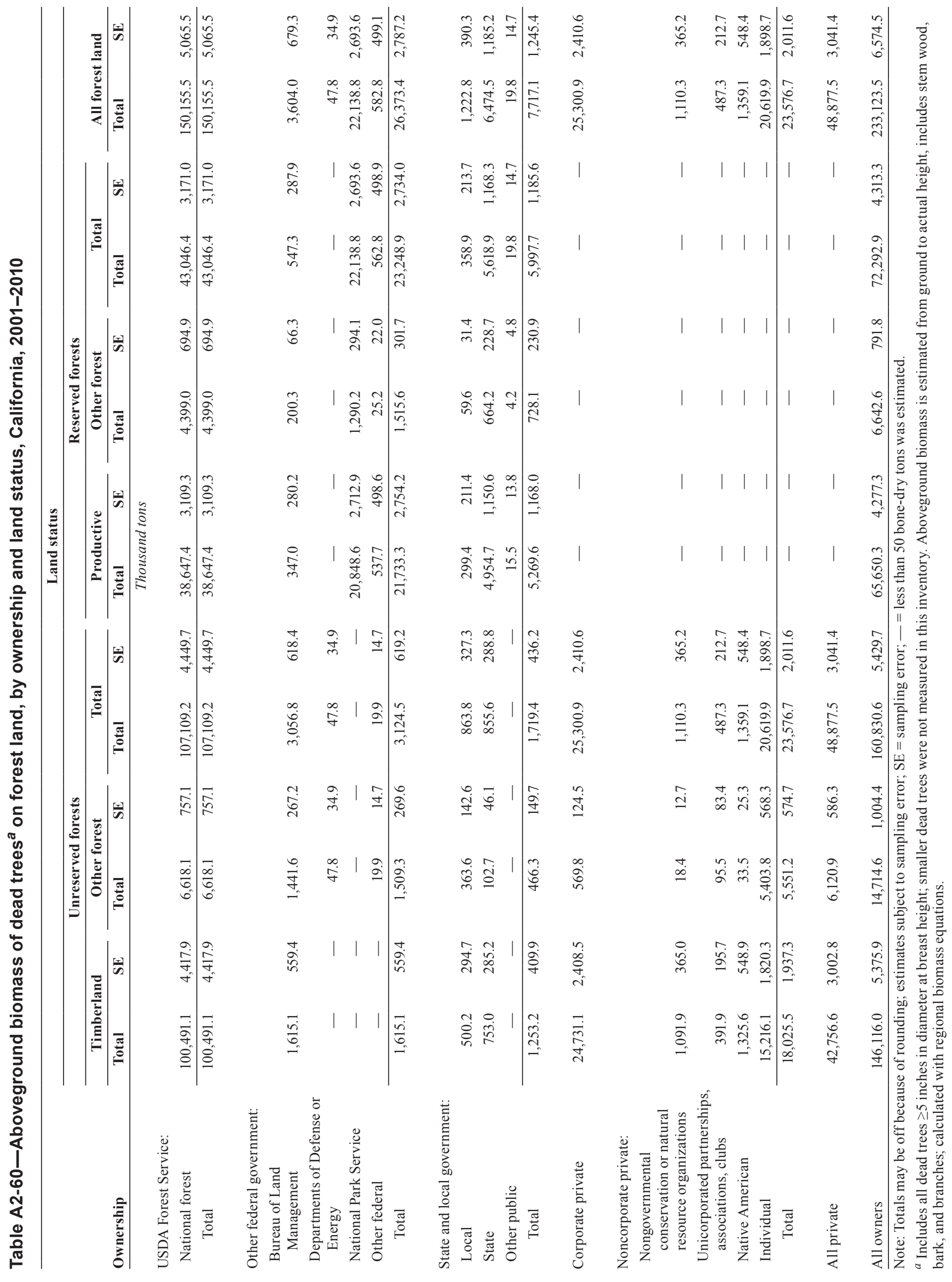




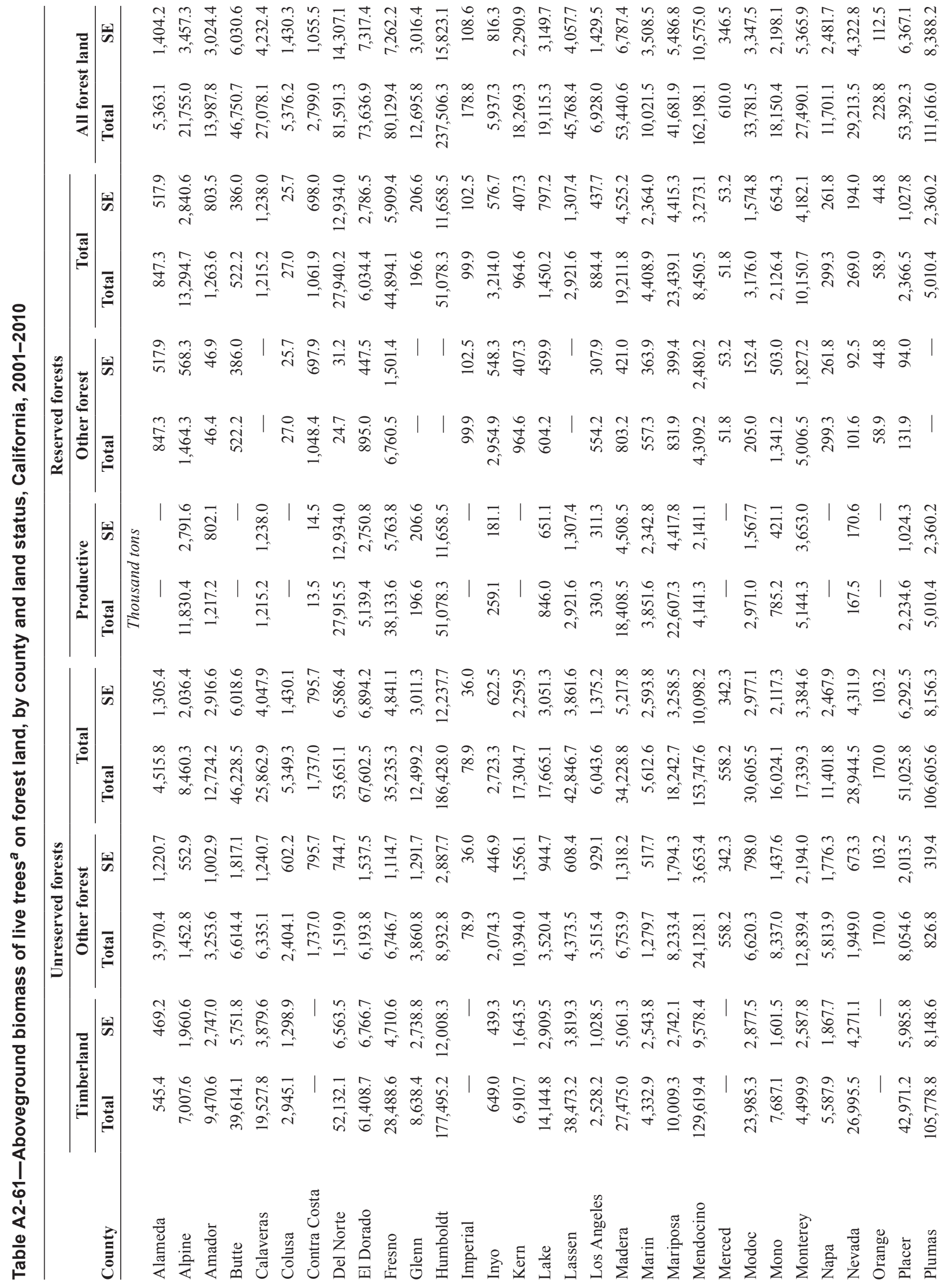




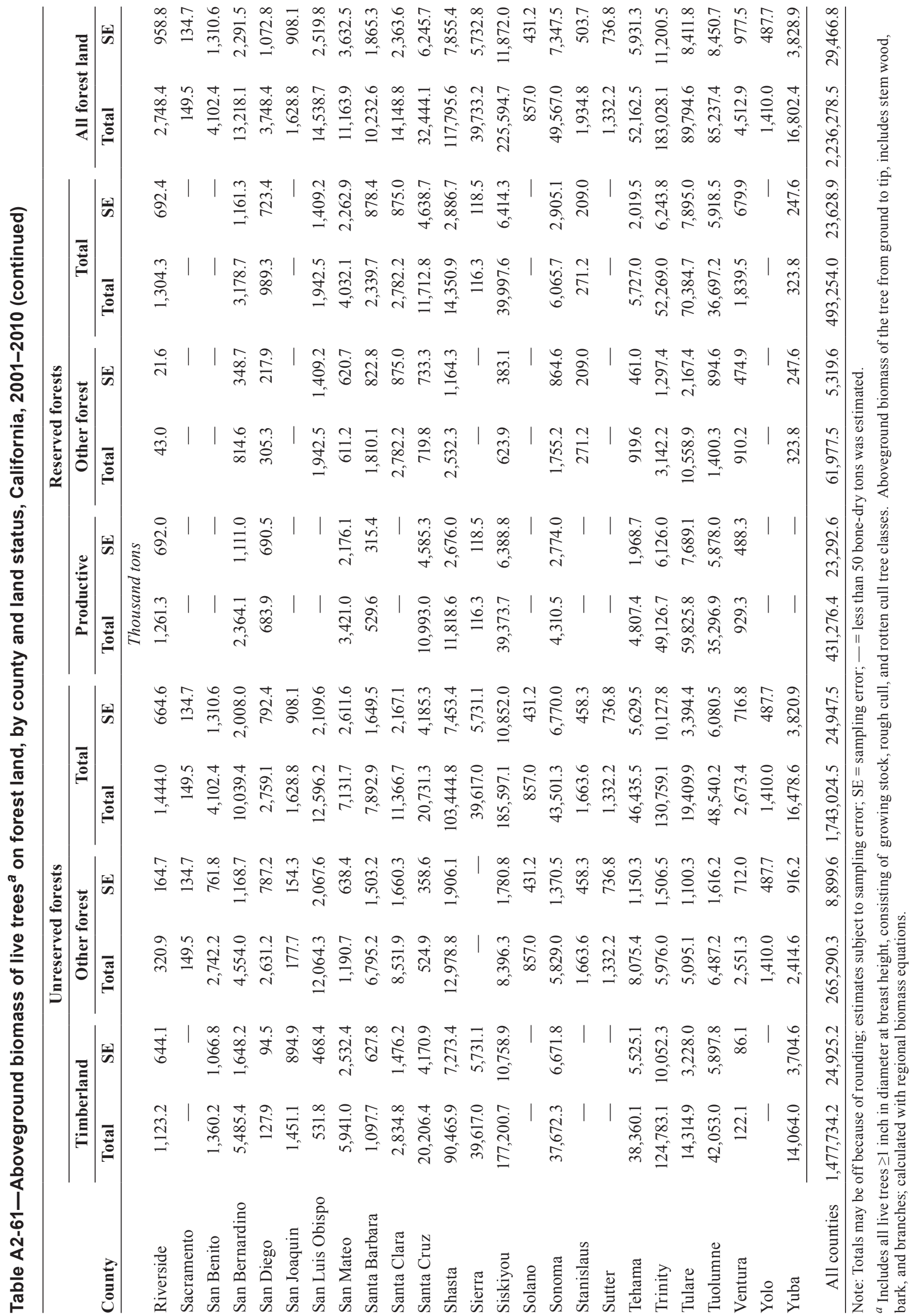




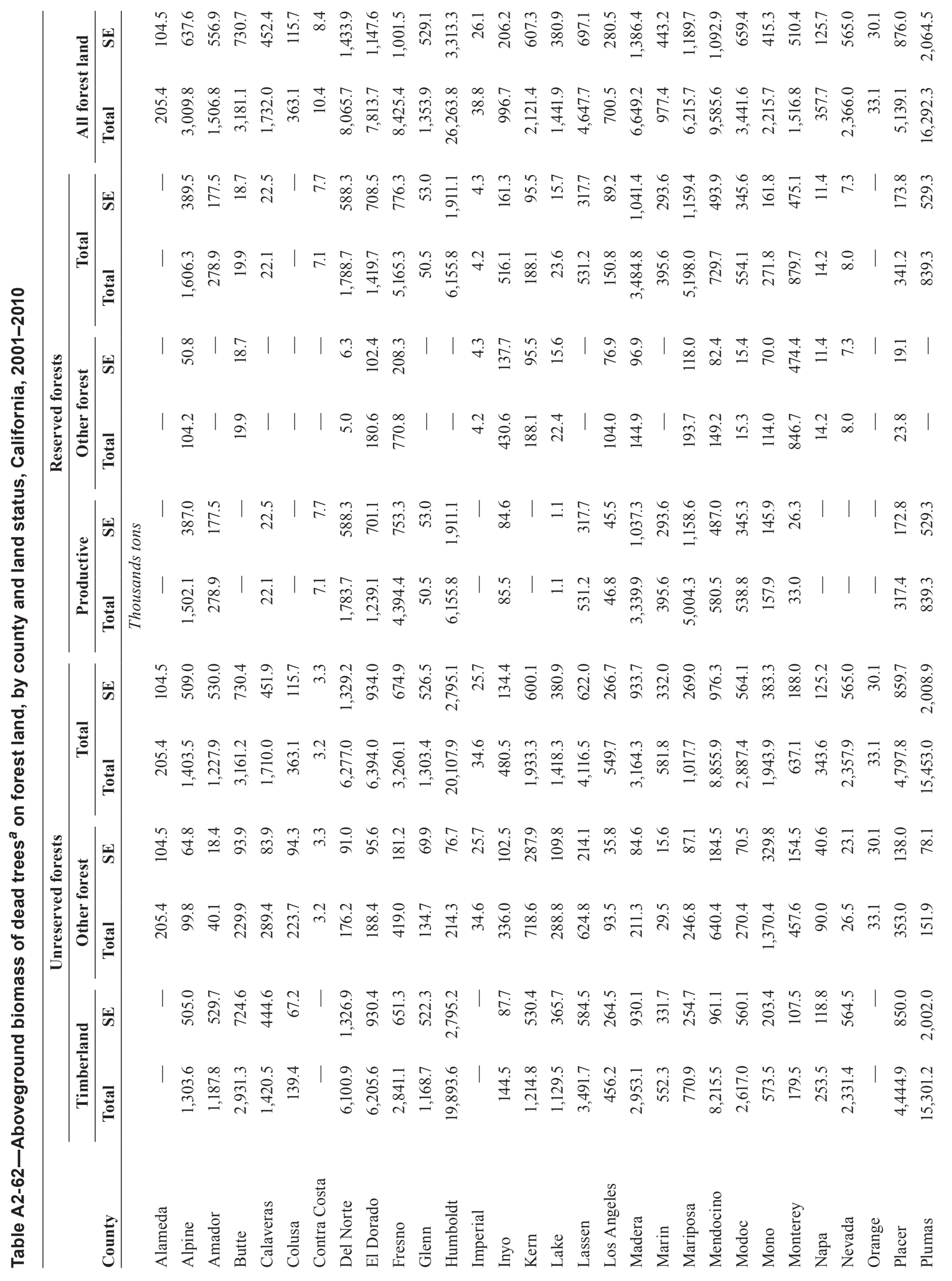




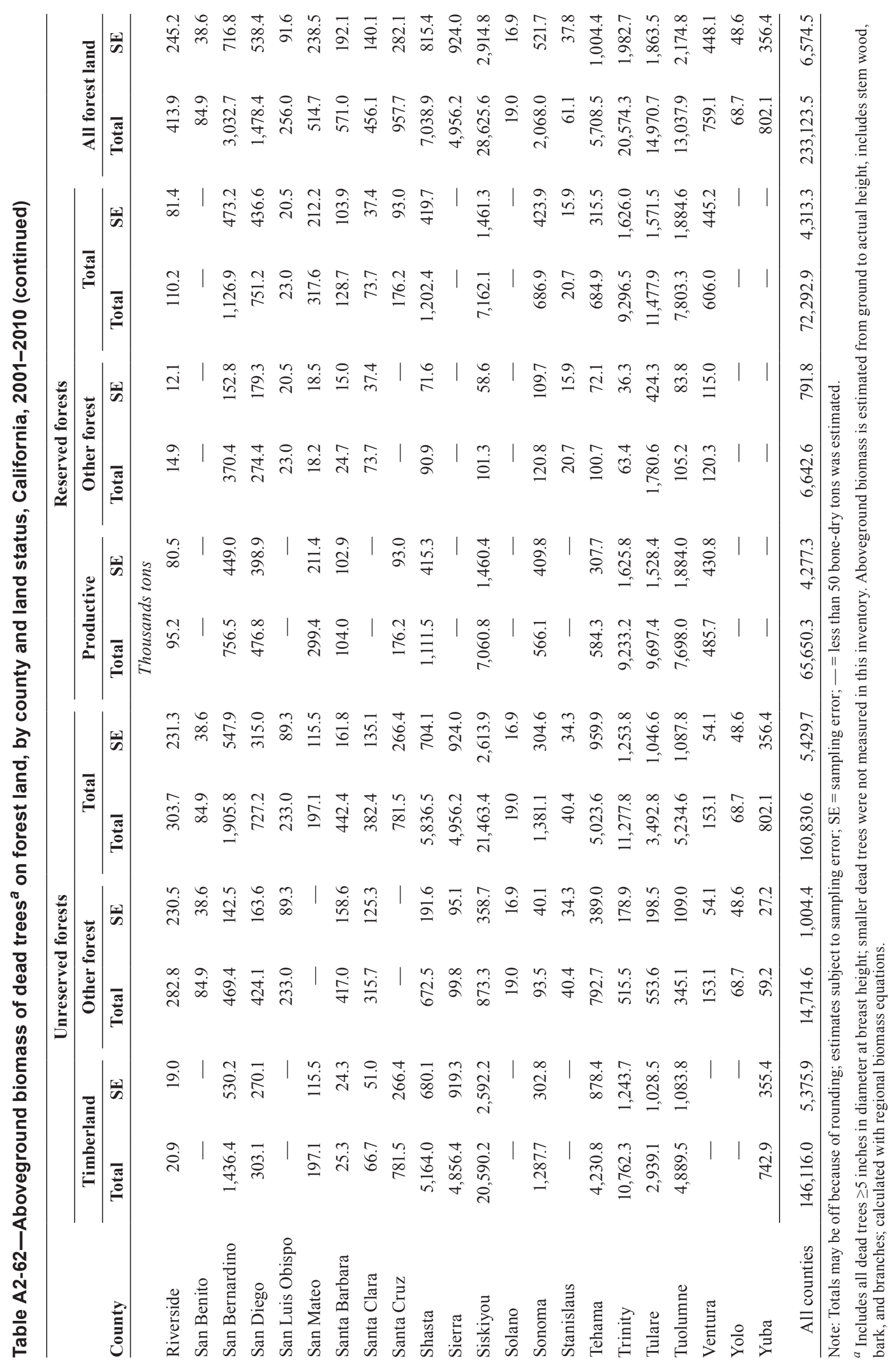




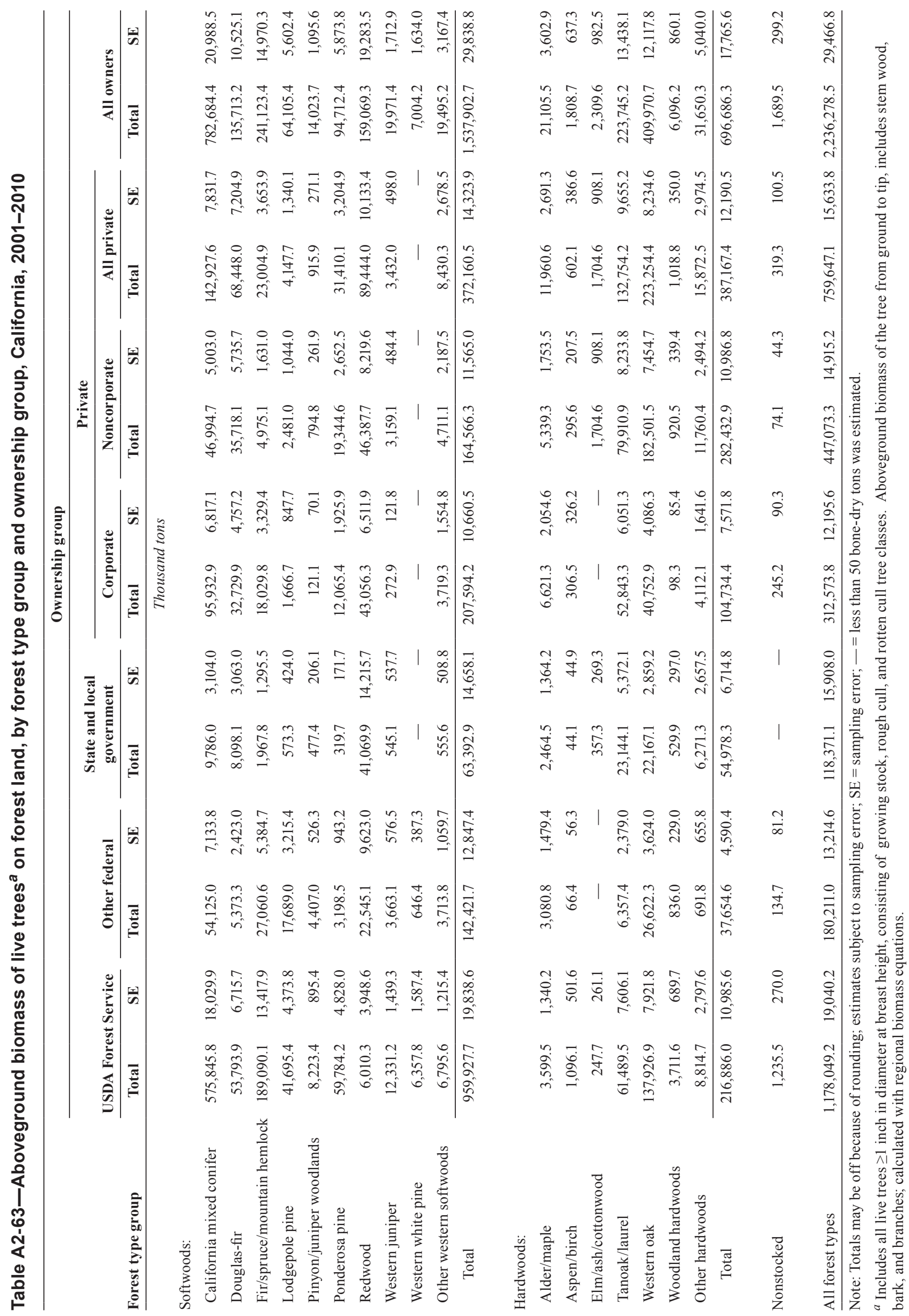




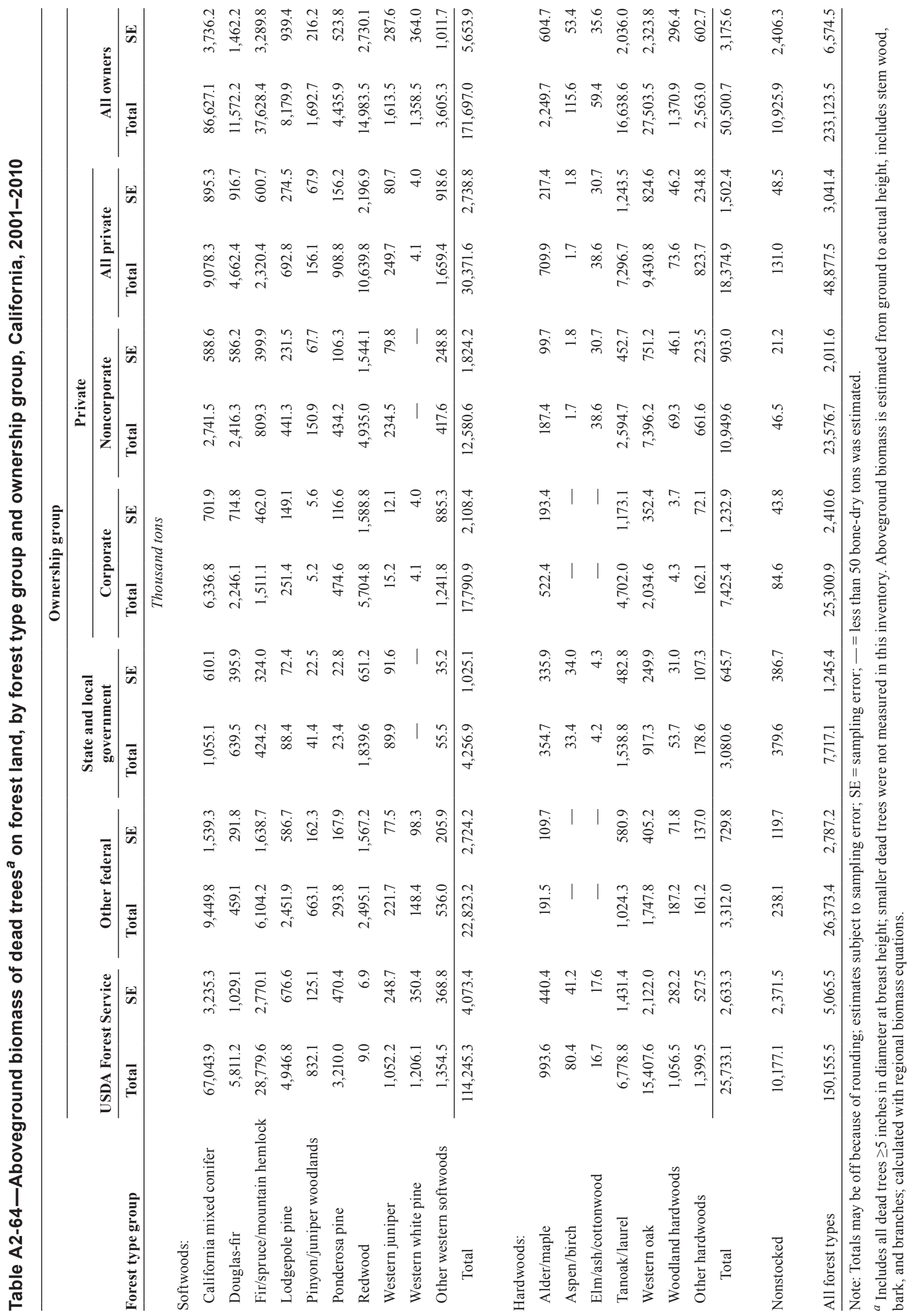


Table A2-65-Aboveground biomass of live trees ${ }^{a}$ on forest land, by forest type group and stand size class, California, 2001-2010

\begin{tabular}{|c|c|c|c|c|c|c|c|c|}
\hline \multirow[b]{3}{*}{ Forest type group } & \multicolumn{6}{|c|}{ Stand size class } & & \\
\hline & \multicolumn{2}{|c|}{$\begin{array}{c}\text { Large-diameter } \\
\text { stands }\end{array}$} & \multicolumn{2}{|c|}{$\begin{array}{l}\text { Medium-diameter } \\
\text { stands }\end{array}$} & \multicolumn{2}{|c|}{$\begin{array}{c}\text { Small-diameter } \\
\text { stands }\end{array}$} & \multicolumn{2}{|c|}{ All classes } \\
\hline & Total & SE & Total & SE & Total & SE & Total & SE \\
\hline & \multicolumn{8}{|c|}{ Thousand tons } \\
\hline \multicolumn{9}{|l|}{ Softwoods: } \\
\hline California mixed conifer & $773,954.4$ & $21,025.7$ & $5,295.7$ & $1,176.6$ & $3,434.3$ & 617.6 & $782,684.4$ & $20,988.5$ \\
\hline Douglas-fir & $131,043.9$ & $10,492.8$ & $2,739.9$ & $1,000.9$ & $1,929.5$ & 645.6 & $135,713.2$ & $10,525.1$ \\
\hline Fir/spruce/mountain hemlock & $239,018.9$ & $14,955.2$ & 724.1 & 384.6 & $1,380.5$ & 394.7 & $241,123.4$ & $14,970.3$ \\
\hline Lodgepole pine & $61,363.1$ & $5,501.0$ & $2,295.2$ & $1,006.4$ & 447.2 & 280.9 & $64,105.4$ & $5,602.4$ \\
\hline Pinyon/juniper woodlands & $13,477.2$ & $1,098.7$ & 376.4 & 153.7 & 170.1 & 58.2 & $14,023.7$ & $1,095.6$ \\
\hline Ponderosa pine & $91,879.8$ & $5,866.0$ & $2,164.7$ & 453.0 & 667.9 & 213.7 & $94,712.4$ & $5,873.8$ \\
\hline Redwood & $158,248.9$ & $19,285.3$ & 463.8 & 259.8 & 356.6 & 185.8 & $159,069.3$ & $19,283.5$ \\
\hline Western juniper & $18,930.6$ & $1,704.3$ & 733.9 & 206.7 & 307.0 & 113.6 & $19,971.4$ & $1,712.9$ \\
\hline Western white pine & $6,523.2$ & $1,613.4$ & 56.2 & 55.6 & 424.7 & 254.1 & $7,004.2$ & $1,634.0$ \\
\hline Other western softwoods & $18,037.9$ & $3,142.3$ & 797.9 & 303.4 & 659.4 & 204.8 & $19,495.2$ & $3,167.4$ \\
\hline Total & $1,512,477.9$ & $29,980.7$ & $15,647.7$ & $1,991.3$ & $9,777.1$ & $1,100.6$ & $1,537,902.7$ & $29,838.8$ \\
\hline
\end{tabular}

Hardwoods:

\begin{tabular}{|c|c|c|c|c|c|c|c|c|}
\hline Alder/maple & $16,618.5$ & $3,333.4$ & $3,963.2$ & $1,358.9$ & 523.8 & 327.0 & $21,105.5$ & $3,602.9$ \\
\hline Aspen/birch & 720.6 & 455.7 & 655.9 & 404.4 & 432.2 & 188.2 & $1,808.7$ & 637.3 \\
\hline Elm/ash/cottonwood & $2,213.5$ & 979.3 & 9.2 & 7.2 & 86.9 & 79.0 & $2,309.6$ & 982.5 \\
\hline Tanoak/laurel & $184,146.0$ & $13,054.9$ & $34,913.9$ & $4,382.8$ & $4,685.3$ & $1,019.2$ & $223,745.2$ & $13,438.1$ \\
\hline Western oak & $276,559.4$ & $11,082.7$ & $116,476.3$ & $6,066.8$ & $16,935.0$ & $1,665.1$ & $409,970.7$ & $12,117.8$ \\
\hline Woodland hardwoods & $5,381.7$ & 818.4 & 654.4 & 264.0 & 60.1 & 35.2 & $6,096.2$ & 860.1 \\
\hline Other hardwoods & $24,739.4$ & $4,816.2$ & $5,635.7$ & $1,488.6$ & $1,275.2$ & 315.8 & $31,650.3$ & $5,040.0$ \\
\hline Total & $510,379.2$ & $17,292.1$ & $162,308.6$ & $7,622.4$ & $23,998.5$ & $2,012.5$ & $696,686.3$ & $17,765.6$ \\
\hline Nonstocked & - & - & - & - & - & - & $1,689.5$ & 299.2 \\
\hline All forest types & $2,022,857.1$ & $30,527.8$ & $177,956.3$ & $7,830.4$ & $33,775.6$ & $2,277.1$ & $2,236,278.5$ & $29,466.8$ \\
\hline
\end{tabular}


Table A2-66-Aboveground biomass of dead trees ${ }^{a}$ on forest land, by forest type group and stand size class, California, 2001-2010

\begin{tabular}{|c|c|c|c|c|c|c|c|c|}
\hline \multirow[b]{3}{*}{ Forest type group } & \multicolumn{8}{|c|}{ Stand size class } \\
\hline & \multicolumn{2}{|c|}{$\begin{array}{c}\text { Large-diameter } \\
\text { stands }\end{array}$} & \multicolumn{2}{|c|}{$\begin{array}{l}\text { Medium-diameter } \\
\text { stands }\end{array}$} & \multicolumn{2}{|c|}{$\begin{array}{c}\text { Small-diameter } \\
\text { stands }\end{array}$} & \multicolumn{2}{|c|}{ All classes } \\
\hline & Total & SE & Total & SE & Total & SE & Total & SE \\
\hline & \multicolumn{8}{|c|}{ Thousand tons } \\
\hline \multicolumn{9}{|l|}{ Softwoods: } \\
\hline California mixed conifer & $84,249.8$ & $3,692.3$ & 784.8 & 296.0 & $1,592.5$ & 606.5 & $86,627.1$ & $3,736.2$ \\
\hline Douglas-fir & $10,508.9$ & $1,376.1$ & 80.2 & 51.0 & 983.1 & 495.8 & $11,572.2$ & $1,462.2$ \\
\hline Fir/spruce/mountain hemlock & $34,539.6$ & $2,928.1$ & 371.7 & 273.0 & $2,717.0$ & $1,519.4$ & $37,628.4$ & $3,289.8$ \\
\hline Lodgepole pine & $7,830.8$ & 910.7 & 340.2 & 230.3 & 9.0 & 9.1 & $8,179.9$ & 939.4 \\
\hline Pinyon/juniper woodlands & $1,312.9$ & 159.9 & 262.6 & 138.8 & 117.1 & 61.9 & $1,692.7$ & 216.2 \\
\hline Ponderosa pine & $4,160.2$ & 513.2 & 101.5 & 47.4 & 174.2 & 96.0 & $4,435.9$ & 523.8 \\
\hline Redwood & $14,664.8$ & $2,717.1$ & 184.0 & 110.9 & 134.7 & 78.0 & $14,983.5$ & $2,730.1$ \\
\hline Western juniper & $1,586.0$ & 287.2 & 22.5 & 16.4 & 5.0 & 2.8 & $1,613.5$ & 287.6 \\
\hline Western white pine & $1,262.2$ & 359.0 & 11.5 & 11.4 & 84.9 & 59.2 & $1,358.5$ & 364.0 \\
\hline Other western softwoods & $3,238.2$ & 988.4 & 94.1 & 50.9 & 273.0 & 209.0 & $3,605.3$ & $1,011.7$ \\
\hline Total & $163,353.5$ & $5,426.1$ & $2,253.1$ & 504.5 & $6,090.4$ & $1,727.9$ & $171,697.0$ & $5,653.9$ \\
\hline \multicolumn{9}{|l|}{ Hardwoods: } \\
\hline Alder/maple & $1,648.3$ & 551.9 & 456.4 & 227.5 & 145.0 & 97.3 & $2,249.7$ & 604.7 \\
\hline Aspen/birch & 40.0 & 33.2 & 66.7 & 41.3 & 8.9 & 7.0 & 115.6 & 53.4 \\
\hline Elm/ash/cottonwood & 26.3 & 19.0 & - & - & 33.1 & 30.1 & 59.4 & 35.6 \\
\hline Tanoak/laurel & $12,105.0$ & $1,541.2$ & $1,598.1$ & 531.0 & $2,935.6$ & $1,244.3$ & $16,638.6$ & $2,036.0$ \\
\hline Western oak & $15,190.2$ & $1,179.1$ & $5,613.2$ & 556.7 & $6,700.1$ & $1,945.4$ & $27,503.5$ & $2,323.8$ \\
\hline Woodland hardwoods & $1,044.8$ & 222.5 & 326.1 & 195.2 & - & - & $1,370.9$ & 296.4 \\
\hline Other hardwoods & $1,665.1$ & 443.6 & 309.7 & 116.8 & 588.2 & 387.2 & $2,563.0$ & 602.7 \\
\hline Total & $31,719.7$ & $2,046.7$ & $8,370.2$ & 832.7 & $10,410.8$ & $2,339.4$ & $50,500.7$ & $3,175.6$ \\
\hline Nonstocked & - & - & - & - & - & - & $10,925.9$ & $2,406.3$ \\
\hline All forest types & $195,073.2$ & $5,613.8$ & $10,623.3$ & 970.9 & $16,501.2$ & $2,904.8$ & $233,123.5$ & $6,574.5$ \\
\hline
\end{tabular}

Note: Totals may be off because of rounding; estimates subject to sampling error; SE = sampling error; - = less than 50 bone-dry tons was estimated.

${ }^{a}$ Includes all dead trees $\geq 5$ inches in diameter at breast height; smaller dead trees were not measured in this inventory. Aboveground biomass is estimated from ground to actual height, includes stem wood, bark, and branches; calculated with regional biomass equations. 


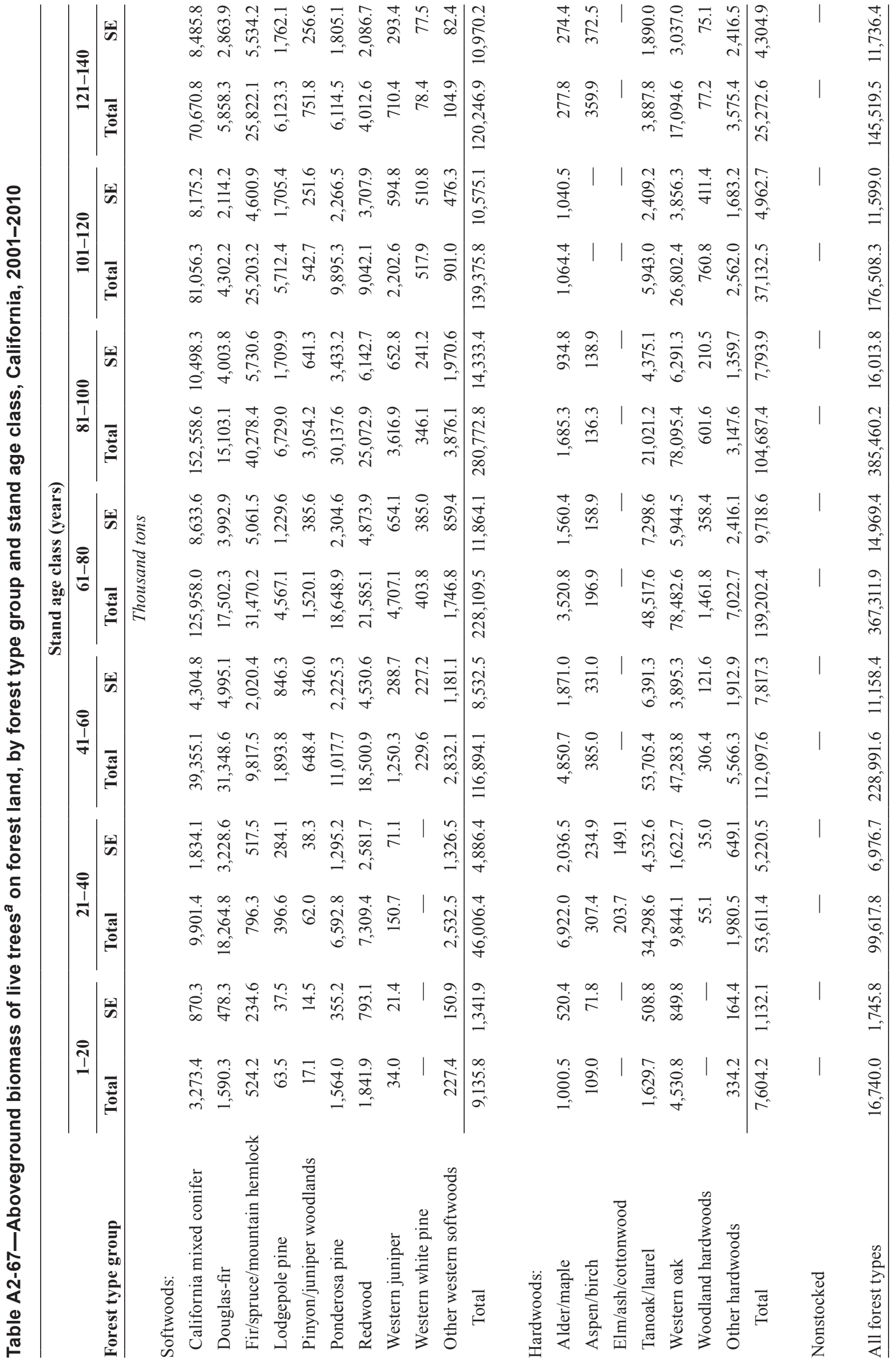




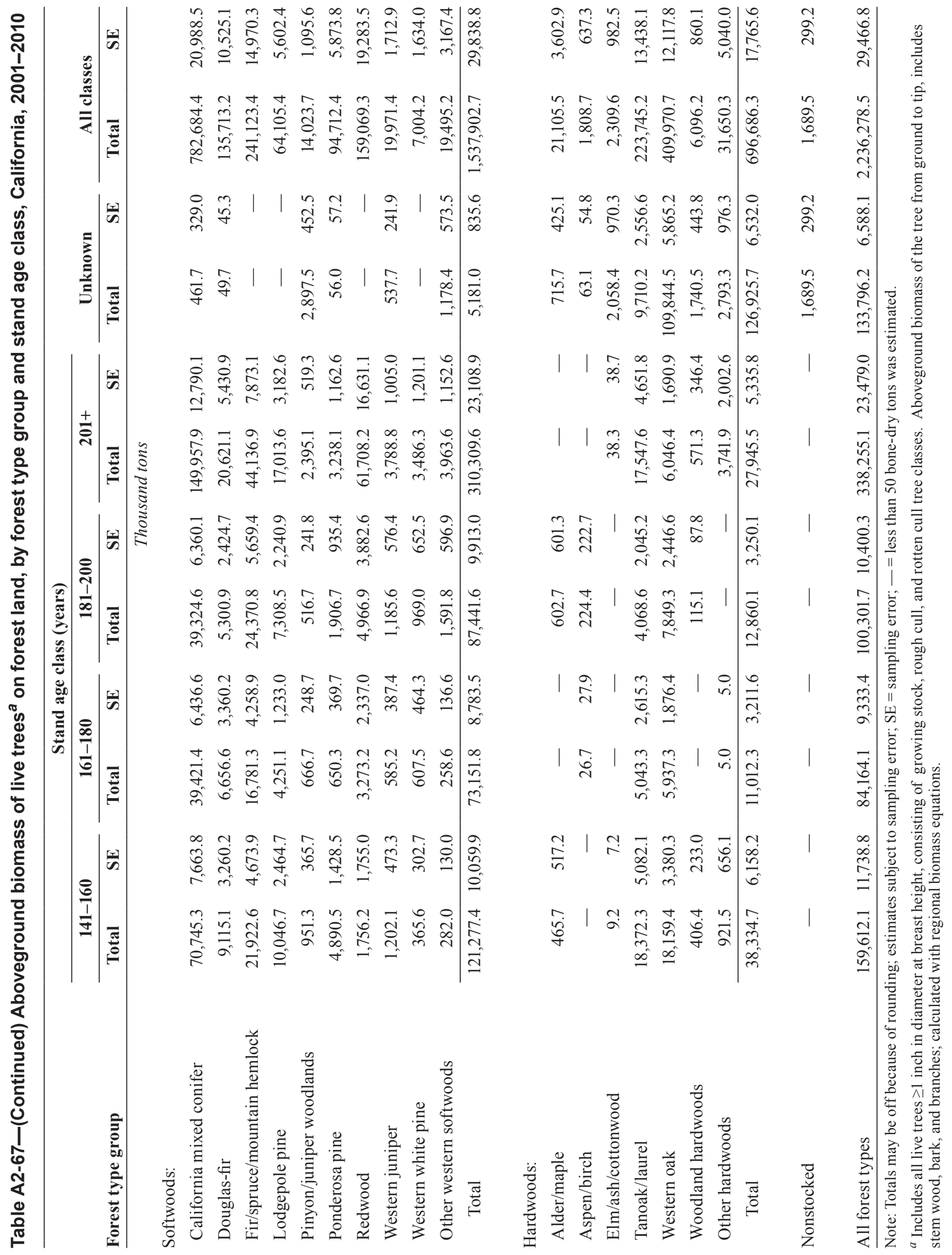




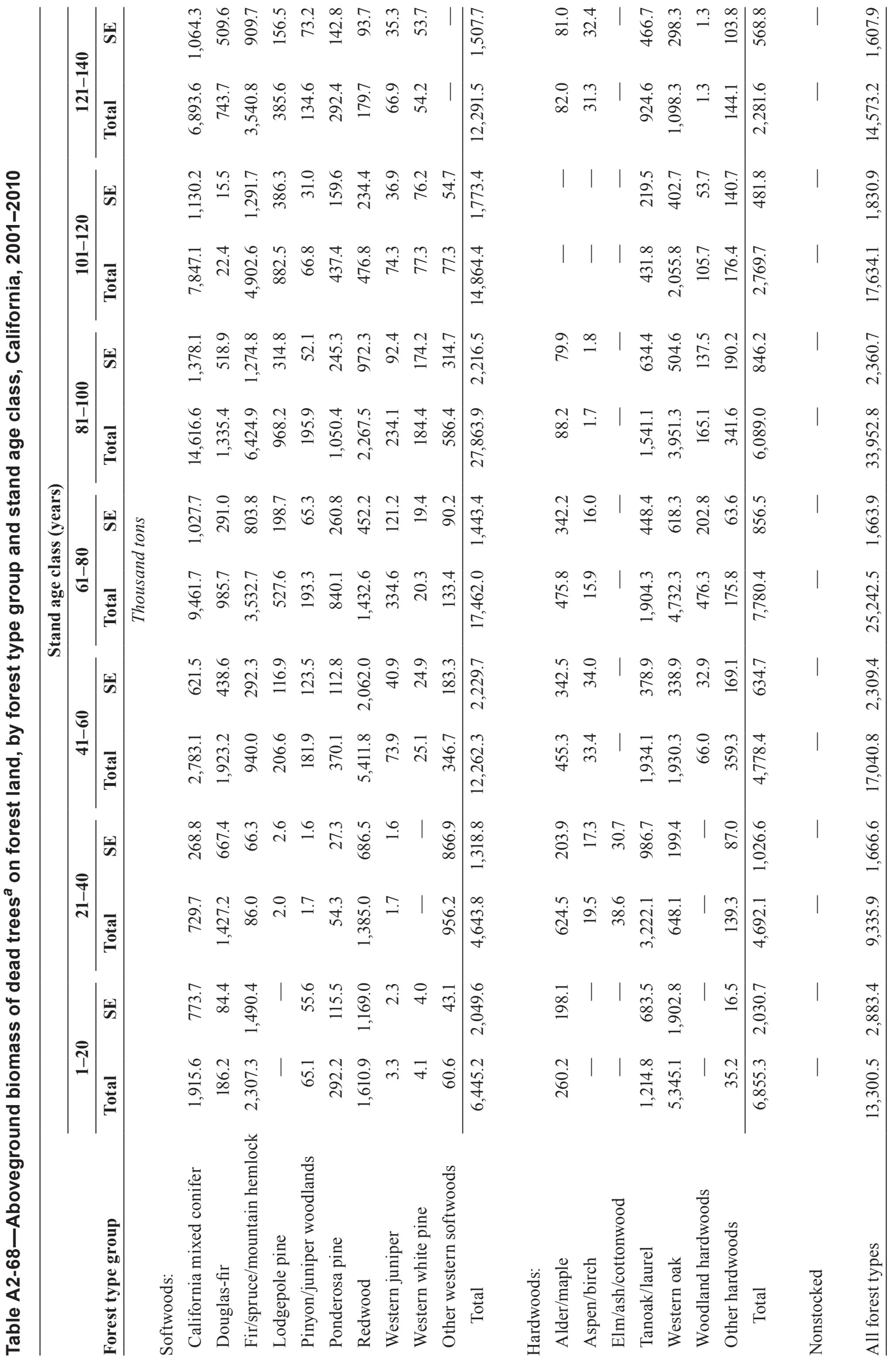




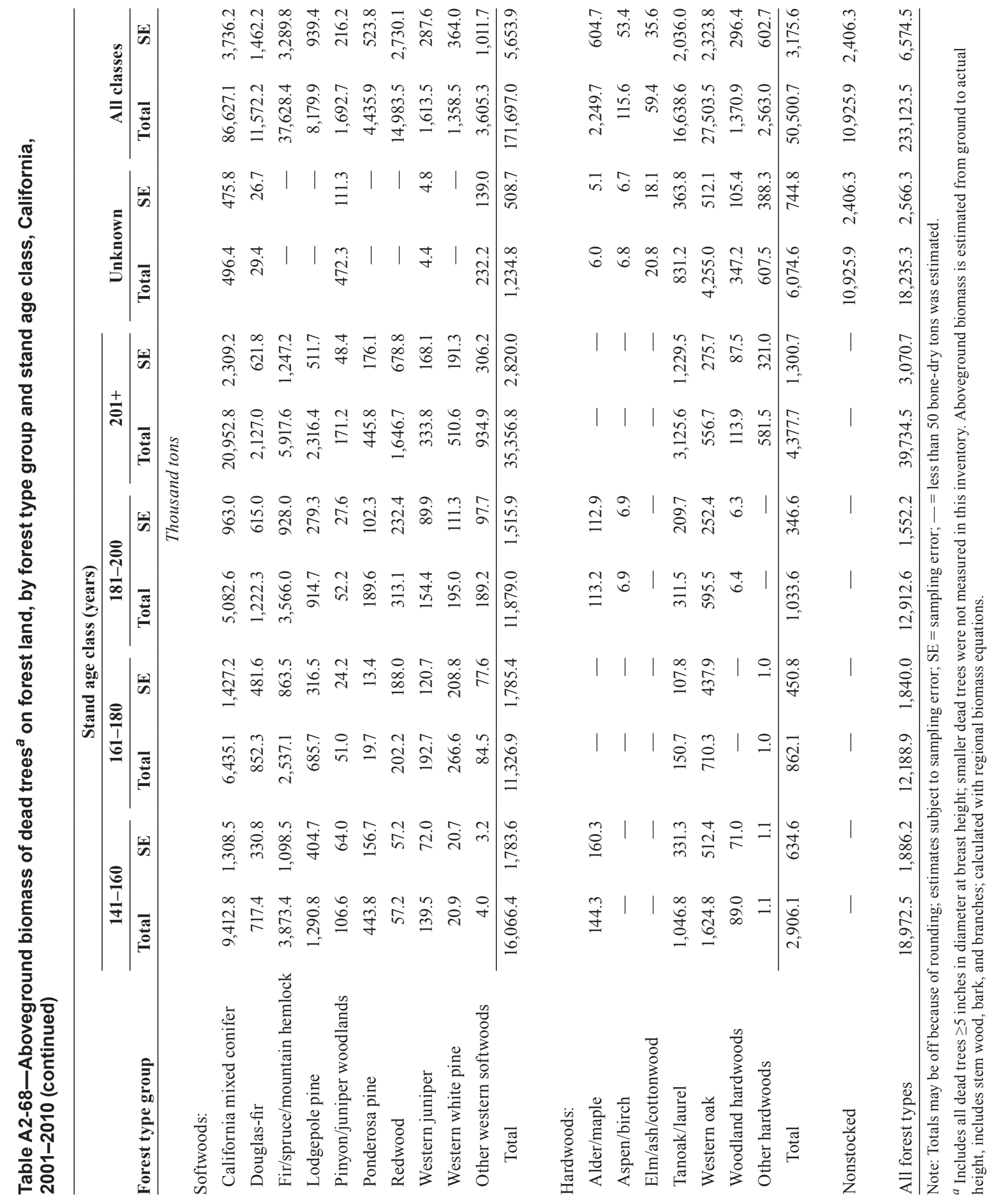




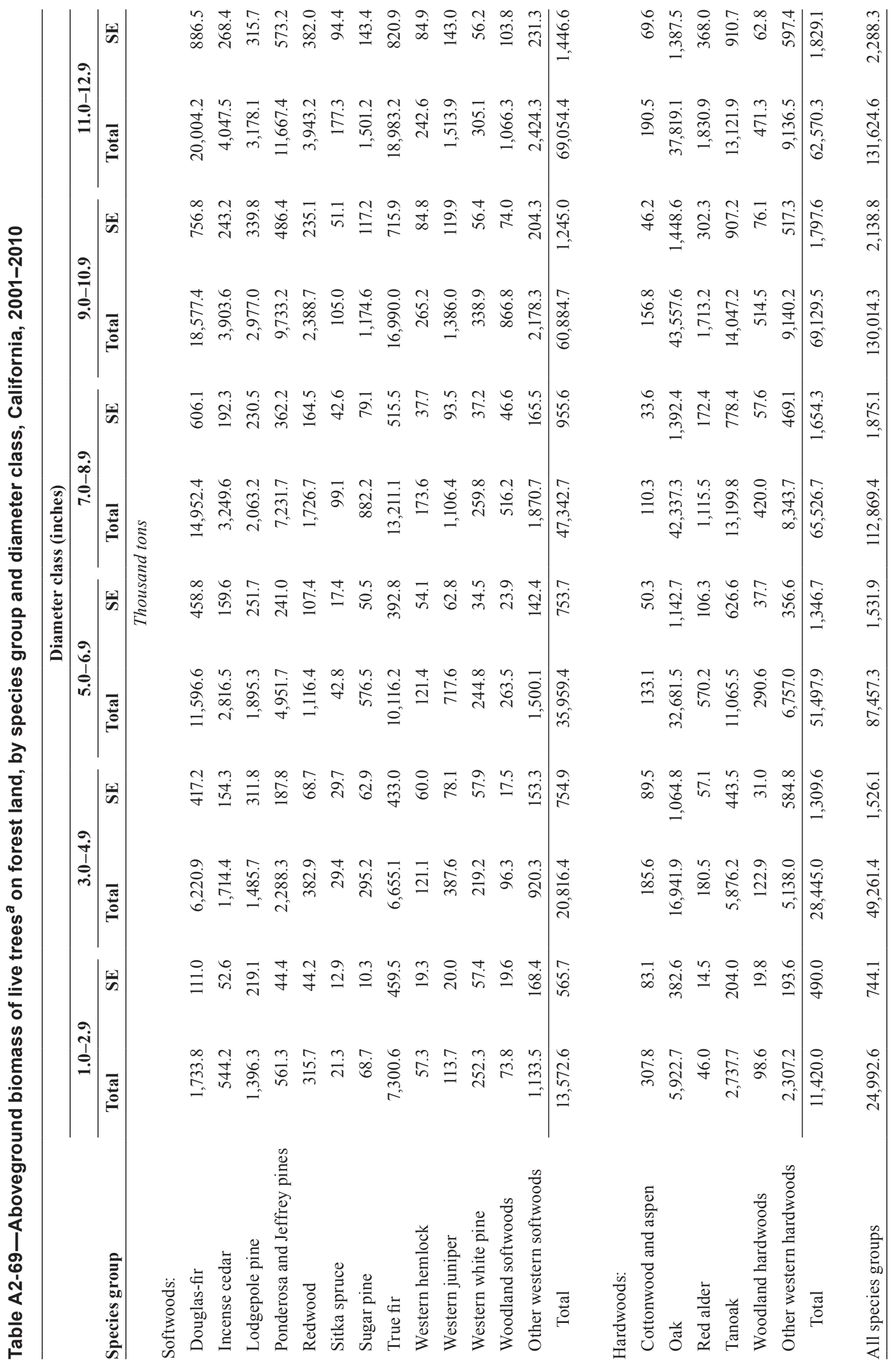




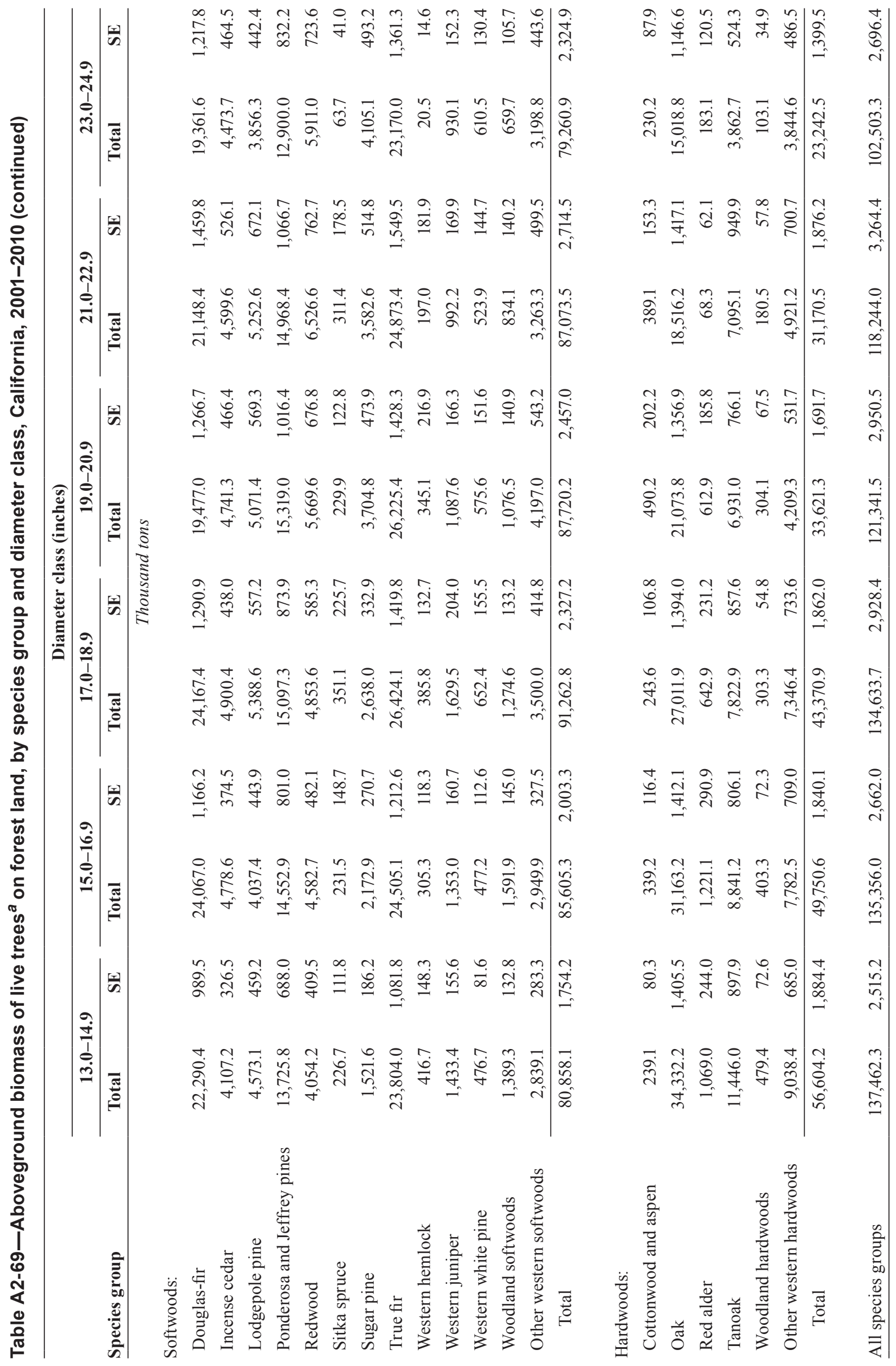




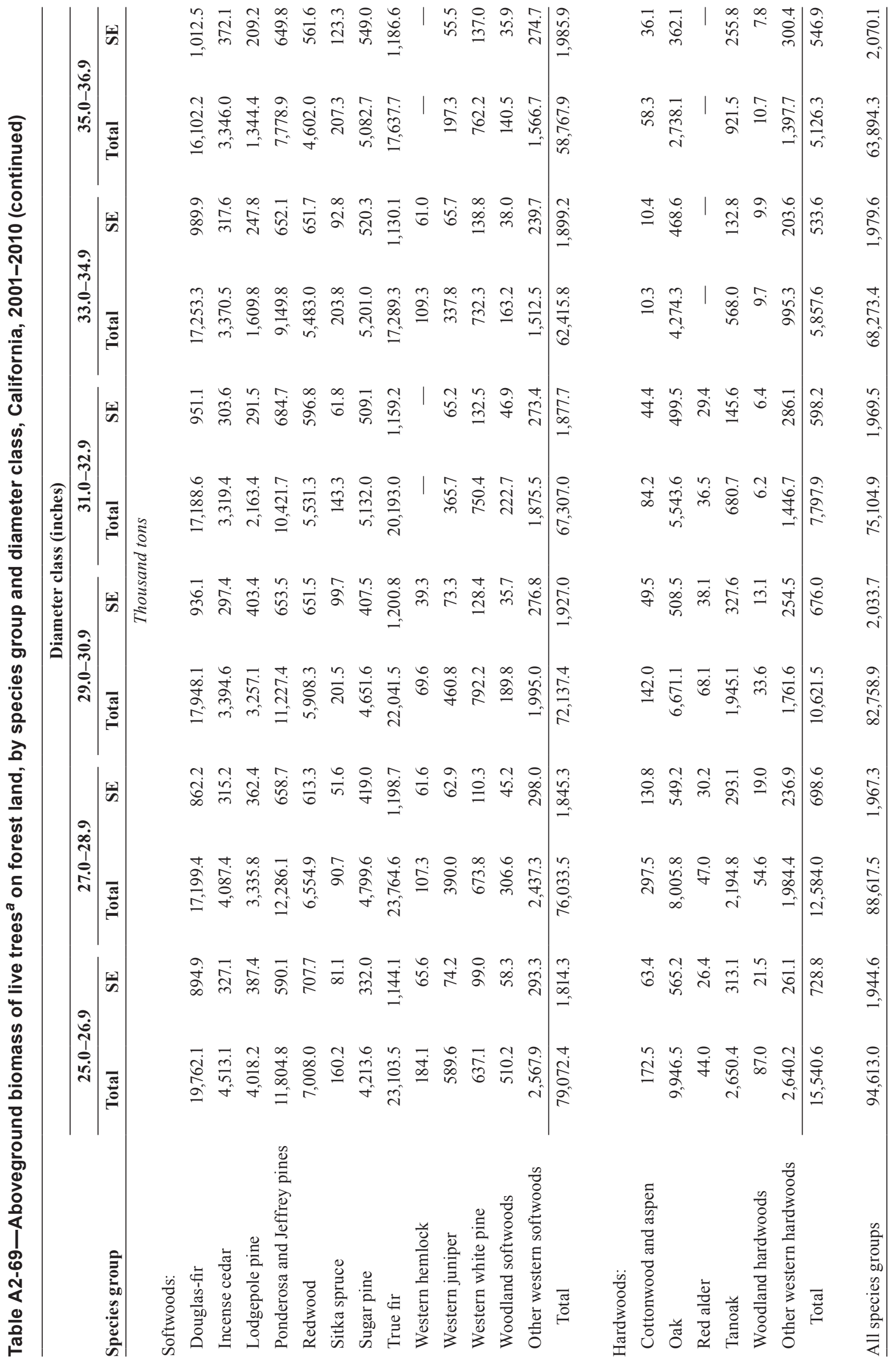




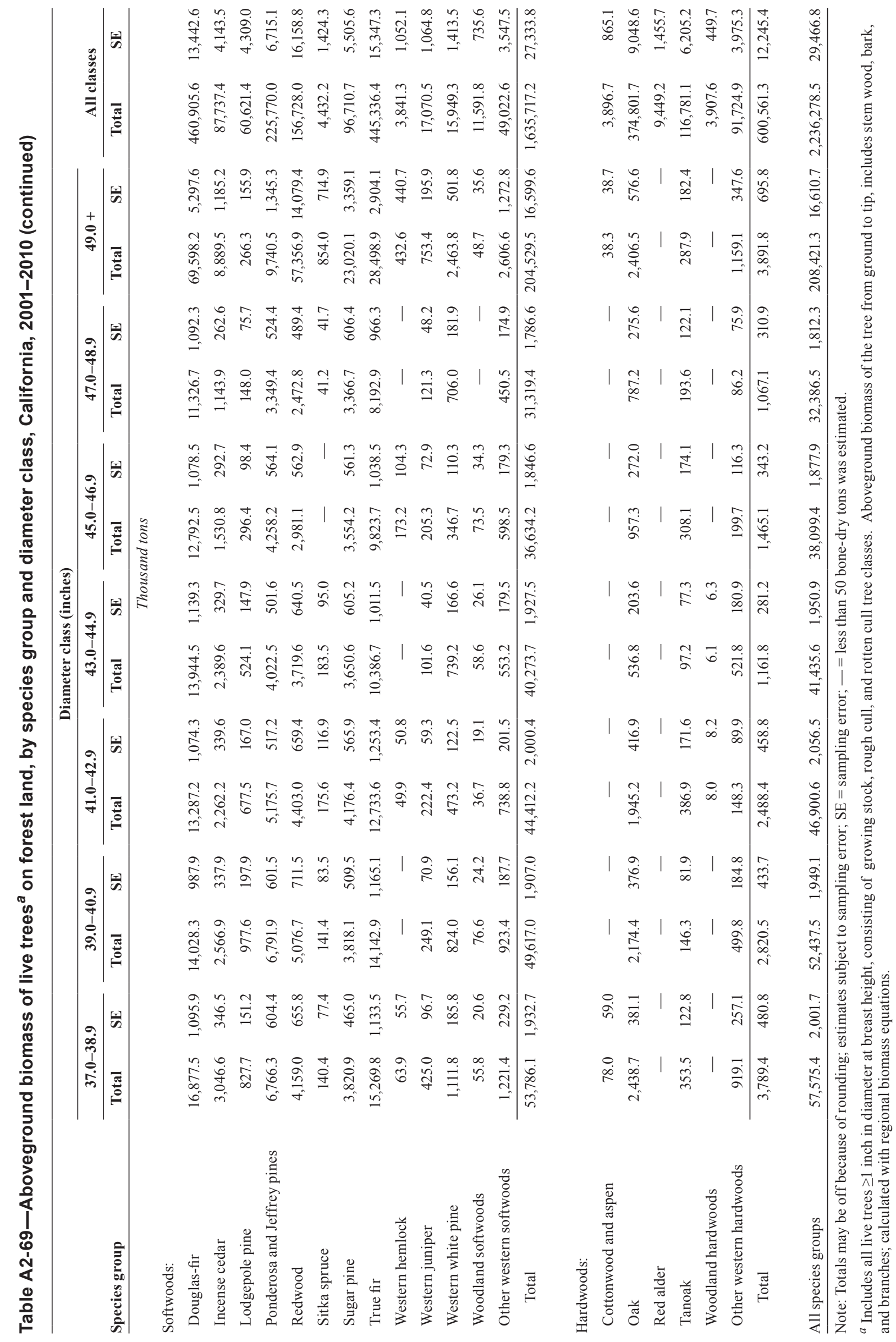




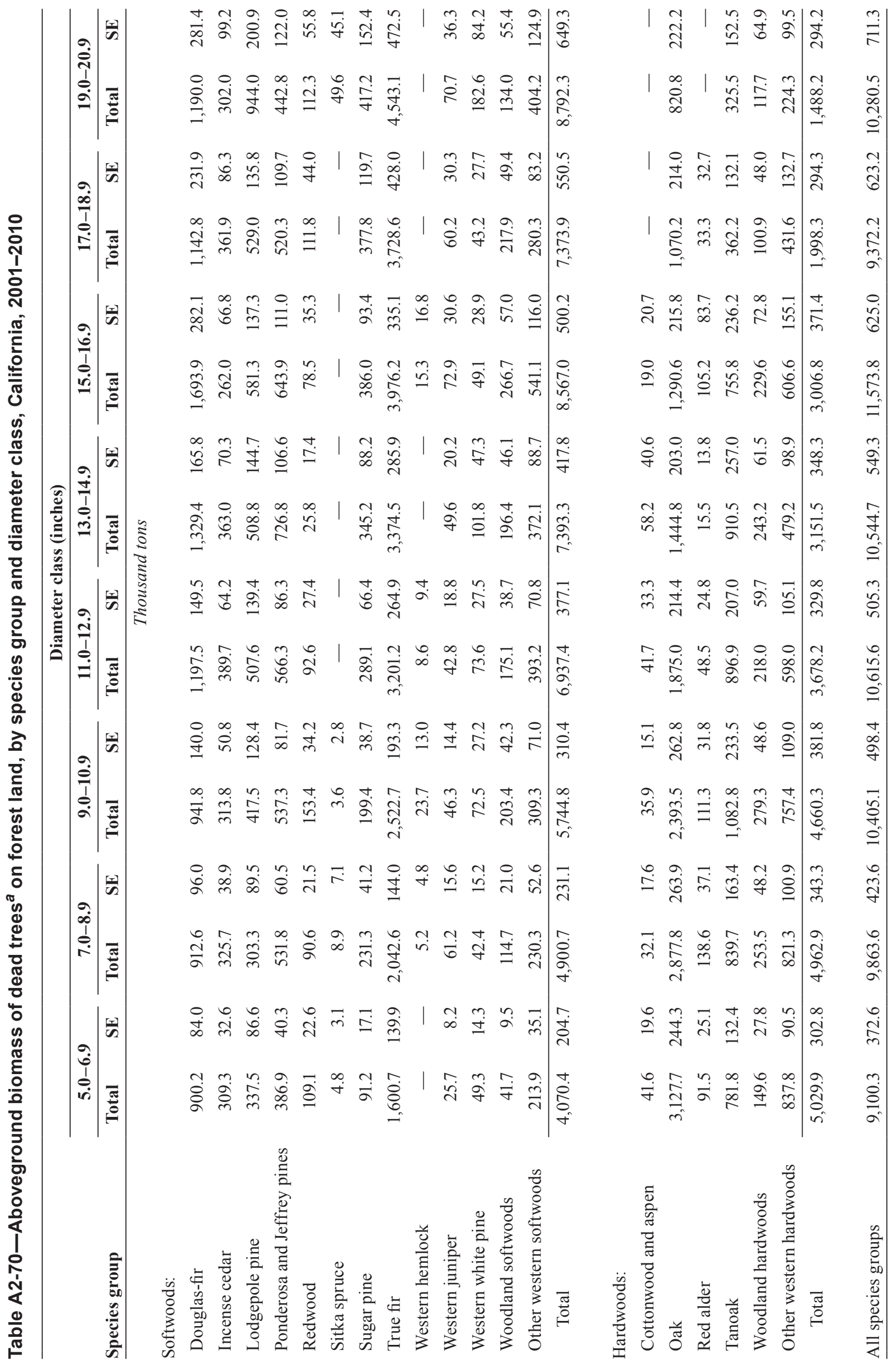




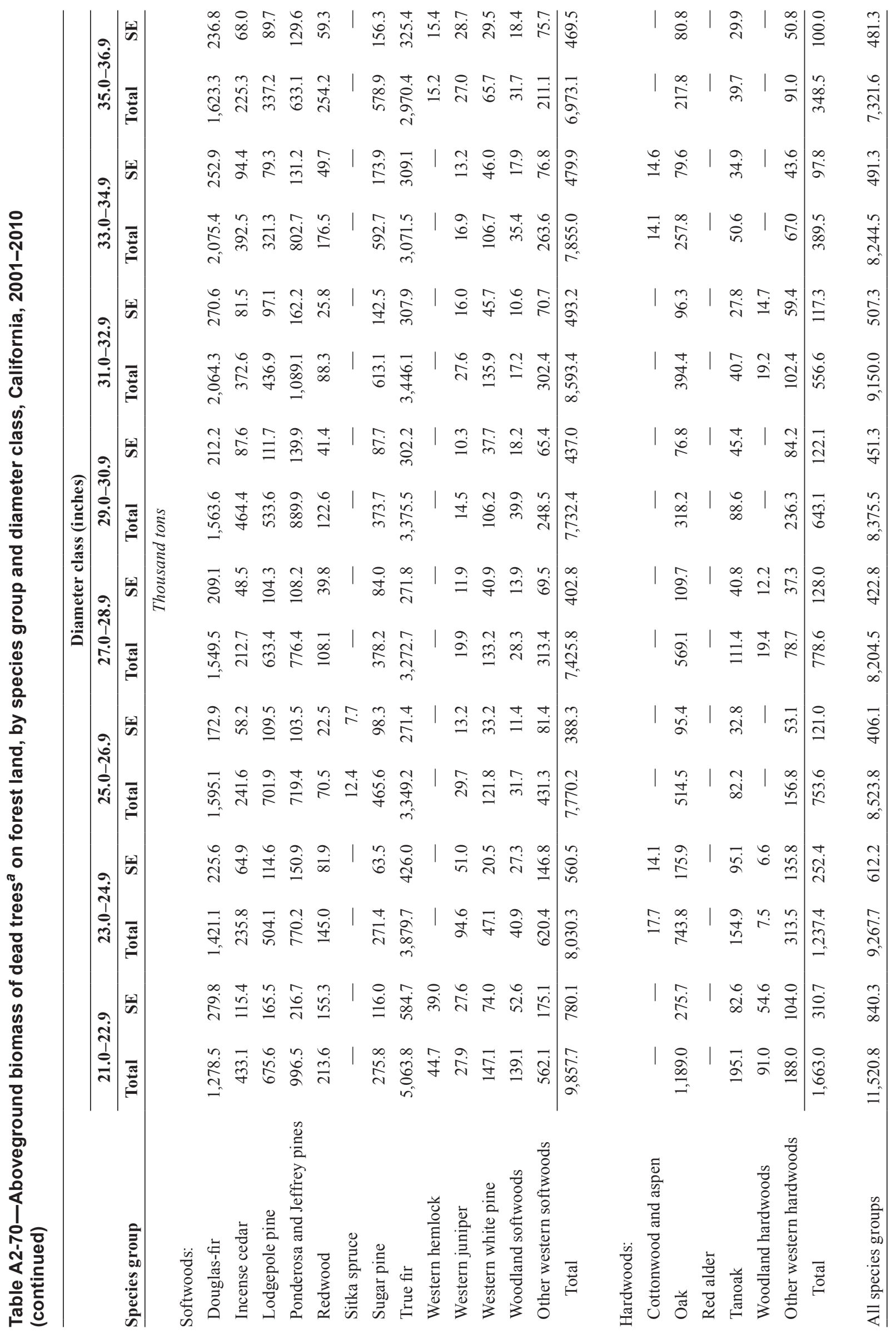




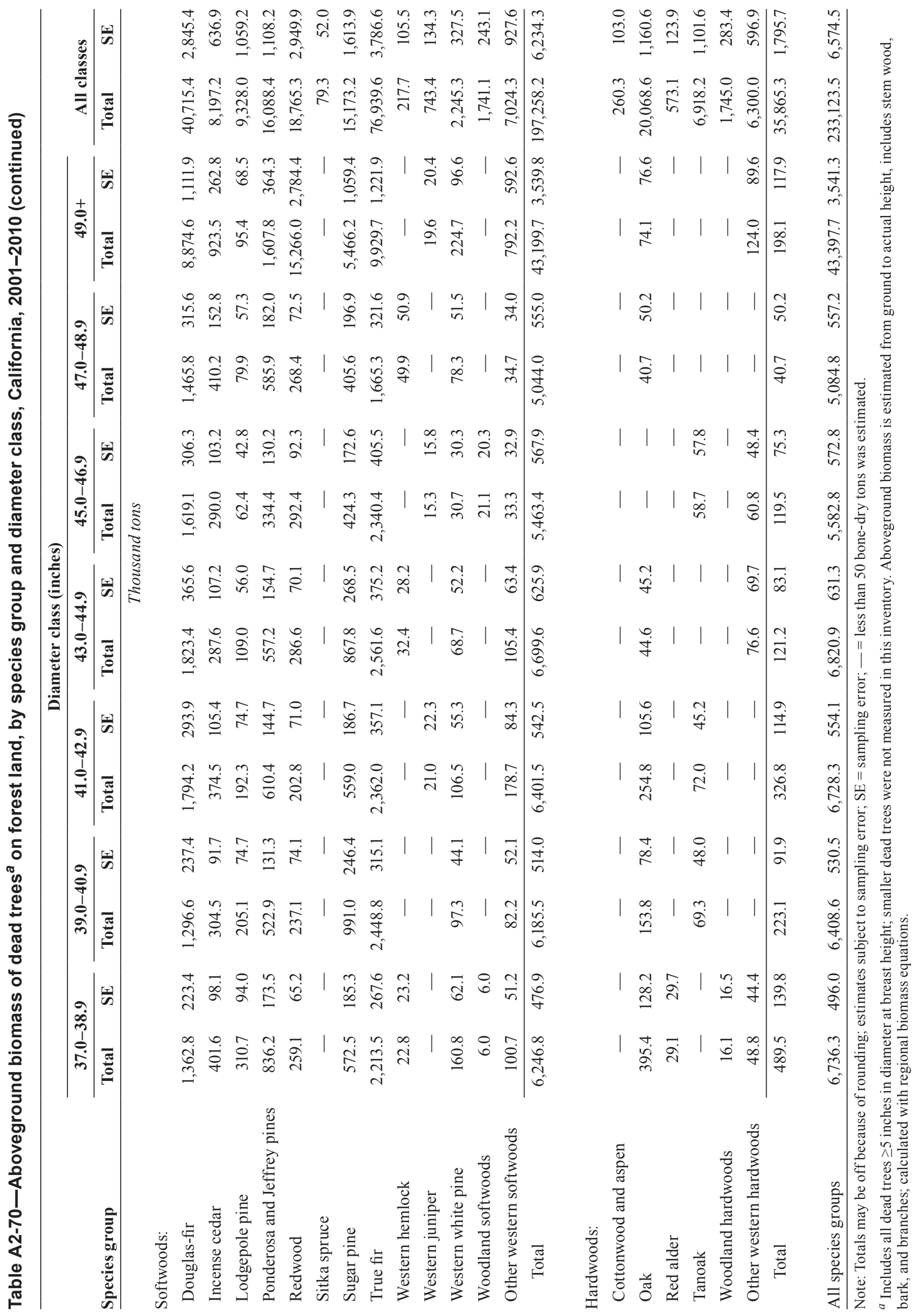


California's Forest Resources: Forest Inventory and Analysis, 2001-2010

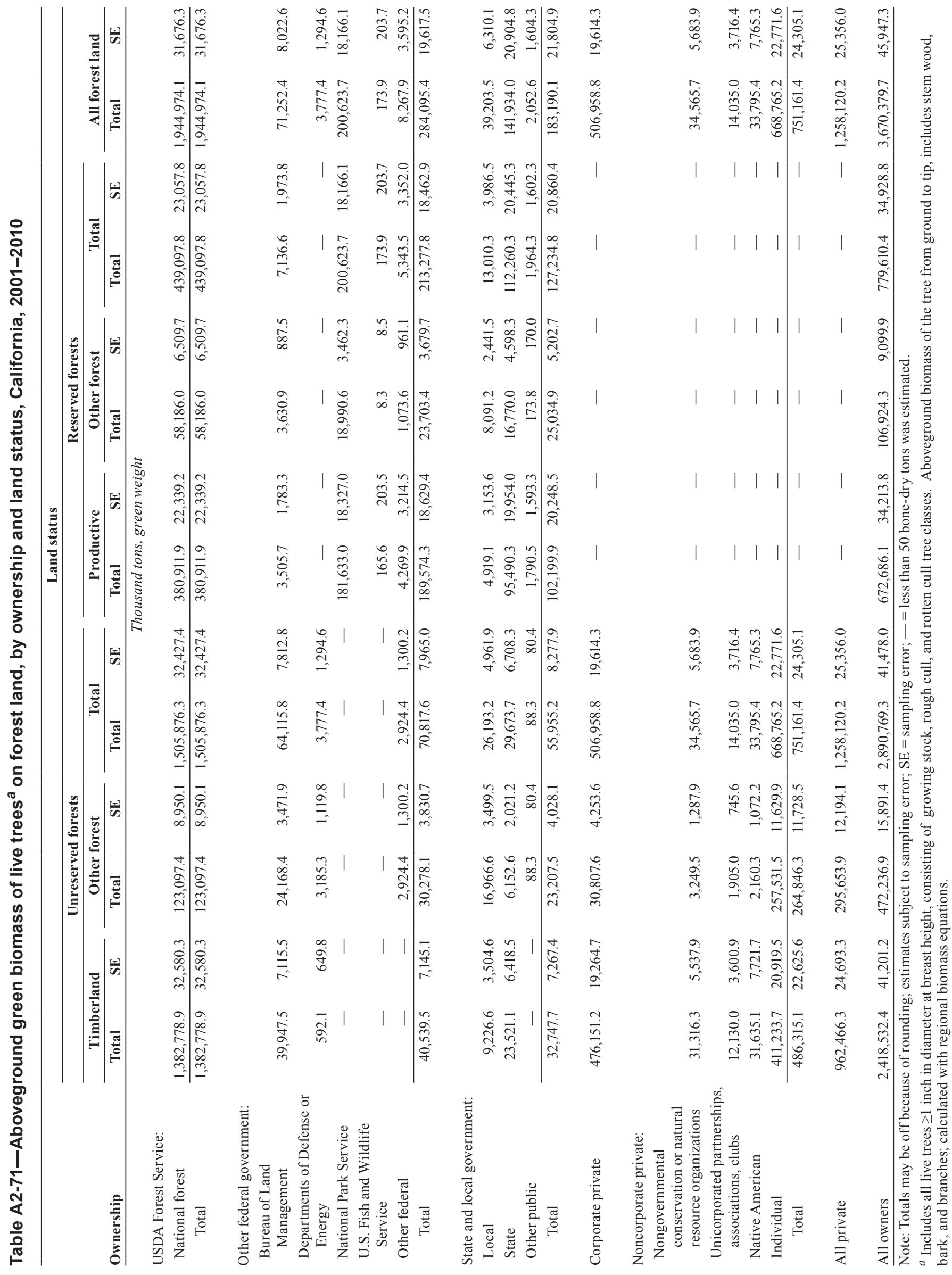




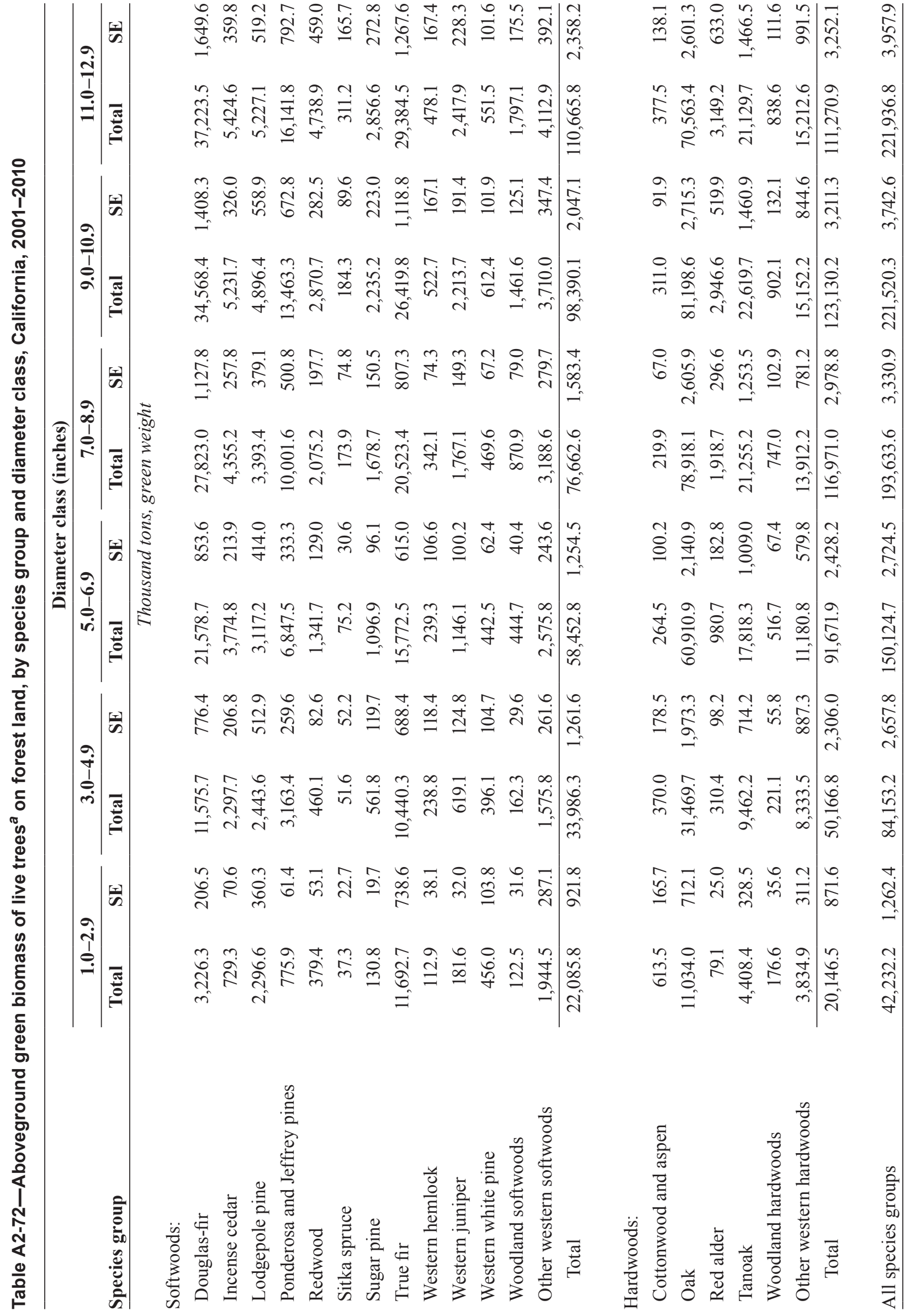




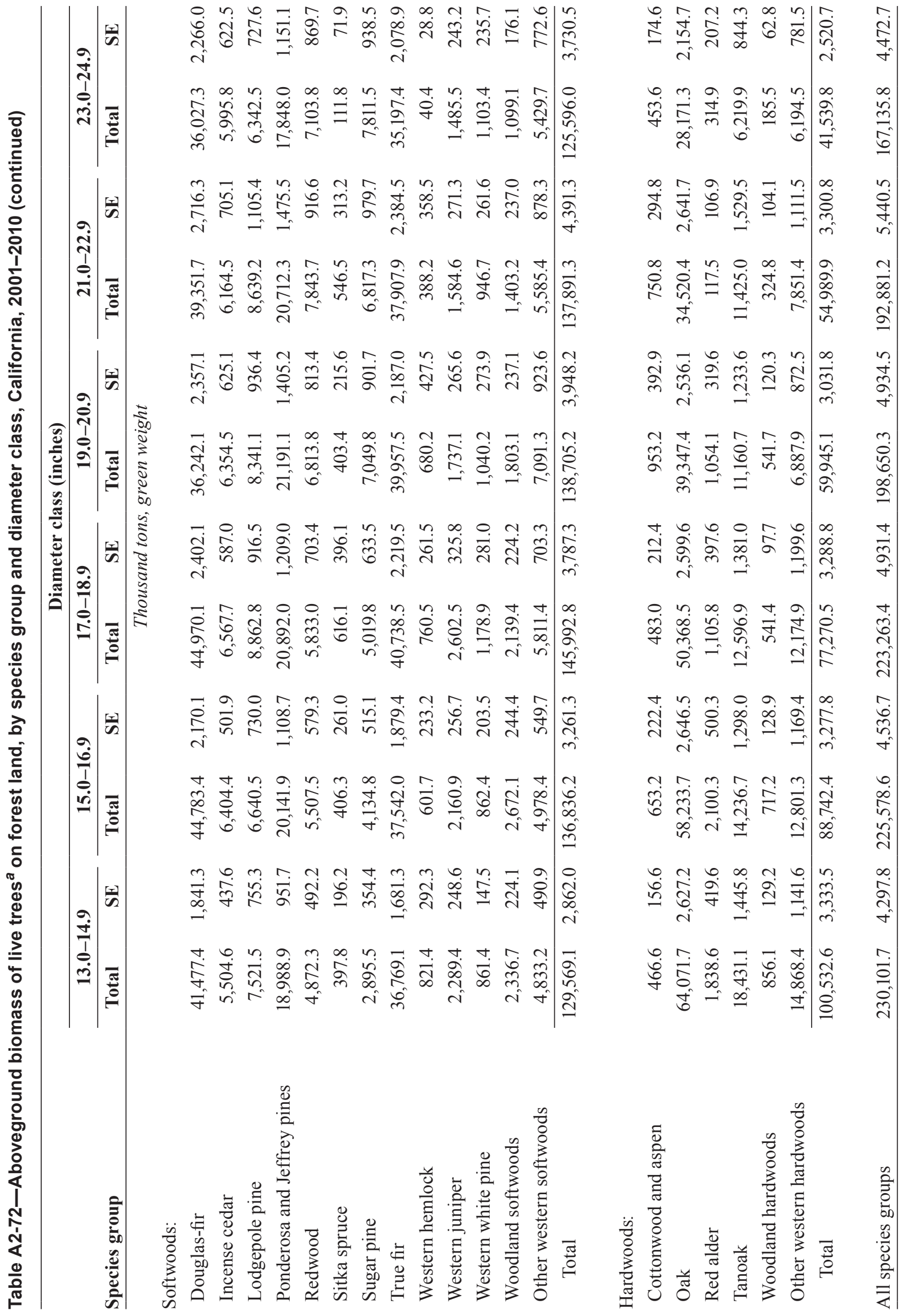




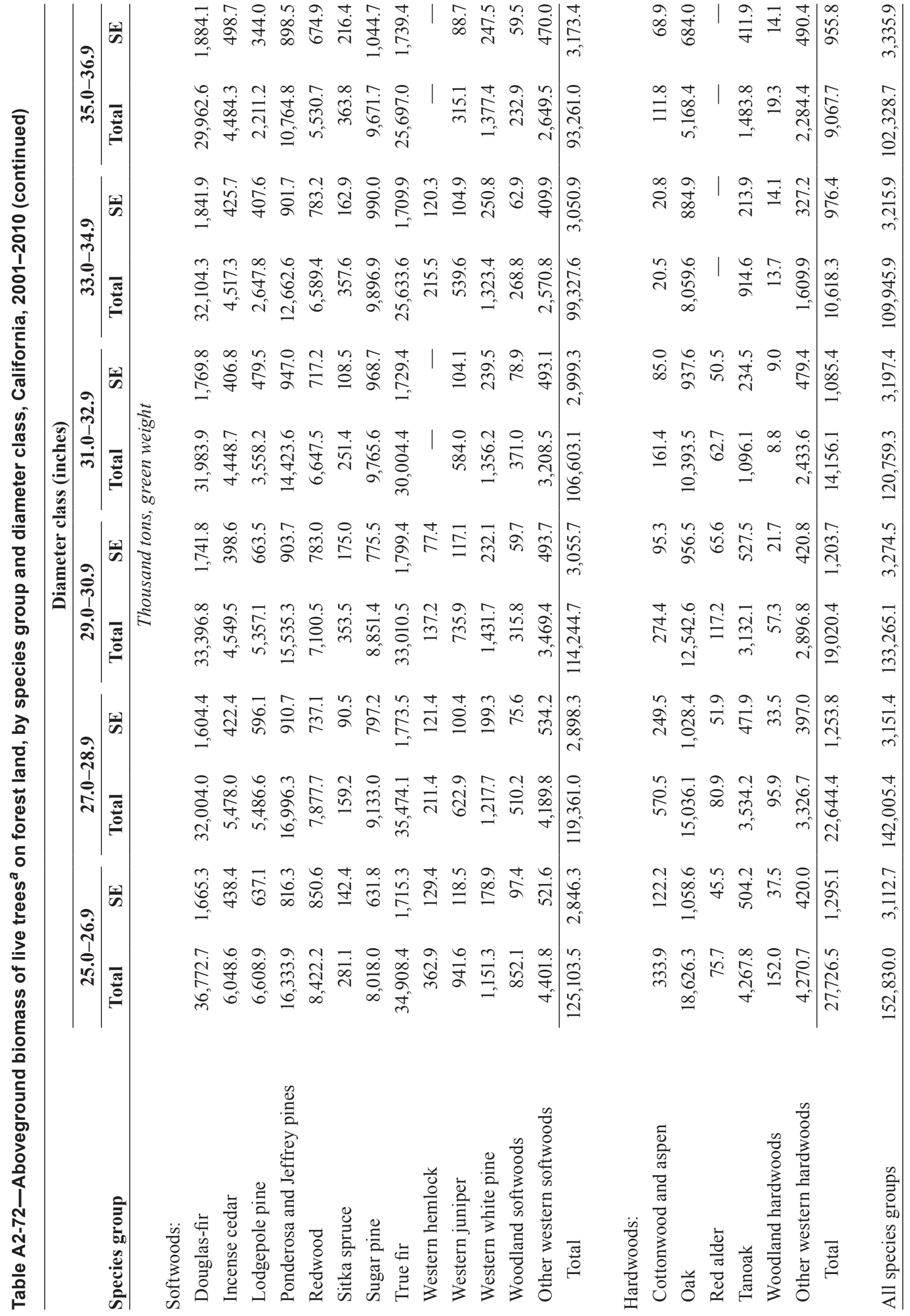




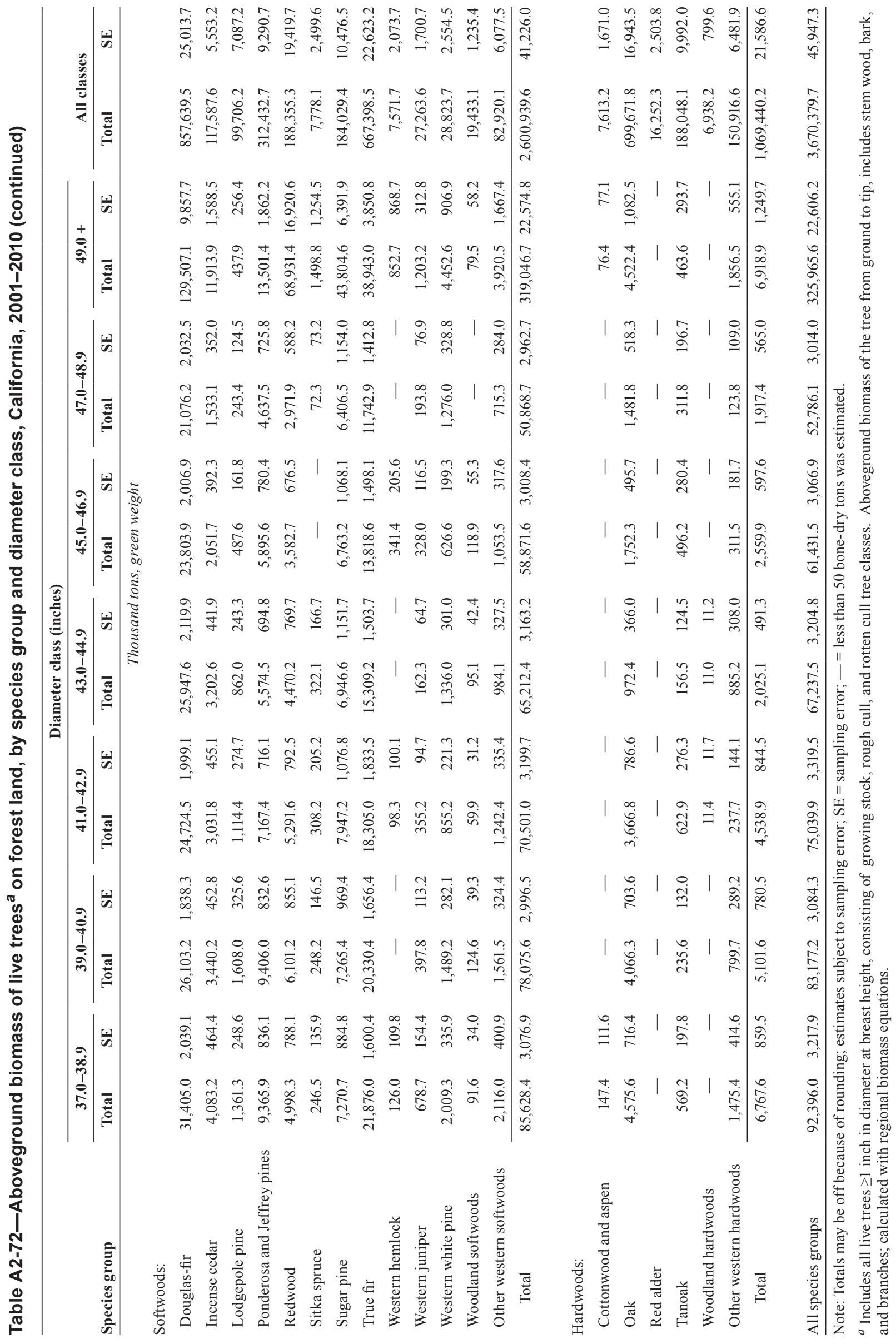




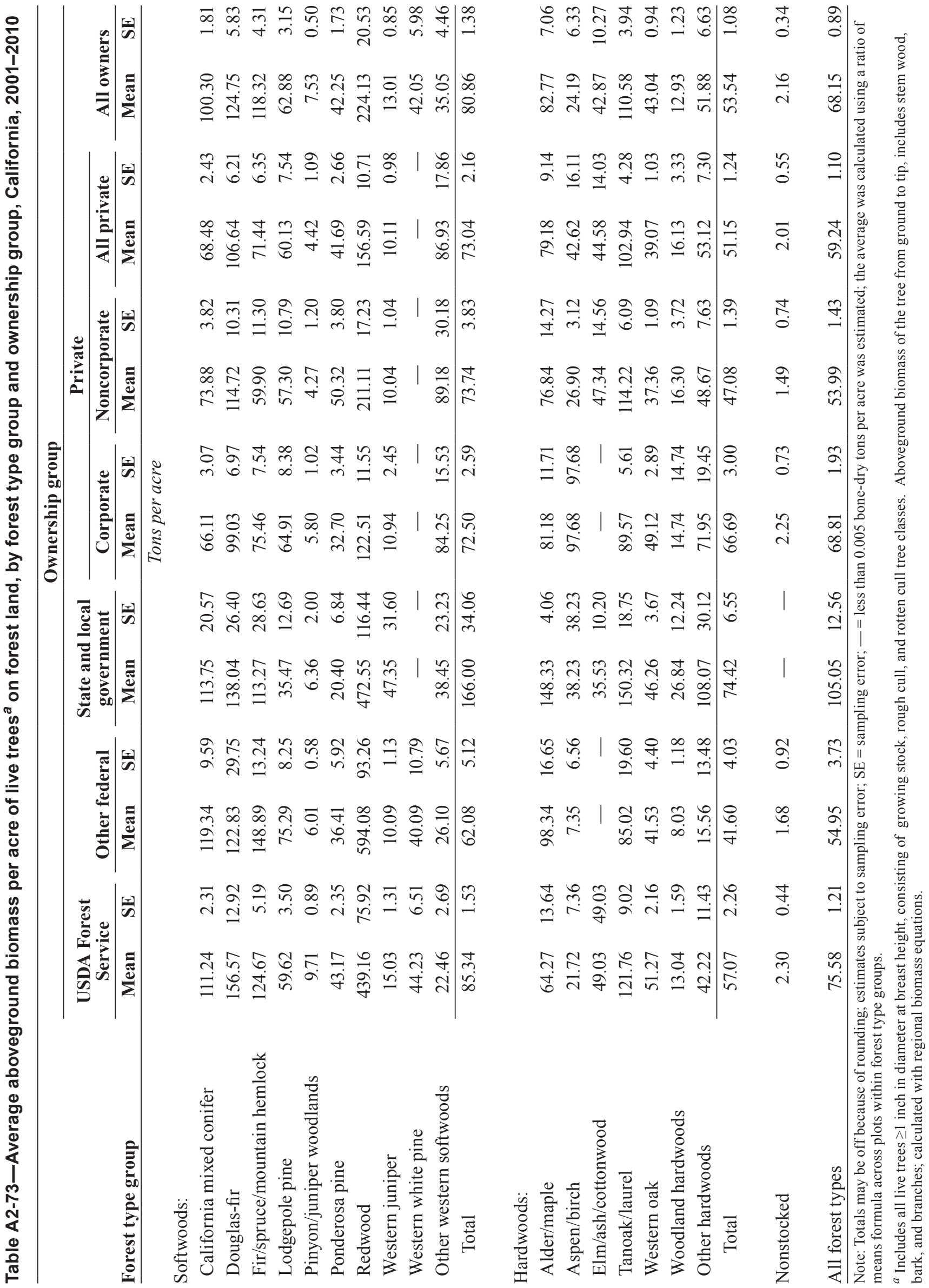




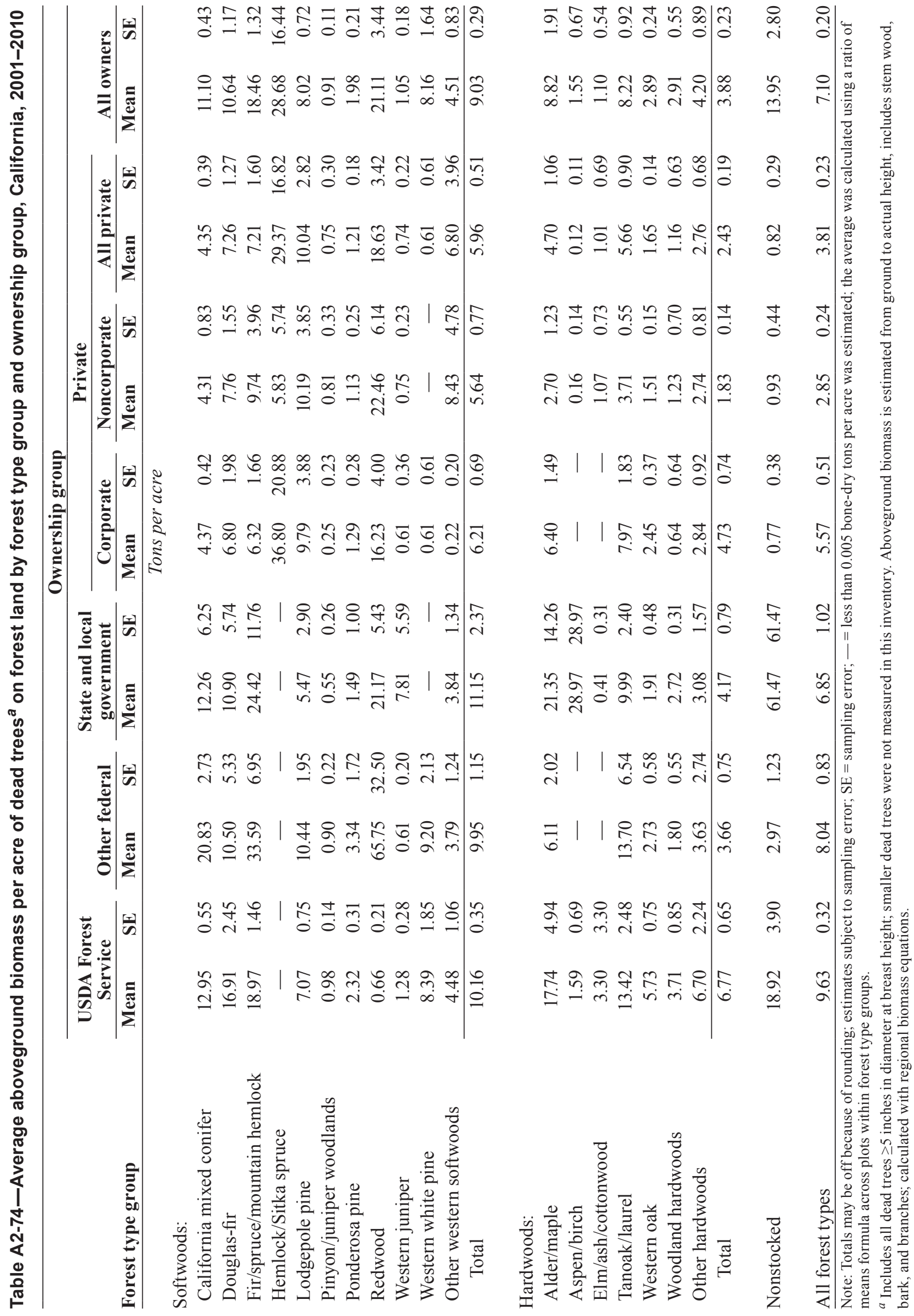


Table A2-75-Average aboveground biomass per acre of live trees ${ }^{a}$ on forest land, by forest type group and stand size class, California, 2001-2010

\begin{tabular}{|c|c|c|c|c|c|c|c|c|}
\hline \multirow[b]{3}{*}{ Forest type group } & \multicolumn{6}{|c|}{ Stand size class } & & \\
\hline & \multicolumn{2}{|c|}{$\begin{array}{c}\text { Large-diameter } \\
\text { stands }\end{array}$} & \multicolumn{2}{|c|}{$\begin{array}{c}\text { Medium-diameter } \\
\text { stands }\end{array}$} & \multicolumn{2}{|c|}{$\begin{array}{c}\text { Small-diameter } \\
\text { stands }\end{array}$} & \multicolumn{2}{|c|}{ All classes } \\
\hline & Mean & SE & Mean & $\mathbf{S E}$ & Mean & SE & Mean & SE \\
\hline & \multicolumn{8}{|c|}{ Tons per acre } \\
\hline \multicolumn{9}{|l|}{ Softwoods: } \\
\hline California mixed conifer & 106.45 & 1.85 & 29.76 & 3.99 & 9.68 & 1.30 & 100.30 & 1.81 \\
\hline Douglas-fir & 138.93 & 6.03 & 49.44 & 9.08 & 21.63 & 4.53 & 124.75 & 5.83 \\
\hline Fir/spruce/mountain hemlock & 125.77 & 4.38 & 31.89 & 8.22 & 12.03 & 2.14 & 118.32 & 4.31 \\
\hline Lodgepole pine & 65.72 & 3.32 & 54.54 & 10.84 & 10.23 & 5.28 & 62.88 & 3.15 \\
\hline Pinyon/juniper woodlands & 8.82 & 0.57 & 1.90 & 0.71 & 1.25 & 0.34 & 7.53 & 0.50 \\
\hline Ponderosa pine & 48.00 & 1.85 & 12.27 & 1.84 & 4.42 & 1.10 & 42.25 & 1.73 \\
\hline Redwood & 239.80 & 21.65 & 21.46 & 6.00 & 12.64 & 3.16 & 224.13 & 20.53 \\
\hline Western juniper & 14.82 & 0.97 & 6.11 & 1.43 & 2.23 & 0.67 & 13.01 & 0.85 \\
\hline Western white pine & 51.02 & 6.59 & 7.71 & 1.58 & 13.52 & 5.62 & 42.05 & 5.98 \\
\hline Other western softwoods & 48.25 & 6.03 & 11.01 & 2.59 & 6.00 & 1.27 & 35.05 & 4.46 \\
\hline Total & 89.35 & 1.50 & 17.51 & 1.79 & 8.17 & 0.74 & 80.86 & 1.38 \\
\hline
\end{tabular}

Hardwoods:

Alder/maple
Aspen/birch
Elm/ash/cottonwood
Tanoak/laurel
Western oak
Woodland hardwoods
Other hardwoods
$\quad$ Total

Nonstocked

\begin{tabular}{rrrrrrrr}
105.61 & 7.39 & 58.36 & 11.24 & 17.62 & 6.83 & 82.77 & 7.06 \\
51.12 & 11.94 & 42.93 & 14.22 & 9.52 & 2.75 & 24.19 & 6.33 \\
51.71 & 10.58 & 3.28 & 3.03 & 10.54 & 1.05 & 42.87 & 10.27 \\
139.84 & 4.64 & 77.03 & 4.35 & 18.50 & 3.06 & 110.58 & 3.94 \\
56.75 & 1.47 & 34.70 & 1.22 & 13.09 & 0.98 & 43.04 & 0.94 \\
14.02 & 1.32 & 10.74 & 3.83 & 2.25 & 0.84 & 12.93 & 1.23 \\
113.93 & 12.74 & 34.75 & 6.35 & 5.53 & 1.04 & 51.88 & 6.63 \\
\hline 72.85 & 1.72 & 39.40 & 1.25 & 12.71 & 0.83 & 53.54 & 1.08
\end{tabular}

\section{All forest types}

${ }^{a}$ Includes all live trees $\geq 1$ inch in diameter at breast height, consisting of growing stock, rough cull, and rotten cull tree classes. Aboveground biomass of the tree from ground to tip, includes stem wood, bark, and branches; calculated with regional biomass equations. 
Table A2-76-Average aboveground biomass per acre of dead trees ${ }^{a}$ on forest land, by forest type group and stand size class, California, 2001-2010

\begin{tabular}{|c|c|c|c|c|c|c|c|c|}
\hline \multirow[b]{3}{*}{ Forest type group } & \multicolumn{6}{|c|}{ Stand size class } & & \\
\hline & \multicolumn{2}{|c|}{$\begin{array}{c}\text { Large-diameter } \\
\text { stands }\end{array}$} & \multicolumn{2}{|c|}{$\begin{array}{c}\text { Medium-diameter } \\
\text { stands }\end{array}$} & \multicolumn{2}{|c|}{$\begin{array}{c}\text { Small-diameter } \\
\text { stands }\end{array}$} & \multicolumn{2}{|c|}{ All classes } \\
\hline & Mean & SE & Mean & SE & Mean & SE & Mean & SE \\
\hline & \multicolumn{8}{|c|}{ Tons per acre } \\
\hline \multicolumn{9}{|l|}{ Softwoods: } \\
\hline California mixed conifer & 11.59 & 0.45 & 4.41 & 1.45 & 4.49 & 1.62 & 11.10 & 0.43 \\
\hline Douglas-fir & 11.14 & 1.27 & 1.45 & 0.79 & 11.02 & 5.01 & 10.64 & 1.17 \\
\hline Fir/spruce/mountain hemlock & 18.17 & 1.21 & 16.37 & 9.02 & 23.68 & 12.01 & 18.46 & 1.32 \\
\hline Hemlock/Sitka spruce & 31.90 & 17.92 & - & - & 0.76 & 0.76 & 28.68 & 16.44 \\
\hline Lodgepole pine & 8.39 & 0.75 & 8.08 & 4.49 & 0.21 & 0.19 & 8.02 & 0.72 \\
\hline Pinyon/juniper woodlands & 0.86 & 0.10 & 1.33 & 0.66 & 0.86 & 0.41 & 0.91 & 0.11 \\
\hline Ponderosa pine & 2.17 & 0.24 & 0.58 & 0.26 & 1.15 & 0.60 & 1.98 & 0.2 \\
\hline Redwood & 22.22 & 3.67 & 8.51 & 2.02 & 4.78 & 2.43 & 21.11 & \\
\hline Western juniper & 1.24 & 0.21 & 0.19 & 0.14 & 0.04 & 0.02 & 1.05 & 0.1 \\
\hline Western white pine & 9.87 & 1.99 & 1.57 & 0.32 & 2.70 & 1.47 & 8.16 & \\
\hline Other western softwoods & 5.82 & 1.08 & 1.30 & 0.62 & 2.56 & 1.87 & 4.51 & 0.83 \\
\hline Total & 9.65 & 0.31 & 2.52 & 0.53 & 5.09 & 1.40 & 9.03 & 0.2 \\
\hline
\end{tabular}

Hardwoods:

\begin{tabular}{|c|c|c|c|c|c|c|c|c|}
\hline Alder/maple & 10.47 & 2.84 & 6.72 & 2.50 & 4.88 & 2.25 & 8.82 & 1.91 \\
\hline Aspen/birch & 2.84 & 1.67 & 4.36 & 2.40 & 0.20 & 0.14 & 1.55 & 0.67 \\
\hline Elm/ash/cottonwood & 0.61 & 0.40 & - & - & 4.01 & 0.40 & 1.10 & 0.54 \\
\hline Tanoak/laurel & 9.19 & 1.01 & 3.53 & 1.10 & 11.59 & 4.59 & 8.22 & 0.92 \\
\hline Western oak & 3.12 & 0.22 & 1.67 & 0.15 & 5.18 & 1.46 & 2.89 & 0.24 \\
\hline Woodland hardwoods & 2.72 & 0.47 & 5.35 & 2.92 & - & - & 2.91 & 0.55 \\
\hline Other hardwoods & 7.67 & 1.58 & 1.91 & 0.62 & 2.55 & 1.62 & 4.20 & 0.89 \\
\hline Total & 4.53 & 0.27 & 2.03 & 0.19 & 5.51 & 1.20 & 3.88 & 0.23 \\
\hline Nonstocked & - & - & - & - & - & - & 13.95 & 2.80 \\
\hline All forest types & 8.15 & 0.23 & 2.12 & 0.18 & 5.35 & 0.91 & 7.10 & 0.20 \\
\hline
\end{tabular}

Note: Totals may be off because of rounding; estimates subject to sampling error; $\mathrm{SE}=$ sampling error; $-=$ less than 0.005 bone-dry tons per acre was estimated; the average was calculated using a ratio of means formula across plots within forest type groups.

${ }^{a}$ Includes all dead trees $\geq 5$ inches in diameter at breast height; smaller dead trees were not measured in this inventory. Aboveground biomass is estimated from ground to actual height, includes stem wood, bark, and branches; calculated with regional biomass equations. 


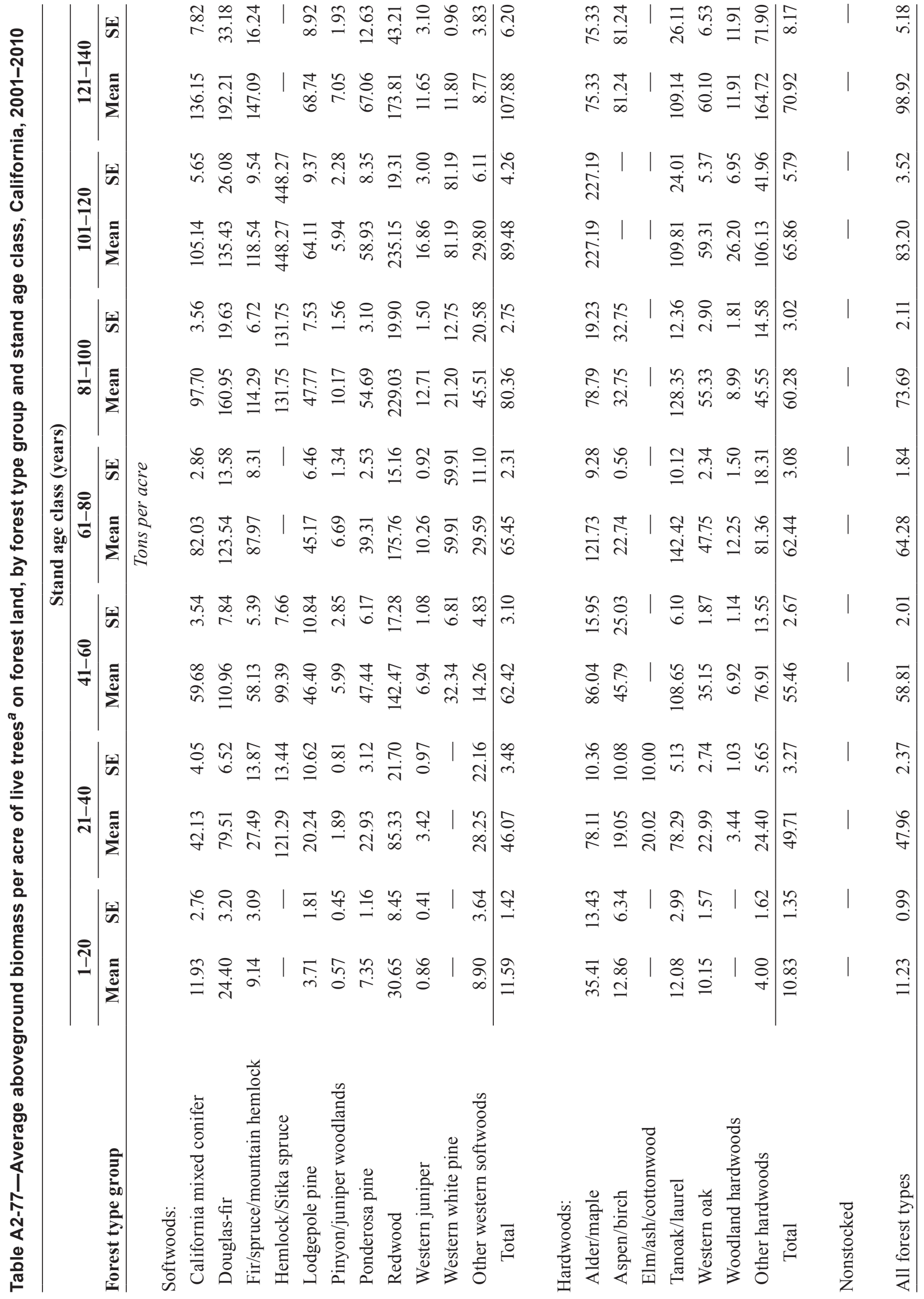




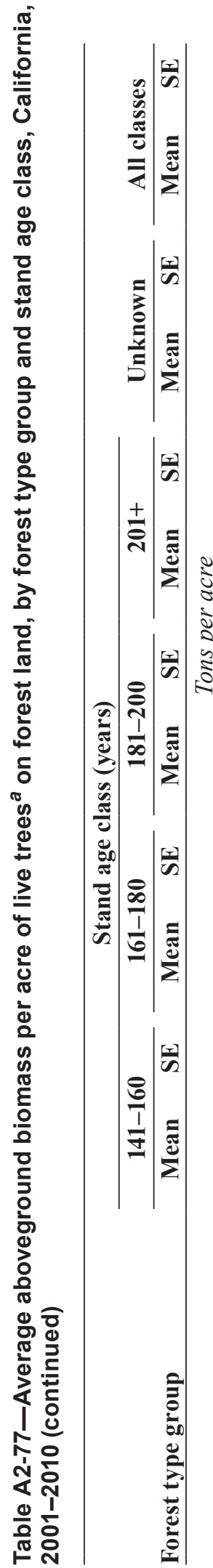

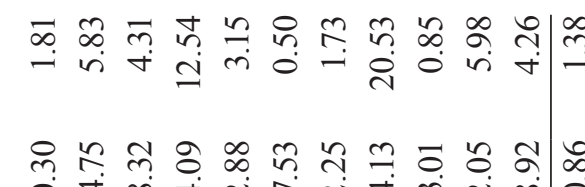

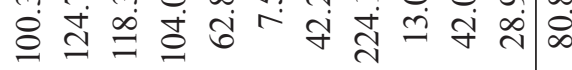

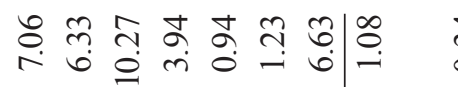

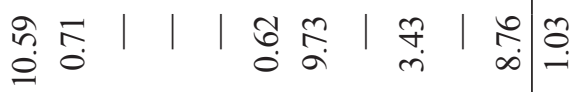

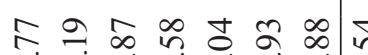

तं

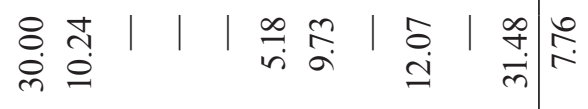

กิ่

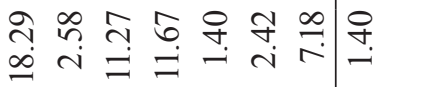

苯

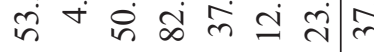

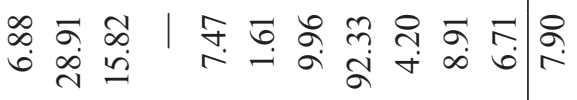

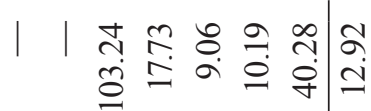

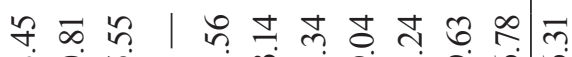

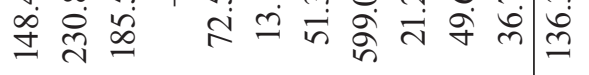

| | ț

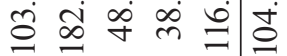

끌둥

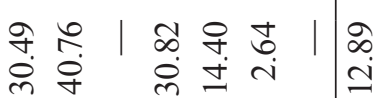

กิ mี

વิ

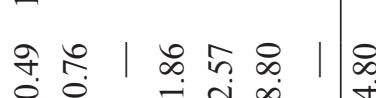

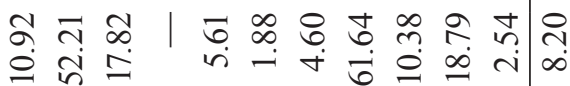

Oे

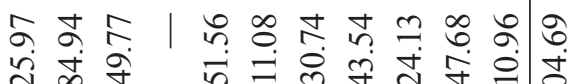

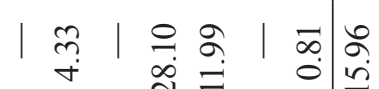

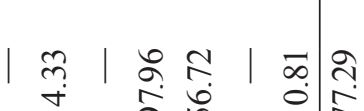

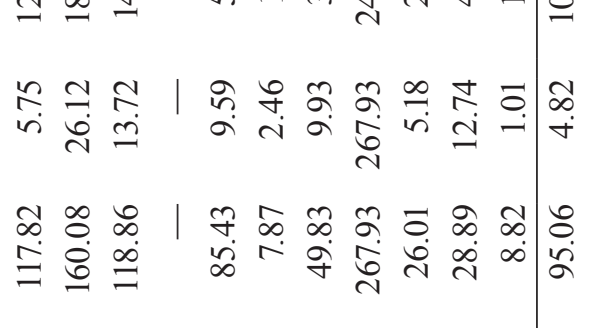

分

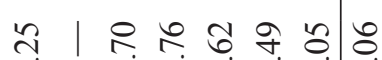

๙

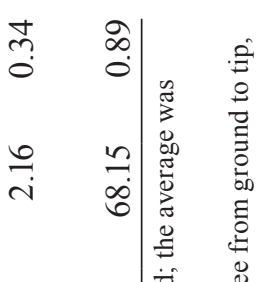




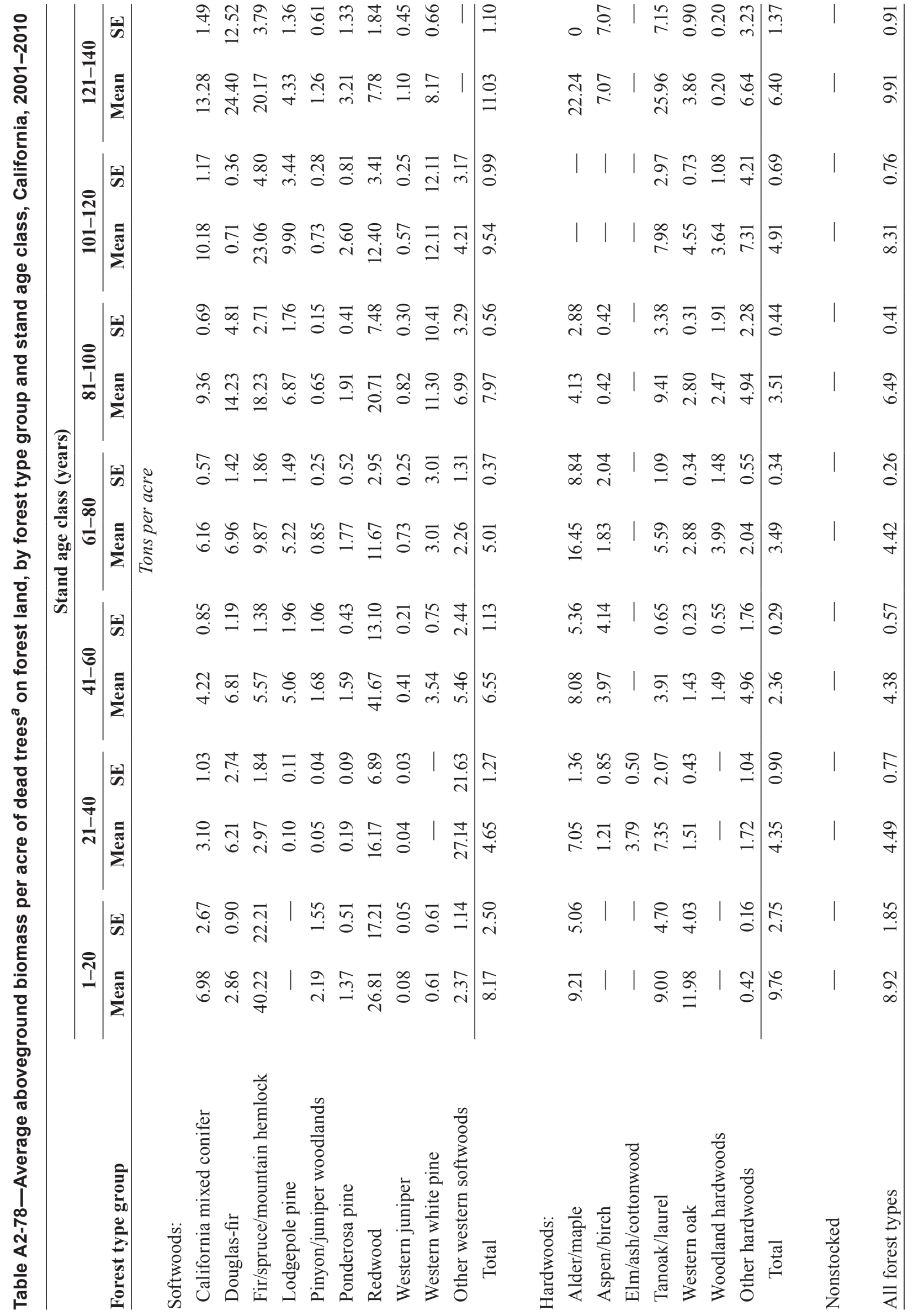




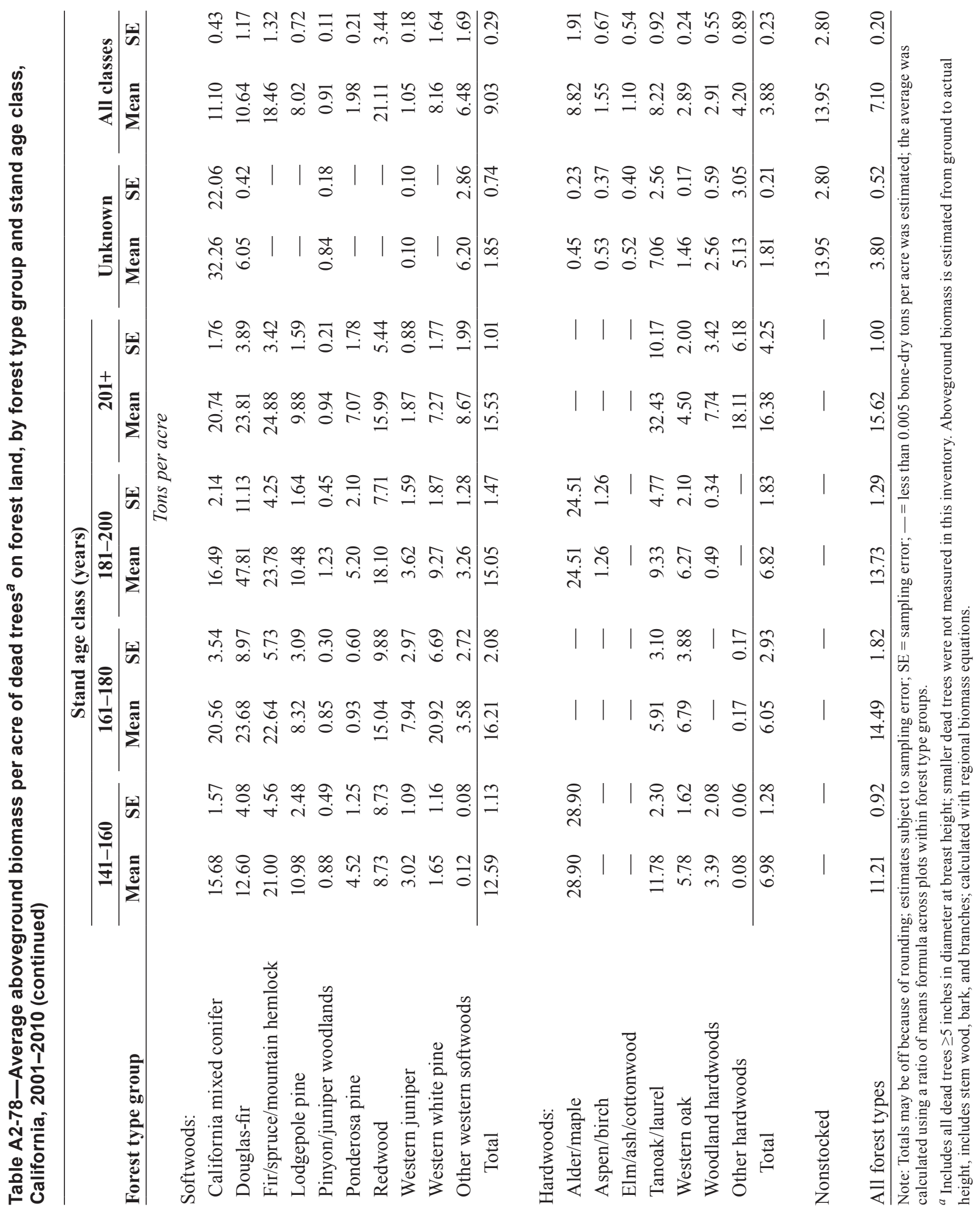




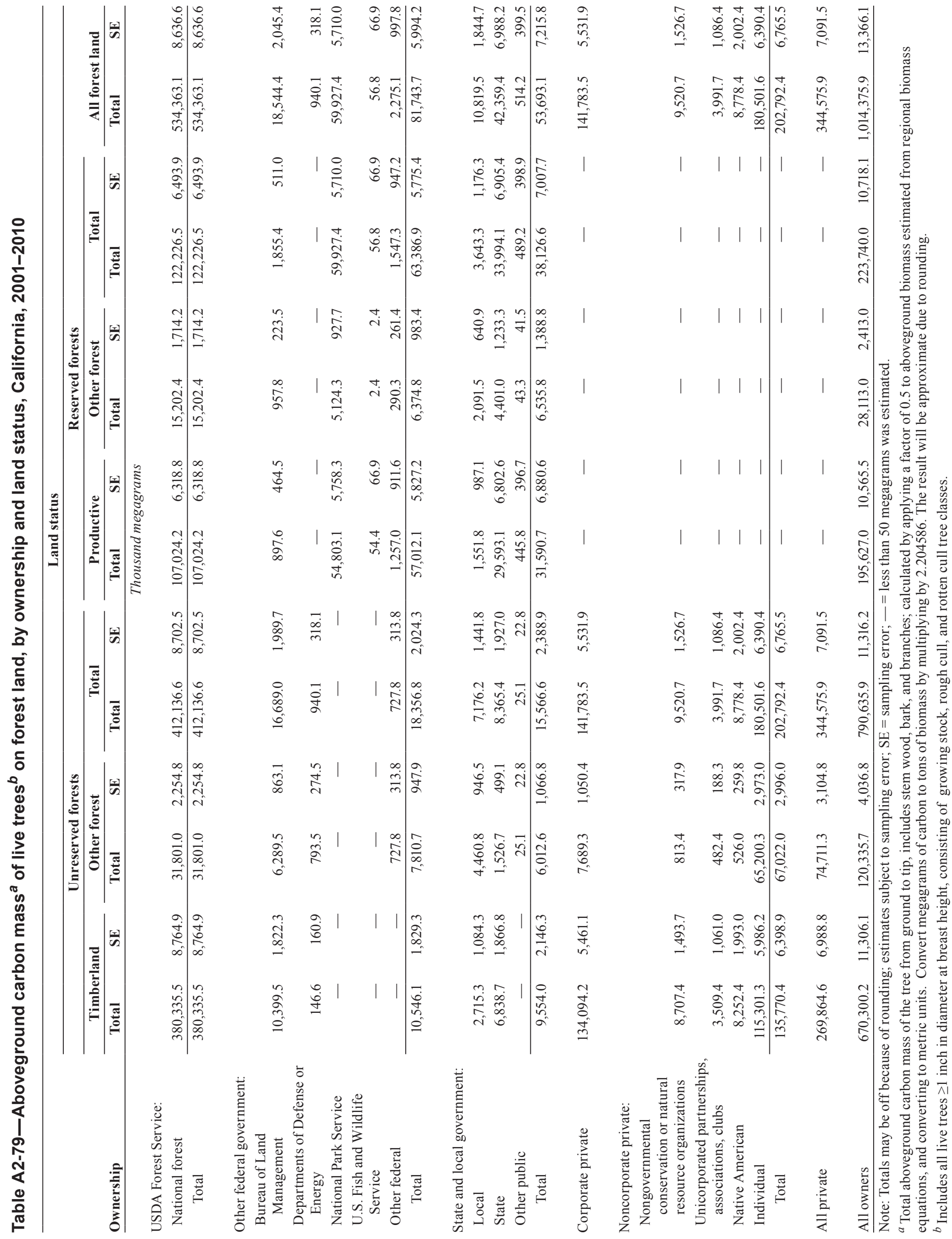


California's Forest Resources: Forest Inventory and Analysis, 2001-2010

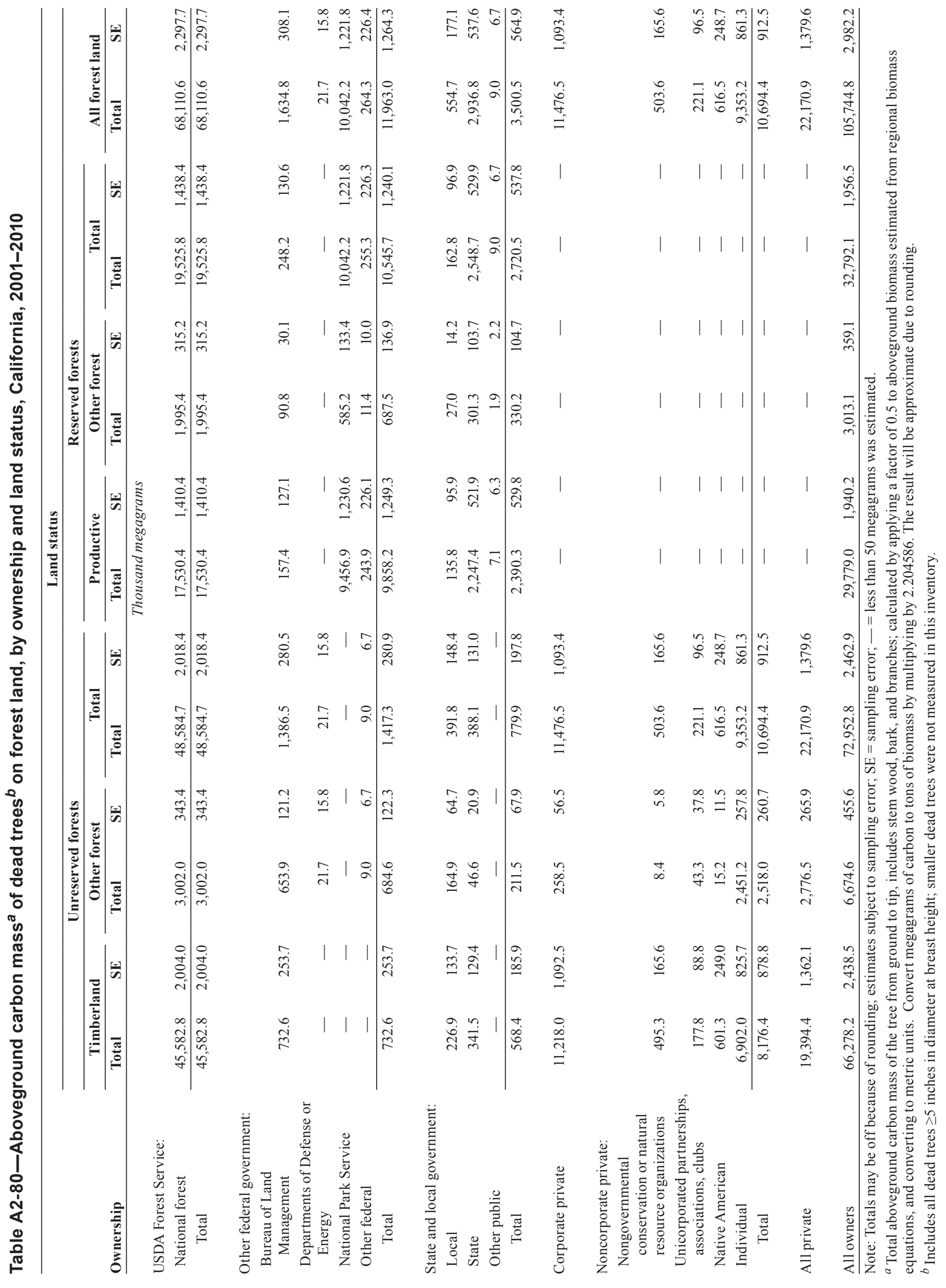




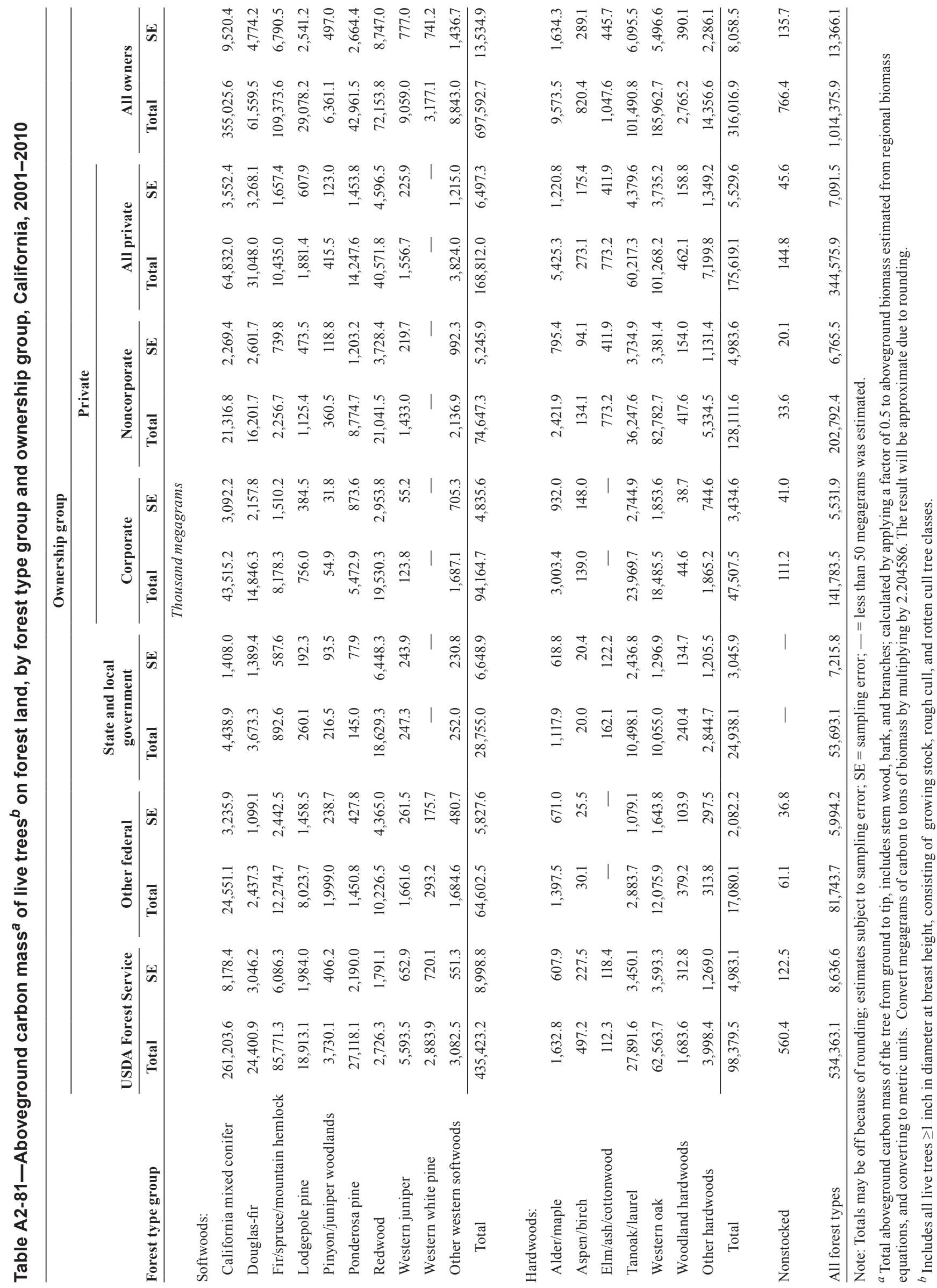




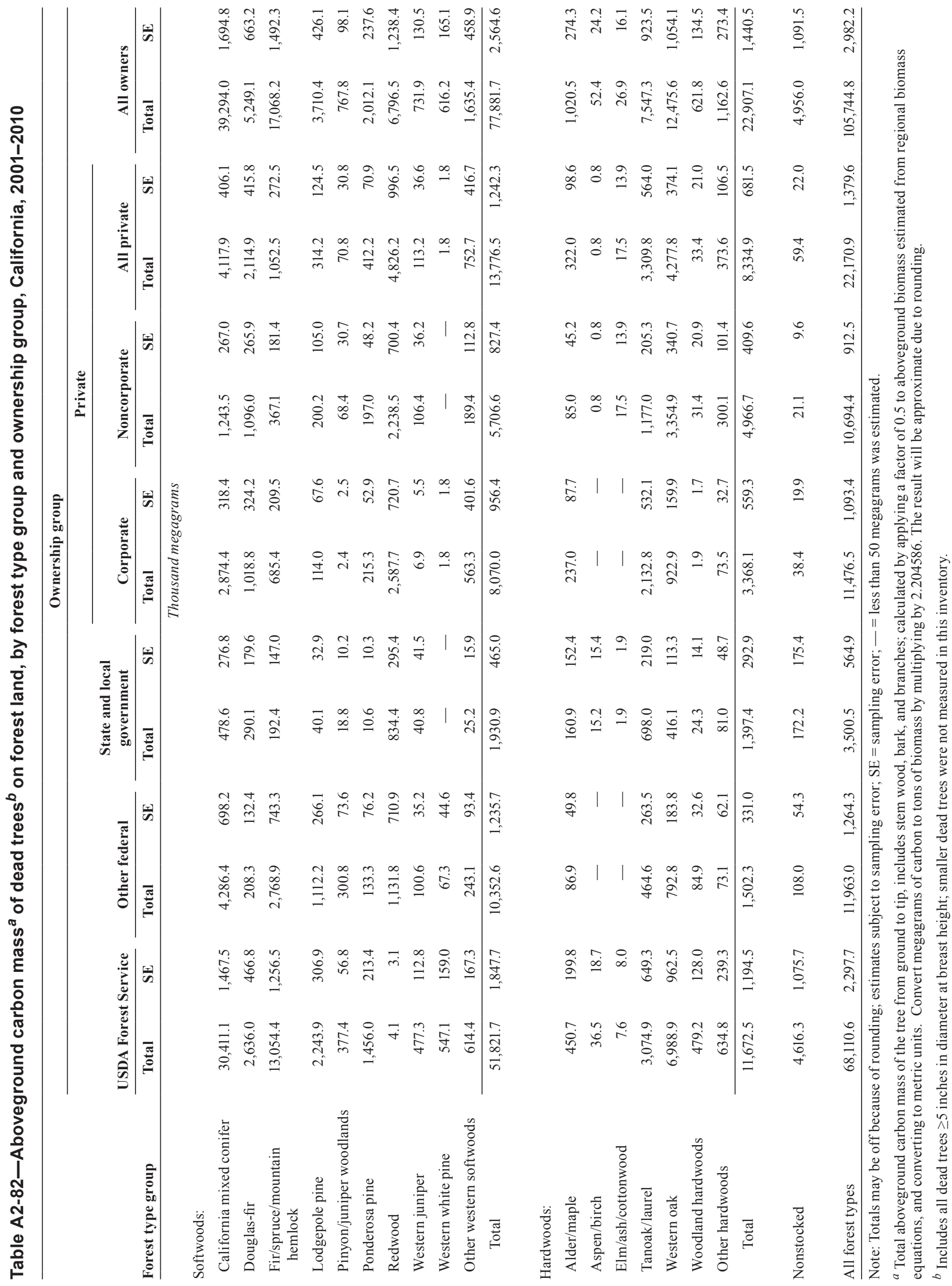


Table A2-83-Aboveground carbon mass ${ }^{a}$ of live trees ${ }^{b}$ on forest land, by forest type group and stand size class, California, 2001-2010

\begin{tabular}{|c|c|c|c|c|c|c|c|c|}
\hline \multirow[b]{3}{*}{ Forest type group } & \multicolumn{6}{|c|}{ Stand size class } & & \\
\hline & \multicolumn{2}{|c|}{$\begin{array}{c}\text { Large-diameter } \\
\text { stands }\end{array}$} & \multicolumn{2}{|c|}{$\begin{array}{c}\text { Medium-diameter } \\
\text { stands }\end{array}$} & \multicolumn{2}{|c|}{$\begin{array}{c}\text { Small-diameter } \\
\text { stands }\end{array}$} & \multicolumn{2}{|c|}{ All classes } \\
\hline & Total & SE & Total & SE & Total & SE & Total & SE \\
\hline & \multicolumn{8}{|c|}{ Thousand megagrams } \\
\hline \multicolumn{9}{|l|}{ Softwoods: } \\
\hline California mixed conifer & $351,065.7$ & $9,537.3$ & $2,402.1$ & 533.7 & $1,557.8$ & 280.1 & $355,025.6$ & $9,520.4$ \\
\hline Douglas-fir & $59,441.5$ & $4,759.5$ & $1,242.8$ & 454.0 & 875.2 & 292.9 & $61,559.5$ & $4,774.2$ \\
\hline Fir/spruce/mountain hemlock & $108,419.0$ & $6,783.7$ & 328.4 & 174.4 & 626.2 & 179.1 & $109,373.6$ & $6,790.5$ \\
\hline Lodgepole pine & $27,834.3$ & $2,495.3$ & $1,041.1$ & 456.5 & 202.8 & 127.4 & $29,078.2$ & $2,541.2$ \\
\hline Pinyon/juniper woodlands & $6,113.3$ & 498.4 & 170.7 & 69.7 & 77.2 & 26.4 & $6,361.1$ & 497.0 \\
\hline Ponderosa pine & $41,676.7$ & $2,660.8$ & 981.9 & 205.5 & 303.0 & 96.9 & $42,961.5$ & $2,664.4$ \\
\hline Redwood & $71,781.7$ & $8,747.8$ & 210.4 & 117.8 & 161.7 & 84.3 & $72,153.8$ & $8,747.0$ \\
\hline Western juniper & $8,586.9$ & 773.1 & 332.9 & 93.7 & 139.2 & 51.5 & $9,059.0$ & 777.0 \\
\hline Western white pine & $2,958.9$ & 731.9 & 25.5 & 25.2 & 192.7 & 115.3 & $3,177.1$ & 741.2 \\
\hline Other western softwoods & $8,182.0$ & $1,425.3$ & 361.9 & 137.6 & 299.1 & 92.9 & $8,843.0$ & $1,436.7$ \\
\hline Total & $686,060.0$ & $13,599.3$ & $7,097.8$ & 903.3 & $4,434.9$ & 499.2 & $697,592.7$ & $13,534.9$ \\
\hline
\end{tabular}

Hardwoods:

\begin{tabular}{lrrrrrrrr} 
Alder/maple & $7,538.2$ & $1,512.0$ & $1,797.7$ & 616.4 & 237.6 & 148.3 & $9,573.5$ & $1,634.3$ \\
Aspen/birch & 326.9 & 206.7 & 297.5 & 183.4 & 196.0 & 85.4 & 820.4 & 289.1 \\
Elm/ash/cottonwood & $1,004.1$ & 444.2 & 4.2 & 3.3 & 39.4 & 35.9 & $1,047.6$ & 445.7 \\
Tanoak/laurel & $83,528.6$ & $5,921.7$ & $15,836.9$ & $1,988.0$ & $2,125.2$ & 462.3 & $101,490.8$ & $6,095.5$ \\
Western oak & $125,447.4$ & $5,027.1$ & $52,833.6$ & $2,751.9$ & $7,681.7$ & 755.3 & $185,962.7$ & $5,496.6$ \\
Woodland hardwoods & $2,441.1$ & 371.2 & 296.8 & 119.7 & 27.3 & 16.0 & $2,765.2$ & 390.1 \\
Other hardwoods & $11,221.8$ & $2,184.6$ & $2,556.3$ & 675.2 & 578.4 & 143.3 & $14,356.6$ & $2,286.1$ \\
\cline { 2 - 8 }$\quad$ Total & $231,508.0$ & $7,843.7$ & $73,623.2$ & $3,457.5$ & $10,885.7$ & 912.9 & $316,016.9$ & $8,058.5$
\end{tabular}

Nonstocked

$\begin{array}{r}05.7 \\ 41.5 \\ 419.0 \\ 834.3 \\ 13.3 \\ 676.7 \\ 81.7 \\ 86.9 \\ 88.9 \\ 82.0 \\ \hline 60.0 \\ \\ \hline 8.2 \\ \hline 26.9 \\ \hline 2.1 \\ \hline\end{array}$

$766.4 \quad 135.7$

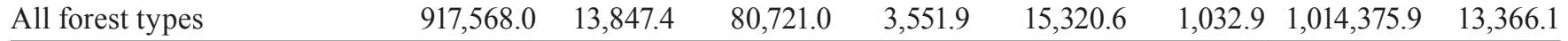

Note: Totals may be off because of rounding; estimates subject to sampling error; SE = sampling error; — = less than 50 megagrams was estimated.

${ }^{a}$ Total aboveground carbon mass of the tree from ground to tip, includes stem wood, bark, and branches; calculated by applying a factor of 0.5 to aboveground biomass estimated from regional biomass equations, and converting to metric units. Convert megagrams of carbon to tons of biomass by multiplying by 2.204586 . The result will be approximate due to rounding.

${ }^{b}$ Includes all live trees $\geq 1$ inch in diameter at breast height, consisting of growing stock, rough cull, and rotten cull tree classes. 
Table A2-84-Aboveground carbon mass ${ }^{a}$ of dead trees ${ }^{b}$ on forest land, by forest type group and stand size class, California, 2001-2010

\begin{tabular}{|c|c|c|c|c|c|c|c|c|}
\hline \multirow[b]{3}{*}{ Forest type group } & \multicolumn{8}{|c|}{ Stand size class } \\
\hline & \multicolumn{2}{|c|}{$\begin{array}{c}\text { Large-diameter } \\
\text { stands }\end{array}$} & \multicolumn{2}{|c|}{$\begin{array}{c}\text { Medium-diameter } \\
\text { stands }\end{array}$} & \multicolumn{2}{|c|}{$\begin{array}{c}\text { Small-diameter } \\
\text { stands }\end{array}$} & \multicolumn{2}{|c|}{ All classes } \\
\hline & Total & SE & Total & $\mathbf{S E}$ & Total & SE & Total & SE \\
\hline & \multicolumn{8}{|c|}{ Thousand megagrams } \\
\hline \multicolumn{9}{|l|}{ Softwoods: } \\
\hline California mixed conifer & $38,215.7$ & $1,674.8$ & 356.0 & 134.3 & 722.3 & 275.1 & $39,294.0$ & $1,694.8$ \\
\hline Douglas-fir & $4,766.9$ & 624.2 & 36.4 & 23.2 & 445.9 & 224.9 & $5,249.1$ & 663.2 \\
\hline Fir/spruce/mountain hemlock & $15,667.2$ & $1,328.2$ & 168.6 & 123.8 & $1,232.4$ & 689.2 & $17,068.2$ & $1,492.3$ \\
\hline Lodgepole pine & $3,552.0$ & 413.1 & 154.3 & 104.5 & 4.1 & 4.1 & $3,710.4$ & 426.1 \\
\hline Pinyon/juniper woodlands & 595.5 & 72.6 & 119.1 & 62.9 & 53.1 & 28.1 & 767.8 & 98.1 \\
\hline Ponderosa pine & $1,887.1$ & 232.8 & 46.0 & 21.5 & 79.0 & 43.5 & $2,012.1$ & 237.6 \\
\hline Redwood & $6,651.9$ & $1,232.5$ & 83.5 & 50.3 & 61.1 & 35.4 & $6,796.5$ & $1,238.4$ \\
\hline Western juniper & 719.4 & 130.3 & 10.2 & 7.4 & 2.3 & 1.3 & 731.9 & 130.5 \\
\hline Western white pine & 572.5 & 162.8 & 5.2 & 5.1 & 38.5 & 26.8 & 616.2 & 165.1 \\
\hline Other western softwoods & $1,468.8$ & 448.3 & 42.7 & 23.1 & 123.8 & 94.8 & $1,635.4$ & 458.9 \\
\hline Total & $74,097.1$ & $2,461.3$ & $1,022.0$ & 228.8 & $2,762.6$ & 783.8 & $77,881.7$ & $2,564.6$ \\
\hline
\end{tabular}

Hardwoods:

\begin{tabular}{|c|c|c|c|c|c|c|c|c|}
\hline Alder/maple & 747.7 & 250.4 & 207.0 & 103.2 & 65.8 & 44.1 & $1,020.5$ & 274. \\
\hline Aspen/birch & 18.1 & 15.1 & 30.2 & 18.7 & 4.0 & 3.2 & 52.4 & 24. \\
\hline Elm/ash/cottonwood & 11.9 & 8.6 & 一 & - & 15.0 & 13.6 & 26.9 & 16 \\
\hline Tanoak/laurel & $5,490.8$ & 699.1 & 724.9 & 240.9 & $1,331.6$ & 564.4 & $7,547.3$ & 923. \\
\hline Western oak & $6,890.3$ & 534.8 & $2,546.1$ & 252.5 & $3,039.2$ & 882.4 & $12,475.6$ & 1,054 \\
\hline Woodland hardwoods & 473.9 & 100.9 & 147.9 & 88.6 & - & - & 621.8 & 134. \\
\hline Other hardwoods & 755.3 & 201.2 & 140.5 & 53.0 & 266.8 & 175.6 & $1,162.6$ & 273. \\
\hline Total & $14,388.1$ & 928.4 & $3,796.7$ & 377.7 & $4,722.3$ & $1,061.2$ & $22,907.1$ & 1,440 \\
\hline Nonstocked & - & - & - & - & - & 一 & $4,956.0$ & 1,09 \\
\hline All forest types & $88,485.2$ & $2,546.4$ & $4,818.7$ & 440.4 & $7,484.9$ & $1,317.6$ & $105,744.8$ & 2,98 \\
\hline
\end{tabular}




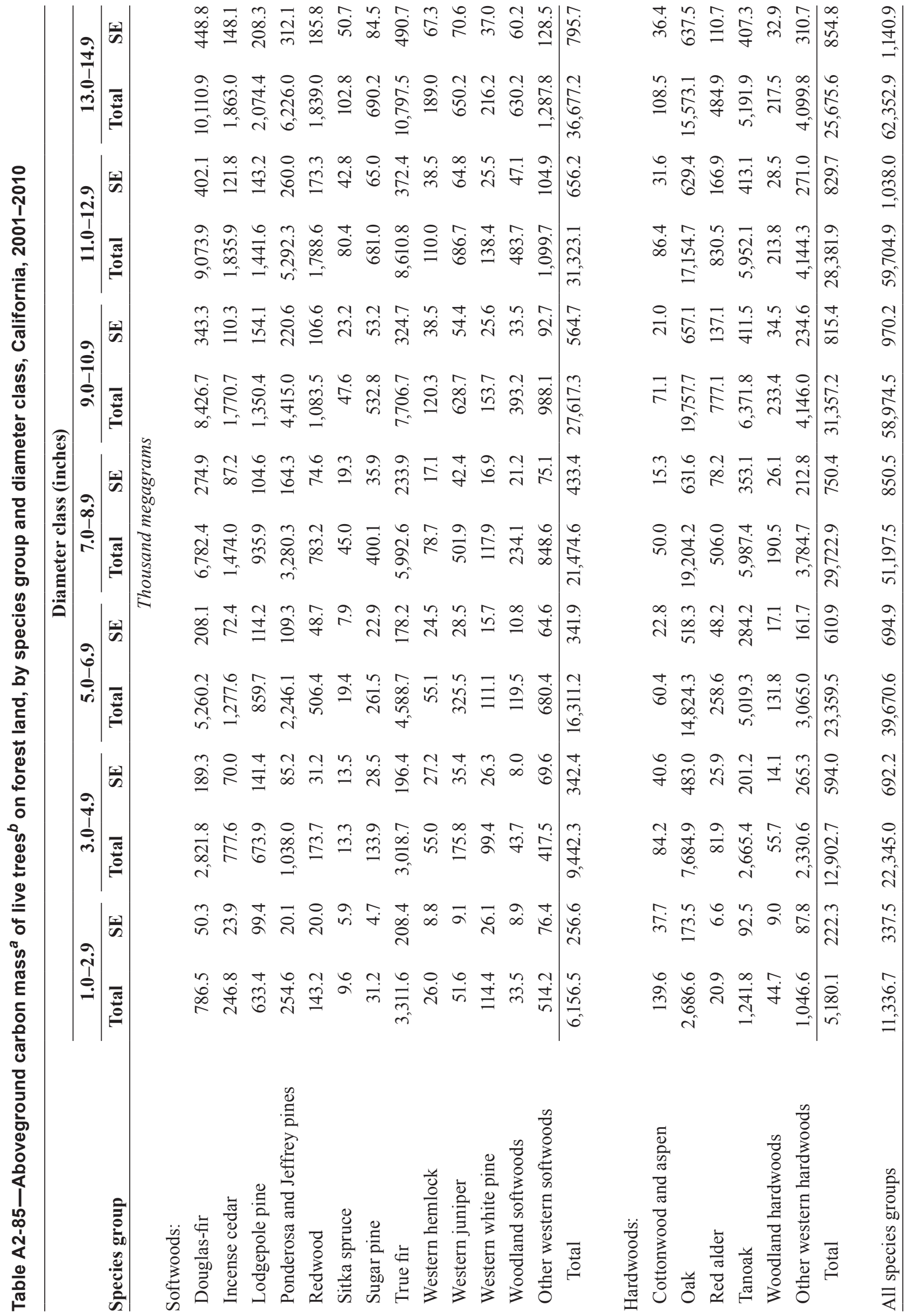




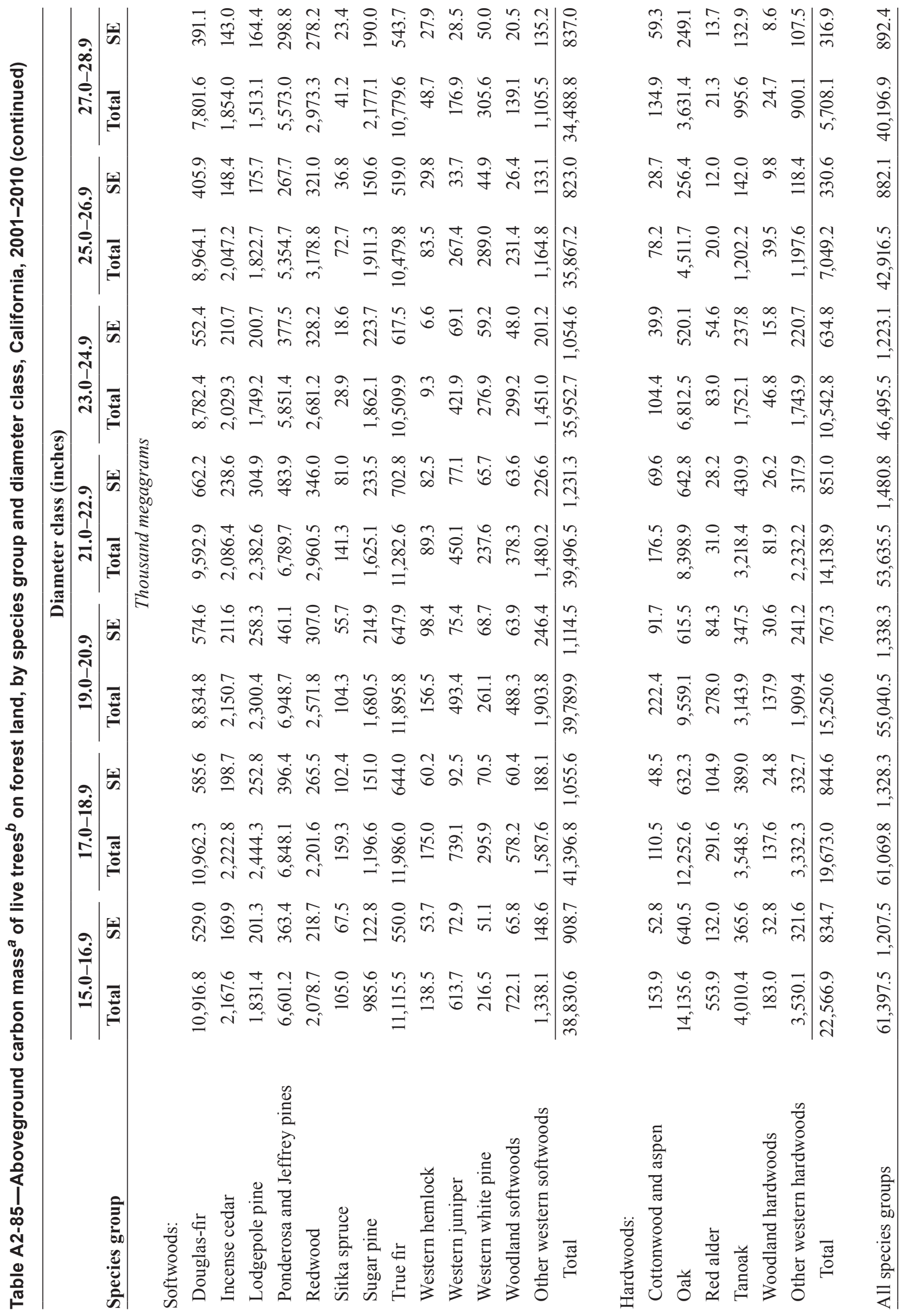




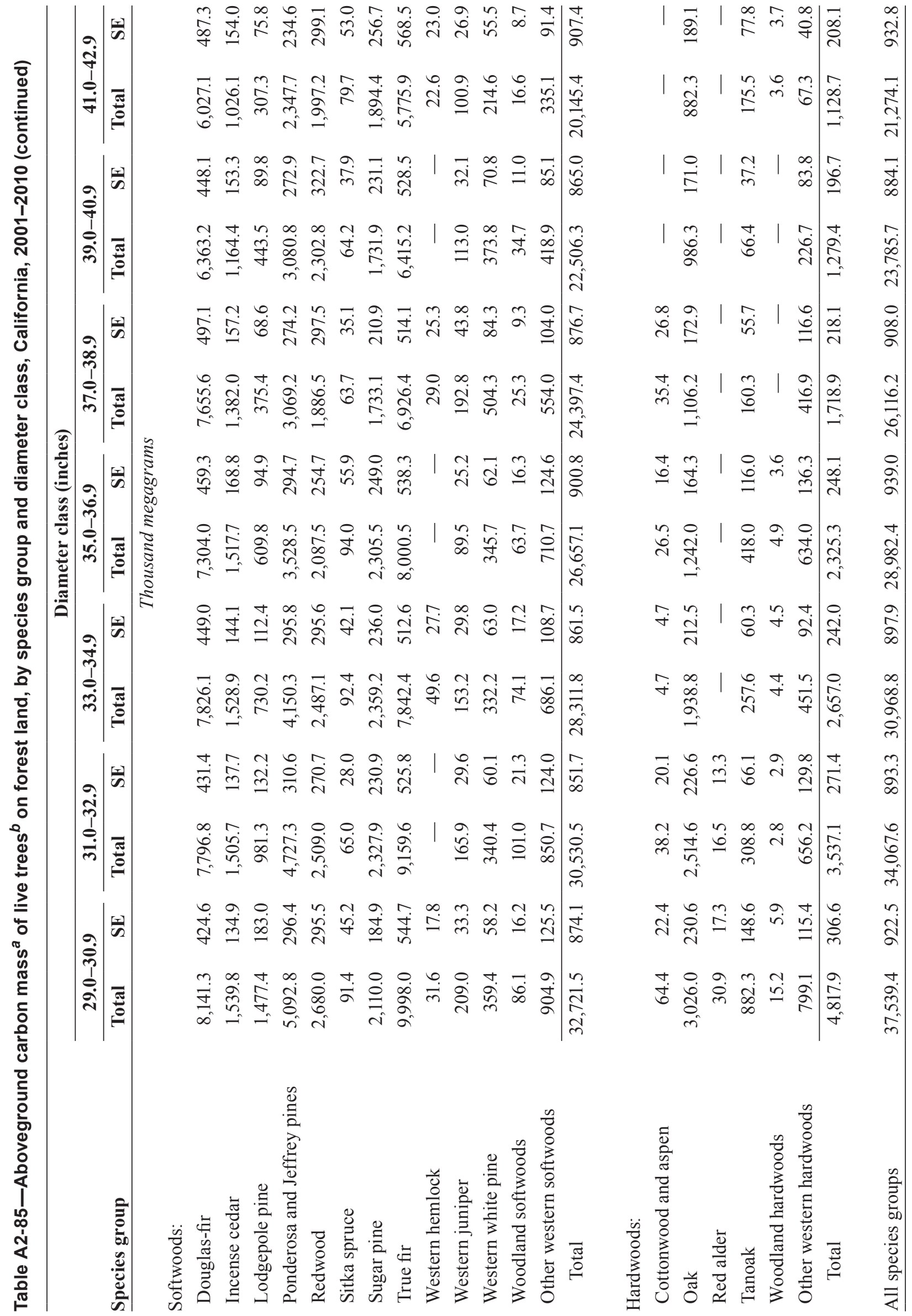




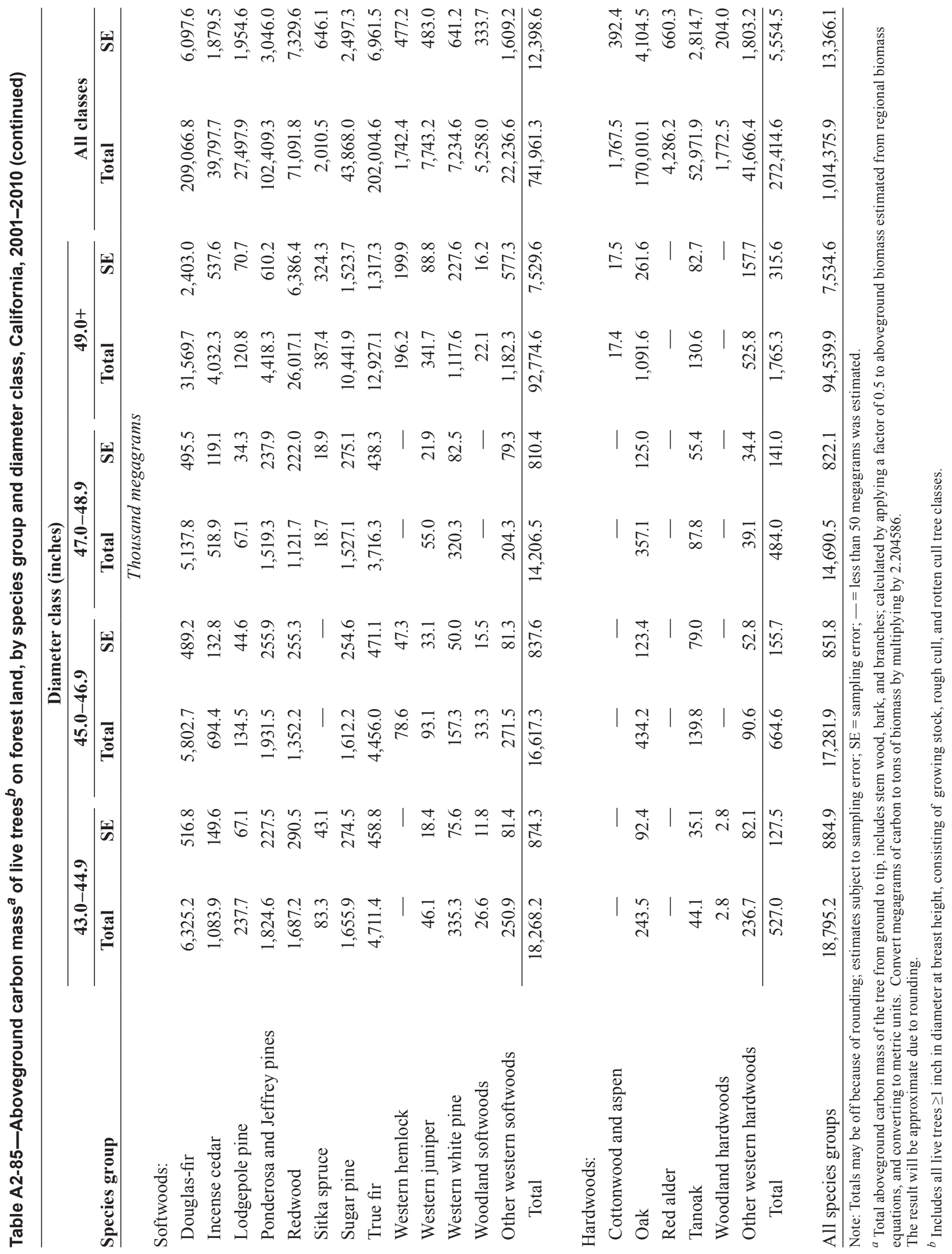




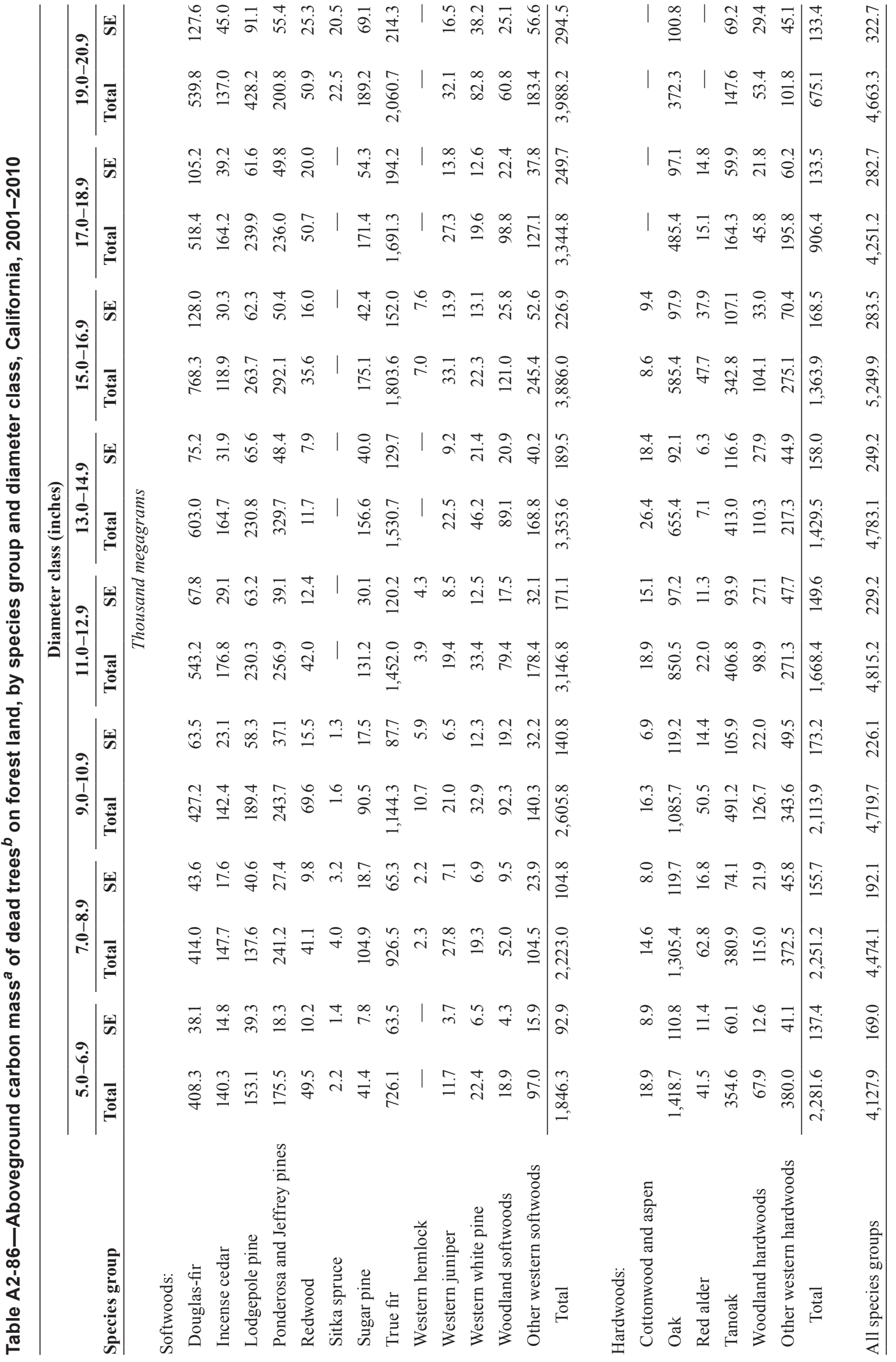




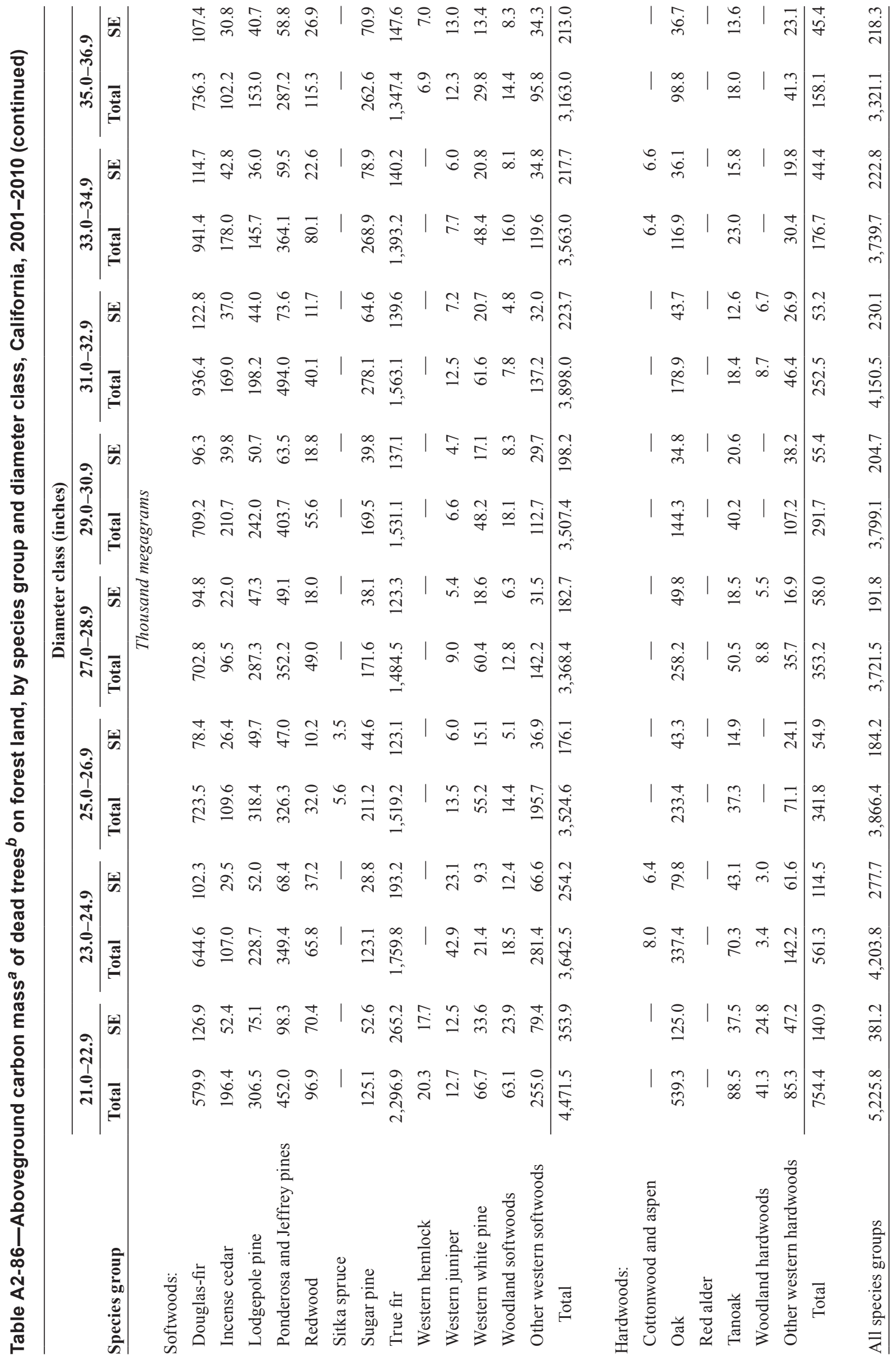




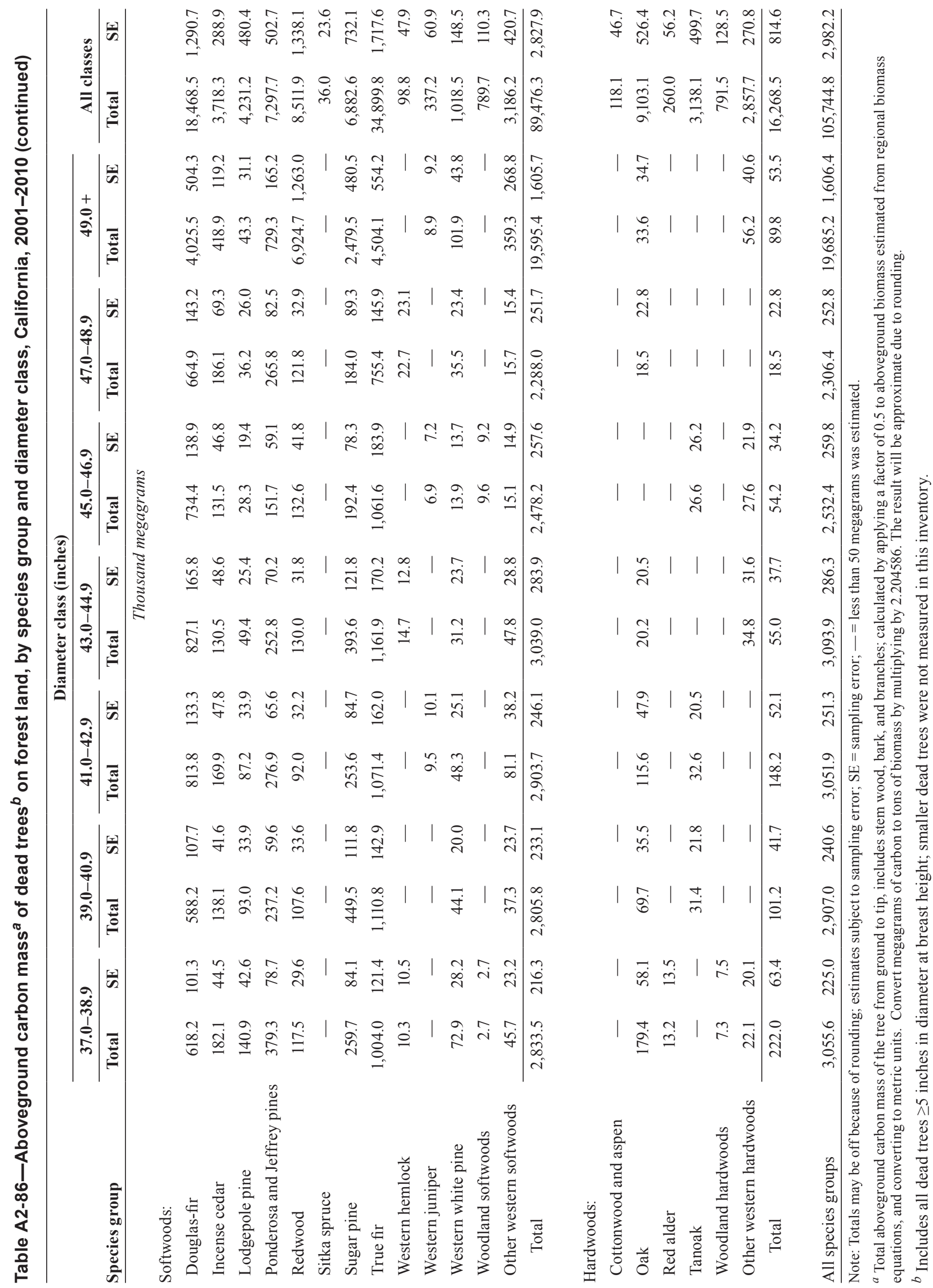


California's Forest Resources: Forest Inventory and Analysis, 2001-2010

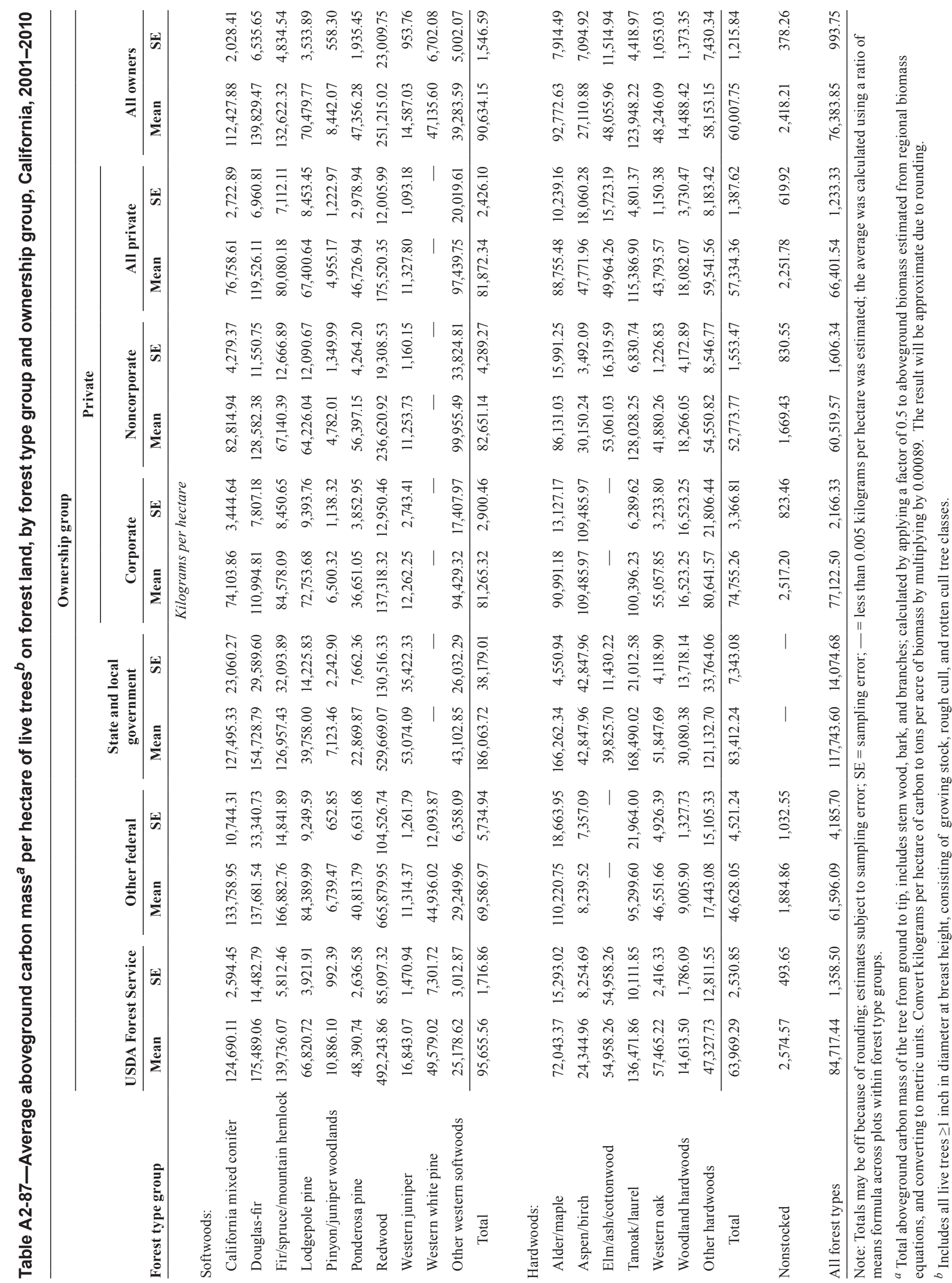




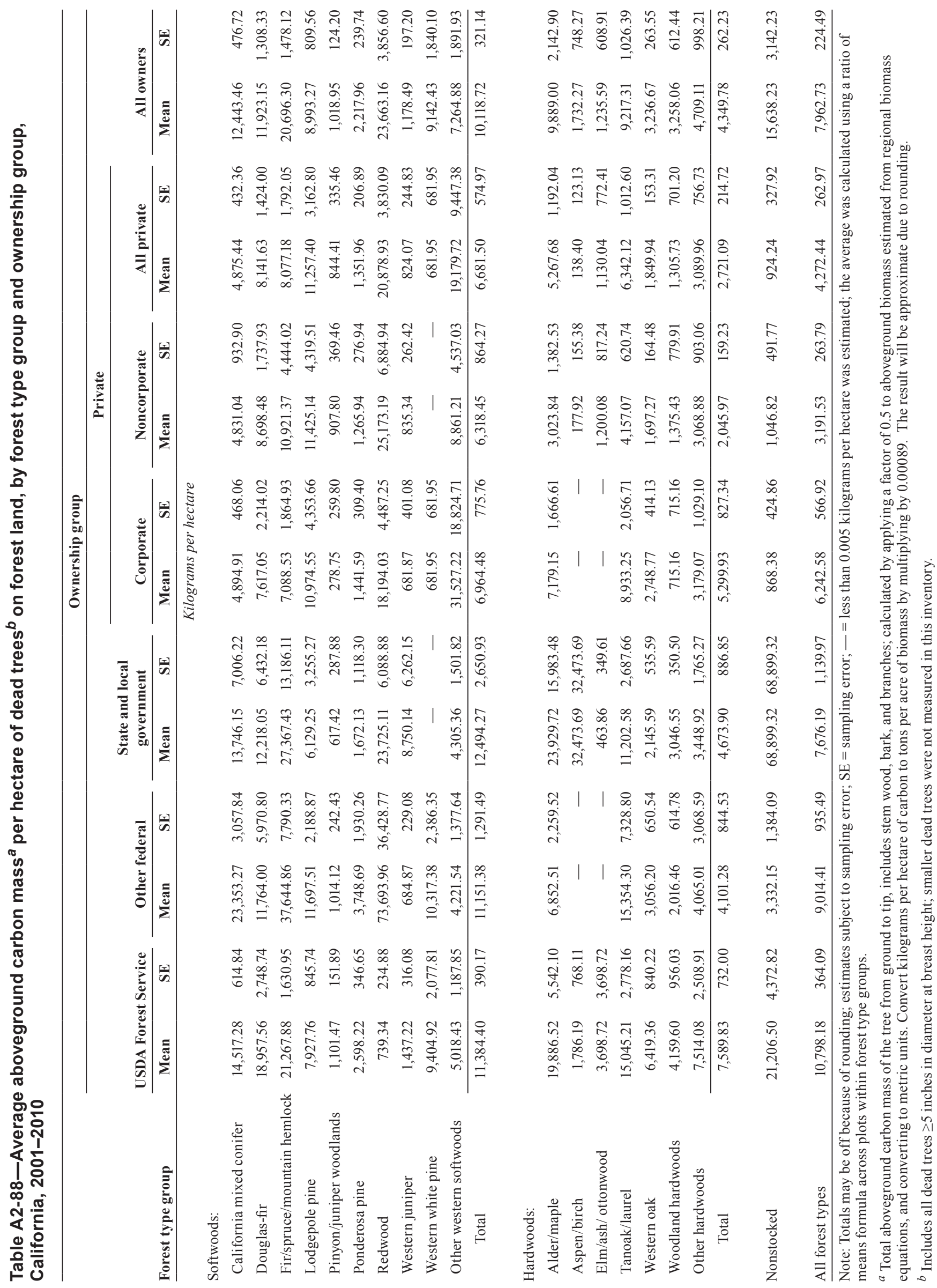


Table A2-89-Average aboveground carbon mass ${ }^{a}$ per hectare of live trees ${ }^{b}$ on forest land, by forest type group and stand size class, California, 2001-2010

\begin{tabular}{|c|c|c|c|c|c|c|c|c|}
\hline \multirow[b]{3}{*}{ Forest type group } & \multicolumn{6}{|c|}{ Stand size class } & & \\
\hline & \multicolumn{2}{|c|}{$\begin{array}{c}\text { Large-diameter } \\
\text { stands }\end{array}$} & \multicolumn{2}{|c|}{$\begin{array}{c}\text { Medium-diameter } \\
\text { stands }\end{array}$} & \multicolumn{2}{|c|}{$\begin{array}{c}\text { Small-diameter } \\
\text { stands }\end{array}$} & \multicolumn{2}{|c|}{ All size classes } \\
\hline & Mean & SE & Mean & $\mathbf{S E}$ & Mean & SE & Mean & SE \\
\hline & \multicolumn{8}{|c|}{ Kilograms per hectare } \\
\hline \multicolumn{9}{|l|}{ Softwoods: } \\
\hline California mixed conifer & $119,320.99$ & $2,075.07$ & $33,355.16$ & $4,476.65$ & $10,848.62$ & $1,457.00$ & $112,427.88$ & $2,028.41$ \\
\hline Douglas-fir & $155,718.12$ & $6,756.28$ & $55,414.45$ & $10,178.32$ & $24,247.52$ & $5,075.22$ & $139,829.47$ & $6,535.65$ \\
\hline Fir/spruce/mountain hemlock & $140,972.86$ & $4,910.79$ & $35,749.58$ & $9,209.70$ & $13,484.99$ & $2,401.63$ & $132,622.32$ & $4,834.54$ \\
\hline Lodgepole pine & $73,664.76$ & $3,724.78$ & $61,132.99$ & $12,152.81$ & $11,463.27$ & $5,912.74$ & $70,479.77$ & $3,533.89$ \\
\hline Pinyon/juniper woodlands & $9,886.92$ & 644.48 & $2,134.29$ & 799.11 & $1,397.89$ & 376.43 & $8,442.07$ & 558.30 \\
\hline Ponderosa pine & $53,801.91$ & $2,075.29$ & $13,756.46$ & $2,062.59$ & $4,951.24$ & $1,236.47$ & $47,356.28$ & $1,935.45$ \\
\hline Redwood & $268,787.39$ & $24,268.52$ & $24,048.79$ & $6,727.14$ & $14,171.52$ & $3,545.94$ & $251,215.02$ & $23,009.75$ \\
\hline Western juniper & $16,616.45$ & $1,081.76$ & $6,847.34$ & $1,602.02$ & $2,502.22$ & 748.95 & $14,587.03$ & 953.76 \\
\hline Western white pine & $57,190.27$ & $7,381.55$ & $8,638.24$ & $1,770.21$ & $15,155.53$ & $6,296.98$ & $47,135.60$ & $6,702.08$ \\
\hline Other western softwoods & $54,087.43$ & $6,761.71$ & $12,339.73$ & $2,903.58$ & $6,720.85$ & $1,420.15$ & $39,283.59$ & $5,002.07$ \\
\hline Total & $100,145.39$ & $1,686.56$ & $19,624.86$ & $2,003.69$ & $9,153.88$ & 831.42 & $90,634.15$ & $1,546.59$ \\
\hline
\end{tabular}

Hardwoods:

\begin{tabular}{lrrrrrrrr} 
Alder/maple & $118,370.76$ & $8,281.13$ & $65,413.01$ & $12,601.00$ & $19,753.26$ & $7,651.06$ & $92,772.63$ & $7,914.49$ \\
Aspen/birch & $57,296.36$ & $13,386.10$ & $48,121.49$ & $15,933.81$ & $10,669.45$ & $3,085.90$ & $27,110.88$ & $7,094.92$ \\
Elm/ash/cottonwood & $57,962.62$ & $11,856.91$ & $3,671.14$ & $3,393.71$ & $11,810.26$ & $1,180.40$ & $48,055.96$ & $11,514.94$ \\
Tanoak/laurel & $156,740.30$ & $5,203.82$ & $86,336.57$ & $4,872.83$ & $20,739.61$ & $3,429.59$ & $123,948.22$ & $4,418.97$ \\
Western oak & $63,604.35$ & $1,647.31$ & $38,888.82$ & $1,365.90$ & $14,671.76$ & $1,093.00$ & $48,246.09$ & $1,053.03$ \\
Woodland hardwoods & $15,709.16$ & $1,481.95$ & $12,042.21$ & $4,297.63$ & $2,522.43$ & 946.21 & $14,488.42$ & $1,373.35$ \\
Other hardwoods & $127,701.83$ & $14,283.64$ & $38,948.95$ & $7,115.73$ & $6,195.33$ & $1,167.92$ & $58,153.15$ & $7,430.34$ \\
\multicolumn{1}{c}{ Total } & $81,654.91$ & $1,922.99$ & $44,162.34$ & $1,401.87$ & $14,248.98$ & 925.17 & $60,007.75$ & $1,215.84$
\end{tabular}

Nonstocked

\begin{tabular}{rrrrrrrr}
- & - & - & - & - & - & $2,418.21$ & 378.26 \\
& & & & & & & \\
32.94 & $1,283.65$ & $39,787.99$ & $1,245.61$ & $12,271.73$ & 661.09 & $76,383.85$ & 993.75 \\
\hline
\end{tabular}

All forest types $94,732.94 \quad 1,283.65 \quad 39,787.99 \quad 1,245.61 \quad 12,271.73$

Note: Totals may be off because of rounding; estimates subject to sampling error; SE = sampling error; — = less than 0.005 kilograms per hectare was estimated; the average was calculated using a ratio of means formula across plots within forest type groups.

${ }^{a}$ Total aboveground carbon mass of the tree from ground to tip, includes stem wood, bark, and branches; calculated by applying a factor of 0.5 to aboveground biomass estimated from regional biomass equations, and converting to metric units. Convert kilograms per hectare of carbon to tons per acre of biomass by multiplying by 0.00089 . The result will be approximate due to rounding.

${ }^{b}$ Includes all live trees $\geq 1$ inch in diameter at breast height, consisting of growing stock, rough cull, and rotten cull tree classes. 
Table A2-90_Average aboveground carbon mass ${ }^{a}$ per hectare of dead trees ${ }^{b}$ on forest land, by forest type group and stand size class, California, 2001-2010

\begin{tabular}{|c|c|c|c|c|c|c|c|c|}
\hline \multirow[b]{3}{*}{ Forest type group } & \multicolumn{6}{|c|}{ Stand size class } & & \\
\hline & \multicolumn{2}{|c|}{$\begin{array}{c}\text { Large-diameter } \\
\text { stands }\end{array}$} & \multicolumn{2}{|c|}{$\begin{array}{c}\text { Medium-diameter } \\
\text { stands }\end{array}$} & \multicolumn{2}{|c|}{$\begin{array}{c}\text { Small-diameter } \\
\text { stands }\end{array}$} & \multicolumn{2}{|c|}{ All size classes } \\
\hline & Mean & SE & Mean & SE & Mean & SE & Mean & SE \\
\hline & \multicolumn{8}{|c|}{ Kilograms per hectare } \\
\hline \multicolumn{9}{|l|}{ Softwoods: } \\
\hline California mixed conifer & $12,988.84$ & 500.76 & $4,943.14$ & $1,629.03$ & $5,030.40$ & $1,810.91$ & $12,443.46$ & 476.72 \\
\hline Douglas-fir & $12,487.68$ & $1,419.01$ & $1,621.21$ & 882.11 & $12,354.04$ & $5,614.09$ & $11,923.15$ & $1,308.33$ \\
\hline Fir/spruce/mountain hemlock & $20,371.41$ & $1,359.29$ & $18,352.30$ & $10,108.24$ & $26,540.96$ & $13,460.15$ & $20,696.30$ & $1,478.12$ \\
\hline Lodgepole pine & $9,400.64$ & 841.48 & $9,060.19$ & $5,037.24$ & 229.93 & 213.26 & $8,993.27$ & 809.56 \\
\hline Pinyon/juniper woodlands & 963.14 & 108.59 & $1,489.39$ & 740.86 & 962.53 & 463.45 & $1,018.95$ & 124.20 \\
\hline Ponderosa pine & $2,436.10$ & 272.23 & 644.89 & 293.95 & $1,291.31$ & 671.95 & $2,217.96$ & 239.74 \\
\hline Redwood & $24,908.28$ & $4,114.23$ & $9,543.02$ & $2,265.90$ & $5,352.53$ & $2,722.54$ & $23,663.16$ & $3,856.60$ \\
\hline Western juniper & $1,392.15$ & 233.73 & 209.85 & 155.23 & 40.49 & 21.25 & $1,178.49$ & 197.20 \\
\hline Western white pine & $11,065.81$ & $2,234.86$ & $1,763.12$ & 361.31 & $3,028.40$ & $1,650.06$ & $9,142.43$ & $1,840.10$ \\
\hline Other western softwoods & $9,709.84$ & $2,699.84$ & $1,455.83$ & 691.13 & $2,782.64$ & $2,010.14$ & $7,264.88$ & $1,891.93$ \\
\hline Total & $10,816.09$ & 342.96 & $2,825.76$ & 592.27 & $5,702.13$ & $1,570.88$ & $10,118.72$ & 321.14 \\
\hline
\end{tabular}

Hardwoods:

\begin{tabular}{lrrrrrrrr} 
Alder/maple & $11,740.59$ & $3,181.57$ & $7,533.48$ & $2,802.35$ & $5,467.60$ & $2,517.81$ & $9,889.00$ & $2,142.90$ \\
Aspen/birch & $3,180.23$ & $1,873.98$ & $4,892.58$ & $2,692.38$ & 219.32 & 152.82 & $1,732.27$ & 748.27 \\
Elm/ash/cottonwood & 689.12 & 445.29 & - & - & $4,496.12$ & 449.37 & $1,235.59$ & 608.91 \\
Tanoak/laurel & $10,303.45$ & $1,129.03$ & $3,951.80$ & $1,228.00$ & $12,994.41$ & $5,148.96$ & $9,217.31$ & $1,026.39$ \\
Western oak & $3,493.51$ & 245.58 & $1,874.12$ & 170.98 & $5,804.70$ & $1,639.54$ & $3,236.67$ & 263.55 \\
Woodland hardwoods & $3,049.69$ & 531.02 & $6,001.17$ & $3,269.54$ & - & - & $3,258.06$ & 612.44 \\
Other hardwoods & $8,595.03$ & $1,768.37$ & $2,140.25$ & 699.78 & $2,857.58$ & $1,816.84$ & $4,709.11$ & 998.21 \\
\cline { 2 - 8 }$\quad$ Total & $5,074.79$ & 299.30 & $2,277.43$ & 211.79 & $6,181.37$ & $1,346.92$ & $4,349.78$ & 262.23
\end{tabular}

Nonstocked

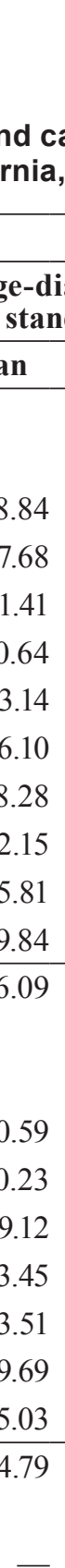

$5,638.23 \quad 3,142.23$

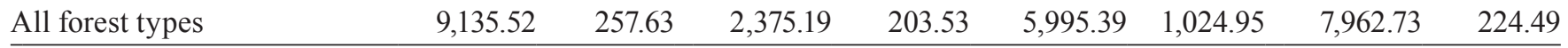

Note: Totals may be off because of rounding; estimates subject to sampling error; $\mathrm{SE}=$ sampling error; — = less than 0.005 kilograms per hectare was estimated; the average was calculated using a ratio of means formula across plots within forest type groups.

${ }^{a}$ Total aboveground carbon mass of the tree from ground to tip, includes stem wood, bark, and branches; calculated by applying a factor of 0.5 to aboveground biomass estimated from regional biomass equations, and converting to metric units. Convert kilograms per hectare of carbon to tons per acre of biomass by multiplying by 0.00089 . The result will be approximate due to rounding.

${ }^{b}$ Includes all dead trees $\geq 5$ inches in diameter at breast height; smaller dead trees were not measured in this inventory. 


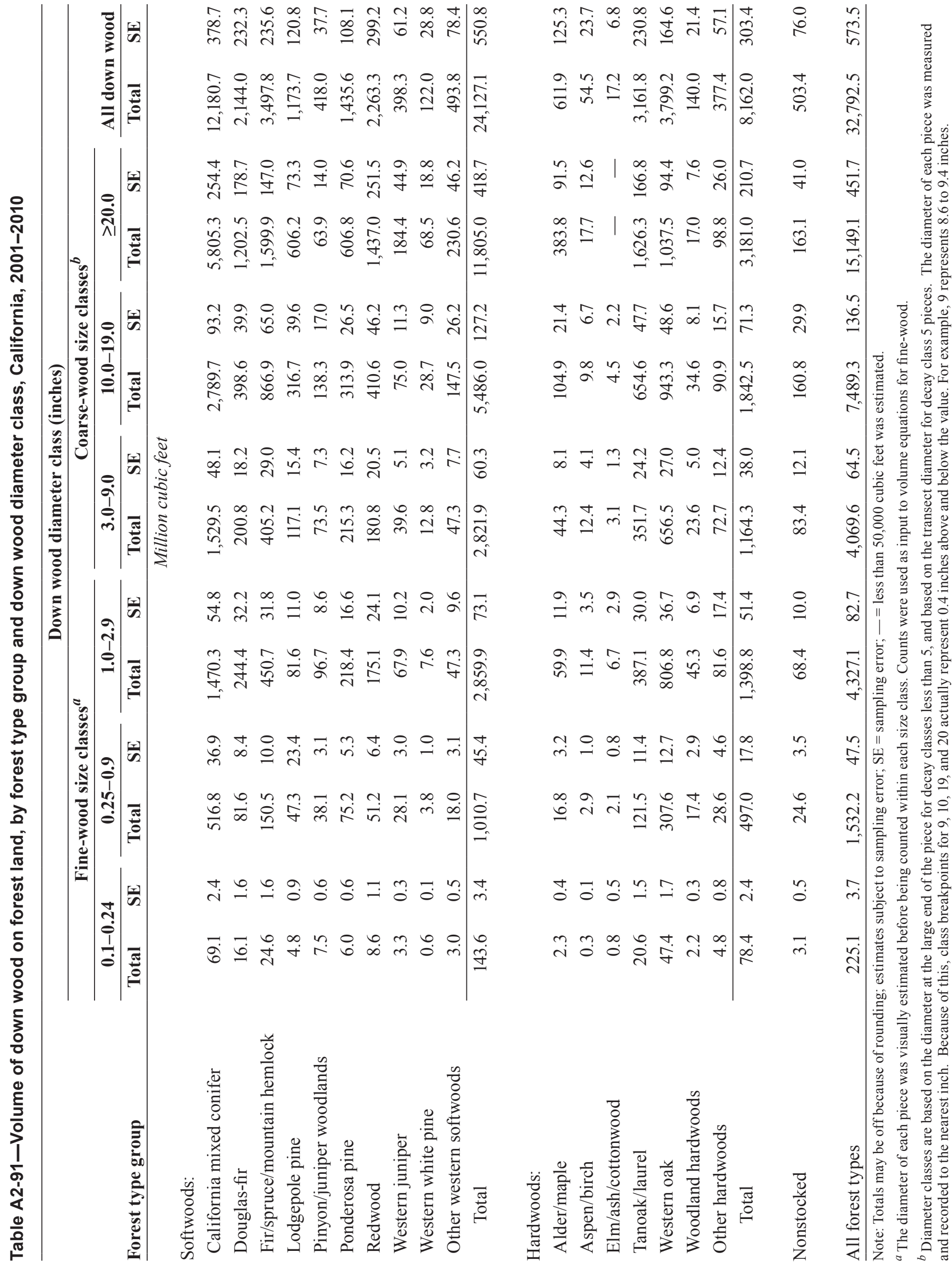




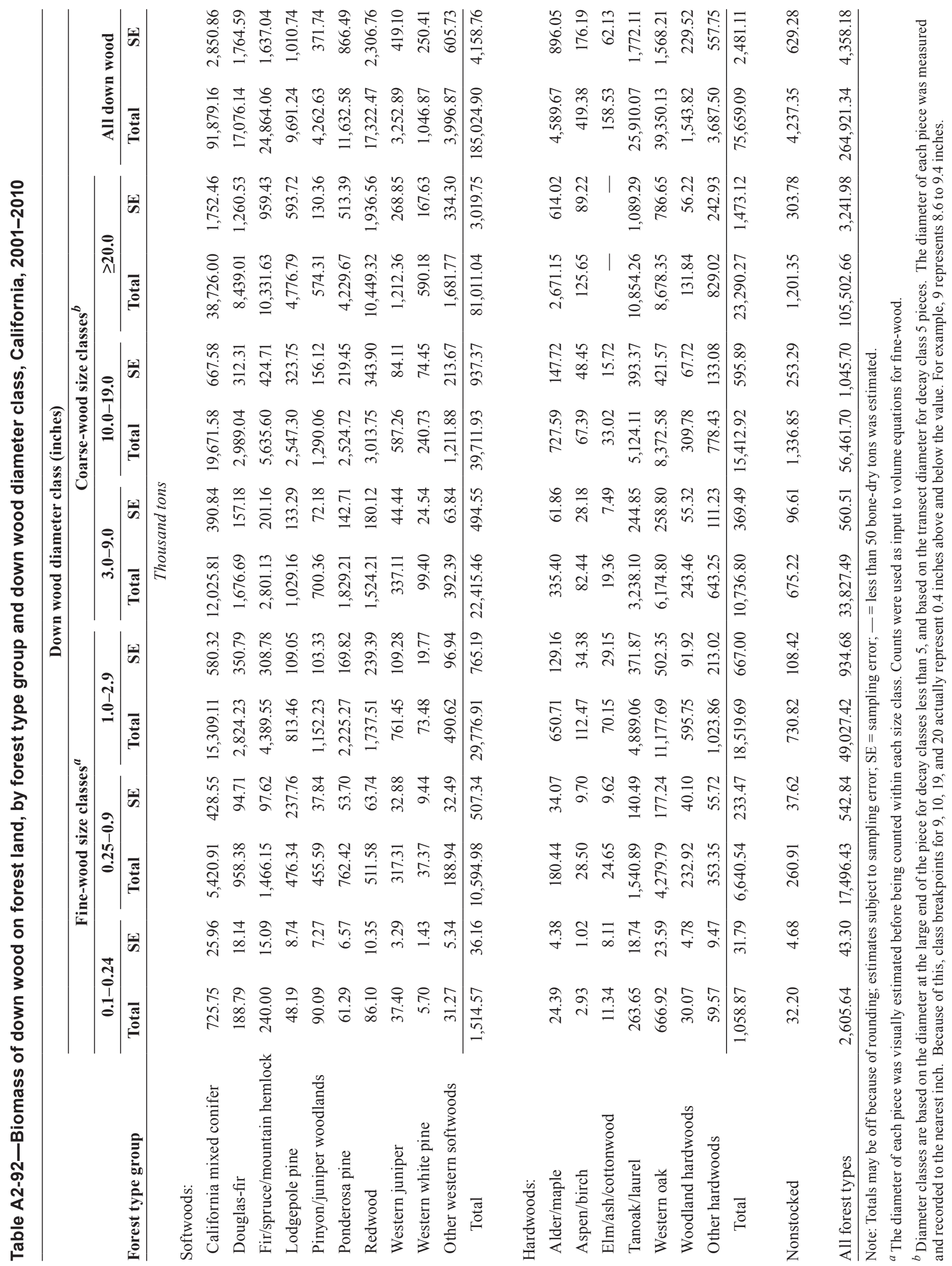




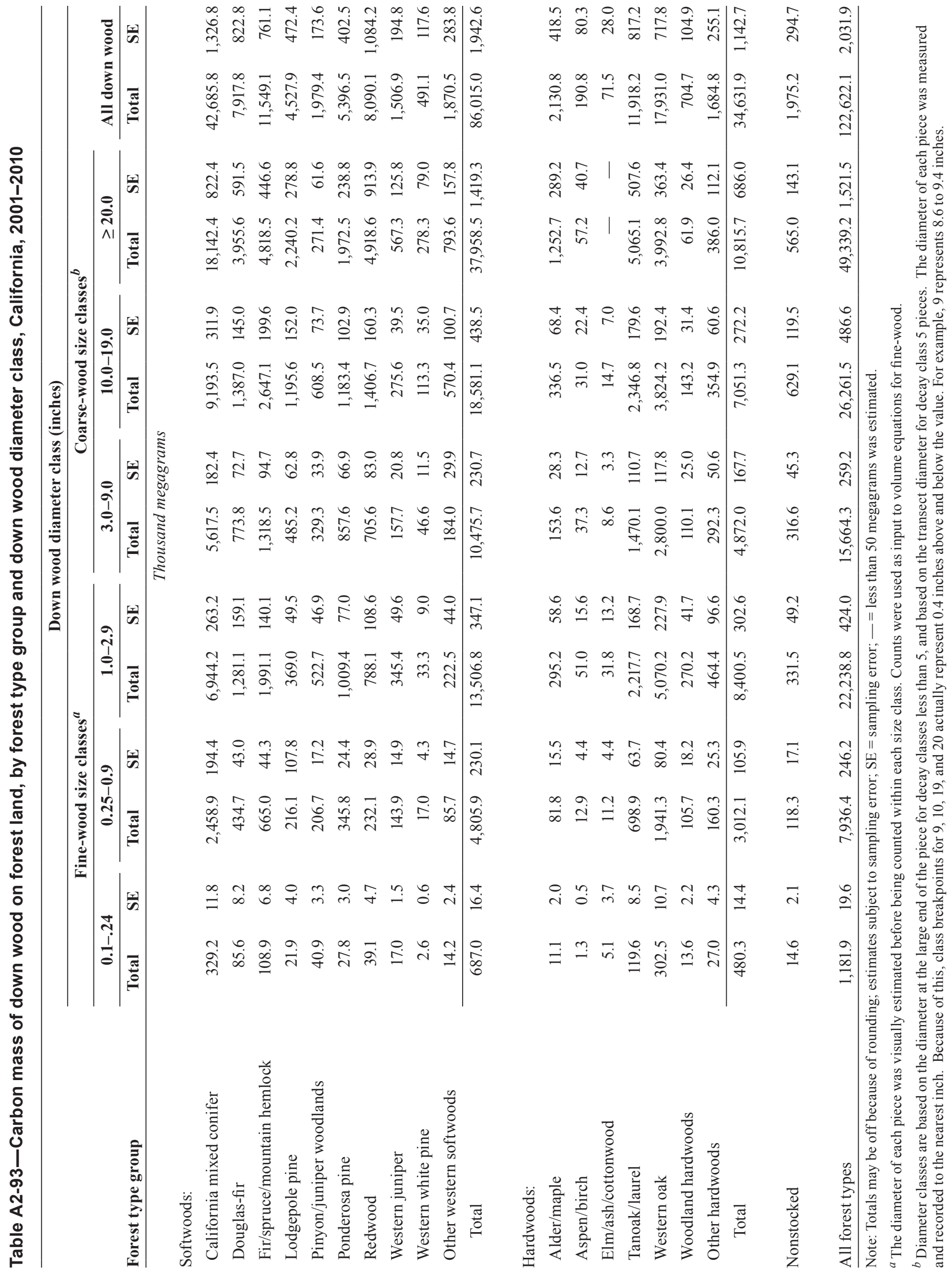


Table A2-94-Number of pieces of down wood ${ }^{a}$ on forest land, by forest type group and down wood diameter class, California, 2001-2010

\begin{tabular}{|c|c|c|c|c|c|c|c|c|}
\hline \multirow[b]{3}{*}{ Forest type group } & \multicolumn{6}{|c|}{ Down wood diameter class (inches) ${ }^{b}$} & & \\
\hline & \multicolumn{2}{|c|}{$3.0-9.0$} & \multicolumn{2}{|c|}{$10.0-19.0$} & \multicolumn{2}{|c|}{$\geq 20.0$} & \multicolumn{2}{|c|}{ All classes } \\
\hline & Total & SE & Total & SE & Total & SE & Total & SE \\
\hline & \multicolumn{8}{|c|}{ Thousand pieces } \\
\hline \multicolumn{9}{|l|}{ Softwoods: } \\
\hline California mixed conifer & $1,449,257.7$ & $44,627.1$ & $312,053.2$ & $11,005.8$ & $73,637.4$ & $3,536.7$ & $1,834,948.3$ & $53,698.7$ \\
\hline Douglas-fir & $168,133.7$ & $14,804.9$ & $43,494.0$ & $4,787.6$ & $17,610.4$ & $2,459.5$ & $229,238.0$ & $19,468.1$ \\
\hline Fir/spruce/mountain hemlock & $376,476.0$ & $25,868.2$ & $92,161.4$ & $6,967.7$ & $23,244.9$ & $2,200.7$ & $491,882.3$ & $32,283.1$ \\
\hline Lodgepole pine & $101,410.3$ & $13,128.9$ & $28,301.2$ & $3,155.4$ & $8,639.6$ & $1,110.2$ & $138,351.1$ & $15,836.5$ \\
\hline Pinyon/juniper woodlands & $95,280.5$ & $9,211.9$ & $22,897.0$ & $2,980.6$ & $2,461.8$ & 568.9 & $120,639.2$ & $11,398.6$ \\
\hline Ponderosa pine & $227,852.6$ & $16,447.1$ & $36,862.8$ & $3,413.1$ & $9,016.6$ & $1,140.7$ & $273,732.0$ & $19,122.2$ \\
\hline Redwood & $147,191.9$ & $16,615.6$ & $47,532.3$ & $5,706.2$ & $17,615.0$ & $2,318.4$ & $212,339.2$ & $22,260.0$ \\
\hline Western juniper & $51,198.4$ & $6,867.0$ & $9,768.1$ & $1,544.5$ & $2,440.0$ & 536.0 & $63,406.5$ & $7,794.5$ \\
\hline Western white pine & $13,797.6$ & $3,365.5$ & $3,184.3$ & $1,050.6$ & $1,328.7$ & 507.4 & $18,310.6$ & $4,187.7$ \\
\hline Other western softwoods & $43,504.6$ & $6,648.8$ & $16,789.3$ & $2,917.5$ & $3,752.3$ & 730.0 & $64,046.2$ & $9,207.7$ \\
\hline Total & $2,674,103.2$ & $54,403.9$ & $613,043.6$ & $14,932.7$ & $159,746.6$ & $5,418.3$ & $3,446,893.5$ & $65,781.5$ \\
\hline
\end{tabular}

Hardwoods:

\begin{tabular}{lrrrrrrrr} 
Alder/maple & $35,183.8$ & $6,509.6$ & $10,708.5$ & $2,176.9$ & $6,206.1$ & $1,559.2$ & $52,098.3$ & $9,355.0$ \\
Aspen/birch & $14,515.5$ & $4,929.9$ & 711.1 & 445.7 & 187.0 & 136.5 & $15,413.6$ & $5,274.9$ \\
Elm/ash/cottonwood & $3,888.0$ & $1,657.8$ & 320.3 & 192.7 & - & - & $4,208.3$ & $1,756.0$ \\
Tanoak/laurel & $314,029.4$ & $20,779.5$ & $67,691.2$ & $5,181.3$ & $22,275.8$ & $2,154.8$ & $403,996.4$ & $25,580.7$ \\
Western oak & $695,041.0$ & $26,863.3$ & $105,628.7$ & $5,555.9$ & $15,896.8$ & $1,310.2$ & $816,566.4$ & $30,794.5$ \\
Woodland hardwoods & $31,071.5$ & $6,564.7$ & $5,928.7$ & $1,483.7$ & 459.4 & 247.6 & $37,459.6$ & $7,533.3$ \\
Other hardwoods & $70,571.0$ & $11,611.8$ & $10,773.1$ & $2,208.0$ & $1,610.1$ & 563.5 & $82,954.2$ & $13,212.4$ \\
\multicolumn{1}{c}{ Total } & $1,164,300.1$ & $35,845.3$ & $201,761.5$ & $8,062.3$ & $46,635.1$ & $2,965.1$ & $1,412,696.7$ & $42,058.2$ \\
& & & & & & & \\
Nonstocked & $72,591.1$ & $9,916.8$ & $15,498.3$ & $2,529.1$ & $2,369.1$ & 632.7 & $90,458.5$ & $11,995.4$ \\
& & & & & & & \\
All forest types & $3,910,994.5$ & $58,398.4$ & $830,303.4$ & $15,903.2$ & $208,750.8$ & $5,954.1$ & $4,950,048.7$ & $68,987.9$ \\
\hline
\end{tabular}

Note: Estimates subject to sampling error; $\mathrm{SE}=$ sampling error.

${ }^{a}$ Includes down wood pieces $\geq 3$ inches in diameter at the transect intersection. Number of pieces can not be estimated for down wood with smaller diameters (fine woody material) because pieces were not individually measured.

${ }^{b}$ Diameter classes are based on the diameter at the large end of the piece for decay class less than 5 , and based on the transect diameter for decay class 5 pieces. The diameter of each piece was measured and recorded to the nearest inch. Because of this, class breakpoints for 9, 10, 19, and 20 actually represent 0.4 inches above and below the value. For example, 9 represents 8.6 to 9.4 inches. 


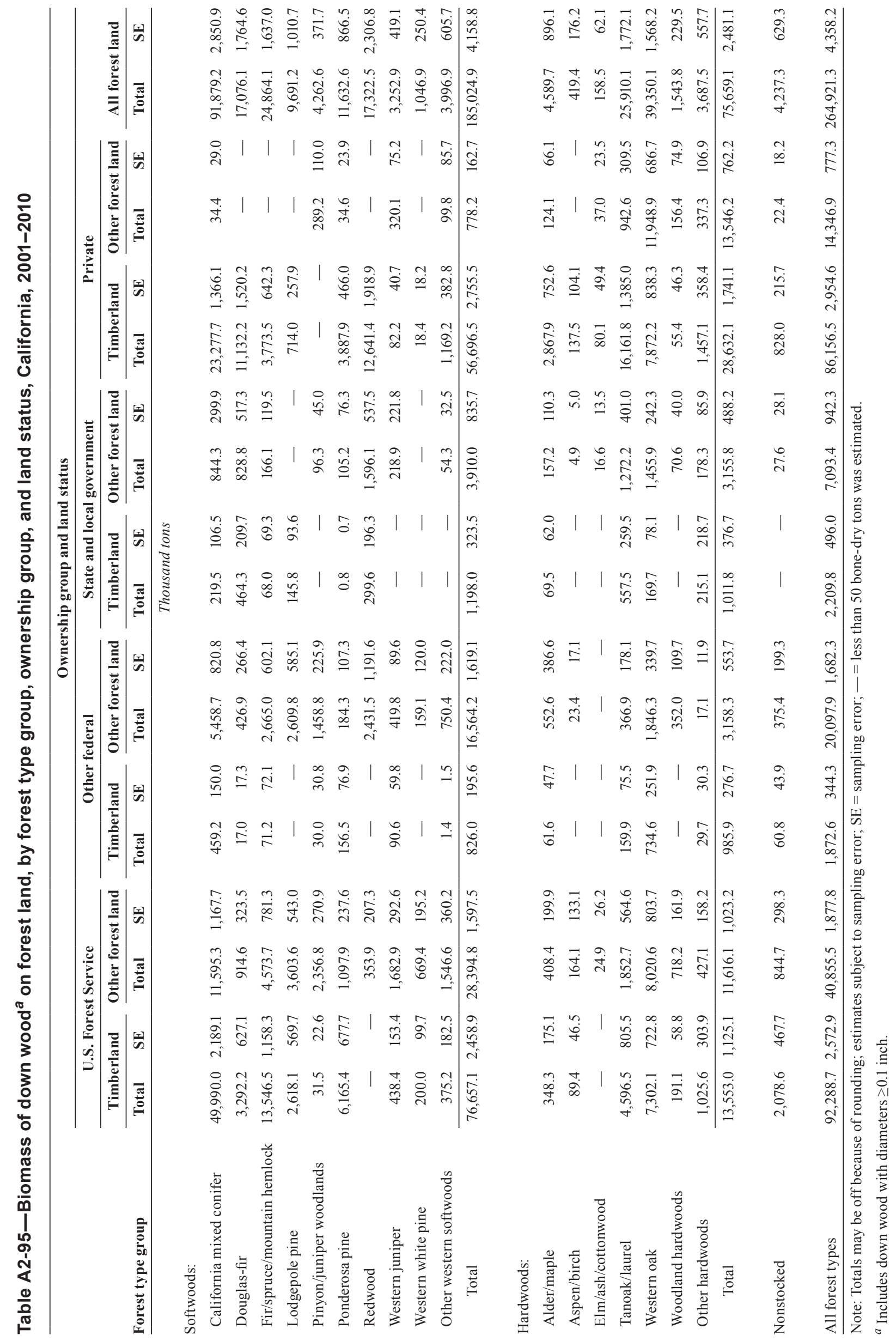




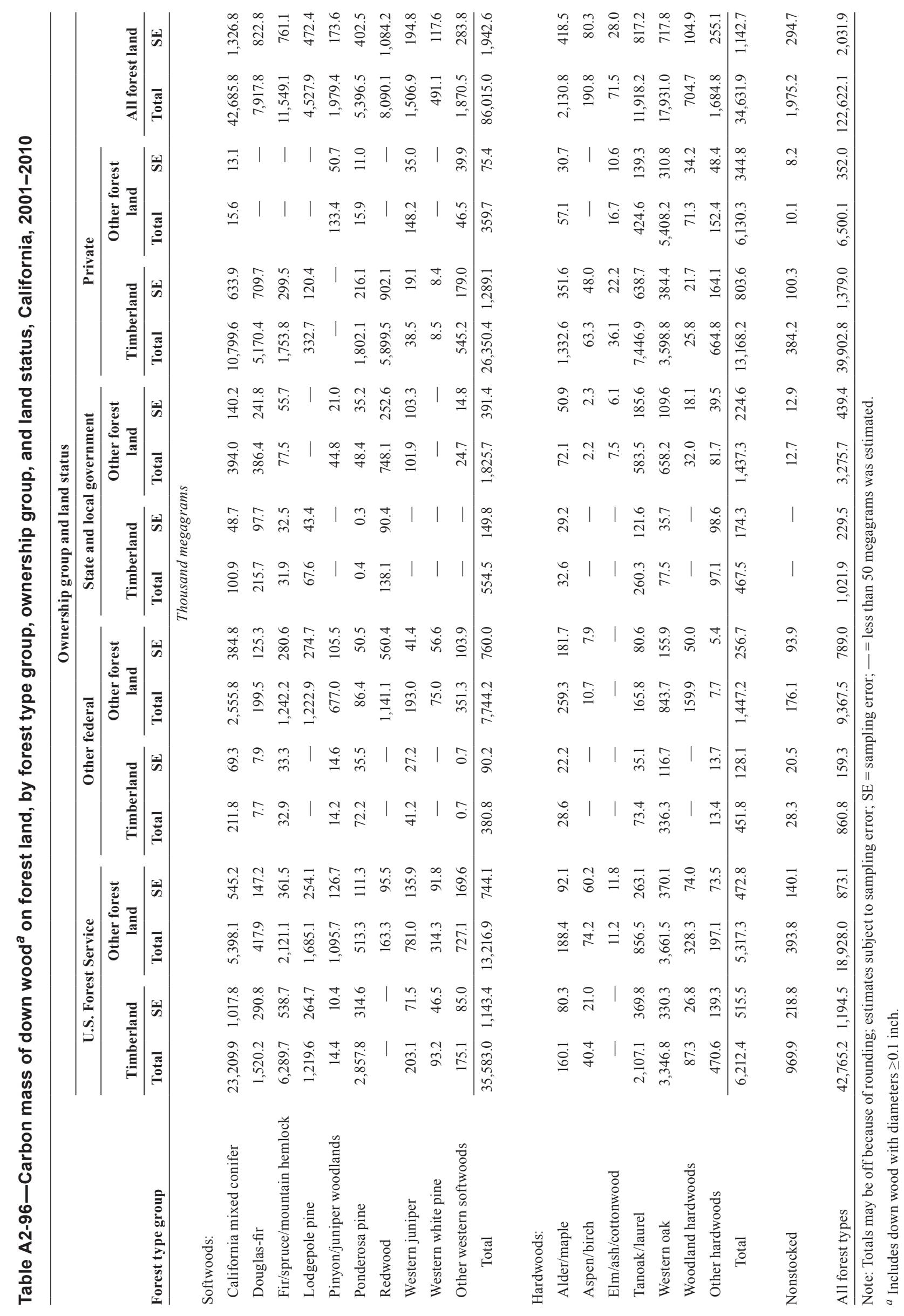




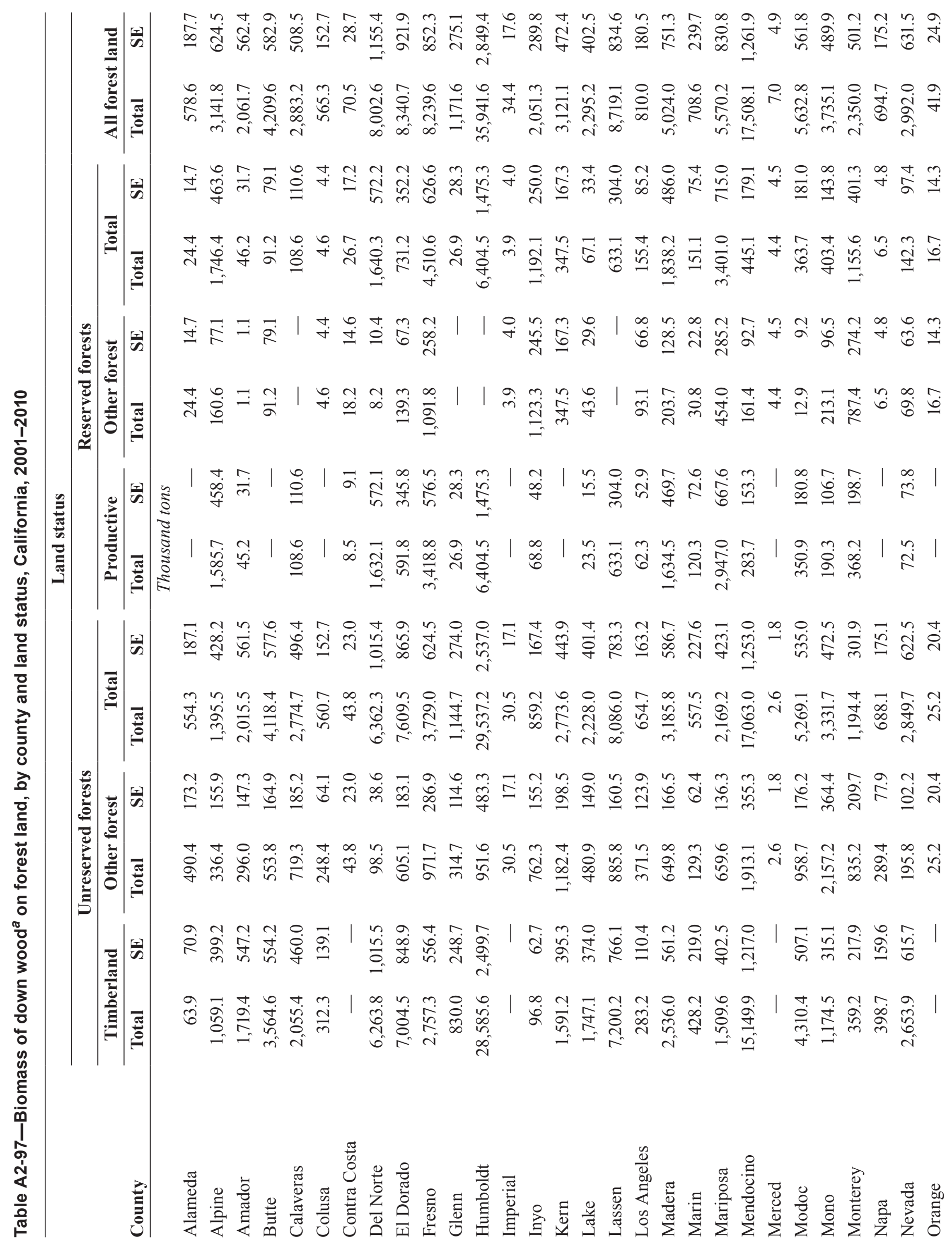




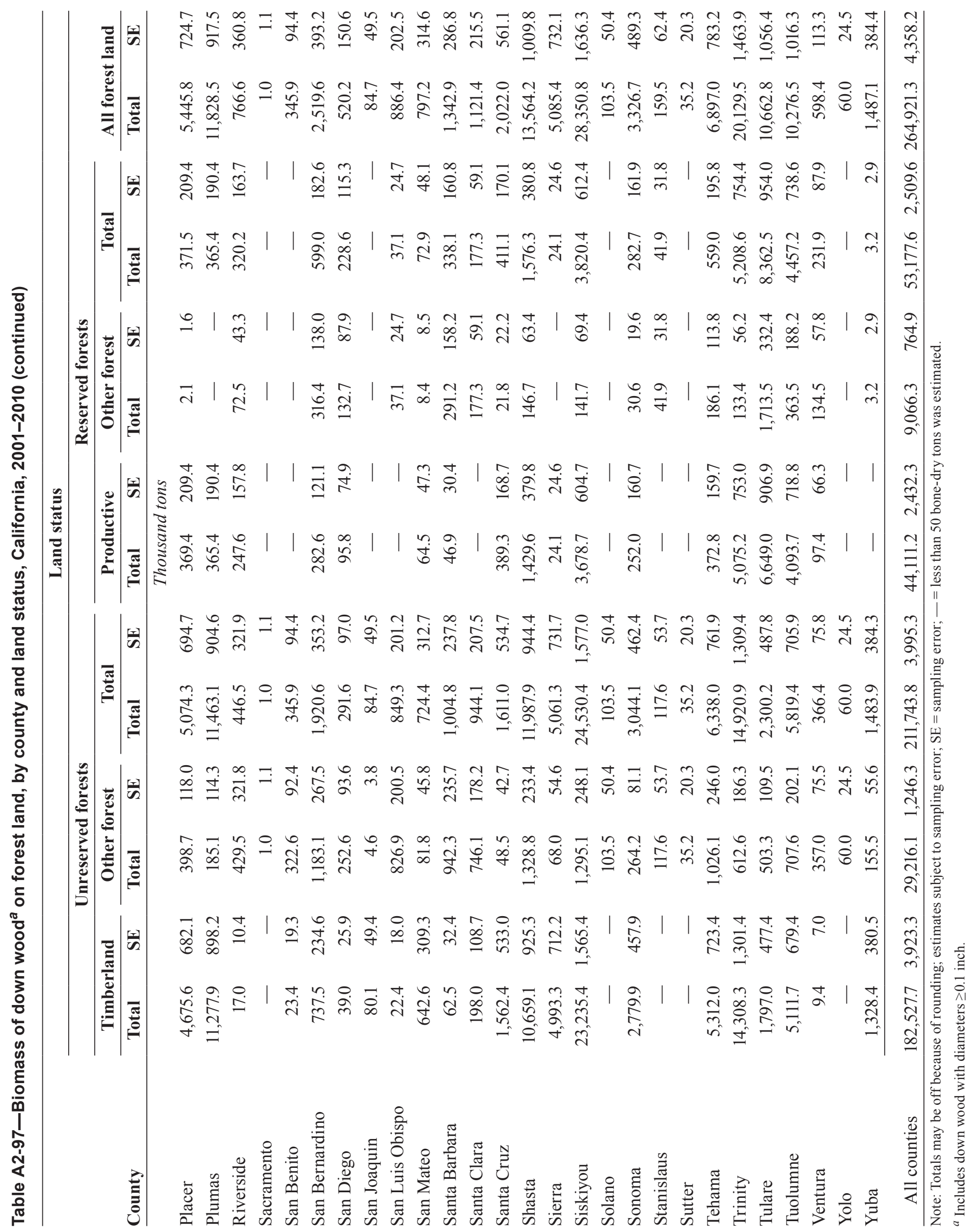




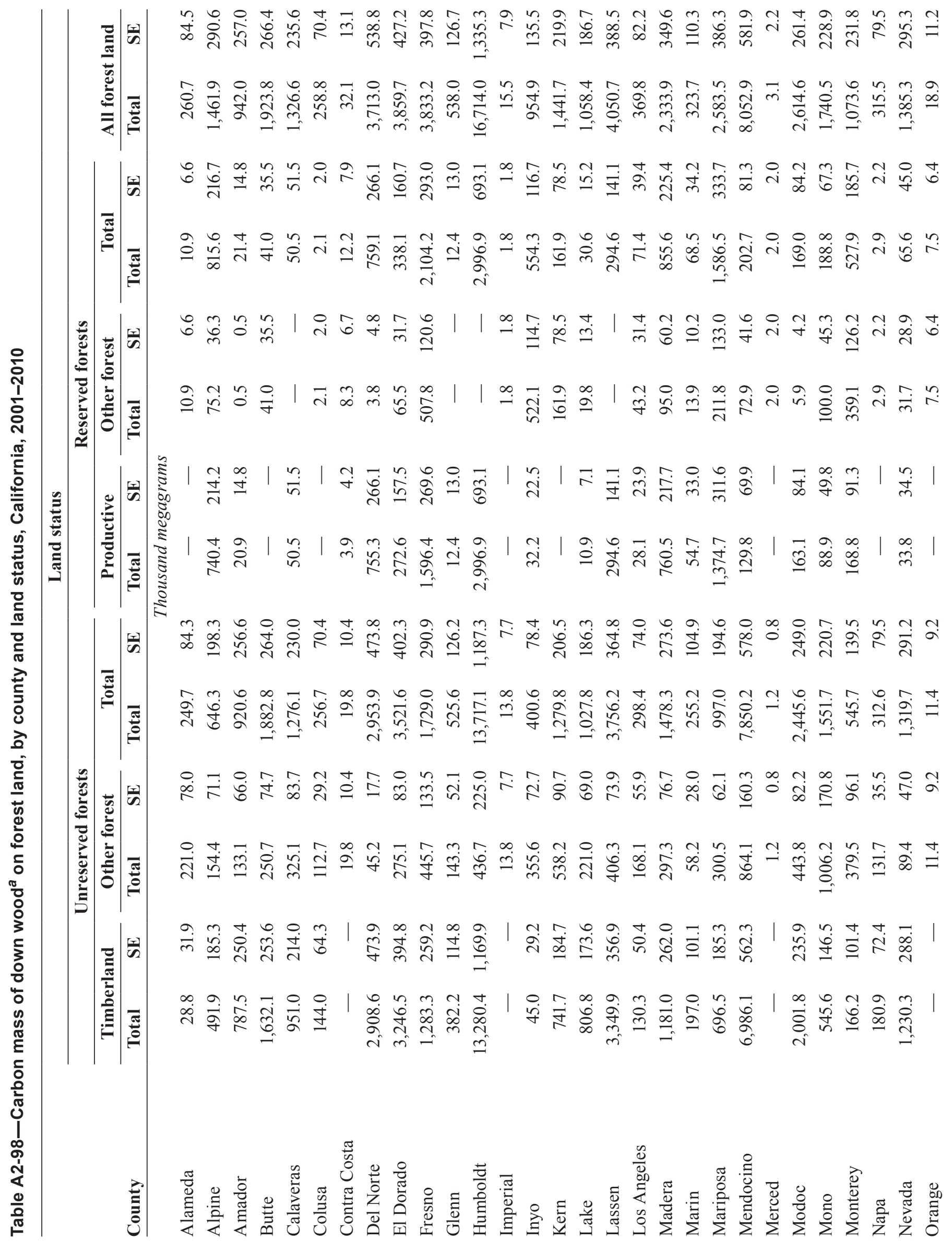




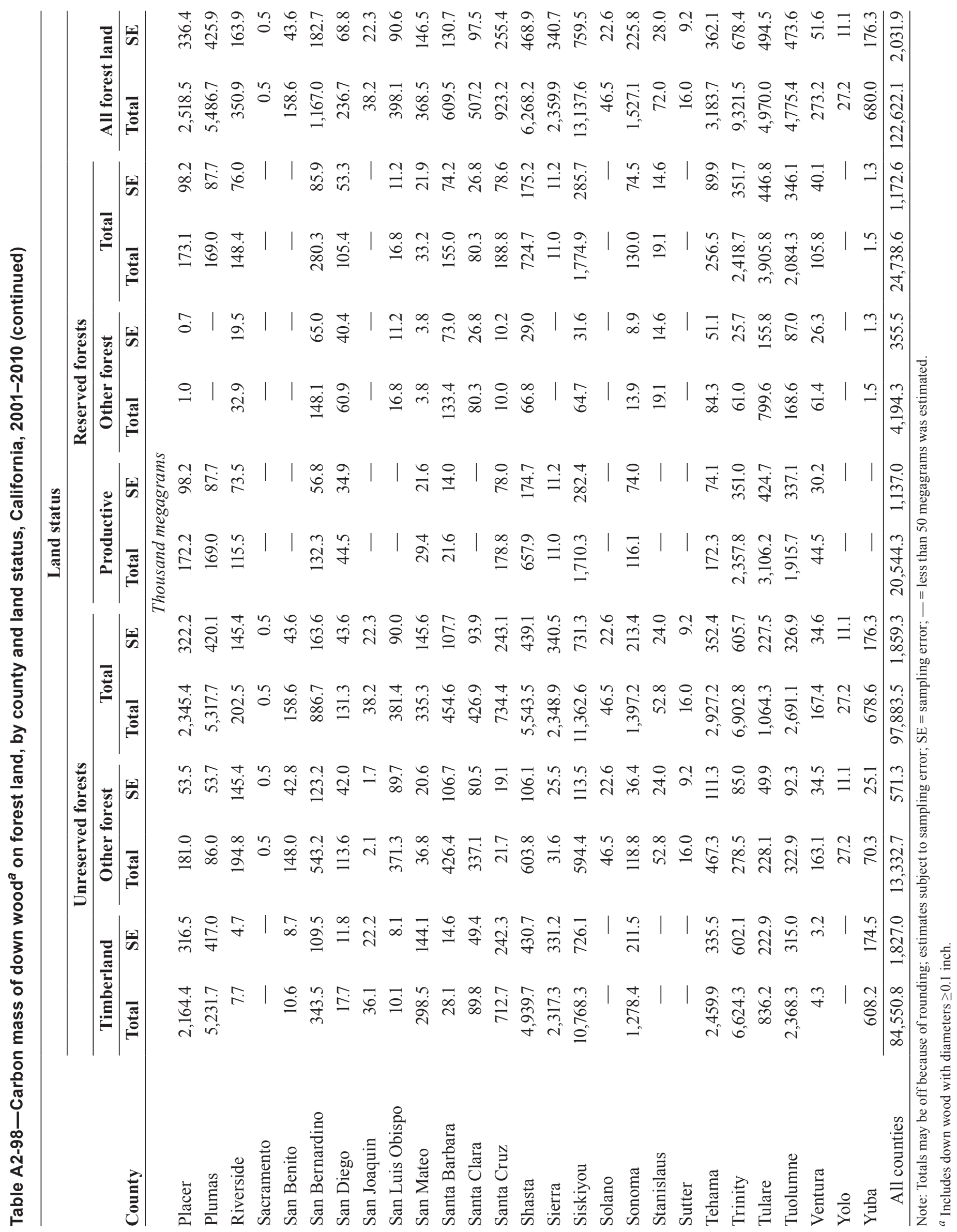




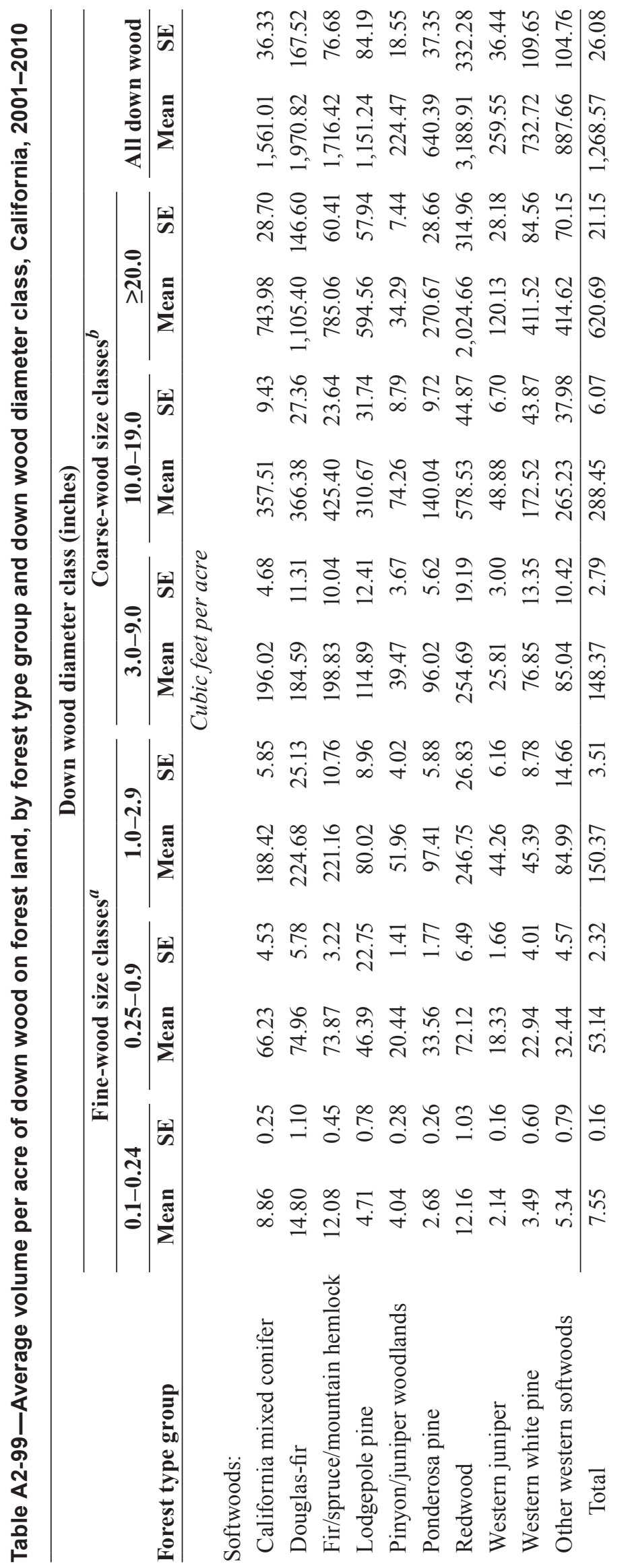

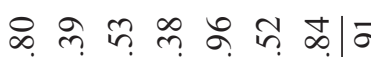
तु่

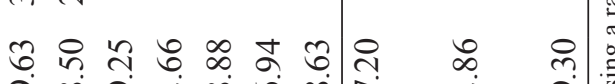

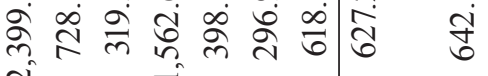

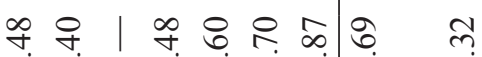

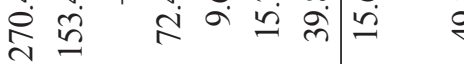

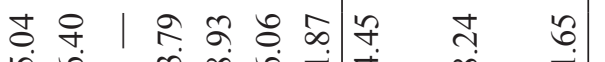
命

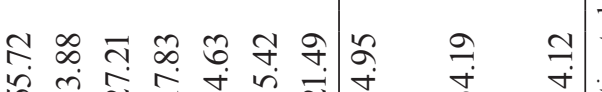

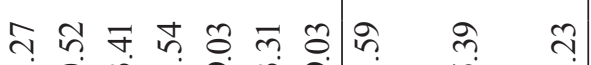

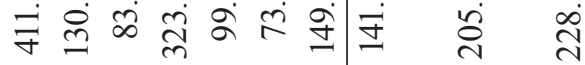
告.

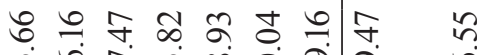

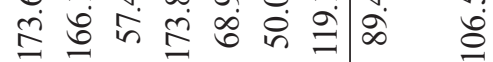

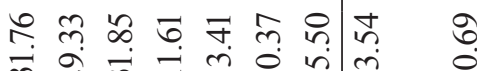

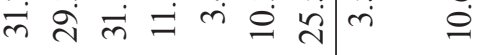
苨

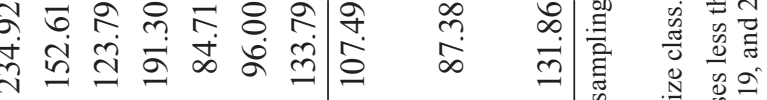
도원

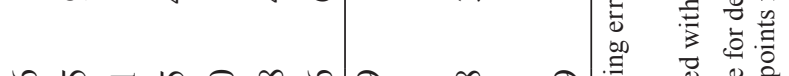

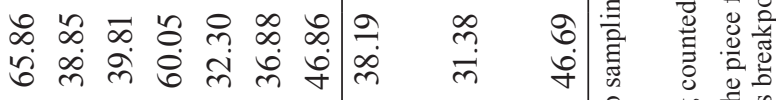

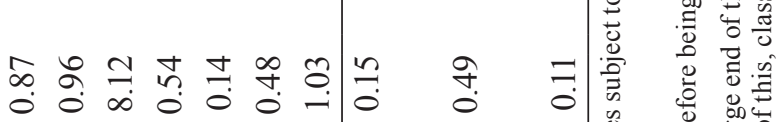

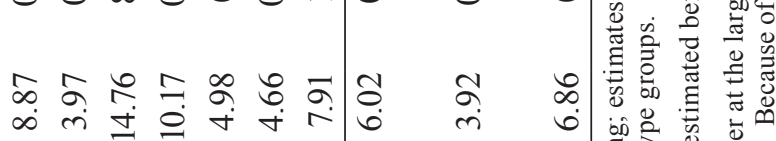

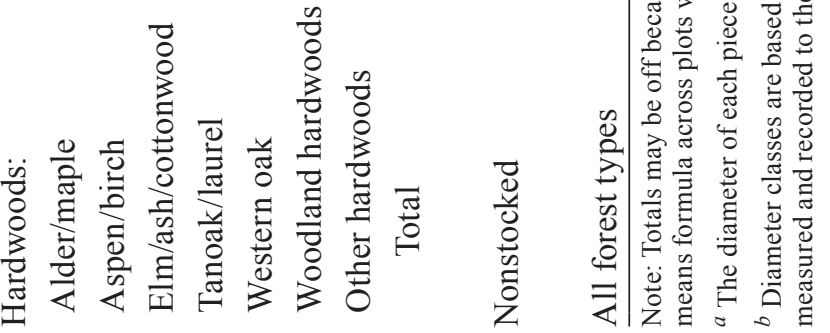




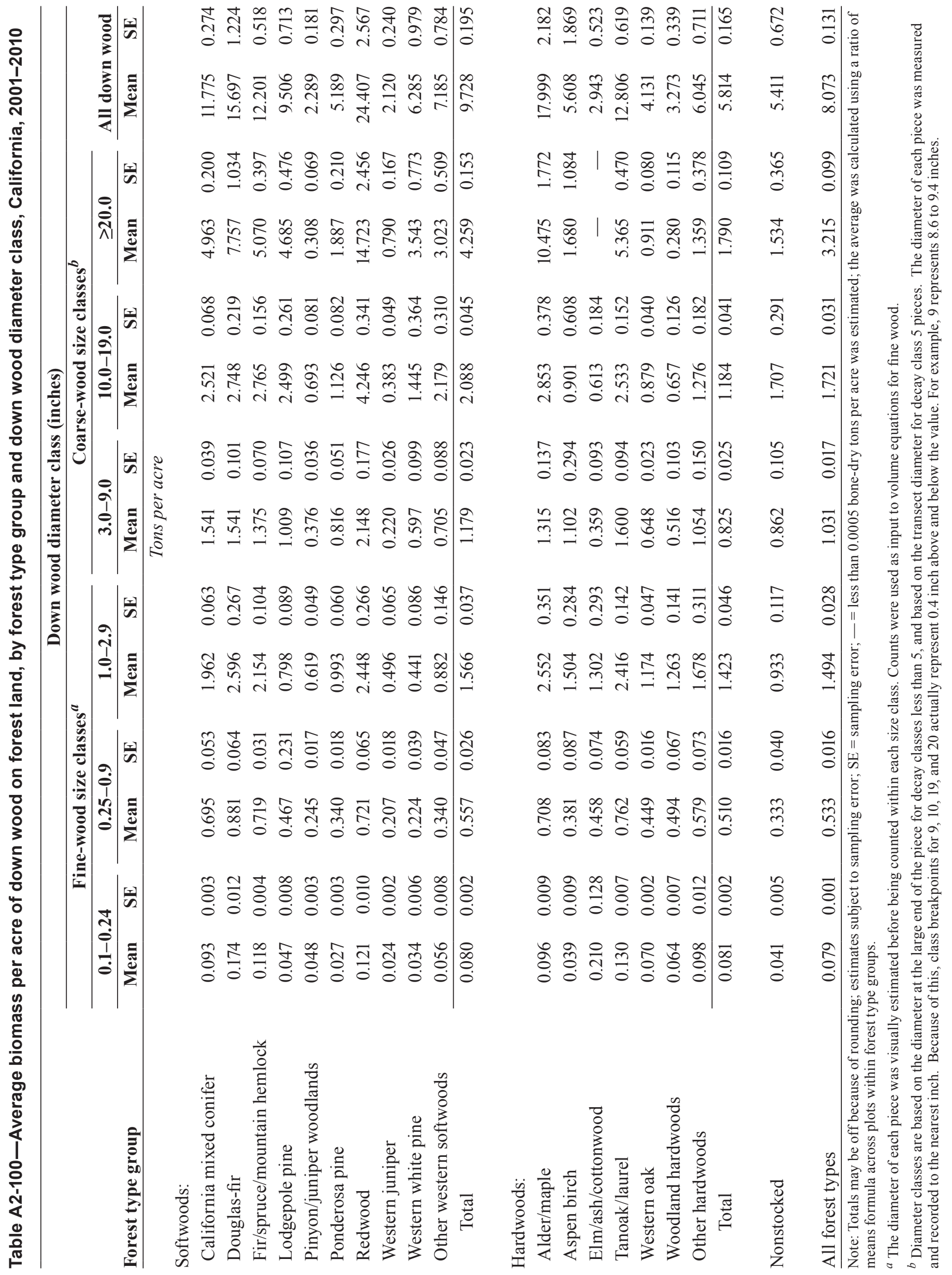




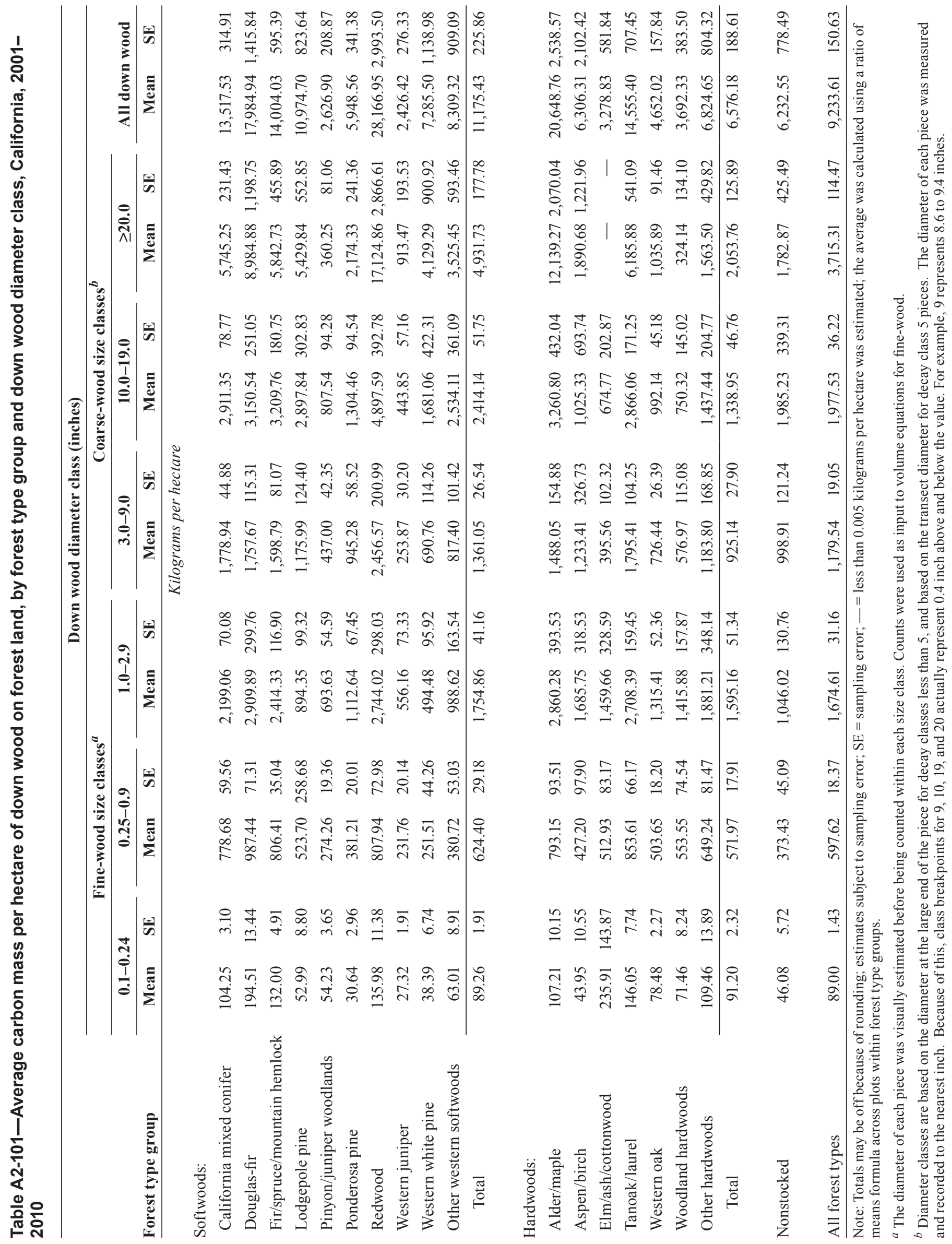


Table A2-102-Average number of pieces per acre of down wood ${ }^{a}$ on forest land, by forest type group and down wood diameter class, California, 2001-2010

\begin{tabular}{|c|c|c|c|c|c|c|c|c|}
\hline \multirow[b]{3}{*}{ Forest type group } & \multicolumn{6}{|c|}{ Down wood diameter class (inches) } & & \\
\hline & \multicolumn{2}{|c|}{$3.0-9.0$} & \multicolumn{2}{|c|}{$10.0-19.0$} & \multicolumn{2}{|c|}{$\geq 20.0$} & \multicolumn{2}{|c|}{ All classes } \\
\hline & Mean & SE & Mean & SE & Mean & SE & Mean & SE \\
\hline & \multicolumn{8}{|c|}{ Pieces per acre } \\
\hline \multicolumn{9}{|l|}{ Softwoods: } \\
\hline California mixed conifer & 185.7 & 4.2 & 40.0 & 1.1 & 9.4 & 0.4 & 235.2 & 4.9 \\
\hline Douglas-fir & 154.6 & 9.1 & 40.0 & 3.4 & 16.2 & 2.0 & 210.7 & 11.2 \\
\hline Fir/spruce/mountain hemlock & 184.7 & 8.6 & 45.2 & 2.5 & 11.4 & 0.9 & 241.4 & 10.1 \\
\hline Lodgepole pine & 99.5 & 10.5 & 27.8 & 2.3 & 8.5 & 0.9 & 135.7 & 11.8 \\
\hline Pinyon/juniper woodlands & 51.2 & 4.6 & 12.3 & 1.6 & 1.3 & 0.3 & 64.8 & 5.7 \\
\hline Ponderosa pine & 101.6 & 5.5 & 16.4 & 1.3 & 4.0 & 0.5 & 122.1 & 6.3 \\
\hline Redwood & 207.4 & 15.4 & 67.0 & 5.9 & 24.8 & 2.6 & 299.2 & 18.8 \\
\hline Western juniper & 33.4 & 4.1 & 6.4 & 0.9 & 1.6 & 0.3 & 41.3 & 4.5 \\
\hline Western white pine & 82.8 & 13.6 & 19.1 & 5.3 & 8.0 & 2.8 & 109.9 & 15.7 \\
\hline Other western softwoods & 78.2 & 8.8 & 30.2 & 4.2 & 6.7 & 1.1 & 115.1 & 11.5 \\
\hline Total & 140.6 & 2.5 & 32.2 & 0.7 & 8.4 & 0.3 & 181.2 & 2.9 \\
\hline
\end{tabular}

Hardwoods:

\begin{tabular}{lrrrrrrrr} 
Alder/maple & 138.0 & 15.0 & 42.0 & 5.6 & 24.3 & 4.6 & 204.3 & 19.5 \\
Aspen/birch & 194.1 & 48.7 & 9.5 & 5.3 & 2.5 & 1.7 & 206.1 & 51.7 \\
Elm/ash/cottonwood & 72.2 & 20.1 & 5.9 & 3.0 & - & - & 78.1 & 20.6 \\
Tanoak/laurel & 155.2 & 6.9 & 33.5 & 2.0 & 11.0 & 0.9 & 199.7 & 8.1 \\
Western oak & 73.0 & 2.4 & 11.1 & 0.5 & 1.7 & 0.1 & 85.7 & 2.7 \\
Woodland hardwoods & 65.9 & 11.9 & 12.6 & 2.9 & 1.0 & 0.5 & 79.4 & 13.4 \\
Other hardwoods & 115.7 & 15.2 & 17.7 & 3.2 & 2.6 & 0.9 & 136.0 & 17.1 \\
$\quad$ & 89.5 & 2.3 & 15.5 & 0.6 & 3.6 & 0.2 & 108.6 & 2.7 \\
$\quad$ Total & & & & & & & & 12.5 \\
Nonstocked & 92.7 & 10.5 & 19.8 & 2.8 & 3.0 & 0.8 & 115.5 & 12.5 \\
& & & & & & & & 2.0 \\
All forest types & 119.2 & 1.7 & 25.3 & 0.5 & 6.4 & 0.2 & 150.8 & 2.0 \\
\hline
\end{tabular}

Note: Totals may be off because of rounding; estimates subject to sampling error; $\mathrm{SE}=$ sampling error; — $=$ less than 0.05 pieces per acre was estimated; the average was calculated using a ratio of means formula across plots within forest type groups.

${ }^{a}$ Includes down wood pieces $\geq 3$ inches in diameter at the transect intersection. Number of pieces can not be estimated for down wood with smaller diameters (fine woody material) because pieces were not individually measured. 


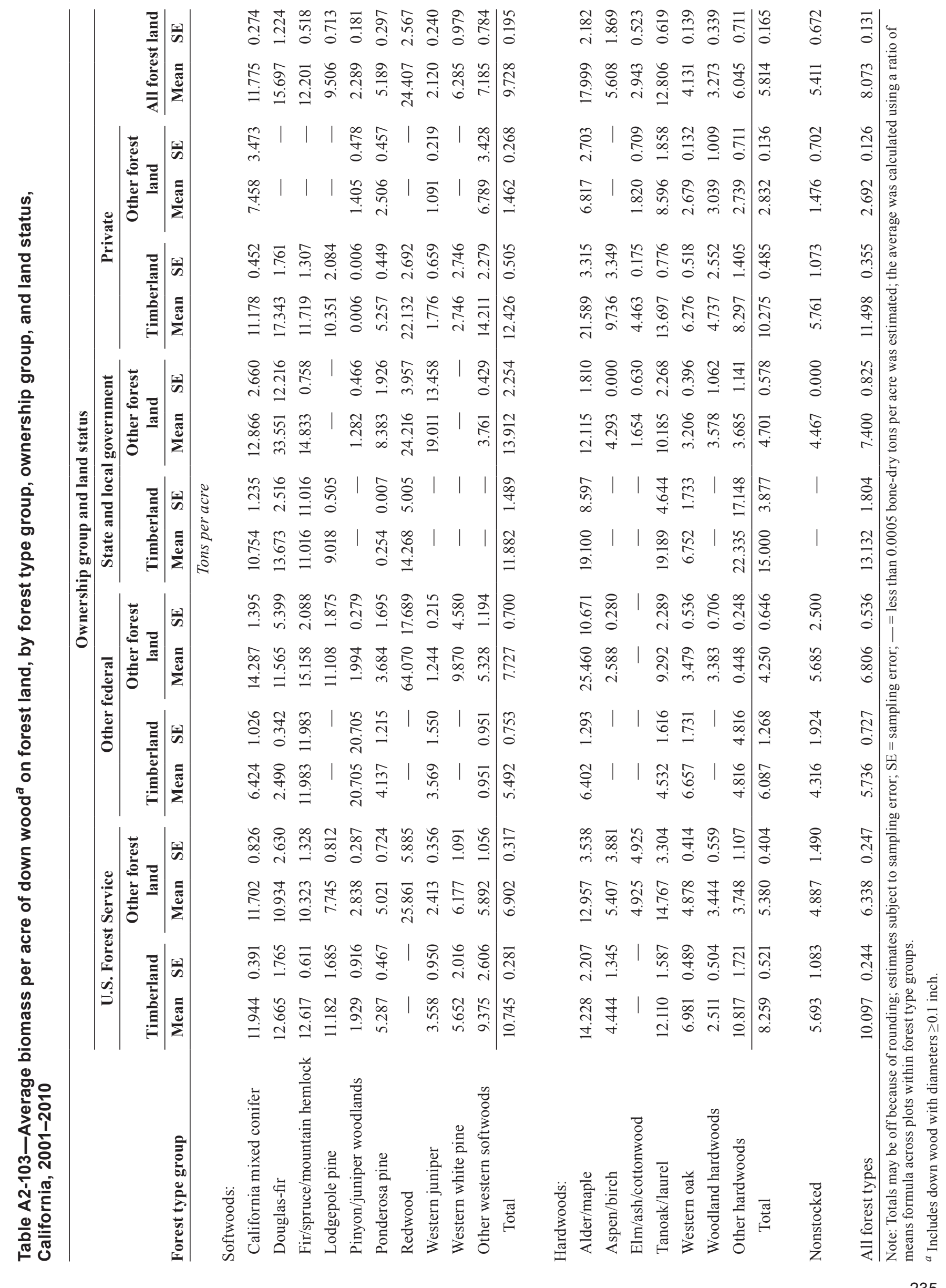




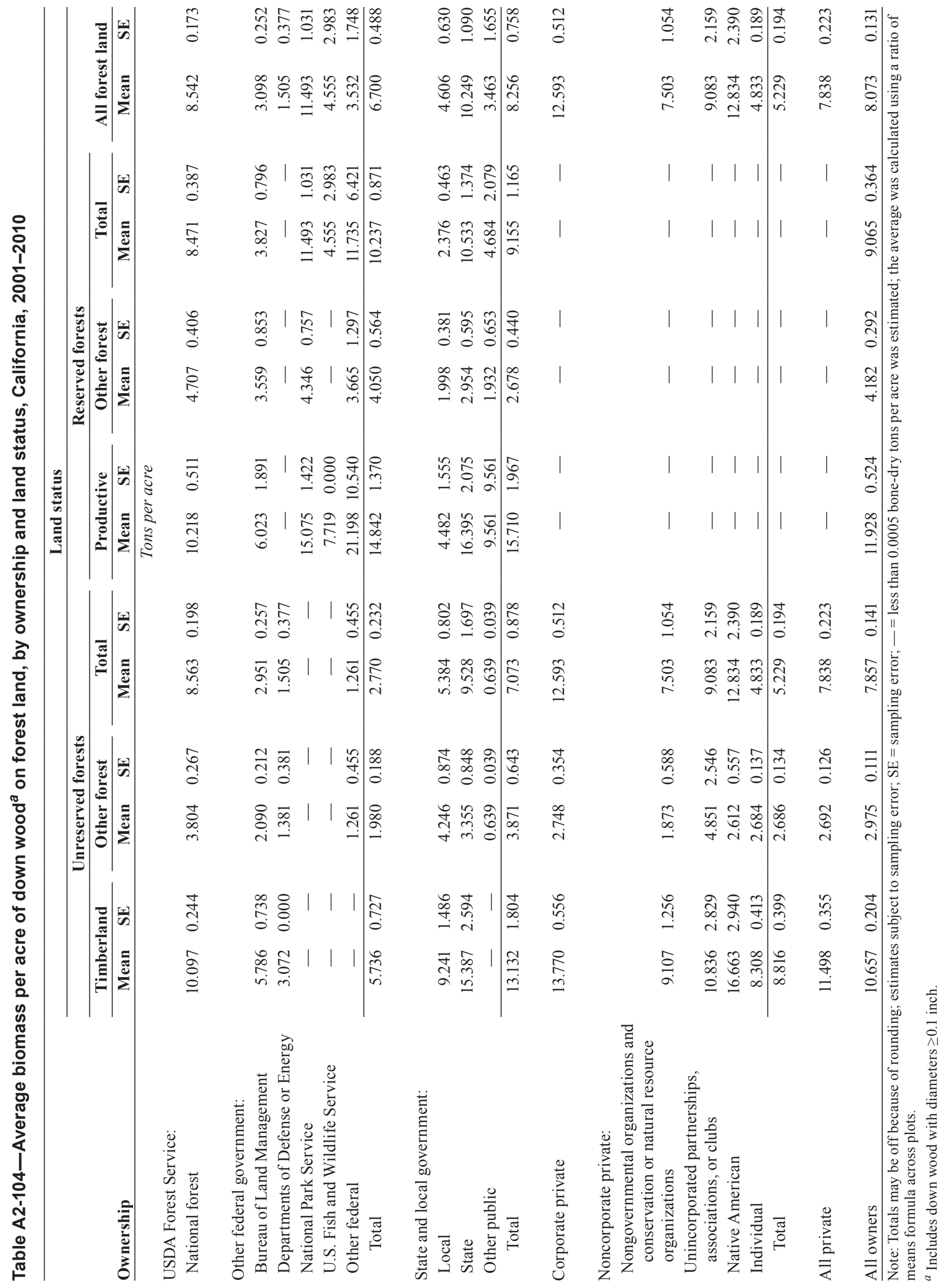


Table A2-105-Average biomass per acre of down wood ${ }^{a}$ on forest land, by county and land status, California, 2001-2010

\begin{tabular}{|c|c|c|c|c|c|c|c|c|c|c|c|c|c|c|}
\hline \multirow[b]{4}{*}{ County } & \multicolumn{14}{|c|}{ Land status } \\
\hline & \multicolumn{6}{|c|}{ Unreserved forests } & \multicolumn{6}{|c|}{ Reserved forests } & & \\
\hline & \multicolumn{2}{|c|}{ Timberland } & \multicolumn{2}{|c|}{ Other forest } & \multicolumn{2}{|c|}{ Total } & \multicolumn{2}{|c|}{ Productive } & \multicolumn{2}{|c|}{ Other forest } & \multicolumn{2}{|c|}{ Total } & \multicolumn{2}{|c|}{ All forest land } \\
\hline & Mean & SE & Mean & SE & Mean & SE & Mean & SE & Mean & SE & Mean & SE & Mean & SE \\
\hline & \multicolumn{14}{|c|}{ Tons per acre } \\
\hline Alameda & 9.970 & 2.725 & 6.118 & 1.322 & 6.403 & 1.288 & - & - & 1.241 & 0.272 & 1.241 & 0.272 & 5.448 & 1.162 \\
\hline Alpine & 8.980 & 2.723 & 4.803 & 1.691 & 7.423 & 1.862 & 10.095 & 2.159 & 2.685 & 0.952 & 8.051 & 1.679 & 7.760 & 1.244 \\
\hline Amador & 13.851 & 3.251 & 3.809 & 1.532 & 9.984 & 2.232 & 2.797 & 1.404 & 0.174 & 0.174 & 2.077 & 1.138 & 9.199 & 2.055 \\
\hline Butte & 9.181 & 0.900 & 4.245 & 0.837 & 7.940 & 0.734 & — & - & 7.335 & 3.391 & 7.335 & 3.391 & 7.925 & 0.721 \\
\hline Calaveras & 10.182 & 1.557 & 3.158 & 0.668 & 6.458 & 0.913 & 17.581 & 17.581 & - & - & 17.581 & 17.581 & 6.615 & 0.915 \\
\hline Colusa & 6.427 & 1.724 & 1.531 & 0.284 & 2.660 & 0.575 & - & - & 0.689 & 0.689 & 0.689 & 0.689 & 2.599 & 0.560 \\
\hline Contra Costa & - & - & 1.831 & 0.343 & 1.831 & 0.343 & 1.532 & 1.532 & 1.324 & 0.584 & 1.384 & 0.415 & 1.631 & 0.276 \\
\hline Del Norte & 12.946 & 1.584 & 1.983 & 0.358 & 11.926 & 1.475 & 18.179 & 4.186 & 2.037 & 2.037 & 17.485 & 4.096 & 12.757 & 1.406 \\
\hline El Dorado & 10.932 & 0.897 & 4.866 & 1.032 & 9.946 & 0.791 & 11.537 & 5.456 & 5.341 & 0.844 & 9.448 & 3.703 & 9.900 & 0.792 \\
\hline Fresno & 9.865 & 1.464 & 2.847 & 0.751 & 6.006 & 0.840 & 8.796 & 1.050 & 4.243 & 0.789 & 6.983 & 0.736 & 6.504 & 0.553 \\
\hline Glenn & 8.369 & 1.414 & 3.325 & 0.862 & 5.906 & 0.930 & 4.576 & 4.576 & - & - & 4.576 & 4.576 & 5.867 & 0.904 \\
\hline Humboldt & 18.913 & 1.322 & 10.550 & 4.574 & 18.442 & 1.277 & 32.208 & 5.303 & - & - & 32.208 & 5.303 & 19.962 & 1.296 \\
\hline Imperial & - & - & 0.564 & 0.252 & 0.564 & 0.252 & - & - & 0.921 & 0.921 & 0.921 & 0.921 & 0.591 & 0.235 \\
\hline Inyo & 5.715 & 1.409 & 2.774 & 0.480 & 2.944 & 0.471 & 5.482 & 0.332 & 4.135 & 0.718 & 4.194 & 0.687 & 3.561 & 0.420 \\
\hline Kern & 10.076 & 1.611 & 2.086 & 0.282 & 3.827 & 0.507 & - & - & 4.781 & 1.908 & 4.781 & 1.908 & 3.914 & 0.492 \\
\hline Lake & 9.098 & 1.260 & 2.959 & 0.701 & 6.284 & 0.829 & 2.151 & 0.182 & 2.387 & 0.864 & 2.299 & 0.543 & 5.981 & 0.777 \\
\hline Lassen & 8.431 & 0.607 & 1.808 & 0.272 & 6.017 & 0.442 & 17.275 & 3.862 & - & - & 17.275 & 3.862 & 6.316 & 0.458 \\
\hline Los Angeles & 4.610 & 1.069 & 2.478 & 0.695 & 3.097 & 0.614 & 5.649 & 3.380 & 3.543 & 1.766 & 4.166 & 1.598 & 3.258 & 0.574 \\
\hline Madera & 11.358 & 1.779 & 2.052 & 0.445 & 5.900 & 0.907 & 11.129 & 2.241 & 5.633 & 2.610 & 10.043 & 1.899 & 6.948 & 0.846 \\
\hline Marin & 18.072 & 2.413 & 3.730 & 1.134 & 9.553 & 2.471 & 6.403 & 0.737 & 2.746 & 0.333 & 5.035 & 0.909 & 8.019 & 1.754 \\
\hline Mariposa & 10.071 & 1.795 & 2.585 & 0.367 & 5.356 & 0.829 & 15.018 & 2.324 & 10.646 & 5.288 & 14.237 & 2.144 & 8.651 & 1.029 \\
\hline Mendocino & 11.628 & 0.662 & 5.298 & 0.743 & 10.254 & 0.561 & 11.193 & 2.308 & 5.310 & 1.842 & 7.985 & 1.752 & 10.181 & 0.546 \\
\hline Merced & - & - & 0.103 & 0.073 & 0.103 & 0.073 & - & - & 0.641 & 0.057 & 0.641 & 0.057 & 0.219 & 0.126 \\
\hline Modoc & 6.247 & 0.478 & 1.437 & 0.233 & 3.883 & 0.306 & 10.588 & 3.275 & 1.050 & 0.126 & 8.014 & 2.830 & 4.016 & 0.312 \\
\hline Mono & 5.714 & 1.205 & 3.907 & 0.546 & 4.397 & 0.518 & 7.844 & 1.814 & 3.086 & 1.041 & 4.323 & 1.045 & 4.389 & 0.475 \\
\hline Monterey & 11.219 & 4.771 & 2.316 & 0.505 & 3.042 & 0.673 & 11.144 & 4.544 & 7.604 & 2.190 & 8.460 & 2.400 & 4.441 & 0.832 \\
\hline Napa & 6.745 & 1.625 & 2.556 & 0.411 & 3.993 & 0.733 & - & - & 0.866 & 0.244 & 0.866 & 0.244 & 3.862 & 0.712 \\
\hline Nevada & 7.698 & 1.490 & 3.234 & 1.380 & 7.031 & 1.296 & 26.558 & 26.558 & 8.627 & 8.627 & 13.148 & 4.619 & 7.190 & 1.266 \\
\hline Orange & - & - & 2.263 & 0.606 & 2.263 & 0.606 & - & - & 6.028 & 2.931 & 6.028 & 2.931 & 3.014 & 0.869 \\
\hline Placer & 9.999 & 0.982 & 3.454 & 0.706 & 8.703 & 0.842 & 9.671 & 4.036 & 0.182 & 0.063 & 7.464 & 3.365 & 8.606 & 0.821 \\
\hline Plumas & 8.431 & 0.464 & 3.808 & 1.981 & 8.269 & 0.454 & 10.086 & 3.176 & - & - & 10.086 & 3.176 & 8.315 & 0.450 \\
\hline Riverside & 1.119 & 0.133 & 8.551 & 5.833 & 6.823 & 4.547 & 10.422 & 3.917 & 1.691 & 0.852 & 4.802 & 2.001 & 5.803 & 2.475 \\
\hline Sacramento & - & - & 0.109 & 0.051 & 0.108 & 0.051 & - & - & - & - & - & - & 0.108 & 0.051 \\
\hline San Benito & 2.470 & 0.471 & 2.292 & 0.491 & 2.303 & 0.462 & - & - & - & - & - & - & 2.303 & 0.462 \\
\hline San Bernardino & 7.844 & 1.531 & 4.944 & 0.814 & 5.762 & 0.747 & 7.464 & 1.145 & 2.007 & 0.775 & 3.063 & 0.757 & 4.764 & 0.559 \\
\hline San Diego & 2.747 & 2.747 & 3.163 & 0.804 & 3.101 & 0.685 & 7.631 & 2.344 & 3.276 & 1.759 & 4.306 & 1.577 & 3.536 & 0.728 \\
\hline San Joaquin & 4.630 & 4.630 & 0.635 & 0.220 & 3.443 & 0.788 & - & - & - & - & - & - & 3.443 & 0.788 \\
\hline
\end{tabular}


Table A2-105-Average biomass per acre of down wood ${ }^{a}$ on forest land, by county and land status, California, 2001-2010 (continued)

\begin{tabular}{|c|c|c|c|c|c|c|c|c|c|c|c|c|c|c|}
\hline \multirow[b]{4}{*}{ County } & \multicolumn{14}{|c|}{ Land status } \\
\hline & \multicolumn{6}{|c|}{ Unreserved forests } & \multicolumn{6}{|c|}{ Reserved forests } & & \\
\hline & \multicolumn{2}{|c|}{ Timberland } & \multicolumn{2}{|c|}{ Other forest } & \multicolumn{2}{|c|}{ Total } & \multicolumn{2}{|c|}{ Productive } & \multicolumn{2}{|c|}{ Other forest } & \multicolumn{2}{|c|}{ Total } & \multicolumn{2}{|c|}{ All forest land } \\
\hline & Mean & SE & Mean & SE & Mean & SE & Mean & SE & Mean & SE & Mean & SE & Mean & SE \\
\hline & \multicolumn{14}{|c|}{ Tons per acre } \\
\hline San Luis Obispo & 1.902 & 1.115 & 3.213 & 0.629 & 3.156 & 0.607 & - & - & 1.284 & 0.579 & 1.284 & 0.579 & 2.974 & 0.556 \\
\hline San Mateo & 14.099 & 4.824 & 4.212 & 1.635 & 11.144 & 3.674 & 4.482 & 1.555 & 1.700 & 1.700 & 3.773 & 1.329 & 9.456 & 2.952 \\
\hline Santa Barbara & 2.925 & 0.443 & 4.482 & 0.873 & 4.338 & 0.797 & 2.427 & 0.820 & 5.032 & 2.200 & 4.380 & 1.687 & 4.349 & 0.732 \\
\hline Santa Clara & 6.951 & 2.012 & 4.021 & 0.648 & 4.411 & 0.652 & - & - & 2.688 & 0.556 & 2.688 & 0.556 & 4.005 & 0.529 \\
\hline Santa Cruz & 12.921 & 3.790 & 5.009 & 1.690 & 12.334 & 3.527 & 8.960 & 1.969 & 3.532 & 3.532 & 8.284 & 1.838 & 11.219 & 2.623 \\
\hline Shasta & 8.556 & 0.508 & 2.987 & 0.405 & 7.091 & 0.414 & 10.417 & 1.753 & 2.850 & 0.765 & 8.353 & 1.403 & 7.218 & 0.398 \\
\hline Sierra & 10.692 & 0.982 & 7.177 & 5.891 & 10.622 & 1.007 & 5.218 & 5.218 & - & - & 5.218 & 5.218 & 10.570 & 1.001 \\
\hline Siskiyou & 10.366 & 0.529 & 2.825 & 0.440 & 9.086 & 0.461 & 10.028 & 1.119 & 4.030 & 1.455 & 9.503 & 1.049 & 9.140 & 0.422 \\
\hline Solano & - & - & 3.908 & 1.152 & 3.908 & 1.152 & - & - & - & - & - & - & 3.698 & 1.096 \\
\hline Sonoma & 8.694 & 0.847 & 2.230 & 0.454 & 6.947 & 0.704 & 11.982 & 3.754 & 1.207 & 0.547 & 6.090 & 2.624 & 6.865 & 0.684 \\
\hline Stanislaus & - & - & 1.370 & 0.518 & 1.370 & 0.518 & - & - & 2.365 & 1.063 & 2.365 & 1.063 & 1.541 & 0.477 \\
\hline Sutter & - & - & 1.281 & 0.317 & 1.281 & 0.317 & - & - & - & - & - & - & 1.281 & 0.317 \\
\hline Tehama & 11.636 & 0.909 & 2.329 & 0.504 & 7.065 & 0.638 & 7.366 & 1.986 & 5.441 & 3.112 & 6.590 & 1.779 & 7.024 & 0.603 \\
\hline Trinity & 11.854 & 0.757 & 4.583 & 0.976 & 11.129 & 0.702 & 10.895 & 1.138 & 2.190 & 0.610 & 9.888 & 1.044 & 10.779 & 0.586 \\
\hline Tulare & 9.045 & 1.833 & 1.669 & 0.283 & 4.599 & 0.847 & 11.421 & 1.095 & 5.859 & 0.794 & 9.561 & 0.801 & 7.756 & 0.616 \\
\hline Tuolumne & 11.013 & 0.858 & 3.978 & 0.883 & 9.064 & 0.723 & 12.176 & 1.456 & 8.174 & 2.869 & 11.709 & 1.337 & 10.048 & 0.681 \\
\hline Ventura & 0.784 & 0.228 & 2.131 & 0.315 & 2.041 & 0.301 & 2.635 & 1.455 & 2.628 & 0.811 & 2.631 & 0.771 & 2.236 & 0.325 \\
\hline Yolo & - & - & 0.901 & 0.278 & 0.901 & 0.278 & - & - & - & - & - & - & 0.901 & 0.278 \\
\hline Yuba & 11.388 & 2.052 & 2.520 & 0.494 & 8.320 & 1.549 & - & - & 0.449 & 0.028 & 0.449 & 0.028 & 8.016 & 1.513 \\
\hline All counties & 10.657 & 0.204 & 2.975 & 0.111 & 7.857 & 0.141 & 11.928 & 0.524 & 4.182 & 0.292 & 9.065 & 0.364 & 8.073 & 0.131 \\
\hline
\end{tabular}

Note: Totals may be off because of rounding; estimates subject to sampling error; SE = sampling error; $-=$ less than 0.0005 bone-dry tons per acre was estimated; the average was calculated using a ratio of means formula across plots.

${ }^{a}$ Includes down wood with diameters $\geq 0.1$ inch. 
Table A2-106-Average biomass per acre of all dead wood (dead trees and down wood) ${ }^{a}$ on forest land, by county and land status, California, 2001-2010

\begin{tabular}{|c|c|c|c|c|c|c|c|c|c|c|c|c|c|c|}
\hline \multirow[b]{4}{*}{ County } & \multicolumn{14}{|c|}{ Land status } \\
\hline & \multicolumn{6}{|c|}{ Unreserved forests } & \multicolumn{7}{|c|}{ Reserved forests } & \\
\hline & \multicolumn{2}{|c|}{ Timberland } & \multicolumn{2}{|c|}{ Other forest } & \multicolumn{2}{|c|}{ Total } & \multicolumn{2}{|c|}{ Productive } & \multicolumn{2}{|c|}{ Other forest } & \multicolumn{2}{|c|}{ Total } & \multicolumn{2}{|c|}{ All forest land } \\
\hline & Mean & SE & Mean & $\overline{S E}$ & Mean & $\overline{\text { SE }}$ & Mean & $\overline{\text { SE }}$ & Mean & $\overline{S E}$ & Mean & SE & Mean & $\overline{S E}$ \\
\hline & \multicolumn{14}{|c|}{ Tons per acre } \\
\hline Alameda & 9.97 & 2.73 & 8.68 & 2.01 & 8.78 & 1.87 & - & - & 1.24 & 0.27 & 1.24 & 0.27 & 7.38 & 1.69 \\
\hline Alpine & 20.03 & 5.43 & 6.23 & 2.45 & 14.89 & 3.72 & 19.66 & 3.41 & 4.43 & 1.36 & 15.46 & 2.75 & 15.19 & 2.26 \\
\hline Amador & 23.42 & 5.47 & 4.33 & 1.58 & 16.07 & 3.77 & 20.06 & 4.06 & 0.17 & 0 & 14.61 & 5.39 & 15.92 & 3.44 \\
\hline Butte & 16.73 & 1.95 & 6.01 & 1.00 & 14.03 & 1.56 & - & - & 8.94 & 4.33 & 8.94 & 4.33 & 13.91 & 1.53 \\
\hline Calaveras & 17.22 & 3.00 & 4.43 & 0.83 & 10.44 & 1.65 & 21.16 & 21.16 & - & - & 21.16 & 21.16 & 10.59 & 1.63 \\
\hline Colusa & 9.30 & 2.36 & 2.91 & 0.60 & 4.38 & 0.86 & - & - & 0.69 & 0.69 & 0.69 & 0.69 & 4.27 & 0.84 \\
\hline Contra Costa & - & - & 1.97 & 0.22 & 1.97 & 0.22 & 2.83 & 2.83 & 1.32 & 0.58 & 1.75 & 0.53 & 1.87 & 0.27 \\
\hline Del Norte & 25.56 & 3.16 & 5.53 & 1.68 & 23.69 & 2.92 & 38.05 & 6.17 & 3.27 & 3.27 & 36.55 & 6.17 & 25.62 & 2.68 \\
\hline El Dorado & 20.62 & 1.75 & 6.38 & 1.62 & 18.30 & 1.54 & 35.69 & 16.16 & 12.26 & 3.01 & 27.79 & 11.12 & 19.18 & 1.74 \\
\hline Fresno & 20.03 & 2.82 & 4.07 & 1.19 & 11.26 & 1.60 & 20.10 & 2.01 & 7.24 & 1.16 & 14.98 & 1.43 & 13.16 & 1.07 \\
\hline Glenn & 20.16 & 5.10 & 4.75 & 1.36 & 12.63 & 2.98 & 13.16 & 0 & - & - & 13.16 & 0 & 12.65 & 2.89 \\
\hline Humboldt & 32.07 & 2.42 & 12.93 & 4.65 & 31.00 & 2.31 & 63.17 & 10.18 & - & - & 63.17 & 10.18 & 34.55 & 2.38 \\
\hline Imperial & - & - & 1.21 & 0.61 & 1.21 & 0.61 & - & - & 1.90 & 1.90 & 1.90 & 0 & 1.26 & 0.57 \\
\hline Inyo & 14.24 & 2.34 & 4.00 & 0.60 & 4.59 & 0.67 & 12.29 & 4.44 & 5.72 & 0.95 & 6.01 & 0.95 & 5.29 & 0.58 \\
\hline Kern & 17.77 & 3.88 & 3.35 & 0.57 & 6.50 & 1.08 & - & - & 7.37 & 2.02 & 7.37 & 2.02 & 6.58 & 1.00 \\
\hline Lake & 14.98 & 2.47 & 4.74 & 1.02 & 10.28 & 1.53 & 2.26 & 0.13 & 3.62 & 0.50 & 3.11 & 0.41 & 9.74 & 1.43 \\
\hline Lassen & 12.52 & 0.99 & 3.08 & 0.51 & 9.08 & 0.71 & 31.77 & 8.61 & - & - & 31.77 & 8.61 & 9.68 & 0.77 \\
\hline Los Angeles & 12.04 & 3.76 & 3.10 & 0.71 & 5.70 & 1.40 & 9.89 & 6.73 & 7.50 & 2.67 & 8.21 & 2.72 & 6.08 & 1.26 \\
\hline Madera & 24.58 & 4.91 & 2.72 & 0.50 & 11.76 & 2.32 & 33.87 & 7.14 & 9.64 & 4.60 & 29.08 & 6.01 & 16.15 & 2.41 \\
\hline Marin & 41.38 & 8.70 & 4.58 & 1.23 & 19.52 & 6.71 & 27.46 & 9.81 & 2.75 & 0.33 & 18.22 & 8.27 & 19.08 & 5.26 \\
\hline Mariposa & 15.21 & 2.50 & 3.55 & 0.55 & 7.87 & 1.20 & 40.52 & 5.26 & 15.19 & 6.15 & 36.00 & 4.73 & 18.30 & 2.28 \\
\hline Mendocino & 17.93 & 1.01 & 7.07 & 1.06 & 15.58 & 0.86 & 34.10 & 17.56 & 10.22 & 2.65 & 21.08 & 8.99 & 15.76 & 0.88 \\
\hline Merced & - & - & 0.10 & 0.07 & 0.10 & 0.07 & - & - & 0.64 & 0.06 & 0.64 & 0.06 & 0.22 & 0.13 \\
\hline Modoc & 10.04 & 1.01 & 1.84 & 0.30 & 6.01 & 0.59 & 26.85 & 9.73 & 2.30 & 1.02 & 20.23 & 8.17 & 6.47 & 0.65 \\
\hline Mono & 8.50 & 1.62 & 6.39 & 0.83 & 6.96 & 0.75 & 14.35 & 6.78 & 4.74 & 1.51 & 7.24 & 2.38 & 6.99 & 0.72 \\
\hline Monterey & 16.82 & 5.95 & 3.59 & 0.78 & 4.67 & 0.98 & 12.14 & 4.55 & 15.78 & 5.28 & 14.90 & 4.32 & 7.31 & 1.41 \\
\hline Napa & 11.03 & 2.47 & 3.35 & 0.61 & 5.99 & 1.19 & - & - & 2.75 & 0.43 & 2.75 & 0.43 & 5.85 & 1.15 \\
\hline Nevada & 14.46 & 2.29 & 3.67 & 1.60 & 12.85 & 2.01 & 26.56 & 26.56 & 9.62 & 9.62 & 13.89 & 4.36 & 12.88 & 1.96 \\
\hline Orange & - & - & 5.24 & 1.68 & 5.24 & 1.68 & - & - & 6.03 & 2.93 & 6.03 & 2.93 & 5.40 & 1.43 \\
\hline Placer & 19.51 & 1.91 & 6.51 & 1.31 & 16.93 & 1.64 & 17.98 & 6.33 & 2.24 & 0.61 & 14.32 & 5.35 & 16.73 & 1.57 \\
\hline Plumas & 19.87 & 1.50 & 6.93 & 2.99 & 19.42 & 1.46 & 33.25 & 12.22 & - & - & 33.25 & 12.22 & 19.77 & 1.46 \\
\hline Riverside & 2.50 & 1.01 & 14.18 & 10.04 & 11.47 & 7.82 & 14.43 & 6.29 & 2.04 & 0.82 & 6.46 & 2.96 & 8.94 & 4.18 \\
\hline Sacramento & - & - & 0.11 & 0.05 & 0.11 & 0.05 & - & - & - & - & - & - & 0.11 & 0.05 \\
\hline San Benito & 2.47 & 0.47 & 2.90 & 0.64 & 2.87 & 0.60 & - & - & - & - & - & - & 2.87 & 0.60 \\
\hline San Bernardino & 23.12 & 4.76 & 6.91 & 1.08 & 11.48 & 1.83 & 27.44 & 8.59 & 4.36 & 1.55 & 8.83 & 2.61 & 10.50 & 1.50 \\
\hline San Diego & 24.08 & 12.36 & 8.48 & 2.27 & 10.83 & 3.07 & 45.59 & 13.93 & 10.05 & 5.33 & 18.46 & 7.36 & 13.58 & 3.40 \\
\hline San Joaquin & 4.63 & 4.63 & 0.64 & 0.22 & 3.44 & 0.79 & - & - & - & - & - & - & 3.44 & 0.79 \\
\hline
\end{tabular}


Table A2-106-Average biomass per acre of all dead wood (dead trees and down wood) ${ }^{a}$ on forest land, by county and land status, California, 2001-2010 (continued)

\begin{tabular}{|c|c|c|c|c|c|c|c|c|c|c|c|c|c|c|}
\hline \multirow[b]{4}{*}{ County } & \multicolumn{14}{|c|}{ Land status } \\
\hline & \multicolumn{6}{|c|}{ Unreserved forests } & \multicolumn{7}{|c|}{ Reserved forests } & \\
\hline & \multicolumn{2}{|c|}{ Timberland } & \multicolumn{2}{|c|}{ Other forest } & \multicolumn{2}{|c|}{ Total } & \multicolumn{2}{|c|}{ Productive } & \multicolumn{2}{|c|}{ Other forest } & \multicolumn{2}{|c|}{ Total } & \multicolumn{2}{|c|}{ All forest land } \\
\hline & Mean & SE & Mean & SE & Mean & SE & Mean & $\overline{\text { SE }}$ & Mean & SE & Mean & SE & Mean & SE \\
\hline & \multicolumn{14}{|c|}{ Tons per acre } \\
\hline San Luis Obispo & 1.90 & 1.12 & 4.12 & 0.75 & 4.02 & 0.73 & - & - & 2.08 & 0.89 & 2.08 & 0.89 & 3.83 & 0.67 \\
\hline San Mateo & 18.42 & 5.48 & 4.21 & 1.64 & 14.18 & 4.32 & 25.29 & 8.94 & 5.40 & 5.40 & 20.22 & 7.77 & 15.56 & 3.82 \\
\hline Santa Barbara & 4.11 & 1.22 & 6.47 & 1.29 & 6.25 & 1.18 & 7.81 & 4.94 & 5.46 & 2.19 & 6.05 & 2.08 & 6.20 & 1.03 \\
\hline Santa Clara & 9.29 & 2.79 & 5.72 & 1.08 & 6.20 & 1.04 & - & - & 3.81 & 0.77 & 3.81 & 0.77 & 5.63 & 0.83 \\
\hline Santa Cruz & 19.38 & 4.30 & 5.01 & 1.69 & 18.32 & 4.04 & 13.01 & 2.02 & 3.53 & 3.53 & 11.83 & 2.09 & 16.53 & 3.03 \\
\hline Shasta & 12.70 & 0.78 & 4.50 & 0.70 & 10.54 & 0.63 & 18.52 & 3.59 & 4.62 & 1.80 & 14.73 & 2.86 & 10.96 & 0.64 \\
\hline Sierra & 21.09 & 2.18 & 17.71 & 4.37 & 21.02 & 2.15 & 5.22 & 5.22 & - & - & 5.22 & 5.22 & 20.87 & 2.14 \\
\hline Siskiyou & 19.55 & 1.29 & 4.73 & 1.08 & 17.04 & 1.11 & 29.28 & 3.75 & 6.91 & 2.19 & 27.32 & 3.48 & 18.37 & 1.07 \\
\hline Solano & - & - & 4.63 & 1.71 & 4.63 & 1.71 & - & - & - & - & - & - & 4.38 & 1.62 \\
\hline Sonoma & 12.72 & 1.22 & 3.02 & 0.61 & 10.10 & 1.02 & 38.90 & 16.23 & 5.97 & 3.35 & 20.89 & 9.63 & 11.13 & 1.36 \\
\hline Stanislaus & - & - & 1.84 & 0.61 & 1.84 & 0.61 & - & - & 3.53 & 0.69 & 3.53 & 0.69 & 2.13 & 0.55 \\
\hline Sutter & - & - & 1.28 & 0.32 & 1.28 & 0.32 & - & - & - & - & - & - & 1.28 & 0.32 \\
\hline Tehama & 20.90 & 2.26 & 4.13 & 1.11 & 12.67 & 1.43 & 18.91 & 5.64 & 8.38 & 3.69 & 14.66 & 4.03 & 12.84 & 1.35 \\
\hline Trinity & 20.77 & 1.24 & 8.44 & 1.83 & 19.54 & 1.16 & 30.72 & 3.49 & 3.23 & 0.94 & 27.54 & 3.21 & 21.80 & 1.24 \\
\hline Tulare & 23.84 & 5.50 & 3.51 & 0.63 & 11.58 & 2.47 & 28.08 & 2.61 & 11.95 & 1.52 & 22.68 & 1.91 & 18.65 & 1.55 \\
\hline Tuolumne & 21.55 & 2.46 & 5.92 & 1.13 & 17.22 & 1.91 & 35.07 & 5.51 & 10.54 & 4.23 & 32.21 & 4.99 & 22.80 & 2.28 \\
\hline Ventura & 0.78 & 0.23 & 3.05 & 0.45 & 2.89 & 0.43 & 15.78 & 9.98 & 4.98 & 2.00 & 9.51 & 4.57 & 5.07 & 1.59 \\
\hline Yolo & - & - & 1.93 & 0.85 & 1.93 & 0.85 & - & - & - & - & - & - & 1.93 & 0.85 \\
\hline Yuba & 17.76 & 4.21 & 3.48 & 0.68 & 12.82 & 3.03 & - & - & 0.45 & 0.03 & 0.45 & 0.03 & 12.34 & 2.94 \\
\hline All counties & 19.19 & 0.41 & 4.47 & 0.17 & 13.83 & 0.27 & 29.68 & 1.26 & 7.25 & 0.50 & 21.39 & 0.87 & 15.18 & 0.27 \\
\hline
\end{tabular}

Note: Totals may be off because of rounding; estimates subject to sampling error; SE = sampling error; - $=$ less than 0.005 bone-dry tons per acre was estimated; the average was calculated using a ratio of means formula across plots.

${ }^{a}$ Includes dead trees $\geq 5$ inches in diameter at breast height and down wood $\geq 0.1$ inch diameter at the transect intersection. 
Table A2-107-Volume of live trees, dead trees, ${ }^{a}$ and down wood on forest land, by stand age class, California, 2001-2010

\begin{tabular}{|c|c|c|c|c|c|c|c|}
\hline \multirow[b]{2}{*}{ Stand age class } & \multicolumn{2}{|c|}{ Live trees (1) } & \multicolumn{2}{|c|}{ Dead trees (1) } & \multicolumn{2}{|c|}{ Down wood (2) } & \multirow[b]{2}{*}{ All volum } \\
\hline & Total & SE & Total & SE & Total & SE & \\
\hline & \multicolumn{7}{|c|}{ Million cubic feet } \\
\hline \multicolumn{8}{|l|}{ Classes in years: } \\
\hline $1-20$ & 857.2 & 92.9 & $1,393.4$ & 372.0 & $1,623.5$ & 145.8 & $3,874.2$ \\
\hline $21-40$ & $5,404.3$ & 386.0 & $1,314.9$ & 295.8 & $2,751.6$ & 254.0 & $9,470.8$ \\
\hline $41-60$ & $12,244.2$ & 609.4 & $2,210.2$ & 411.6 & $3,969.3$ & 296.7 & $18,423.7$ \\
\hline $61-80$ & $19,249.8$ & 793.6 & $2,102.3$ & 148.3 & $4,946.6$ & 251.9 & $26,298.7$ \\
\hline $81-100$ & $20,455.8$ & 857.5 & $2,843.9$ & 237.8 & $4,809.1$ & 236.0 & $28,108.9$ \\
\hline $101-120$ & $9,373.8$ & 621.9 & $1,338.2$ & 130.0 & $2,389.5$ & 184.0 & $13,101.4$ \\
\hline $121-140$ & $7,805.8$ & 629.7 & $1,066.3$ & 114.2 & $1,790.5$ & 160.7 & $10,662.6$ \\
\hline $141-160$ & $8,468.3$ & 626.6 & $1,450.1$ & 136.0 & $2,111.1$ & 189.9 & $12,029.6$ \\
\hline $161-180$ & $4,500.0$ & 500.3 & 890.2 & 127.9 & $1,194.5$ & 145.2 & $6,584.8$ \\
\hline $181-200$ & $5,346.3$ & 551.6 & 950.3 & 107.4 & $1,528.5$ & 158.9 & $7,825.2$ \\
\hline $201+$ & $18,276.4$ & $1,268.7$ & $2,964.6$ & 220.2 & $4,024.6$ & 273.4 & $25,265.6$ \\
\hline Unknown & $6,540.0$ & 320.0 & $1,243.1$ & 156.6 & $1,653.5$ & 111.6 & $9,436.6$ \\
\hline All classes & $118,522.0$ & $1,589.8$ & $19,767.5$ & 728.6 & $32,792.5$ & 573.5 & $171,082.0$ \\
\hline
\end{tabular}

Note: Totals may be off because of rounding; data subject to sampling error; $\mathrm{SE}=$ standard error.

${ }^{a}$ Live and dead tree volume is aboveground volume of the tree from ground to tip (live trees) and to actual height (dead trees), including stem volume and stem bark. 
Table A2-108-Biomass of live trees, dead trees ${ }^{a}$, and down wood on forest land, by stand age class, California, 2001-2010

\begin{tabular}{|c|c|c|c|c|c|c|c|}
\hline \multirow[b]{2}{*}{ Stand age class } & \multicolumn{2}{|c|}{ Live trees } & \multicolumn{2}{|c|}{ Dead trees } & \multicolumn{2}{|c|}{ Down wood } & \multirow[b]{2}{*}{ All biomass } \\
\hline & Total & SE & Total & SE & Total & SE & \\
\hline & \multicolumn{7}{|c|}{ Thousand tons } \\
\hline \multicolumn{8}{|l|}{ Classes in years: } \\
\hline $1-20$ & $16,740.0$ & $1,745.8$ & $13,300.5$ & $2,883.4$ & $14,032.8$ & $1,222.1$ & $44,073.3$ \\
\hline $21-40$ & $99,617.8$ & $6,976.7$ & $9,335.9$ & $1,666.6$ & $21,003.4$ & $1,830.8$ & $129,957.1$ \\
\hline $41-60$ & $228,991.6$ & $11,158.4$ & $17,040.8$ & $2,309.4$ & $32,613.9$ & $2,300.2$ & $278,646.3$ \\
\hline $61-80$ & $367,311.9$ & $14,969.4$ & $25,242.5$ & $1,663.9$ & $41,204.5$ & $2,019.7$ & $433,758.9$ \\
\hline $81-100$ & $385,460.2$ & $16,013.8$ & $33,952.8$ & $2,360.7$ & $39,510.0$ & $1,894.3$ & $458,923.0$ \\
\hline $101-120$ & $176,508.3$ & $11,599.0$ & $17,634.1$ & $1,830.9$ & $18,805.1$ & $1,436.0$ & $212,947.5$ \\
\hline $121-140$ & $145,519.5$ & $11,736.4$ & $14,573.2$ & $1,607.9$ & $14,187.1$ & $1,257.1$ & $174,279.8$ \\
\hline $141-160$ & $159,612.1$ & $11,738.8$ & $18,972.5$ & $1,886.2$ & $15,969.7$ & $1,397.9$ & $194,554.3$ \\
\hline $161-180$ & $84,164.1$ & $9,333.4$ & $12,188.9$ & $1,840.0$ & $9,063.6$ & $1,112.1$ & $105,416.6$ \\
\hline $181-200$ & $100,301.7$ & $10,400.3$ & $12,912.6$ & $1,552.2$ & $11,844.1$ & $1,217.6$ & $125,058.4$ \\
\hline $201+$ & $338,255.1$ & $23,479.0$ & $39,734.5$ & $3,070.7$ & $30,004.1$ & $1,965.7$ & $407,993.7$ \\
\hline Unknown & $133,796.2$ & $6,588.1$ & $18,235.3$ & $2,566.3$ & $16,682.8$ & $1,013.5$ & $168,714.3$ \\
\hline All classes & $2,236,278.5$ & $29,466.8$ & $233,123.5$ & $6,574.5$ & $264,921.3$ & $4,358.2$ & $2,734,323.3$ \\
\hline
\end{tabular}

Note: Totals may be off because of rounding; data subject to sampling error; $\mathrm{SE}=$ standard error.

${ }^{a}$ Live and dead tree biomass is total aboveground biomass of the tree from ground to tip (live trees) and to actual height (dead trees), including stem wood, bark, and branches. 
Table A2-109-Carbon mass of live trees, dead trees ${ }^{a}$, and down wood on forest land, by stand age class, California, 2001-2010

\begin{tabular}{|c|c|c|c|c|c|c|c|}
\hline \multirow[b]{2}{*}{ Stand age class } & \multicolumn{2}{|c|}{ Live trees } & \multicolumn{2}{|c|}{ Dead trees } & \multicolumn{2}{|c|}{ Down wood } & \multirow[b]{2}{*}{$\begin{array}{c}\text { All carbon } \\
\text { mass }\end{array}$} \\
\hline & Total & SE & Total & SE & Total & SE & \\
\hline & \multicolumn{7}{|c|}{ Thousand megagrams } \\
\hline \multicolumn{8}{|l|}{ Classes in years: } \\
\hline $1-20$ & $7,593.2$ & 791.9 & $6,033.1$ & $1,307.9$ & $6,487.3$ & 565.4 & $20,113.6$ \\
\hline $21-40$ & $45,186.6$ & $3,164.6$ & $4,234.8$ & 756.0 & $9,737.2$ & 853.5 & $59,158.6$ \\
\hline $41-60$ & $103,870.6$ & $5,061.5$ & $7,729.7$ & $1,047.5$ & $15,111.2$ & $1,077.2$ & $126,711.5$ \\
\hline $61-80$ & $166,612.7$ & $6,790.1$ & $11,450.0$ & 754.7 & $19,004.6$ & 934.0 & $197,067.3$ \\
\hline $81-100$ & $174,844.7$ & $7,263.9$ & $15,401.0$ & $1,070.8$ & $18,241.5$ & 875.8 & $208,487.2$ \\
\hline $101-120$ & $80,064.2$ & $5,261.3$ & $7,998.8$ & 830.5 & $8,706.8$ & 666.1 & $96,769.8$ \\
\hline $121-140$ & $66,007.7$ & $5,323.7$ & $6,610.4$ & 729.4 & $6,564.2$ & 581.9 & $79,182.3$ \\
\hline $141-160$ & $72,400.0$ & $5,324.7$ & $8,605.9$ & 855.6 & $7,406.9$ & 650.4 & $88,412.8$ \\
\hline $161-180$ & $38,176.8$ & $4,233.6$ & $5,528.9$ & 834.6 & $4,215.6$ & 518.1 & $47,921.3$ \\
\hline $181-200$ & $45,496.8$ & $4,717.6$ & $5,857.2$ & 704.1 & $5,521.1$ & 568.6 & $56,875.1$ \\
\hline $201+$ & $153,432.5$ & $10,650.1$ & $18,023.6$ & $1,392.9$ & $13,980.4$ & 917.4 & $185,436.5$ \\
\hline Unknown & $60,690.0$ & $2,988.4$ & $8,271.5$ & $1,164.1$ & $7,645.3$ & 468.7 & $76,606.8$ \\
\hline All classes & $1,014,375.9$ & $13,366.1$ & $105,744.8$ & $2,982.2$ & $122,622.1$ & $2,031.9$ & $1,242,742.8$ \\
\hline
\end{tabular}

Note: Totals may be off because of rounding; data subject to sampling error; $\mathrm{SE}=$ standard error.

${ }^{a}$ Live and dead tree carbon mass is total aboveground carbon mass of the tree from ground to tip (live trees) and to actual height (dead trees), including stem wood, bark, and branches. 
Table A2-110-Average volume per acre of live trees, dead trees ${ }^{a}$, and down wood on forest land, by stand age class, California, 2001-2010

\begin{tabular}{|c|c|c|c|c|c|c|c|}
\hline \multirow[b]{2}{*}{ Stand age class } & \multicolumn{2}{|c|}{ Live trees } & \multicolumn{2}{|c|}{ Dead trees } & \multicolumn{2}{|c|}{ Down wood } & \multirow[b]{2}{*}{ All volume } \\
\hline & Total & SE & Total & SE & Total & SE & \\
\hline & \multicolumn{7}{|c|}{ Cubic feet per acre } \\
\hline \multicolumn{8}{|l|}{ Classes in years: } \\
\hline $1-20$ & 575.05 & 53.20 & 934.79 & 242.58 & $1,089.13$ & 75.56 & $2,599.0$ \\
\hline $21-40$ & $2,601.99$ & 133.51 & 633.08 & 138.75 & $1,324.80$ & 102.67 & $4,559.9$ \\
\hline $41-60$ & $3,144.58$ & 112.54 & 567.63 & 103.79 & $1,019.41$ & 67.63 & $4,731.6$ \\
\hline $61-80$ & $3,368.60$ & 98.49 & 367.90 & 23.68 & 865.62 & 36.36 & $4,602.1$ \\
\hline $81-100$ & $3,910.59$ & 113.86 & 543.68 & 42.19 & 919.38 & 35.39 & $5,373.7$ \\
\hline $101-120$ & $4,418.56$ & 190.72 & 630.78 & 52.36 & $1,126.36$ & 65.05 & $6,175.7$ \\
\hline $121-140$ & $5,306.41$ & 277.87 & 724.89 & 63.59 & $1,217.16$ & 79.17 & $7,248.5$ \\
\hline $141-160$ & $5,004.17$ & 236.18 & 856.93 & 64.08 & $1,247.53$ & 86.59 & $7,108.6$ \\
\hline $161-180$ & $5,349.23$ & 396.14 & $1,058.18$ & 124.19 & $1,419.94$ & 126.01 & $7,827.4$ \\
\hline $181-200$ & $5,683.27$ & 384.12 & $1,010.17$ & 85.01 & $1,624.88$ & 112.49 & $8,318.3$ \\
\hline $201+$ & $7,184.45$ & 389.69 & $1,165.37$ & 70.54 & $1,582.09$ & 82.57 & $9,931.9$ \\
\hline Unknown & $1,363.01$ & 52.75 & 259.07 & 31.35 & 344.62 & 20.46 & $1,966.7$ \\
\hline All classes & $3,611.77$ & 47.91 & 602.38 & 22.18 & 999.30 & 17.32 & $70,443.3$ \\
\hline
\end{tabular}

Note: Totals may be off because of rounding; estimates subject to sampling error; $\mathrm{SE}=$ sampling error; the average was calculated using a ratio of means formula across plots.

${ }^{a}$ Live and dead tree volume is aboveground volume of the tree from ground to tip (live trees) and to actual height (dead trees), including stem volume and stem bark. 
Table A2-111-Average biomass per acre of live trees, dead trees ${ }^{a}$, and down wood on forest land, by stand age class, California, 2001-2010

\begin{tabular}{|c|c|c|c|c|c|c|c|}
\hline \multirow[b]{2}{*}{ Stand age class } & \multicolumn{2}{|c|}{ Live trees } & \multicolumn{2}{|c|}{ Dead trees } & \multicolumn{2}{|c|}{ Down wood } & \multirow[b]{2}{*}{ All biomass } \\
\hline & Total & SE & Total & SE & Total & SE & \\
\hline & \multicolumn{7}{|c|}{ Tons per acre } \\
\hline \multicolumn{8}{|l|}{ Classes in years: } \\
\hline $1-20$ & 11.23 & 0.99 & 8.92 & 1.85 & 9.41 & 0.62 & 29.6 \\
\hline $21-40$ & 47.96 & 2.37 & 4.49 & 0.77 & 10.11 & 0.72 & 62.6 \\
\hline $41-60$ & 58.81 & 2.01 & 4.38 & 0.57 & 8.38 & 0.52 & 71.6 \\
\hline $61-80$ & 64.28 & 1.84 & 4.42 & 0.26 & 7.21 & 0.29 & 75.9 \\
\hline $81-100$ & 73.69 & 2.11 & 6.49 & 0.41 & 7.55 & 0.28 & 87.7 \\
\hline $101-120$ & 83.20 & 3.52 & 8.31 & 0.76 & 8.86 & 0.50 & 100.4 \\
\hline $121-140$ & 98.92 & 5.18 & 9.91 & 0.91 & 9.64 & 0.61 & 118.5 \\
\hline $141-160$ & 94.32 & 4.39 & 11.21 & 0.92 & 9.44 & 0.62 & 115.0 \\
\hline $161-180$ & 100.05 & 7.38 & 14.49 & 1.82 & 10.77 & 0.97 & 125.3 \\
\hline $181-200$ & 106.62 & 7.27 & 13.73 & 1.29 & 12.59 & 0.85 & 132.9 \\
\hline $201+$ & 132.97 & 7.20 & 15.62 & 1.00 & 11.79 & 0.58 & 160.4 \\
\hline Unknown & 27.88 & 1.10 & 3.80 & 0.52 & 3.48 & 0.18 & 35.2 \\
\hline All classes & 68.15 & 0.89 & 7.10 & 0.20 & 8.07 & 0.13 & 83.3 \\
\hline
\end{tabular}

Note: Totals may be off because of rounding; estimates subject to sampling error; $\mathrm{SE}=$ sampling error; the average was calculated using a ratio of means formula across plots.

${ }^{a}$ Live and dead tree biomass is total aboveground biomass of the tree from ground to tip (live trees) and to actual height (dead trees), including stem wood, bark, and branches. 
Table A2-112-Average carbon mass per hectare of live trees, dead trees ${ }^{a}$, and down wood on forest land, by stand age class, California, 2001-2010

\begin{tabular}{|c|c|c|c|c|c|c|c|}
\hline \multirow[b]{2}{*}{ Stand age class } & \multicolumn{2}{|c|}{ Live trees } & \multicolumn{2}{|c|}{ Dead trees } & \multicolumn{2}{|c|}{ Down wood } & \multirow{2}{*}{$\begin{array}{c}\text { All carbon } \\
\text { mass }\end{array}$} \\
\hline & Total & SE & Total & SE & Total & SE & \\
\hline & \multicolumn{7}{|c|}{ Kilograms per hectare } \\
\hline \multicolumn{8}{|l|}{ Classes in years: } \\
\hline $1-20$ & $12,587.34$ & $1,104.74$ & $10,001.10$ & $2,075.11$ & $10,753.96$ & 704.78 & $33,342.4$ \\
\hline $21-40$ & $53,759.57$ & $2,653.03$ & $5,038.20$ & 861.89 & $11,584.58$ & 832.12 & $70,382.4$ \\
\hline $41-60$ & $65,918.66$ & $2,257.66$ & $4,905.44$ & 640.45 & $9,589.89$ & 598.11 & $80,414.0$ \\
\hline $61-80$ & $72,046.42$ & $2,058.81$ & $4,951.19$ & 293.87 & $8,217.96$ & 327.56 & $85,215.6$ \\
\hline $81-100$ & $82,596.02$ & $2,361.42$ & $7,275.38$ & 454.81 & $8,617.24$ & 319.65 & $98,488.6$ \\
\hline $101-120$ & $93,258.15$ & $3,942.82$ & $9,316.98$ & 847.33 & $10,141.57$ & 576.41 & $112,716.7$ \\
\hline $121-140$ & $110,881.17$ & $5,805.92$ & $11,104.28$ & $1,015.77$ & $11,026.71$ & 700.53 & $133,012.2$ \\
\hline $141-160$ & $105,719.52$ & $4,915.28$ & $12,566.49$ & $1,027.82$ & $10,815.68$ & 719.20 & $129,101.7$ \\
\hline $161-180$ & $112,138.67$ & $8,273.07$ & $16,240.31$ & $2,045.03$ & $12,382.70$ & $1,123.44$ & $140,761.7$ \\
\hline $181-200$ & $119,510.33$ & $8,147.28$ & $15,385.46$ & $1,441.26$ & $14,502.63$ & 980.76 & $149,398.4$ \\
\hline $201+$ & $149,039.81$ & $8,070.74$ & $17,507.55$ & $1,124.29$ & $13,580.17$ & 666.84 & $180,127.5$ \\
\hline Unknown & $31,255.14$ & $1,229.46$ & $4,259.81$ & 580.46 & $3,937.33$ & 206.39 & $39,452.3$ \\
\hline All classes & $76,383.85$ & 993.75 & $7,962.73$ & 224.49 & $9,233.61$ & 150.63 & $93,580.2$ \\
\hline
\end{tabular}

Note: Totals may be off because of rounding; estimates subject to sampling error; SE = sampling error; the average was calculated using a ratio of means formula across plots.

${ }^{a}$ Live and dead tree carbon mass is total aboveground carbon mass of the tree from ground to tip (live trees) and to actual height (dead trees), including stem wood, bark, and branches. 


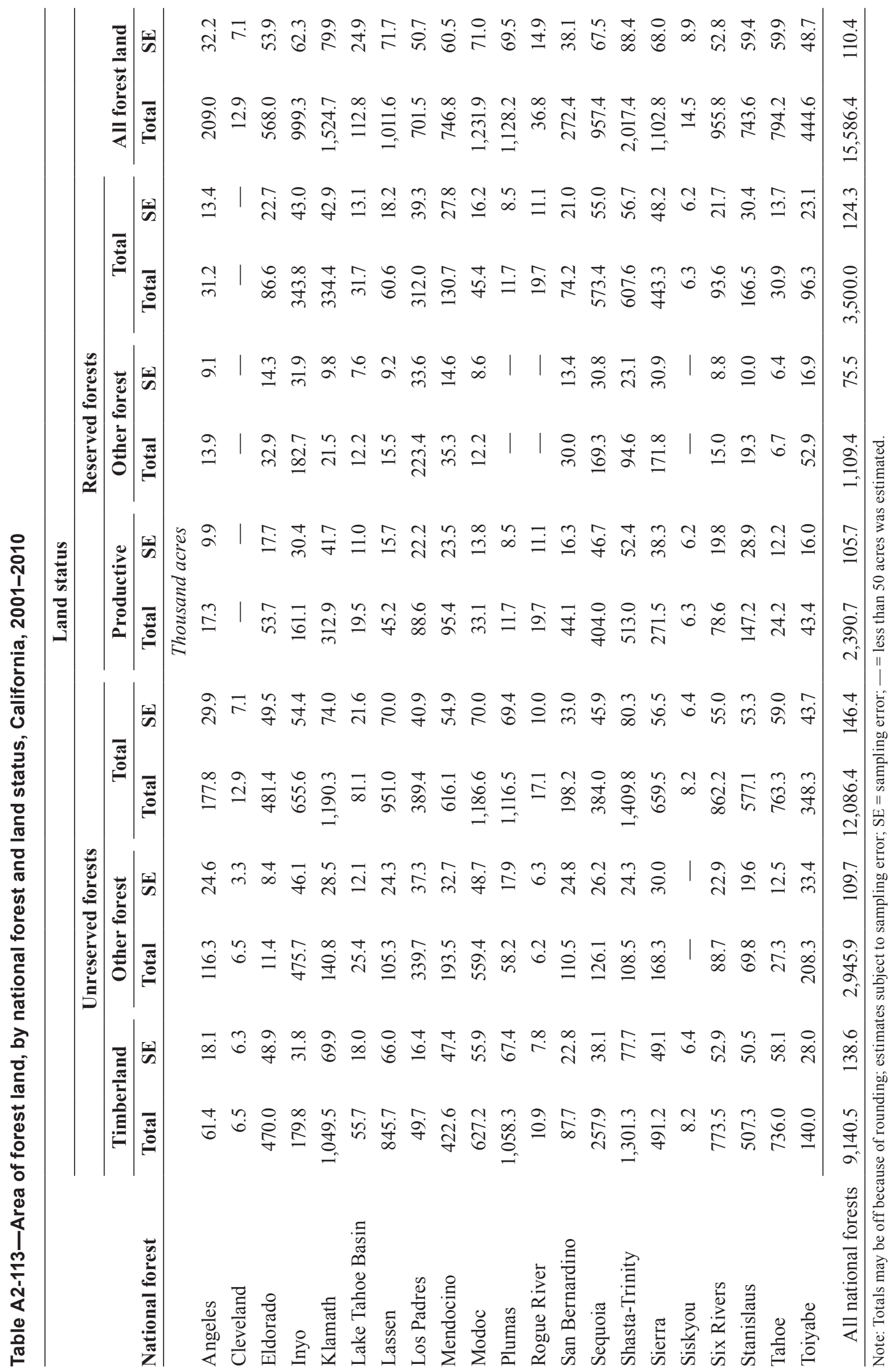




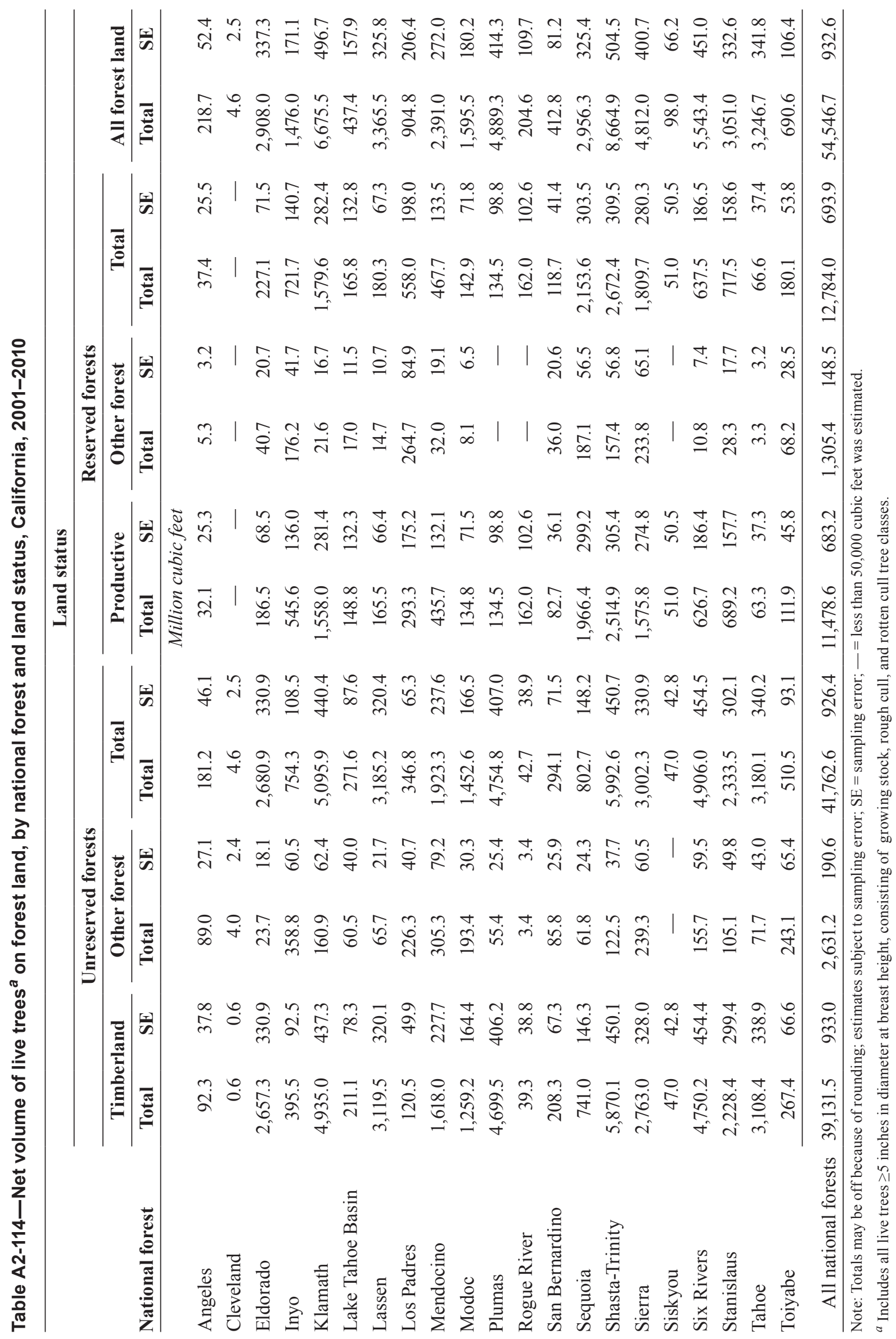




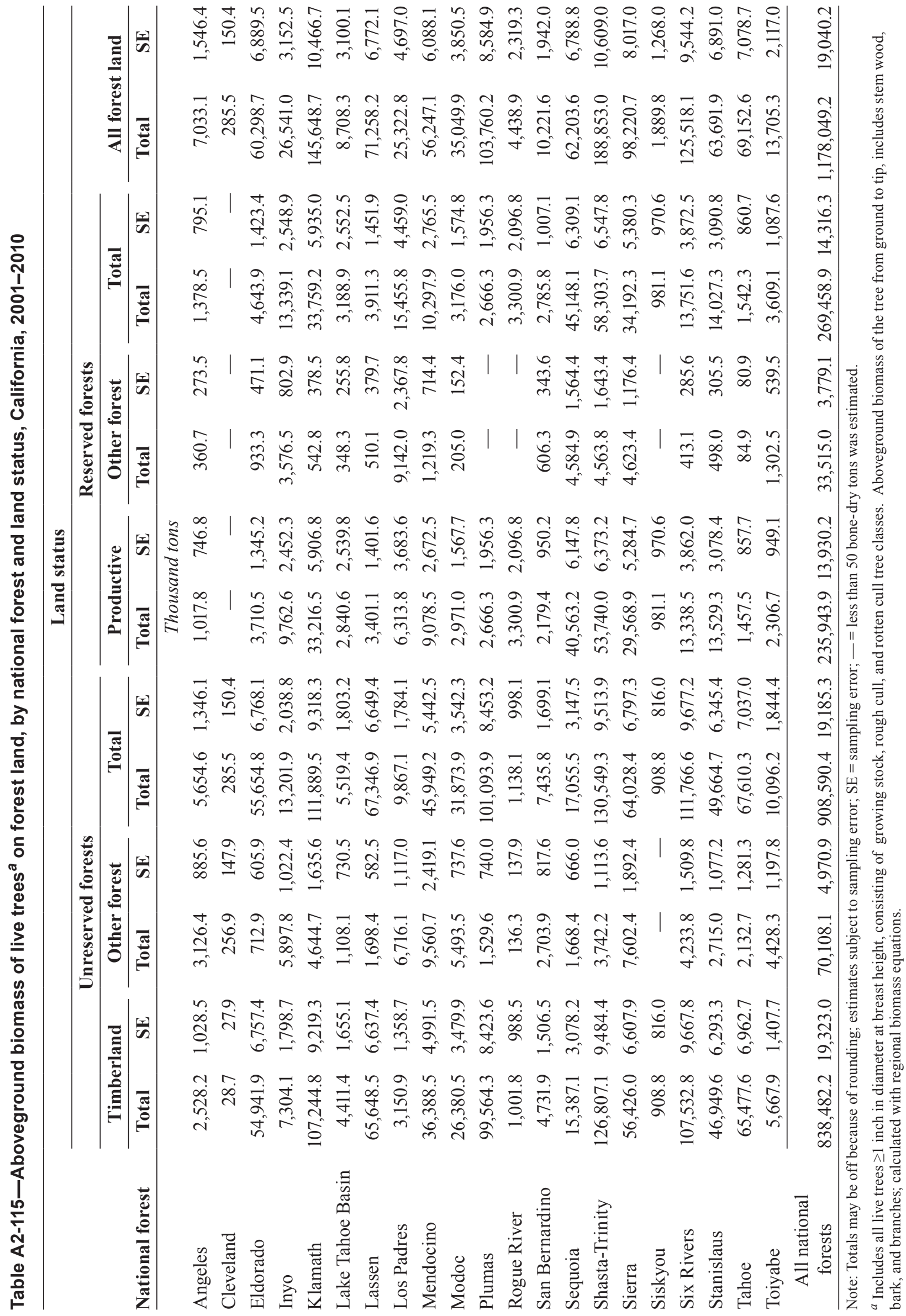




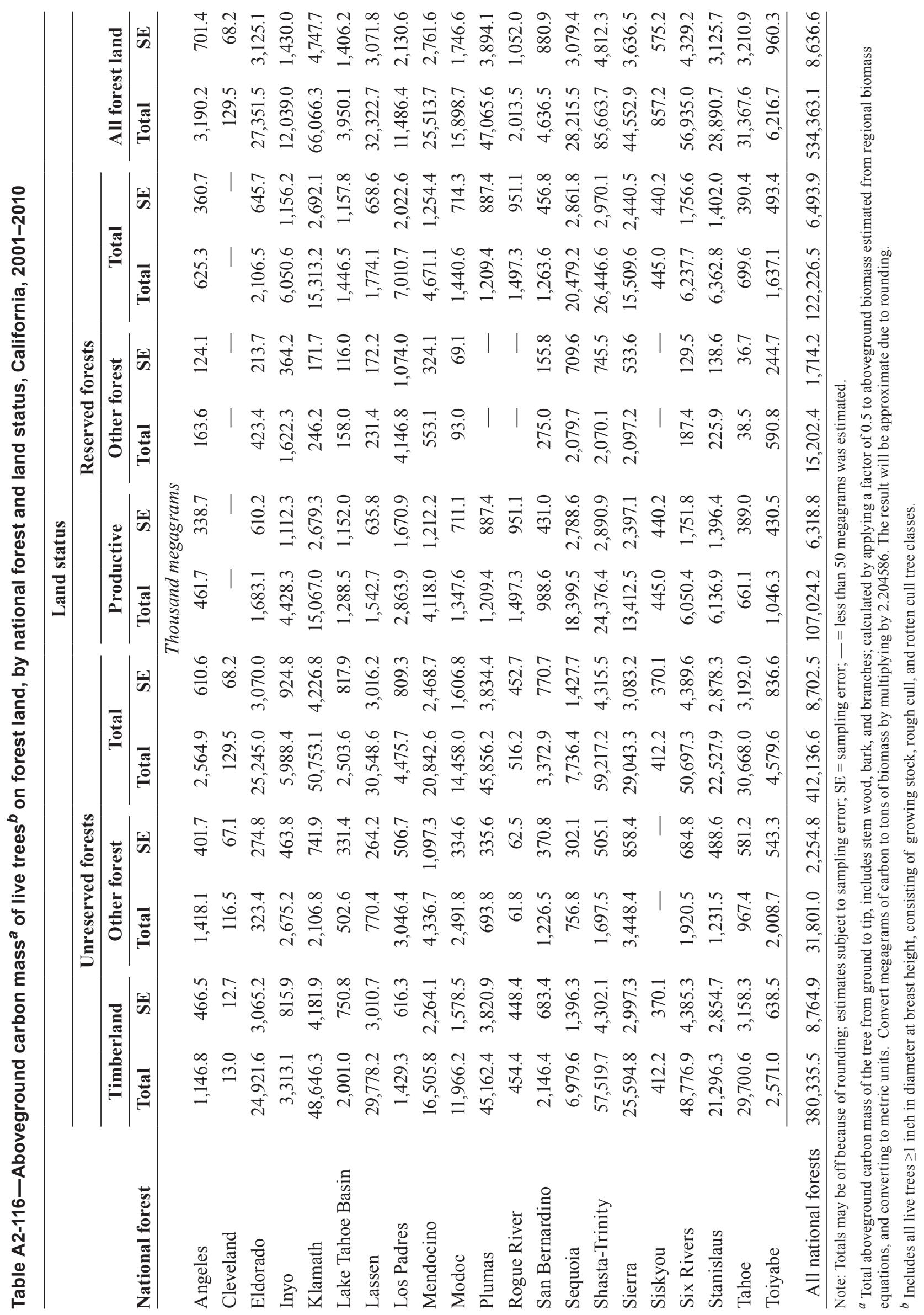




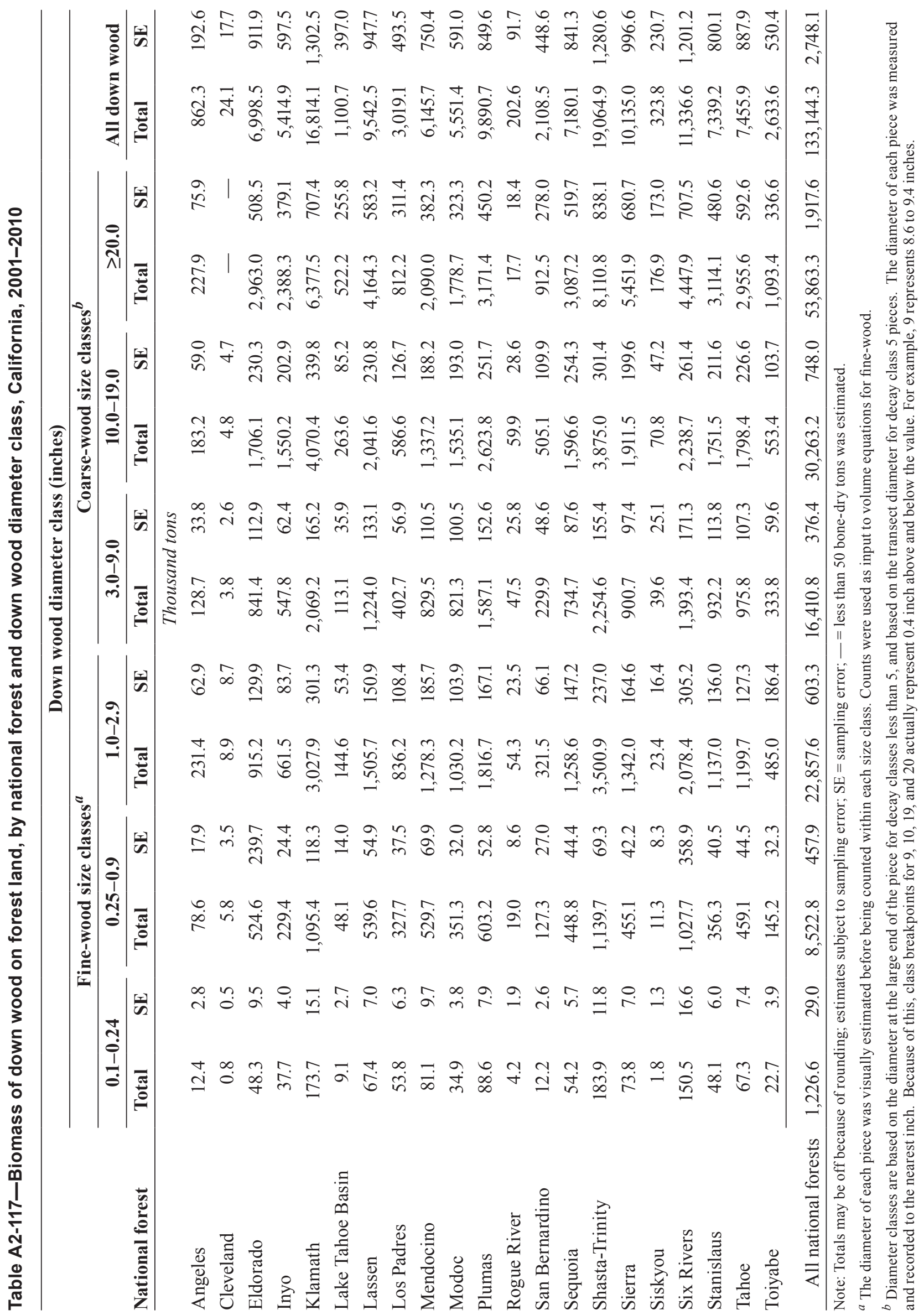




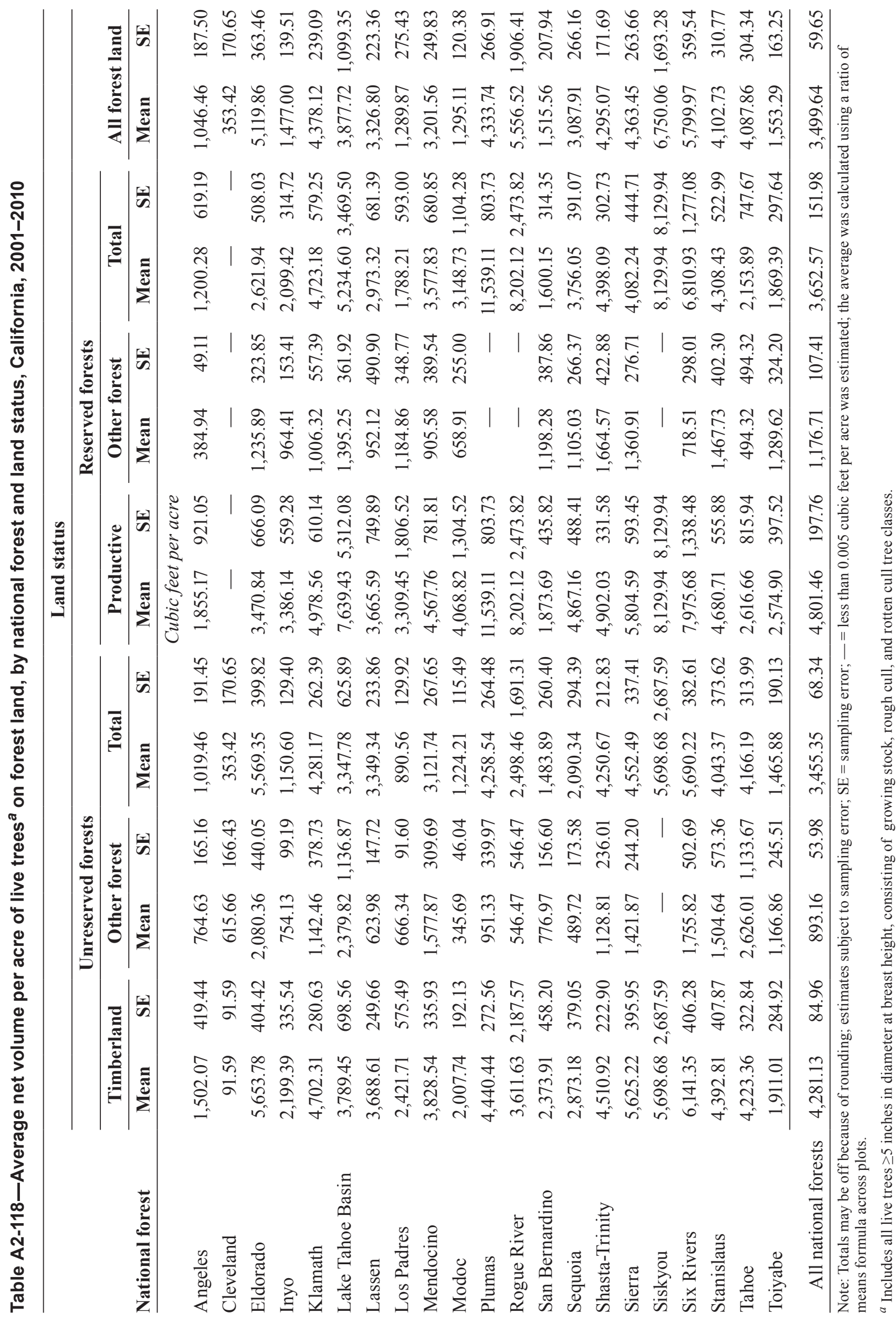




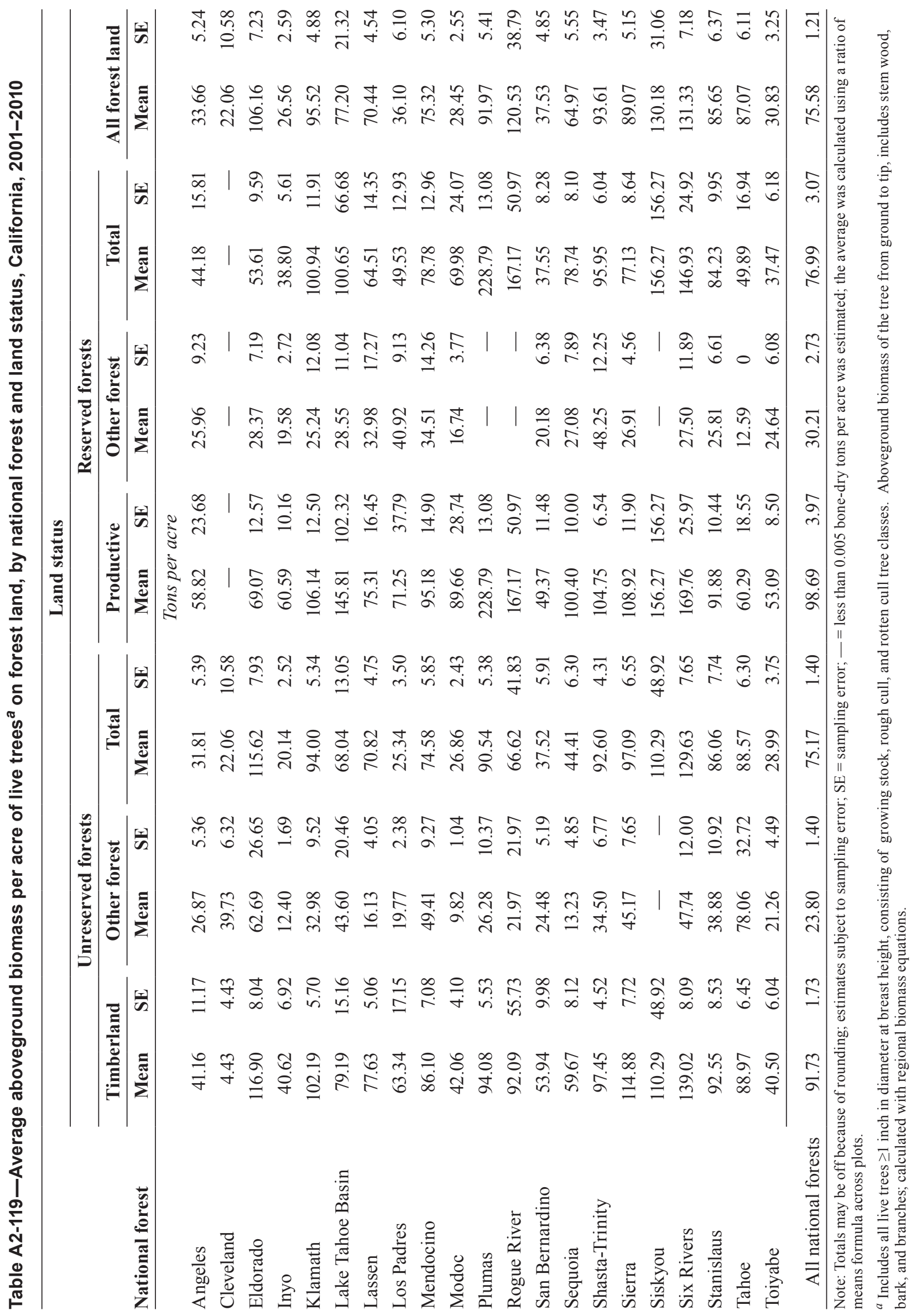




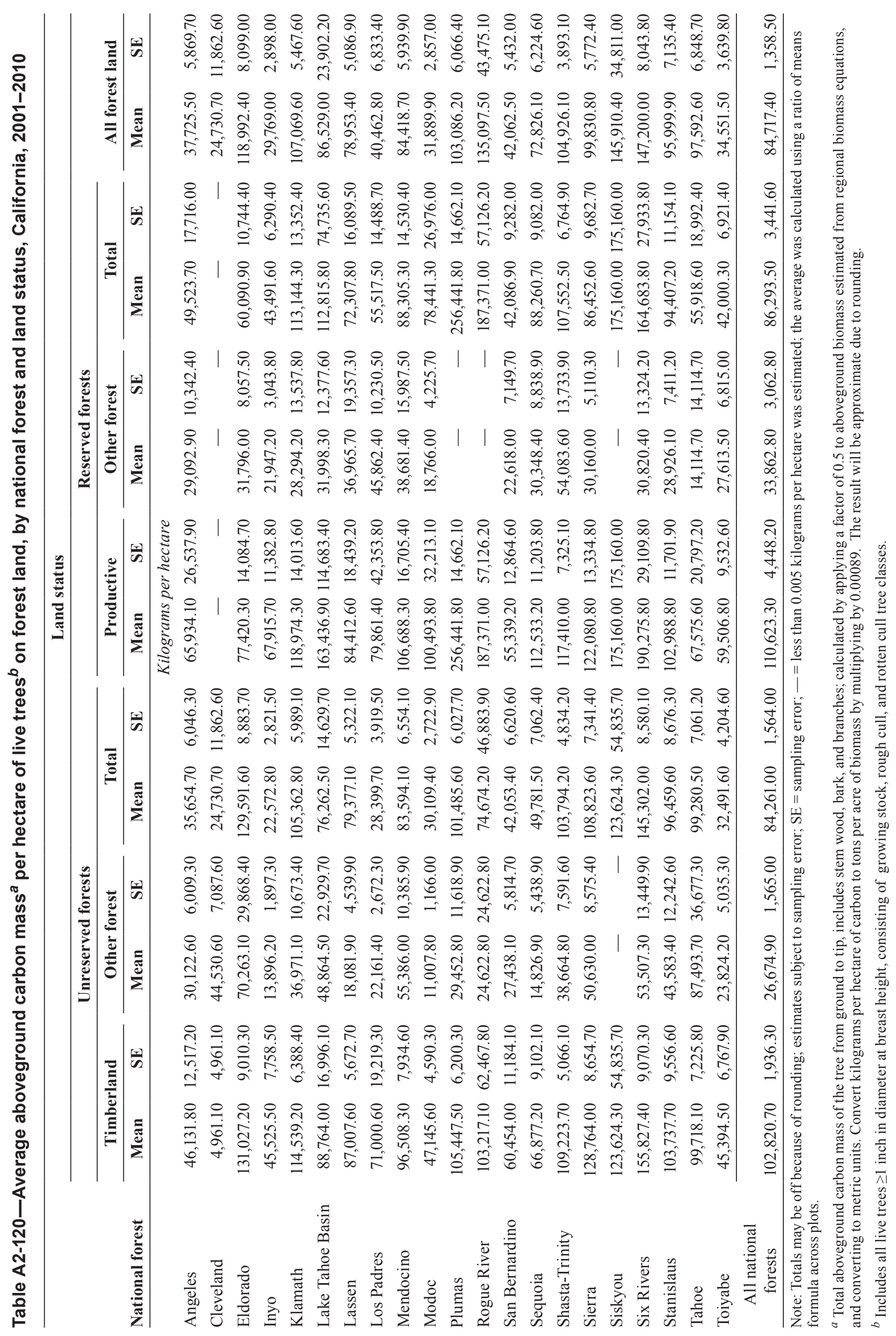




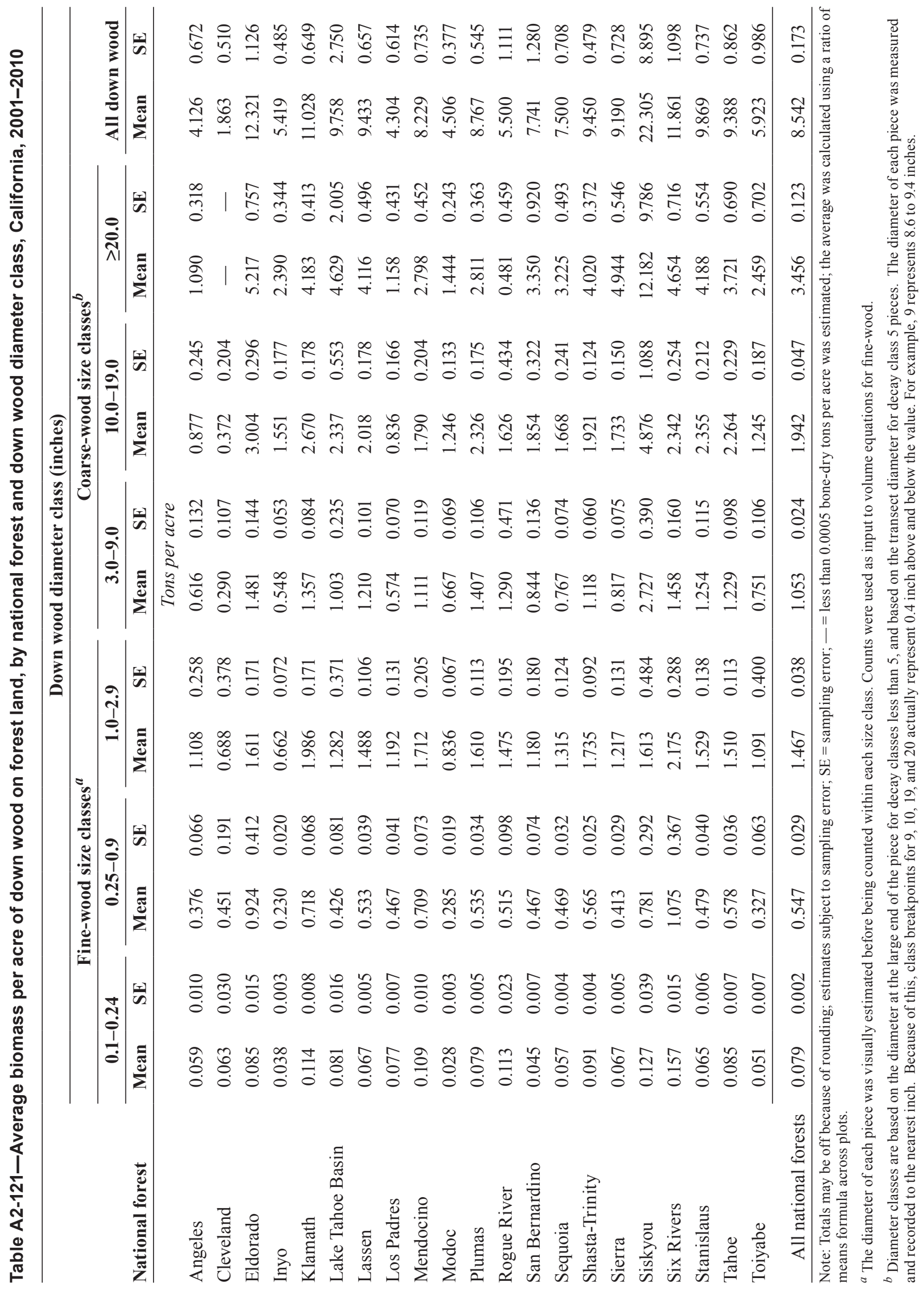




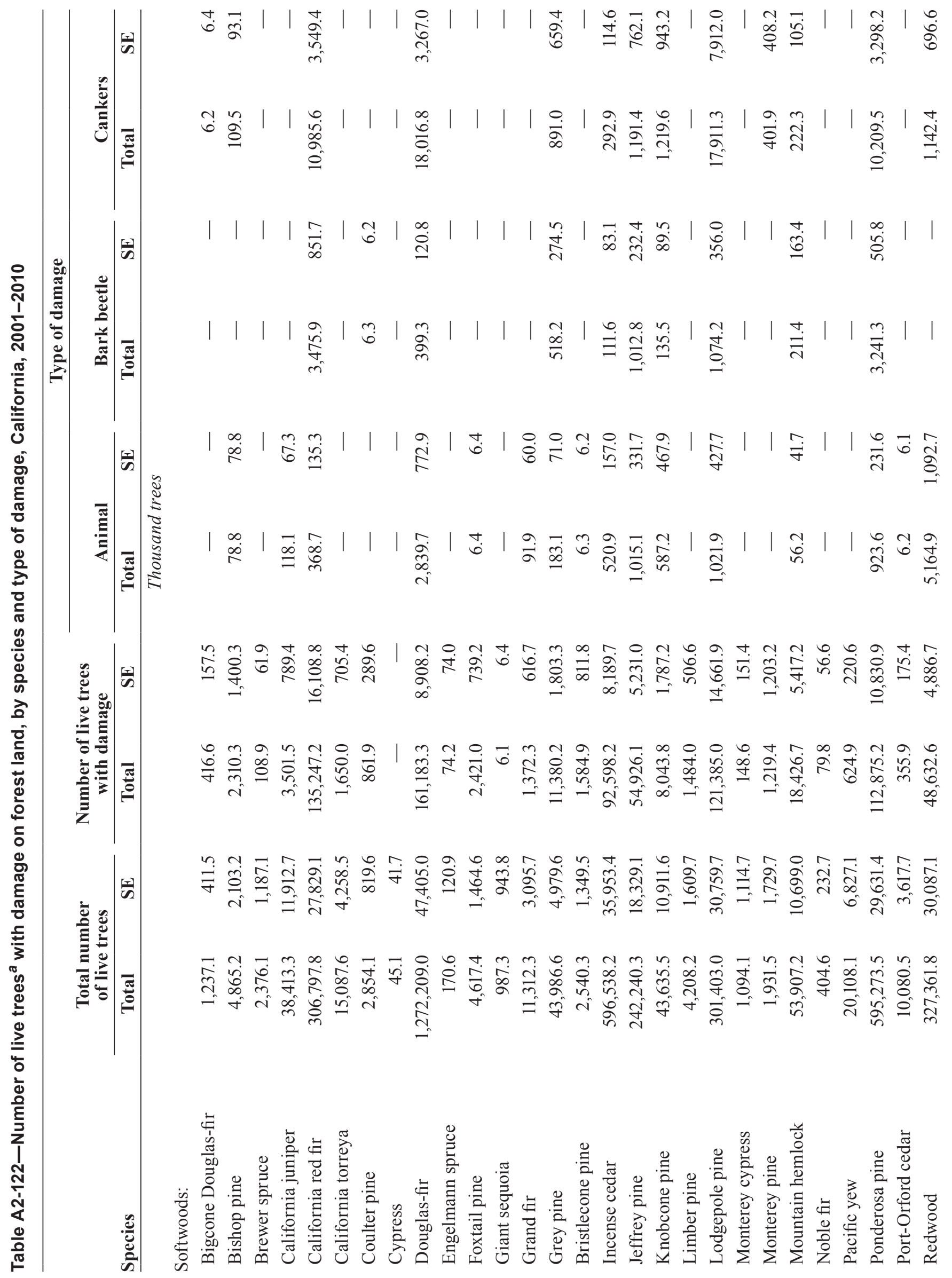




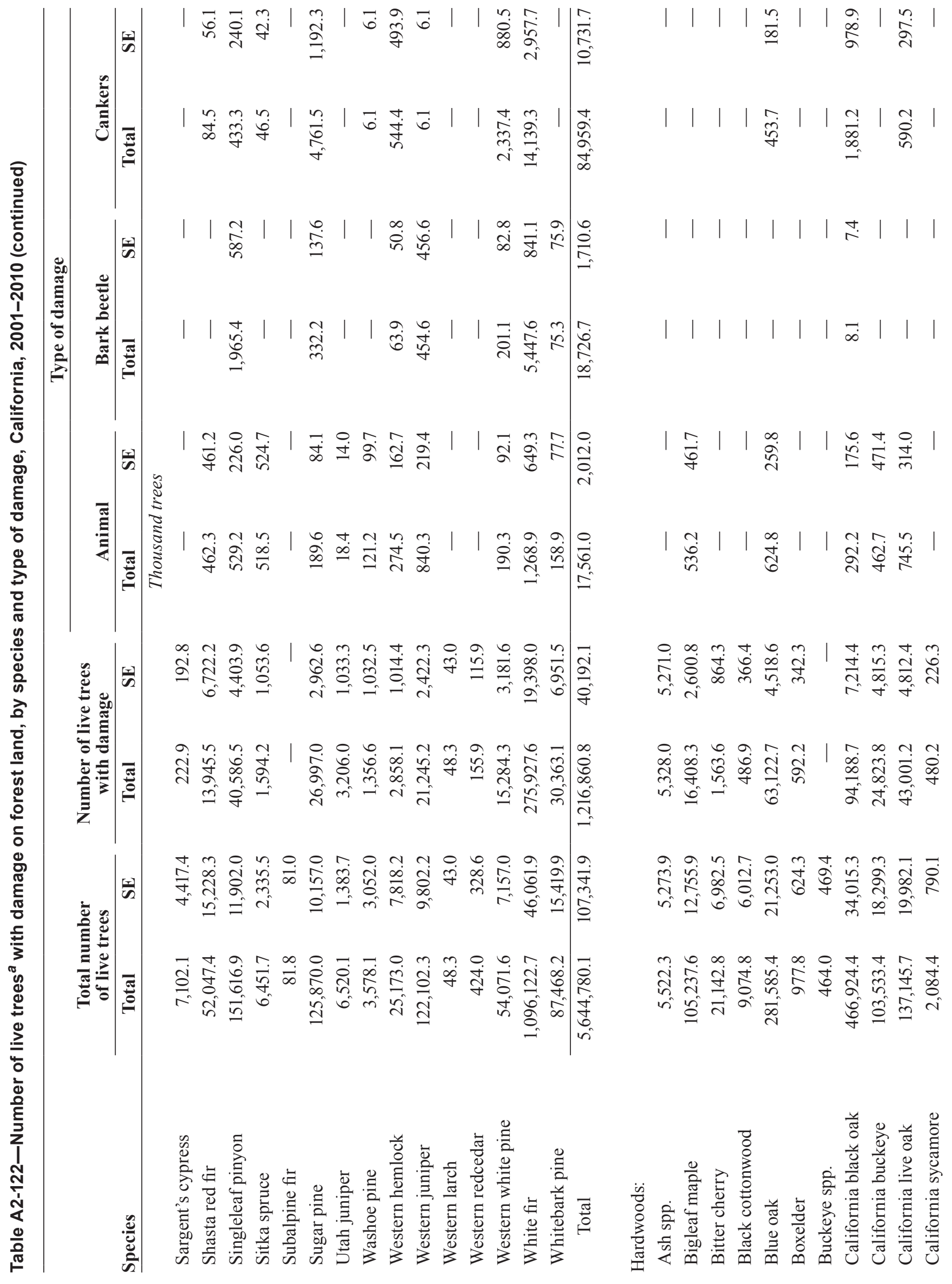




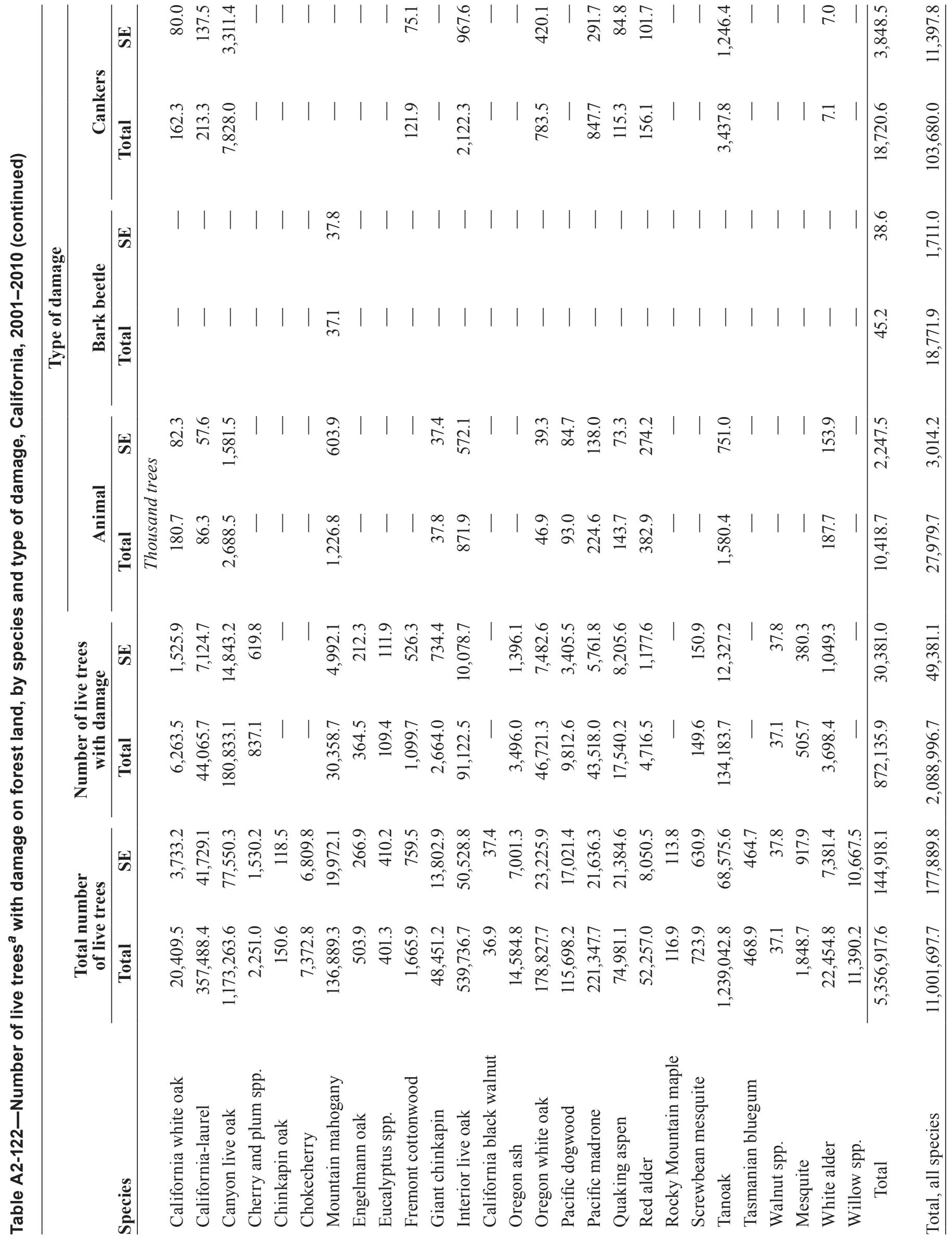




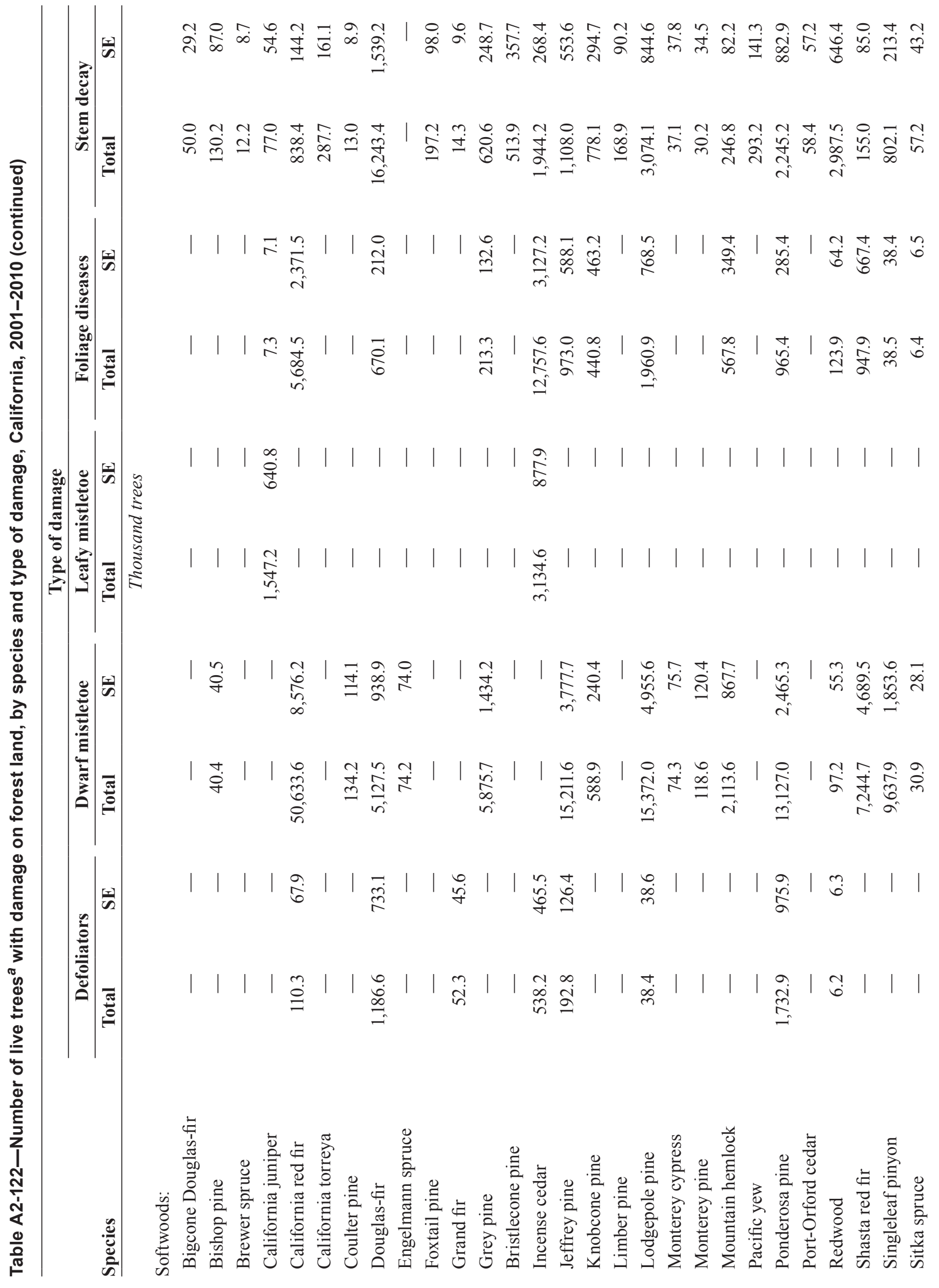




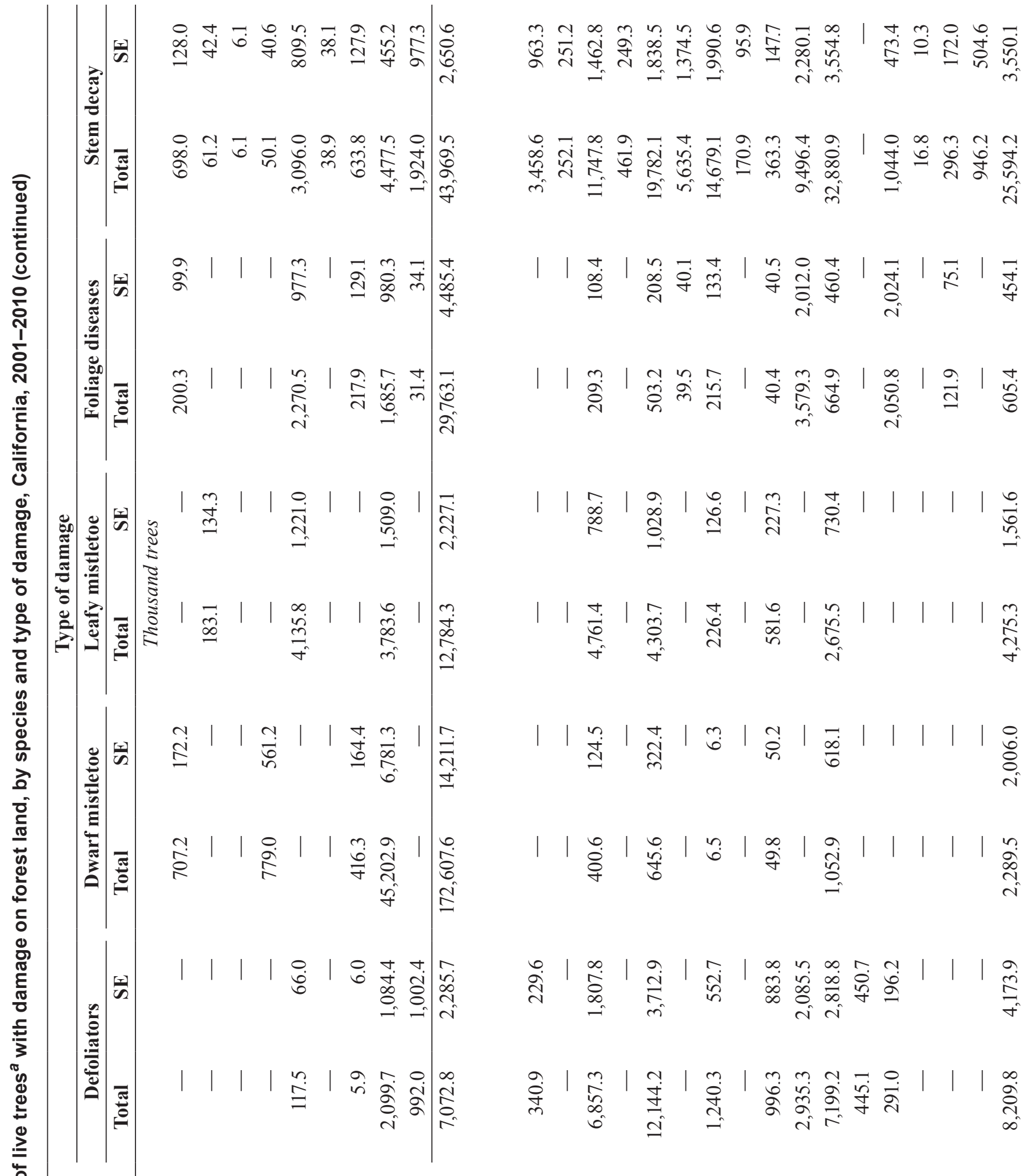




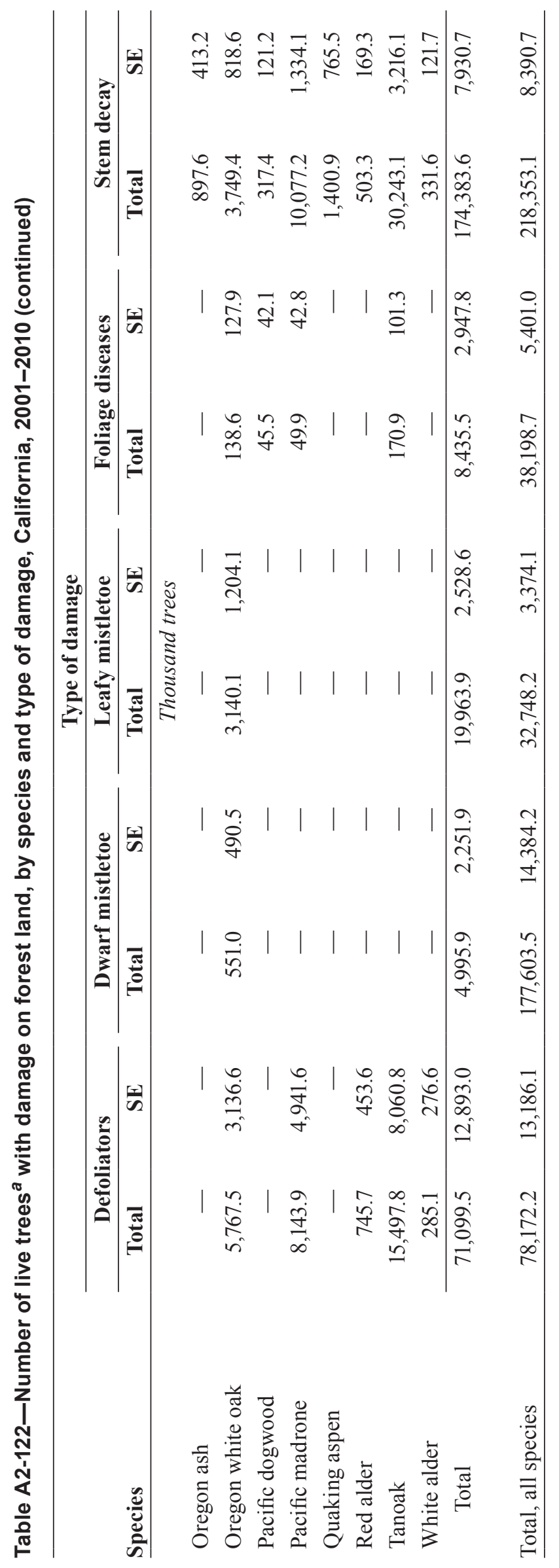




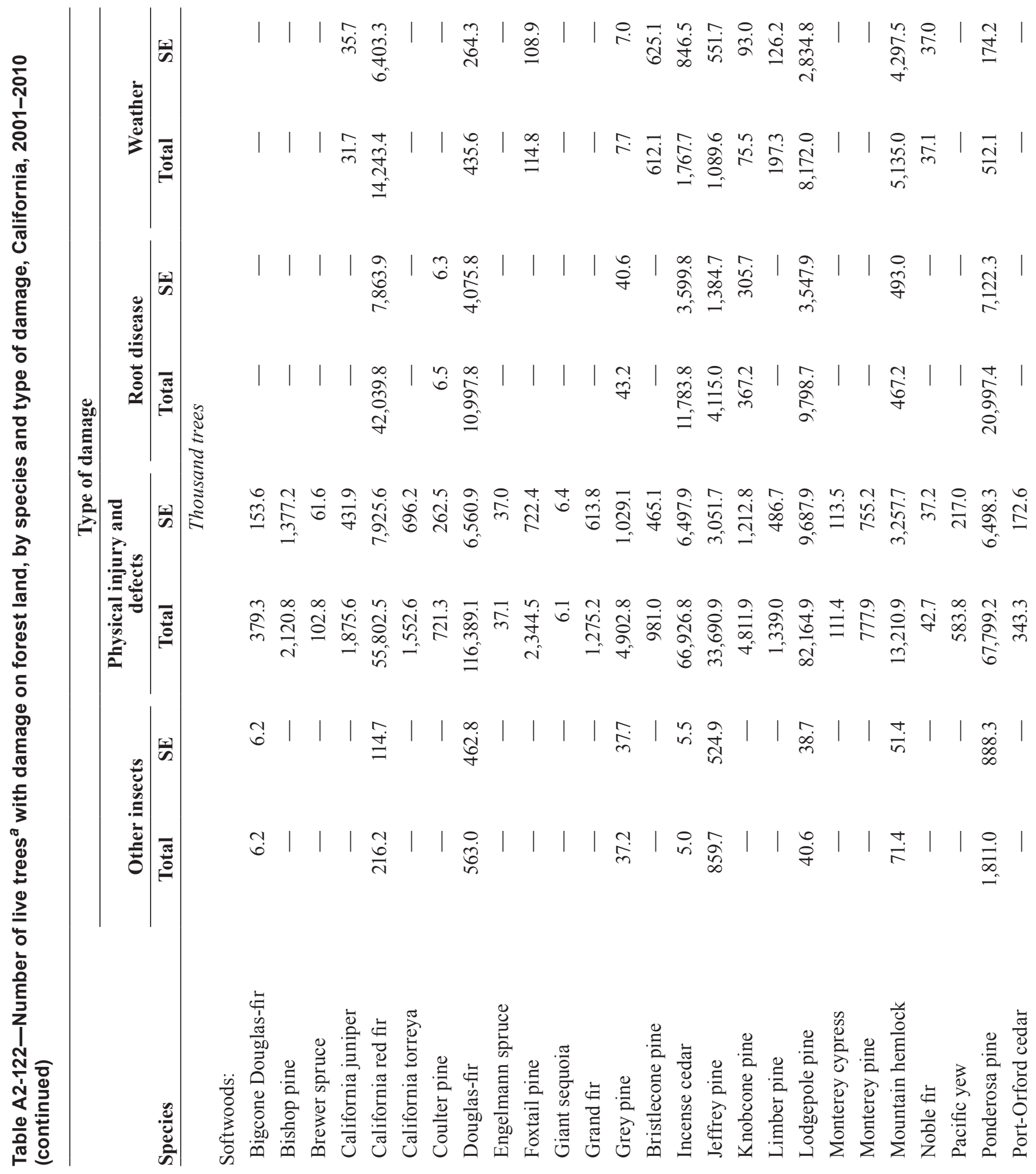




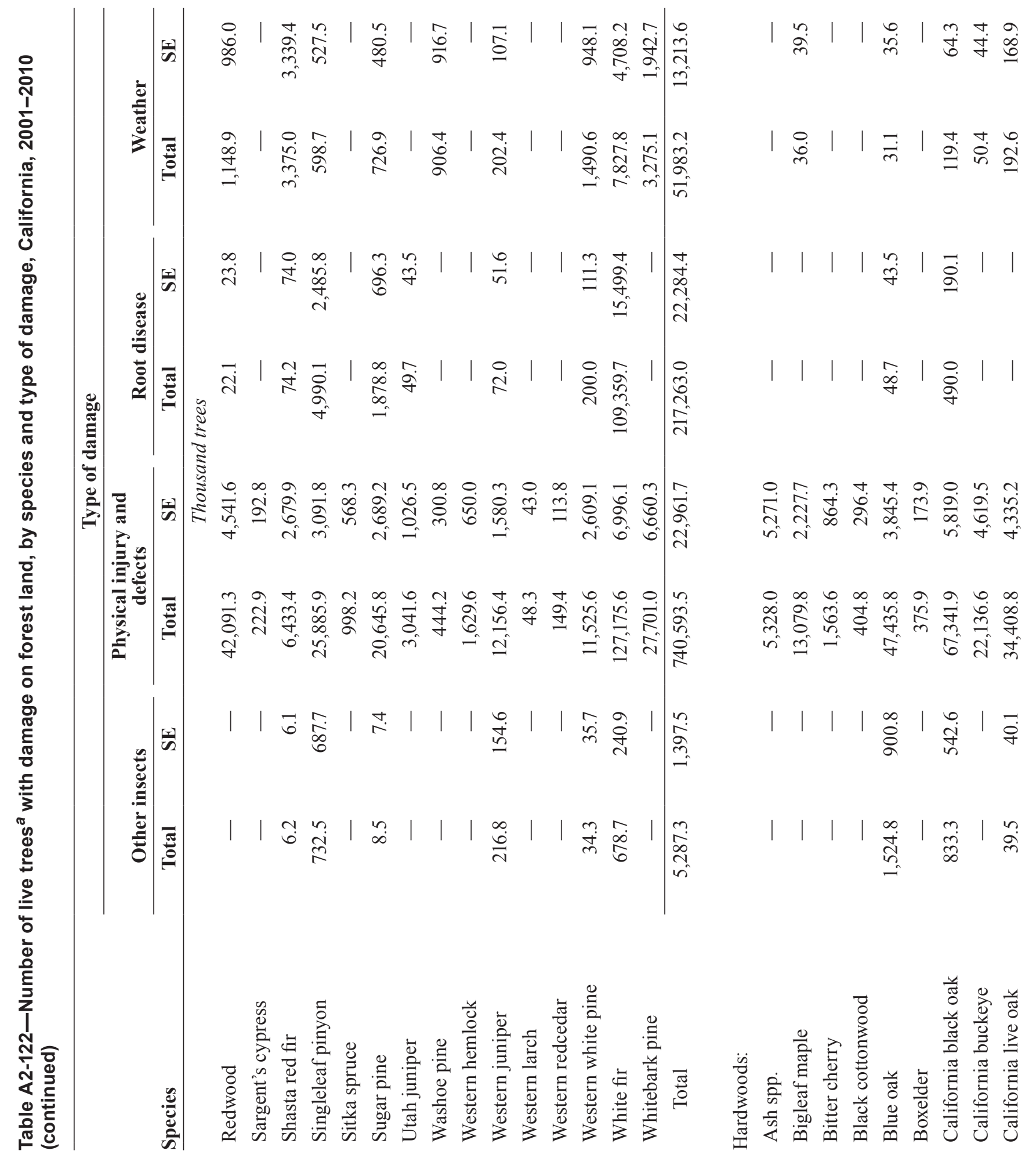




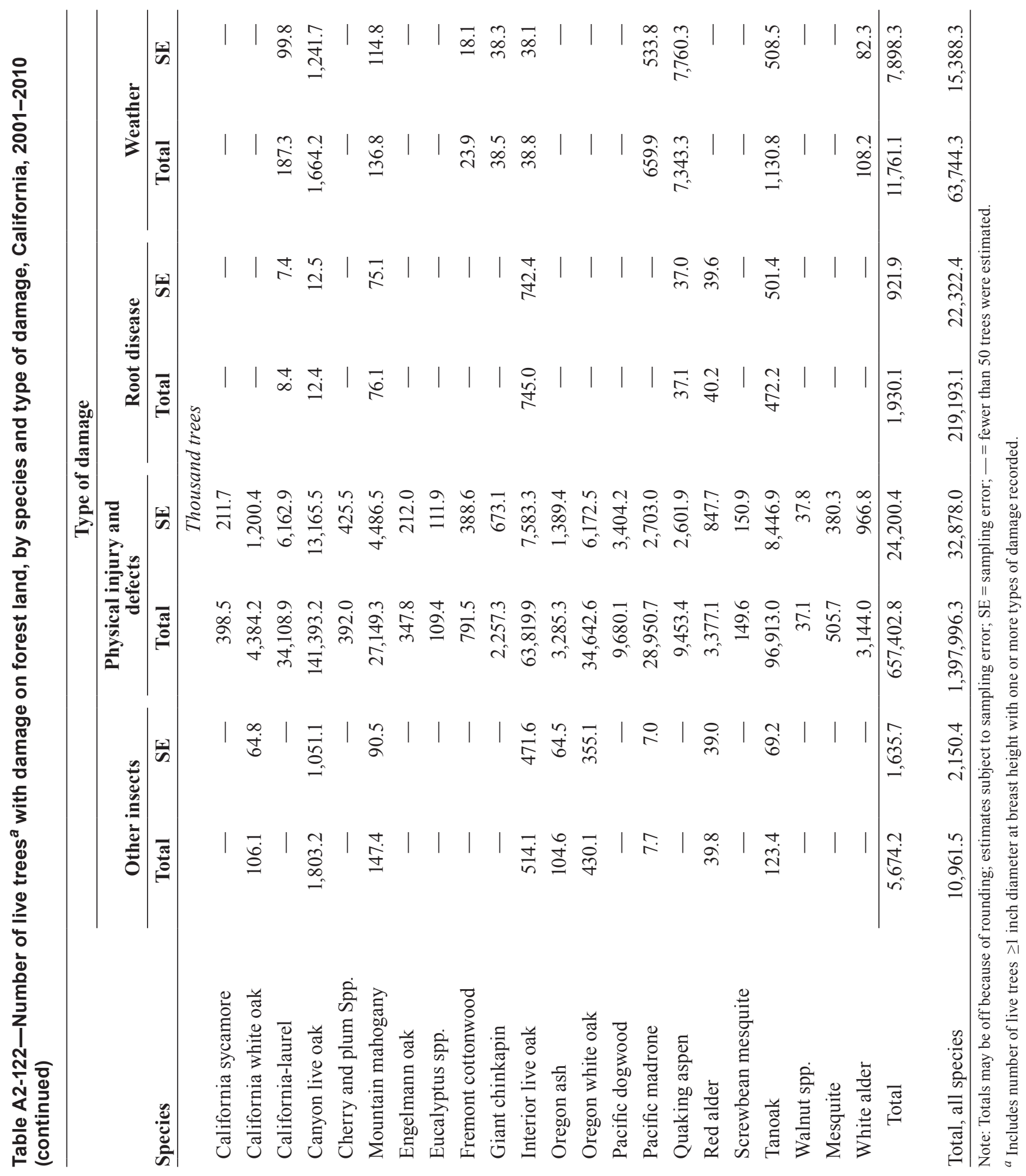


California's Forest Resources: Forest Inventory and Analysis, 2001-2010

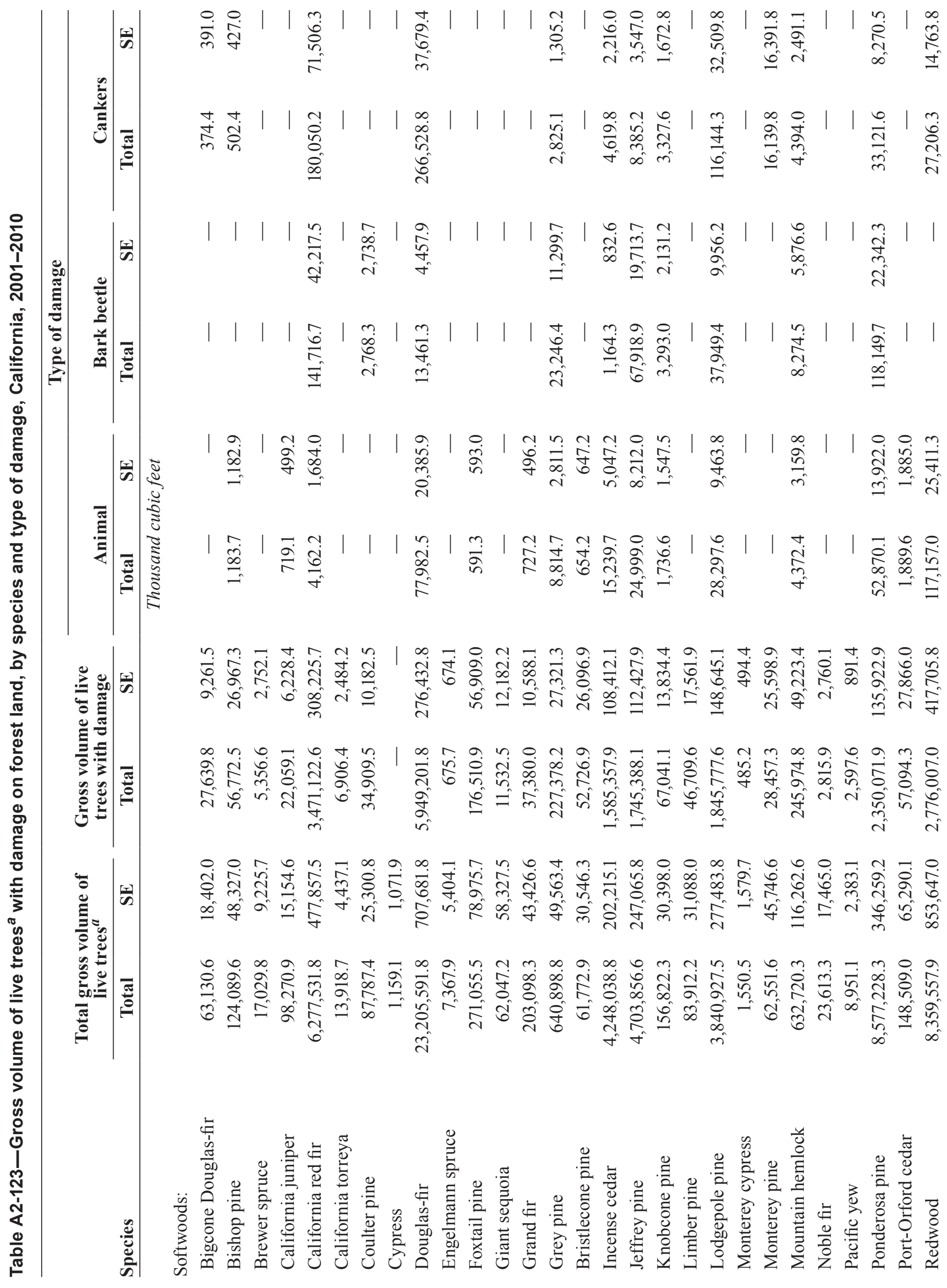




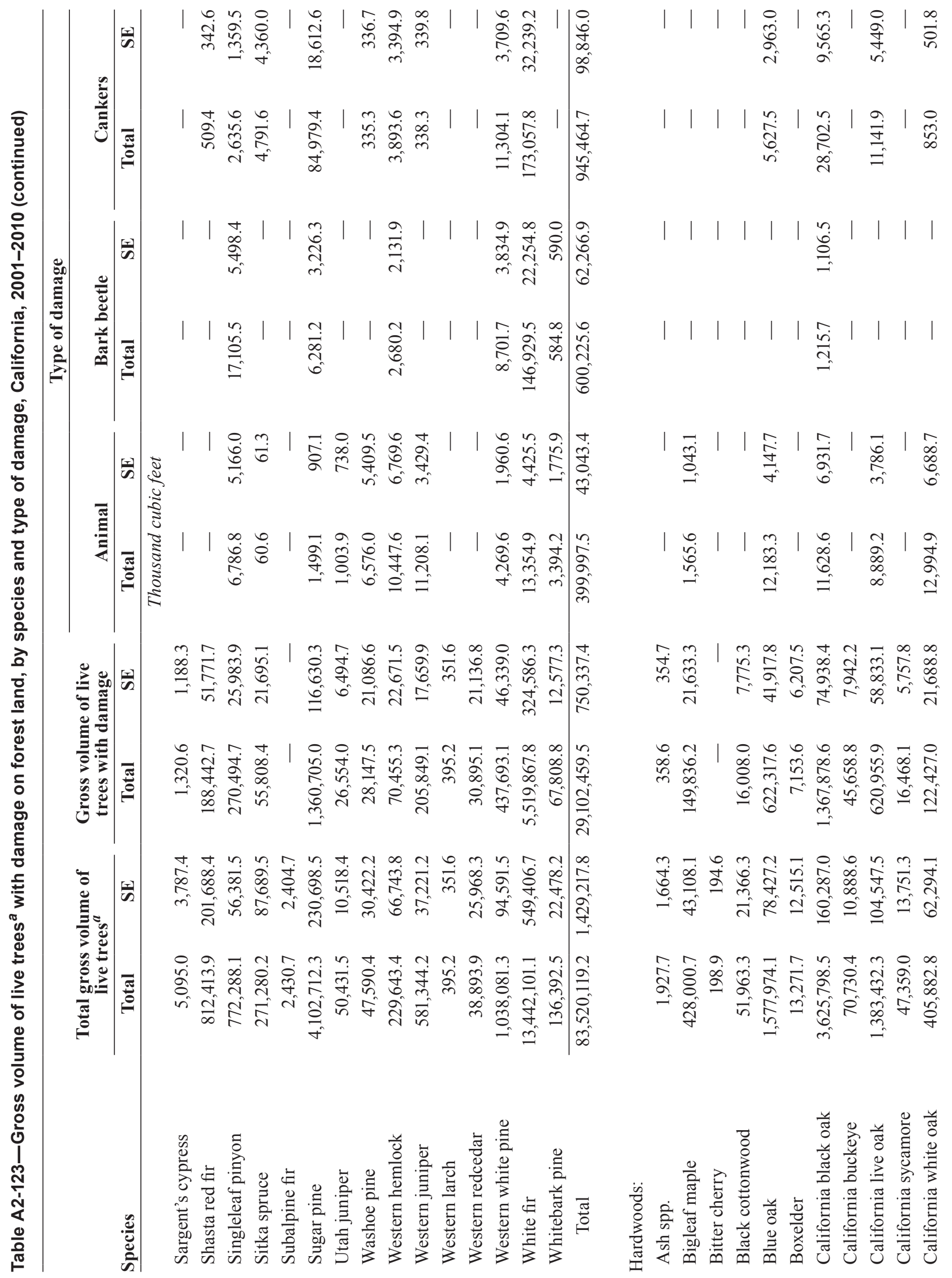




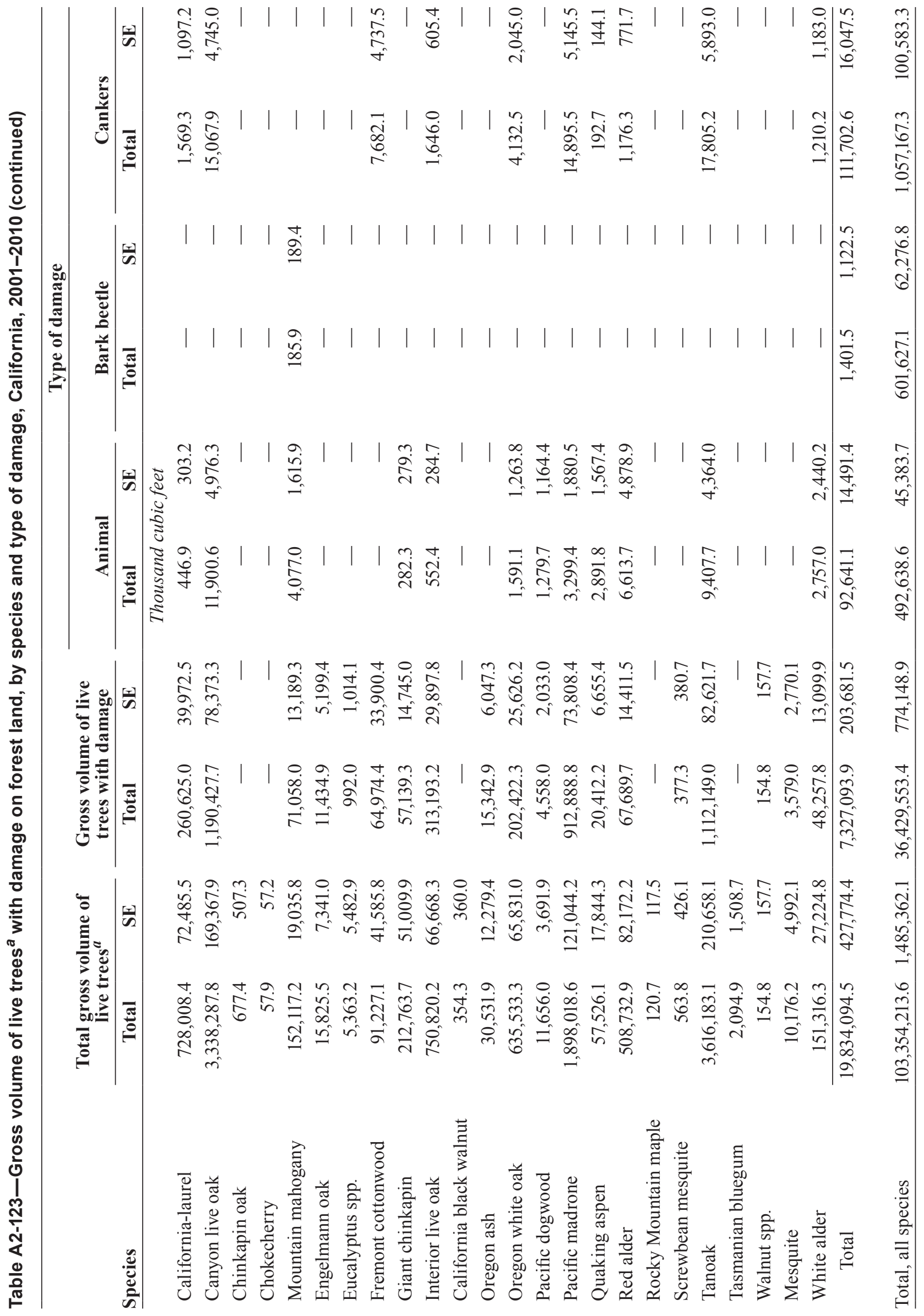




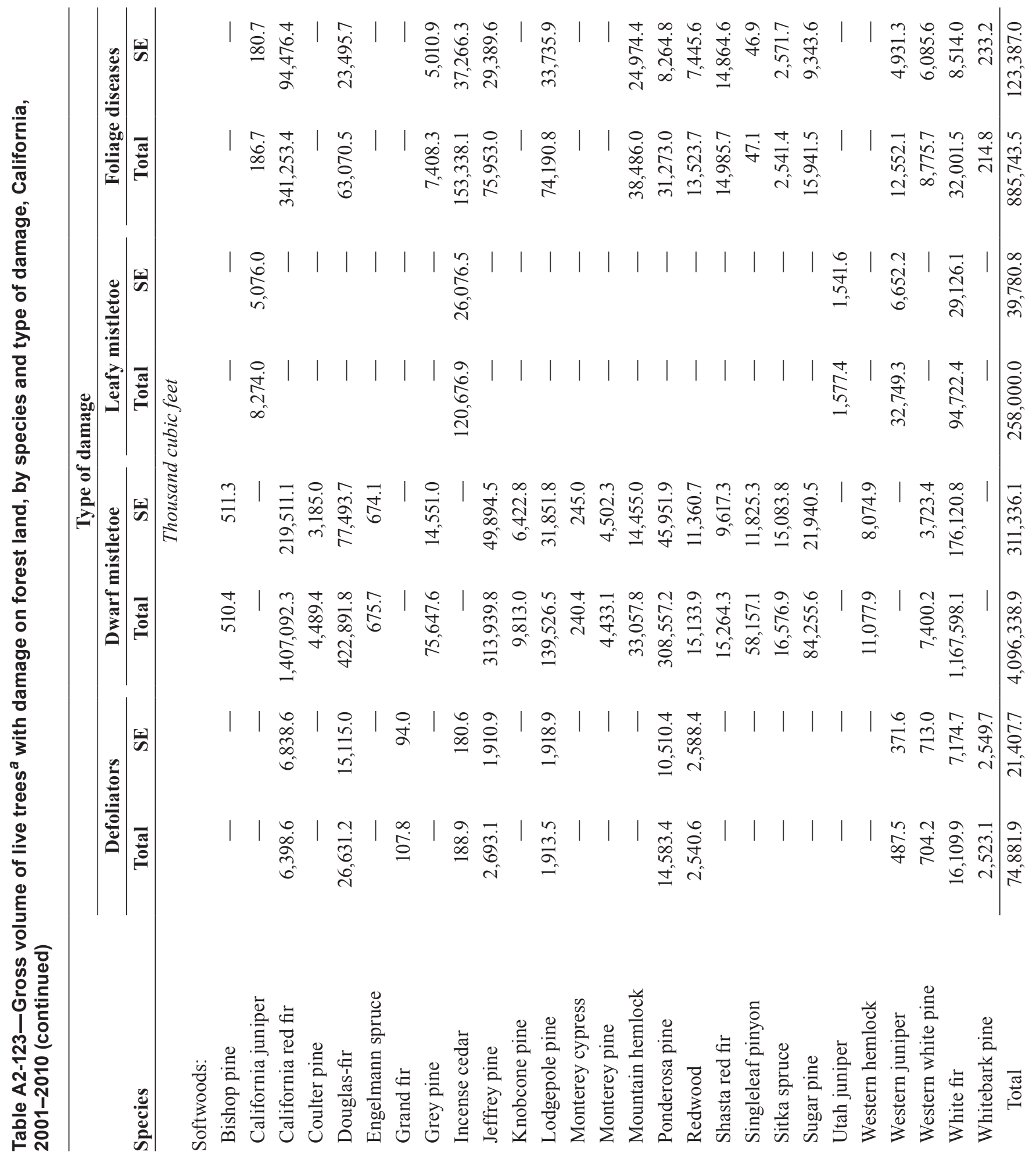




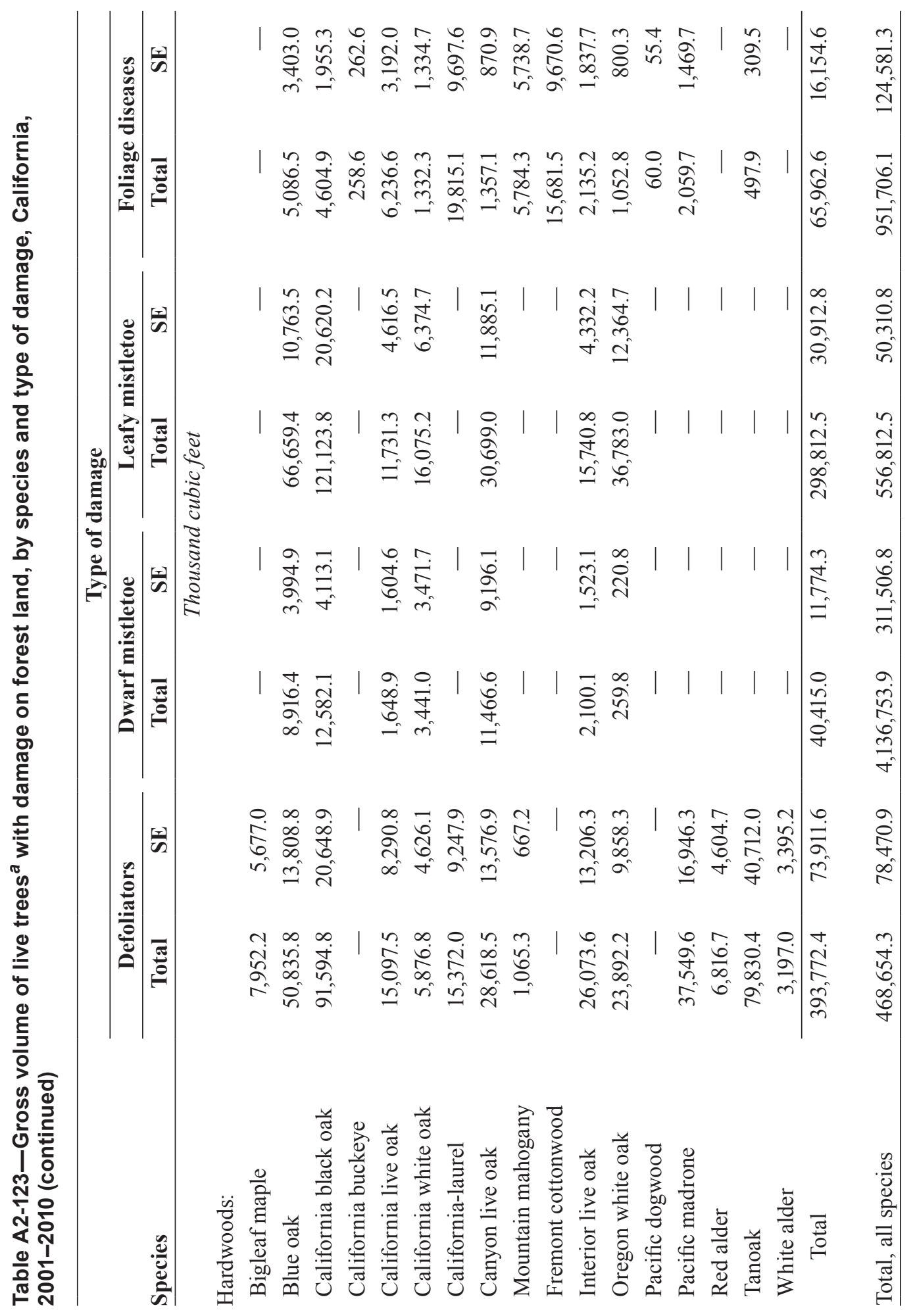




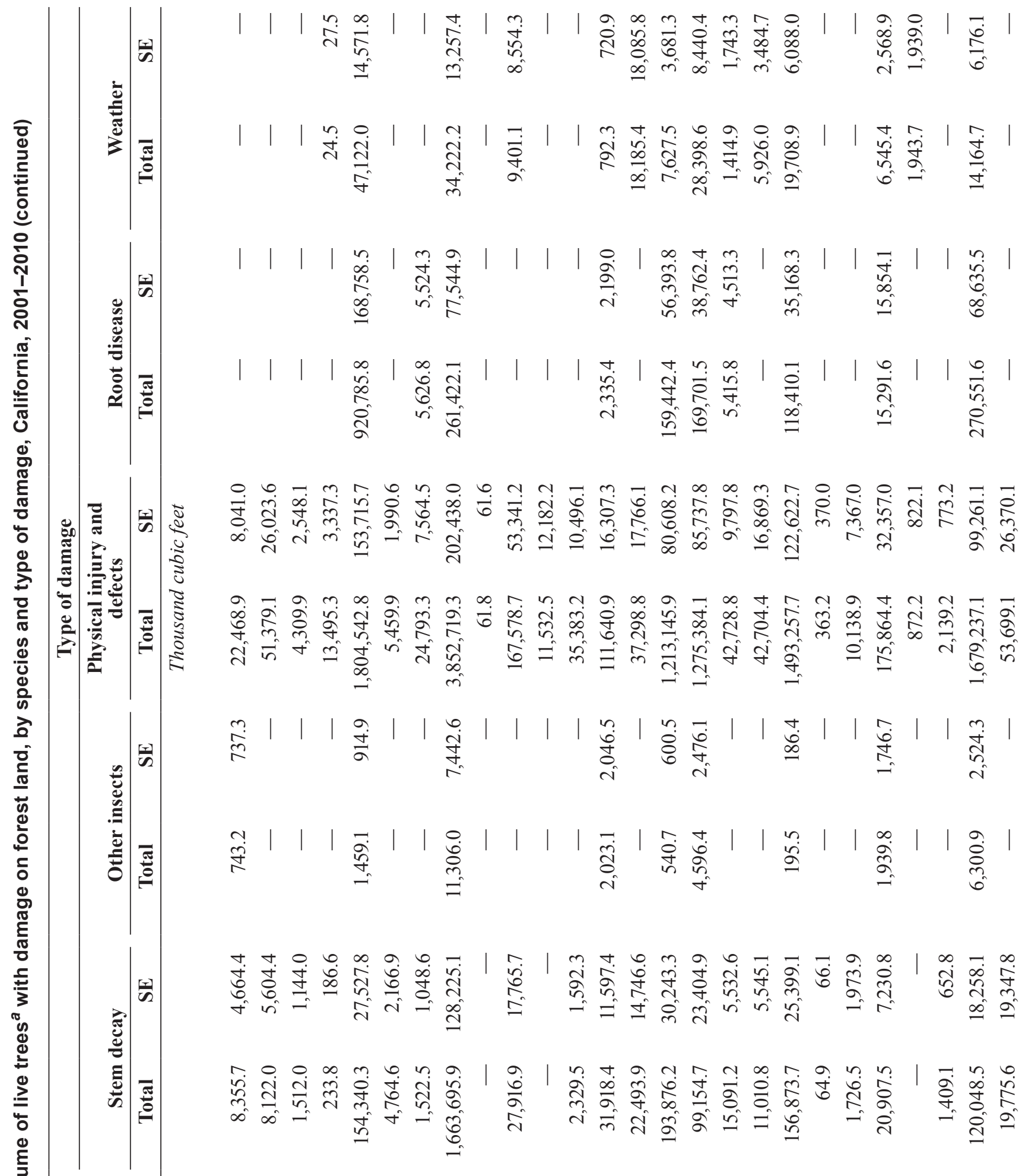

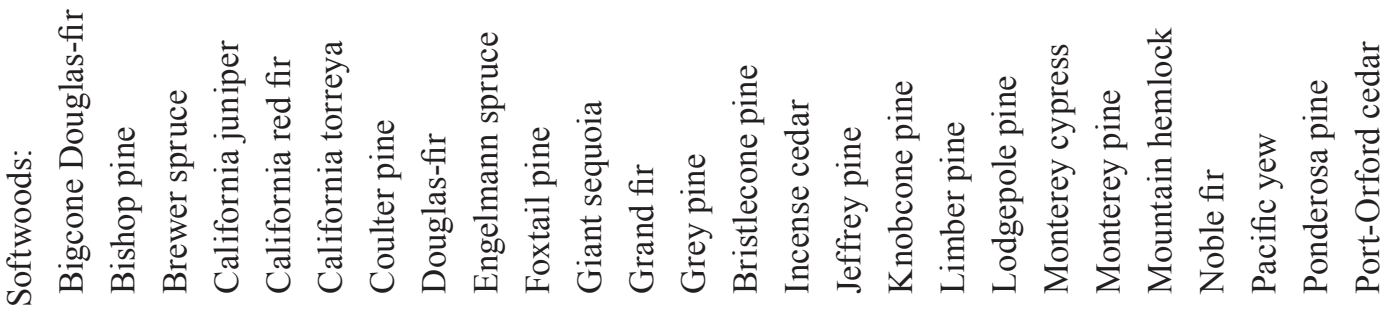




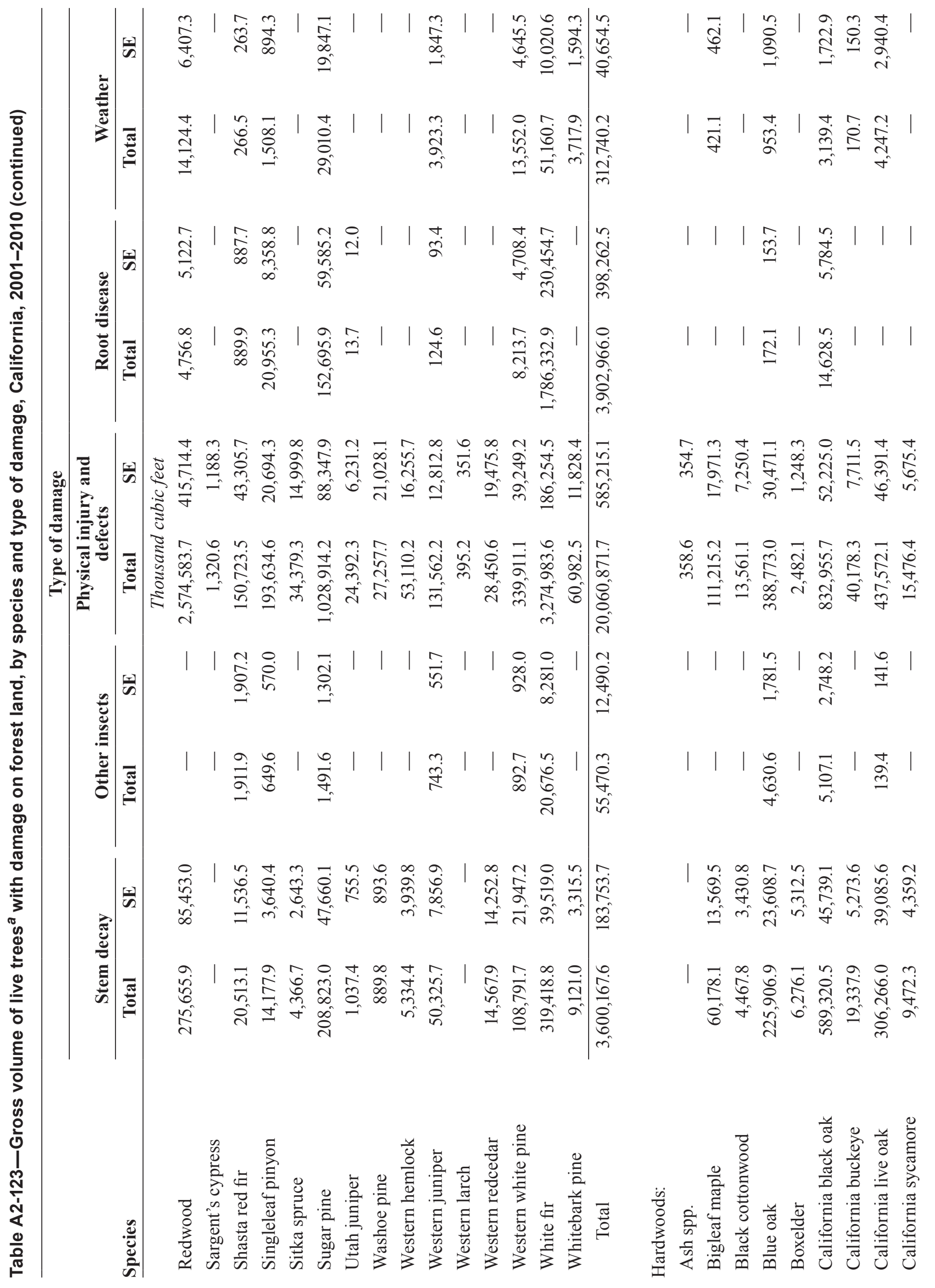




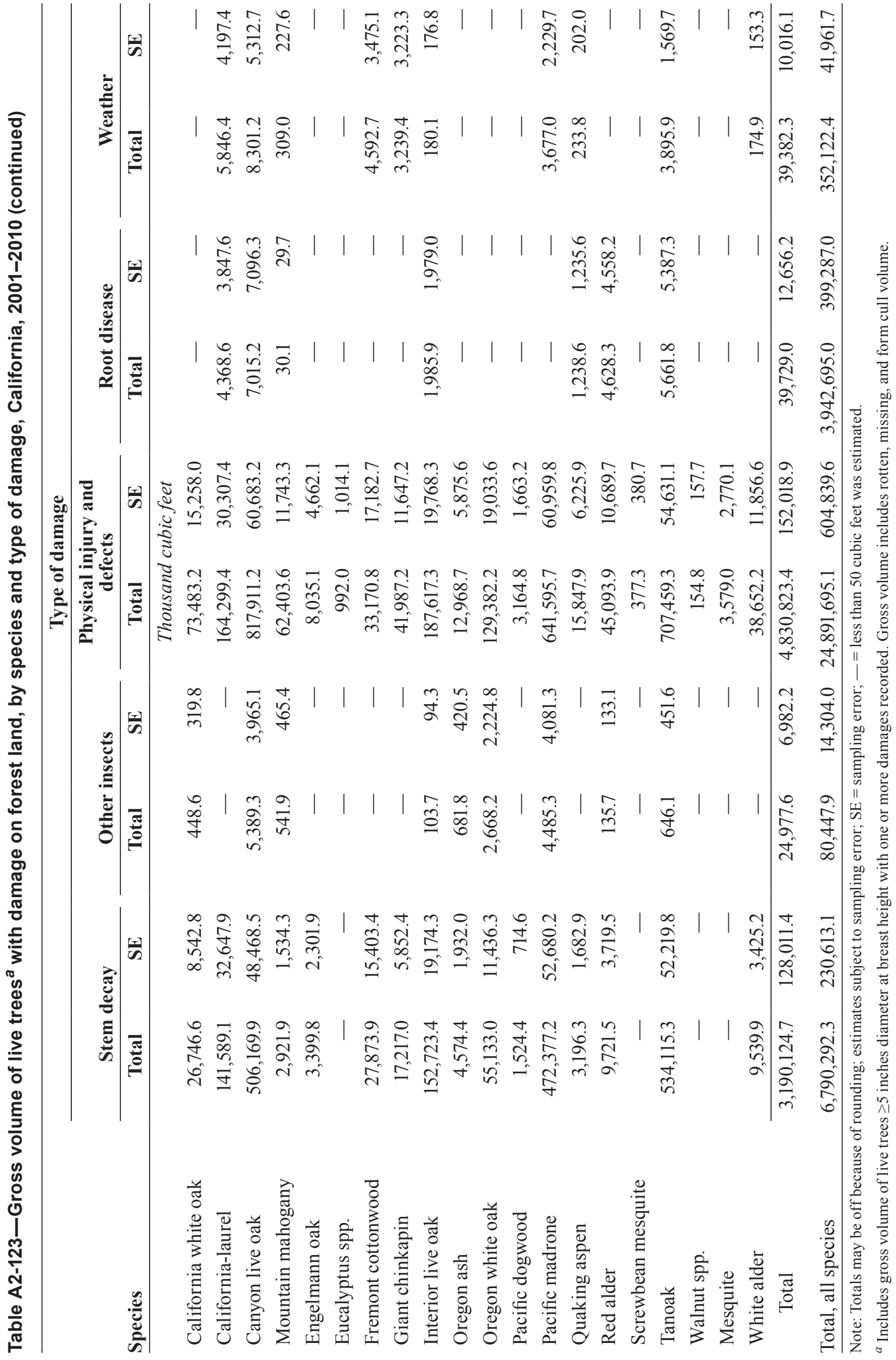




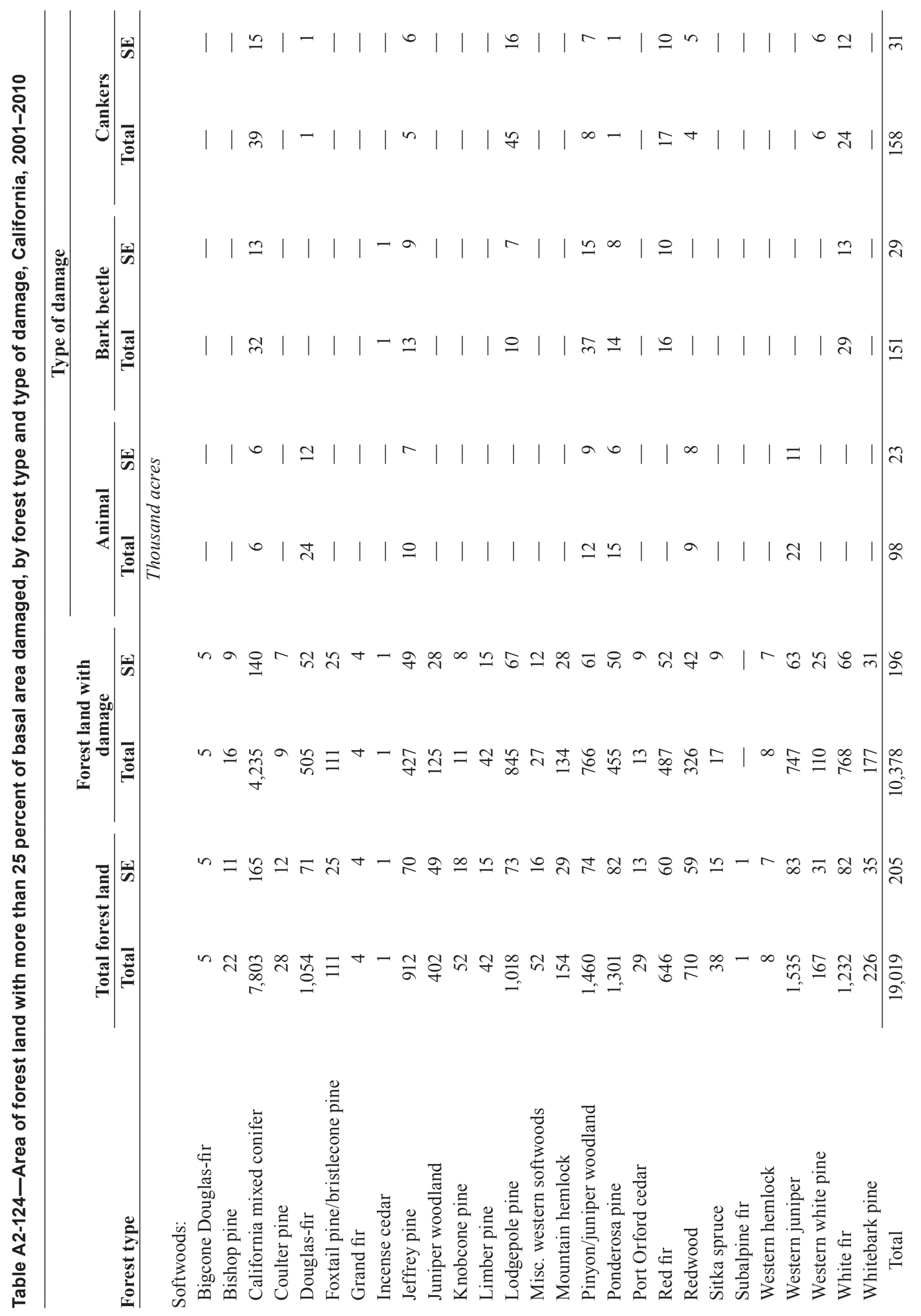


GENERAL TECHNICAL REPORT PNW-GTR-913

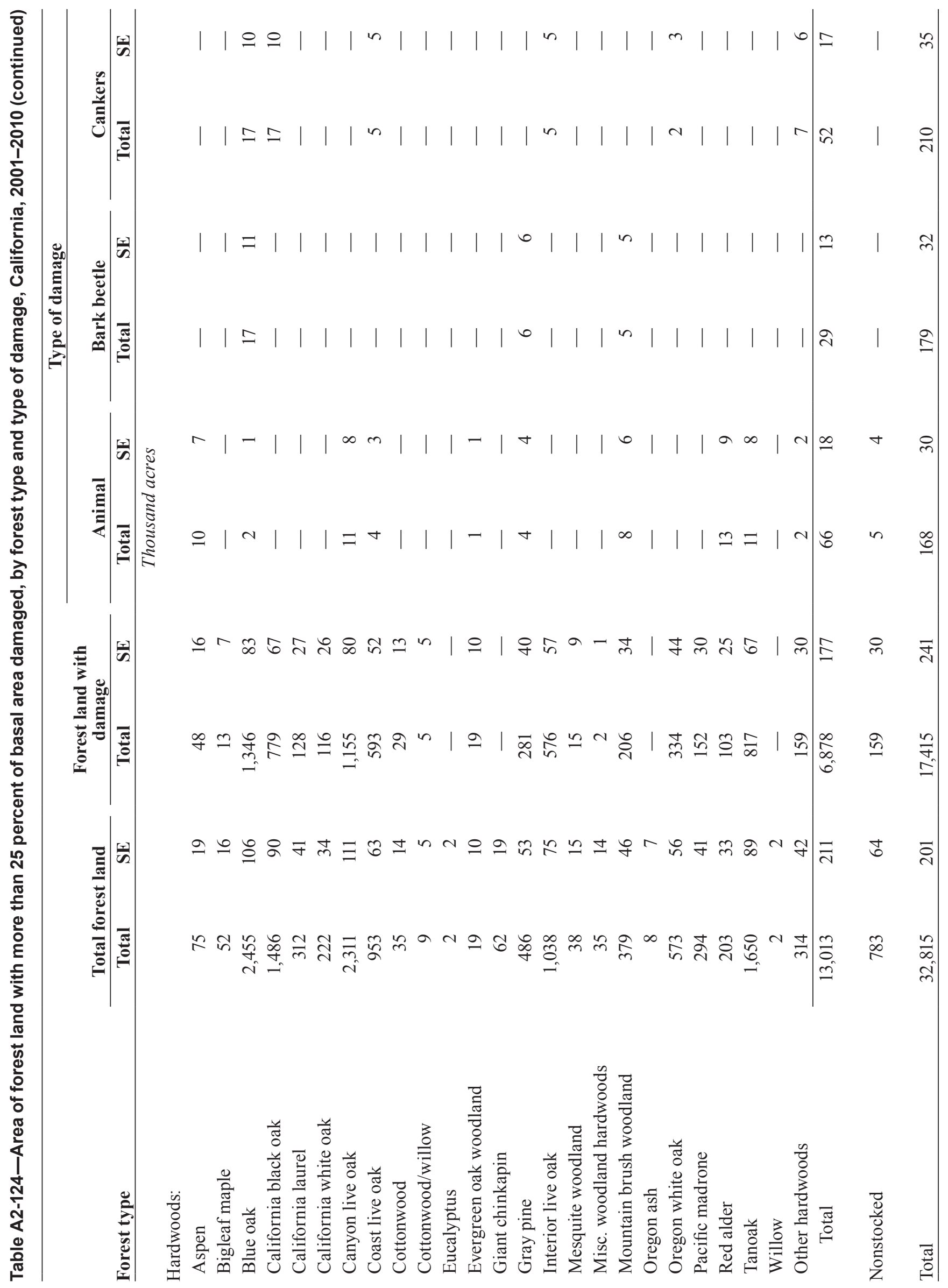




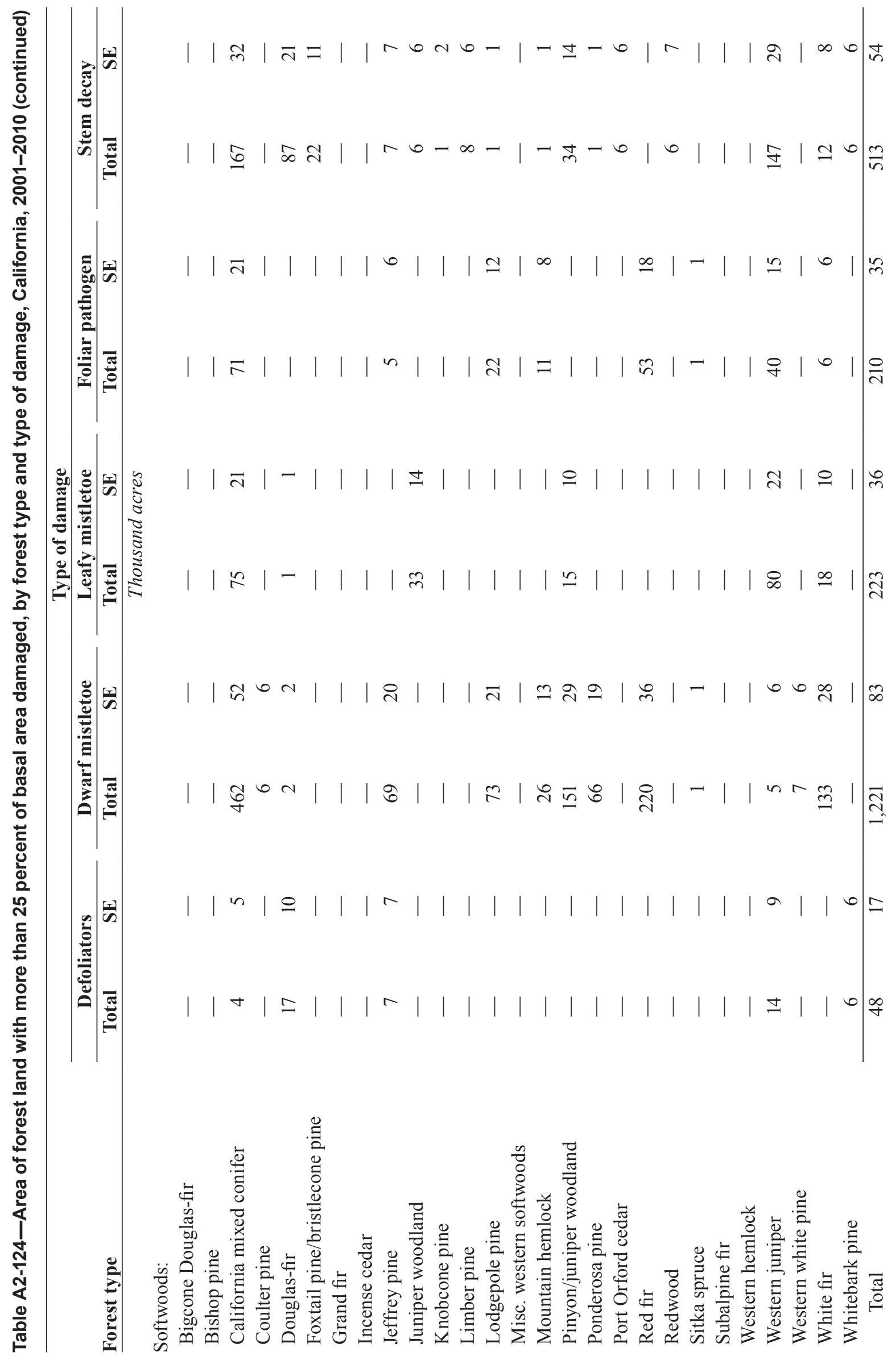




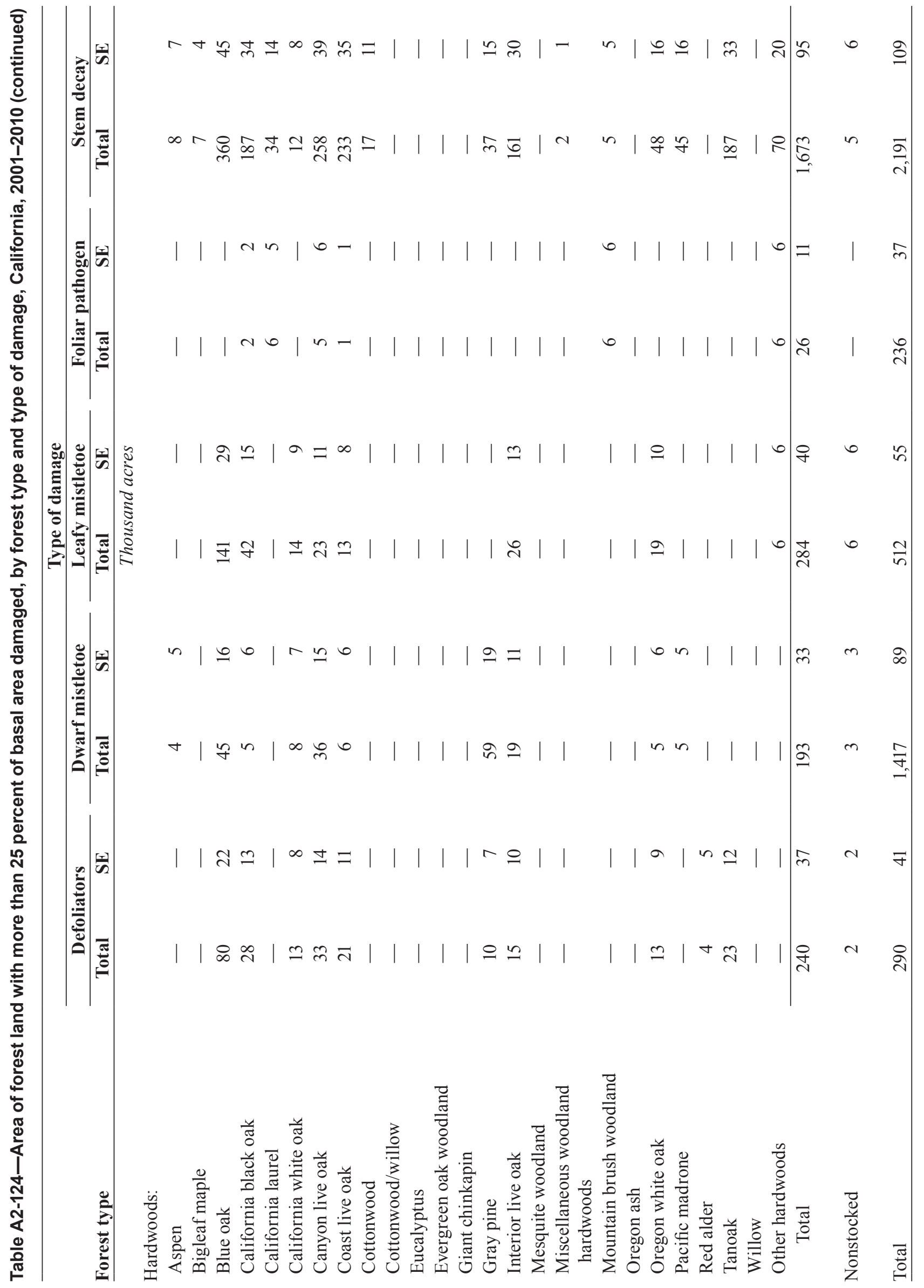




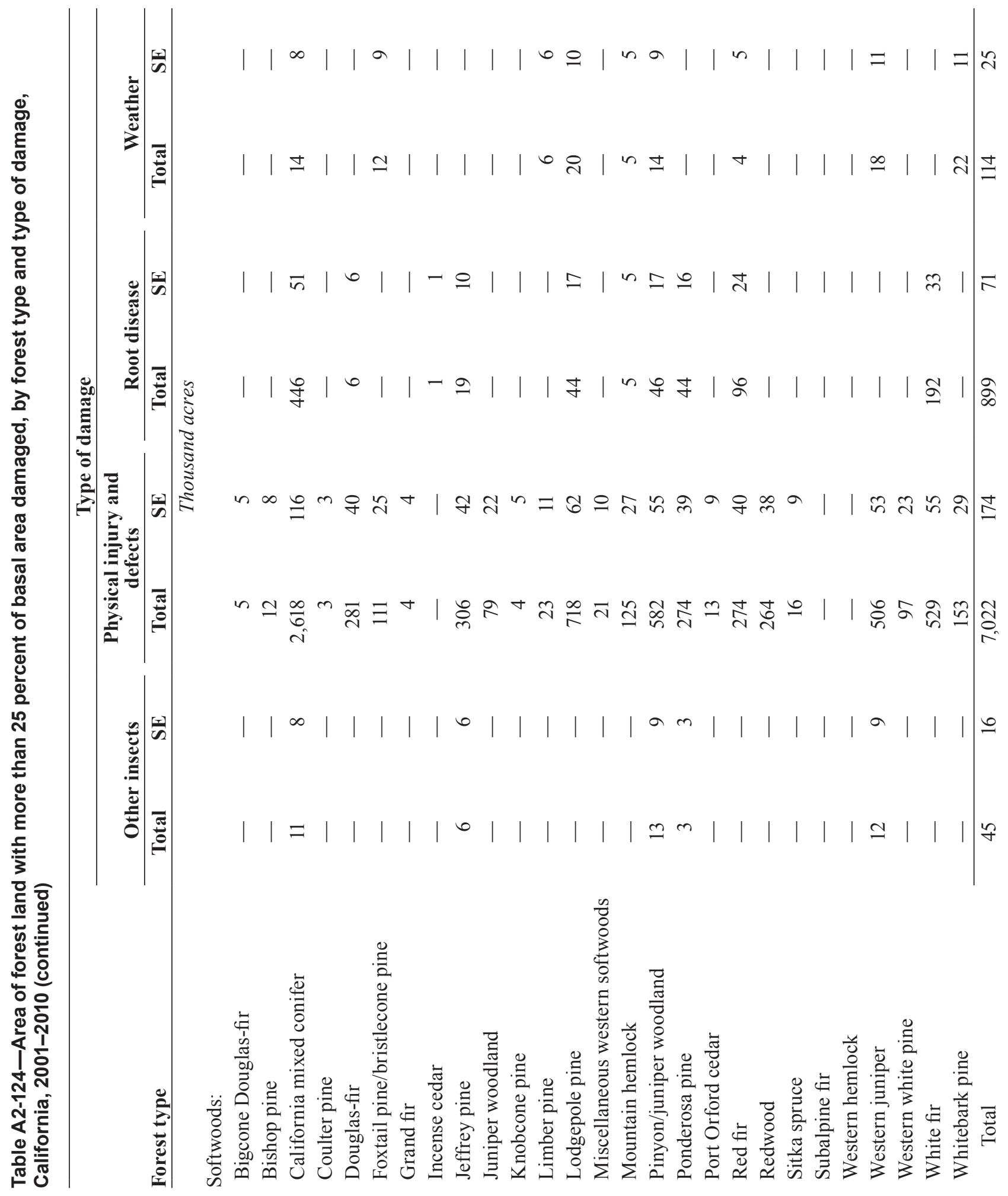




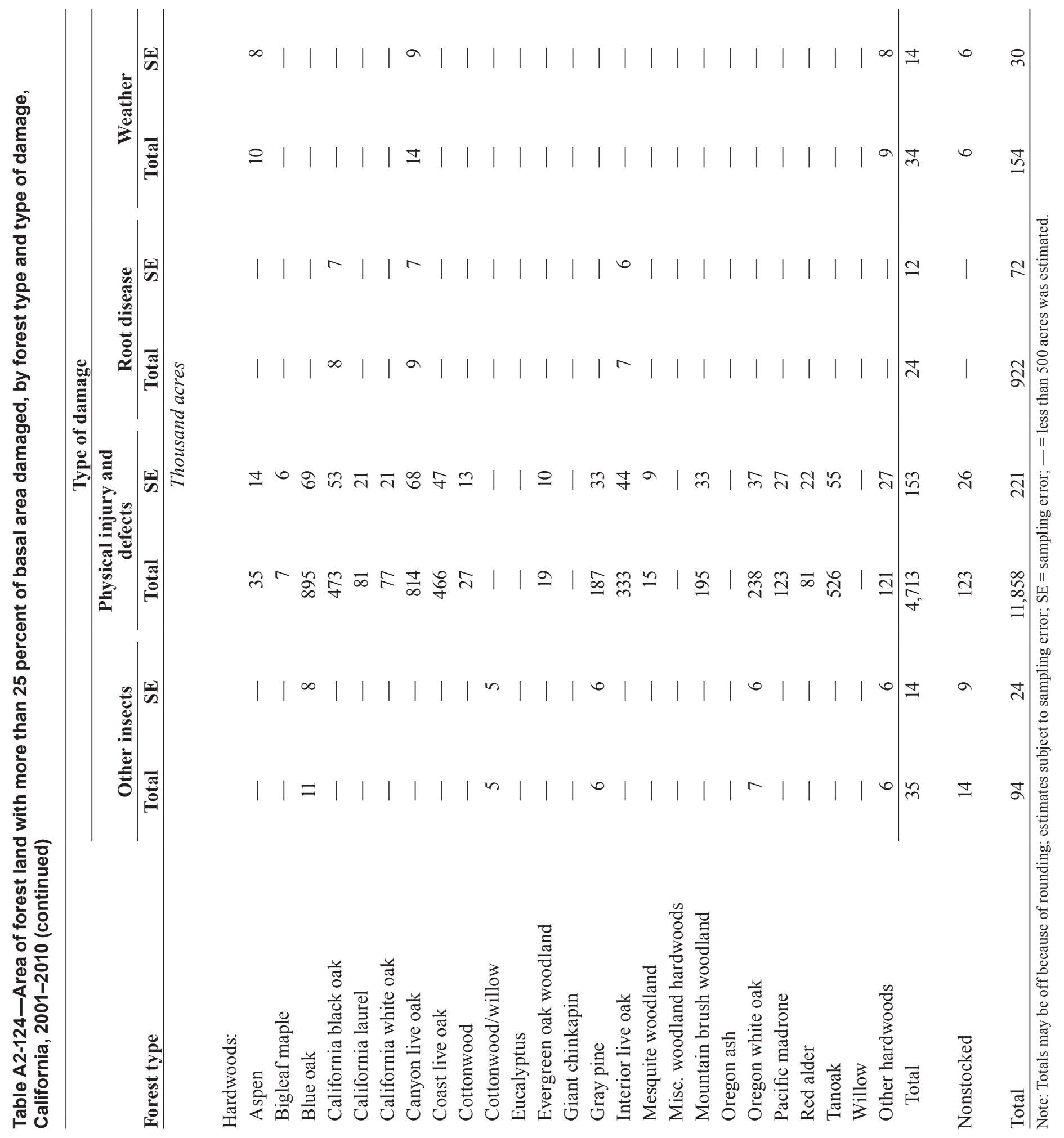


Table A2-125-Total roundwood output by product, species group, and source of material, California, 2006

\begin{tabular}{|c|c|c|c|c|}
\hline \multirow[b]{3}{*}{ Product and species group } & \multicolumn{3}{|c|}{ Source of material } & \multirow[b]{3}{*}{ All sources } \\
\hline & \multicolumn{2}{|c|}{ Growing-stock trees } & \multirow[b]{2}{*}{ Other sources } & \\
\hline & Sawtimber & Poletimber & & \\
\hline & \multicolumn{4}{|c|}{ Thousand cubic feet } \\
\hline \multicolumn{5}{|l|}{ Sawlogs: } \\
\hline Softwood & 249,174 & 924 & 35,405 & 285,503 \\
\hline Hardwood & 0 & 0 & 68 & 68 \\
\hline Total & 249,174 & 924 & 35,473 & 285,571 \\
\hline \multicolumn{5}{|l|}{ Veneer logs: } \\
\hline Softwood & 26,000 & 96 & 1,459 & 27,555 \\
\hline Hardwood & 0 & 0 & 0 & 0 \\
\hline Total & 26,000 & 96 & 1,459 & 27,555 \\
\hline \multicolumn{5}{|l|}{ Pulpwood: } \\
\hline Softwood & 0 & 0 & 0 & 0 \\
\hline Hardwood & 0 & 0 & 0 & 0 \\
\hline Total & 0 & 0 & 0 & 0 \\
\hline \multicolumn{5}{|l|}{ Composite panels: } \\
\hline Softwood & 1,496 & 6 & 49 & 1,551 \\
\hline Hardwood & 0 & 0 & 0 & 0 \\
\hline Total & 1,496 & 6 & 49 & 1,551 \\
\hline \multicolumn{5}{|l|}{ Poles and posts: } \\
\hline Softwood & 1,055 & 0 & 11 & 1,066 \\
\hline Hardwood & 0 & 0 & 0 & 0 \\
\hline Total & 1,055 & 0 & 11 & 1,066 \\
\hline \multicolumn{5}{|l|}{ Other miscellaneous: } \\
\hline Softwood & 6 & 0 & 0 & 6 \\
\hline Hardwood & 0 & 0 & 0 & 0 \\
\hline Total & 6 & 0 & 0 & 6 \\
\hline \multicolumn{5}{|l|}{ Total industrial products: } \\
\hline Softwood & 277,731 & 1,026 & 36,924 & 315,681 \\
\hline Hardwood & 0 & 0 & 68 & 68 \\
\hline Total & 277,731 & 1,026 & 36,992 & 315,749 \\
\hline \multicolumn{5}{|l|}{ Fuelwood $^{a}$ : } \\
\hline Softwood & 58,557 & 217 & 87,903 & 146,677 \\
\hline Hardwood & 0 & 1 & 4,854 & 4,855 \\
\hline Total & 58,557 & 218 & 92,757 & 151,532 \\
\hline \multicolumn{5}{|l|}{ All products: } \\
\hline Softwood & 336,288 & 1,243 & 124,827 & 462,358 \\
\hline Hardwood & 0 & 1 & 4,922 & 4,923 \\
\hline Total & 336,288 & 1,244 & 129,749 & 467,281 \\
\hline
\end{tabular}




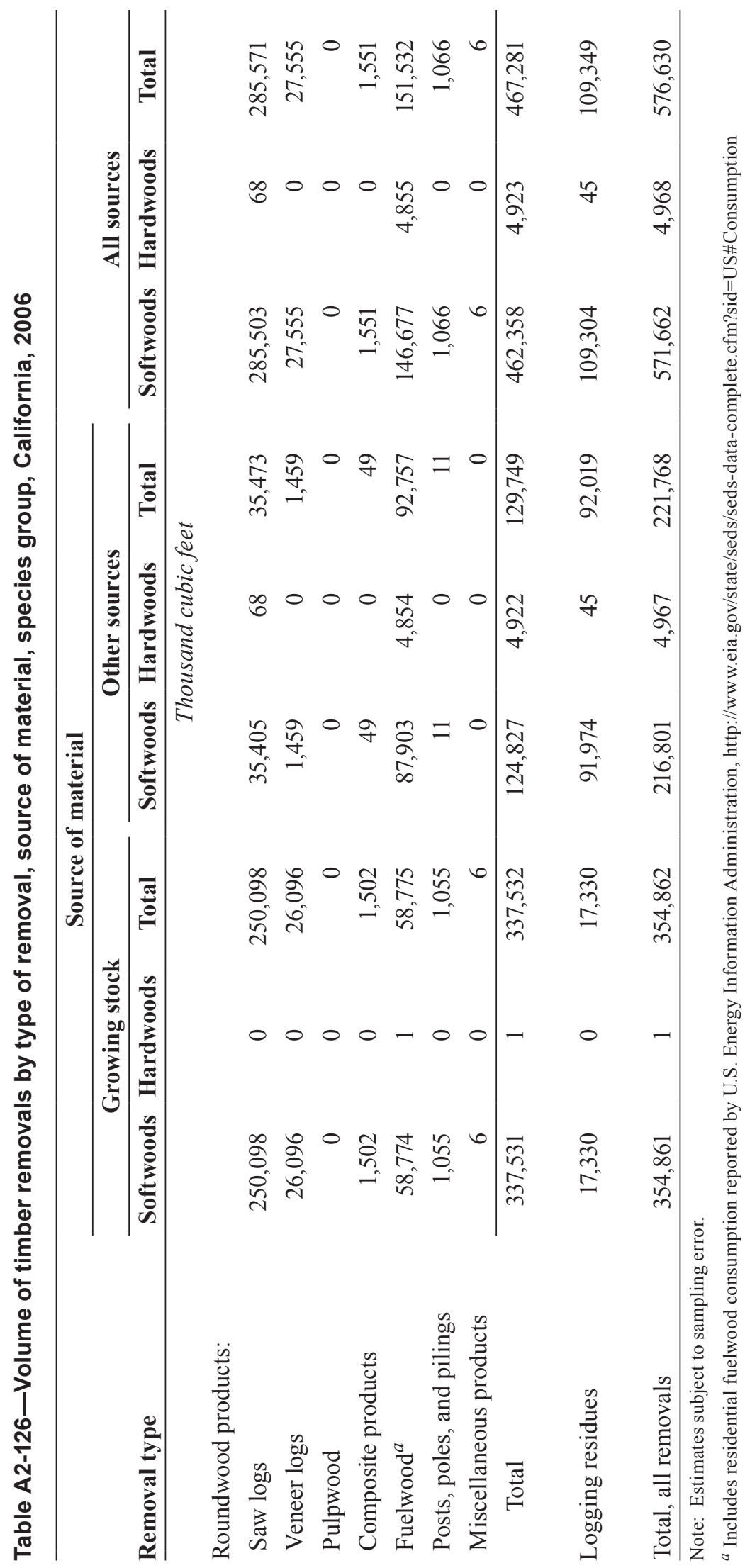


Table A2-127-Estimated area of forest land covered by the most abundant vascular plant nontimber forest products, by plant group and species, California, 2001-2010

\begin{tabular}{|c|c|c|c|}
\hline Plant group and scientific name & Common name & Total & SE \\
\hline Tree seedlings and saplings: & & \multicolumn{2}{|c|}{$\ldots \ldots-\ldots$ Acres $\ldots \ldots$} \\
\hline Abies concolor & White fir & 130,071 & 5,434 \\
\hline Abies magnifica & California red fir & 44,545 & 3,444 \\
\hline Arbutus menziesii & Pacific madrone & 30,232 & 2,975 \\
\hline Calocedrus decurrens & Incense cedar & 104,686 & 6,117 \\
\hline Lithocarpus densiflorus & Tanoak & 315,007 & 17,294 \\
\hline Pseudotsuga menziesii & Douglas-fir & 135,729 & 6,682 \\
\hline Quercus kelloggii & California black oak & 90,067 & 8,789 \\
\hline Sequoia sempervirens & Redwood & 31,689 & 2,266 \\
\hline \multicolumn{4}{|l|}{ Shrubs: } \\
\hline Amelanchier alnifolia & Saskatoon serviceberry & 30,496 & 5,833 \\
\hline Arctostaphylos & Manzanita & 77,177 & 10,189 \\
\hline Arctostaphylos manzanita & Whiteleaf manzanita & 45,636 & 7,814 \\
\hline Arctostaphylos nevadensis & Pinemat manzanita & 120,376 & 12,771 \\
\hline Arctostaphylos patula & Greenleaf manzanita & 389,048 & 23,060 \\
\hline Arctostaphylos viscida & Sticky whiteleaf manzanita & 266,870 & 23,106 \\
\hline Artemisia tridentata & Big sagebrush & 249,510 & 15,135 \\
\hline Ceanothus & Ceanothus & 28,529 & 5,082 \\
\hline Ceanothus velutinus & Snowbrush ceanothus & 120,888 & 14,286 \\
\hline Chimaphila umbellata & Pipsissewa & 28,062 & 3,470 \\
\hline Corylus cornuta & Beaked hazelnut & 70,546 & 7,427 \\
\hline Gaultheria shallon & Salal & 106,523 & 13,004 \\
\hline Holodiscus discolor & Oceanspray & 46,552 & 5,739 \\
\hline Mahonia nervosa & Cascade barberry & 34,438 & 4,957 \\
\hline Quercus vacciniifolia & Huckleberry oak & 356,321 & 27,088 \\
\hline Rubus parviflorus & Thimbleberry & 29,620 & 5,046 \\
\hline Rubus spectabilis & Salmonberry & 31,673 & 9,974 \\
\hline Rubus ursinus & California blackberry & 58,534 & 7,973 \\
\hline Salix & Willow & 60,342 & 5,524 \\
\hline Symphoricarpos albus & Common snowberry & 30,752 & 3,945 \\
\hline Umbellularia californica & California laurel & 55,450 & 5,603 \\
\hline Vaccinium ovatum & California huckleberry & 304,289 & 22,272 \\
\hline
\end{tabular}


Table A2-127-Estimated area of forest land covered by the most abundant vascular plant nontimber forest products, by plant group and species, California, 2001-2010 (continued)

\begin{tabular}{llcc}
\hline Plant group and scientific name & Common name & Total & SE \\
\hline Herbs: & & ---- Acres - - - - \\
Achillea millefolium & Common yarrow & 19,332 & 3,103 \\
Apocynum androsaemifolium & Spreading dogbane & 12,658 & 1,786 \\
Arnica & Arnica & 4,354 & 1,382 \\
Athyrium filix-femina & Common ladyfern & 13,294 & 2,581 \\
Balsamorhiza sagittata & Arrowleaf balsamroot & 10,647 & 2,042 \\
Blechnum spicant & Deer fern & 5,483 & 1,427 \\
Claytonia perfoliata & Miner's lettuce & 14,886 & 4,929 \\
Clinopodium douglasii & Yerba buena & 3,621 & 1,006 \\
Fragaria & Strawberry & 6,325 & 930 \\
Polystichum munitum & Western swordfern & 234,691 & 19,159 \\
Pteridium aquilinum & Western brackenfern & 137,884 & 9,745 \\
Rubus & Blackberry & 28,067 & 4,851 \\
Taraxacum & Dandelion & 12,962 & 4,299 \\
Xerophyllum tenax & Common beargrass & 18,972 & 4,819 \\
\hline
\end{tabular}

Note: Estimates subject to sampling error; $\mathrm{SE}=$ sampling error.

Table A2-128-Percentage of forested plots with selected lichen nontimber forest products present, by species, California, 2001-2005

\begin{tabular}{llr}
\hline Scientific name & Common name & Percent \\
\hline Alectoria sarmentosa & Witch's hair lichen & 13.5 \\
Bryoria fremontii & Old man's beard & 13.1 \\
Letharia vulpina & Wolf lichen & 53.8 \\
Lobaria pulmonaria & Lungwort & 6.9 \\
Parmelia saxatilis & Crottle & 1.5 \\
Ramalina menziesii & Lace lichen & 2.9 \\
Usnea & Beard lichens & 38.2 \\
Usnea hirta & Beard lichen & 1.1 \\
Vulpicida canadensis & Brown-eyed sunshine lichen & 8.4 \\
\hline
\end{tabular}

Note: Estimates subject to sampling error.

292 forested plots were sampled. 
Table A2-129-Estimated mean crown density and other statistics for live trees ${ }^{a}$ on forest land, by species group, California, 2001-2010

\begin{tabular}{|c|c|c|c|c|c|}
\hline \multirow[b]{2}{*}{ Species group } & \multirow[b]{2}{*}{ Plots } & \multirow[b]{2}{*}{ Trees } & \multicolumn{3}{|c|}{ Crown density } \\
\hline & & & Mean & Minimum & Maximum \\
\hline & $-\cdots--1$ & $r-\ldots-\ldots$ & $-\ldots$ & - Percent - & $-\ldots$ \\
\hline \multicolumn{6}{|l|}{ Softwoods: } \\
\hline Douglas-fir & 89 & 701 & 40 & 0 & 85 \\
\hline Incense cedar & 51 & 302 & 39 & 5 & 80 \\
\hline Lodgepole pine & 21 & 269 & 40 & 10 & 70 \\
\hline Ponderosa and Jeffrey pines & 103 & 743 & 42 & 5 & 95 \\
\hline Redwood & 11 & 155 & 35 & 5 & 70 \\
\hline Sugar pine & 38 & 132 & 39 & 0 & 75 \\
\hline True fir & 93 & 1,145 & 44 & 0 & 99 \\
\hline Western hemlock & 3 & 9 & 49 & 40 & 70 \\
\hline Western white pine & 16 & 68 & 42 & 15 & 65 \\
\hline Woodland softwoods & 15 & 89 & 50 & 10 & 90 \\
\hline Other western softwoods & 63 & 326 & 41 & 0 & 85 \\
\hline Total & 503 & 3,939 & & & \\
\hline \multicolumn{6}{|l|}{ Hardwoods: } \\
\hline Cottonwood and aspen & 4 & 38 & 39 & 10 & 85 \\
\hline Oak & 152 & 1,949 & 35 & 0 & 85 \\
\hline Red alder & 6 & 52 & 44 & 5 & 70 \\
\hline Woodland hardwoods & 18 & 171 & 39 & 5 & 85 \\
\hline Other western hardwoods & 72 & 956 & 36 & 0 & 85 \\
\hline Total & 252 & 3,166 & & & \\
\hline All species & 755 & 7,105 & & & \\
\hline
\end{tabular}

Note: Estimates subject to sampling error.

${ }^{a}$ Includes all live trees $\geq 5$ inches in diameter at breast height, consisting of growing stock, rough cull, and rotten cull tree classes. 
Table A2-130-Estimated mean foliage transparency and other statistics for live trees ${ }^{a}$ on forest land, by species group, California, 2001-2010

\begin{tabular}{|c|c|c|c|c|c|}
\hline \multirow[b]{2}{*}{ Species group } & \multirow[b]{2}{*}{ Plots } & \multirow[b]{2}{*}{ Trees } & \multicolumn{3}{|c|}{ Foliage transparency } \\
\hline & & & Mean & Minimum & Maximum \\
\hline & - - - - - - & er- - - - - - & $-\cdots$ & - - Percent - - & 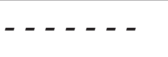 \\
\hline \multicolumn{6}{|l|}{ Softwoods: } \\
\hline Douglas-fir & 89 & 701 & 19 & 0 & 70 \\
\hline Incense cedar & 51 & 302 & 19 & 5 & 40 \\
\hline Lodgepole pine & 21 & 269 & 19 & 5 & 55 \\
\hline Ponderosa and Jeffrey pines & 103 & 743 & 22 & 5 & 70 \\
\hline Redwood & 11 & 155 & 22 & 10 & 45 \\
\hline Sugar pine & 38 & 132 & 19 & 5 & 35 \\
\hline True fir & 93 & 1,145 & 17 & 0 & 99 \\
\hline Western hemlock & 3 & 9 & 17 & 15 & 20 \\
\hline Western white pine & 16 & 68 & 17 & 10 & 30 \\
\hline Woodland softwoods & 15 & 89 & 19 & 5 & 30 \\
\hline Other western softwoods & 63 & 326 & 19 & 5 & 85 \\
\hline Total & 503 & 3,939 & & & \\
\hline \multicolumn{6}{|l|}{ Hardwoods: } \\
\hline Cottonwood and aspen & 4 & 38 & 30 & 5 & 60 \\
\hline Oak & 152 & 1,949 & 24 & 5 & 99 \\
\hline Red alder & 6 & 52 & 30 & 15 & 60 \\
\hline Woodland hardwoods & 18 & 171 & 26 & 15 & 80 \\
\hline Other western hardwoods & 72 & 956 & 23 & 5 & 99 \\
\hline Total & 252 & 3,166 & & & \\
\hline All species & 755 & 7,105 & & & \\
\hline
\end{tabular}

Note: Estimates subject to sampling error.

${ }^{a}$ Includes all live trees $\geq 5$ inches in diameter at breast height, consisting of growing stock, rough cull, and rotten cull tree classes. 
Table A2-131-Estimated mean crown dieback and other statistics for live trees ${ }^{a}$ on forest land, by species group, California, 2001-2010

\begin{tabular}{|c|c|c|c|c|c|}
\hline \multirow[b]{2}{*}{ Species group } & \multirow[b]{2}{*}{ Plots } & \multirow[b]{2}{*}{ Trees } & \multicolumn{3}{|c|}{ Crown dieback } \\
\hline & & & Mean & Minimum & Maxim \\
\hline & $-\ldots$ & er- - - - - - & $-\ldots$ & - Percent - & $-\ldots$ \\
\hline \multicolumn{6}{|l|}{ Softwoods: } \\
\hline Douglas-fir & 89 & 701 & 1.1 & 0 & 90 \\
\hline Incense cedar & 51 & 302 & 1.8 & 0 & 90 \\
\hline Lodgepole pine & 21 & 269 & 3.1 & 0 & 45 \\
\hline Ponderosa and Jeffrey pines & 103 & 743 & 1.5 & 0 & 80 \\
\hline Redwood & 11 & 155 & 2.6 & 0 & 60 \\
\hline Sugar pine & 38 & 132 & 1.7 & 0 & 50 \\
\hline True fir & 93 & 1,145 & 2.2 & 0 & 99 \\
\hline Western hemlock & 3 & 9 & 0.0 & 0 & 0 \\
\hline Western white pine & 16 & 68 & 3.7 & 0 & 20 \\
\hline Woodland softwoods & 15 & 89 & 4.8 & 0 & 40 \\
\hline Other western softwoods & 63 & 326 & 2.5 & 0 & 95 \\
\hline Total & 503 & 3,939 & & & \\
\hline
\end{tabular}

Hardwoods:

Cottonwood and aspen

Oak

Red alder

Woodland hardwoods

Other western hardwoods Total

\begin{tabular}{rr}
4 & 38 \\
152 & 1,949 \\
6 & 52 \\
18 & 171 \\
72 & 956 \\
\hline 252 & 3,166
\end{tabular}

8.9

5.5

3.1

11.9

2.8
90

90

45

80

60

50

99

0

20

40

95

All species 755 7,105

Note: Estimates subject to sampling error.

${ }^{a}$ Includes all live trees $\geq 5$ inches in diameter at breast height, consisting of growing stock, rough cull, and rotten cull tree classes. 
Table A2-132-Estimated area of forest land covered by selected nonnative vascular plant species and number of sample plots ${ }^{a}$, by life form and species, California, 2001-2010

\begin{tabular}{|c|c|c|c|c|c|}
\hline \multirow[b]{2}{*}{ Life form } & \multirow[b]{2}{*}{ Scientific name } & \multirow[b]{2}{*}{ Common name } & \multicolumn{2}{|c|}{ Area covered } & \multirow[b]{2}{*}{ Number of plots } \\
\hline & & & Total & SE & \\
\hline & & & - - - - & $-\ldots$ & \\
\hline \multicolumn{6}{|l|}{ Shrubs } \\
\hline & Cytisus scoparius & Scotch broom & 9,504 & 4,349 & 19 \\
\hline & Erica lusitanica & Spanish heath & 4,480 & 1,579 & 34 \\
\hline & Genista monspessulana & French broom & 3,710 & 1,816 & 9 \\
\hline & Rubus armeniacus & Himalayan blackberry & 42,016 & 6,861 & 101 \\
\hline & Rubus laciniatus & Cutleaf blackberry & 1,645 & 789 & 7 \\
\hline
\end{tabular}

Forbs

$\begin{array}{llrrr}\text { Carduus pycnocephalus } & \text { Italian plumeless thistle } & 4,338 & 1,502 & 17 \\ \text { Centaurea solstitialis } & \text { Yellow star-thistle } & 22,476 & 4,402 & 84 \\ \text { Cirsium } & \text { Thistle } & 26,502 & 3,690 & 295 \\ \text { Cirsium arvense } & \text { Canada thistle } & 2,660 & 1,553 & 10 \\ \text { Cirsium vulgare } & \text { Bull thistle } & 2,547 & 690 & 42 \\ \text { Digitalis purpurea } & \text { Purple foxglove } & 1,027 & 680 & 7 \\ \text { Hypericum perforatum } & \text { Common St. Johnswort } & 2,535 & 687 & 51 \\ \text { Hypochaeris radicata } & \text { Hairy cat's ear } & 1,150 & 595 & 23 \\ \text { Torilis arvensis } & \text { Spreading hedgeparsley } & 87,833 & 8,743 & 276\end{array}$

Grasses

\begin{tabular}{llrrr} 
Aira caryophyllea & Silver hairgrass & 7,754 & 1,897 & 38 \\
Arundo donax & Giant reed & 1,555 & 896 & 8 \\
Avena barbata & Slender oat & 19,412 & 4,693 & 31 \\
Avena fatua & Wild oat & 42,575 & 9,109 & 55 \\
Briza maxima & Big quakinggrass & 19,393 & 6,623 & 36 \\
Bromus diandrus & Ripgut brome & 111,163 & 13,230 & 202 \\
Bromus hordeaceus & Soft brome & 84,514 & 11,845 & 128 \\
Bromus madritensis & Compact brome & 14,954 & 3,547 & 46 \\
Bromus tectorum & Cheatgrass & 185,304 & 13,273 & 630 \\
Cynosurus echinatus & Bristly dogstail grass & 123,435 & 12,907 & 227 \\
Taeniatherum caput-medusae & Medusahead & 64,487 & 9,384 & 135 \\
\hline
\end{tabular}

Note: Estimates subject to sampling error; $\mathrm{SE}=$ sampling error. Estimates are likely low for all species because only plants present at $>3$ percent cover on a subplot were recorded, and because most grasses and some forbs can be difficult to identify to species outside of their short flowering seasons.

${ }^{a}$ Total number of sample plots with accessible forest land was 5,575. 
Table A2-133-Mean cover of understory vegetation on forest land, by forest type group and life form, California 2001-2010

\begin{tabular}{|c|c|c|c|c|c|c|c|c|c|c|c|c|}
\hline \multirow[b]{3}{*}{ Forest type group } & \multicolumn{10}{|c|}{ Life form } & & \\
\hline & \multicolumn{2}{|c|}{$\begin{array}{l}\text { Seedlings + } \\
\text { saplings }\end{array}$} & \multicolumn{2}{|c|}{ Shrubs } & \multicolumn{2}{|c|}{ Forbs } & \multicolumn{2}{|c|}{ Graminoids } & \multicolumn{2}{|c|}{$\begin{array}{c}\text { All } \\
\text { understory } \\
\text { plants }\end{array}$} & \multicolumn{2}{|c|}{ Bare soil } \\
\hline & Mean & SE & Mean & SE & Mean & SE & Mean & SE & Mean & SE & Mean & SE \\
\hline & \multicolumn{12}{|c|}{ Percent } \\
\hline \multicolumn{13}{|l|}{ Softwoods: } \\
\hline California mixed conifer & 6.1 & 0.2 & 17.2 & 0.5 & 5.4 & 0.2 & 3.4 & 0.2 & 30.6 & 0.6 & 3.9 & 0.2 \\
\hline Douglas-fir & 8.6 & 0.6 & 24.9 & 1.5 & 9.6 & 1.0 & 5.8 & 0.7 & 45.3 & 1.8 & 2.8 & 0.4 \\
\hline $\begin{array}{l}\text { Fir/spruce/mountain } \\
\text { hemlock }\end{array}$ & 3.3 & 0.2 & 17.0 & 1.1 & 6.5 & 0.5 & 3.0 & 0.3 & 28.4 & 1.2 & 4.2 & 0.4 \\
\hline Hemlock/Sitka spruce & 1.7 & 0.4 & 23.6 & 4.9 & 28.2 & 7.4 & 9.7 & 3.7 & 56.6 & 12.4 & 1.3 & 0.9 \\
\hline Lodgepole pine & 3.4 & 0.3 & 12.1 & 1.3 & 8.4 & 0.8 & 10.4 & 1.0 & 31.8 & 1.8 & 4.9 & 0.6 \\
\hline Other western softwoods & 1.8 & 0.2 & 14.2 & 0.9 & 6.7 & 0.4 & 14.3 & 0.8 & 34.9 & 1.2 & 10.4 & 0.7 \\
\hline Pinyon/juniper & 0.9 & 0.1 & 17.6 & 0.7 & 4.5 & 0.4 & 8.1 & 0.6 & 29.5 & 1.0 & 12.7 & 0.8 \\
\hline Ponderosa pine & 2.9 & 0.2 & 23.3 & 1.1 & 6.4 & 0.4 & 7.9 & 0.5 & 38.7 & 1.2 & 4.6 & 0.3 \\
\hline Redwood & 7.4 & 0.6 & 24.4 & 1.9 & 19.4 & 1.8 & 3.6 & 0.5 & 50.2 & 2.1 & 2.0 & 0.4 \\
\hline Western white pine & 6.5 & 2.5 & 14.6 & 3.7 & 7.9 & 1.6 & 4.6 & 0.9 & 32.1 & 4.5 & 8.8 & 3.0 \\
\hline Total & 4.5 & 0.1 & 18.0 & 0.3 & 6.7 & 0.2 & 6.1 & 0.2 & 33.4 & 0.4 & 5.5 & 0.2 \\
\hline \multicolumn{13}{|l|}{ Hardwoods: } \\
\hline Alder/maple & 6.1 & 1.0 & 36.1 & 3.8 & 20.6 & 2.9 & 3.4 & 0.8 & 59.3 & 3.7 & 1.6 & 0.5 \\
\hline Aspen/birch & 13.4 & 2.9 & 23.9 & 3.8 & 11.1 & 2.3 & 9.5 & 1.6 & 54.1 & 6.8 & 3.0 & 1.0 \\
\hline Elm/ash/cottonwood & 3.1 & 1.3 & 38.8 & 7.0 & 7.4 & 2.8 & 29.2 & 6.7 & 68.7 & 6.2 & 4.6 & 2.6 \\
\hline Exotic hardwoods & 10.0 & 10.0 & 0.0 & 0.0 & 0.0 & 0.0 & 0.0 & 0.0 & 10.0 & 10.0 & 0.0 & 0.0 \\
\hline Other hardwoods & 7.8 & 0.9 & 20.2 & 2.1 & 9.2 & 1.0 & 17.0 & 2.4 & 50.3 & 2.7 & 5.9 & 1.2 \\
\hline Tanoak/laurel & 11.3 & 0.6 & 19.4 & 1.1 & 7.9 & 0.6 & 3.5 & 0.5 & 40.0 & 1.3 & 2.7 & 0.3 \\
\hline Western oak & 3.9 & 0.1 & 20.2 & 0.5 & 13.4 & 0.4 & 28.1 & 0.7 & 58.5 & 0.7 & 3.5 & 0.2 \\
\hline Woodland hardwoods & 2.3 & 0.5 & 19.0 & 1.8 & 6.1 & 0.8 & 9.2 & 1.4 & 35.3 & 2.5 & 14.0 & 2.4 \\
\hline Total & 5.3 & 0.2 & 20.4 & 0.5 & 12.2 & 0.3 & 22.5 & 0.5 & 54.4 & 0.6 & 3.9 & 0.2 \\
\hline Nonstocked & 1.2 & 0.2 & 27.0 & 2.2 & 9.5 & 1.1 & 11.1 & 1.4 & 46.3 & 2.4 & 12.8 & 1.5 \\
\hline All forest types & 4.7 & 0.1 & 19.2 & 0.3 & 8.9 & 0.2 & 12.7 & 0.2 & 42.0 & 0.3 & 5.0 & 0.1 \\
\hline Chaparral on national forest & 0.4 & 0.1 & 37.3 & 1.8 & 4.1 & 0.4 & 3.9 & 0.4 & 44.3 & 2.0 & 5.6 & 0.5 \\
\hline
\end{tabular}

Note: Estimates subject to sampling error; $\mathrm{SE}=$ sampling error. 
Table A2-134-Mean of understory vegetation by forest type class, age class group, and lifeform, California 2001-2010

\begin{tabular}{|c|c|c|c|c|c|c|c|c|c|c|c|c|}
\hline \multirow{3}{*}{$\begin{array}{l}\text { Forest type class }^{a} \\
\text { Age class group }\end{array}$} & \multicolumn{10}{|c|}{ Lifeform } & & \\
\hline & \multicolumn{2}{|c|}{$\begin{array}{l}\text { Seedlings + } \\
\text { saplings }\end{array}$} & \multicolumn{2}{|c|}{ Shrubs } & \multicolumn{2}{|c|}{ Forbs } & \multicolumn{2}{|c|}{ Graminoids } & \multicolumn{2}{|c|}{$\begin{array}{c}\text { All understory } \\
\text { plants }\end{array}$} & \multicolumn{2}{|c|}{ Bare soil } \\
\hline & Mean & SE & Mean & SE & Mean & SE & Mean & SE & Mean & SE & Mean & SE \\
\hline & \multicolumn{12}{|c|}{ Percent } \\
\hline \multicolumn{13}{|l|}{ Dry conifer: } \\
\hline $0-19$ & 1.7 & 0.2 & 27.0 & 1.8 & 8.9 & 0.9 & 11.3 & 1.2 & 46.2 & 2.0 & 12.3 & 1.3 \\
\hline $20-39$ & 3.1 & 0.6 & 32.7 & 2.8 & 6.8 & 1.0 & 10.4 & 1.7 & 49.1 & 3.0 & 6.3 & 1.1 \\
\hline $40-79$ & 2.5 & 0.3 & 19.7 & 1.1 & 6.7 & 0.5 & 11.4 & 0.8 & 38.7 & 1.3 & 9.4 & 0.7 \\
\hline $80-159$ & 2.1 & 0.2 & 16.0 & 0.6 & 6.0 & 0.3 & 10.4 & 0.5 & 32.6 & 0.9 & 8.5 & 0.5 \\
\hline $160+$ & 1.8 & 0.2 & 12.0 & 0.9 & 6.2 & 0.6 & 6.7 & 0.6 & 25.2 & 1.3 & 6.9 & 0.7 \\
\hline All ages & 2.1 & 0.1 & 18.4 & 0.5 & 6.6 & 0.2 & 10.1 & 0.3 & 35.3 & 0.6 & 8.8 & 0.3 \\
\hline
\end{tabular}

Wet conifer:

$\begin{array}{lrlllllllllll}0-19 & 7.1 & 0.8 & 28.6 & 3.1 & 12.5 & 1.7 & 11.9 & 2.0 & 54.9 & 3.6 & 9.5 & 1.7 \\ 20-39 & 7.0 & 0.7 & 30.1 & 2.1 & 11.5 & 1.4 & 4.7 & 0.8 & 49.7 & 2.4 & 4.4 & 0.8 \\ 40-79 & 6.0 & 0.3 & 20.1 & 0.9 & 6.4 & 0.4 & 3.6 & 0.2 & 34.4 & 1.0 & 3.6 & 0.2 \\ 80-159 & 5.7 & 0.2 & 15.4 & 0.6 & 6.6 & 0.3 & 3.6 & 0.2 & 29.4 & 0.7 & 3.4 & 0.2 \\ 160+ & 5.7 & 0.3 & 18.5 & 1.0 & 6.5 & 0.4 & 2.4 & 0.2 & 31.4 & 1.1 & 3.6 & 0.3 \\ \text { All ages } & 5.9 & 0.2 & 18.3 & 0.4 & 6.9 & 0.2 & 3.6 & 0.1 & 32.9 & 0.5 & 3.7 & 0.1\end{array}$

Dry hardwood:

\begin{tabular}{|c|c|c|c|c|c|c|c|c|c|c|c|}
\hline 0-19 & 8.3 & 0.7 & 27.5 & 1.9 & 14.9 & 1.4 & 24.0 & 2.1 & 66.7 & 1.9 & 6.7 \\
\hline $20-39$ & 6.2 & 0.6 & 23.8 & 1.5 & 12.1 & 1.0 & 22.4 & 1.8 & 58.5 & 1.7 & 2.8 \\
\hline $40-79$ & 5.4 & 0.2 & 20.8 & 0.8 & 10.0 & 0.5 & 18.2 & 0.9 & 49.7 & 1.1 & 3.3 \\
\hline $80-159$ & 4.1 & 0.2 & 18.0 & 0.7 & 13.3 & 0.5 & 27.3 & 0.9 & 55.9 & 0.9 & 3.9 \\
\hline $160+$ & 7.5 & 1.0 & 12.9 & 1.4 & 8.1 & 1.4 & 11.1 & 2.2 & 37.4 & 2.7 & 6.0 \\
\hline All ages & 5.2 & 0.2 & 20.0 & 0.5 & 12.0 & 0.3 & 22.9 & 0.6 & 54.2 & 0.6 & 3.9 \\
\hline
\end{tabular}

Wet hardwood:

\begin{tabular}{|c|c|c|c|c|c|c|c|c|c|c|c|c|}
\hline $0-19$ & 10.3 & 2.6 & 24.2 & 6.2 & 10.9 & 3.4 & 12.3 & 6.7 & 52.1 & 8.1 & 2.6 & 1.8 \\
\hline $20-39$ & 9.1 & 2.3 & 41.2 & 6.4 & 27.7 & 5.2 & 4.7 & 1.6 & 70.5 & 5.1 & 1.2 & 0.5 \\
\hline $40-79$ & 5.6 & 1.3 & 36.5 & 3.7 & 13.0 & 2.1 & 10.5 & 2.9 & 60.8 & 3.7 & 2.3 & 0.8 \\
\hline $80-159$ & 6.7 & 1.3 & 23.2 & 6.4 & 12.8 & 3.8 & 6.7 & 2.5 & 46.3 & 8.9 & 3.3 & 2.0 \\
\hline $160+$ & 3.9 & 0.5 & 13.5 & 5.0 & 5.6 & 1.9 & 5.9 & 1.0 & 28.2 & 5.2 & 6.0 & 3.0 \\
\hline All ages & 7.1 & 1.0 & 34.1 & 2.9 & 16.9 & 2.1 & 8.1 & 1.5 & 59.6 & 3.0 & 2.3 & 0.5 \\
\hline
\end{tabular}

All classes:

\begin{tabular}{lrrrrrrrrrrrr}
$0-19$ & 5.2 & 0.4 & 27.4 & 1.2 & 11.9 & 0.7 & 16.5 & 1.1 & 55.8 & 1.3 & 9.5 & 0.7 \\
$20-39$ & 6.0 & 0.4 & 27.5 & 1.1 & 11.8 & 0.7 & 15.7 & 1.1 & 55.5 & 1.3 & 3.7 & 0.3 \\
$40-79$ & 5.0 & 0.2 & 20.6 & 0.5 & 8.2 & 0.3 & 11.8 & 0.5 & 42.5 & 0.7 & 4.6 & 0.2 \\
$80-159$ & 4.2 & 0.1 & 16.6 & 0.4 & 9.1 & 0.3 & 14.6 & 0.4 & 40.7 & 0.5 & 4.8 & 0.2 \\
$160+$ & 4.7 & 0.2 & 15.7 & 0.6 & 6.6 & 0.3 & 4.8 & 0.4 & 30.2 & 0.8 & 4.9 & 0.3 \\
\multicolumn{1}{c}{ All ages } & 4.7 & 0.1 & 19.2 & 0.3 & 8.9 & 0.2 & 12.7 & 0.2 & 42.0 & 0.3 & 5.0 & 0.1 \\
\hline
\end{tabular}

Note: Estimates subject to sampling error; SE = sampling error.

${ }^{a}$ Dry conifer includes the pinyon/juniper; ponderosa, western white, and lodgepole pines; other softwoods, mixed conifer, and nonstocked forest type groups. Wet conifer includes the Douglas-fir, fir/spruce/mountain hemlock, hemlock/Sitka spruce, and redwood forest type groups. Dry hardwood includes the western oak, tanoak/laurel, other hardwoods, and exotic forest type groups. Wet hardwood includes the elm/ash/cottonwood, aspen/birch, and alder/maple forest type groups. 
Table A2-135-Summary of lichen community indicator species richness on forest land, by location, California, 1998-2001, 2003

\begin{tabular}{|c|c|c|c|c|}
\hline \multirow[b]{2}{*}{ Parameter } & \multicolumn{4}{|c|}{ Location } \\
\hline & California & $\begin{array}{c}\text { Greater Central } \\
\text { Valley }\end{array}$ & $\begin{array}{c}\text { Greater Sierra } \\
\text { Nevada }\end{array}$ & $\begin{array}{c}\text { Northwest } \\
\text { Coast }^{a}\end{array}$ \\
\hline Number of plots ${ }^{b}$ & 288 & 76 & 133 & 68 \\
\hline \multicolumn{5}{|l|}{ Number of plots by lichen species richness category: } \\
\hline 0 to 6 species & 61 & 7 & 43 & 3 \\
\hline 7 to 15 species & 141 & 41 & 67 & 31 \\
\hline 16 to 25 species & 62 & 19 & 18 & 24 \\
\hline$>25$ species & 24 & 9 & 5 & 10 \\
\hline Median & 12 & 13 & 9 & 16 \\
\hline Range of species richness per plot (low-high) & $0-39$ & $2-31$ & $0-34$ & $1-39$ \\
\hline Average lichen species richness per plot (alpha diversity) & 12.59 & 14.38 & 9.87 & 17.21 \\
\hline Standard deviation of lichen species richness per plot & 7.97 & 6.82 & 7.06 & 8.05 \\
\hline Species turnover rate (beta diversity) ${ }^{c}$ & 16.52 & 9.11 & 16.92 & 9.36 \\
\hline Total number of species per area (gamma diversity) & 208 & 131 & 167 & 161 \\
\hline
\end{tabular}

${ }^{a}$ The Northwest Coast borders the Greater Central Valey and covers northwestern California.

${ }^{b}$ Plot totals do not include quality assurance surveys or plots without lichens present.

${ }^{c}$ Beta diversity is calculated as gamma diversity divided by alpha diversity.

Table A2-136-Summary of lichen community indicator climate index parameters on forest land, for the moisture gradient of Jovan and McCune's (2004) model, California, 1998-2001, 2003

\begin{tabular}{|c|c|c|c|c|}
\hline Parameter & Total & $\begin{array}{c}\text { Greater Central } \\
\text { Valley }^{a}\end{array}$ & $\begin{array}{c}\text { Greater Sierra } \\
\text { Nevada }^{a}\end{array}$ & Northwest Coast ${ }^{b}$ \\
\hline Number of plots surveyed $^{c}$ & 264 & 76 & 121 & 67 \\
\hline \multicolumn{5}{|c|}{ Number of plots by climate index category ${ }^{d}$ : } \\
\hline Wettest $(-2.28$ to -0.71$)$ & 66 & 5 & 16 & 45 \\
\hline Wet (-0.71 to 0.13$)$ & 66 & 11 & 39 & 16 \\
\hline Dry $(0.13$ to 0.89$)$ & 68 & 25 & 40 & 3 \\
\hline Driest (0.89 to 2.22$)$ & 64 & 35 & 26 & 3 \\
\hline Climate index extremes & -2.28 to 2.22 & -1.17 to 2.22 & -2.20 to 2.13 & -2.28 to 1.57 \\
\hline Average score on climate index & 0.08 & 0.77 & 0.21 & -0.92 \\
\hline Standard deviation on climate index & 1.04 & 0.83 & 0.82 & 0.83 \\
\hline
\end{tabular}

${ }^{a}$ The greater Central Valley (GCV) and greater Sierra Nevada are mapped in figure 7.

${ }^{b}$ The Northwest Coast borders the GCV and covers northwestern California.

${ }^{c}$ Plot totals do not include quality assurance surveys or plots without lichens present.

${ }^{d}$ Categories are based on data quartiles. 
Table A2-137-Summary of lichen community indicator climate index parameters on forest land, for the temperature gradient of Jovan and McCune's (2004) model. California, 1998-2001, 2003

\begin{tabular}{|c|c|c|c|c|}
\hline Parameter & Total & $\begin{array}{c}\text { Greater Central } \\
\text { Valley }^{a}\end{array}$ & $\begin{array}{c}\text { Greater Sierra } \\
\text { Nevada }^{a}\end{array}$ & $\begin{array}{l}\text { Northwest } \\
\text { Coast }^{b}\end{array}$ \\
\hline Number of plots surveyed ${ }^{c}$ & 264 & 76 & 121 & 67 \\
\hline \multicolumn{5}{|c|}{ Number of plots by climate index category ${ }^{d}$ : } \\
\hline Warmest $(-2.59$ to -1.04$)$ & 67 & 44 & 6 & 17 \\
\hline Warm $(-1.04$ to 0.01$)$ & 65 & 25 & 15 & 25 \\
\hline Cool (0.01 to 0.87$)$ & 66 & 5 & 43 & 18 \\
\hline Coolest (0.87 to 2.14$)$ & 66 & 2 & 57 & 7 \\
\hline Climate index extremes & -2.59 to 2.14 & -2.59 to 2.10 & -2.07 to 2.14 & -2.46 to 1.27 \\
\hline Average score on climate index & -0.02 & -0.96 & 0.73 & -0.32 \\
\hline Standard deviation on climate index & 1.13 & 0.79 & 0.88 & 0.92 \\
\hline
\end{tabular}

${ }^{a}$ The greater Central Valley (GCV) and greater Sierra Nevada are mapped in figure 7.

${ }^{b}$ The Northwest Coast borders the GCV and covers northwestern California.

${ }^{c}$ Plot totals do not include quality assurance surveys or plots without lichens present.

${ }^{d}$ Categories are based on data quartiles.

Table A2-138-Summary of lichen community indicator air quality index parameters on forest land, by type of parameter, Greater Central Valley, California, 1998-2001, 2003

\begin{tabular}{|c|c|c|c|}
\hline Parameter & $\begin{array}{c}\text { Greater } \\
\text { Central Valley }\end{array}$ & On-frame ${ }^{a}$ & Off-frame ${ }^{b}$ \\
\hline Number of plots surveyed ${ }^{c}$ & 108 & 76 & 32 \\
\hline \multicolumn{4}{|c|}{ Number of plots by air quality index category: $d$} \\
\hline 1 (Worst) : -0.99 to 0.13 & 45 & 19 & 26 \\
\hline 2: 0.13 to 0.55 & 23 & 19 & 4 \\
\hline 3: 0.55 to 0.85 & 22 & 20 & 2 \\
\hline 4 (Best): 0.85 to 1.58 & 18 & 18 & 0 \\
\hline Air quality index extremes & -0.99 to 1.58 & -0.86 to 1.58 & -0.99 to 0.70 \\
\hline Average score on air quality index & 0.28 & 0.52 & -0.27 \\
\hline Standard deviation on air quality index & 0.61 & 0.50 & 0.46 \\
\hline
\end{tabular}

${ }^{a}$ On-frame plots are on the Forest Inventory and Analysis sampling grid.

${ }^{b}$ Off-frame plots were located in cities, agricultural areas, and/or near air quality monitors.

${ }^{c}$ Plot totals do not include quality assurance surveys or plots without lichens present.

${ }^{d}$ Categories are based on the data quartiles for on-frame data. 
Table A2-139-Summary of lichen community indicator air quality index parameters on forest land, by type of parameter, Greater Sierra Nevada, California, 1998-2001, 2003

\begin{tabular}{|c|c|c|c|}
\hline Parameter & $\begin{array}{c}\text { Greater Sierra } \\
\text { Nevada }\end{array}$ & On-frame ${ }^{a}$ & Off-frame ${ }^{b}$ \\
\hline Number of plots surveyed $^{c}$ & 146 & 122 & 24 \\
\hline \multicolumn{4}{|c|}{ Number of plots by air quality index category: ${ }^{d}$} \\
\hline 1 (Best): -43.36 to -15.88 & 35 & 31 & 4 \\
\hline 2: -15.88 to -8.22 & 31 & 30 & 1 \\
\hline $3:-8.22$ to 4.35 & 33 & 30 & 3 \\
\hline 4 (Worst): 4.35 to 66.49 & 47 & 31 & 16 \\
\hline Air quality index extremes & -43.36 to 66.49 & -43.36 to 66.49 & -32.38 to 41.61 \\
\hline Average score on air quality index & -2.77 & -5.13 & 10.27 \\
\hline Standard deviation on air quality index & 19.28 & 18.32 & 19.60 \\
\hline
\end{tabular}

${ }^{a}$ On-frame plots are on the Forest Inventory and Analysis sampling grid.

${ }^{b}$ Off-frame plots were located in cities, agricultural areas, and/or near air quality monitors.

${ }^{c}$ Plot totals do not include quality assurance surveys or plots without lichens present.

${ }^{d}$ Categories are based on the data quartiles for on-frame data. 


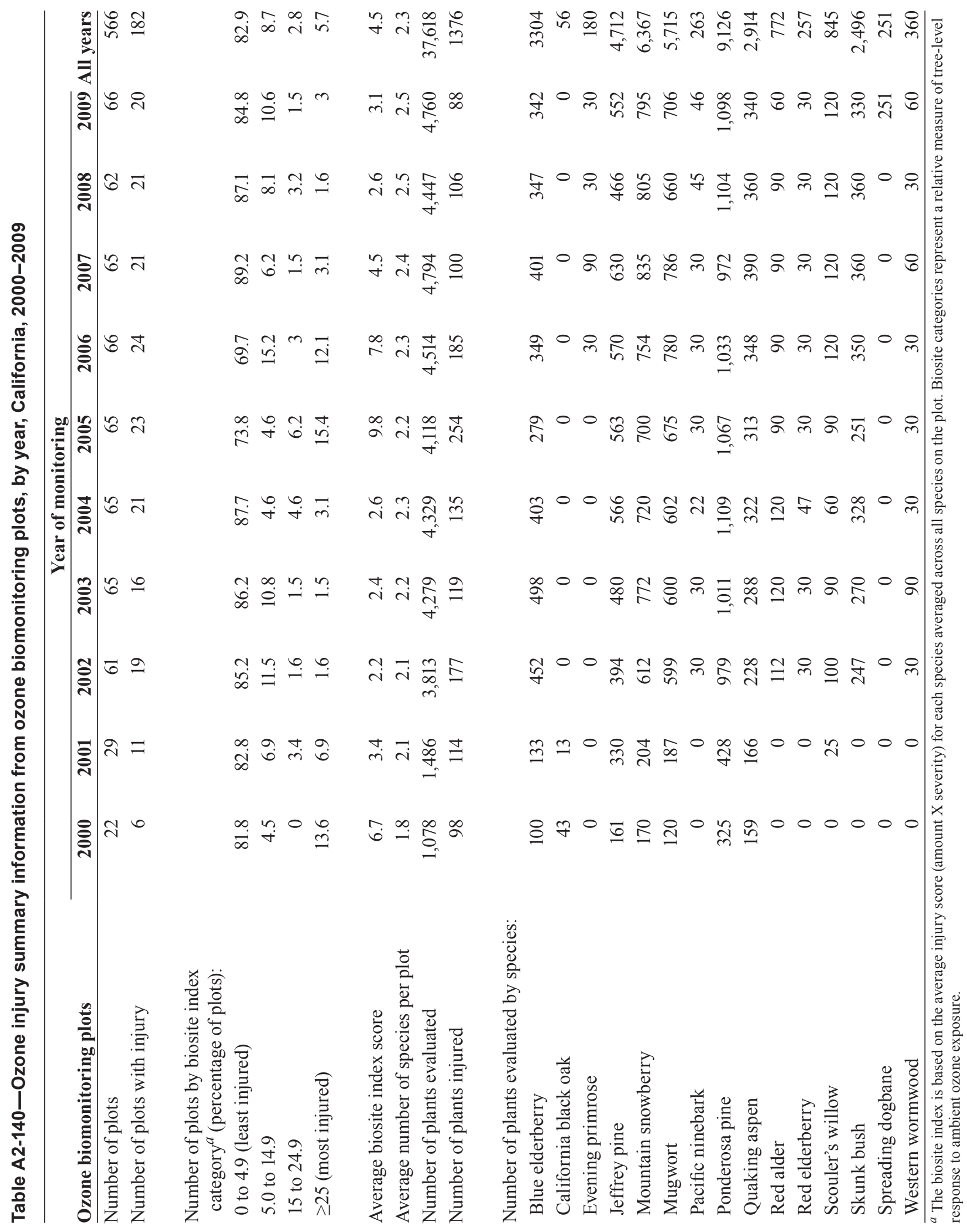




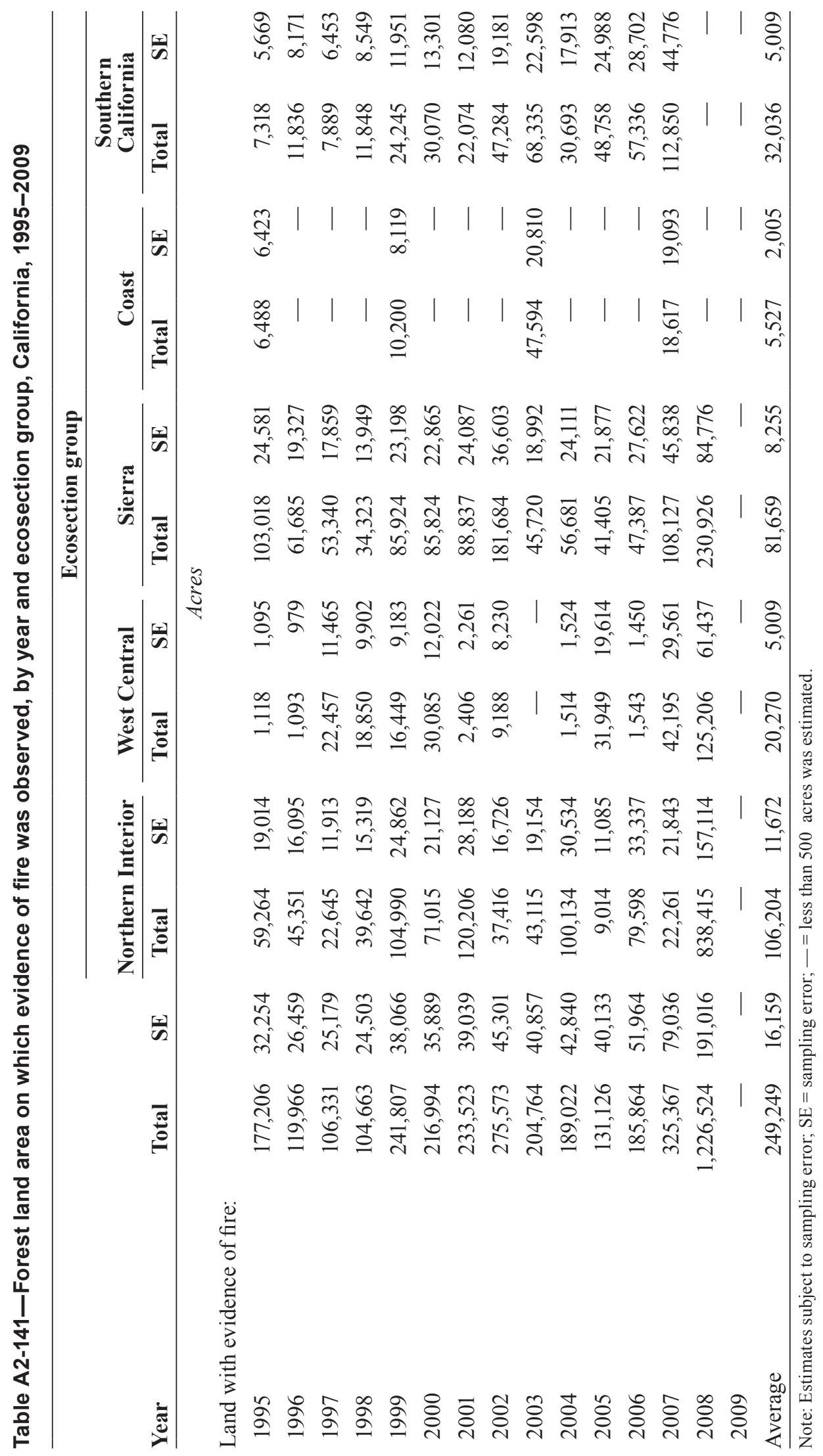





\begin{tabular}{|ll|}
\hline \multicolumn{2}{|c|}{ Pacific Northwest Research Station } \\
Website & http://www.fs.fed.us/pnw/ \\
Telephone & $(503) 808-2592$ \\
Publication requests & $(503) 808-2138$ \\
FAX & $(503) 808-2130$ \\
E-mail & pnw_pnwpubs@fs.fed.us \\
Mailing address & Publications Distribution \\
& Pacific Northwest Research Station \\
& P.O. Box 3890 \\
& Portland, OR 97208-3890 \\
\hline
\end{tabular}

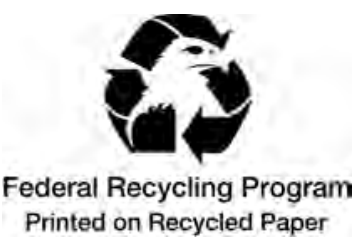


U.S. Department of Agriculture

Pacific Northwest Research Station 1220 SW $3^{\text {rd }}$ Ave.

P.O. Box 3890

Portland, OR 97208-3890

Official Business

Penalty for Private Use, $\$ 300$ 